\title{
Kruppel-like factor 4 (KLF4) regulates protumorigenic signaling in triple-negative breast cancer (TNBC) cells
}

\author{
Sriganesh B. Sharma
}

Follow this and additional works at: https://researchrepository.wvu.edu/etd

\section{Recommended Citation}

Sharma, Sriganesh B., "Kruppel-like factor 4 (KLF4) regulates protumorigenic signaling in triple-negative breast cancer (TNBC) cells" (2015). Graduate Theses, Dissertations, and Problem Reports. 6619.

https://researchrepository.wvu.edu/etd/6619

This Dissertation is protected by copyright and/or related rights. It has been brought to you by the The Research Repository @ WVU with permission from the rights-holder(s). You are free to use this Dissertation in any way that is permitted by the copyright and related rights legislation that applies to your use. For other uses you must obtain permission from the rights-holder(s) directly, unless additional rights are indicated by a Creative Commons license in the record and/ or on the work itself. This Dissertation has been accepted for inclusion in WVU Graduate Theses, Dissertations, and Problem Reports collection by an authorized administrator of The Research Repository @ WVU.

For more information, please contact researchrepository@mail.wvu.edu. 


\title{
Krüppel-like factor 4 (KLF4) regulates protumorigenic signaling in triple-negative breast cancer (TNBC) cells
}

\author{
Sriganesh B. Sharma
}

Dissertation submitted to the School of Medicine at West Virginia University in partial fulfillment of the requirements for the degree of

\author{
Doctor of Philosophy \\ in \\ Cancer Cell Biology
}

Scott A. Weed, Ph.D., Chair

Laura F. Gibson, Ph.D. Lan Guo, Ph.D.

Michael D. Schaller, Ph.D. William Tse, Ph.D.

J. Michael Ruppert, M.D., Ph.D., Mentor

Cancer Cell Biology Program

Morgantown, West Virginia

2015

Key Words: KLF4, microRNAs, miR-206, miR-21, breast cancer, RAS-ERK signaling, RAS-GTP, cancer stem cell, MEK 1/2 inhibitor, PDGFR $\beta$, drug resistance

Copyright 2015 Sriganesh B. Sharma 


\title{
ABSTRACT \\ Krüppel-like factor 4 (KLF4) regulates protumorigenic signaling in triple- negative breast cancer (TNBC) cells
}

\author{
Sriganesh B. Sharma
}

The zinc-finger pluripotency factor Krüppel-like factor 4 (KLF4) exerts context dependent roles in the maintenance of tissue homeostasis and the pathobiology of many malignancies, including breast cancer. Despite the multitude of studies focused on the role of KLF4 across these contexts, the signaling programs that are regulated by this factor in breast cancer cells remain unclear. In this dissertation we deliniate three KLF4-dependent protumorigenic signaling axes that endow triple-negative breast cancer (TNBC) cells with enhanced capacity to resist cell stress.

In the first study (Chapter 2), we found that KLF4 promotes the expression of two microRNAs (miRs), miR-206 and miR-21 (miR-206/21), to positively regulate RAS-ERK signaling and dependent cell phenotypes in TNBC cells. The collaborative action of miR-206/21 suppressed RAS-inhibitory GTPase activating protein (GAP) activity by repressing the translation of the GAP RASA1 and the Neurofibromatosis-1 (NF-1) GAP associated protein SPRED1. This attenuation of GAP activity resulted in increased levels of wild-type RAS-GTP (WT-RAS-GTP) levels and RAF-ERK activation. Interestingly, KLF4-miR-206/21 promoted RAS-ERK signaling in cells harboring activating RAS-mutations, and this regulation of pathway activity was attributed to the selective action of GAPs on WT-RAS proteins. This study uncovered a role for GAP proteins in regulating RAS-ERK signaling in RAS-mutant and RASWT cells alike and is consistent with the emerging paradigm in which the output of RAS signaling in RAS-mutant cells is dependent on the levels of WT-RAS-GTP.

In the second study (Chapter 3), we identified that KLF4 and miR-206 are functional markers of TNBC mammary cancer stem-like cells (MaCSCs) which promote cell survival against cell stresses including matrix detatchment, growth in immunocompromised mice, and against cytotoxic chemotherapy. Interestingly, the modulation of endogenous KLF4 and miR206 only had subtle effects on MaCSC population. miR-206 repressed the translation of the proapoptotic molecules CX43 and the well-established miR-21 target PDCD4. Suppression of CX43 and PDCD4 promoted TNBC cell survival, and reduced levels of both proteins was observed in MaCSCs compared to non stem-like cells. These results suggest that low PDCD4 and CX43 contribute to the enhanced chemo- and radio-resistance of MaCSCs, and implicate KLF4-miR206 signaling in enforcing tumor cell survival.

Finally, in the third study (Chapter 4), we found that KLF4 mediates TNBC cell resistance toward MEK 1/2 inhibition by promoting the transcription of the receptor tyrosine kinase (RTK), PDGFR $\beta$. This RTK has been functionally implicated as a critical resistance factor that protects cells from the cytostatic effects of RAS-ERK pathway inhibition. KLF4 
antagonized cMYC mediated repression of PDGFR $\beta$, and either KLF4 or PDGFR $\beta$ was sufficient to promote MEK 1/2 inhibitor resistant TNBC cell proliferation. Surprisingly, the time dependent re-emergence of RAS-ERK signaling upon MEK 1/2 inhibitor treatment was not dependent on KLF4 or PDGFR $\beta$, and thus our results suggest that alternative pathways may be involved in endowing TNBC cell resistance to MEK 1/2 inhibition.

Collectively, the studies presented in dissertation suggest that KLF4 is a critical mediator of cell survival in TNBC cells. In agreement with this role for KLF4, in a recently published study, we found that KLF4 collaborates with a related KLF member, KLF5, to promote lapatinib resistance in HER2+ breast cancer cells by regulating the expression of the anti-apoptotic proteins MCL1 and BCL-XL. Our results point to the targeting of KLF4 and/or KLF4-dependent effectors in the treatment of TNBC. 


\section{DEDICATION}

This dissertation is dedicated to my parents, Mrs. Brindha Sharma and Dr. Balasubramanian Sharma, and my brother Mr. Sripadh Sharma, for their unconditional love and support in all my ventures. My parents instilled in me the importance of life-long learning and self-discipline, and these important values empower me to achieve my goals. 


\section{ACKNOWLEDGEMENTS}

The work in this dissertation is not only an individual acheivement, but also the result of the tremendous encouragement and support that I receive from my colleagues, friends, and family.

I would like to show my gratitude to my mentor Dr. J. Michael (Mike) Ruppert for training me for the past four years. His mentorship has allowed me to mature as a scientist and his leadership has given me great insight into how to succeed in the next phase of my training. I am very grateful to my fellow lab members - Dr. Chen-Chung Lin, Dr. Wentao Deng, Mark Farrugia, Daniel Vanderbilt, and Michael Mckinstry - for their camaraderie, support, and encouragement.

I am very thankful for the advice and guidance of my dissertation committee members Drs. Scott Weed, Laura Gibson, Michael (Mike) Schaller, Lan Guo, and William Tse. I also thank the labs of Drs. Wysolmerski, Vona-Davis, Gibson, Pugacheva, Ivanov, and Frisch for their help and support.

Furthermore, I am grateful for the help I have received from the various core facilities (AMIF - Sarah McLaughlin and Emily Ellis; MIF - Drs. Amamda Ammer and Karen Martin; Flow cytometry - Dr. Kathleen Brundage) and the administration of the Mary Babb Cancer Center, the Office of Graduate Education, and the MD-PhD program.

Finally, I would also like to express my heartfelt gratitude for the student advocacy of Drs. Fred Minnear and David Siderovski, who were instrumental for my successes during my my tenure as an MD-PhD student. 


\section{TABLE OF CONTENTS}

TITLE PAGE

ABSTRACT

DEDICATION

ACKNOWLEDGEMENTS $\quad \mathrm{v}$

TABLE OF CONTENTS Ni vi vi

LIST OF FIGURES $\quad$ xii

LIST OF TABLES $\quad$ XV

LIST OF ABBREVIATIONS _ Xvi

CHAPTER 1: INTRODUCTION AND LITERATURE REVIEW 1

KRÜPPEL-LIKE FACTOR 4 (KLF4) 2

A general overview of Krüppel-like Factors $\quad 2$

The identification, distribution, and structure of KLF4 4

Regulation of KLF4 transcription, and KLF4-mediated gene transcriptional activation $\quad 6$ and repression

Regulation of KLF4 transcription $\quad 6$

$\begin{array}{ll}\text { KLF4-mediated transcriptional activation } & 7\end{array}$

KLF4-mediated transcriptional repression $\quad 8$

Roles of KLF4 in Normal Cells and Tissues $\quad 9$

Proliferation and programmed cell death (apoptosis) $\quad 9$

Differentiation and the maintenance of tissue homeostatsis 10

Functions of KLF4 as a stress response factor $\quad 12$

Maintenance and establishment of pluripotency 13 
The role of KLF4 in tumor biology 14

$\begin{array}{ll}\text { KLF4 as a tumor suppressor } & 15\end{array}$

$\begin{array}{ll}\text { KLF4 as an oncogene } & 17\end{array}$

The role of KLF4 in breast cancer $\quad 18$

Interpreting studies analyzing KLF4 in tumor biology 19

Molecular mechanisms of KLF4-dependent phenotypes 21

$\begin{array}{ll}\text { MICRORNAS (miRs) } & 22\end{array}$

$\begin{array}{ll}\text { A brief overview of microRNAs (miRs) } & 22\end{array}$

$\begin{array}{ll}\text { miR biogenesis and nomenclature } & 23\end{array}$

$\begin{array}{ll}\text { Biogenesis } & 23\end{array}$

$\begin{array}{ll}\text { Nomenclature } & 24\end{array}$

miR-mediated gene regulation $\quad 25$

A brief overview of eukaryotic mRNA translation initiation 26

Cap-dependent translation initiation 26

$\begin{array}{ll}\text { Cap-independent translation initiation } & 27\end{array}$

$\begin{array}{ll}\text { Regulation of eukaryotic initiation and post-initiation } & 28\end{array}$

Regulation of translation at initiation $\quad 28$

$\begin{array}{ll}\text { Regulation of translation post-initiation } & 29\end{array}$

miR-mediated mRNA translation repression 30

miR-mediated mRNA translation activation 32

Identifying and validating mRNA targets of miRs 33

The functions of miRs in tumor cell biology 34

Tumor suppressive miRs $\quad 35$ 
Oncogenic miRs (oncomiRs)

$\begin{array}{ll}\text { Signaling by KLF4 and miRs } & 37\end{array}$

THE RAS-ERK/MAPK SIGNALING PATHWAY 39

A brief overview of RAS-ERK signaling 39

The discovery of RAS proto-oncogenes and effector signaling pathways $\quad 40$

$\begin{array}{ll}\text { Organization of MAPK signaling cascades } & 41\end{array}$

Activation of RAS-ERK signaling $\quad 42$

$\begin{array}{ll}\text { RAS structure and activation } & 42\end{array}$

$\begin{array}{ll}\text { RAF structure and activation } & 44\end{array}$

Activation of MEK 1/2 and ERK 1/2 46

Substrates of ERK 1/2 46

$\begin{array}{ll}\text { Regulation of RAS-ERK pathway activity } & 47\end{array}$

$\begin{array}{ll}\text { GTPase Activating Proteins (GAPs) } & 48\end{array}$

$\begin{array}{lr}\text { Adaptor, docking, and scaffolding proteins } & 49\end{array}$

Negative regulators/modulators of RAS-ERK signaling 51

MAPK phosphatases $\quad 53$

ERK 1/2 dependent feedback regulation of RAS-ERK pathway activity 53

The roles of RAS-ERK signaling in human disease 55

RAS-ERK signaling in developmental disorders $\quad 55$

The role of RAS-ERK signaling in cancer 56

$\begin{array}{ll}\text { Therapeutic inhibition of RAS-ERK signaling } & 58\end{array}$

$\begin{array}{lr}\text { Strategies to target mutant RAS proteins } & 59\end{array}$

$\begin{array}{ll}\text { Strategies targeting proteins that regulate RAS activity } & 60\end{array}$ 
The role of RAS-ERK signaling in breast cancer

A brief overview of breast cancer

RAS-ERK signaling in breast cancer

CHAPTER 2: microRNAs-206 and -21 cooperate to promote RAS-Extracellular Signal Regulated Kinase (ERK) signaling by suppressing the translation of RASAI and SPREDI

ABSTRACT

INTRODUCTION

MATERIALS AND METHODS

RESULTS

DISCUSSION

ACKNOWLEDGEMENTS

REFERENCES

161

FIGURE LEGENDS

TABLES

FIGURES

CHAPTER 3: Krüppel-like factor 4 signals through microRNA-206 to promote tumor initiation and cell survival

ABSTRACT 
$\begin{array}{ll}\text { INTRODUCTION } & 203\end{array}$

$\begin{array}{ll}\text { MATERIALS AND METHODS } & 205\end{array}$

$\begin{array}{ll}\text { RESULTS } & 210\end{array}$

$\begin{array}{ll}\text { DISCUSSION } & 217\end{array}$

$\begin{array}{ll}\text { ACKNOWLEDGEMENTS } & 219\end{array}$

$\begin{array}{ll}\text { REFERENCES } & 220\end{array}$

$\begin{array}{ll}\text { FIGURE LEGENDS } & 229\end{array}$

$\begin{array}{ll}\text { FIGURES } & 236\end{array}$

TABLES (SUPPLEMENT) 243

CHAPTER 4: Krüppel-like Factor 4 (KLF4) promotes the expression of plateletderived growth factor receptor beta polypeptide (PDGFRß) and resistance to MEK 1/2 inhibition in triple-negative breast cancers (TNBCs) 245

$\begin{array}{ll}\text { CAPSULE } & 246\end{array}$

$\begin{array}{ll}\text { ABSTRACT } & 247\end{array}$

$\begin{array}{ll}\text { INTRODUCTION } & 248\end{array}$

$\begin{array}{ll}\text { MATERIALS AND METHODS } & 250\end{array}$

$\begin{array}{ll}\text { RESULTS } & 255\end{array}$

$\begin{array}{ll}\text { DISCUSSION } & 261\end{array}$

$\begin{array}{ll}\text { ACKNOWLEDGEMENTS } & 264\end{array}$

$\begin{array}{ll}\text { CONFLICTS OF INTEREST } & 264\end{array}$

$\begin{array}{ll}\text { FOOTNOTES } & 264\end{array}$

$\begin{array}{ll}\text { REFERENCES } & 266\end{array}$

$\begin{array}{ll}\text { FIGURE LEGENDS } & 273\end{array}$

$\begin{array}{ll}\text { FIGURES } & 279\end{array}$ 
CHAPTER 5: SUMMARY \& DISCUSSION 285

INHIBITION OF miR-206/21 FOR THE TREATMENT OF TNBC 287

$\begin{array}{ll}\text { The expanding roles of GAPs in cancer } & 288\end{array}$

Targeting RAS GAPs and RAS-ERK signaling in the treatment of cancers 290

Emerging paradigms in miR-regulation of RAS-ERK signaling 291

The potential of in vivo inhibition of miR-206/21 for the treatment of cancer 292

Design of effective miR-based therapeutic strategies for targeting miRs in vivo 295

Obstacles that must be overcome for the successful utilization of anti-miR

$\begin{array}{ll}\text { therapeutics } & 296\end{array}$

THE USE OF SMALL MOLECULE INHIBITION OF RAS-ERK SIGNALING IN

$\begin{array}{ll}\text { THE TREATMENT OF CANCER } & 298\end{array}$

Concerns for co-targeting resistance mechanisms along with RAS-ERK signaling

299

Use of RAS-ERK inhibitors in combinatorial anti-cancer therapies 300

The evaluation of efficacy of RAS-ERK inhibitors in clinical trials 301

$\begin{array}{ll}\text { CONCLUSIONS } & 302\end{array}$

$\begin{array}{ll}\text { FIGURES } & 303\end{array}$

$\begin{array}{ll}\text { REFERENCES } & 306\end{array}$

$\begin{array}{ll}\text { APPENDIX } & 321\end{array}$

PART I: MicroRNA-based therapeutic strategies to target mutant and wild type 323 RAS in cancer

PART II: Curriculum Vitae (CV) 363 


\section{LIST OF FIGURES}

\section{CHAPTER 1:}

Figure 1: Schematic representation of Krüppel-like factor 4 (KLF4)

Figure 2: Structural organization of human KLF4

Figure 3: Localization of Klf4 and Klf5 in mouse colonic epithelium

Figure 4: Canonical miR biogenesis

Figure 5: Schematic of alternative miR biosynthetic pathways

Figure 6: Cap-dependent translation initiation

Figure 7: Mechanisms of miR function in plants and animals

Figure 8: The general structure of mammalian MAPK pathways

Figure 9: RAS activation at the plasma membrane

Figure 10: Mechanism of GEF mediated catalysis

Figure 11: Mechanism of GAP mediated GTP hydrolysis

Figure 12: The C-terminal regions of human RAS isoforms

Figure 13: RAS-CAAX box processing

Figure 14: The structure of RAF enzymes

Figure 15: The cRAF activation cycle

Figure 16: Modulation of RAS-ERK signaling by various regulatory proteins

\section{CHAPTER 2:}

Figure 1: KLF4 is bound to the MIR206 promoter region and induces miR-206 expression

Figure 2: KLF4 rapidly induces miR-206 and RAS-ERK signaling

Figure 3: KLF4 and miR-206 regulate the levels of two RAS-ERK pathway suppressors, RASA1 and SPRED1

Figure 4: miR-206 represses the translation of RASA1 and SPREDI by directly targeting the 3 ' UTRs 
Figure 5: Endogenous KLF4 is bound to the MIR21 promoter region and maintains miR21 expression in TNBC cells

Figure 6: miR-21 directly represses the translation of RASA1 and SPRED1

Figure 7: KLF4 promotes activated ERK 1/2 levels, miR-206, and miR-21 expression in a panel composed of human mammary epithelial cells and TNBC cell lines

Figure 8: Endogenous miR-206 and miR-21 cooperate to promote RAS-ERK signaling

Figure 9: Inhibition of miR-206 and miR-21 cooperatively suppresses ERK 1/2 dependent phenotypes in TNBC cells

Figure 10: RASA1 and SPRED1 are limiting factors for RAS-ERK signaling in TNBC cells

Figure 11: RASA1 and SPRED1 mediate the regulation of RAS-ERK pathway signaling by miR-206 and miR-21

Figure 12: Exogenous miR-206 and miR-21 cooperate to promote RAS-ERK signaling and cell survival in KLF4-depleted cells

Figure 13: KLF4-dependent miRs cooperatively promote RAS-ERK pathway activity by co-targeting of pathway inhibitors

\section{CHAPTER 3:}

Figure 1: KLF4 and miR-206 are selectively expressed in basal-like mammary cancers and in the MaCSC population

Figure 2: KLF4 and miR-206 are enriched in ALDH ${ }^{\mathrm{High}}$ MaCSCs derived from human patient-derived xenografts (PDXs) and the C3(1)/TAg GEMM

Figure 3: KLF4 and miR-206 promote MaCSC abundance

Figure 4: Endogenous KLF4-miR-206 signaling promotes in vivo tumorigenesis and cell survival

Figure 5: miR-206 suppresses the translation of the tumor suppressor PDCD4

Figure 6: KLF4-miR-206 signaling suppresses CX43 in MaCSCs

Figure 7: miR-206 promotes chemoresistance in TNBC cells 


\section{CHAPTER 4:}

Figure 1: KLF4 regulates PDGFR $\beta$ expression in human and murine models of TNBC

Figure 2: KLF4 is required for the time-dependent induction of PDGFR $\beta$ in response to MEK 1/2 inhibition

Figure 3: KLF4 opposes cMYC mediated repression of PDGFR $\beta$

Figure 4: KLF4 promotes resistance to MEK 1/2 inhibition in TNBC cells

Figure 5: KLF4-PDGFR $\beta$ regulation is critical for MEK 1/2 inhibitor resistance

Figure 6: KLF4 promotes MEK 1/2 inhibitor resistance through PDGFR $\beta$

\section{CHAPTER 5:}

Figure 1: Overview of the studies presented in this dissertation

Figure 2: An emerging paradigm in RAS-signaling

Figure 3: Chemical modifications of anti-miR therapeutics

\section{APPENDIX:}

\section{PART I:}

Figure 1: miRs regulate RAS-ERK pathway activity by regulation of RAS-GTP

PART II: (none) 


\section{LIST OF TABLES}

CHAPTER 1: (none)

\section{CHAPTER 2:}

Table 1: Pathway enrichment analysis of putative miR-206-regulated genes

Table 2: Putative miR-206 target genes related to the MAPK/ERK signaling pathway

Table 3: Pathway enrichment analysis of putative miR-21-regulated genes

Table 4: Intersection of the pathways targeted by miR-206 and miR-21

\section{CHAPTER 3:}

Table 1: Oligonucleotides for PCR mutagenesis

Table 2: Oligonucleotides for real-time quantitative PCR analysis

CHAPTER 4: (none)

CHAPTER 5: (none)

\section{APPENDIX:}

PART I:

Table 1: miRs that regulate RAS-ERK pathway activity in a variety of cancer contexts

PART II: (none) 


\section{LIST OF ABBREVIATIONS}

4E-BPs

4-OHT

A

AGO

ALL

AML

APC

ATP

AUG

Bax

C

CBP

CD11d

CDK

cDNA

CLL

CREB

CX43

CYP1A1

DCIS

DCR1

DGCR8

$\mathrm{E}$

eIF

EKLF

EMT

ER

ERK

ES

EZF

$\mathrm{F}$

FXR1

G

GI

GKLF

GDP

GTP

GW182
4E-binding proteins

4-hydroxytamoxifen

Alanine

Argonaute

Acute Lymphocytic Leukemia

Acute Myelogenous Leukemia

Adenomatous Polyposis Coli

Adenosine-5'-triphosphate

Translation start codon

BCL2-associated $X$ protein

Cysteine

CREB-binding protein

Integrin, alpha D

Cyclin-dependent kinases

Complementary DNA

Chronic lymphocytic leukemia

cAMP response element binding

Connexin 43

Cytochrome P450, family 1, subfamily A, polypeptide 1

Ductal carcinoma of the breast

Dicer 1

DiGeoge syndrome critical region gene 8

Glutamic acid

Eukaryotic initiation factor

Erythroid Krüppel-like factor

Epithelial-Mesenchymal Transition

Estrogen Receptor

Extracellular Signal-Regulated Kinase

Embryonic stem

Epithelial Zinc Finger

Phenylalanine

Fragile X-Related protein 1

Glycine

Gastrointestinal

Gut-enriched Krüppel-like factor

Guanosine-5'-diphosphate

Guanosine-5'-triphosphate

Trinucleotide repeat containing 6A 


\begin{tabular}{|c|c|}
\hline $\mathrm{H}$ & Histidine \\
\hline HAT & Histone acetyltransferase \\
\hline $\mathrm{HCV}$ & Hepatitis C virus \\
\hline HDAC & Histone deacetylase \\
\hline HDC & Histidine decarboxylase \\
\hline HMEC & human mammary epithelial cells \\
\hline iPS & Inducible pluripotent stem \\
\hline IRES & Internal ribosome entry sites \\
\hline $\mathrm{K}$ & Lysine \\
\hline KLF & Krüppel-like factor \\
\hline LATS2 & LATS, large tumor suppressor, homolog 2 \\
\hline Lefty 1 & Left-right determination factor 1 \\
\hline LIF & Leukemia inhibitory factor \\
\hline $\mathrm{m}^{7} \mathrm{G}$ & 7-methylguanylate \\
\hline MaCsC & Mammary Cancer Stem Cell \\
\hline MAPK & Mitogen Activated Protein Kinase \\
\hline MEK & MAP/ERK Kinase \\
\hline MET & Mesenchymal-Epithelial Transition \\
\hline $\operatorname{miR}$ & microRNA \\
\hline miRNP & miR-protein complex; micro-ribonucleoprotein \\
\hline MMLV & Moloney Murine Leukemia Virus \\
\hline mRNA & Messenger RNA \\
\hline $\mathrm{mRNP}$ & mRNA-protein complex; messenger-ribonucleoprotein \\
\hline Nanog & Nanog homeobox \\
\hline NLS & Nuclear localization signals \\
\hline NMD & Nonsense-mediated decay \\
\hline $\mathrm{P}$ & Proline \\
\hline PABP & Poly (A)-binding protein \\
\hline PCNA & Proliferating cell nuclear antigen \\
\hline PEST & Proline, Glutamic acid, Serine, Threonine \\
\hline PDGFR $\beta$ & Platelet-derived Growth Factor beta receptor \\
\hline Pou5f1 & POU class 5 homeobox 1 \\
\hline pre-miR & Precursor miR \\
\hline pri-miR & Primary microRNAs \\
\hline RISC & RNA-induced silencing complex \\
\hline RNA & Ribonucleic acid \\
\hline RTK & Receptor tyrosine kinase \\
\hline $\mathrm{S}$ & Serine \\
\hline SCC & Squamous cell carcinoma \\
\hline shRNA & Short hairpin Ribonucleic Acid \\
\hline
\end{tabular}




$\begin{array}{cc}\text { siRNA } & \text { Small interfering RNA } \\ \text { SMC } & \text { Smooth muscle cell } \\ \text { Sox2 } & \text { SRY (sex determining region Y)-box 2 } \\ \text { SP1 } & \text { Sp1 transcription factor } \\ \text { T } & \text { Threonine } \\ \text { TARBP2 } & \text { TAR binding protein } 2 \\ \text { TBP } & \text { TATA-box binding protein } \\ \text { TCF4 } & \text { Transcription factor } 4 \\ \text { TGF } 1 & \text { Transforming growth factor } \beta 1 \\ \text { TNBC } & \text { Triple-negative breast cancer } \\ \text { TNF } \alpha & \text { Tumor necrosis factor alpha } \\ \text { TOP } & \text { Terminal oligopyrimidine tract } \\ \text { tRNA } & \text { Transfer RNA } \\ \text { u-PAR } & \text { Plasminogen activator, urokinase receptor } \\ \text { UPS } & \text { Ubiquitin Proteasome System } \\ \text { UTR } & \text { Untranslated region } \\ \text { VSMC } & \text { Vascular smooth muscle cell } \\ \text { Wnt } & \text { Wingless } \\ X & \text { Any amino acid residue } \\ \text { Y } & \text { Tyrosine }\end{array}$




\section{CHAPTER 1}

\section{INTRODUCTION AND LITERATURE REVIEW}

The studies presented in this dissertation describe how the zinc finger pluripotency factor Krüppel-like factor 4 (KLF4) promotes protumorigenic signaling in triple-negative breast cancer (TNBC) cells. The study presented in Chapter 2 describes how KLF4 promotes signaling through the RAS - extracellular signal regulated kinase/mitogen activated protein kinase (RASERK/MAPK) pathway in TNBC cells. KLF4 signals through two microRNAs (miRs), miR-206 and miR-21, to suppress RAS GTPase activating protein (GAP) activity and promote wild-type RAS-GTP (WT-RAS-GTP) levels and steady state RAS-ERK signaling. The study presented in Chapter 3, shows that KLF4 and miR-206 are functional markers of mammary cancer stem cells (MaCSCs) and potential effectors of chemoresistance that suppress pro-apoptotic molecules in TNBC cells. Collectively, these studies indicate that KLF4 is a potent regulator of oncogenic signaling and drug resistance in TNBC cells. The last study, presented in Chapter 4, connects these two subjects and investigates how KLF4 promotes TNBC cell survival against pharmacological inhibition of the RAS-ERK pathway. To this end, KLF4 regulates the expression of platelet-derived growth factor beta polypeptide (PDGFR $\beta$ ), which is a key receptor tyrosine kinase (RTK) that is functionally implicated in mediating cellular escape from MEK 1/2 inhibition across various cancer contexts. Finally, a discussion of the implications of the presented studies as well as the outlook for future studies in the field of KLF4, miR therapeutics, and kinase-inhibition strategies is presented in Chapter 5. 


\section{KRÜPPEL-LIKE FACTOR 4 (KLF4)}

\section{A general overview of Krüppel-like Factors:}

Krüppel-like factors (KLFs) are zinc-finger transcription factors that regulate numerous cellular physiological processes such as development, growth, proliferation, and apoptosis. Deregulation of KLFs can result in developmental defects and are implicated in the pathogenesis of cardiovascular disease, metabolic disturbances, and cancer. KLFs share homology to the Drosophila melanogaster Krüppel protein, member of the "gap" subset segmentation genes. Gap genes regulate segmentation of the thorax and anterior abdomen in the Drosphila embryo (1). Loss of Krüppel results in developmental defects that result in a "crippled" phenotype in Drosophila, indicating that Krüppel is necessary for proper embryonic development (2).

KLFs share homology to the transcription factor Sp1, one of the first mammalian transcription factor to be cloned (1) (Fig. 1). Sp1 and the KLFs share similar carboxy-terminal (C-terminal) regions that contain three $\mathrm{C}_{2} \mathrm{H}_{2}$ zinc-finger motifs and bind GC-rich DNA regions, but sequence similarity for both $\mathrm{Sp} 1$ and the KLFs diverges at the amino-terminus (N-terminus) $(3,4)$. Thus, the KLFs are grouped as members of the Sp1/KLF family of transcription factors. $\mathrm{C}_{2} \mathrm{H}_{2}$-zinc fingers are the most common types of zinc finger motifs found in mammalian transcription factors $(5,6)$. In this motif, a zinc atom is tetrahedrally coordinated between two cysteine $(\mathrm{C})$ and two histidine $(\mathrm{H})$ residues that are found in the general structure: $\mathrm{CX}_{2-}$ ${ }_{4} \mathrm{CX}_{12} \mathrm{HX}_{2-6} \mathrm{H}$ [X represents any amino acid]. Each zinc-finger contains two $\mathrm{N}$-terminal $\beta$-strands and one C-terminal $\alpha$-helix. This $\alpha$-helix determines the specificity of the zinc finger for binding particular DNA sequences (5). 
A distinguishing feature of the KLFs is a highly conserved spacer that connects each of the zinc-fingers called the "Krüppel-link", which consists of seven amino acids: TGEKP(Y/F)X (7). The zinc-fingers of both Sp1 and KLF transcription factors can recognize the consensus sequence 5'-CACCC-3' (4,8-10). However, though Sp1 and the KLFs recognize similar sequence elements, these factors regulate the transcription of non-overlapping sets of genes resulting in distinct and context dependent roles in numerous physiological processes $(4,11,12)$.

KLFs are widely conserved among mammals and many KLF family members have homologs in Gallus gallus (chicken), Danio rerio (zebrafish), and Xenopus laevis (frog) (12). The KLF family of transcription factors consists of 17 members, that share homology in the Cterminal regions that allow each factor to bind to GC-rich DNA sequences $(3,7)$. However, the structures of KLF N-terminal regions, which can contain various protein-protein interaction domains, significantly vary between family members. These N-terminal regions of the KLFs interact with transcriptional co-activators, co-repressors, and chromatin-modifying proteins, which enable KLFs to either activate or repress the translation of target genes $(4,11,12)$. Thus, structural similarities among KLF family members can often translate to overlap in the function of these factors. These similarities allow certain KLFs to bind overlapping sets of transcriptional targets and suggest potential functional redundance of some KLFs. For example, KLF2, KLF4, and KLF5 share many transcriptional targets to maintain pluripotency in embryonic stem cells (ES cells) (13).

By the differential regulation of the transcription of numerous genes, KLFs and KLFdependent effector molecules are important in regulating cell fate in many organ systems including immune, cardiovascular, respiratory, hematological, and digestive systems $(4,11,12)$. KLFs can act as stress response and plasticity factors in numerous cellular contexts $(10,12,14)$. 
More recently, KLFs have been established in inducing and maintaining pluripotency in adult somatic cells and ES cells (13,15-18). Along with these roles in normal tissues, KLFs have been implicated in tumor biology, with important context dependent oncogenic and tumor suppressive roles defined for many KLFs $(11,12,14)$.

Krüppel-like factor 4 (KLF4) is one of the best studied KLF family member that has a multitude of roles in both normal and cancerous tissues, including being a critical mediator of pluripotency in adult somatic cells. In breast cancer, KLF4 protein is consistently upregulated $(19,20)$. Furthermore, increased KLF4 protein and KLF4 promoter demethylation are indicators of poor prognosis (21). Unraveling the signaling pathways impacted by KLF4 has the potential to reveal new signaling pathways relevant to various aspects of the malignant phenotype and can lead to new avenues of therapeutic intervention. This dissertation will focus on how KLF4 can impact signaling through receptor tyrosine kinases (RTKs), the RAS-ERK pathway, and proapoptotic proteins to promote tumorigenic properties in breast cancer.

\section{The identification, distribution, and structure of KLF4:}

The Krüppel-like factor 4 (KLF4) gene was initially identified in a low-stringency cDNA screen using a probe encoding the zinc-finger region of the immediate-early transcription factor zif268 (22). Concurrently, KLF4 was also identified in primary mouse fibroblast cDNA libraries of E13.5 embryos (23). Early studies showed that KLF4 is highly expressed in the epithelial cells of the gut and the skin, and therefore it was initially named Gut-enriched Krüppel-like factor (GKLF) and Epithelial Zinc Finger (EZF) (22,23). Human GKLF/EZF was first identified in a human umbilical vein endothelial cell (HUVEC) cDNA library using a probe containing the zinc finger region of erythroid KLF (EKLF/KLF1) (24). As GKLF/EZF was the fourth KLF to be 
identified, it was later renamed KLF4. KLF4 was subsequently detected in numerous other adult tissues including in the lung, skin, testis (23,25-27), thymus (28), cornea (29), lymphocytes (30), vascular endothelial cells, and cardiac myocytes (31). Enhanced expression of KLF4 protein in the more superficial layers of the skin, gut, and other tissues suggested a role in terminal differentiation.

The human KLF4 gene is located on chromosome 9q31 (Fig. 2). KLF4 encodes a transcript that is approximately $3.5 \mathrm{~kb}$ and contains five exons. The resulting major gene product is a 470 amino acid (aa) protein with a predicted molecular weight of approximately $55 \mathrm{kDa}$ (24). Several functional domains of KLF4 have been described including an N-terminal acidic amino acid rich transcription activation domain, a centrally located repressor domain, and three C-terminal $\mathrm{C}_{2} \mathrm{H}_{2}$-zinc fingers $(22,24,32)$. KLF4 has two nuclear localization signal (NLS) sequences, of which one is located in a basic amino acid stretch towards the $\mathrm{N}$-terminus from the zinc-finger domains, and the other within the zinc-finger containing region (33). Additonally, two PEST sequences are present between the transcriptional activation and repression domain, suggesting that KLF4 may be degraded by the ubiquitin proteasome (UPS) pathway (Fig. 2) (34). Finally, the minimum essential binding sequence for KLF4 was identified as 5'-(G/A)(G/A)GG(C/T)G(C/T)-3' and is similar to that of other KLFs, including EKLF/KLF1 (9). 


\section{Regulation of KLF4 transcription, and KLF4-mediated gene transcriptional activation and repression:}

\section{Regulation of KLF4 transcription:}

Although a variety of stimuli can regulate KLF4 expression in cells, the molecular mechanisms of how KLF4 is induced are not well known. Several transcription factors induce the transcription of KLF4. Notably, upon DNA damage, p53 promotes the transcription of the KLF4 gene $(35,36)$. CDX2, a transcription factor that plays a role in intestinal epithelial differentiation, can induce the activity of a KLF4 promoter-reporter (37). Furthermore, other KLF family members can regulate KLF4 transcription. Whereas KLF4 can promote its own transcription, KLF5 may inhibit KLF4 transcription by binding the KLF4 promoter $(38,39)$. Though this proposed antagonistic relationship between these closely related KLFs remains to be explored further in vivo, immunostaining of colonic epithelium for both transcription factors supports this idea as KLF4 and KLF5 are enriched in distinct epithelial compartments. Whereas KLF4 is readily detectable in the post-mitotic terminally differentiated colonic epithelium, KLF5 is enriched in actively dividing intestinal crypt cells. These results are interpreted as support for functionally antagonistic relationship between KLF4 and KLF5 (Fig. 3) $(12,40)$.

Transcription factors, such as KLF4, can either promote or repress the transcription of their target genes by recruiting co-activators or co-repressors. These co-factors can recruit two classes of chromatin regulators: chromatin modifiers, enzymes that alter the acetylation, methylation, phosphorylation, ubiquination; and chromatin remodelers, ATP dependent enzymes that can reposition nucleosomes. Furthermore, KLF4 interacts with other transcription factors to coordinate gene expression. Additionally, KLF4 associates with chromatin regulating proteins 
that can induce either an open or closed chromatin state, and thereby facilitate either transcription activation or repression respectively. The following sections describe the molecular mechanisms of how KLF4 promotes and represses target gene transcription.

\section{KLF4-mediated transcriptional activation:}

KLF4 can promote the transcription of its target genes by interacting with co-activator proteins via its $\mathrm{N}$-terminal transcription activation domain. When fused to the $\mathrm{C}$-terminal zinc fingers, this N-terminal region can activate a synthetic KLF4-responsive reporter construct, suggesting that KLF4 activity is positively regulated by this region (32). KLF4 interacts with the transcriptional co-activators, CREB binding protein and the closely related p300 histone acetyltransferase (HAT), and this interaction is important for KLF4 mediated gene transcription (32). Briefly, HATs transfer acetyl groups, which carry negative charges, onto critical positively charged residues (predominantly lysines and histidines) on histone proteins (41). Therefore, HATs neutralize the positive charge on these histones required for interaction of these proteins with negatively charged DNA, thereby decreasing DNA compaction, and allow access of the transcriptional machinery to the DNA. The reverse process of deacetylation of histones is catalyzed by histone deacetylases (HDAC), which facilitate a closed chromatin conformation, and consequently transcriptional repression (42). KLF4 is acetylated on residues 225 and 229 by p300/CBP and this acetylation is critical for KLF4 function (43). Mutations that disrupt the interaction between KLF4 and HATs neutralize the ability of KLF4 to activate target gene transcription.

Moreover, KLF4 can interact with Tip60, which possesses HAT activity and can recruit HDAC7 (44). Additional co-activators that associate with KLF4 include the zinc finger protein 
Krox20 and the NFкB subunit p65/RelA $(45,46)$. Target genes that can be transactivated by KLF4 include: CYP1A1 (47), DLK2 (48), E-cadherin (49,50), intestinal alkaline phosphatase (51,52), inducible nitric oxide synthase (iNOS) (46), keratin 4 (53), keratin19 (54), KLF4 (38,39), Laminin-1 (55), Laminin- $\alpha$ 3A (56), Laminin- $\gamma 1$ (57), Lefty 1 (58), miR-206 (59), Nanog (13,58), Notch1 (60), Oct4 (13), p21 ${ }^{\text {Cip1/Waf1 }}(35,38,61)$, p27 $7^{\text {Kip1 }}(61)$, p57 ${ }^{\text {Kip2 }}$ (62), Rb (61), Sox2 (13), SPRR1A (63), SPRR2A (63), Tbx-3 (13), and u-PAR (64).

\section{KLF4-mediated transcriptional repression:}

There are two mechanisms by which KLF4 may repress target gene transcription. Firstly, as KLF4 recognizes a similar target consensus sequence as other transcription factors (i.e., Sp1), KLF4 could compete for binding to target sites with these transcription factors, via a method of transcriptional repression termed passive repression $(4,47,65,66)$. Secondly, KLF4 possesses a centrally located repressor domain within can actively repress target gene transcription by recruiting and interacting with co-repressors. KLF4 interacts with HDAC enzymes to repress the expression of genes including CD11d, cyclin B1 (CCNB1), and p53 (TP53) $(43,67,68)$.

KLF4 also represses transcriptional targets of Wnt signaling by interacting with $\beta$ catenin/TCF4 (69,70). Additional targets that are repressed by KLF4 include BAX (71), Cyclin D1 (25,72), Cyclin D2 (73), Cyclin E (74), Fibroblast growth factor 5 (FGF5) (13), Histone decarboxylase (HDC) (44), KLF2 (13,58), Laminin a1 (52), MAPK8 (75), N-Cadherin (75), Ornithine decarboxylase (ODC) (76), SM22 $\alpha$ (77), Smooth muscle $\alpha$-actin (78), and Sp1 (65), Vimentin (75). 


\section{Roles of KLF4 in Normal Cells and Tissues:}

In humans, KLF4 is expressed in wide variety of tissues, and plays a critical role in regulating numerous cell physiologic processes including proliferation, programmed cell death (apoptosis), differentiation, and maintaining tissue homeostasis in the context of various organ systems. Related to these roles, KLF4 acts as a stress response factor in response to cellular injury. Although dispensible for normal embryonic development, KLF4 is critical for the formation of colonic goblet (mucous-producing) cells, and for the formation of the skin permeability barrier. Consequently, KLF4-deficient mice die soon after birth due to dehydration. Finally, KLF4 plays a role in the induction and maintenance of pluripotency in inducible pluripotent stem cells (iPSCs) and embryonic stem cells (ESCs). The following sections address each of these roles of KLF4 in normal cells and tissues.

\section{Proliferation and programmed cell death (apoptosis):}

KLF4, particularly enforced expression of the exogenous protein, generally inhibits cell proliferation and may even induce growth arrest $(22,39,40)$. In contrast, when the endogenous factor is suppressed, there are only subtle effects on cell proliferation, and there is typically a modest increase in the growth rate $(79,80)$. Consistent with a role in cell proliferation, KLF4 expression is low in actively dividing cells, but is induced upon growth inhibition by various stimuli including serum starvation and contact inhibition $(22,40)$. In the gut and skin epithelia, KLF4 is highly expressed in the post-mitotic cellular compartment $(12,40,81-83)$.

In normal cells and tissues, studies analyzing KLF4 upon treatment of cells with DNA damaging agents or $\gamma$-irradiation provide strong evidence that KLF4 slows cell proliferation $(35,36,74)$ KLF4 mediates its growth inhibitory effects by transcriptional regulation of cell cycle 
specific genes including cyclins and cyclin dependent kinase inhibitors. Whereas KLF4 represses the transcription of positive regulators of the cell cycle including cyclin B1 (CCNB1), cyclin D1 (CCND1), and cyclin E (CCNE1), KLF4 promotes the transcription of numerous inhibitors of the

cell cycle progression including $\mathrm{p} 21^{\mathrm{Cip} 1 / \mathrm{Waf} 1}(C D K N 1 A), \mathrm{p} 27^{\mathrm{Kip} 1}(C D K N 1 B)$, and $\mathrm{p} 57^{\mathrm{Kip} 2}$ (CDKN1C) $(35,36,72,74,84,85)$.

In addition to modulating the expression of cell cycle regulatory genes, KLF4 impacts cell proliferation by other means. In the intestinal epithelium, KLF4 may antagonize canonical Wnt signaling by interacting with the $\beta$-catenin/TCF complex $(69,70)$. Furthermore, KLF4 represses the translation of ornithine decarboxylase (ODC), a key enzyme in the synthesis of polyamines, which are important for cell proliferation (76). Thus, these studies suggest that KLF4 plays a cell proliferation suppressive role in normal tissues and cells.

In addition to suppressing cell proliferation in response to DNA damage and $\gamma$ irradiation, KLF4 plays an anti-apoptotic role. Upon $\gamma$-irradiation, KLF4 represses the transcription of the pro-apoptotic gene $B A X$ by inhibiting p53-mediated transactivation at the $B A X$ promoter (86). Furthermore, KLF4 binds the TP53 promoter to repress its transcription, and prevent apoptosis (68). Additional evidence suggests that KLF4 may influence p53-dependent pro-apoptotic signaling following DNA damage, though these mechanisms remain to be fully elucidated (87).

\section{Differentiation and the maintenance of tissue homeostasis:}

Much like the other KLFs, KLF4 plays an important role in the development, differentiation, and maintenance of tissue homeostasis in a wide variety of epithelial and nonepithelial contexts $(4,11,12)$. In intestinal and esophageal epithelia, KLF4 expression is restricted 
to the terminal differentiated epithelium $(12,40)$. Upon conditional knockout of $K l f 4$, the gastric epithelium displays aberrant and hyperplastic growth indicating that KLF4 is necessary for the establishment of proper gastrointestinal epithelial architecture (88). In a cell culture context, butyrate (a natural byproduct of bacterial fermentation in the colon that is thought to function as an HDAC inhibitor) induces KLF4 expression, which in turn activates the transcription of intestinal alkaline phosphatase (IAP) and laminin-1 (Lama1), and promotes a differentiated phenotype $(25,51,55,89)$.

The significant role of KLF4 in promoting proper epithelial differentiation can be seen in Klf4-null $\left(K l f 4^{-1-}\right)$ mice, which die of dehydration soon after birth due to defects in epidermal barrier formation of the skin (83). Consistent with this role, immunohistochemical staining reveals that KLF4 expression is enriched in the differentiated and post-mitotic growth arrested granular and spinous layers of the skin, but not in the mitotically active undifferentiated basal layers. Interestingly, overexpression of Klf4 combined with maternally administered glucocorticoids accelerates skin barrier formation, highlighting a synergistic relationship between the glucocorticoid receptor and KLF4 (90). Interestingly, the morphology of KLF4deficient skin is essentially normal, with no increase in epithelial cellularity and no apparent alteration of several differentiation markers (83), and thus argues against a major role for endogenous KLF4 in the regulation of cell proliferation.

In the gut, $\mathrm{Klf4}^{-/-}$mice also display a largely normal morphology, however there is a reduction in the number of colonic goblet cells. Furthermore, these cells display aberrant morphology and the loss of the differentiation marker MUC2 (82). Finally, targeted deletion of KLF4 in the corneal epithelium results in corneal fragility and a loss of goblet cells in the 
conjunctiva (91). These results identify a cell fate or pro-differentiation function of KLF4 during development of the skin and gut.

KLF4 regulates development and differentiation in a variety of non-epithelial cell contexts as well. Klf4 promotes the maturation of monocytes from hematopoetic stem cells $(46,73,92,93)$. In vascular smooth muscle cells (VSMCs) the role of KLF4 is discordant with its pro-differentiation role in epithelial cells. KLF4 is present at low levels in VSMCs and normally represses the differentiation of these cells in response to TGF $\beta(77,94,95)$. Similarly, in rat lung fibroblasts, KLF4 suppresses differentiation by interacting with Smad3 (96). In airway smooth muscle cells, KLF4 regulates the airway response to inflammatory stimuli (97). In endothelial cells, KLF4 is rapidly upregulated upon shear stress injury and proinflammatory stimuli, and promotes the expression of anti-thrombotic and anti-inflammatory genes including endothelial Nitric Oxide synthase (eNOS) $(77,97,98)$. Finally, siRNA mediated knockdown of KLF4 in differentiated adipocytes represses the levels of differentiation specific markers (45). These results suggest that KLF4 is critical in the development, differentiation, and maintenance of tissue homeostasis in a wide variety of contexts.

\section{Functions of KLF4 as a stress response factor:}

Many of the observed functions of KLF4 support a hypothesis for a role in the response to cell stress (99). A multitude of cell stresses including DNA damage and RAS induced senesence induce the expression of KLF4, and in several contexts, KLF4 has been found critical for the survival of cells or animals that were placed under stress $(36,71,95,97,100-104)$. In the cardiovascular system, shear stress induces the expression of both KLF4 and KLF5 in the 
vascular endothelium, and deletion of KLF4 in vascular endothelial cells renders mice intolerant of vascular stress induced by aortic banding (97).

The hypothesis that KLF4 is a stress response factor, particularly within epithelial cells, appears to provide a rationale for many of its effects in cancer cells. Indeed, several of the effects of KLF4 on cancer cells could be viewed favorable or adaptive responses in the context of normal epithelial cells exposed to stress. For example, reduced cell proliferation rates, increased

resistance to cell death, and altered migration rates could all support reconstitution of a normal tissue following injury $(4,11,12)$. As such, KLF4 joins TGF $\beta$ and other cancer-relevant molecules that have critical roles in the response to cell stress.

\section{Maintenance and establishment of pluripotency:}

Recent advances in the field of stem cell biology offer the hope of novel clinicaltherapeutic applications in regenerative medicine. The ability to reprogram somatic cells into pluripotent stem cells (termed induced pluripotent stem [iPS] cells) represents a major achievement that would enable this hope. KLFs, especially KLF4, in addition to OCT3/4, SOX2, and cMYC (termed Yamanaka factors) were identified as four critical transcription factors that could endow pluripotency to somatic cells including mouse embryonic fibroblasts and human dermal fibroblasts $(16,17)$. The role of KLF4 in inducing and maintaining pluripotency was further substantiated by studies that showed that KLF4 is an upstream regulator of the transcription of these Yamanaka factors (105). The ability of KLF4 to exert this role in iPS cells may stem from its co-operative action with cMYC, which may counteract the slow-growth phenotype of KLF4 (106). Thus far, the requirement for KLF4 in reprogramming adult somatic cells has been shown by multiple studies $(107,108)$. 
KLF4 has also been implicated as a critical factor in the self renewal of embryonic stem (ES) cells, which are cultured pluripotent stem cells derived from the inner cell mass of blastocysts (the early stage of an embryo prior to implantation). In these cells, Leukemia inhibitory factor (LIF) promotes self renewal through the induction of Klf4 (109). In turn, Klf4 is thought to maintain pluripotency by promoting levels of Oct3/4 (109). Recently, Klf2, Klf4, and Klf5 were identified as being functionally redundant in maintaining pluripotency in mouse ES cells, suggesting a broader role for KLFs in pluripotency (13). Though having overlapping functions in this context, these KLFs may possess non-redundant roles as well. Whereas Klf4 inhibits differentiation of ES cells to mesoderm, Klf5 inhibits the differentiation to endoderm (18). These studies identify a novel role for KLF4 in regulating cell fate and renewal in undifferentiated embryonic tissues.

\section{The role of KLF4 in tumor biology:}

The role of KLF4 in the tumor pathogenesis may result from the deregulation of its functions in normal cells and tissues $(4,11,12)$. KLF4 plays complex context-dependent roles in the development and progression of numerous cancers. Though some of the functions of KLF4 lend it to exert a tumor suppressive role in cancers including in esophageal, gastric, colon, lung, and hematopoetic malignancies, many other KLF4-dependent phenotypes may predominate to promote protumorigenic properties in head and neck, skin, and breast cancers. Even within specific cancers, studies report discordant findings on the role of KLF4 in regards to whether it is a tumor suppressor or tumor promoter (oncogene). These conflicting observations may be a result of the cell and experimental context-dependent functions of KLF4 $(68,110)$. A consistent theme is that endogenous KLF4 promotes resistance to cell death, particularly within cancer stem cells, while having only minor effects on cell proliferation, and this appears to hold across tumor 
types, including colorectal carcinoma where KLF4 is typically considered a tumor suppressor $(37,86,111)$. The following sections review the evidence for its dual tumor suppressive and oncogenic roles of KLF4 in various cancers.

\section{KLF4 as a tumor suppressor:}

Consistent with its growth inhibitory role, KLF4 is considered a tumor suppressor for a variety of cancer types (12). The tumor suppressor effects may reflect the the role of KLF4 in a normal context as a stress response and/or plasticity factor $(36,71,97,100)$.

Studies that analyzed gastrointestinal cancers strongly suggest this role for KLF4. In primary human colon adenocarcinoma samples, KLF4 mRNA is reduced compared with the normal colon (112). In a panel of 30 colon cancer specimens, the mean level of KLF4 mRNA was reduced by $50 \%$ compared to matched normal samples (112). Immunohistochemical staining analysis indicated that KLF4 protein expression was downregulated in colon cancer specimens (113). Many genetic alterations involving the KLF4 gene in colon cancer appear to inhibit KLF4 tumor suppressor functions, and include loss of heterozygosity (LOH) and, promoter hypermethylation (112-114). However, KLF4 mutations that disrupt protein function that have been reported in colon cancer cell lines have not been confirmed (112). In cell culture models, KLF4 mRNA levels are downregulated in colon cancer cells compared to normal colonic epithelial cells $(112,114)$. Overexpression of KLF4 promotes cell cycle arrest by inducing $\mathrm{p} 21^{\mathrm{Cip} 1 / \text { Waf1 }}$ in RKO colon cancer cells (86). Furthermore, ectopic expression of KLF4 in these cells also reduces colony formation, cell migration, and in vivo tumor growth.

In colon cancer cells, KLF4 expression is regulated by adenematous polyposis coli (APC), which is the most frequently mutated tumor suppressor gene in these cancers (115). APC 
maintains intestinal epithelial homeostasis through modulating the activity of the Wnt- $\beta$-catenin signaling pathway (116). KLF4 is reported to inhibit Wnt signaling induced colon cancer cell proliferation by downregulating $\beta$-catenin mRNA and protein, and interacting with the $\beta$ catenin/TCF4 complex to inhibit Wnt-mediated transcriptional activity $(69,70)$. Compared to control tissues, KLF4 mRNA is lower in colon cancer specimens from patients with familial adenomatous polyposis (FAP), which result from germline mutations in APC (115). This observation is also made in colon cancer specimens from $A p c^{\min }$ mice, which carry point mutations in the $A P C$ gene. Furthermore, $A p c^{\min }$ mice with hemizygous loss of Klf4 (Klf4 ${ }^{+/-}$ $I A p c^{\text {min }}$ ) loss display a 55\% increased incidence of intestinal adenomas compared to $A p c^{\text {min }}$ mice (117). Furthermore, the levels of $\beta$-catenin and cyclin D1 are increased in the intestinal tissues of $K l f 4^{+-} / A p c^{\text {min }}$ mice. In both $K l f 4^{+-} / A p c^{\text {min }}$ and $A p c^{\min }$, Klf4 protein levels are further decreased in adenomas compared to the normal intestinal mucosa, and are inversely correlated with adenoma size (117).

Additionally, KLF4 is thought to suppress intestinal tumorigenesis by antagonizing Notch signaling $(118,119)$. In intestinal tissues, Notch signaling suppresses goblet cell differentiation and is upregulated in adenomas (120). In cell culture models, pharmacologic inhibition of Notch signaling upregulates KLF4 expression and reduces proliferation. Treatment of $A p c^{\min }$ with the $\gamma$-secretase inhibitor (Notch signaling antagonist) dibenzazepine (DBZ) reduces intestinal adenoma formation compared to mice treated with a drug vehicle control (118). Taken together, these results suggest a tumor suppressive role for KLF4 in intestinal tumorigenesis.

However, a recent study that analyzed the role for KLF4 in colon cancer stem-like cells (CSCs) shows an oncogenic role for KLF4 in this context (111). Consistent results were 
observed in studies that showed an anti-apoptotic role in colorectal cancer cell lines (71). CSCs are a low abundance population of cancer cells that are thought to be responsible for cancer recurrence, metastasis, and drug recurrence (121-124). KLF4 expression was found to be enriched in isolated colon CSCs and KLF4 was shown to mediate chemoresistance, migration, invasion, and in vivo tumorigenicity (111). Furthermore, knockdown of KLF4 resulted in a decrease in the CSC-fraction. This contradicting result suggests that KLF4 may exert a context dependent role depending on whether its function is analyzed in CSC or non-CSCs and may in fact indicate a protumorigenic role for KLF4 in colon cancer.

Putative tumor suppressive roles for KLF4 have also been identified in cancers that arise in other areas of the gastrointestinal tract including in the esophagus $(63,125)$, stomach $(65,126,127)$, liver (128) and pancreas (85). Consistent with this role, KLF4 has been documented to be a tumor suppressor in other cancer contexts including in lung $(129,130)$, prostate $(131,132)$, urinary bladder (133), and hematological malignancies (134).

\section{KLF4 as an oncogene:}

Much like its tumor suppressive effects, the protumorigenic effects of KLF4 may reflect its normal role as a stress response factor and ability to promote cellular plasticity $(36,71,97,100)$. The oncogenic activity of KLF4 was first identified by our laboratory (135). Using an unbiased screen, we identified KLF4 as a major transforming activity in human tumor cell cDNA library (135). We found that KLF4 induced transformation in E1A-immortalized rat kidney epithelial cells (RK3E) cells (135). These KLF4 transformed cells produced subcutaneous tumors in immunocompromised mice (135). The role for KLF4 as an oncogene is supported by the increased expression of KLF4 mRNA and protein in oral dysplastic epithelium, in squamous 
cell carcinoma (SCC) of the skin, and in breast cancer, in comparison to normal tissues $(131,136,137)$.

The Klf4 promoter was identified as a common retroviral integration site by Moloney murine leukemia virus (MMLV) leading to retrovirus induced leukemia (138). Furthermore, the role of KLF4 in inducing pluripotency in somatic cells may suggest that KLF4 could promote a cancer stem cell phenotype. In numerous contexts, loss-/gain-of-function studies show that KLF4 may promote additional protumorigenic properties, including escape from RAS-induced senescence and enhanced cell survival following $\gamma$-radiation-induced DNA damage $(68,71,135,136)$

Modeling the upregulated expression KLF4 in basal keratinocytes of human dysplastic skin and oral mucosa, conditional expression of KLF4 in the basal layers of the skin of transgenic mice results in the development of cutaneous SCC (136). In these transgenic mice, expression of KLF4 and proliferating cell nuclear antigen (PCNA) is restricted to mutually exclusive compartments in both the normal and hyperplastic skin of these transgenic mice (137). However, both KLF4 and PCNA colocalize in dysplastic and cancerous epithelium showing that successive increases of KLF4 in the nuclei of basal keratinocytes is associated development of superficially invasive SCC (137). Furthermore, our lab also showed that KLF4 promotes the expression of several retinoic acid receptors, including retinoic acid receptor- $\gamma(\mathrm{RAR}-\gamma)$ and retinoid X receptor- $\alpha$ (RXR- $\alpha)$, which can be pharamacologically targeted (139).

\section{The role of KLF4 in breast cancer:}

There have been conflicting reports about the function of KLF4 in breast cancers. Many studies have suggested a tumor suppressor role for KLF4 in breast cancer. For example, KLF4 
was shown to promote epithelial features in breast cancer cells and reduce the ability of these cells to invade, migrate, and metastasize $(49,50,75,140,141)$. Furthermore, KLF4 was shown to suppress ER $\alpha$ signaling and ER $\alpha$-dependent breast cancer cell proliferation in vitro (142). Finally, KLF4 was shown to promote apoptosis in HER2-positive breast cancer cells (143).

However, studies have consistently shown a role for KLF4 in promoting protumorigenic phenotypes in breast cancer cells. In human breast cancers, there is typically higher expression of KLF4 in tumor cells compared with the adjacent, uninvolved epithelium. This elevated protein level, or else demethylation of the KLF4 promoter, portends a poor prognosis $(20,21,144)$. In contrast with a study that analyzed ER $\alpha$-signaling, KLF4 stabilizes ER $\alpha$ and promoted breast cancer proliferation (145). KLF4 also promotes the expression of the platelet isoform of phosphofructokinase in breast cancer cells and promotes cell growth (146). KLF4 knockdown leads to reduced migration and invasion, increased rates of apoptosis, and reduced rates of tumor initiation in a mouse xenograft model (Chapter 3) $(59,79,80,147,148)$. KLF4 may activate Notch signaling in mammalian epithelial cell culture models and may promote this signaling in breast cancer cells (149). These results support the identification of KLF4 as an oncogene, and provided initial clues as to its function in these contexts. However, the precise mechanisms of how KLF4 exerts its effects in breast cancer cells are not well known. Identification of KLF4 regulated signaling pathways may help elucidate the function of this transcription factor in breast cancer.

\section{Interpreting studies analyzing KLF4 in tumor biology:}

The development of experimental model systems has allowed us to gain better understanding of the function of many genes in a multitude of physiological and pathological contexts. Cancer models serve as surrogates to spontaneous human tumors, and provide a setting 
in which individual genes, or sets of genes, may be manipulated and the role of these gene(s) can be deduced from resulting phenotypes. Though these experimental settings are powerful tools in analyzing gene function, cancer models are limited in the ability to recapitulate the complexity of human cancer. For example, in vitro cell culture models and in vivo tumor xenograft models in immunocompromised mice analyze relatively homogeneous tumor cell populations and often do not account for tumor cell interaction with stromal elements which are critical in cancer biology. Also, genetically engineered animal models of cancers may not accurately depict human cancers which often do not display uniform genetic alterations in tumor cells. Furthermore, gene loss/gain-of-function studies in vitro may alter gene expression to a level that may not be representative in the physiological/disease state. Thus, careful interpretation of experimental results obtained in any model system is required, and investigation of gene function across model systems is required for the accurate analysis of the context dependent function of any gene.

The study of a protein such as KLF4, which has a multitude of cell physiological functions, using model systems can be challenging. The ability to accurately model a slowgrowth factor such as KLF4 in vitro and in vivo is difficult as tumor growth in these models is much faster than spontaneous human tumors. For example, wheras human breast cancers double in size in approximately 130 days (99), cell culture models and the xenografted tumors that are used for functional studies double much more rapidly.

Also, potentially, adaptive mechanisms or altered differentiation or barrier formation within KLF4-deficient epithelium could account for enhanced tumor formation in KLF4 deficient tissues. Although uniform or organ-wide epithelial depletion or hemizygosity of KLF4 in colorectal mucosa clearly promotes neoplasia in the $A p c^{\text {min }}$ mouse model, such uniform depletion does not necessarily model the development of spontaneous tumors, which are much 
more focal in their origin. Providing a clear example of this phenomenon, organ-wide Notch depletion in cutaneous keratinoctyes of genetically engineered mice promotes skin tumorigenesis by disrupting the skin permeability barrier and triggering an inflammatory response, rather than through a cell autonomous effect within tumor cells, and this study therefore argued against a tumor suppressor role of Notch in the skin (150). The inability to clearly delineate whether a gene exerts its effects through either cell autonomous or non-cell autonomous effects further serves as a reason why studies in cancer model systems should be interpreted with care.

If KLF4 evolved to enable organisms to adapt to environmental or cellular stress, then the potential for a complex role in cancer, potentially including both protumorigenic and antitumorigenic effects across many cancer types, seems plausible. If so, then a better understanding of the signaling involving this critical factor could serve to shed new light upon specific signaling mechanisms involved in cancer suppression, and other mechanisms that promote tumor development, with potential new avenues for therapeutic intervention.

\section{Molecular mechanisms of KLF4-dependent phenotypes:}

Though studies have delineated numerous roles for KLF4 in diverse cancer contexts, the molecular mechanisms that underlie many of these processes remain to be fully explored. Furthermore, how certain KLF4-dependent phenotypes may enable KLF4 to play contextdependent roles remains a significant quandary. Many of the KLF4-dependent phenotypes depend upon p53-mediated signaling and the modulation of cell cycle specific genes including p21 ${ }^{\text {Cip1/Waf1 }}(68,110)$. Whether KLF4 functions upstream, downstream, or both of p53 and $\mathrm{p} 21^{\mathrm{Cip} 1 / \mathrm{Waf} 1}$, requires further investigation $(68,110)$. 
Additionally, little is known about the binding partners, targets, and downstream effectors of KLF4. It is also not known whether KLF4 coordinately signals with other tumor suppressive or protumorigenic pathways. Therefore, analysis of KLF4-dependent signaling may enable a more thorough understanding of how KLF4 functions in diverse contexts.

One group of putative KLF4-effector molecules are microRNAs (miRs). miRs associate with the RNA-induced silencing complexes (RISC) to regulate the translation of mRNAs (151154). Much like in other cancer relevant genes, deregulation of miR expression occurs in multiple cancer types, including in breast cancer, and distinct miRs can mediate either oncogenic or tumor suppressive effects (151-154). Of relevance, our lab identified microRNA-206 (miR206) as a potential downstream effectors of KLF4 in breast cancer cells that also regulates KLF4 translation in a negative feedback loop (59). Chapters 2 and 3 of this dissertation focus on KLF4miR dependent signaling.

\section{MICRORNAS (miRs)}

\section{A brief overview of microRNAs (miRs)}

MicroRNAs (miRs) are genomically encoded single stranded noncoding RNAs that are typically 19-25 nt in length and result from extensive processing of endogenous hairpin-shaped structures (155-158). miRs were initially identified in Caenorhabidits elegans as genes required

for the regulation of the proper developmental timing $(159,160)$. Subsequently, thousands of miRs and putative miR-encoding genes have been identified in a wide variety of organisms, 
including plants, invertebrates, and metazoans (155-158). Since their discovery, miRs have emerged as important regulators of gene expression and are functionally implicated in numerous contexts including in cancer (161-163). miRs typically repress the translation of mRNA transcripts by associating with mutiprotein complexes termed RNA-induced silencing complexes (RISCs). miR selectivity for target mRNAs arises from miR sequence complementarity to portions of target mRNAs. Though miRs typically repress the translation of their target mRNAs, few miRs have been documented to promote the translation of their transcripts, suggesting an additional level of complexity in this modality of regulating gene expression.

\section{miR biogenesis and nomenclature}

\section{Biogenesis}

$\mathrm{miR}$ biogenesis is a multi-step process involving the transcription of $\mathrm{miR}$ genes and the subsequent post-transcriptional processing of the transcripts by class III ribonucleases (RNAseIII) and their accessory double strand RNA (dsRNA) binding proteins to yield mature miRs of 19-25 nt length (Fig. 4) (155-158). miR genes can be transcribed by RNA polymerase II to yield primary transcripts called pri-miRs $(164,165)$. miR genes that are present proximal to tRNA coding genes or Alu and other such repeated elements may be transcribed by RNA polymerase III (166). The transcribed pri-miRs may arise from individual miR genes, from the introns of protein coding regions, or from polycistronic genes that may encode a cluster of multiple miRs $(156,167)$. In animals, pri-miRs are subsequently cleaved in the nucleus to form stem loop structures of 60-70 nt length called pre-miRs. This processing step is mediated by the heterodimeric nuclear complex containing the RNAse-III enzyme Drosha and the non-catalytic 
dsRNA binding protein, DiGeorge Syndrome Critical Region 8 (DGCR8; also called Pasha in other organisms) (168-173).

These pre-miRs are then recognized by the Ran-GTPase/exportin-5 complex and transported out of the nucleus, where they are processed by the cytoplasmic RNAse-III enzyme Dicer to yield short (19-25 nt) miR-miR* (miR* exhibits near complete complementarity to miR) duplexes $(168,174-178)$. In mammals, mature miRs can then be incorporated into large multiprotein complexes, termed RNA-induced silencing complexes (RISC), that can contain Argonaute (AGO) family endonucleases $(179,180)$. miRs in these RISC complexes can recognize target mRNAs by sequence complementarity (typically between bases $2-8$ at the 5 'end of the miR [termed the miR seed sequence] and the mRNA 3' UTR) and typically repress protein translation through a variety of means. In plants, miRs exhibit near perfect complementarity to target mRNA, and miR-mRNA interactions result in the cleavage of the target mRNA molecules, and consequently translation repression.

Though the majority of miRs are thought to be derived from the mentioned Drosha/Dicer dependent process, it is important to note that many miRs can arise from alternative/noncanonical biosynthetic pathways (Fig. 5) (181). Biogenesis of miRs may occur through Drosha/DGCR8 independent (miR genes located in introns of protein coding genes [miRtrons]) or through Dicer independent pathways (direct cleavage of pre-miR stem loops into miR-miR* duplexes by AGO proteins) (181).

\section{Nomenclature}

The systematic nomenclature for miRs is defined by miRbase, which serves as the repository for miR sequence data and target validation $(182,183)$. Mature miRs are designated by 
a three letter prefix such as "miR" or "let", followed by a number (e.g. miR-1), and both the gene and the stem-loop precursor are designated as "mir" (note: lower case 'r'; mir-1). Identical miRs that arise from distinct primary sequences are denoted with additional numbers that are added to the mature miR designation (e.g. miR-1-1, miR-1-2, etc.). Distinct miRs that arise from a common primary sequence are designated with a letter after the miR name (e.g. miR-16-a, miR16-b, etc.).

Finally, in the case of miRs that may arise from both arms of the stem loop precursor: miRs that arise from the 5'-arm of the stem loop precursor are designated as $-5 \mathrm{p}$, and those that arise from the 3 '-arm are designated as $-3 p$ (e.g. miR-21-5p, miR-21-3p). Of these two miRs, the less abundant species can also be denoted as miR* (e.g. miR-21-3p is often designated as miR$21 *)$.

\section{miR-mediated gene regulation}

Though the results of miR-mediated gene regulation have been extensively documented, the molecular mechanisms of how miRs repress the translation of target mRNA requires further study $(155,157,158)$. It is generally accepted that miRs function to regulate gene expression at the post-transcriptional/translational level, though the mechanistic details of this process are still being uncovered.

The process of mRNA translation includes three major steps: initiation, elongation, and termination. Initiation is the process by which the translation machinery (the ribosome and associated proteins) is recruited to the translation start site in the mRNA. Elongation involves the assembly of a polypeptide chain through the action of the translation machinery. And finally, in the termination step, the newly synthesized polypeptide chain is released from the ribosome 
(184). Various lines of evidence suggest that miR regulation of mRNA translation can occur at both initiation and post-initiation steps. In eukaryotic cells, the rate-limiting step of translation is initiation. Thus, the initiation step is a common target for translational control. The following section briefly reviews the initiation of translation.

\section{A brief overview of eukaryotic mRNA translation initiation}

Translation initiation can occur through two distinct processes termed cap-dependent and cap-independent translation initiation (184-186).

\section{Cap-dependent Translation Initiation:}

A distinguishing feature of eukaryotic mRNAs that are transcribed in the nucleus is the $\mathrm{m}^{7} \mathrm{GpppN}\left(\mathrm{m}^{7} \mathrm{G}=7\right.$-methylguanylate, $\mathrm{p}=$ phosphate group, $\mathrm{N}=$ any nucleotide) structure that is present at the 5'-terminus of the exported mRNA called the 5'-cap. The 5'-cap is added to mRNAs in a series of enzyme catalyzed steps, and occurs along with intron splicing and the addition of a 3'-poly(A) tail, prior to export out of the nucleus. Capping at the 5'-end serves to protect mRNA from degradation and allows ribosome recruitment to the mRNA to initiate translation. Translation initiation that involves recognition of this 5 '-cap by the ribosome is termed cap-dependent initiation.

Cap-dependent translational initiation represents the most common mechanism of initiation (Fig. 6) (185). In eukaryotes, this method of initiation involves recruitment of the 80S ribosome (composed of the large [60S] and small [40S] ribosomal subunits) to the mRNA translation start site. Initially, a $43 \mathrm{~S}$ pre-initiation complex is formed. This complex consists of the small ribsomal subunit, the eukaryotic initiation factors (eIFs) 1, 1A, 3 (and probably 5), and a ternary complex containing GTP bound eIF2 which is also bound to tRNA ${ }^{\text {Met }}$ (GTP-eIF2- 
tRNA $^{\mathrm{Met}}$ ). This pre-initiation complex recognizes and attaches to the capped 5'-proximal region with the help of eIF4A (DEAD-box RNA helicase), eIF4B, eIF4E (cap binding protein) and eIF4F (a coordinating scaffold), which serve to unwind the mRNA $5^{\prime}$ untranslated region (5' UTR) secondary structure. The complex then scans the 5' UTR of the mRNA and recognizes the initiation codon (AUG) in an ATP-dependent manner (187-189).

Recognizing and binding the initiation codon by the initiator tRNA (tRNA ${ }^{\mathrm{Met}}$ ), results in the eIF5B mediated hydrolysis of the GTP bound to eIF2, and allows the displacement of the eIFs and the joining of the 60S subunit. The scaffolding protein, eIF4G may concurrently interact with eIF4E and the poly(A)-binding protein (PABP) and allow the formation of a circular nucleoprotein structure upon translation initiation. This circular structure may allow the regulation of translation initiation by elements that interact with the 3' UTR of mRNA (184186).

Cap-independent translation initiation:

The most well-characterized method of Cap-independent translation initiation is mediated via internal ribosome entry sites (IRES) and involves interactions between the translation machinery and the mRNA. This method of translation initiation bypasses the requirement for the 5'-cap structure and therefore does not require eIF4E (186). IRESs can interact directly with the p50 domain of eIF4G, which can recruit the $43 \mathrm{~S}$ pre-initiation complex to start translation initiation (186). 


\section{Regulation of eukaryotic translation initiation and post-initiation}

\section{Regulation of translation at initiation}

As mentioned above, the initiation of translation is a complex, rate-limiting, multi-step process for mRNA translation and thus is a critical target for translational control (186). Regulation at this step can occur in two ways: by modulating the availability of eIFs (global control: the translation of all mRNAs can be affected) or modulating the scanning of the mRNA by the ribosome by the action of RNA binding proteins (selective control: the translation of only targeted mRNAs is affected) (186).

Global regulation of translation initiation can be regulated by altering the availability of active eIFs. The activity of the eIFs can be modulated by phosphorylation. The exchange of GTP for GDP on eIF2 by eIF2B (a guanine nucleotide exchange factor; GEF) is necessary for reconstitution of the ternary complex described above. Phosphorylation of eIF2-GTP enhances its affinity towards eIF2B and stabilizes the eIF2-eIF2B complex, and thereby prevents subsequent formation of eIF2-ternary complexes $(185,186)$. Phosphorylation of several other eIFs including eIF4E can activate the initiation of translation. Phosphorylation also indirectly affects the availability of the eIF4E proteins by modulating interaction with binding proteins $(190,191)$.

Selective regulation of initiation can be modulated by RNA binding proteins that recognize particular RNA sequence motifs, and typically act in an inhibitory manner. Regulation of initiation can occur at the 5' UTR as in the case of the regulation of mRNAs encoding ribosomal proteins (192). The initiation of translation of these mRNAs proceeds with poor efficiency when nutrient availability is scarce and the need for protein translation is low. This 
inhibition of mRNA translation initiation is modulated by interaction of RNA binding proteins (which remain to be conclusively elucidated) with the $5^{\prime}$ terminal oligopyrimidine tract (5' TOP) that is found in the 5' UTR of many of these mRNAs $(186,192)$. Furthermore, RNA binding proteins may also interact with the 3' UTR of target mRNAs. Numerous studies suggest that the simultaneous interaction of the PABP with the poly(A) tail and the eIF4G complex, forming a circular structure, is indispensible in promoting the initiation of translation $(186,193,194)$. The formation of this circular structure may serve as the functional basis of the regulation (typically inhibition) of translation initiation by proteins, such as RISC complexes containing miRs, that typically bind sequence elements in the 3' UTR $(155,157,158,186)$.

\section{Regulation of translation after initiation (post-initiation)}

Post-initiation steps include elongation and termination. Regulation of translation at these steps affects translational efficiency. Furthermore mRNA stability can be impacted concurrently through the processes of mRNA decay $(195,196)$. mRNA decay can be modulated by two major pathways: mRNA turn-over, which involves the degradation of functional polyadenylated transcripts and mRNA surveillance, which serves as a quality control mechanism for preventing the translation of defective mRNAs $(195,196)$.

In eukaryotic mRNA turnover, functional mRNA transcripts are typically degraded via one of two pathways: the exonucleolytic and endonucleolytic pathways. The exonucleolytic pathway is initiated by deadenylation of the poly(A) tail followed by decapping at the 5' end. These transcripts can then be degraded by exonucleases at both the 3 ' and the 5 ' ends (197). In the endonucleolytic pathway, transcripts can bypass deadenylation and be degraded by RNAseIII enzymes mediated cleavage without deadenylation. This method of endonucleocytic cleavage 
typically occurs during miR mediated translation repression in plants $(198,199)$. The initiated endonucleolytic cleavage of mRNA transcripts can be subsequently coupled by exonucleocytic cleavage as well.

mRNA surveillance serves as a quality control mechansim to ensure that defective, truncated, or generally faulty transcripts are destroyed (197). mRNA surveillance can occur through nonsense-mediated decay (NMD), which is used to eliminate truncated transcripts containing premature stop codons, and non-stop decay (NSD), which is used to degrade transcripts without stop codons (200-202). Recently, a new mechanism of mRNA surveillance called no-go decay (NGD) has been described, through which transcripts with stalled ribosomes are degraded (203).

\section{miR-mediated mRNA translation repression}

miRs often repress the translation of mRNAs. As mentioned earlier, miRs confer target specificity to RISC complexes which contain argonaute (AGO) proteins. Of the four mammalian AGO isoforms (AGO1-4), only AGO2 possesses RNAse-III activity (204). AGO2 can cleave mRNAs that posses sequence complementarity to the miRs with which they associate in the RISC complex. In animal cells, miR-mediated translation repression can result in the downregulation of target mRNAs by degradation. However, this mRNA destruction occurs via mechanisms that are independent of endonucleocytic mRNA cleavage (Fig. 7) (185). Whether miR-mediated translation repression occurs at the initiation or post-initiation steps is controversial (186). Regardless of the mode of translation repression, target mRNAs may ultimately be degraded $(155,157,158,186)$. 
miRs and their targets can associate with the free messenger ribonucleoprotein (mRNP) pool in mammalian cells, rather than mRNAs in polysomes, indicating that translation inhibition may occur at an early step (205-207). A concordant role for miRs in blocking translation initiation was the finding that AGO2 exhibits sequence similarities to the cytoplasmic capbinding protein eIF4E, and can bind to 7-methylguanylate triphosphate in vitro (208). This suggests that miRs may recruit AGO2 protein containing RISC to the cap structure by competing the binding site of eIF4E, therefore inhibiting translation at the cap-recognition step. Furthermore, studies show that mRNAs translated through IRES mediated cap-independent mechanisms were not suppressed by miRs, further supporting the proposal that miRs inhibit capdependent translation initiation $(205,206,209,210)$. miRs in RISC complexes can also inhibit translation post-initiation by impacting ribosome assembly, recruiting protein co-factors that interfere with translation elongation, and by poly(A) tail shortening $(155,157,158,186)$.

Though early studies reported that animal miRs repress translation without significantly reducing the levels of target mRNAs, many studies have demonstrated that miR-mediated degradation of target mRNAs can occur as well (211-215). A well documented outcome of translation repression at both the initiation and post-initiation steps is destruction of the target mRNA (216-220). Whereas depletion of either Dicer or AGO proteins increases the levels of predicted and validated miR target mRNAs, overexpression of exogenous miRs decreases the transcripts containing binding sites for those ectopic miRs (221-223).

In contrast to plants, where AGO-dependent endonucleocytic cleavage of target mRNAs is the predominant result of miR-mediated gene regulation, in animals, destruction of the target mRNA occurs via deadenylation followed by exonucleocytic cleavage by general mRNA degradation machinery. In fact, miRs can accelerate the deadenylation and decapping of their 
target transcripts in many contexts $(213,221,224)$. Furthermore, in animals, though the degradation of target mRNA transcripts can proceed in the absence of the catalytic activity of AGO2, miR-mediated mRNA decay nonetheless requires AGO proteins $(225,226)$.

A critical component of the RISC complex is the accessory protein GW182, which interacts with AGO, and is crucial in recruiting the CAF1-CCR4-NOT deadenylase complex, the decapping enzyme DCP2, and several decapping activators to accelerate miR-mediated mRNA decay $(213,224)$. Indeed, GW182 also functions to interact with PABP, and thus may repress translation initiation, thereby identifying GW182 as an effector of miR-mediated gene repression $(225,226)$.

\section{miR-mediated mRNA translation activation}

miRs generally repress gene expression, but some studies have found that miRs can promote the translation of specific mRNAs (158). Indirectly, miRs can promote gene expression by repressing the translation of cellular components that inhibit mRNA translation (227). Translation activation by miRs has been documented in numerous contexts, but precise molecular mechanisms of these processes are currently unclear. miR-122 promotes the efficient IRES mediated translation of mRNAs encoded by the Hepatitis C virus (HCV) and its expression is important for the pathogenesis of Hepatitis C (228-230). Interestingly, a switch in the activity of some miRs can be induced in response to external stimuli. In cells growth arrested following serum starvation, miR-369-3p activates the translation of the tumor necrosis factor $\alpha(T N F \alpha)$ mRNA, whereas under normal conditions, this miR represses $T N F \alpha$ translation (231-235). A similar phenomenon is observed in the regulation of mRNAs encoding ribosomal proteins by miR-10a (236). Finally, our lab identified that miR-206 could both promote and repress the 
translation of KLF4 depending on the cellular context, e.g. in normal and cancerous mammary epithelial cells respectively (59). Generally, mechanisms of miR-mediated mRNA translation activation are even more poorly understood compared to miR-mediated translation repression, and this activity of miRs and the roles it exerts in cell biology remain to be further elucidated.

\section{Identifying and validating $m R N A$ targets of miRs}

In order to uncover functional roles for miRs, identification of their target mRNAs is necessary. To this end, numerous target prediction software programs are available and identify putative miR-target mRNA interactions in silico (237). Several algorithms are used by these programs to identify candidate mRNAs for miRs. Since most (but not all) functional miR-mRNA interactions occur in the 3' UTR of the mRNA transcript, these algorithms search for complementarity between the miR seed sequence (approximately bases 2-8 from the 5'-end of the miR) and candidate 3' UTRs (238-241). Some algorithms then assign scores and rank the putative transcripts based on the degree of complementarity between the miR and 3' UTR of the mRNA. Furthermore, certain algorithms try to identify functional interactions based on evolutionary conservation of each site in candidate targets. Finally, computational models can be used to predict miR-targeted mRNAs by calculating high affinity interactions between the miR and the cognate mRNA $(242,243)$.

Experimental validation of miR-targeted mRNAs is necessary to implicate miRs in the regulation of identified candidate mRNAs. Using loss and gain of function experiments, the protein or mRNA levels of the candidate miR target can be analyzed, though changes in the levels of either are not indicative of direct miR-mediated regulation. Translation reporter assays can be utilized to more directly validate candidate miRNA targets and identify functional miR 
interaction sites within transcripts. In these translation reporter assays, the 3 ' UTR is cloned downstream of a reporter ORF, such as firefly luciferase. Modulating miR levels in the experimental system can be achieved by inhibiting the activity of endogenous miRs (e.g. by antisense molecules targeting the mature miR) or by delivering exogenous miRs may either upregulate or downregulate reporter activity respectively, indicating that a miR responsive element may be present in the transcript analyzed. Functional interaction of the miR with a particular site in this transcript may then be deduced if these changes to reporter activity are abolished in a reporter containing miR-target site mutations. It must be noted that for proper interpretation of these translational reporter experiments, it is critical to ensure that mutagenesis of the target site does not alter the overall secondary structure of the transcript. Other approaches include pulse-chase metabolic labeling of nascent, endogenous transcripts instead of using a translational reporter and functional miR-mRNA interactions can be analyzed by RNAimmunoprecipitation (RIP) of components of the RISC complex and by sequencing mRNA transcripts associated with the miR of interest $(152,154,157,237)$.

\section{The functions of miRs in tumor cell biology}

By repressing the expression of target mRNAs, miRs play key roles in diverse cell processes, such as control of cell differentiation, apoptosis, proliferation, division, protein secretion, and viral infection $(155,157,158,186)$. By regulating these processes, miRs play critical roles in the development and differentiation, and maintenance of homeostasis of tissues in virtually every organ system $(153,154,237,244)$. Additionally, miRs play important roles in maintaining the regenerative capacity of adult stem cells and regulating metabolic processes (244). Therefore, it is not surprising that the deregulation of miR expression is critical in cancer pathogenesis $(151,153,154,245,246)$. Human cancers display differential expression of miRs 
suggesting that miRs may play oncogenic or tumor suppressive roles $(245,246)$. Indeed, miRs can function in tumor biology as either tumor suppressors or oncogenes. Oncogenic miRs (oncomiRs) can exert their influence by suppressing the translation of tumor suppressor mRNAs, and tumor suppressive miRs can suppress translation of the transcripts of oncogenes. Furthermore, miRs can exert either tumor suppressive or oncogenic effects by regulating the activity of signaling pathways through repression of pathway components $(151,153,154,245,246)$. Thus, whether by individual or co-operative action, miRs can play important roles in tumor biology. The following section reviews both tumor suppressor and oncogenic miRs.

\section{Tumor suppressive miRs}

As described above, miRs can function as tumor suppressors by targeting and repressing the translation of transcripts encoding oncoproteins that play critical roles in regulating numerous pathways such as cell proliferation, survival, migration, invasion, and angiogenesis $(153,154,247)$. The initial evidence for the involvement of miRs in cancer pathogenesis came from studies that analyzed the loss of chromosome $13 q 14$ in B-cell chronic lymphocytic leukemia (CLL) $(248,249)$. This genomic region was found to contain the miR-15a/miR-16-1 cluster locus, thereby leading to loss of expression of these miRs $(248,249)$. Consequently, in CLL patient samples, the levels of miR-15a/miR-16-1 and the anti-apoptotic protein BCL2 are inversely correlated (250). Furthermore, studies showed that miR-15a/miR-16-1 regulated BCL2 expression at the post-translational level, and thereby supported a tumor suppressive role for these miRs (250). 
Another example of a tumor suppressor miR is let-7. Downregulation of let-7 is frequently seen in lung cancers and loss of let-7 expression is significantly correlated with reduced post-operative survival in lung cancer patients (251). Overexpression of let-7 in lung cancer cells reduces cell proliferation suggesting that let-7 can function as a tumor suppressor (252). Subsequent studies indicate that let-7 exerts its tumor suppressive effects by targeting and repressing the translation of RAS and cMYC proto-oncogenes (252).

The expression of miR-34 family members has been reported to be downregulated in a variety of cancers (253). miR-34a and p53 form a positive feedback loop. Whereas p53 promotes the expression of miR-34a, miR-34a targets and represses SIRT1 translation to consequently upregulate the expression of both p53 and the pro-apoptotic protein PUMA (254). Therefore loss of this miR results in the indirect loss of both p53 and its proapoptotic target, PUMA (254). Finally, consistent downregulation of miR-143 and miR-145 has been observed in numerous tumor types and has led to their identification as putative tumor suppressive miRs $(245,246)$. The levels of miR-143 and miR-145 inversely correlate with tumor size in colon cancer patient samples (255). These miRs participate in negative feedback loops and repress components of oncogenic signaling pathways (256). In turn, these miRs are also downregulated by oncogenic RAS and epidermal growth factor receptor (EGFR) signaling in numerous contexts $(257,258)$. Expression of both miRs inhibits cell migration and invasion, suggesting that miR-143 and miR145 may indeed function as tumor suppressors in a variety of contexts (259).

\section{Oncogenic miRs (oncomiRs)}

miRs that are increased in tumors have the potential to function as oncogenes. Some of these miRs can exert net oncogenic roles by negatively regulating the expression of tumor 
suppressors that function to inhibit cell proliferation, survival, migration, invasion, and angiogenesis $(151,153,154,245-247)$. Many oncomiRs (and tumor suppressor targets) have been characterized in a many of tumor contexts, including the miR-17-92 cluster (lung cancer) (260), miR-372/miR-373 (testicular germ cell tumors) (261), miR-155 (B-cell lymphomas) (262), and miR-221/miR-222 (numerous tumor types) (263).

Of note in this context is miR-21. miR-21 is one of the most well-characterized oncomiR that is consistently upregulated in numerous tumor types $(245,246,264)$. Initially identified as an anti-apoptotic factor in glioblastoma cells, studies show that miR-21 consistently plays an oncogenic roles in a variety of malignancies including those affecting the breast $(265,266)$, CNS (267-269), liver and bile-duct (268,270,271), pancreas (272,273), lung (274-276), head and neck (277), gastrointestinal tract (278,279), hematopoetic and immune systems (280-282), and genitourinary systems $(283,284)$. In these cancers, miR-21 promotes cell proliferation, survival, invasion, migration, and chemoresistance by suppressing negative regulators of many oncogenic pathways including RAS-effector signaling (RAS-ERK and RAS-AKT), JAK-STAT, and the TGF $\beta$ pathways $(154,245-247)$. In turn, the expression of miR-21 can be regulated by the activity of these oncogenic signaling pathways it regulates, suggesting that feed-forward loops maintain high levels of miR-21 in cancer cells and that miR-21 is a major regulator of oncogenic signaling in cancer cells $(154,245-247)$.

\section{Signaling by KLF4 and miRs}

miRs directly regulate the translation of KLF4 in a variety of contexts, including in cancer. miR-145 induces the differentiation of human embryonic stem cells (hESCs) by repressing the translation of KLF4 (285). Furthermore, miR-1 represses KLF4 expression to

promote differentiation of mouse ESCs to smooth muscle cells (286). In an epithelial- 
mesenchymal transition (EMT) context, miR-200c and miR-203, both of which are inducers of epithelial differentiation, were shown to repress KLF4 translation $(287,288)$. In a cancer related context, miR-10b regulates cell invasion and migration by repressing KLF4 expression in human esophageal SCC cell lines (289). Recently, miR-7 was shown to suppress KLF4 translation in a study that analyzed brain metastasis of breast CSCs (140).

Conversely, few studies have documented KLF4 mediated regulation of miRs, and KLF4-miR signaling continues to be charactrerized. One study identified that KLF4 repressed the transcription of miR-146a, which in turn, suppressed the translation of KLF4 (290). Our lab identified miR-206 and miR-344 as two miRs that can regulate the expression of KLF4 (59). miR-206 is termed a "muscle specific miR", indicating its higher expression in this tissue, which mediates skeletal muscle regeneration in response to injury (291-293). In ER $\alpha$-positive breast cancers, miR-206 represses ER $\alpha$ expression, suggesting a tumor suppressive or anti-proliferative role for this miR in luminal subtype tumors (294). Alternatively, miR-206 may induce insensitivity to estrogen analogs in the treatment of ER $\alpha$-positive breast cancers, through the repression of ERa, and thus may not truly function as a tumor suppressor (294-296). Furthermore, in ER-negative breast tumors miR-206 expression is high, and suppression of miR206 activity in breast carcinoma cells mirrors several of the phenotypic alterations that result from KLF4 suppression, suggesting that miR-206 may in fact play an oncogenic role (Chapters 2-3)(297).

Interestingly, we showed that miR-206 could either promote or repress $K L F 4$ translation depending on the cell context. Whereas in normal mammary epithelial cells, miR-206 promotes KLF4 translation, miR-206 represses KLF4 translation in breast carcinoma cells. Furthermore, 
our study identified that miR-206 may be a KLF4-dependent effector in promoting tumorigenic signaling in triple-negative breast cancer (TNBC) cells (chapters 2-3) (59).

\section{THE RAS-ERK/MAPK SIGNALING PATHWAY}

\section{A brief overview of RAS-ERK signaling}

During the course of the evolution, adaptations were required to support intercellular communication in multicellular organisms, and necessitated eukaryotic cells to develop specialized signaling pathways to respond to external stimuli. RAS GTPase proteins are essential components of these pathways that function as binary switches that can serve as transducers of extracellular signals (298-300). RAS-effector pathways predominantly function downstream of cell membrane associated molecules including receptor tyrosine kinases (RTKs), integrins, and ion channels. Signaling downstream of RAS proteins can occur through phosphoinositide 3kinase - protein kinase B/AKT (RAS-PI3K-AKT) pathway and the extracellular signal-regulated kinase/mitogen activated protein kinase (RAS-ERK/MAPK) signaling cascade. RAS-ERK signaling is crucial in the regulation of a variety of cell physiologic processes including meiosis, mitosis, differentiation, motility, and survival (300-306). Aberrant signaling through the RASERK pathway (and RAS-AKT) disrupts tissue homeostasis, and is critical in the development and progression of congenital developmental disorders, and a wide variety of cancers (298300,306-311). 


\section{The discovery of RAS proto-oncogenes and effector signaling pathways}

The identification of $R A S$ genes emerged from the study of potently oncogenic retroviruses (312-315). Through a series of seminal studies, mammalian $R A S$ genes were found to encode GTP binding proteins that are cellular counterparts to the oncogenes encoded by these viruses (316-318). Studies also showed that RAS proteins that were isolated from cancer cells were similar to virally encoded RAS proteins, and could potently induced the transformation of NIH3T3 fibroblasts (319-324). Furthermore, both cancer and retrovirus derived $R A S$ genes were found to differ from their cellular counterparts by a single critical missense mutation (325-327). Interestingly, such alterations in $R A S$ genes were observed in patient tumor samples, indicating that RAS mutations were not artifacts of in vitro cell passaging and suggesting that RAS genes played a major role in tumor biology (328-330). Further studies identified a plethora of virally encoded oncogenes and their cellular counterparts, some of which play major roles as downstream effectors of RAS mediated signaling in human cells (299).

The human genome contains three RAS genes (HRAS, NRAS, and KRAS) that encode four RAS GTPase proteins (HRAS, NRAS, and two alternatively spliced KRAS isoforms: KRAS4A, and KRAS4B) of approximately $21 \mathrm{kDa}$ molecular weight. These proteins are virtually ubiquitously expressed in all human tissues (298-300). These RAS proteins belong to the RAS super family of GTPase proteins that contain RHO, RAN, RAB, and ARF family members, all of which function as binary switches that can alternate between GTP and GDP bound states. Furthermore, the RAS super family is related to heterotrimeric G-proteins that also exhibit GTPase activity. Finally, RAS proteins can localize to many subcellular compartments. Localization to the plasma membrane, as a consequence of extensive post-translational modifications such as acylation of key C-terminal residues (299). Differential regulation of 
localization and other post-translational modifications may serve as the biochemical basis for the non-redundant roles of RAS isoforms that has been documented in several studies $(299,331)$.

\section{Organization of MAPK signaling cascades}

The RAS-ERK pathway belongs to a set multi-tiered signaling cascades that are termed classical (conventional) mitogen activated protein kinase (MAPK) signaling pathways. This set of pathways also incldues the p38, JNK, and ERK5 MAPK cascades (Fig. 8) $(301,332,333)$. Classical MAPK signaling occurs through the sequential activation of multiple components (kinases) that are arranged in a hierarchical order, thus the term signaling cascades. Pathway stimulation ultimately results in the activation of effector kinases, termed MAPKs. In general, the activation of classical MAPKs is regulated by MAPK kinases (MAPKKs or MAP2Ks), which are in turn stimulated by MAPKK kinases (MAPKKKs or MAP3Ks) (301). The MAPK superfamily of signaling pathway also contains atypical MAPKs (ERK 3, ERK4, ERK 7, NLK), which differ from classical MAPKs in structure and regulation (333). However, precise details

on the regulation, function, and substrate specificity of these kinases is currently not well known (333).

A hallmark of classical MAPK signaling is that activation of MAPKs occurs by the phosphorylation of conserved threonine and tyrosine residues in a classical T-X-Y motif (where $\mathrm{X}$ represents any amino acid; T-E-Y in ERK 1/2 MAPKs, T-P-Y in JNK MAPKs, T-G-Y in p38 MAPKs, and T-D-Y in ERK5 MAPK) in the activation loop of these kinases. Phosphorylation at both residues is required for maximal MAPK activity (301). Though the activation of both MAP2Ks and MAPKs is relatively straightforward, the regulation of MAP3K activity is often a complex multi-step process. In RAS-ERK signaling, ERK 1 and ERK 2 (ERK 1/2; MAPK) are 
activated by MEK 1 and MEK 2 (MEK 1/2; MAP2K), which in turn can be stimulated by the RAF family of serine/thereonine kinases (ARAF, BRAF, and CRAF/RAF1) of MAP3Ks. Activation of RAF proteins is mediated by the activity of RAS GTPases through direct interaction of RAS-GTP with RAF (301).

\section{Activation of RAS-ERK signaling}

\section{RAS structure and activation}

As mentioned above, RAS proteins are GTPase enzymes that can function as binary switches by alternating between GTP-bound (active) or GDP-bound (inactive) states. Transition between these states is mediated by guanine nucleotide exchange factors (GEFs), which promote RAS activation (i.e. promote the formation of RAS-GTP), and GTPase activating proteins (GAPs), that quench RAS activity (i.e. promote the formation of RAS-GDP) (Fig. 9) (298300,302,308). The structure of RAS proteins and the regulation of RAS activity by these proteins is discussed in subsequent sections.

RAS-ERK signaling predominantly occurs in response to the activity of receptor molecules (e.g. RTKs) that are located at the cell membrane $(301,334,335)$. GEFs are recruited to sites of receptor activation (generally at the plasma membrane) and stimulate RAS $(299,308)$. As a result, GEF mediated formation of RAS-GTP is thought to be the predominant mode of RAS activation in response to RTK signaling (301,308,334-336). GEFs accelerate GDP release from RAS molecules and catalyze the formation of the GTP bound state of RAS. This process occurs through a series of fast reactions, and the equilibrium of these reactions is shifted to favor the formation of RAS-GTP due to the high intracellular [GTP]/[GDP] ratio (Fig. 10) (308). GEFs interact with RAS proteins at critical regions termed switch I (residues 30-38) and switch II 
(residues 60-76), and orient the phosphate-binding loop (P-loop; residues 10-17) and the $\mathrm{Mg}^{2+}$ binding area $(337,338)$. This perturbation of RAS structure is thought to be the main cause for the decreased affinity between RAS proteins and nucleotides which results in the release of GDP $(299,308,336)$. These conformational changes in RAS structure allow downstream molecules to preferentially interact with active RAS through RAS-binding domains (RBDs) and RASassociation (RA) domains $(299,308,336)$. Transitions between the GTP- and GDP-loaded RAS are described to occur through a "loaded-spring" mechanism, where the release of the terminal phosphate after GTP-hydrolysis allows the RAS switch regions to relax and allow the RAS molecule to adopt an inactive confirmation $(339,340)$.

Conversely, GAPs accelerate the conversion of RAS-GTP to RAS-GDP. GAPs interact with the catalytic site of RAS proteins by inserting an arginine side chain and neutralizing electrostatic charges that develop during the hydrolysis of the $\gamma$-phosphate of GTP. GAPs stabilize the switch II domain and allow a conserved glutamine (Q61) to participate in GTP hydrolysis (Fig. 11) (308). These observations signify the critical requirement of the switch and P-loop regions for both the activation and attenuation of RAS molecules. Furthermore, these structural studies shed light as to how consistent mutation of residues (at positions 12, 13, and 61) that lie in these important domains result in RAS proteins that are virtually insensitive to GAP catalytic activity $(341,342)$.

In addition to the switch and P-loop regions, RAS proteins require extensive processing of the C-terminal plasma membrane interacting hypervariable region (HVR) for proper subcellular localization and activity (343). Firstly, all three RAS proteins contain a key cysteine residue (C185 in KRAS-4A and KRAS-4B; C186 in NRAS and HRAS) which is present in a structure called the CAAX-box and is farnesylated under normal conditions (Fig. 12) (336). The 
addition of the farnesyl group is catalyzed by the enzyme farnesyl-transferase (FT), which attaches the 15-carbon farnesyl group via a stable thioether linkage in an irreversible reaction (Fig. 13) (336,344). Early pharmacological strategies to inhibit RAS signaling exploited this step of RAS processing by targeting (FT). FT-inhibitors largely failed in the treatment of RAS-driven cancer as RAS proteins can undergo alternative processing at the key cysteine residue, including prenylation by geranylgeranyl transferase I (GGTase I) (345). Furthermore, RAS proteins possess additional upstream cysteine residues which can become palmitoylated (346). RAS palmitoylation is reversible and is carried out by palmitoyl transferases. This reversible palmitoylation is thought to be an efficient way of shuttling RAS proteins (NRAS, HRAS, and KRAS4A) between the endoplasmic reticulum/golgi apparatus and the plasma membrane (347,348). In contrast, KRAS4B contains a C-terminal stretch of lysine residues (termed, polybasic lysine stretch), which allows it to interact with acidic phospholipids in the plasma membrane (349). Furthermore, S181 of KRAS4B has been found to be phosphorylated by protein kinase $\mathrm{C}(\mathrm{PKC})$, and this phosphorylation impacts the plasma membrane localization of KRAS4B (350). Secondly, in the endoplasmic reticulum, the last three amino acids of the CAAX box are subject to proteolytic cleavage by RAS-converting enzyme 1 (RCE1) $(351,352)$. Lastly, the key cysteine residue is carboxymethylated by the action of isoprenylcysteine-carboxylmethyltransferase (ICMT) (353). After this last processing step, the fully modified RAS protein consists of a methyl-esterified farnesyl or geranylgeranyl cysteine (Fig. 13) (336). This proper localization is required before GEF mediated activation in response to RTK stimulation.

\section{RAF structure and activation}

RAF family serine/threonine kinases (ARAF, BRAF, and CRAF/RAF1) are MAP3Ks that recognize GTP-RAS through their N-terminal RBD and are thereby recruited to sites of RAS 
activation. RAF proteins are subsequently activated in a complex multi-step process (354-356). Human RAF genes were originally identified as homologs of retrovirally encoded oncogenes, and the name RAF corresponds to the rapidly accelerated fibrosarcoma that was observed upon infection with these viruses (357-360). Each RAF family kinase shares three conserved regions (CR1, 2, and 3) and a cysteine rich domain (CRD), which are critical in regulating RAF activation (Fig. 14) (355). At the N-terminus is CR1, which contains the RBD and therefore is indispensible for proper RAS-RAF interaction. The CRD is located adjacent to CR1 and may function as a secondary site for the interaction with RAS. CR2 contains important regulatory phosphorylation sites that allow the adaptor protein 14-3-3 to bind and inhibit RAF activation. Finally the C-terminal CR3 contains the kinase domain, including the activation loop, which must be phosphorylated for kinase activity (354-356).

Briefly, RAF activation involves the following steps (all residues correspond to positions on CRAF/RAF1) (Fig. 15) (354-356).

1. Binding to RAS via the RBD.

2. Dephosphorylation of a critical residue (S259) by a protein phosphatase within CR2 to release the inhibitory 14-3-3 adaptor protein.

3. Phosphorylation of residues in the negative charge region (N-region; S338 and Y341) upstream of CR3 and on the activation segment (T491 and S494). Phosphorylation of the Nregion residues is critical for maximal RAF activity and interaction with MEK 1/2. It is not conclusively known which kinases phosphorylate S338 and Y341 (and their analogous residues on ARAF and BRAF). Interestingly, the residue corresponding to Y341 on BRAF (Y446) is constitutively phosphorylated, suggesting that BRAF is primed for activation.

4. Homo- or heterodimerization with another RAF molecule. 
Deactivation of CRAF occurs by specific dephosphorylation of S338 by protein phosphatase 5 (PP5). The kinase responsible for the rephosphorylation of S259 has not yet been conclusively determined (356).

\section{Activation of MEK 1/2 and ERK 1/2}

RAF kinases have restricted substrate specificity and catalyze the phosphorylation on two critical residues in the activation loop of the MAP2Ks: MEK 1 and MEK 2 (MEK 1/2) (355,361363). MEK $1 / 2$ phosphorylation by RAF occurs with the help of the scaffolding protein kinase suppressor of RAS (KSR). Phosphorylation of these sites (site 1: S218/S222 and site 2: S222/S226 on MEK1/MEK2 respectively) by RAF is indispensible for MEK $1 / 2$ activity $(361,364)$.

MEK $1 / 2$ are dual specificity kinases that phosphorylate both threonine and tyrosine residues $(363,365,366)$. Activated (phoshphorylated) MEK 1/2 can in turn phosphorylate a key threonine and tyrosine residue on the activation segment on the MAPKs, ERK 1 and ERK 2 (ERK 1/2) (367,368). These residues compose the MAPK hallmark T-X-Y motif: T(202/185)-EY(204/187) on ERK 1/2 respectively (368). Activated (phosphorylated) ERK 1/2 can localize to may subcellular compartments including the nucleus and phosphorylate and consequently activate its many targets to elicit many of the observed RAS-ERK phenotypes $(366,369)$.

\section{Substrates of ERK 1/2}

RAS-ERK signaling promotes various cell physiologic process including cell proliferation, motility, protein synthesis, and survival through the activation of ERK 1/2 substrates (366,369). Over 200 ERK 1/2 substrates have been identified across numerous subcellular compartments (369). Furthermore, RAS-ERK signaling can be directed to many of 
these subcellular compartments through the action of scaffolding and adaptor proteins (discussed in the next section). Upon RAS-ERK pathway stimulation, ERK $1 / 2$ can translocate to the nucleus (370-372). The nuclear effects of ERK 1/2 are executed by a large number of substrates, that include the transcription factors cMYC, cFOS, ELK, and ETS (373-375). Furthermore, ERK 1/2 can activate transcription by ER $\alpha$ (376), PPAR $\gamma(377), \operatorname{RXR} \alpha$ (378), and the glucocorticoid receptor (GR) (379). Many of these transcription factors promote the transcription of immediate early genes (IEGs) and are indispensible in promoting RAS-ERK dependent phenotypes such as proliferation, protein synthesis, and survival (380). Furthermore, ERK 1/2 phosphorylate and activate ribosomal s6 family kinases (RSKs) and MAPK interacting kinases (MNKs), which belong to the family of MAPK activated protein kinases (MAPKAPKs) that directly promote protein synthesis by upregulating mRNA translation (381). Finally, active ERK 1/2 can directly phosphorylate pathway components such as the RASGEF SOS, cRAF, and MEK1 to inhibit pathway activity $(335,366,369,382,382-384)$. Thus, ERK $1 / 2$ serve as the effector kinases of the RAS-ERK signaling pathway.

\section{Regulation of RAS-ERK pathway activity}

The importance of RAS-ERK signaling in the regulation of major cell physiologic processes requires its pathway activity to be tightly regulated. Spatiotemporal regulation of RASERK signaling is required to achieve specificity in responding to each of the diverse stimuli that activate this pathway (Fig. 16) (385). Studies that analyzed nerve growth factor (NGF) and epidermal growth factor (EGF) stimulation in PC12 rat pheochromocytoma cells indicate that both duration and magnitude of pathway activity determine the cellular response to stimuli $(334,335,386,387)$. To this end, numerous mechanisms that act at various hierarchical levels of RAS-ERK signaling are required for proper modulation of pathway activity. These mechanisms 
include the regulation of pathway activity by the action of GAPs, docking, adaptor, and scaffolding proteins, direct antagonists/negative modulators and protein phosphatases $(299,308,363,366,369,385,388-390)$. Furthermore the RAS-ERK activity can be controlled by ERK 1/2 dependent feedback phosphorylation of pathway components (334).

\section{GTPase Activating Proteins (GAPs)}

GTPase activating proteins promote the formation of inactive RAS (i.e. GDP-RAS) by catalyzing GTP hydrolysis (Figs. 9 and 11) (299,308,388,390). RAS proteins are GTPase enzymes, however they possess a low intrinsic GTPase catalytic activity (391). Strikingly, mutated RAS proteins derived from oncogenic retroviruses and cancer cells, which almost always differ from their non-mutated (wild-type) counterparts by alterations at positions including 12, 13, or 61, were found to display even lower intrinsic GTPase activities. Indeed, GAPs were found to be necessary for efficient and timely inactivation of wild-type RAS proteins by accelerating GTP hydrolysis of RAS proteins by up to 300 fold (391). In contrast, mutated RAS proteins are virtually resistant to the action of GAPs (392-395).

The biochemical basis for GAP-mediated GTP hydrolysis was uncovered by a seminal crystallographic and molecular modeling study that analyzed the structure of the GAP catalytic domain of p120 RASGAP (RASA1) interacting with RAS (342). This study showed that GAPs function by associating with the switch I region of RAS proteins, and by positioning a critical arginine residue (termed the arginine finger) proximal to the Q61 residue of RAS that coordinates the water molecule necessary for GTP hydrolysis (Fig. 11). This arginine finger neutralizes the negative charge from the terminal phosphate group of GTP and stabilizes the transition state of the hydrolysis reaction (342). Furthermore, the glycine residues at positions 12 
and 13 were found to be critical for GAP mediated GTP hydrolysis as well, as mutations of these residues sterically blocked the position of the GAP-arginine finger. Thus, this study explains the structural basis for the GAP resistance of mutated (at positions 12, 13, or 61) RAS proteins.

The first RAS GAP to be identified was p120 RASGAP (RASA1) (396-398). Concurrent studies identified a second GAP, neurofibromin 1 (NF1), the mutation of which was uncovered to be the causative lesion of Neurofibromatosis type I (399-401). Subsequently, additional RAS GAP genes have identified in the human genome (402).

Analysis of both RASA1 and NF1 suggested distinct non-overlapping functions for both GAPs (403-405). These studies indicated that whereas, NF1 activity affects basal (serum starved) RAS activity, RASA1 was responsible for deactivation of RAS following serum stimulation. Though NF1 was found to possess a higher affinity for GTP-RAS than RASA1, RASA1 possessed a much greater GAP catalytic activity (403-405). Congenital defects in RASA1 and NF1 produce distinct phenotypes, further suggesting diverse roles for GAPs. Additionally, these results may be a consequence of the structure dissimilarity of these two proteins as NF1 and RASA1 share little homology besides their GAP catalytic domains (390).

\section{Adaptor, docking, and scaffolding proteins}

Proper interaction of the core components of the RAS-ERK pathways is required to achieve signaling efficiency and specificity (Fig. 16) (385). To this end, three classes of proteins participate in the spatiotemporal regulation of signal duration, magnitude, and localization to subcellular compartments. Adaptor proteins are recruited to sites of RTK activation and serve to functionally link required signaling components to these sites (385). These proteins contain domains that can recognize various motifs that are present on RTKs and their immediate 
downstream effectors such as GEFs and GAPs. These domains include (but are not limited to) SRC homology 2 (SH2), which recognize phosphotyrosine residues, SRC homology 3 (SH3), which interact with proline rich sequences, and general phosphotyrosine binding (PTB) domains (385). One example of an adaptor protein critical to RTK-RAS signaling is GRB2. The GRB2 protein structure consists of an SH2 domain flanked by two SH3 domains (406). Upon activation, the cytoplasmic tyrosine residues of RTKs are phosphorylated and serve as recognition sites for GRB2 binding. GRB2 can then recruit the RAS GEF, SOS, through its Nterminal SH3 domain. The C-terminal SH3 domain can interact with docking proteins, which function to tether adaptor proteins to the plasma membranes $(385,406,407)$.

Scaffolding proteins play critical roles in the regulation of ERK/MAPK activity (385). These proteins act as platforms that physically coordinate core components of RAS-ERK signaling and facilitate efficient signaling. Furthermore, scaffolding proteins allow signaling specificity by insulating RAS-ERK components from interacting with undesired substrates.

The kinase suppressor of RAS (KSR) protein is a mutli-domain scaffold that facilitates ERK activation at the plasma membrane. Much like RAF proteins, KSR is sequestered from the plasma membrane by the inhibitory action of 14-3-3 adaptor proteins. KSR is constitutively associated with MEK 1/2, casein kinase 2 (CK2), and protein phosphatase 2 catalytic subunit A (PP2A) (408-410) Furthermore, in quiescent cells KSR associates with the E3 ubiquitin ligase impeding mitogenic signal propagation (IMP) $(408,409)$. Upon RTK stimulation, KSR translocates the plasma membrane and associates with RAS-RAF complexes. Through the activity of the regulatory subunit of the protein phosphatase PP2 (PP2B) at the plasma membrane on a regulatory phosphorylation site, KSR is freed from the inhibitory 14-3-3 (411). Finally, 
KSR associated CK2 promotes RAF activation, and RAF-MEK 1/2-ERK 1/2 signaling proceeds (408-412).

Additional scaffolding proteins that are critical in spatiotemporal regulation of RAS-ERK signaling include MEK partner 1 (MP1) $(413,414)$, which directs ERK signaling to late endosomes, Paxillin (415), a critical component of focal adhesions, and hSef (human honmolog of the drosophila gene Sef: similar expression to fgf genes) (416), which targets this signaling to the golgi apparatus.

\section{Negative regulators/modulators of RAS-ERK signaling}

Modulators that specifically antagonize RAS-ERK signaling by interfering proteinprotein interactions have been identified and include Erbin, RAF kinase inhibitory protein (RKIP), Sprouty (SPRY), and the Sprouty related EVH1 domain containing (SPRED) family of signaling regulators (Fig. 16). Erbin is a member of the LAP (leucine rich-repeat domain and PDZ [post synaptic density protein] domain containing) family that inhibits RAS-RAF interaction (417). RKIP interacts with the kinase domain of both MEK 1/2 and RAF proteins and inhibits signaling through these molecules (418).

The Drosophila Sprouty protein was identified in studies that analyzed developmental branching morphogenesis of tracheal and endothelial tubes (419). Sprouty was found to inhibit RAS-ERK pathway activation downstream of stimulation of numerous RTKs. Subsequently, four human sprouty (SPRY) proteins were identified (420). The protein structure of SPRY proteins is poorly characterized, but all family members contain a conserved cysteine-rich domain (CRD) and a critical tyrosine residue (Y55 on SPRY2) that is phosphorylated in response to RTK activation (421). Studies suggest that SPRY proteins interact with the protein 
phosphatase PP2A and the E3 ubiquitin ligase cbl, but how these interactions function to antagonize RAS-ERK signaling is unclear (422-425). SPRY1 and SPRY2 have been documented to interact and sequester GRB2/SOS complexes in a context dependent manner (421). Furthermore, SPRY proteins are induced in response to acute RAS-ERK signaling in an AP-1dependent manner, and thus may participate in a negative feedback. However, the precise molecular mechanisms of the action of SPRY proteins remain to be elucidated (422-425).

Sprouty related EVH1 containing (SPRED) proteins were identified as inhibitors of RASERK signaling that contain an N-terminal enabled (Ena) domain and a vasodilator-stimulated phosphoprotein (VASP) [EVH1] domain, a centrally located cKIT binding domain, and a Cterminal CRD reminiscent of SPRY proteins $(389,426)$. Studies identified four mammalian SPRED genes (SPRED1-3, and EVE3, an alternative splice isoform of SPRED3) (426). Of proteins in this family, SPRED1 remains the best characterized member. Studies identified that SPRED1 interacts with RAS-RAF complexes and specifically inhibit signaling downstream of RAF (426). Furthermore, SPRED1 can associate with caveolin-rich microdomains in the plasma membrane, and inhibit RAS-ERK signaling at these locations (427). Consequences of SPRED1mediated RAS-ERK signaling include inhibition of IL-3 mediated ERK activation in hematopoetic cells and inhibition of Rho-mediated cell contractility (428-430). A recent study described a role for SPRED1 in the plasma membrane localization of NF1, indicating that NF1 is a catalytic partner for SPRED1 mediated inhibition of RAS-ERK signaling (431). Taken together, these results suggest that SPRED1 may inhibit RAS-ERK signaling at more than one hierarchical level (422-425). 


\section{MAPK phosphatases}

The dephosphorylation of activated MAPKs by protein phosphatases plays a major role in regulating the duration and magnitude of signaling $(366,432)$. Removal of one phosphate from either of the two phosphorylated residues on ERK 1/2 results in deactivation of the kinase (433). Three major classes of protein phosphatases regulate RAS-ERK activity. These include tyrosine specific MAPK phosphatases (MKPs), consisting of PTP-SL (protein tyrosine phosphatase, receptor type R [PTPRR]), STEP (protein tyrosine phosphatase, non-receptor type 5 [PTPN5]), and HePTP (protein tyrosine phosphatase, non-receptor type 7 [PTPN7]); serine/threonine phosphatases, including PP2A and PP2C; and dual specificity phosphatases (DUSPs), that are composed of ten family members $(366,432)$. DUSPs can be classified into three groups based on their subcellular localization. DUSP 1, 2, 4, and 5 are predominantly found in the cytoplasm; DUSP 6, 7 and 9 are predominantly found in the nucleus; and DUSP 8, 10 and 16 can be found in both the cytoplasm and the nucleus. DUSP 1, 2, 4, 5, 6, 7, 8, and 9 possess phosphatase activity towards ERK 1/2 and are apparent negative regulators of RAS-ERK signaling $(366,432)$.

\section{ERK 1/2 dependent feedback regulation of RAS-ERK pathway activity}

Given the critical roles that RAS-ERK signaling plays in numerous cell physiological processes, the maintenance of pathway activity within acceptable ranges is required. Dynamic regulation of RAS-ERK activity, as well as the activation of upstream regulators such as RTKs is mediated by ERK 1/2 dependent feedback mechanisms (334,335). Activated ERK $1 / 2$ (phosphoERK 1/2 - pERK 1/2) can phosphorylate positive regulators of the RAS-ERK cascade and consequently suppress the activity of these proteins. Direct phosphorylation of SOS by ERK 1/2 impairs SOS interaction with GRB2, reduces SOS recruitment to the plasma membrane, and 
attenuates RAS activation (383). Furthermore, ERK 1/2 phosphorylates RAF which leads to the reduction of RAF kinase activity and a subsequent decrease in MEK 1/2 activation $(384,434)$. pERK 1/2 can also further inhibit MEK1 activity, but not that of MEK2 through direct phosphorylation at T292 of MEK1 (435,436). Activated ERK 1/2 can also phosphorylate docking proteins. RAS-ERK activation leads to GAB1 phosphorylation and reduces its ability to recruit PI3-K and activate the AKT pathway $(437,438)$. ERK 1/2 similarly regulates signaling downstream of insulin and FGF receptors by phosphorylating IRS1 and FRS2 docking proteins in response to receptor activation $(439,440)$. Finally, ERK $1 / 2$ can directly phosphorylate RTKs, such as EGFR, and may be involved in controlling the molecular mechanisms which mediate RTK internalization and degradation and diminish the activity of upstream pathways (441-443).

As an additional mode of pathway regulation, ERK 1/2 is also involved in more indirect feedback mechanisms which involve signal dependent transcription of inhibitors of RAS-ERK pathway activity. Often, this type of ERK dependent pathway activity regulation involves the stabilization and activation of certain transcription factors $(335,444)$. For example, cMYC protein is transiently stabilized by ERK 1/2 phosphorylation at a critical serine residue (S62) (445). An acute reduction in ERK activation following pharmacological MEK 1/2 inhibition in numerous contexts results in loss of cMYC mediated transcriptional activity $(436,445)$. Thus, ERK 1/2 dependent factors such as cMYC serve as "sensors" of RAS-ERK signaling that can modulate pathway activity at a transcriptional level. In summary, these ERK dependent feedback loops are critical in maintenance of RAS-ERK signaling homeostasis, and disruption of this regulation can lead to rapid reactivation of signaling (436). 


\section{The roles of RAS-ERK signaling in human disease}

The precise control of RAS-ERK signaling is critical to regulate cell physiologic processes. Whereas proper signaling through this pathway allows cells to proliferate, differentiate, migrate, and survive in an appropriate manner so as to maintain tissue homeostasis, aberrant signaling results in the development and progression of human disease. Particularly, inappropriate activation of this signaling through alteration of pathway components is critical in the pathogenesis of a spectrum of developmental disorders and a wide variety of cancers.

\section{RAS-ERK signaling in developmental disorders}

Germline mutations in RAS-ERK pathway components results in increased pathway activity and in hereditary familial congenital disorders that feature defects in the development of multiple organ systems including the cardiovascular, CNS, immune, musculoskeletal, and integumentary systems $(309,311,446)$. Though the resulting developmental disorders arise from distinct genetic lesions, these syndromes nonetheless share overlapping phenotypic features, indicating that regulated RAS-ERK signaling is required for proper development. Collectively, these disorders are termed RASopathies and/or cardiofaciocutaneous spectrum of developmental disorders $(309,311,446)$.

Neurofibromatosis type I (NF1) is an autosomal dominant disorder that arises from either genetic deletions or inactivating mutations in the $N F 1$ gene $(309,311,446)$. Neurofibromatosis I was the first identified congenital RASopathy, and patients with NF1 present with neurofibromas, multiple café-au-lait spots, axillary/inguinal freckling, osseous lesions, and optic nerve tumors $(447,448)$. Furthermore, patients with NF1 have reduced life expectancy, as they have an increased likelihood of developing gliomas, astrocytomas, leukemias, and 
neurofibrosarcomas (449). Though the cells of NF1 patients display increased RAS-ERK activity, the NF1 phenotype in $N f 1^{-/}$mouse embryos is only partially restored by re-expressing the GAP catalytic domain of the Nf1 protein, supporting the potential for GAP-independent roles of NF1 in development (390).

A similar disorder to NF1, NF1-like syndrome (Leguis syndrome), results from mutations in the SPRED1 gene (310). Germline mutations of RASA1, result in congenital capillary malformation - arteriovenous malformations (CM-AVMs) and predispose patients to developing CNS tumors $(450,451)$. Alterations of the protein phosphatase PTPN11 (SHP2) gene produce two similar syndromes: LEOPARD syndrome and Noonan syndrome (452,453). Additional RASopathies include Costello syndrome, which is caused by HRAS mutations (454), and cardiofaciocutaneous (CFC) syndromes which can be produced by germline $B R A F, M E K 1$, or MEK2 mutations $(455,456)$.

\section{The role of RAS-ERK signaling in cancer}

Early studies that identified human $R A S$ genes as counterparts to viral encoded oncogenes forged the link between RAS signaling and cancer biology. Subsequent studies uncovered that the dysregulation of RAS-ERK signaling plays a pivotal role in the pathogenesis of a variety of cancers $(298-300,302,306,457,458)$. Somatic alterations in RAS-ERK pathway components are a major cause of the aberrant pathway activation observed in cancers.

Mutational activation of $R A S$ genes can occur at positions 12,13 , or 61 , and confers resistance to GAP-mediated GTP hydrolysis (298-300,302,306,308,457,458). Such activating point mutations are found in $20-30 \%$ of all human cancers and represent one of the most common genetic alterations in cancers. These mutations drive sustained RAS-ERK signaling and 
are critical in the pathobiology of numerous cancers. Among activating $R A S$ mutations, KRAS mutations are the most common ( $85 \%$ of all cancers), followed by $N R A S(12 \%)$, and $H R A S(3 \%)$ (300). Furthermore, most cancers seem to display preference for the mutation of one RAS isoform over the others. Whereas KRAS mutations are found most commonly in pancreatic $(57 \%)$, biliary $(31 \%)$, colorectal $(33 \%)$, and non-small cell lung $(17 \%)$ cancers, HRAS is most commmonly mutated in urinary bladder $(11 \%)$ and salivary gland (15\%) cancers, and NRAS most commonly mutated in melanomas (18\%) and hematopoetic malignancies (11\%) (300). Furthermore, specific activating mutations are found to be preferentially enriched in each mutated RAS isoform. Though the G12D mutation is most common in KRAS (85\%) and HRAS (55\%), the Q61L mutation occurs most commonly occurs in NRAS (62\%) (300). These results warrant further studies to understand the seemingly nonredundant roles of each RAS isoform.

The second most common cause of inappropriate activation of RAS-ERK signaling in cancers is by activating point mutations in $B R A F$, which occur in roughly $7 \%$ of all human cancers (459). Mutation at position 600 (V600E) of BRAF results in constitutive activation of the kinase and promotes signaling through the ERK pathway. BRAF mutations are most commonly found in malignant melanoma and papillary thyroid cancers (459).

Additional genetic alterations contribute to the activation of RAS-ERK signaling in cancers. Gene amplification and/or mutation of RTKs, cognate ligands, and RAS-ERK pathway components are frequently observed in various cancers and drive inappropriate RAS signaling $(334,335,460,461)$. Furthermore, the loss/mutational inactivation of RAS-ERK inhibitory molecules can lead to the enhanced RAS-ERK signaling as well as is seen with somatic NF1 loss various CNS tumors and the inactivation of other GAP proteins in liver cancers $(449,462)$. 
Additional mechanisms could activate RAS-ERK signaling in cancers. It is possible that diverse oncogenic signaling pathways may converge on RAS-ERK signaling and produce the pathway activation that is seen in numerous cancers, including in breast carcinoma. The mechanisms of RAS-ERK activation in breast cancer are currently not well understood $(463,464)$. The characteristic activating $R A S$ and $R A F$ mutations that are prevalent in other cancers occur only infrequently in breast cancer (465). Thus, identifying how RAS-ERK signaling may be upregulated in breast cancer could yield new targeted therapeutic strategies.

\section{Therapeutic inhibition of RAS-ERK signaling}

Given the important role of RAS-ERK signaling in development and progression of many cancers, the successful therapeutic inhibition of this signaling has been a long standing goal of the targeted chemotherapy era $(298,300,302,336,466)$. Numerous strategies targeting the various hierarchical tiers of this pathway have been employed to block RAS-ERK signaling but these strategies have only displayed limited success (467). These strategies have been developed in parallel with agents targeting upstream drivers of RAS-ERK and PI3-K-AKT signaling, such as RTKs $(334,335)$. However, the use of agents targeting RAS-ERK signaling or upstream input pathways as single agents has largely failed due to the rapid emergence of cellular resistance, usually through adaptive reprogramming of cellular signaling (467). Nonetheless, the development and the effective use of RAS-ERK inhibitory agents holds great promise for the treatment of cancers (and developmental disorders) that rely on aberrant activation of this signaling. These therapeutic strategies can be grouped into three broad categories: those that directly target RAS, modulate factors that regulate RAS activity, and those that target downstream kinases (e.g. RAF, MEK, and ERK). 


\section{Strategies to target mutant RAS proteins:}

Pharmacological targeting of RAS proteins has been attempted using a variety of means. Several of these methods have attempted to selectively inhibit the activity of mutated (activated) RAS proteins. Since RAS proteins are intracellular enzymes, agents targeting these molecules need to be cell membrane permeable, thus rendering small molecule agents desirable. The majority of small molecule therapeutic agents function by blocking the activity, and often the active site, of the intendend target. Unfortunatley, a loss of RAS GTPase function, including a loss of sensitivity to GAP proteins, leads to a phenotypic gain function (increased signaling activity). Thus, a small molecule that blocked the active site of RAS would not function to reduce RAS signaling, and may likely be oncogenic $(336,466)$. Instead, pharmacological agents would need to restore the GTPase function of RAS protein and the sensitivity of these proteins to GAPs. However, structural studies have indicated that it may not be feasible to design small molecule agents to rescue either of these two functions in mutant RAS proteins $(341,342)$.

As the loss of GAP inhibitory action leads to an increased proportion of RAS-GTP molecules, the development of GTP competitive molecules was pursued. This work was encouraged by the successful development of potent ATP competitive small molecule kinase inhibitors (468). Unfortunately, this approach is not promising for targeting RAS molecules due to kinetics of RAS:GTP interaction, which is favored by the high affinity for GTP by RAS proteins (pM [picomolar] range) and the high intracellular concentration of GTP $(\mu \mathrm{M}$ [micromolar] range) (469).

Therapeutic strategies have also tried to exploit RAS processing which involves a series of enzyme catalyzed reactions directed at the -CAAX motif (Figs. 12 and 13). As mentioned 
previously, -CAAX processing is critical for the proper subcellular localization of RAS proteins. The first step of this reaction is the prenylation of a critical cysteine residue of the -CAAX box by the enzyme farnesyl transferase (FT) (470). Preclinical studies indicated that farnesyl transferase inhibitors (FTIs) could be efficacious in the treatment of RAS-mutant cancers (471). However, clinical trials of two FTIs (Tipfarinib and Lonafarnib) in lung, colorectal, and pancreatic cancers showed that these agents did not possess the anticipated anti-tumor activity $(472,473)$.

Several factors have been proposed for the failure of these agents in vivo. Importantly, in preclinical trials, the efficacy of FTIs was only consistently observed in HRAS-mutant cancers, and the development of these agents hinged on the assumption that all NRAS-and KRAS-driven cancers would be susceptible to FTIs (474-476). Furthermore, in vitro studies suggest that NRAS and KRAS are susbtrates for alternative prenylation, i.e. geranylgeranylation, at this critical cysteine residue by the enzyme geranylgeranyltransferase I (GGTase I) (477-479). This alternative modification may enable these RAS isoforms to bypass farnesylation and allow protein maturation and localization to occur. Strategies to target the pool of prenylation substrates in addition to targeting specific prenylation enzymes have been considered, including combinatorial inhibition of HMG CoA reductase (using statins) and/or FT-inhibition or GGTase inhibition (480-486). Whether these combinatiorial approaches will yield clinical benefit is still to be seen.

\section{Strategies targeting proteins that regulate RAS activity}

The RAS-GTP interaction allows RAS proteins to interact with downstream targets and drive oncogenic signaling. Though the development of pharmacological strategies to disrupt 
protein-protein interactions is in its infancy, studies utilizing antibody fragments specific to RAS-effector interactions have demonstrated the potential value of this strategy (487). Small molecule agents against the HRAS-cRAF interaction show inhibition of multiple RAS driven pathways in vitro and in vivo and are orally active against KRAS mutation positive colon carcinoma xenografts (488). Agents have also been developed to disrupt the SOS-RAS interaction and abrogate GTP-GDP exchange (489). More direct strategies of modulating SOS action are currently being investigated as well $(490,491)$. How this approach will translate to the benefit of patients afflicted with RAS driven cancers is yet to be seen.

Though there has been a consistent and concerted effort to develop mutant RAS specific therapeutic strategies, the rationale for focusing solely on mutant RAS may need to be reevaluated. Recently, the critical role of wild-type RAS signaling in RAS-mutant cells has been uncovered (331). Studies have shown that mutant RAS proteins rely upon the wild-type (RASWT) counterparts to endow cells with oncogenic signaling and phenotypes (492-494). Analysis of RAS and SOS proteins in RAS-mutant cells indicate that the total ratio of [RAS-GTP]/[RASGDP] may determine the output from RAS proteins regardless of mutation status. In RASmutant and RAS-WT cells alike, wild-type RAS proteins are dependent on the action of GEFs and GAPs, and the activity of WT-RAS proteins may contribute a significant proportion of the signal through downstream pathways (492-494). Indeed, concordant effects are observed when GAP proteins are modulated in RAS-mutant cells, with GAP activity suppression implicated in increased WT-RAS-GTP and RAS-ERK signaling in WT- and mutant-RAS cells alike (Chapter 2) (80). Therefore, the development of RAS inhibitory strategies, rather than mutant-RAS specific strategies, may ultimately benefit RAS-driven cancers. 


\section{Strategies that target downstream pathways (RAF-MEK-ERK):}

Unlike for RAS-GTPases, the development of small molecule agents targeting protein kinases has yielded potent ATP-competitive and allosteric inhibitors (468). These kinase inhibitory strategies provide an excellent experimental tool for studying cell signaling and their effective use in a clinical setting holds great promise for the treatment of cancers. Inhibitors of downstream pathways include agents that target RAF (vemurafinib, dabrafenib, etc.) $(495,496)$, MEK 1/2 (selumetinib; AZD6244, trametinib, etc.) (497-499), and ERK 1/2 (FR180204) (500). However, in contrast to the potent action of these compounds in vitro, therapeutic resistance emerges rapidly and hampers the successful use of these agents as single agents $(436,467,501-$ 504). Acute loss of RAS-ERK signaling results in adaptive changes in the activation of the kinome (436). Pathway inhibition also disrupts ERK 1/2 dependent negative feedback regulation of positive pathway regulators including MEK1 and BRAF $(383,384,434)$. Furthermore, long term treatment with RAS-ERK inhibitory compounds results in tumor cells acquiring somatic mutations in NRAS, MEK2, or AKT1 to counteract sustained inhibited signaling (505-507). Finally, the induction of multiple RTKs and the activation of alternative signaling pathways (e.g., PI3-K-AKT) can compensate for the inhibited RAS-ERK signaling (436,507-510). Either singly or in combination, these adaptive changes ultimately circumvent blocked signaling and promote therapeutic resistance.

Care must be taken in using RAF inhibitors, as therapy with these agents results in the paradoxical activation of RAS-ERK signaling. Three seminal studies have investigated this phenomenon. First, it was found that BRAF inhibition in NRAS-mutant/BRAF-WT melanoma cells induces the formation of BRAF-CRAF heterodimers leading to increased downstream ERK phosphorylation (511). A second study analyzing ATP-competitive RAF inhibitors supported the 
critical role of BRAF-CRAF heterodimers in inducing ERK activation in cells containing wildtype RAF (512). In addition, this study found that the formation of CRAF-CRAF homodimers was also mediated the induction in MEK 1/2 and ERK 1/2 phosphorylation upon RAF-inhibition. Furthermore, this study found that RAS was required for MEK-ERK activation upon RAFinhibition. Surprisingly, BRAF inhibitors were nonetheless effectively inhibit RAS-ERK signaling in cells containing activated BRAF (BRAF-V600E). A third study supported the importance of RAF dimerization (including all RAF isoforms) in mediating resistance to RAFinhibitors, and found that drug treatment increased the kinase activity of all RAF isoforms in RAF-wild-type cells (513). Collectively, these studies highlight the critical role for WT-RAF proteins in mediating resistance to RAS-ERK inhibitory strategies, however, the reason why RAF-inhibitors are efficacious in BRAF-mutant cells is still not clear.

\section{The role of RAS-ERK signaling in breast cancer}

\section{A brief overview of breast cancer}

Breast cancer consists of a heterogenous set of malignancies that can arise from the lactiferous ducts and lobules of the mammary gland (514-516). Breast ducts are lined with columnar epithelium (luminal cells) supported by a layer of basal epithelial and myoepithelial cells. The majority of breast cancers are of ductal origin, with these ductal carcinomas comprising approximately $85 \%$ of all cases (514-516).

Breast cancers can be classified based on the expression of hormonal markers ER $\alpha$, PR, and HER2 gene amplification (by immunohistostaining [IHS] and/or fluorescence in situ hybridization [FISH]) or by the analysis of gene expression. mRNA expression profiling classifies breast cancer into molecular subtypes consisting of the luminal A and luminal B, HER2 
enriched, basal-like, and claudin-low categories (514-518). Initial analyses identified an additional breast cancer molecular subtype that resembled the normal breast (514). However, subsequent studies have indicated that the samples that were clustered in this subtype might have been contaminated with noncancerous breast tissue $(515,516)$. There is considerable overlap between breast cancers classified using either method. ER $\alpha /$ PR-positive cancers often display a luminal A or luminal B gene expression profile, and HER2-amplified cancers display HER2enriched profile (516). A third group that display ER $\alpha / \mathrm{PR} / H E R 2$-negativity (15\% of all breast cancers) are termed triple-negative breast cancers (TNBCs) and are majorly comprised of the basal-like (BL) and claudin-low (CL) molecular subtypes. BL and CL compose approximately $80 \%$ and $15 \%$ of all TNBCs respectively $(516,518-521)$.

Subtyping by the expression of hormonal markers identifies breast cancers that are candidates for targeted neoadjuvant hormone based therapies such as anti-estrogen therapies for ER $\alpha$-positive cancers, and Herceptin/trastuzumab for HER2-amplified breast cancers. Furthermore, the distinct breast cancer subtypes identified by either method also feature significant differences in prognosis $(514,516,517)$. Whereas, luminal A cancers, which are often candidates for anti-estrogen therapies, have the best prognosis, HER2-amplified and BL cancers display poor prognoses $(514,516,517)$.

\section{RAS-ERK signaling in breast cancer}

Though numerous studies that analyzed ERK signaling in breast cancer cell culture models suggest a general protumorigenic role for this pathway, early studies that analyzed patient specimens did not correlate poor survival with increased RAS-ERK signaling in breast cancer $(463,464)$. Nevertheless, the upregulation of ERK $1 / 2$ activity and phosphorylation of its 
substrates is consistently identified in breast cancer specimens compared to adjacent uninvolved mammary tissue $(522,523)$. Interestingly, the activation of ERK signaling in breast cancers occurs despite the low frequency of $R A S$ and $R A F$ mutations in breast cancers, suggesting that alternative mechanisms play a critical role in pathway regulation $(307,465)$. However, in contrast to early studies that correlated ERK $1 / 2$ activation with lymph node-positivity and tamoxifen resistance, and suggested that RAS-ERK signaling could impact disease outcome and metastasis (524), some studies associated the increased activation of ERK 1/2 with a good prognosis $(525,526)$.

Indeed, comprehensive analyses of breast tumors reveal that though genetic alterations that favor increased RAS signaling occur in a variety of breast tumor subtypes, TNBCs, especially basal-like breast cancers, display gene expression profiles that mirror RAS-ERK activation, and genomic alterations including copy number gain of $K R A S(32 \%)$ and $B R A F$ (30\%), and reduced gene copy number for pathway inhibitors such as RASA1 and DUSP4 (515,519,527-533). Furthermore, TNBC cells display an increased sensitivity to MEK 1/2 inhibition compared to breast cancer cells of other subtypes and basal-like breast cancers display an activated RAS-ERK signaling signature, suggesting a critical role for RAS-ERK signaling in these cancers $(528,529)$. Currently, clinical trials of MEK 1/2 inhibitors in combination with other agents are underway for the treatment of TNBCs $(336,436,534,535)$.

In general, TNBCs display a poor prognosis compared to hormone receptor positive breast cancers $(514,515,517,521)$. The treatment of TNBCs relies majorly on cytotoxic chemotherapy, as the lack of expression of hormonal markers in these cancers, precludes treatment with currently established targeted therapies (536-538). The enrichment of genetic alterations that favor increased RAS-ERK signaling and the critical role of this pathway in 
TNBC make pharmacologic targeting of this pathway an attractive therapeutic strategy. However, targeting this pathway is challenging due to rapid development of resistance to pathway inhibition, which can occur through dynamic reprogramming of the kinome, leading to compensatory upregulation of the RAS-ERK signaling pathway and other RAS-effector pathways $(306,436)$. Identification of additional mechanisms of pathway upregulation may lead to the development of therapeutic strategies that could effectively inhibit this pathway, and improve the outcomes in TNBC. 


\section{QUESTIONS ADDRESSED IN THIS DISSERTATION}

KLF4 is a critical factor that maintains tissue homeostasis and plays context dependent roles in a multitude of pathological states. KLF4 dependent phenotypes arise from its ability to promote epithelial differentiation and act a stress response factor. However, how this transcription factor functions in cancer cells, especially in breast cancer, remains unclear. Analysis of KLF4 signaling may help to better understand cancer cell signaling and to identify new therapeutic approaches. This dissertation addresses how KLF4 can exert an oncogenic role in breast cancer cells and identifies one of the first detailed mechanisms for promotion of tumorigenic signaling by this pluripotency factor.

The first study (chapter 2) identifies two miRs, miR-206 and the oncogenic miR-21, as KLF4-dependent effectors in regulating RAS-ERK signaling (80). miR-206 and miR-21 cooperate to suppress the translation of RASA1 and SPREDI, and promote RAS-ERK signaling in both RAS-mutant and RAS-wild-type TNBC cells. We identify RASA1 and SPRED1 as limiting factors for RAS-ERK pathway activity in breast cancer cells. Whereas other tumor suppressors may be permanently inactivated by genetic alteration, the translation of these two pathway inhibitors is repressed as a consequence of miR-206/21. Thus, reactivation of RASA1 and SPRED1 through anti-miR-206/21 therapy is now a possibility. This study also identified a novel role for GAP proteins in regulating RAS-ERK signaling in RAS-mutant cells by impacting the activity of wild-type RAS proteins. The role of GAPs and WT RAS proteins as targets of therapeutic anti-miRs as proposed in this study is consistent with seminal articles describing critical roles for wild-type RAS proteins as effectors of pathway activity in RAS-mutant cancer cells $(493,494)$. 
The second study (chapter 3) describes the role of KLF4-miR-206 signaling in promoting TNBC cell survival, anoikis resistance, and chemoresistance. This study also identifies the wellestablished tumor suppressor PDCD4 as a miR-206 targeted transcript. Interestingly, PDCD4 has been reported to be a negative regulator of RAS-ERK-AP1 signaling, thereby correlating the observations of this study with the results presented in chapter 2.

The final study (chapter 4) presented in this dissertation identifies the role of KLF4 in promoting resistance to MEK 1/2 inhibition in TNBC cells. RAS-ERK pathway inhibition results in adaptive reprogramming of cell signaling which includes the induction of multiple RTKs. These changes to cell signaling result in the reemergence of RAS-ERK signaling, though the precise mechanism of how this happens is not currently known. For example, it is not known if the predominant driver of ERK $1 / 2$ activation is through the derepression of ERK 1/2 mediated feedback loops or the increased RTK-RAS signaling. Also it is not known whether other RTK dependent pathways are activated when these receptors are induced.

In this study, KLF4 was found to allow cells to escape MEK 1/2 inhibition by promoting the expression of PDGFR $\beta$, an RTK that has been functionally implicated in mediating resistance to RAS-ERK pathway inhibitory therapies. However, both KLF4 and PDGFR $\beta$ were dispensible for the partial restoration of RAS-ERK signaling. Instead, KLF4 promoted steady state RASERK signaling, and this effect was attributed to the regulation of GAPs (Chapter 2). Furthermore, KLF4 opposed cMYC mediated repression of PDGFRB transcription to regulate the expression of this RTK. This study provides an example of the concerted action of two pluripotency factors, KLF4 and cMYC, in a non-IPS/non-ES context. 
Finally, the summary (chapter 5) offers a discussion of the implications of these findings and options for future directions. Topics of discussion within this chapter include: the therapeutic potential for anti-miR-206/21 in the treatment of TNBCs and the effective use of small molecule inhibition of RAS-ERK signaling in the treatment of cancers. 


\section{FIGURES}

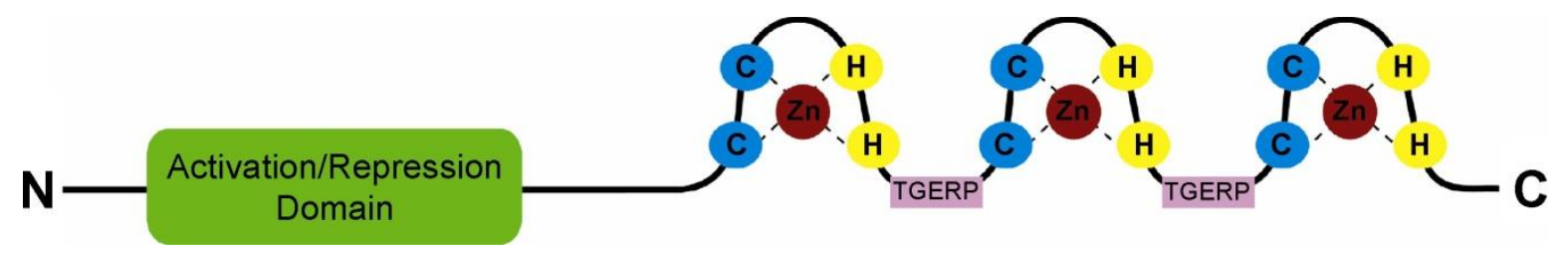

Figure 1: Schematic representation of Krüppel-like factor 4 (KLF4). Adapted from Pearson R., et al., Int. J. Biochem.Cell.Biol, 2008 (539) 


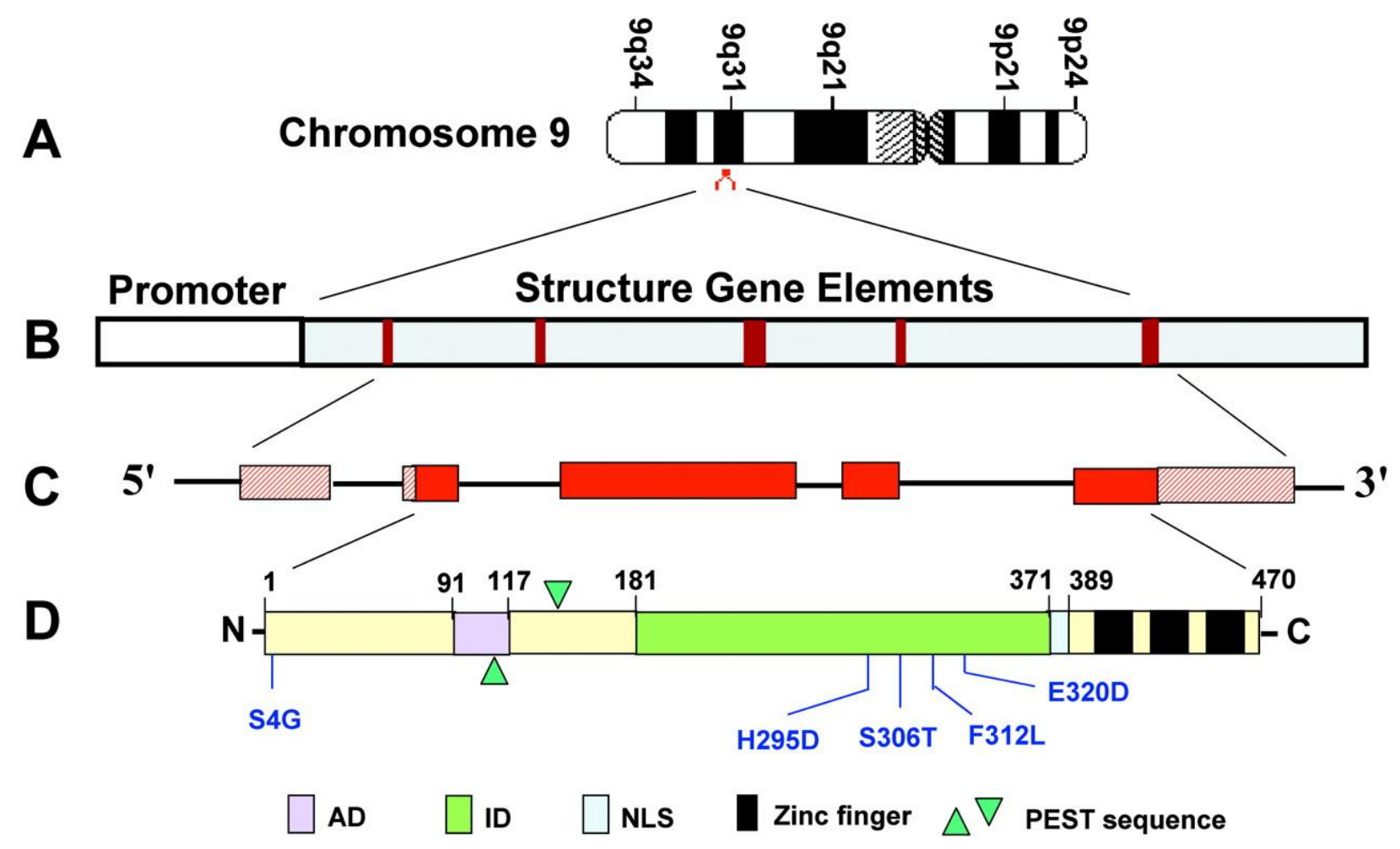

Figure 2: Structural organization of human KLF4: (A) Location of the KLF4 gene on chromosome 9q31; (B) Schematic of the KLF4 gene. The position of exons are indicated in red; (C) Schematic of the primary KLF4 mRNA transcript. The ORF is indicated in dark red; (D) Diagram of the KLF4 protein showing relative positions of the activating domain (AD), inhibitory/repressor domain (ID), nuclear localization signal (NLS), zinc-fingers, and the PEST sequence. The indicated mutations have been identified in tumor cells. Adapted from Wei D., et al., Carcinogenesis, 2006 (540). 

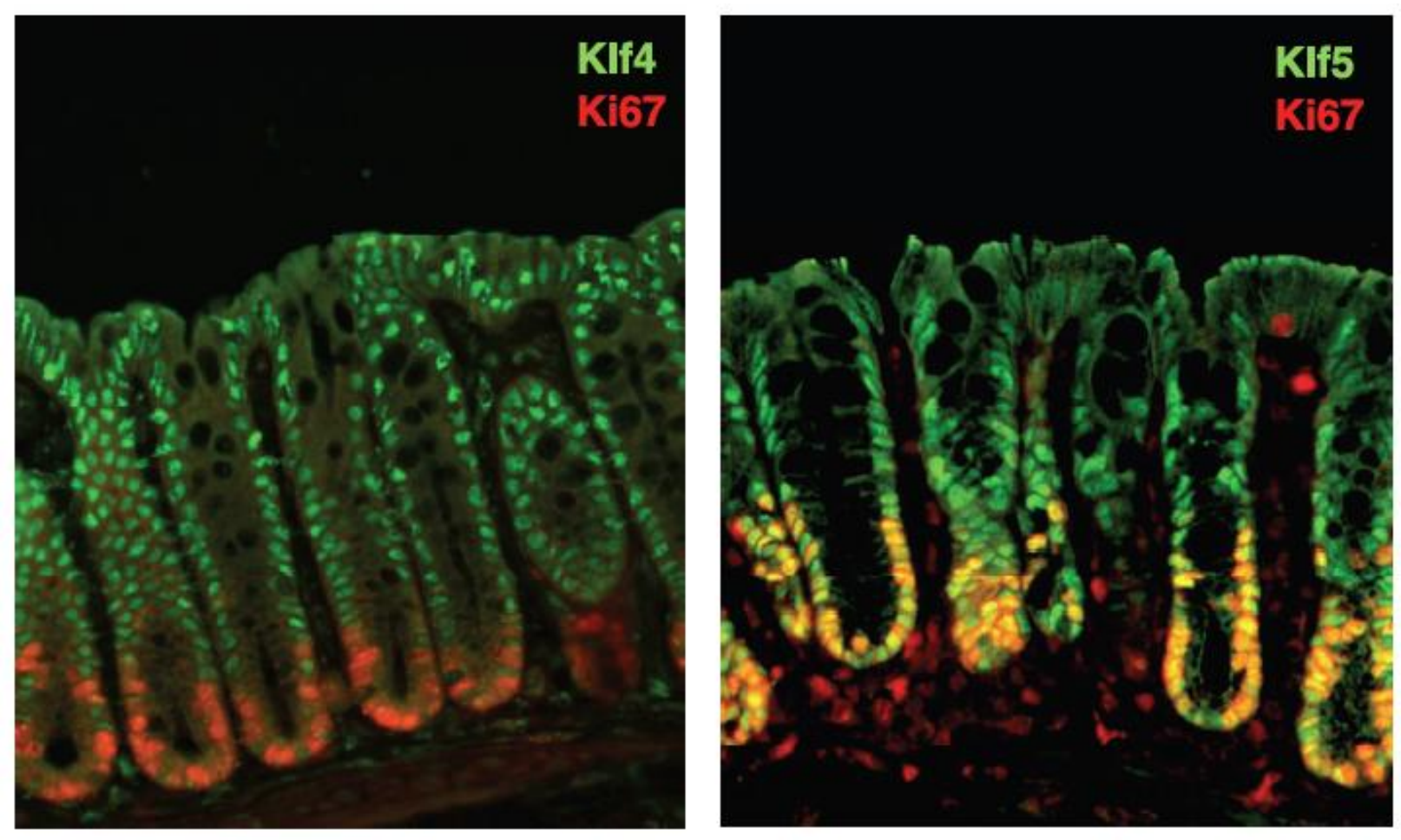

Figure 3: Localization of Klf4 (left; green) and Klf5 (right; green) in mouse colonic epithelium as visualized by immunoflourescence staining. Staining for the proliferation marker Ki67 is shown (left and right; red). Adapted from McConnell B.B., et al, Physiol. Reviews, 2010 (12). 


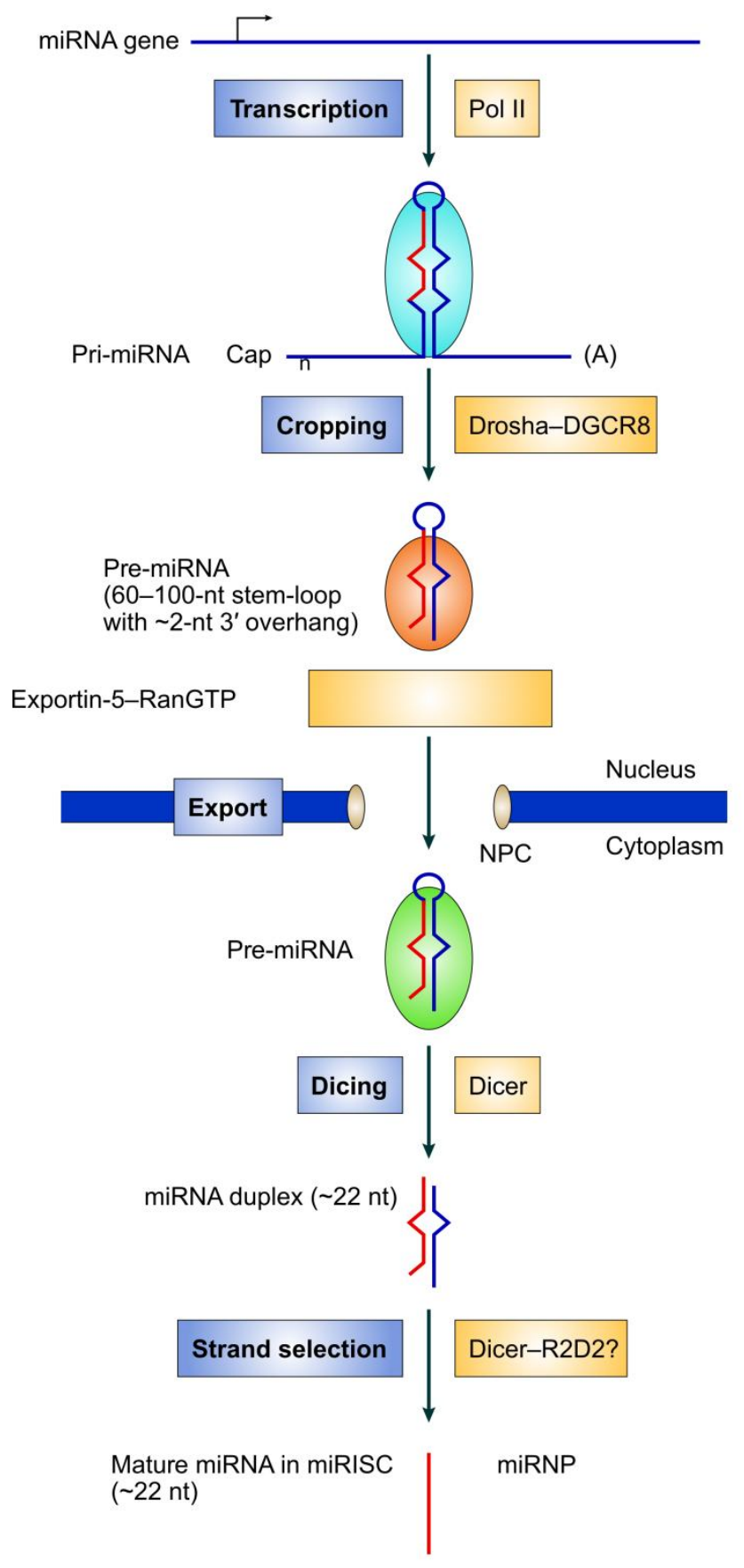

Figure 4: Canonical miR biogenesis. Adapted from Kim, V.N., Nat. Rev. Mol. Cell Biol., 2005 (156) 


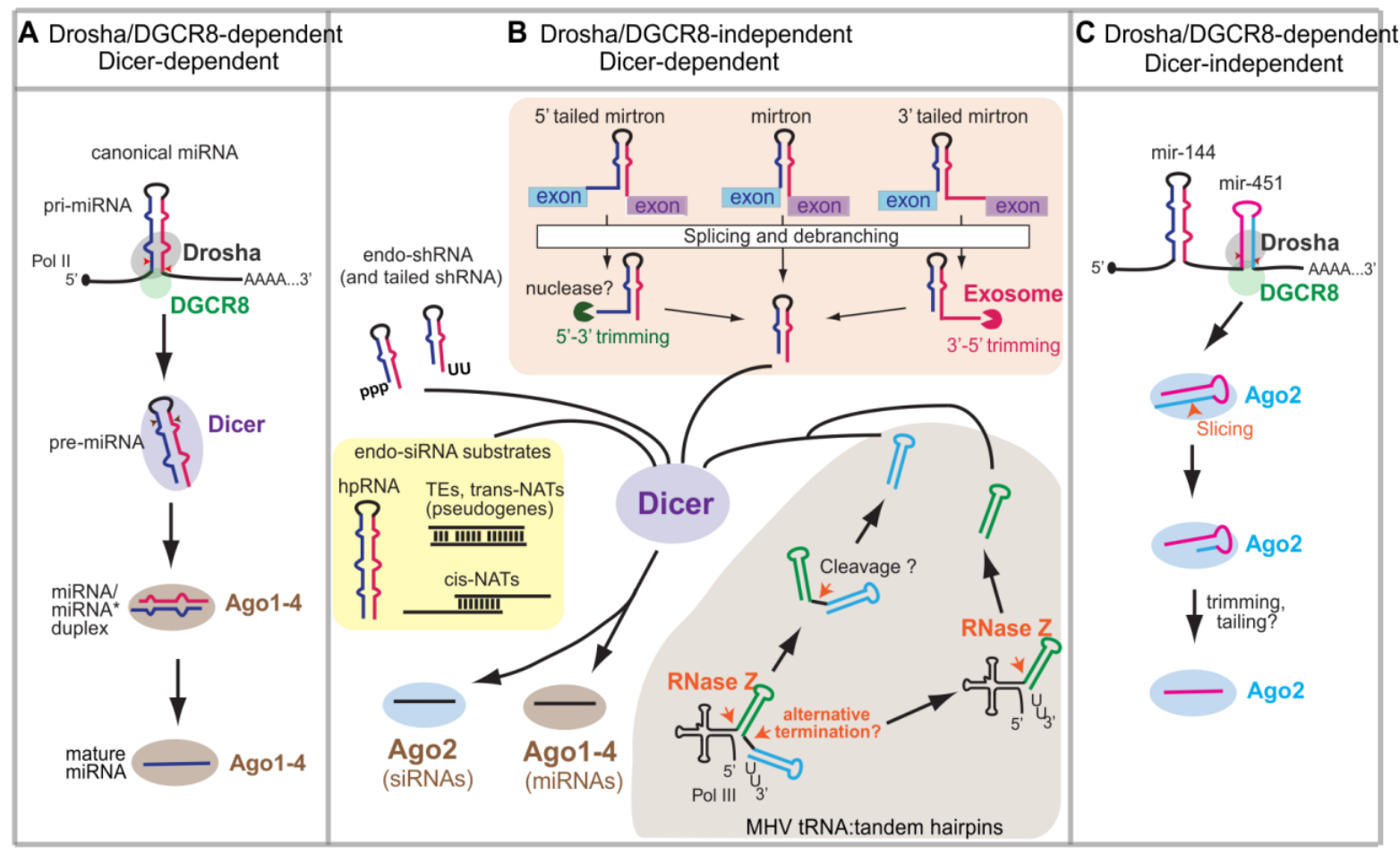

Figure 5: Schematic of alternative miR biosynthetic pathways. (A) Canonical Drosha/DGCR8 and Dicer dependent miR synthesis; (B) miRtron derived miR synthesis: Drosha/DGCR8independent and Dicer-dependent miR synthesis; (C) AGO dependent miR synthesis: Drosha/DGCR8-dependent, Dicer-independent. Adapted from Yang, J-S. et al., Mol. Cell, 2011 (181). 

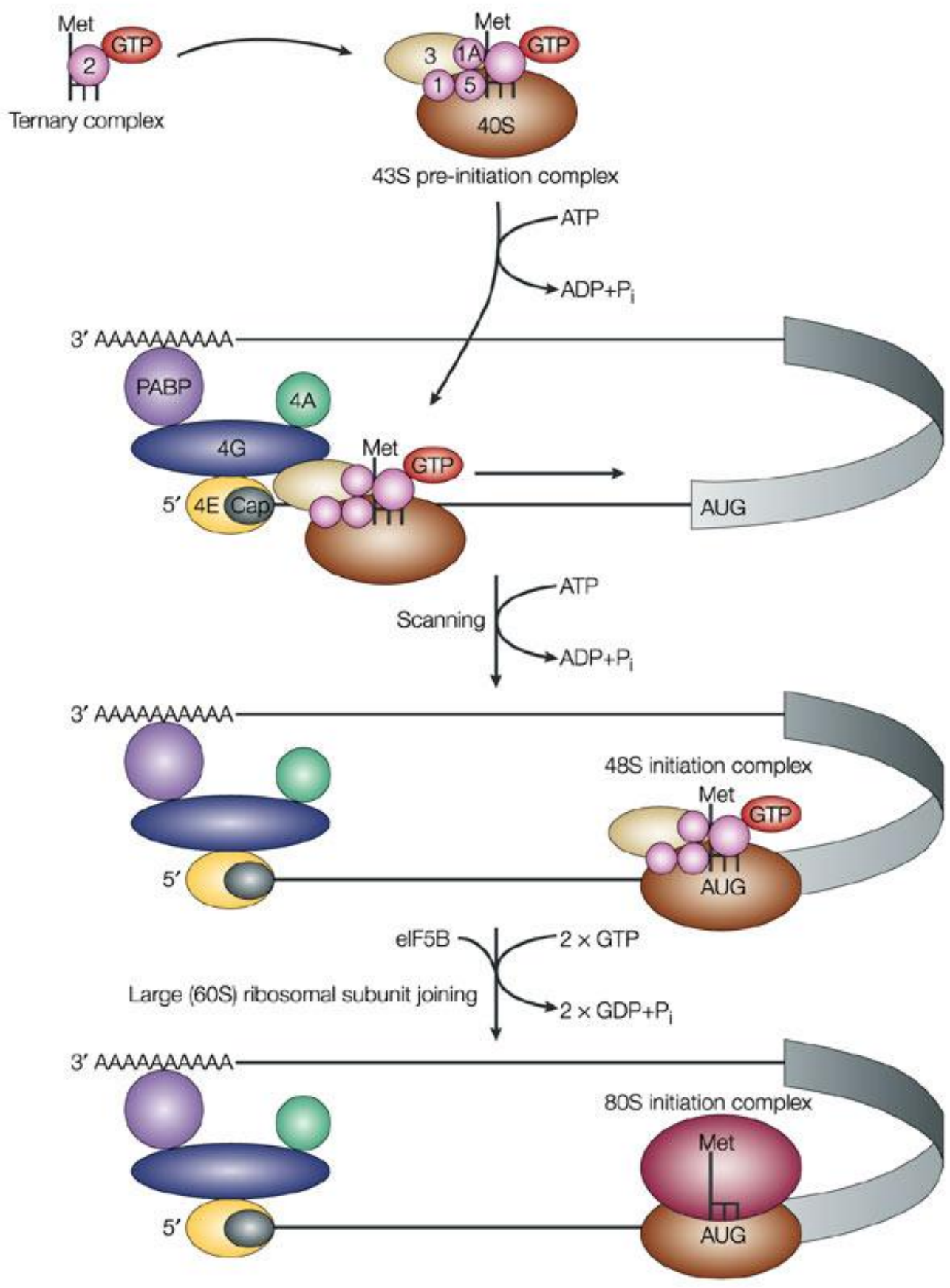

Nature Reviews | Molecular Cell Biology

Figure 6: Cap-dependent translation initiation. Adapted from Gebauer, F. et al., Nat.Rev.Mol.Cell Biol. 5, 827-835 (185) 
a Endonucleolytic cleavage

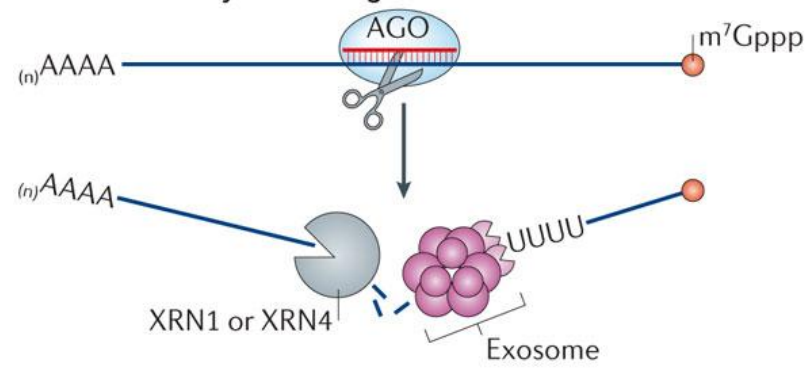

c Translational repression
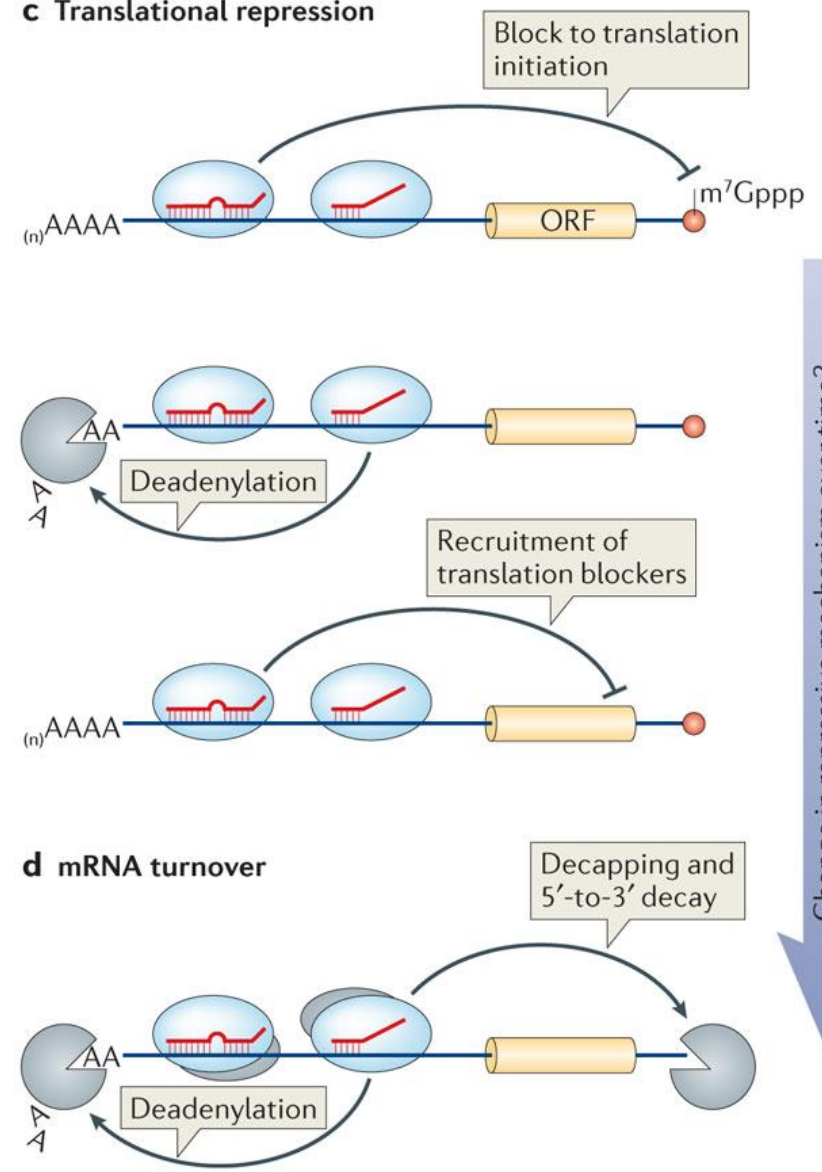

b

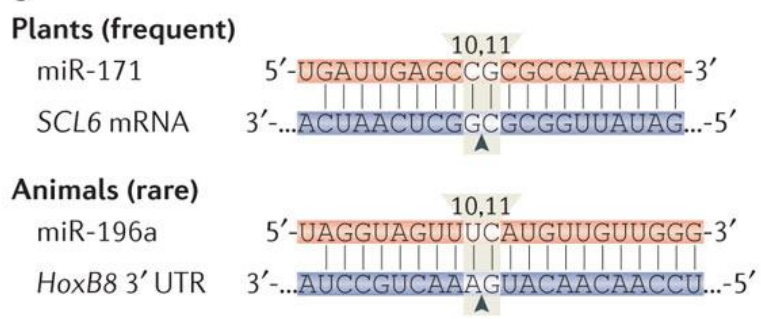

e

Plants (rare?)

miR-172a/c

SCL6 mRNA

5'-AGAAUCUUGAUGAUGCUGCAU-3'

$3^{\prime}$-...UCUUAGGACUACUACGACGUC...-5'

Animals (canonical seed match site; most frequent)

$$
\begin{aligned}
& \text { lin-4 } \\
& \text { 5' } 2 \text {-UCCUGAGACCUCAAGUGUGA-3' } \\
& \text { lin-14 3' UTR } \\
& \text { 3'-..AGGGACUC }
\end{aligned}
$$

Animals (G-bulge site; less frequent?)

$$
\begin{array}{lc}
2 & 8 \\
\text { miR-124 } & \text { 5'-UAAGG-CACGCGGUGAAUGCC-3' } \\
\text { Mink1 3' UTR } & \text { 3'-...GUUCC } \text { SUUG }_{\text {AUGUAACUCUUU...-5' }}
\end{array}
$$

Animals (3' supplementary site; less frequent?)

$$
\begin{aligned}
& \text { miR-2 5'-UAUCACA GCCA } 1316 \text { GCUUUGA GGAGC-3' } \\
& \text { grim 3' UTR 3'-...UUAGUGU UACGCGAAACU }{ }_{\text {AACUC...-5' }}
\end{aligned}
$$

Animals ( $3^{\prime}$ compensatory site; rare)

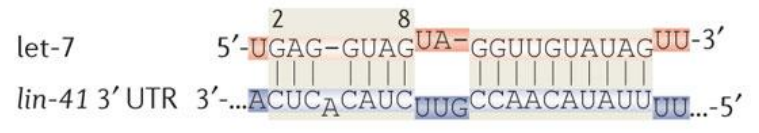

Figure 7: Mechanisms of miR function in plants and animals. (A and B) Perfect complementarity of miR sequence to mRNA target results in endonucleocytic cleavage of target transcripts (occurs most commonly in plant cells); (C-E) In animal cells, translation repression and mRNA turnover are the most common mechanisms of miR-mediated gene repression. Adapted from Ameres, S.L. et al., Nat.Rev.Mol.Cell Biol., 2013 (185). 


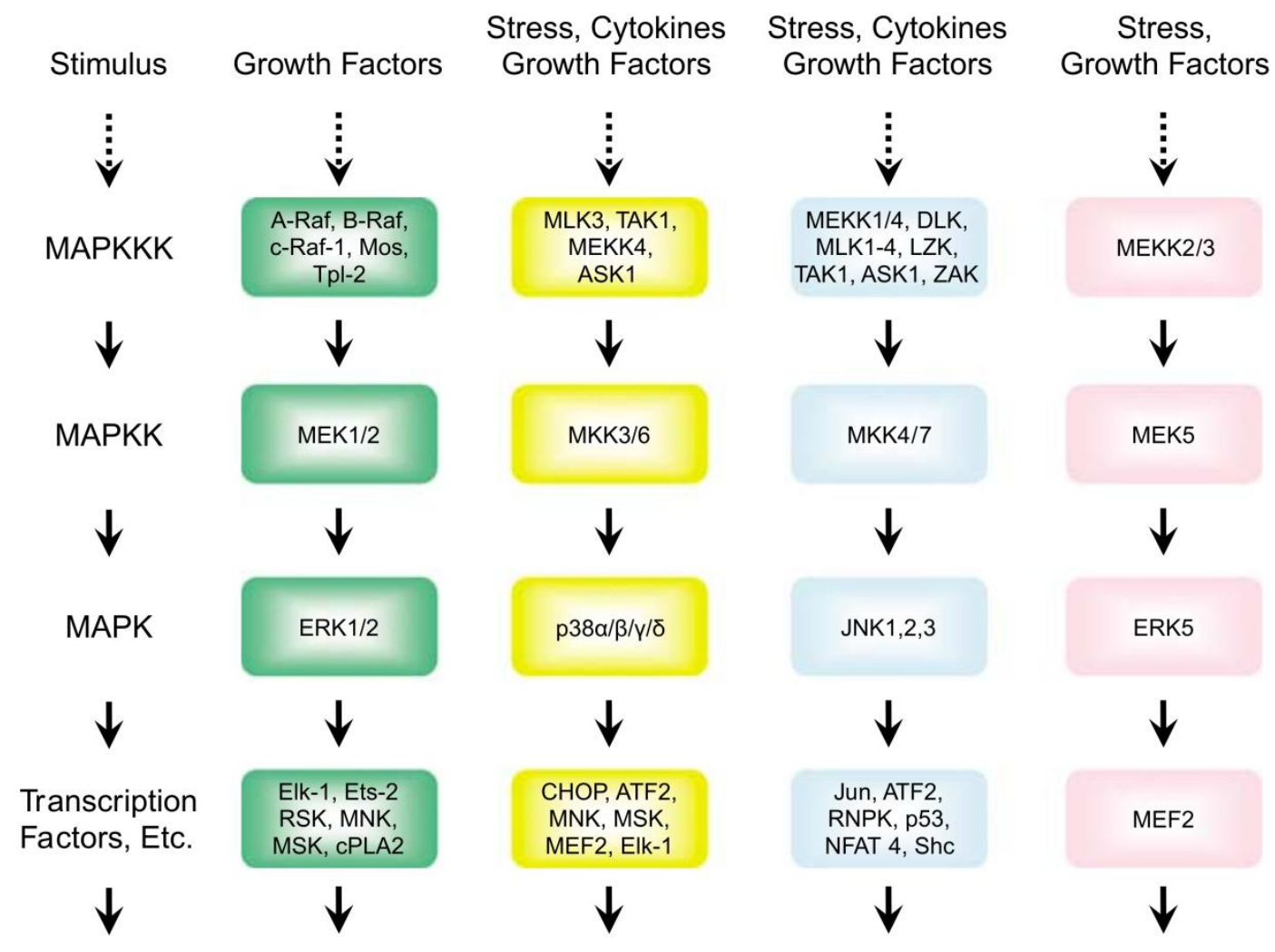

Figure 8: The general structure of mammalian MAPK pathways. Adapted from Roberts, P.J. and Der, C.J., Oncogene, 2007 (458) 


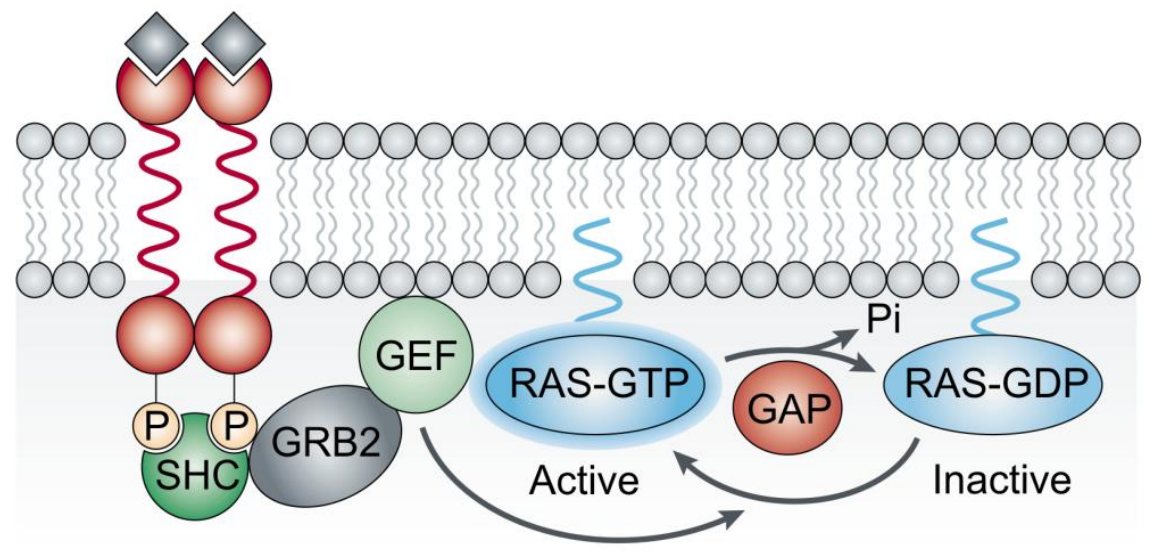

Figure 9: RAS activation at the plasma membrane in response to RTK stimulation. Activation of RAS (formation of RAS-GTP) occurs through the action of guanine nucleotide exchange factors (GEFs). The deactivation of RAS occurs by the action of GTPase activating proteins (GEFs). Adapted from Downward, J., Nat Rev Cancer, 2003 (302). 


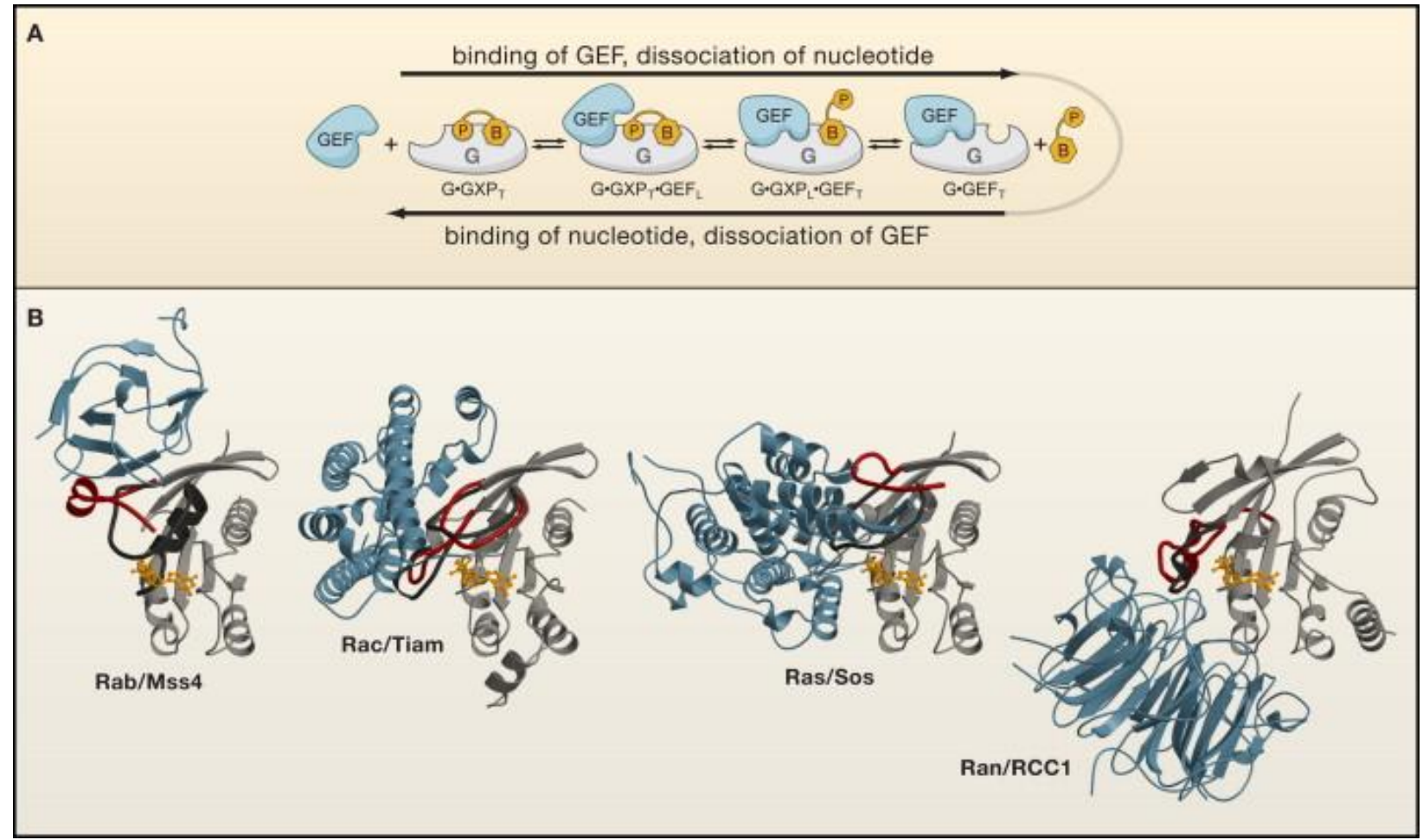

Figure 10: Mechanism of GEF mediated catalysis (A) The guanine nucleotide exchange reach occurs in successive reversible steps. GEFs induce conformational changes in the GTPase and facilitate nucleotide binding. (B) Ribbon structures of numerous GEF-GTPase:nucleotide complexes. Despite their common GEF functions, GEFs are structurally unrelated. Adapted from Bos, J. L., Cell, 2007 (308) 


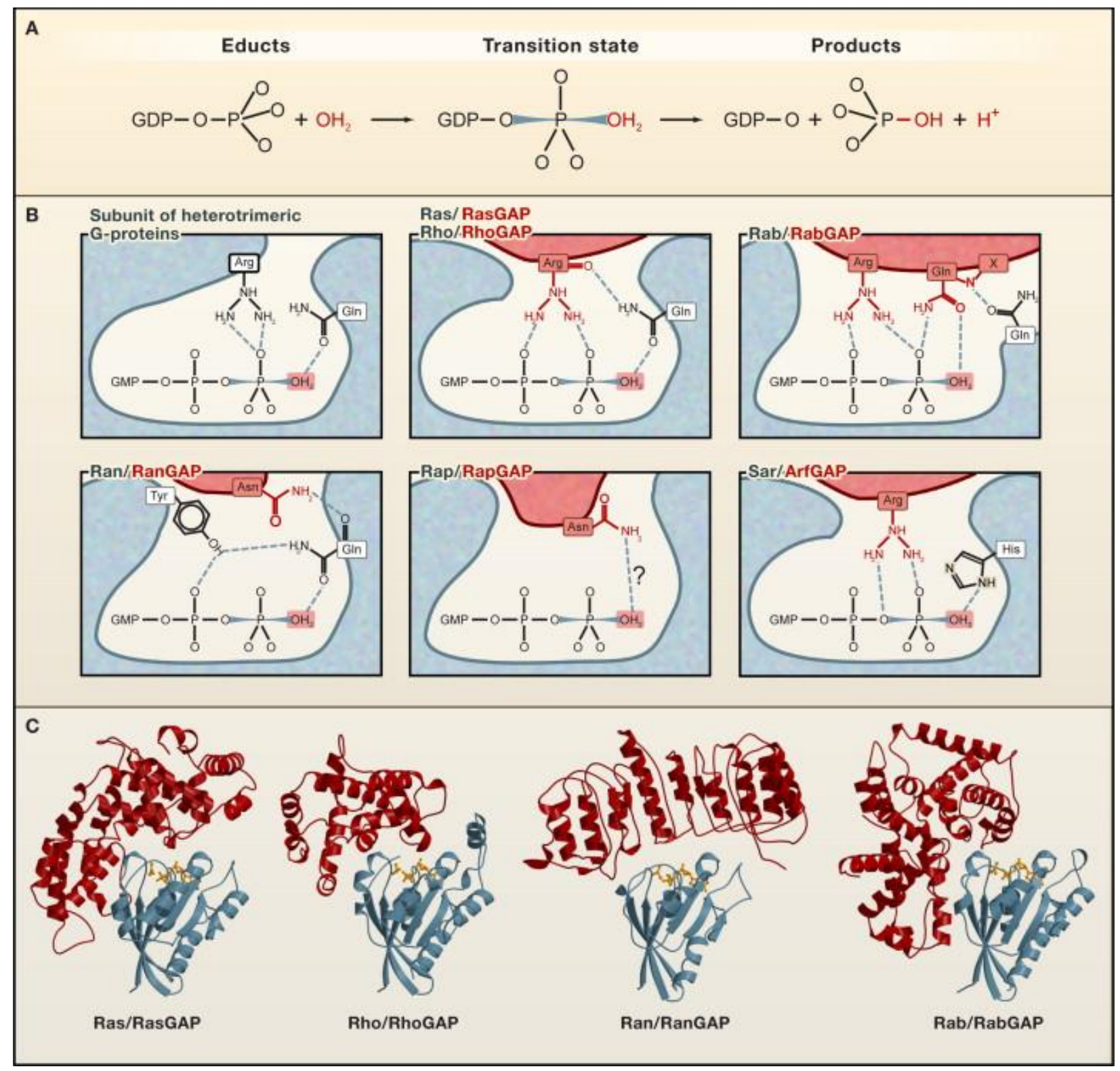

Figure 11: Mechanism of GAP mediated GTP hydrolysis. (A) Schematic representation of GTP hydrolysis. (B) GAP mediated catalysis occurs through distinct mechanisms. Critical residues involved in catalysis are shown (Red: GAP residue, White: GTPase residue). (C) Despite their common GAP catalytic functions, GAPs are structurally unrelated. Adapted from Bos, J. L., Cell, 2007 (308) 


\section{C-termini of Ras proteins}

HVR linker domain minimal lipid anchor

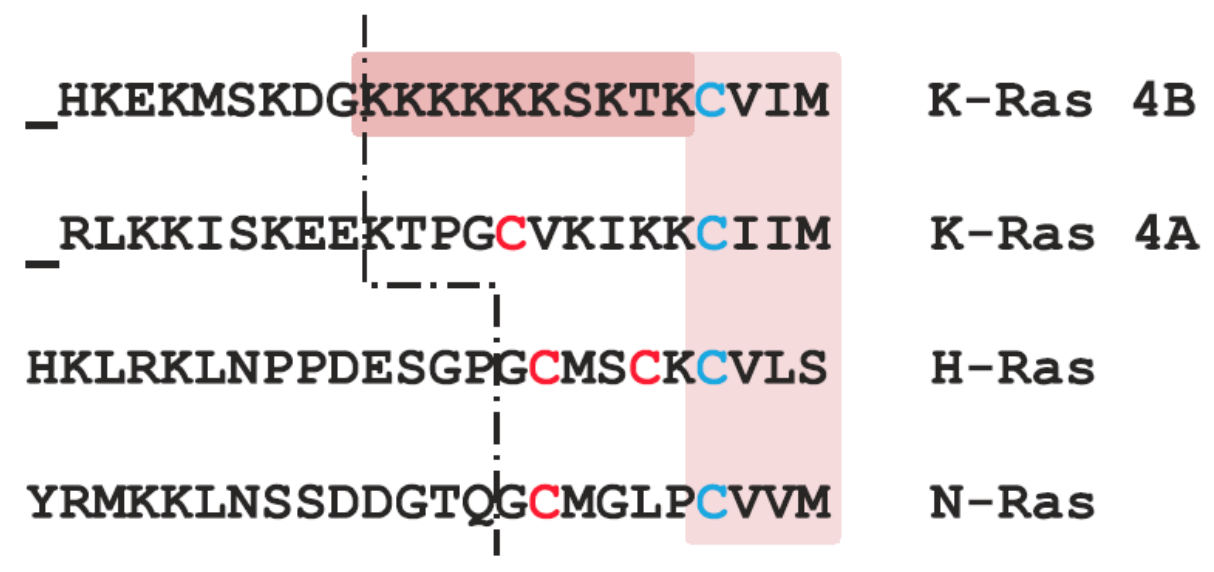

Polylysine motif

C : farnesylated Cys

CAAX box

C : palmitoylated Cys

Figure 12: The C-terminal regions of human RAS isoforms. Adapted from Gysin, S. Genes and Cancer, 2011 (336) 


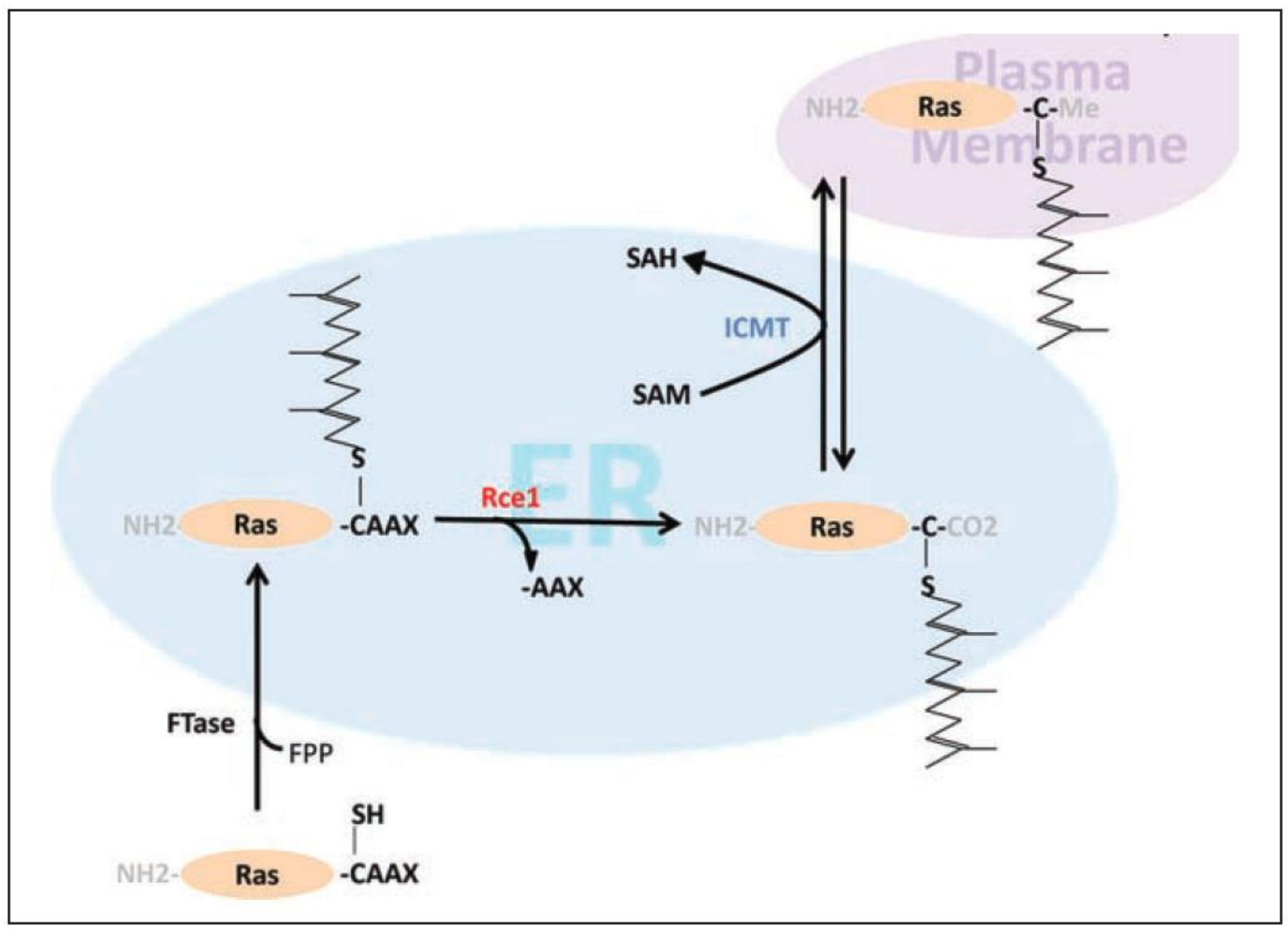

Figure 13: RAS-CAAX box processing. The key cysteine residue of the -CAAX box is farnesylated by the action of farnesyl transferase (FTase). Alternatively this residue can be prenylated by the action of other enzymes, including geranylgeranyltransferase I (GGTase I; not shown). The last three residues of the -CAAX box are cleaved by Ras converting enzyme 1 (RCE1). Finally the key cysteine is carboxymethylated by isoprenyl cysteine carboxymethyltransferase (ICMT). Adapted from Gysin, S. Genes and Cancer, 2011 (336) 


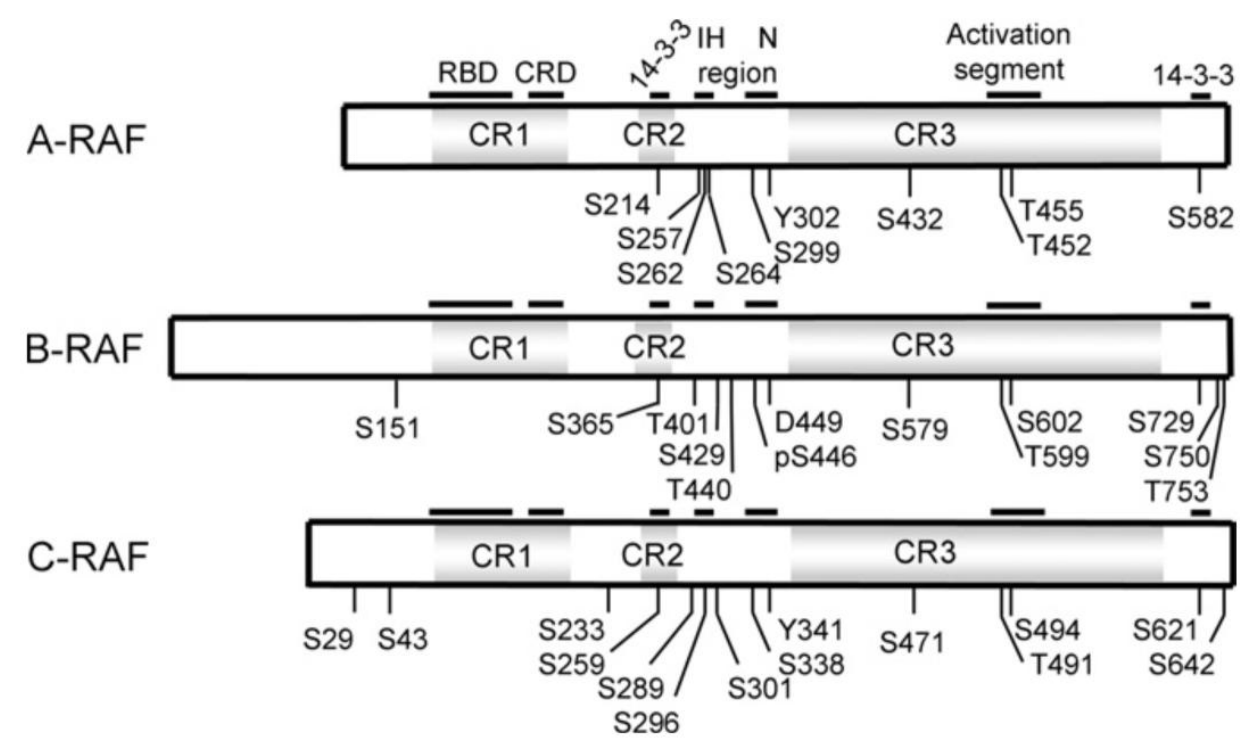

Figure 14: The structure of RAF enzymes. The domains and key regulatory phosphoryation sites in RAF proteins is shown. Conserved Region 1 (CR1), Conserved Region 2 (CR2), Conserved Region 3 (CR3), RAS-binding domain (RBD), cysteine rich domain (CRD). Adapted from Roskoski Jr., R. Biochem. Biophys. Res. Comm., 2010 (355). 


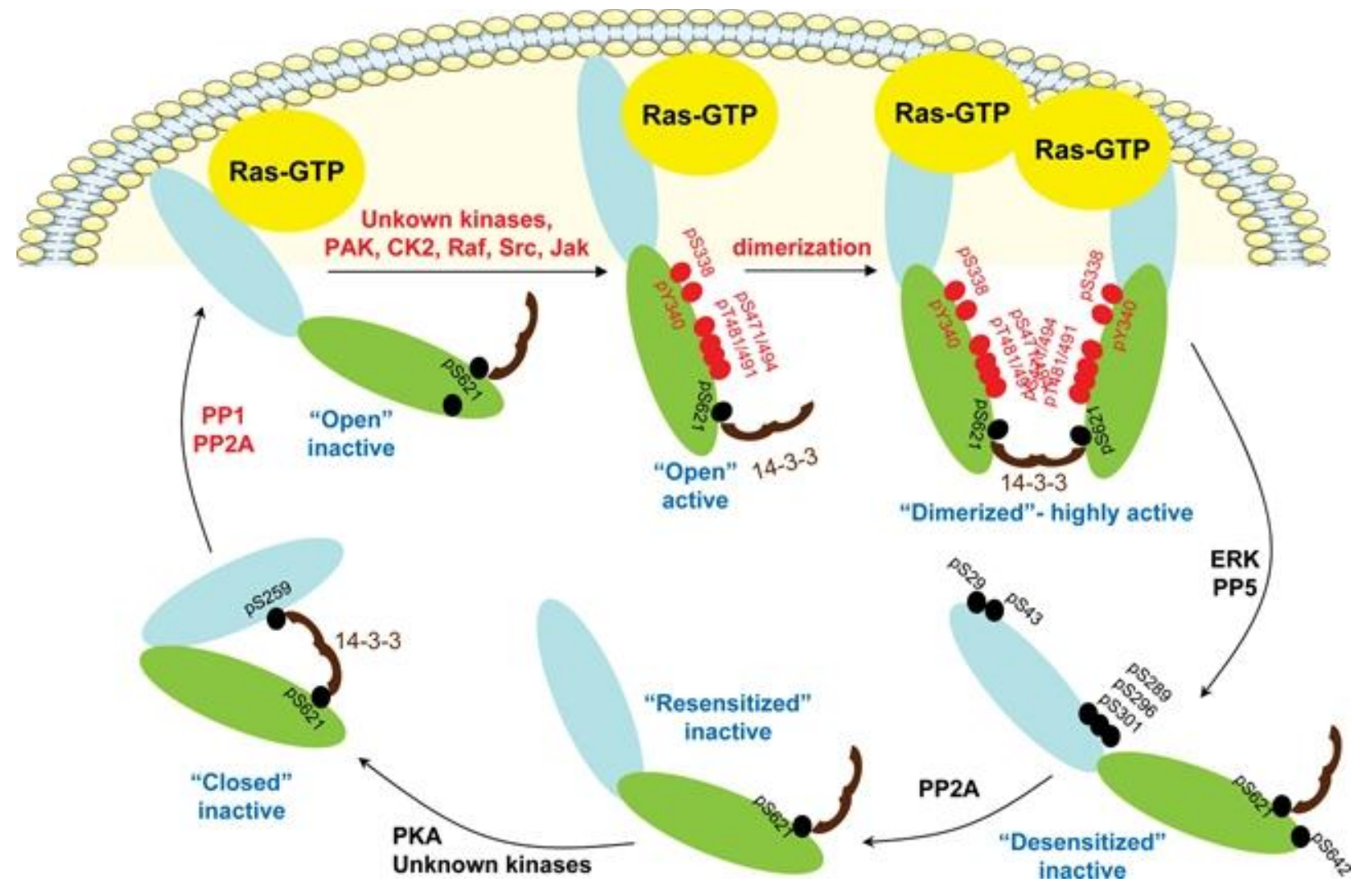

Figure 15: The cRAF activation cycle. Activating steps are shown in red. Adapted from Matallanas, D., Genes and Cancer, 2011 (356) 


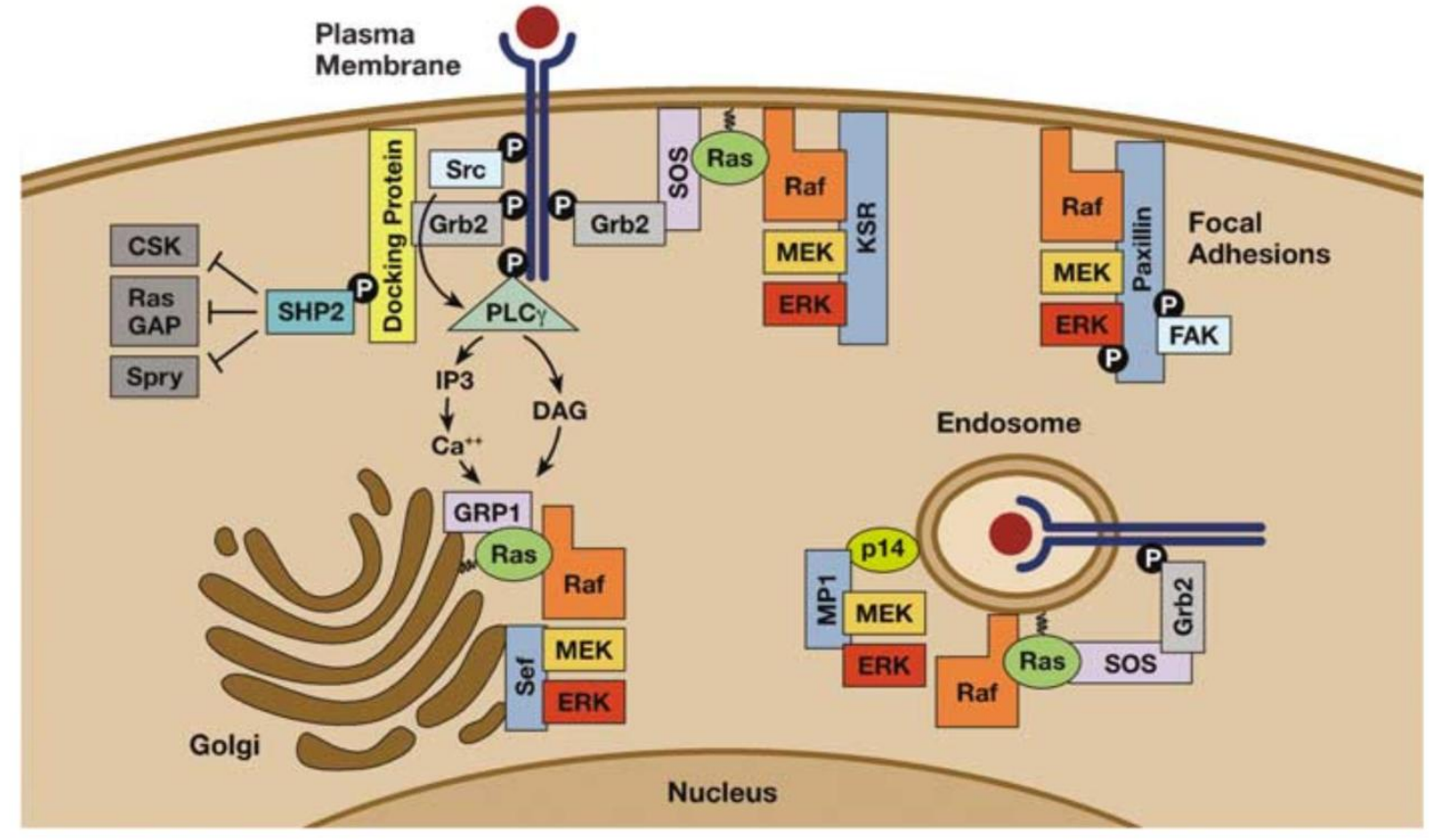

Figure 16: Modulation of RAS-ERK signaling by various regulatory proteins. Adapted from McKay, M.M. and Morrison, D.K., Oncogene, 2007 (385). 


\section{REFERENCES}

1. Schuh, R., W. Aicher, U. Gaul, S. Cote, A. Preiss, D. Maier, E. Seifert, U. Nauber, C. Schroder, R. Kemler, and . 1986. A conserved family of nuclear proteins containing structural elements of the finger protein encoded by Krüppel, a Drosophila segmentation gene. Cell 47:1025-1032.

2. Preiss, A., U. B. Rosenberg, A. Kienlin, E. Seifert, and H. Jackle. 1985. Molecular genetics of Krüppel, a gene required for segmentation of the Drosophila embryo. Nature 313:27-32.

3. Kadonaga, J. T., K. R. Carner, F. R. Masiarz, and R. Tjian. 1987. Isolation of cDNA encoding transcription factor Sp1 and functional analysis of the DNA binding domain. Cell 51:1079-1090.

4. Black, A. R., J. D. Black, and J. zizkhan-Clifford. 2001. Sp1 and krüppel-like factor family of transcription factors in cell growth regulation and cancer. J Cell Physiol 188:143-160.

5. Luchi, S. 2001. Three classes of C2H2 zinc finger proteins. Cell Mol. Life Sci. 58:625635.

6. Brayer, K. J. and D. J. Segal. 2008. Keep your fingers off my DNA: protein-protein interactions mediated by $\mathrm{C} 2 \mathrm{H} 2$ zinc finger domains. Cell Biochem. Biophys. 50:111131.

7. Dang, D. T., J. Pevsner, and V. W. Yang. 2000. The biology of the mammalian Krüppel-like family of transcription factors. Int. J. Biochem. Cell Biol. 32:1103-1121.

8. Miller, I. J. and J. J. Bieker. 1993. A novel, erythroid cell-specific murine transcription factor that binds to the CACCC element and is related to the Krueppel family of nuclear proteins. Mol. Cell. Biol. 13:2776-2786.

9. Shields, J. M. and V. W. Yang. 1998. Identification of the DNA sequence that interacts with the gut-enriched Krüppel-like factor. Nucleic Acids Res. 26:796-802.

10. Kaczynski, J., T. Cook, and R. Urrutia. 2003. Sp1- and Krüppel-like transcription factors. Genome Biol. 4:206.

11. Evans, P. M. and C. Liu. 2008. Roles of Krupel-like factor 4 in normal homeostasis, cancer and stem cells. Acta Biochim. Biophys. Sin. (Shanghai) 40:554-564.

12. McConnell, B. B. and V. W. Yang. 2010. Mammalian Krüppel-like factors in health and diseases. Physiol Rev. 90:1337-1381. 
13. Jiang, J., Y. S. Chan, Y. H. Loh, J. Cai, G. Q. Tong, C. A. Lim, P. Robson, S. Zhong, and $\mathrm{H}$. H. Ng. 2008. A core Klf circuitry regulates self-renewal of embryonic stem cells. Nat. Cell Biol. 10:353-360.

14. Tetreault, M. P., Y. Yang, and J. P. Katz. 2013. Krüppel-like factors in cancer. Nat. Rev. Cancer 13:701-713.

15. Nandan, M. O. and V. W. Yang. 2009. The role of Krüppel-like factors in the reprogramming of somatic cells to induced pluripotent stem cells. Histol. Histopathol. 24:1343-1355.

16. Takahashi, K. and S. Yamanaka. 2006. Induction of pluripotent stem cells from mouse embryonic and adult fibroblast cultures by defined factors. Cell 126:663-676.

17. Takahashi, K., K. Tanabe, M. Ohnuki, M. Narita, T. Ichisaka, K. Tomoda, and S. Yamanaka. 2007. Induction of pluripotent stem cells from adult human fibroblasts by defined factors. Cell 131:861-872.

18. Aksoy, I., V. Giudice, E. Delahaye, F. Wianny, M. Aubry, M. Mure, J. Chen, R. Jauch, G. K. Bogu, T. Nolden, H. Himmelbauer, D. M. Xavier, A. Sachinidis, H. Schulz, O. Hummel, P. Martinelli, N. Hubner, L. W. Stanton, F. X. Real, P. Y. Bourillot, and P. Savatier. 2014. Klf4 and Klf5 differentially inhibit mesoderm and endoderm differentiation in embryonic stem cells. Nat. Commun. 5:3719.

19. Pandya, A. Y., L. I. Talley, A. R. Frost, T. J. Fitzgerald, V. Trivedi, M. Chakravarthy, D. C. Chhieng, W. E. Grizzle, J. A. Engler, H. Krontiras, K. I. Bland, A. F. Lobuglio, S. M. Lobo-Ruppert, and J. M. Ruppert. 2004. Nuclear localization of KLF4 is associated with an aggressive phenotype in early-stage breast cancer. Clin Cancer Res 10:27092719.

20. Chen, C. J., S. E. Lin, Y. M. Lin, S. H. Lin, D. R. Chen, and C. L. Chen. 2012. Association of Expression of Krüppel-like Factor 4 and Krüppel-like Factor 5 with the Clinical Manifestations of Breast Cancer. Pathol. Oncol Res 18:161-168.

21. Kamalakaran, S., V. Varadan, H. E. Giercksky Russnes, D. Levy, J. Kendall, A. Janevski, M. Riggs, N. Banerjee, M. Synnestvedt, E. Schlichting, R. Karesen, P. K. Shama, H. Rotti, R. Rao, L. Rao, M. H. Eric Tang, K. Satyamoorthy, R. Lucito, M. Wigler, N. Dimitrova, B. Naume, A. L. Borresen-Dale, and J. B. Hicks. 2011. DNA methylation patterns in luminal breast cancers differ from non-luminal subtypes and can identify relapse risk independent of other clinical variables. Mol Oncol 5:77-92.

22. Shields, J. M., R. J. Christy, and V. W. Yang. 1996. Identification and characterization of a gene encoding a gut-enriched Krüppel-like factor expressed during growth arrest. J Biol Chem 271:20009-20017.

23. Garrett-Sinha, L. A., H. Eberspaecher, M. F. Seldin, and B. de Crombrugghe. 1996. A gene for a novel zinc-finger protein expressed in differentiated epithelial cells and transiently in certain mesenchymal cells. J Biol Chem 271:31384-31390. 
24. Yet, S. F., M. M. Mcanulty, S. C. Folta, H. W. Yen, M. Yoshizumi, C. M. Hsieh, M. D. Layne, M. T. Chin, H. Wang, M. A. Perrella, M. K. Jain, and M. E. Lee. 1998. Human EZF, a Krüppel-like zinc finger protein, is expressed in vascular endothelial cells and contains transcriptional activation and repression domains. J. Biol. Chem. 273:10261031.

25. Shie, J. L., Z. Y. Chen, M. J. O'Brien, R. G. Pestell, M. E. Lee, and C. C. Tseng. 2000. Role of gut-enriched Krüppel-like factor in colonic cell growth and differentiation. Am. J Physiol Gastrointest. Liver Physiol 279:G806-G814.

26. Jenkins, T. D., O. G. Opitz, J. Okano, and A. K. Rustgi. 1998. Transactivation of the human keratin 4 and Epstein-Barr virus ED-L2 promoters by gut-enriched Krüppel-like factor. J. Biol. Chem. 273:10747-10754.

27. Ton-That, H., K. H. Kaestner, J. M. Shields, C. S. Mahatanankoon, and V. W. Yang. 1997. Expression of the gut-enriched Krüppel-like factor gene during development and intestinal tumorigenesis. FEBS Lett. 419:239-243.

28. Panigada, M., S. Porcellini, F. Sutti, L. Doneda, O. Pozzoli, G. G. Consalez, M. Guttinger, and F. Grassi. 1999. GKLF in thymus epithelium as a developmentally regulated element of thymocyte-stroma cross-talk. Mechanisms of Development 81:103-113.

29. Chiambaretta, F., G. F. De, G. Turet, G. Marceau, P. Gain, B. Dastugue, D. Rigal, and V. Sapin. 2004. Cell and tissue specific expression of human Krüppel-like transcription factors in human ocular surface. Mol. Vis. 10:901-909.

30. Fruman, D. A., G. Z. Ferl, S. S. An, A. C. Donahue, A. B. Satterthwaite, and O. N. Witte. 2002. Phosphoinositide 3-kinase and Bruton's tyrosine kinase regulate overlapping sets of genes in B lymphocytes. Proc. Natl. Acad. Sci. U. S. A 99:359-364.

31. Cullingford, T. E., M. J. Butler, A. K. Marshall, e. L. Tham, P. H. Sugden, and A. Clerk. 2008. Differential regulation of Krüppel-like factor family transcription factor expression in neonatal rat cardiac myocytes: effects of endothelin-1, oxidative stress and cytokines. Biochim. Biophys. Acta 1783:1229-1236.

32. Geiman, D. E., T. T. Han, J. M. Johnson, and V. W. Yang. 2000. Transactivation and growth suppression by the gut-enriched Krüppel-like factor (Krüppel-like factor 4) are dependent on acidic amino acid residues and protein-protein interaction. Nucleic Acids Res. 28:1106-1113.

33. Shields, J. M. and V. W. Yang. 1997. Two potent nuclear localization signals in the gut-enriched Krüppel-like factor define a subfamily of closely related Krüppel proteins. J. Biol. Chem. 272:18504-18507.

34. Chen, Z. Y., X. Wang, Y. Zhou, G. Offner, and C. C. Tseng. 2005. Destabilization of Krüppel-like factor 4 protein in response to serum stimulation involves the ubiquitinproteasome pathway. Cancer Res. 65:10394-10400. 
35. Zhang, W., D. E. Geiman, J. M. Shields, D. T. Dang, C. S. Mahatan, K. H. Kaestner, J. R. Biggs, A. S. Kraft, and V. W. Yang. 2000. The gut-enriched Krüppel-like factor (Krüppel-like factor 4) mediates the transactivating effect of p53 on the p21WAF1/Cip1 promoter. J. Biol. Chem. 275:18391-18398.

36. Yoon, H. S., X. Chen, and V. W. Yang. 2003. Krüppel-like factor 4 mediates p53dependent G1/S cell cycle arrest in response to DNA damage. J Biol Chem 278:21012105.

37. Dang, D. T., C. S. Mahatan, L. H. Dang, I. A. Agboola, and V. W. Yang. 2001. Expression of the gut-enriched Krüppel-like factor (Krüppel-like factor 4) gene in the human colon cancer cell line RKO is dependent on CDX2. Oncogene 20:4884-4890.

38. Mahatan, C. S., K. H. Kaestner, D. E. Geiman, and V. W. Yang. 1999. Characterization of the structure and regulation of the murine gene encoding gut-enriched Krüppel-like factor (Krüppel-like factor 4). Nucleic Acids Res. 27:4562-4569.

39. Dang, D. T., W. Zhao, C. S. Mahatan, D. E. Geiman, and V. W. Yang. 2002. Opposing effects of Krüppel-like factor 4 (gut-enriched Krüppel-like factor) and Krüppel-like factor 5 (intestinal-enriched Krüppel-like factor) on the promoter of the Krüppel-like factor 4 gene. Nucleic Acids Res 30:2736-2741.

40. McConnell, B. B., A. M. Ghaleb, M. O. Nandan, and V. W. Yang. 2007. The diverse functions of Krüppel-like factors 4 and 5 in epithelial biology and pathobiology. Bioessays 29:549-557.

41. Ogryzko, V. V., R. L. Schiltz, V. Russanova, B. H. Howard, and Y. Nakatani. 1996. The transcriptional coactivators p300 and cbp are histone acetyltransferases. Cell 87:953-959.

42. Strahl, B. D. and C. D. Allis. 2000. The language of covalent histone modifications. Nature 403:41-45.

43. Evans, P. M., W. Zhang, X. Chen, J. Yang, K. K. Bhakat, and C. Liu. 2007. Krüppellike factor 4 is acetylated by $\mathrm{p} 300$ and regulates gene transcription via modulation of histone acetylation. J Biol. Chem. 282:33994-34002.

44. Ai, W., H. Zheng, X. Yang, Y. Liu, and T. C. Wang. 2007. Tip60 functions as a potential corepressor of KLF4 in regulation of HDC promoter activity. Nucleic Acids Res 35:6137-6149.

45. Birsoy, K., Z. Chen, and J. Friedman. 2008. Transcriptional regulation of adipogenesis by KLF4. Cell Metab 7:339-347.

46. Feinberg, M. W., Z. Cao, A. K. Wara, M. A. Lebedeva, S. Senbanerjee, and M. K. Jain. 2005. Krüppel-like factor 4 is a mediator of proinflammatory signaling in macrophages. J Biol. Chem. 280:38247-38258. 
47. Zhang, W., J. M. Shields, K. Sogawa, Y. Fujii-Kuriyama, and V. W. Yang. 1998. The gut-enriched Krüppel-like factor suppresses the activity of the CYP1A1 promoter in an Sp1-dependent fashion. J. Biol. Chem. 273:17917-17925.

48. Rivero, S., M. J. az-Guerra, E. M. Monsalve, J. Laborda, and J. J. Garcia-Ramirez. 2012. DLK2 is a transcriptional target of KLF4 in the early stages of adipogenesis. J. Mol. Biol. 417:36-50.

49. Yori, J. L., E. Johnson, G. Zhou, M. K. Jain, and R. A. Keri. 2010. Krüppel-like Factor 4 Inhibits Epithelial-to-Mesenchymal Transition through Regulation of E-cadherin Gene Expression. J Biol. Chem. 285:16854-16863.

50. Yori, J. L., D. D. Seachrist, E. Johnson, K. L. Lozada, F. W. bdul-Karim, L. A. Chodosh, W. P. Schiemann, and R. A. Keri. 2011. Krüppel-like factor 4 inhibits tumorigenic progression and metastasis in a mouse model of breast cancer. Neoplasia 13:601-610.

51. Hinnebusch, B. F., A. Siddique, J. W. Henderson, M. S. Malo, W. Zhang, C. P. Athaide, M. A. Abedrapo, X. Chen, V. W. Yang, and R. A. Hodin. 2004. Enterocyte differentiation marker intestinal alkaline phosphatase is a target gene of the gutenriched Krüppel-like factor. Am. J. Physiol Gastrointest. Liver Physiol 286:G23-G30.

52. Siddique, A., M. S. Malo, L. M. Ocuin, B. F. Hinnebusch, M. A. Abedrapo, J. W. Henderson, W. Zhang, M. Mozumder, V. W. Yang, and R. A. Hodin. 2003. Convergence of the thyroid hormone and gut-enriched Krüppel-like factor pathways in the context of enterocyte differentiation. J Gastrointest. Surg. 7:1053-1061.

53. Okano, J., O. G. Opitz, H. Nakagawa, T. D. Jenkins, S. L. Friedman, and A. K. Rustgi. 2000. The Krüppel-like transcriptional factors Zf9 and GKLF coactivate the human keratin 4 promoter and physically interact. FEBS Lett. 473:95-100.

54. Brembeck, F. H. and A. K. Rustgi. 2000. The tissue-dependent keratin 19 gene transcription is regulated by GKLF/KLF4 and Sp1. J. Biol. Chem. 275:28230-28239.

55. Piccinni, S. A., A. L. Bolcato-Bellemin, A. Klein, V. W. Yang, M. Kedinger, P. SimonAssmann, and O. Lefebvre. 2004. Krüppel-like factors regulate the Lama1 gene encoding the laminin alpha1 chain. J Biol. Chem. 279:9103-9114.

56. Miller, K. A., E. A. Eklund, M. L. Peddinghaus, Z. Cao, N. Fernandes, P. W. Turk, B. Thimmapaya, and S. A. Weitzman. 2001. Krüppel-like factor 4 regulates laminin alpha 3A expression in mammary epithelial cells. J. Biol. Chem. 276:42863-42868.

57. Higaki, Y., D. Schullery, Y. Kawata, M. Shnyreva, C. Abrass, and K. Bomsztyk. 2002. Synergistic activation of the rat laminin gammal chain promoter by the gut-enriched Krüppel-like factor (GKLF/KLF4) and Sp1. Nucleic Acids Res 30:2270-2279.

58. Nakatake, Y., N. Fukui, Y. Iwamatsu, S. Masui, K. Takahashi, R. Yagi, K. Yagi, J. Miyazaki, R. Matoba, M. S. Ko, and H. Niwa. 2006. Klf4 cooperates with Oct3/4 and 
Sox 2 to activate the Lefty1 core promoter in embryonic stem cells. Mol. Cell Biol. 26:7772-7782.

59. Lin, C. C., L. Z. Liu, J. B. Addison, A. V. Ivanov, and J. M. Ruppert. 2011. A KLF4miRNA-206 autoregulatory feedback loop can promote or inhibit protein translation depending upon cell context. Mol. Cell Biol. 31:2513-2527.

60. Liu, Z., L. Teng, S. K. Bailey, A. R. Frost, K. I. Bland, A. F. Lobuglio, J. M. Ruppert, and S. M. Lobo-Ruppert. 2009. Epithelial transformation by KLF4 requires Notch1 but not canonical Notch1 signaling. Cancer Biol. Ther 8:1840-1851.

61. Nickenig, G., S. Baudler, C. Muller, C. Werner, N. Werner, H. Welzel, K. Strehlow, and M. Bohm. 2002. Redox-sensitive vascular smooth muscle cell proliferation is mediated by GKLF and Id3 in vitro and in vivo. Faseb J 16:1077-1086.

62. Chen, X., E. M. Whitney, S. Y. Gao, and V. W. Yang. 2003. Transcriptional profiling of Krüppel-like factor 4 reveals a function in cell cycle regulation and epithelial differentiation. J Mol Biol 326:665-677.

63. Luo, A., J. Kong, G. Hu, C. C. Liew, M. Xiong, X. Wang, J. Ji, T. Wang, H. Zhi, M. $\mathrm{Wu}$, and Z. Liu. 2004. Discovery of Ca2+-relevant and differentiation-associated genes downregulated in esophageal squamous cell carcinoma using cDNA microarray. Oncogene 23:1291-1299.

64. Wang, H., L. Yang, M. S. Jamaluddin, and D. D. Boyd. 2004. The Krüppel-like KLF4 transcription factor, a novel regulator of urokinase receptor expression, drives synthesis of this binding site in colonic crypt luminal surface epithelial cells. J Biol. Chem. 279:22674-22683.

65. Kanai, M., D. Wei, Q. Li, Z. Jia, J. Ajani, X. Le, J. Yao, and K. Xie. 2006. Loss of Krüppel-like factor 4 expression contributes to Sp1 overexpression and human gastric cancer development and progression. Clin Cancer Res 12:6395-6402.

66. Ai, W., Y. Liu, M. Langlois, and T. C. Wang. 2004. Krüppel-like factor 4 (KLF4) represses histidine decarboxylase gene expression through an upstream Sp1 site and downstream gastrin responsive elements. J. Biol. Chem. 279:8684-8693.

67. Noti, J. D., A. K. Johnson, and J. D. Dillon. 2005. The leukocyte integrin gene CD11d is repressed by gut-enriched Krüppel-like factor 4 in myeloid cells. J Biol. Chem. 280:3449-3457.

68. Rowland, B. D., R. Bernards, and D. S. Peeper. 2005. The KLF4 tumour suppressor is a transcriptional repressor of p53 that acts as a context-dependent oncogene. Nat. Cell Biol. 7:1074-1082.

69. Zhang, W., X. Chen, Y. Kato, P. M. Evans, S. Yuan, J. Yang, P. G. Rychahou, V. W. Yang, X. He, B. M. Evers, and C. Liu. 2006. Novel cross talk of Krüppel-like factor 4 
and beta-catenin regulates normal intestinal homeostasis and tumor repression. Mol. Cell Biol. 26:2055-2064.

70. Evans, P. M., X. Chen, W. Zhang, and C. Liu. 2010. KLF4 interacts with betacatenin/TCF4 and blocks p300/CBP recruitment by beta-catenin. Mol. Cell Biol. 30:372-381.

71. Ghaleb, A. M., J. P. Katz, K. H. Kaestner, J. X. Du, and V. W. Yang. 2007. Krüppellike factor 4 exhibits antiapoptotic activity following gamma-radiation-induced DNA damage. Oncogene 26:2365-2373.

72. Shie, J. L., Z. Y. Chen, M. Fu, R. G. Pestell, and C. C. Tseng. 2000. Gut-enriched Krüppel-like factor represses cyclin D1 promoter activity through Sp1 motif. Nucleic Acids Res. 28:2969-2976.

73. Klaewsongkram, J., Y. Yang, S. Golech, J. Katz, K. H. Kaestner, and N. P. Weng. 2007. Krüppel-like factor 4 regulates B cell number and activation-induced B cell proliferation. J Immunol. 179:4679-4684.

74. Yoon, H. S., A. M. Ghaleb, M. O. Nandan, I. M. Hisamuddin, W. B. Dalton, and V. W. Yang. 2005. Krüppel-like factor 4 prevents centrosome amplification following gamma-irradiation-induced DNA damage. Oncogene 24:4017-4025.

75. Tiwari, N., N. Meyer-Schaller, P. Arnold, H. Antoniadis, M. Pachkov, N. E. van, and G. Christofori. 2013. Klf4 is a transcriptional regulator of genes critical for EMT, including Jnk1 (Mapk8). PLoS One 8:e57329.

76. Chen, Z. Y., J. L. Shie, and C. C. Tseng. 2002. Gut-enriched Krüppel-like factor represses ornithine decarboxylase gene expression and functions as checkpoint regulator in colonic cancer cells. J. Biol. Chem. 277:46831-46839.

77. Adam, P. J., C. P. Regan, M. B. Hautmann, and G. K. Owens. 2000. Positive- and negative-acting Krüppel-like transcription factors bind a transforming growth factor beta control element required for expression of the smooth muscle cell differentiation marker SM22alpha in vivo. J. Biol. Chem. 275:37798-37806.

78. Liu, Y., S. Sinha, and G. Owens. 2003. A transforming growth factor-beta control element required for SM alpha-actin expression in vivo also partially mediates GKLFdependent transcriptional repression. J Biol. Chem. 278:48004-48011.

79. Farrugia, M. K., S. B. Sharma, C. C. Lin, S. L. McLaughlin, D. B. Vanderbilt, A. G. Ammer, M. A. Salkeni, P. Stoilov, Y. M. Agazie, C. J. Creighton, and J. M. Ruppert. 2015. Regulation of anti-apoptotic signaling by Krüppel-like factors 4 and 5 mediates lapatinib resistance in breast cancer. Cell Death. Dis. 6:e1699.

80. Sharma, S. B., C. C. Lin, M. K. Farrugia, S. L. McLaughlin, E. J. Ellis, K. M. Brundage, M. A. Salkeni, and J. M. Ruppert. 2014. MicroRNAs 206 and 21 cooperate 
to promote RAS-extracellular signal-regulated kinase signaling by suppressing the translation of RASA1 and SPRED1. Mol. Cell Biol. 34:4143-4164.

81. Ghaleb, A. M., M. O. Nandan, S. Chanchevalap, W. B. Dalton, I. M. Hisamuddin, and V. W. Yang. 2005. Krüppel-like factors 4 and 5: the yin and yang regulators of cellular proliferation. Cell Res 15:92-96.

82. Katz, J. P., N. Perreault, B. G. Goldstein, C. S. Lee, P. A. Labosky, V. W. Yang, and K. H. Kaestner. 2002. The zinc-finger transcription factor Klf4 is required for terminal differentiation of goblet cells in the colon. Development 129:2619-2628.

83. Segre, J. A., C. Bauer, and E. Fuchs. 1999. Klf4 is a transcription factor required for establishing the barrier function of the skin. Nat. Genet. 22:356-360.

84. Ky, N., C. B. Lim, J. Li, J. P. Tam, M. S. Hamza, and Y. Zhao. 2009. KLF4 suppresses HDACi induced caspase activation and the SAPK pathway by targeting p57(Kip2). Apoptosis. 14:1095-1107.

85. Wei, D., M. Kanai, Z. Jia, X. Le, and K. Xie. 2008. Krüppel-like factor 4 induces p27Kip1 expression in and suppresses the growth and metastasis of human pancreatic cancer cells. Cancer Res 68:4631-4639.

86. Dang, D. T., X. Chen, J. Feng, M. Torbenson, L. H. Dang, and V. W. Yang. 2003. Overexpression of Krüppel-like factor 4 in the human colon cancer cell line RKO leads to reduced tumorigenecity. Oncogene 22:3424-3430.

87. Zhou, Q., Y. Hong, Q. Zhan, Y. Shen, and Z. Liu. 2009. Role for Krüppel-like factor 4 in determining the outcome of p53 response to DNA damage. Cancer Res. 69:82848292.

88. Katz, J. P., N. Perreault, B. G. Goldstein, L. Actman, S. R. McNally, D. G. Silberg, E. E. Furth, and K. H. Kaestner. 2005. Loss of Klf4 in mice causes altered proliferation and differentiation and precancerous changes in the adult stomach. Gastroenterology 128:935-945.

89. Chen, Z. Y., S. Rex, and C. C. Tseng. 2004. Krüppel-like factor 4 is transactivated by butyrate in colon cancer cells. J Nutr. 134:792-798.

90. Patel, S., Z. F. Xi, E. Y. Seo, D. McGaughey, and J. A. Segre. 2006. Klf4 and corticosteroids activate an overlapping set of transcriptional targets to accelerate in utero epidermal barrier acquisition. Proc. Natl. Acad. Sci. U. S. A 103:18668-18673.

91. Swamynathan, S. K., J. P. Katz, K. H. Kaestner, R. shery-Padan, M. A. Crawford, and J. Piatigorsky. 2007. Conditional deletion of the mouse Klf4 gene results in corneal epithelial fragility, stromal edema, and loss of conjunctival goblet cells. Mol. Cell Biol. 27:182-194. 
92. Alder, J. K., R. W. Georgantas, III, R. L. Hildreth, I. M. Kaplan, S. Morisot, X. Yu, M. McDevitt, and C. I. Civin. 2008. Krüppel-like factor 4 is essential for inflammatory monocyte differentiation in vivo. J Immunol. 180:5645-5652.

93. Good, K. L. and S. G. Tangye. 2007. Decreased expression of Krüppel-like factors in memory B cells induces the rapid response typical of secondary antibody responses. Proc. Natl. Acad. Sci. U. S. A 104:13420-13425.

94. King, K. E., V. P. Iyemere, P. L. Weissberg, and C. M. Shanahan. 2003. Krüppel-like factor 4 (KLF4/GKLF) is a target of bone morphogenetic proteins and transforming growth factor beta 1 in the regulation of vascular smooth muscle cell phenotype. J. Biol. Chem. 278:11661-11669.

95. Liu, Y., S. Sinha, O. G. McDonald, Y. Shang, M. H. Hoofnagle, and G. K. Owens. 2005. Krüppel-like factor 4 abrogates myocardin-induced activation of smooth muscle gene expression. J Biol. Chem. 280:9719-9727.

96. Hu, B., Z. Wu, T. Liu, M. R. Ullenbruch, H. Jin, and S. H. Phan. 2007. Gut-enriched Krüppel-like factor interaction with Smad3 inhibits myofibroblast differentiation. Am. J Respir. Cell Mol. Biol. 36:78-84.

97. Hamik, A., Z. Lin, A. Kumar, M. Balcells, S. Sinha, J. Katz, M. W. Feinberg, R. E. Gerzsten, E. R. Edelman, and M. K. Jain. 2007. Krüppel-like factor 4 regulates endothelial inflammation. J Biol. Chem. 282:13769-13779.

98. Yoshida, T., K. H. Kaestner, and G. K. Owens. 2008. Conditional deletion of Krüppellike factor 4 delays downregulation of smooth muscle cell differentiation markers but accelerates neointimal formation following vascular injury. Circ. Res. 102:1548-1557.

99. Michaelson, J., S. Satija, R. Moore, G. Weber, E. Halpern, A. Garland, D. Puri, and D. B. Kopans. 2002. The pattern of breast cancer screening utilization and its consequences. Cancer 94:37-43.

100. McCormick, S. M., S. G. Eskin, L. V. McIntire, C. L. Teng, C. M. Lu, C. G. Russell, and K. K. Chittur. 2001. DNA microarray reveals changes in gene expression of shear stressed human umbilical vein endothelial cells. Proc. Natl. Acad. Sci. U. S. A 98:89558960 .

101. Pedersen, T. X., C. Leethanakul, V. Patel, D. Mitola, L. R. Lund, K. Dano, M. Johnsen, J. S. Gutkind, and T. H. Bugge. 2003. Laser capture microdissection-based in vivo genomic profiling of wound keratinocytes identifies similarities and differences to squamous cell carcinoma. Oncogene 22:3964-3976.

102. Liu, Y., J. Wang, Y. Yi, H. Zhang, J. Liu, M. Liu, C. Yuan, D. Tang, I. J. Benjamin, and X. Xiao. 2006. Induction of KLF4 in response to heat stress. Cell Stress Chaperones 11:379-389. 
103. Liao, X., S. M. Haldar, Y. Lu, D. Jeyaraj, K. Paruchuri, M. Nahori, Y. Cui, K. H. Kaestner, and M. K. Jain. 2010. Krüppel-like factor 4 regulates pressure-induced cardiac hypertrophy. J Mol. Cell Cardiol. 49:334-338.

104. Lai, J. K., H. C. Wu, Y. C. Shen, H. Y. Hsieh, S. Y. Yang, and C. C. Chang. 2012. Krüppel-like factor 4 is involved in cell scattering induced by hepatocyte growth factor. J Cell Sci 125:4853-4864.

105. Kim, J., J. Chu, X. Shen, J. Wang, and S. H. Orkin. 2008. An extended transcriptional network for pluripotency of embryonic stem cells. Cell 132:1049-1061.

106. Yamanaka, S. 2007. Strategies and new developments in the generation of patientspecific pluripotent stem cells. Cell Stem Cell 1:39-49.

107. Di Stefano B., A. Prigione, and V. Broccoli. 2009. Efficient genetic reprogramming of unmodified somatic neural progenitors uncovers the essential requirement of Oct 4 and Klf4. Stem Cells Dev. 18:707-716.

108. Shi, Y., C. Desponts, J. T. Do, H. S. Hahm, H. R. Scholer, and S. Ding. 2008. Induction of pluripotent stem cells from mouse embryonic fibroblasts by Oct4 and Klf4 with small-molecule compounds. Cell Stem Cell 3:568-574.

109. Li, Y., J. McClintick, L. Zhong, H. J. Edenberg, M. C. Yoder, and R. J. Chan. 2005. Murine embryonic stem cell differentiation is promoted by SOCS-3 and inhibited by the zinc finger transcription factor Klf4. Blood 105:635-637.

110. Rowland, B. D. and D. S. Peeper. 2006. KLF4, p21 and context-dependent opposing forces in cancer. Nat. Rev. Cancer 6:11-23.

111. Leng, Z., K. Tao, Q. Xia, J. Tan, Z. Yue, J. Chen, H. Xi, J. Li, and H. Zheng. 2013. Krüppel-Like Factor 4 Acts as an Oncogene in Colon Cancer Stem Cell-Enriched Spheroid Cells. PLoS One 8:e56082.

112. Zhao, W., I. M. Hisamuddin, M. O. Nandan, B. A. Babbin, N. E. Lamb, and V. W. Yang. 2004. Identification of Krüppel-like factor 4 as a potential tumor suppressor gene in colorectal cancer. Oncogene 23:395-402.

113. Choi, B. J., Y. G. Cho, J. W. Song, C. J. Kim, S. Y. Kim, S. W. Nam, N. J. Yoo, J. Y. Lee, and W. S. Park. 2006. Altered expression of the KLF4 in colorectal cancers. Pathol Res Pract 202:585-589.

114. Xu, J., B. Lu, F. Xu, H. Gu, Y. Fang, Q. Huang, and M. Lai. 2008. Dynamic downregulation of Krüppel-like factor 4 in colorectal adenoma-carcinoma sequence. J. Cancer Res. Clin. Oncol. 134:891-898.

115. Dang, D. T., K. E. Bachman, C. S. Mahatan, L. H. Dang, F. M. Giardiello, and V. W. Yang. 2000. Decreased expression of the gut-enriched Krüppel-like factor gene in 
intestinal adenomas of multiple intestinal neoplasia mice and in colonic adenomas of familial adenomatous polyposis patients. FEBS Lett. 476:203-207.

116. Barker, N., W. M. van de, and H. Clevers. 2008. The intestinal stem cell. Genes Dev 22:1856-1864.

117. Ghaleb, A. M., B. B. McConnell, M. O. Nandan, J. P. Katz, K. H. Kaestner, and V. W. Yang. 2007. Haploinsufficiency of Krüppel-like factor 4 promotes adenomatous polyposis coli dependent intestinal tumorigenesis. Cancer Res 67:7147-7154.

118. Ghaleb, A. M., G. Aggarwal, A. B. Bialkowska, M. O. Nandan, and V. W. Yang. 2008. Notch inhibits expression of the Krüppel-like factor 4 tumor suppressor in the intestinal epithelium. Mol. Cancer Res 6:1920-1927.

119. Zheng, H., D. M. Pritchard, X. Yang, E. Bennett, G. Liu, C. Liu, and W. Ai. 2009. KLF4 gene expression is inhibited by the notch signaling pathway that controls goblet cell differentiation in mouse gastrointestinal tract. Am. J Physiol Gastrointest. Liver Physiol 296:G490-G498.

120. van Es, J. H., M. E. van Gijn, O. Riccio, B. M. van den, M. Vooijs, H. Begthel, M. Cozijnsen, S. Robine, D. J. Winton, F. Radtke, and H. Clevers. 2005. Notch/gammasecretase inhibition turns proliferative cells in intestinal crypts and adenomas into goblet cells. Nature 435:959-963.

121. Nguyen, L. V., R. Vanner, P. Dirks, and C. J. Eaves. 2012. Cancer stem cells: an evolving concept. Nat. Rev. Cancer 12:133-143.

122. Pattabiraman, D. R. and R. A. Weinberg. 2014. Tackling the cancer stem cells - what challenges do they pose? Nat. Rev. Drug Discov. 13:497-512.

123. Clevers, H. 2011. The cancer stem cell: premises, promises and challenges. Nat. Med 17:313-319.

124. Reya, T., S. J. Morrison, M. F. Clarke, and I. L. Weissman. 2001. Stem cells, cancer, and cancer stem cells. Nature 414:105-111.

125. Wang, N., Z. H. Liu, F. Ding, X. Q. Wang, C. N. Zhou, and M. Wu. 2002. Downregulation of gut-enriched Krüppel-like factor expression in esophageal cancer. World J Gastroenterol 8:966-970.

126. Cho, Y. G., J. H. Song, C. J. Kim, S. W. Nam, N. J. Yoo, J. Y. Lee, and W. S. Park. 2007. Genetic and epigenetic analysis of the KLF4 gene in gastric cancer. APMIS 115:802-808.

127. Wei, D., W. Gong, M. Kanai, C. Schlunk, L. Wang, J. C. Yao, T. T. Wu, S. Huang, and K. Xie. 2005. Drastic down-regulation of Krüppel-like factor 4 expression is critical in human gastric cancer development and progression. Cancer Res 65:2746-2754. 
128. Lin, Z. S., H. C. Chu, Y. C. Yen, B. C. Lewis, and Y. W. Chen. 2012. Krüppel-like factor 4, a tumor suppressor in hepatocellular carcinoma cells reverts epithelial mesenchymal transition by suppressing slug expression. PLoS. One. 7:e43593.

129. Bianchi, F., J. Hu, G. Pelosi, R. Cirincione, M. Ferguson, C. Ratcliffe, P. P. di Fiore, K. Gatter, F. Pezzella, and U. Pastorino. 2004. Lung cancers detected by screening with spiral computed tomography have a malignant phenotype when analyzed by cDNA microarray. Clin Cancer Res 10:6023-6028.

130. Hu, W., W. L. Hofstetter, H. Li, Y. Zhou, Y. He, A. Pataer, L. Wang, K. Xie, S. G. Swisher, and B. Fang. 2009. Putative tumor-suppressive function of Krüppel-like factor 4 in primary lung carcinoma. Clin. Cancer Res. 15:5688-5695.

131. Foster, K. W., A. R. Frost, P. McKie-Bell, C. Y. Lin, J. A. Engler, W. E. Grizzle, and J. M. Ruppert. 2000. Increase of GKLF messenger RNA and protein expression during progression of breast cancer. Cancer Res. 60:6488-6495.

132. Luo, J., T. Dunn, C. Ewing, J. Sauvageot, Y. Chen, J. Trent, and W. Isaacs. 2002. Gene expression signature of benign prostatic hyperplasia revealed by cDNA microarray analysis. Prostate 51:189-200.

133. Ohnishi, S., S. Ohnami, F. Laub, K. Aoki, K. Suzuki, Y. Kanai, K. Haga, M. Asaka, F. Ramirez, and T. Yoshida. 2003. Downregulation and growth inhibitory effect of epithelial-type Krüppel-like transcription factor KLF4, but not KLF5, in bladder cancer. Biochem Biophys Res Commun 308:251-256.

134. Yasunaga, J., Y. Taniguchi, K. Nosaka, M. Yoshida, Y. Satou, T. Sakai, H. Mitsuya, and M. Matsuoka. 2004. Identification of aberrantly methylated genes in association with adult T-cell leukemia. Cancer Res 64:6002-6009.

135. Foster, K. W., S. Ren, I. D. Louro, S. M. Lobo-Ruppert, P. McKie-Bell, W. Grizzle, M. R. Hayes, T. R. Broker, L. T. Chow, and J. M. Ruppert. 1999. Oncogene expression cloning by retroviral transduction of adenovirus E1A-immortalized rat kidney RK3E cells: transformation of a host with epithelial features by c-MYC and the zinc finger protein GKLF. Cell Growth Differ. 10:423-434.

136. Foster, K. W., Z. Liu, C. D. Nail, X. Li, T. J. Fitzgerald, S. K. Bailey, A. R. Frost, I. D. Louro, T. M. Townes, A. J. Paterson, J. E. Kudlow, S. M. Lobo-Ruppert, and J. M. Ruppert. 2005. Induction of KLF4 in basal keratinocytes blocks the proliferationdifferentiation switch and initiates squamous epithelial dysplasia. Oncogene 24:14911500 .

137. Huang, C. C., Z. Liu, X. Li, S. K. Bailey, C. D. Nail, K. W. Foster, A. R. Frost, J. M. Ruppert, and S. M. Lobo-Ruppert. 2005. KLF4 and PCNA identify stages of tumor initiation in a conditional model of cutaneous squamous epithelial neoplasia. Cancer Biology and Therapy 4:1401-1408. 
138. Suzuki, T., H. Shen, K. Akagi, H. C. Morse, J. D. Malley, D. Q. Naiman, N. A. Jenkins, and N. G. Copeland. 2002. New genes involved in cancer identified by retroviral tagging. Nat. Genet. 32:166-174.

139. Jiang, W., W. Deng, S. K. Bailey, C. D. Nail, A. R. Frost, W. J. Brouillette, D. D. Muccio, C. J. Grubbs, J. M. Ruppert, and S. M. Lobo-Ruppert. 2009. Prevention of KLF4-mediated tumor initiation and malignant transformation by UAB30 rexinoid. Cancer Biol. Ther 8:287-296.

140. Okuda, H., F. Xing, P. R. Pandey, S. Sharma, M. Watabe, S. K. Pai, Y. Y. Mo, M. Iiizumi-Gairani, S. Hirota, Y. Liu, K. Wu, R. Pochampally, and K. Watabe. 2013. miR7 Suppresses Brain Metastasis of Breast Cancer Stem-Like Cells By Modulating KLF4. Cancer Res.

141. Lee, H. K., D. S. Lee, and J. C. Park. 2015. Nuclear factor I-C regulates E-cadherin via control of KLF4 in breast cancer. BMC Cancer 15:1118.

142. Akaogi, K., Y. Nakajima, I. Ito, S. Kawasaki, S. H. Oie, A. Murayama, K. Kimura, and J. Yanagisawa. 2009. KLF4 suppresses estrogen-dependent breast cancer growth by inhibiting the transcriptional activity of ER $\alpha$. Oncogene 28:2894-2902.

143. Wang, B., M. Z. Zhao, N. P. Cui, D. D. Lin, A. Y. Zhang, Y. Qin, C. Y. Liu, W. T. Yan, J. H. Shi, and B. P. Chen. 2015. Krüppel-like factor 4 induces apoptosis and inhibits tumorigenic progression in SK-BR-3 breast cancer cells. FEBS Open. Bio 5:147-154.

144. Pandya, A. Y., L. I. Talley, A. R. Frost, T. J. Fitzgerald, V. Trivedi, M. Chakravarthy, D. C. Chhieng, W. E. Grizzle, J. A. Engler, H. Krontiras, K. I. Bland, A. F. Lobuglio, S. M. Lobo-Ruppert, and J. M. Ruppert. 2004. Nuclear localization of KLF4 is associated with an aggressive phenotype in early-stage breast cancer. Clin. Cancer Res. 10:27092719.

145. Hu, D., Z. Zhou, N. E. Davidson, Y. Huang, and Y. Wan. 2012. Novel insight into KLF4 proteolytic regulation in estrogen receptor signaling and breast carcinogenesis. $\mathbf{J}$ Biol. Chem. 287:13584-13597.

146. Moon, J. S., H. E. Kim, E. Koh, S. H. Park, W. J. Jin, B. W. Park, S. W. Park, and K. S. Kim. 2011. Krüppel-like factor 4 (KLF4) activates the transcription of the gene for the platelet isoform of phosphofructokinase (PFKP) in breast cancer. J Biol. Chem. 286:23808-23816.

147. Yu, F., J. Li, H. Chen, J. Fu, S. Ray, S. Huang, H. Zheng, and W. Ai. 2011. Krüppellike factor 4 (KLF4) is required for maintenance of breast cancer stem cells and for cell migration and invasion. Oncogene 30:2161-2172.

148. Yu, F., Y. Shi, J. Wang, J. Li, D. Fan, and W. Ai. 2013. Deficiency of Krüppel-like factor KLF4 in mammary tumor cells inhibits tumor growth and pulmonary metastasis 
and is accompanied by compromised recruitment of myeloid-derived suppressor cells. Int J Cancer 133:2872-2883.

149. Liu, Z., L. Teng, S. K. Bailey, A. R. Frost, K. I. Bland, A. F. Lobuglio, J. M. Ruppert, and S. M. Lobo-Ruppert. 2009. Epithelial transformation by KLF4 requires Notch1 but not canonical Notch1 signaling. Cancer Biol. Ther. 8:1840-1851.

150. Demehri, S., A. Turkoz, and R. Kopan. 2009. Epidermal Notch1 loss promotes skin tumorigenesis by impacting the stromal microenvironment. Cancer Cell 16:55-66.

151. Lee, Y. S. and A. Dutta. 2009. MicroRNAs in cancer. Annu. Rev. Pathol. 4:199-227.

152. Iorio, M. V., M. Ferracin, C. G. Liu, A. Veronese, R. Spizzo, S. Sabbioni, E. Magri, M. Pedriali, M. Fabbri, M. Campiglio, S. Menard, J. P. Palazzo, A. Rosenberg, P. Musiani, S. Volinia, I. Nenci, G. A. Calin, P. Querzoli, M. Negrini, and C. M. Croce. 2005. MicroRNA gene expression deregulation in human breast cancer. Cancer Res 65:70657070 .

153. Kasinski, A. L. and F. J. Slack. 2011. Epigenetics and genetics. MicroRNAs en route to the clinic: progress in validating and targeting microRNAs for cancer therapy. Nat. Rev. Cancer 11:849-864.

154. Iorio, M. V. and C. M. Croce. 2012. MicroRNA dysregulation in cancer: diagnostics, monitoring and therapeutics. A comprehensive review. EMBO Mol. Med. 4:143-159.

155. He, L. and G. J. Hannon. 2004. MicroRNAs: small RNAs with a big role in gene regulation. Nat. Rev. Genet. 5:522-531.

156. Kim, V. N. 2005. MicroRNA biogenesis: coordinated cropping and dicing. Nat. Rev. Mol. Cell Biol. 6:376-385.

157. Chen, K. and N. Rajewsky. 2007. The evolution of gene regulation by transcription factors and microRNAs. Nat. Rev. Genet. 8:93-103.

158. Ameres, S. L. and P. D. Zamore. 2013. Diversifying microRNA sequence and function. Nat. Rev. Mol. Cell Biol. 14:475-488.

159. Wightman, B., T. R. Burglin, J. Gatto, P. Arasu, and G. Ruvkun. 1991. Negative regulatory sequences in the lin-14 3'-untranslated region are necessary to generate a temporal switch during Caenorhabditis elegans development. Genes Dev. 5:1813-1824.

160. Lee, R. C., R. L. Feinbaum, and V. Ambros. 1993. The C. elegans heterochronic gene lin-4 encodes small RNAs with antisense complementarity to lin-14. Cell 75:843-854.

161. Calin, G. A. and C. M. Croce. 2006. MicroRNA-cancer connection: the beginning of a new tale. Cancer Res 66:7390-7394. 
162. Calin, G. A. and C. M. Croce. 2006. MicroRNA signatures in human cancers. Nat. Rev. Cancer 6:857-866.

163. Esquela-Kerscher, A. and F. J. Slack. 2006. Oncomirs - microRNAs with a role in cancer. Nat. Rev. Cancer 6:259-269.

164. Lee, Y., M. Kim, J. Han, K. H. Yeom, S. Lee, S. H. Baek, and V. N. Kim. 2004. MicroRNA genes are transcribed by RNA polymerase II. EMBO J 23:4051-4060.

165. Rodriguez, A., S. Griffiths-Jones, J. L. Ashurst, and A. Bradley. 2004. Identification of mammalian microRNA host genes and transcription units. Genome Res 14:1902-1910.

166. Borchert, G. M., W. Lanier, and B. L. Davidson. 2006. RNA polymerase III transcribes human microRNAs. Nat. Struct. Mol. Biol. 13:1097-1101.

167. Kim, V. N., J. Han, and M. C. Siomi. 2009. Biogenesis of small RNAs in animals. Nat. Rev. Mol. Cell Biol. 10:126-139.

168. Lee, Y., C. Ahn, J. Han, H. Choi, J. Kim, J. Yim, J. Lee, P. Provost, O. Radmark, S. Kim, and V. N. Kim. 2003. The nuclear RNase III Drosha initiates microRNA processing. Nature 425:415-419.

169. Denli, A. M., B. B. Tops, R. H. Plasterk, R. F. Ketting, and G. J. Hannon. 2004. Processing of primary microRNAs by the Microprocessor complex. Nature 432:231235.

170. Gregory, R. I., K. P. Yan, G. Amuthan, T. Chendrimada, B. Doratotaj, N. Cooch, and R. Shiekhattar. 2004. The Microprocessor complex mediates the genesis of microRNAs. Nature 432:235-240.

171. Landthaler, M., A. Yalcin, and T. Tuschl. 2004. The human DiGeorge syndrome critical region gene 8 and Its D. melanogaster homolog are required for miRNA biogenesis. Curr. Biol. 14:2162-2167.

172. Han, J., Y. Lee, K. H. Yeom, Y. K. Kim, H. Jin, and V. N. Kim. 2004. The DroshaDGCR8 complex in primary microRNA processing. Genes Dev. 18:3016-3027.

173. Han, J., Y. Lee, K. H. Yeom, J. W. Nam, I. Heo, J. K. Rhee, S. Y. Sohn, Y. Cho, B. T. Zhang, and V. N. Kim. 2006. Molecular basis for the recognition of primary microRNAs by the Drosha-DGCR8 complex. Cell 125:887-901.

174. Wu, H., H. Xu, L. J. Miraglia, and S. T. Crooke. 2000. Human RNase III is a 160-kDa protein involved in preribosomal RNA processing. J. Biol. Chem. 275:36957-36965.

175. Bernstein, E., A. A. Caudy, S. M. Hammond, and G. J. Hannon. 2001. Role for a bidentate ribonuclease in the initiation step of RNA interference. Nature 409:363-366. 
176. Ketting, R. F., S. E. Fischer, E. Bernstein, T. Sijen, G. J. Hannon, and R. H. Plasterk. 2001. Dicer functions in RNA interference and in synthesis of small RNA involved in developmental timing in C. elegans. Genes Dev. 15:2654-2659.

177. Provost, P., D. Dishart, J. Doucet, D. Frendewey, B. Samuelsson, and O. Radmark. 2002. Ribonuclease activity and RNA binding of recombinant human Dicer. EMBO J. 21:5864-5874.

178. Lee, Y., K. Jeon, J. T. Lee, S. Kim, and V. N. Kim. 2002. MicroRNA maturation: stepwise processing and subcellular localization. EMBO J. 21:4663-4670.

179. Bartel, D. P. 2009. MicroRNAs: target recognition and regulatory functions. Cell 136:215-233.

180. Czech, B. and G. J. Hannon. 2011. Small RNA sorting: matchmaking for Argonautes. Nat. Rev. Genet. 12:19-31.

181. Yang, J. S. and E. C. Lai. 2011. Alternative miRNA biogenesis pathways and the interpretation of core miRNA pathway mutants. Mol. Cell 43:892-903.

182. Ambros, V., B. Bartel, D. P. Bartel, C. B. Burge, J. C. Carrington, X. Chen, G. Dreyfuss, S. R. Eddy, S. Griffiths-Jones, M. Marshall, M. Matzke, G. Ruvkun, and T. Tuschl. 2003. A uniform system for microRNA annotation. RNA. 9:277-279.

183. Griffiths-Jones, S., R. J. Grocock, S. van Dongen, A. Bateman, and A. J. Enright. 2006. miRBase: microRNA sequences, targets and gene nomenclature. Nucleic Acids Res. 34:D140-D144.

184. Kapp, L. D. and J. R. Lorsch. 2004. The molecular mechanics of eukaryotic translation. Annu. Rev. Biochem. 73:657-704.

185. Gebauer, F. and M. W. Hentze. 2004. Molecular mechanisms of translational control. Nat. Rev. Mol. Cell Biol. 5:827-835.

186. Jackson, R. J., C. U. Hellen, and T. V. Pestova. 2010. The mechanism of eukaryotic translation initiation and principles of its regulation. Nat. Rev. Mol. Cell Biol. 11:113127.

187. Kozak, M. 1987. An analysis of 5'-noncoding sequences from 699 vertebrate messenger RNAs. Nucleic Acids Res. 15:8125-8148.

188. Kozak, M. 1995. Adherence to the first-AUG rule when a second AUG codon follows closely upon the first. Proc. Natl. Acad. Sci. USA 92:2662-2666.

189. Kozak, M. 2002. Pushing the limits of the scanning mechanism for initiation of translation. Gene 299:1-34. 
190. Raught, B. and A. C. Gingras. 1999. eIF4E activity is regulated at multiple levels. Int. J. Biochem. Cell Biol. 31:43-57.

191. Gingras, A. C., B. Raught, and N. Sonenberg. 2001. Control of translation by the target of rapamycin proteins. Prog. Mol. Subcell. Biol. 27:143-174.

192. Hamilton, T. L., M. Stoneley, K. A. Spriggs, and M. Bushell. 2006. TOPs and their regulation. Biochem. Soc. Trans. 34:12-16.

193. Sachs, A. B. and R. W. Davis. 1989. The poly(A) binding protein is required for poly(A) shortening and 60S ribosomal subunit-dependent translation initiation. Cell 58:857-867.

194. Kahvejian, A., Y. V. Svitkin, R. Sukarieh, M. N. M'Boutchou, and N. Sonenberg. 2005. Mammalian poly(A)-binding protein is a eukaryotic translation initiation factor, which acts via multiple mechanisms. Genes Dev. 19:104-113.

195. Parker, R. and H. Song. 2004. The enzymes and control of eukaryotic mRNA turnover. Nat. Struct. Mol. Biol. 11:121-127.

196. Wilusz, C. J., M. Wormington, and S. W. Peltz. 2001. The cap-to-tail guide to mRNA turnover. Nat. Rev. Mol. Cell Biol. 2:237-246.

197. Fasken, M. B. and A. H. Corbett. 2005. Process or perish: quality control in mRNA biogenesis. Nat. Struct. Mol. Biol. 12:482-488.

198. Tomari, Y. and P. D. Zamore. 2005. MicroRNA biogenesis: drosha can't cut it without a partner. Curr. Biol. 15:R61-R64.

199. Tomari, Y. and P. D. Zamore. 2005. Perspective: machines for RNAi. Genes Dev 19:517-529.

200. Amrani, N., M. S. Sachs, and A. Jacobson. 2006. Early nonsense: mRNA decay solves a translational problem. Nat. Rev. Mol. Cell Biol. 7:415-425.

201. van Hoof, A., P. A. Frischmeyer, H. C. Dietz, and R. Parker. 2002. Exosome-mediated recognition and degradation of mRNAs lacking a termination codon. Science 295:22622264.

202. Frischmeyer, P. A., H. A. van, K. O'Donnell, A. L. Guerrerio, R. Parker, and H. C. Dietz. 2002. An mRNA surveillance mechanism that eliminates transcripts lacking termination codons. Science 295:2258-2261.

203. Doma, M. K. and R. Parker. 2006. Endonucleolytic cleavage of eukaryotic mRNAs with stalls in translation elongation. Nature 440:561-564. 
204. Meister, G., M. Landthaler, A. Patkaniowska, Y. Dorsett, G. Teng, and T. Tuschl. 2004. Human Argonaute2 mediates RNA cleavage targeted by miRNAs and siRNAs. Mol. Cell 15:185-197.

205. Mathonnet, G., M. R. Fabian, Y. V. Svitkin, A. Parsyan, L. Huck, T. Murata, S. Biffo, W. C. Merrick, E. Darzynkiewicz, R. S. Pillai, W. Filipowicz, T. F. Duchaine, and N. Sonenberg. 2007. MicroRNA inhibition of translation initiation in vitro by targeting the cap-binding complex eIF4F. Science 317:1764-1767.

206. Pillai, R. S., S. N. Bhattacharyya, C. G. Artus, T. Zoller, N. Cougot, E. Basyuk, E. Bertrand, and W. Filipowicz. 2005. Inhibition of translational initiation by Let-7 MicroRNA in human cells. Science 309:1573-1576.

207. Pillai, R. S., S. N. Bhattacharyya, and W. Filipowicz. 2007. Repression of protein synthesis by miRNAs: how many mechanisms? Trends Cell Biol. 17:118-126.

208. Kiriakidou, M., G. S. Tan, S. Lamprinaki, M. De Planell-Saguer, P. T. Nelson, and Z. Mourelatos. 2007. An mRNA m7G cap binding-like motif within human Ago2 represses translation. Cell 129:1141-1151.

209. Humphreys, D. T., B. J. Westman, D. I. Martin, and T. Preiss. 2005. MicroRNAs control translation initiation by inhibiting eukaryotic initiation factor 4E/cap and poly(A) tail function. Proc. Natl. Acad. Sci. U. S. A 102:16961-16966.

210. Wakiyama, M., K. Takimoto, O. Ohara, and S. Yokoyama. 2007. Let-7 microRNAmediated mRNA deadenylation and translational repression in a mammalian cell-free system. Genes Dev 21:1857-1862.

211. Bagga, S., J. Bracht, S. Hunter, K. Massirer, J. Holtz, R. Eachus, and A. E. Pasquinelli. 2005. Regulation by let-7 and lin-4 miRNAs results in target mRNA degradation. Cell 122:553-563.

212. Wu, L. and J. G. Belasco. 2005. Micro-RNA regulation of the mammalian lin-28 gene during neuronal differentiation of embryonal carcinoma cells. Mol. Cell Biol. 25:91989208 .

213. Eulalio, A., I. Behm-Ansmant, D. Schweizer, and E. Izaurralde. 2007. P-body formation is a consequence, not the cause, of RNA-mediated gene silencing. Mol. Cell Biol. 27:3970-3981.

214. Chendrimada, T. P., K. J. Finn, X. Ji, D. Baillat, R. I. Gregory, S. A. Liebhaber, A. E. Pasquinelli, and R. Shiekhattar. 2007. MicroRNA silencing through RISC recruitment of eIF6. Nature 447:823-828.

215. Wang, B., T. M. Love, M. E. Call, J. G. Doench, and C. D. Novina. 2006.

Recapitulation of short RNA-directed translational gene silencing in vitro. Mol. Cell 22:553-560. 
216. Lim, L. P., N. C. Lau, P. Garrett-Engele, A. Grimson, J. M. Schelter, J. Castle, D. P. Bartel, P. S. Linsley, and J. M. Johnson. 2005. Microarray analysis shows that some microRNAs downregulate large numbers of target mRNAs. Nature 433:769-773.

217. Jing, Q., S. Huang, S. Guth, T. Zarubin, A. Motoyama, J. Chen, P. F. Di, S. C. Lin, H. Gram, and J. Han. 2005. Involvement of microRNA in AU-rich element-mediated mRNA instability. Cell 120:623-634.

218. Baek, D., J. Villen, C. Shin, F. D. Camargo, S. P. Gygi, and D. P. Bartel. 2008. The impact of microRNAs on protein output. Nature 455:64-71.

219. Selbach, M., B. Schwanhausser, N. Thierfelder, Z. Fang, R. Khanin, and N. Rajewsky. 2008. Widespread changes in protein synthesis induced by microRNAs. Nature 455:5863.

220. Guo, H., N. T. Ingolia, J. S. Weissman, and D. P. Bartel. 2010. Mammalian microRNAs predominantly act to decrease target mRNA levels. Nature 466:835-840.

221. Giraldez, A. J., Y. Mishima, J. Rihel, R. J. Grocock, D. S. Van, K. Inoue, A. J. Enright, and A. F. Schier. 2006. Zebrafish MiR-430 promotes deadenylation and clearance of maternal mRNAs. Science 312:75-79.

222. Rehwinkel, J., P. Natalin, A. Stark, J. Brennecke, S. M. Cohen, and E. Izaurralde. 2006. Genome-wide analysis of mRNAs regulated by Drosha and Argonaute proteins in Drosophila melanogaster. Mol. Cell Biol. 26:2965-2975.

223. Schmitter, D., J. Filkowski, A. Sewer, R. S. Pillai, E. J. Oakeley, M. Zavolan, P. Svoboda, and W. Filipowicz. 2006. Effects of Dicer and Argonaute down-regulation on mRNA levels in human HEK293 cells. Nucleic Acids Res 34:4801-4815.

224. Behm-Ansmant, I., J. Rehwinkel, T. Doerks, A. Stark, P. Bork, and E. Izaurralde. 2006. mRNA degradation by miRNAs and GW182 requires both CCR4:NOT deadenylase and DCP1:DCP2 decapping complexes. Genes Dev 20:1885-1898.

225. Eulalio, A., E. Huntzinger, and E. Izaurralde. 2008. GW182 interaction with Argonaute is essential for miRNA-mediated translational repression and mRNA decay. Nat. Struct. Mol. Biol. 15:346-353.

226. Eulalio, A., F. Tritschler, and E. Izaurralde. 2009. The GW182 protein family in animal cells: new insights into domains required for miRNA-mediated gene silencing. RNA. 15:1433-1442.

227. Bruno, I. G., R. Karam, L. Huang, A. Bhardwaj, C. H. Lou, E. Y. Shum, H. W. Song, M. A. Corbett, W. D. Gifford, J. Gecz, S. L. Pfaff, and M. F. Wilkinson. 2011. Identification of a microRNA that activates gene expression by repressing nonsensemediated RNA decay. Mol. Cell 42:500-510. 
228. Jopling, C. L., M. Yi, A. M. Lancaster, S. M. Lemon, and P. Sarnow. 2005. Modulation of hepatitis C virus RNA abundance by a liver-specific MicroRNA. Science 309:15771581 .

229. Lanford, R. E., E. S. Hildebrandt-Eriksen, A. Petri, R. Persson, M. Lindow, M. E. Munk, S. Kauppinen, and H. Orum. 2010. Therapeutic silencing of microRNA-122 in primates with chronic hepatitis C virus infection. Science 327:198-201.

230. Jangra, R. K., M. Yi, and S. M. Lemon. 2010. DDX6 (Rck/p54) is required for efficient hepatitis $\mathrm{C}$ virus replication but not for internal ribosome entry site-directed translation. J. Virol. 84:6810-6824.

231. Steitz, J. A. and S. Vasudevan. 2009. miRNPs: versatile regulators of gene expression in vertebrate cells. Biochem. Soc. Trans. 37:931-935.

232. Truesdell, S. S., R. D. Mortensen, M. Seo, J. C. Schroeder, J. H. Lee, O. LeTonqueze, and S. Vasudevan. 2012. MicroRNA-mediated mRNA translation activation in quiescent cells and oocytes involves recruitment of a nuclear microRNP. Sci Rep. 2:842.

233. Vasudevan, S. and J. A. Steitz. 2007. AU-rich-element-mediated upregulation of translation by FXR1 and Argonaute 2. Cell 128:1105-1118.

234. Vasudevan, S., Y. Tong, and J. A. Steitz. 2008. Cell-cycle control of microRNAmediated translation regulation. Cell Cycle 7:1545-1549.

235. Vasudevan, S., Y. Tong, and J. A. Steitz. 2007. Switching from repression to activation: microRNAs can up-regulate translation. Science 318:1931-1934.

236. Orom, U. A., F. C. Nielsen, and A. H. Lund. 2008. MicroRNA-10a binds the 5'UTR of ribosomal protein mRNAs and enhances their translation. Mol. Cell 30:460-471.

237. Sayed, D. and M. Abdellatif. 2011. MicroRNAs in development and disease. Physiol Rev. 91:827-887.

238. Betel, D., M. Wilson, A. Gabow, D. S. Marks, and C. Sander. 2008. The microRNA.org resource: targets and expression. Nucleic Acids Res. 36:D149-D153.

239. Friedman, R. C., K. K. Farh, C. B. Burge, and D. P. Bartel. 2009. Most mammalian mRNAs are conserved targets of microRNAs. Genome Res. 19:92-105.

240. Grimson, A., K. K. Farh, W. K. Johnston, P. Garrett-Engele, L. P. Lim, and D. P. Bartel. 2007. MicroRNA targeting specificity in mammals: determinants beyond seed pairing. Mol. Cell 27:91-105.

241. Lewis, B. P., C. B. Burge, and D. P. Bartel. 2005. Conserved seed pairing, often flanked by adenosines, indicates that thousands of human genes are microRNA targets. Cell. 120:15-20. 
242. Ding, Y., C. Y. Chan, and C. E. Lawrence. 2004. Sfold web server for statistical folding and rational design of nucleic acids. Nucleic Acids Res. 32:W135-W141.

243. Long, D., R. Lee, P. Williams, C. Y. Chan, V. Ambros, and Y. Ding. 2007. Potent effect of target structure on microRNA function. Nat. Struct. Mol. Biol. 14:287-294.

244. Sun, K. and E. C. Lai. 2013. Adult-specific functions of animal microRNAs. Nat. Rev. Genet. 14:535-548.

245. Iorio, M. V., M. Ferracin, C. G. Liu, A. Veronese, R. Spizzo, S. Sabbioni, E. Magri, M. Pedriali, M. Fabbri, M. Campiglio, S. Menard, J. P. Palazzo, A. Rosenberg, P. Musiani, S. Volinia, I. Nenci, G. A. Calin, P. Querzoli, M. Negrini, and C. M. Croce. 2005. MicroRNA gene expression deregulation in human breast cancer. Cancer Res. 65:70657070 .

246. Volinia, S., G. A. Calin, C. G. Liu, S. Ambs, A. Cimmino, F. Petrocca, R. Visone, M. Iorio, C. Roldo, M. Ferracin, R. L. Prueitt, N. Yanaihara, G. Lanza, A. Scarpa, A. Vecchione, M. Negrini, C. C. Harris, and C. M. Croce. 2006. A microRNA expression signature of human solid tumors defines cancer gene targets. Proc Natl Acad Sci U S A 103:2257-2261.

247. Zhang, B., X. Pan, G. P. Cobb, and T. A. Anderson. 2007. microRNAs as oncogenes and tumor suppressors. Dev Biol. 302:1-12.

248. Calin, G. A., C. D. Dumitru, M. Shimizu, R. Bichi, S. Zupo, E. Noch, H. Aldler, S. Rattan, M. Keating, K. Rai, L. Rassenti, T. Kipps, M. Negrini, F. Bullrich, and C. M. Croce. 2002. Frequent deletions and down-regulation of micro- RNA genes miR15 and miR16 at 13q14 in chronic lymphocytic leukemia. Proc. Natl. Acad. Sci. U. S. A 99:15524-15529.

249. Calin, G. A., C. Sevignani, C. D. Dumitru, T. Hyslop, E. Noch, S. Yendamuri, M. Shimizu, S. Rattan, F. Bullrich, M. Negrini, and C. M. Croce. 2004. Human microRNA genes are frequently located at fragile sites and genomic regions involved in cancers. Proc. Natl. Acad. Sci. U. S. A 101:2999-3004.

250. Cimmino, A., G. A. Calin, M. Fabbri, M. V. Iorio, M. Ferracin, M. Shimizu, S. E. Wojcik, R. I. Aqeilan, S. Zupo, M. Dono, L. Rassenti, H. Alder, S. Volinia, C. G. Liu, T. J. Kipps, M. Negrini, and C. M. Croce. 2005. miR-15 and miR-16 induce apoptosis by targeting BCL2. Proc. Natl. Acad. Sci. U. S. A 102:13944-13949.

251. Takamizawa, J., H. Konishi, K. Yanagisawa, S. Tomida, H. Osada, H. Endoh, T. Harano, Y. Yatabe, M. Nagino, Y. Nimura, T. Mitsudomi, and T. Takahashi. 2004. Reduced expression of the let-7 microRNAs in human lung cancers in association with shortened postoperative survival. Cancer Res. 64:3753-3756.

252. Johnson, S. M., H. Grosshans, J. Shingara, M. Byrom, R. Jarvis, A. Cheng, E. Labourier, K. L. Reinert, D. Brown, and F. J. Slack. 2005. RAS is regulated by the let-7 microRNA family. Cell 120:635-647. 
253. Misso, G., M. T. Di Martino, R. G. De, A. A. Farooqi, A. Lombardi, V. Campani, M. R. Zarone, A. Gulla, P. Tagliaferri, P. Tassone, and M. Caraglia. 2014. Mir-34: a new weapon against cancer? Mol. Ther Nucleic Acids 3:e194.

254. Yamakuchi, M., M. Ferlito, and C. J. Lowenstein. 2008. miR-34a repression of SIRT1 regulates apoptosis. Proc. Natl. Acad. Sci. U. S. A 105:13421-13426.

255. Akao, Y., Y. Nakagawa, I. Hirata, A. Iio, T. Itoh, K. Kojima, R. Nakashima, Y. Kitade, and T. Naoe. 2010. Role of anti-oncomirs miR-143 and -145 in human colorectal tumors. Cancer Gene Ther. 17:398-408.

256. Pagliuca, A., C. Valvo, E. Fabrizi, M. S. di, M. Biffoni, D. Runci, S. Forte, M. R. De, and L. Ricci-Vitiani. 2013. Analysis of the combined action of miR-143 and miR-145 on oncogenic pathways in colorectal cancer cells reveals a coordinate program of gene repression. Oncogene 32:4806-4813.

257. Zhu, H., U. Dougherty, V. Robinson, R. Mustafi, J. Pekow, S. Kupfer, Y. C. Li, J. Hart, K. Goss, A. Fichera, L. Joseph, and M. Bissonnette. 2011. EGFR signals downregulate tumor suppressors miR-143 and miR-145 in Western diet-promoted murine colon cancer: role of G1 regulators. Mol. Cancer Res. 9:960-975.

258. Kent, O. A., R. R. Chivukula, M. Mullendore, E. A. Wentzel, G. Feldmann, K. H. Lee, S. Liu, S. D. Leach, A. Maitra, and J. T. Mendell. 2010. Repression of the miR-143/145 cluster by oncogenic Ras initiates a tumor-promoting feed-forward pathway. Genes Dev. 24:2754-2759.

259. Kojima, S., H. Enokida, H. Yoshino, T. Itesako, T. Chiyomaru, T. Kinoshita, M. Fuse, R. Nishikawa, Y. Goto, Y. Naya, M. Nakagawa, and N. Seki. 2014. The tumorsuppressive microRNA-143/145 cluster inhibits cell migration and invasion by targeting GOLM1 in prostate cancer. J. Hum. Genet. 59:78-87.

260. Hayashita, Y., H. Osada, Y. Tatematsu, H. Yamada, K. Yanagisawa, S. Tomida, Y. Yatabe, K. Kawahara, Y. Sekido, and T. Takahashi. 2005. A polycistronic microRNA cluster, miR-17-92, is overexpressed in human lung cancers and enhances cell proliferation. Cancer Res. 65:9628-9632.

261. Voorhoeve, P. M., C. le Sage, M. Schrier, A. J. Gillis, H. Stoop, R. Nagel, Y. P. Liu, J. van Duijse, J. Drost, A. Griekspoor, E. Zlotorynski, N. Yabuta, G. De Vita, H. Nojima, L. H. Looijenga, and R. Agami. 2006. A genetic screen implicates miRNA-372 and miRNA-373 as oncogenes in testicular germ cell tumors. Cell 124:1169-1181.

262. Eis, P. S., W. Tam, L. Sun, A. Chadburn, Z. Li, M. F. Gomez, E. Lund, and J. E. Dahlberg. 2005. Accumulation of miR-155 and BIC RNA in human B cell lymphomas. Proc Natl Acad Sci U S A 102:3627-3632.

263. Garofalo, M., C. Quintavalle, G. Romano, C. M. Croce, and G. Condorelli. 2012. miR221/222 in cancer: their role in tumor progression and response to therapy. Curr. Mol. Med. 12:27-33. 
264. Chan, J. A., A. M. Krichevsky, and K. S. Kosik. 2005. MicroRNA-21 is an antiapoptotic factor in human glioblastoma cells. Cancer Res. 65:6029-6033.

265. Qian, B., D. Katsaros, L. Lu, M. Preti, A. Durando, R. Arisio, L. Mu, and H. Yu. 2009. High miR-21 expression in breast cancer associated with poor disease-free survival in early stage disease and high TGF-beta1. Breast Cancer Res. Treat. 117:131-140.

266. Yan, L. X., Q. N. Wu, Y. Zhang, Y. Y. Li, D. Z. Liao, J. H. Hou, J. Fu, M. S. Zeng, J. P. Yun, Q. L. Wu, Y. X. Zeng, and J. Y. Shao. 2011. Knockdown of miR-21 in human breast cancer cell lines inhibits proliferation, in vitro migration and in vivo tumor growth. Breast Cancer Res. 13:R2.

267. Gabriely, G., T. Wurdinger, S. Kesari, C. C. Esau, J. Burchard, P. S. Linsley, and A. M. Krichevsky. 2008. MicroRNA 21 promotes glioma invasion by targeting matrix metalloproteinase regulators. Mol. Cell Biol. 28:5369-5380.

268. Selaru, F. M., A. V. Olaru, T. Kan, S. David, Y. Cheng, Y. Mori, J. Yang, B. Paun, Z. Jin, R. Agarwal, J. P. Hamilton, J. Abraham, C. Georgiades, H. Alvarez, P. Vivekanandan, W. Yu, A. Maitra, M. Torbenson, P. J. Thuluvath, G. J. Gores, N. F. LaRusso, R. Hruban, and S. J. Meltzer. 2009. MicroRNA-21 is overexpressed in human cholangiocarcinoma and regulates programmed cell death 4 and tissue inhibitor of metalloproteinase 3. Hepatology 49:1595-1601.

269. Corsten, M. F., R. Miranda, R. Kasmieh, A. M. Krichevsky, R. Weissleder, and K. Shah. 2007. MicroRNA-21 knockdown disrupts glioma growth in vivo and displays synergistic cytotoxicity with neural precursor cell delivered S-TRAIL in human gliomas. Cancer Res. 67:8994-9000.

270. Meng, F., R. Henson, H. Wehbe-Janek, K. Ghoshal, S. T. Jacob, and T. Patel. 2007. MicroRNA-21 regulates expression of the PTEN tumor suppressor gene in human hepatocellular cancer. Gastroenterology 133:647-658.

271. Meng, F., R. Henson, M. Lang, H. Wehbe, S. Maheshwari, J. T. Mendell, J. Jiang, T. D. Schmittgen, and T. Patel. 2006. Involvement of human micro-RNA in growth and response to chemotherapy in human cholangiocarcinoma cell lines. Gastroenterology 130:2113-2129.

272. Kadera, B. E., L. Li, P. A. Toste, N. Wu, C. Adams, D. W. Dawson, and T. R. Donahue. 2013. MicroRNA-21 in pancreatic ductal adenocarcinoma tumor-associated fibroblasts promotes metastasis. PLoS. One. 8:e71978.

273. Yu, J., A. Li, S. M. Hong, R. H. Hruban, and M. Goggins. 2012. MicroRNA alterations of pancreatic intraepithelial neoplasias. Clin. Cancer Res. 18:981-992.

274. Markou, A., E. G. Tsaroucha, L. Kaklamanis, M. Fotinou, V. Georgoulias, and E. S. Lianidou. 2008. Prognostic value of mature microRNA-21 and microRNA-205 overexpression in non-small cell lung cancer by quantitative real-time RT-PCR. Clin. Chem. 54:1696-1704. 
275. Gao, W., Y. Yu, H. Cao, H. Shen, X. Li, S. Pan, and Y. Shu. 2010. Deregulated expression of miR-21, miR-143 and miR-181a in non small cell lung cancer is related to clinicopathologic characteristics or patient prognosis. Biomed. Pharmacother. 64:399-408.

276. Zhang, J. G., J. J. Wang, F. Zhao, Q. Liu, K. Jiang, and G. H. Yang. 2010. MicroRNA21 (miR-21) represses tumor suppressor PTEN and promotes growth and invasion in non-small cell lung cancer (NSCLC). Clin. Chim. Acta 411:846-852.

277. Li, J., H. Huang, L. Sun, M. Yang, C. Pan, W. Chen, D. Wu, Z. Lin, C. Zeng, Y. Yao, P. Zhang, and E. Song. 2009. MiR-21 indicates poor prognosis in tongue squamous cell carcinomas as an apoptosis inhibitor. Clin. Cancer Res. 15:3998-4008.

278. Yamamichi, N., R. Shimomura, K. Inada, K. Sakurai, T. Haraguchi, Y. Ozaki, S. Fujita, T. Mizutani, C. Furukawa, M. Fujishiro, M. Ichinose, K. Shiogama, Y. Tsutsumi, M. Omata, and H. Iba. 2009. Locked nucleic acid in situ hybridization analysis of miR-21 expression during colorectal cancer development. Clin. Cancer Res. 15:4009-4016.

279. Schmitz, K. J., S. Hey, A. Schinwald, J. Wohlschlaeger, H. A. Baba, K. Worm, and K. W. Schmid. 2009. Differential expression of microRNA 181b and microRNA 21 in hyperplastic polyps and sessile serrated adenomas of the colon. Virchows Arch. 455:49-54.

280. Pan, Y., M. Meng, G. Zhang, H. Han, and Q. Zhou. 2014. Oncogenic MicroRNAs in the Genesis of Leukemia and Lymphoma. Curr. Pharm. Des.

281. Seca, H., R. T. Lima, V. Lopes-Rodrigues, J. E. Guimaraes, G. M. Almeida, and M. H. Vasconcelos. 2013. Targeting miR-21 induces autophagy and chemosensitivity of leukemia cells. Curr. Drug Targets. 14:1135-1143.

282. Tagawa, H., S. Ikeda, and K. Sawada. 2013. Role of microRNA in the pathogenesis of malignant lymphoma. Cancer Sci. 104:801-809.

283. Yao, Q., H. Xu, Q. Q. Zhang, H. Zhou, and L. H. Qu. 2009. MicroRNA-21 promotes cell proliferation and down-regulates the expression of programmed cell death 4 (PDCD4) in HeLa cervical carcinoma cells. Biochem. Biophys. Res. Commun. 388:539-542.

284. Li, T., D. Li, J. Sha, P. Sun, and Y. Huang. 2009. MicroRNA-21 directly targets MARCKS and promotes apoptosis resistance and invasion in prostate cancer cells. Biochem. Biophys. Res. Commun. 383:280-285.

285. Xu, N., T. Papagiannakopoulos, G. Pan, J. A. Thomson, and K. S. Kosik. 2009. MicroRNA-145 Regulates OCT4, SOX2, and KLF4 and Represses Pluripotency in Human Embryonic Stem Cells. Cell 137:647-658. 
286. Xie, C., H. Huang, X. Sun, Y. Guo, M. Hamblin, R. P. Ritchie, M. T. Garcia-Barrio, J. Zhang, and Y. E. Chen. 2010. MicroRNA-1 Regulates Smooth Muscle Cell Differentiation by Repressing KLF4. Stem Cells Dev in press.

287. Burk, U., J. Schubert, U. Wellner, O. Schmalhofer, E. Vincan, S. Spaderna, and T. Brabletz. 2008. A reciprocal repression between ZEB1 and members of the miR-200 family promotes EMT and invasion in cancer cells. EMBO Rep. 9:582-589.

288. Wellner, U., J. Schubert, U. C. Burk, O. Schmalhofer, F. Zhu, A. Sonntag, B. Waldvogel, C. Vannier, D. Darling, A. Z. Hausen, V. G. Brunton, J. Morton, O. Sansom, J. Schuler, M. P. Stemmler, C. Herzberger, U. Hopt, T. Keck, S. Brabletz, and T. Brabletz. 2009. The EMT-activator ZEB1 promotes tumorigenicity by repressing stemness-inhibiting microRNAs. Nat. Cell Biol. 11:1487-1495.

289. Tian, Y., A. Luo, Y. Cai, Q. Su, F. Ding, H. Chen, and Z. Liu. 2010. MicroRNA-10B promotes human esophageal cancer cell migration and invasion through KLF4. J Biol. Chem. 285:7986-7994.

290. Sun, S. G., B. Zheng, M. Han, X. M. Fang, H. X. Li, S. B. Miao, M. Su, Y. Han, H. J. Shi, and J. K. Wen. 2011. miR-146a and Krüppel-like factor 4 form a feedback loop to participate in vascular smooth muscle cell proliferation. EMBO Rep. 12:56-62.

291. Williams, A. H., G. Valdez, V. Moresi, X. Qi, J. McAnally, J. L. Elliott, R. BasselDuby, J. R. Sanes, and E. N. Olson. 2009. MicroRNA-206 delays ALS progression and promotes regeneration of neuromuscular synapses in mice. Science 326:1549-1554.

292. Dey, B. K., J. Gagan, and A. Dutta. 2011. miR-206 and -486 induce myoblast differentiation by downregulating Pax7. Mol. Cell Biol. 31:203-214.

293. Liu, N., A. H. Williams, J. M. Maxeiner, S. Bezprozvannaya, J. M. Shelton, J. A. Richardson, R. Bassel-Duby, and E. N. Olson. 2012. microRNA-206 promotes skeletal muscle regeneration and delays progression of Duchenne muscular dystrophy in mice. $\mathrm{J}$ Clin Invest 122:2054-2065.

294. Adams, B. D., H. Furneaux, and B. A. White. 2007. The micro-ribonucleic acid (miRNA) miR-206 targets the human estrogen receptor $\alpha(E R \alpha)$ and represses ER $\alpha$ messenger RNA and protein expression in breast cancer cell lines. Mol. Endocrinol. 21:1132-1147.

295. Adams, B. D., D. M. Cowee, and B. A. White. 2009. The role of miR-206 in the epidermal growth factor (EGF) induced repression of estrogen receptor $\alpha(E R \alpha)$ signaling and a luminal phenotype in MCF-7 breast cancer cells. Mol. Endocrinol. 23:1215-1230.

296. Guttilla, I. K., B. D. Adams, and B. A. White. 2012. ERalpha, microRNAs, and the epithelial-mesenchymal transition in breast cancer. Trends Endocrinol. Metab 23:73-82. 
297. Kondo, N., T. Toyama, H. Sugiura, Y. Fujii, and H. Yamashita. 2008. miR-206 Expression is down-regulated in estrogen receptor $\alpha$-positive human breast cancer. Cancer Res 68:5004-5008.

298. Karnoub, A. E. and R. A. Weinberg. 2008. Ras oncogenes: split personalities. Nat. Rev. Mol. Cell Biol. 9:517-531.

299. Cox, A. D. and C. J. Der. 2010. Ras history: The saga continues. Small GTPases. 1:227.

300. Pylayeva-Gupta, Y., E. Grabocka, and D. Bar-Sagi. 2011. RAS oncogenes: weaving a tumorigenic web. Nat. Rev. Cancer 11:761-774.

301. Johnson, G. L. and R. Lapadat. 2002. Mitogen-activated protein kinase pathways mediated by ERK, JNK, and p38 protein kinases. Science 298:1911-1912.

302. Downward, J. 2003. Targeting RAS signalling pathways in cancer therapy. Nat. Rev. Cancer 3:11-22.

303. Dhillon, A. S., S. Hagan, O. Rath, and W. Kolch. 2007. MAP kinase signalling pathways in cancer. Oncogene 26:3279-3290.

304. Kolch, W. 2005. Coordinating ERK/MAPK signalling through scaffolds and inhibitors. Nat. Rev. Mol. Cell Biol. 6:827-837.

305. Mebratu, Y. and Y. Tesfaigzi. 2009. How ERK1/2 activation controls cell proliferation and cell death: Is subcellular localization the answer? Cell Cycle 8:1168-1175.

306. Young, A., J. Lyons, A. L. Miller, V. T. Phan, I. R. Alarcon, and F. Mccormick. 2009. Ras signaling and therapies. Adv. Cancer Res 102:1-17.

307. Bos, J. L. 1989. RAS oncogenes in human cancer: a review. Cancer Res. 49:46824689.

308. Bos, J. L., H. Rehmann, and A. Wittinghofer. 2007. GEFs and GAPs: critical elements in the control of small G proteins. Cell 129:865-877.

309. Schubbert, S., K. Shannon, and G. Bollag. 2007. Hyperactive Ras in developmental disorders and cancer. Nat. Rev. Cancer 7:295-308.

310. Brems, H., M. Chmara, M. Sahbatou, E. Denayer, K. Taniguchi, R. Kato, R. Somers, L. Messiaen, S. S. De, J. P. Fryns, J. Cools, P. Marynen, G. Thomas, A. Yoshimura, and E. Legius. 2007. Germline loss-of-function mutations in SPRED1 cause a neurofibromatosis 1-like phenotype. Nat. Genet. 39:1120-1126.

311. Tidyman, W. E. and K. A. Rauen. 2009. The RASopathies: developmental syndromes of Ras/MAPK pathway dysregulation. Curr. Opin. Genet. Dev. 19:230-236. 
312. Harvey, J. J. 1964. An unidentified virus which causes the rapid production of tumors in mice. Nature 204:1104-1105.

313. Scolnick, E. M. and W. P. Parks. 1974. Harvey sarcoma virus: a second murine type C sarcoma virus with rat genetic information. J. Virol. 13:1211-1219.

314. Scolnick, E. M., E. Rands, D. Williams, and W. P. Parks. 1973. Studies on the nucleic acid sequences of Kirsten sarcoma virus: a model for formation of a mammalian RNAcontaining sarcoma virus. J. Virol. 12:458-463.

315. Kirsten, W. H. and L. A. Mayer. 1967. Morphologic responses to a murine erythroblastosis virus. J. Natl. Cancer Inst. 39:311-335.

316. Scolnick, E. M., A. G. Papageorge, and T. Y. Shih. 1979. Guanine nucleotide-binding activity as an assay for src protein of rat-derived murine sarcoma viruses. Proc. Natl. Acad. Sci. U. S. A 76:5355-5359.

317. Shih, T. Y., M. O. Weeks, H. A. Young, and E. M. Scholnick. 1979. Identification of a sarcoma virus-coded phosphoprotein in nonproducer cells transformed by Kirsten or Harvey murine sarcoma virus. Virology 96:64-79.

318. Ellis, R. W., D. Defeo, T. Y. Shih, M. A. Gonda, H. A. Young, N. Tsuchida, D. R. Lowy, and E. M. Scolnick. 1981. The p21 src genes of Harvey and Kirsten sarcoma viruses originate from divergent members of a family of normal vertebrate genes. Nature 292:506-511.

319. Santos, E., S. R. Tronick, S. A. Aaronson, S. Pulciani, and M. Barbacid. 1982. T24 human bladder carcinoma oncogene is an activated form of the normal human homologue of of BALB- and Harvey-MSV transforming genes. Nature 298:343-347.

320. Chang, E. H., M. E. Furth, E. M. Scolnick, and D. R. Lowy. 1982. Tumorigenic transformation of mammalian cells induced by a normal human gene homologous to the oncogene of Harvey murine sarcoma virus. Nature 297:479-483.

321. Parada, L. F., C. J. Tabin, C. Shih, and R. A. Weinberg. 1982. Human EJ bladder carcinoma oncogene is homologue of Harvey sarcoma virus ras gene. Nature 297:474478.

322. Der, C. J., T. G. Krontiris, and G. M. Cooper. 1982. Transforming genes of human bladder and lung carcinoma cell lines are homologous to the ras genes of Harvey and Kirsten sarcoma viruses. Proc. Natl. Acad. Sci. U. S. A 79:3637-3640.

323. Krontiris, T. G. and G. M. Cooper. 1981. Transforming activity of human tumor DNAs. Proc. Natl. Acad. Sci. U. S. A 78:1181-1184.

324. Shih, C., B. Z. Shilo, M. P. Goldfarb, A. Dannenberg, and R. A. Weinberg. 1979. Passage of phenotypes of chemically transformed cells via transfection of DNA and chromatin. Proc. Natl. Acad. Sci. U. S. A 76:5714-5718. 
325. Taparowsky, E., Y. Suard, O. Fasano, K. Shimizu, M. Goldfarb, and M. Wigler. 1982. Activation of the T24 bladder carcinoma transforming gene is linked to a single amino acid change. Nature 300:762-765.

326. Reddy, E. P., R. K. Reynolds, E. Santos, and M. Barbacid. 1982. A point mutation is responsible for the acquisition of transforming properties by the T24 human bladder carcinoma oncogene. Nature 300:149-152.

327. Tabin, C. J., S. M. Bradley, C. I. Bargmann, R. A. Weinberg, A. G. Papageorge, E. M. Scolnick, R. Dhar, D. R. Lowy, and E. H. Chang. 1982. Mechanism of activation of a human oncogene. Nature 300:143-149.

328. Feig, L. A., R. C. Bast, Jr., R. C. Knapp, and G. M. Cooper. 1984. Somatic activation of rasK gene in a human ovarian carcinoma. Science 223:698-701.

329. Santos, E., D. Martin-Zanca, E. P. Reddy, M. A. Pierotti, P. G. Della, and M. Barbacid. 1984. Malignant activation of a K-ras oncogene in lung carcinoma but not in normal tissue of the same patient. Science 223:661-664.

330. Capon, D. J., P. H. Seeburg, J. P. McGrath, J. S. Hayflick, U. Edman, A. D. Levinson, and D. V. Goeddel. 1983. Activation of Ki-ras2 gene in human colon and lung carcinomas by two different point mutations. Nature 304:507-513.

331. Fotiadou, P. P., C. Takahashi, H. N. Rajabi, and M. E. Ewen. 2007. Wild-type NRas and KRas perform distinct functions during transformation. Mol. Cell Biol. 27:67426755 .

332. Manning, G., D. B. Whyte, R. Martinez, T. Hunter, and S. Sudarsanam. 2002. The protein kinase complement of the human genome. Science 298:1912-1934.

333. Coulombe, P. and S. Meloche. 2007. Atypical mitogen-activated protein kinases: structure, regulation and functions. Biochim. Biophys. Acta 1773:1376-1387.

334. Schlessinger, J. 2000. Cell signaling by receptor tyrosine kinases. Cell 103:211-225.

335. Lemmon, M. A. and J. Schlessinger. 2010. Cell signaling by receptor tyrosine kinases. Cell 141:1117-1134.

336. Gysin, S., M. Salt, A. Young, and F. Mccormick. 2011. Therapeutic strategies for targeting ras proteins. Genes Cancer 2:359-372.

337. Lenzen, C., R. H. Cool, H. Prinz, J. Kuhlmann, and A. Wittinghofer. 1998. Kinetic analysis by fluorescence of the interaction between Ras and the catalytic domain of the guanine nucleotide exchange factor Cdc25Mm. Biochem. 37:7420-7430.

338. Klebe, C., H. Prinz, A. Wittinghofer, and R. S. Goody. 1995. The kinetic mechanism of Ran--nucleotide exchange catalyzed by RCC1. Biochem. 34:12543-12552. 
339. Saraste, M., P. R. Sibbald, and A. Wittinghofer. 1990. The P-loop--a common motif in ATP- and GTP-binding proteins. Trends Biochem. Sci. 15:430-434.

340. Vetter, I. R. and A. Wittinghofer. 2001. The guanine nucleotide-binding switch in three dimensions. Science 294:1299-1304.

341. Scheffzek, K., C. Klebe, K. Fritzwolf, W. Kabsch, and A. Wittinghofer. 1995. Crystal structure of the nuclear Ras-related protein Ran in its GDP-bound form. Nature 374:378-381.

342. Scheffzek, K., M. R. Ahmadian, W. Kabsch, L. Wiesmuller, A. Lautwein, F. Schmitz, and A. Wittinghofer. 1997. The Ras-RasGAP complex: structural basis for GTPase activation and its loss in oncogenic Ras mutants. Science 277:333-338.

343. Willumsen, B. M., A. Christensen, N. L. Hubbert, A. G. Papageorge, and D. R. Lowy. 1984. The $\mathrm{p} 21$ ras $\mathrm{C}$-terminus is required for transformation and membrane association. Nature 310:583-586.

344. Casey, P. J., P. A. Solski, C. J. Der, and J. E. Buss. 1989. p21ras is modified by a farnesyl isoprenoid. Proc. Natl. Acad. Sci. U. S. A 86:8323-8327.

345. Fiordalisi, J. J., R. L. Johnson, C. A. Weinbaum, K. Sakabe, Z. Chen, P. J. Casey, and A. D. Cox. 2003. High affinity for farnesyltransferase and alternative prenylation contribute individually to K-Ras4B resistance to farnesyltransferase inhibitors. J Biol. Chem. 278:41718-41727.

346. Hancock, J. F., A. I. Magee, J. E. Childs, and C. J. Marshall. 1989. All ras proteins are polyisoprenylated but only some are palmitoylated. Cell 57:1167-1177.

347. Mitchell, D. A., A. Vasudevan, M. E. Linder, and R. J. Deschenes. 2006. Protein palmitoylation by a family of DHHC protein S-acyltransferases. J Lipid Res 47:11181127.

348. Goodwin, J. S., K. R. Drake, C. Rogers, L. Wright, J. Lippincott-Schwartz, M. R. Philips, and A. K. Kenworthy. 2005. Depalmitoylated Ras traffics to and from the Golgi complex via a nonvesicular pathway. J Cell Biol. 170:261-272.

349. Ghomashchi, F., X. Zhang, L. Liu, and M. H. Gelb. 1995. Binding of prenylated and polybasic peptides to membranes: affinities and intervesicle exchange. Biochem. 34:11910-11918.

350. Ashery, U., O. Yizhar, B. Rotblat, and Y. Kloog. 2006. Nonconventional trafficking of Ras associated with Ras signal organization. Traffic. 7:119-126.

351. Bergo, M. O., H. D. Lieu, B. J. Gavino, P. Ambroziak, J. C. Otto, P. J. Casey, Q. M. Walker, and S. G. Young. 2004. On the physiological importance of endoproteolysis of CAAX proteins: heart-specific RCE1 knockout mice develop a lethal cardiomyopathy. J Biol. Chem. 279:4729-4736. 
352. Boyartchuk, V. L., M. N. Ashby, and J. Rine. 1997. Modulation of Ras and a-factor function by carboxyl-terminal proteolysis. Science 275:1796-1800.

353. Bergo, M. O., G. K. Leung, P. Ambroziak, J. C. Otto, P. J. Casey, A. Q. Gomes, M. C. Seabra, and S. G. Young. 2001. Isoprenylcysteine carboxyl methyltransferase deficiency in mice. J Biol. Chem. 276:5841-5845.

354. Zebisch, A. and J. Troppmair. 2006. Back to the roots: the remarkable RAF oncogene story. Cell Mol. Life Sci. 63:1314-1330.

355. Roskoski, R., Jr. 2010. RAF protein-serine/threonine kinases: structure and regulation. Biochem. Biophys. Res. Commun. 399:313-317.

356. Matallanas, D., M. Birtwistle, D. Romano, A. Zebisch, J. Rauch, K. A. von, and W. Kolch. 2011. Raf family kinases: old dogs have learned new tricks. Genes Cancer 2:232-260.

357. Mark, G. E. and U. R. Rapp. 1984. Primary structure of v-raf: relatedness to the src family of oncogenes. Science 224:285-289.

358. Huleihel, M., M. Goldsborough, J. Cleveland, M. Gunnell, T. Bonner, and U. R. Rapp. 1986. Characterization of murine A-raf, a new oncogene related to the v-raf oncogene. Mol. Cell Biol. 6:2655-2662.

359. Bonner, T. I., H. Oppermann, P. Seeburg, S. B. Kerby, M. A. Gunnell, A. C. Young, and U. R. Rapp. 1986. The complete coding sequence of the human raf oncogene and the corresponding structure of the c-raf-1 gene. Nucleic Acids Res 14:1009-1015.

360. Sithanandam, G., W. Kolch, F. M. Duh, and U. R. Rapp. 1990. Complete coding sequence of a human B-raf cDNA and detection of B-raf protein kinase with isozyme specific antibodies. Oncogene 5:1775-1780.

361. Zheng, C. F. and K. L. Guan. 1994. Activation of MEK family kinases requires phosphorylation of two conserved Ser/Thr residues. EMBO J 13:1123-1131.

362. Zheng, C. F. and K. L. Guan. 1993. Cloning and characterization of two distinct human extracellular signal-regulated kinase activator kinases, MEK1 and MEK2. J Biol. Chem. 268:11435-11439.

363. Roskoski, R., Jr. 2012. MEK1/2 dual-specificity protein kinases: structure and regulation. Biochem. Biophys. Res. Commun. 417:5-10.

364. Alessi, D. R., Y. Saito, D. G. Campbell, P. Cohen, G. Sithanandam, U. Rapp, A. Ashworth, C. J. Marshall, and S. Cowley. 1994. Identification of the sites in MAP kinase kinase-1 phosphorylated by p74raf-1. EMBO J 13:1610-1619.

365. Ferrell, J. E., Jr. and R. R. Bhatt. 1997. Mechanistic studies of the dual phosphorylation of mitogen-activated protein kinase. J Biol. Chem. 272:19008-19016. 
366. Roskoski, R., Jr. 2012. ERK1/2 MAP kinases: structure, function, and regulation. Pharmacol. Res. 66:105-143.

367. Seger, R., N. G. Ahn, J. Posada, E. S. Munar, A. M. Jensen, J. A. Cooper, M. H. Cobb, and E. G. Krebs. 1992. Purification and characterization of mitogen-activated protein kinase activator(s) from epidermal growth factor-stimulated A431 cells. J Biol. Chem. 267:14373-14381.

368. Haystead, T. A., P. Dent, J. Wu, C. M. Haystead, and T. W. Sturgill. 1992. Ordered phosphorylation of p42mapk by MAP kinase kinase. FEBS Lett. 306:17-22.

369. Wortzel, I. and R. Seger. 2011. The ERK Cascade: Distinct Functions within Various Subcellular Organelles. Genes Cancer 2:195-209.

370. Chen, R. H., C. Sarnecki, and J. Blenis. 1992. Nuclear localization and regulation of erk- and rsk-encoded protein kinases. Mol. Cell Biol. 12:915-927.

371. Brunet, A., D. Roux, P. Lenormand, S. Dowd, S. Keyse, and J. Pouyssegur. 1999. Nuclear translocation of p42/p44 mitogen-activated protein kinase is required for growth factor-induced gene expression and cell cycle entry. EMBO J. 18:664-674.

372. Zehorai, E., Z. Yao, A. Plotnikov, and R. Seger. 2010. The subcellular localization of MEK and ERK--a novel nuclear translocation signal (NTS) paves a way to the nucleus. Mol. Cell Endocrinol. 314:213-220.

373. Murphy, L. O., J. P. MacKeigan, and J. Blenis. 2004. A network of immediate early gene products propagates subtle differences in mitogen-activated protein kinase signal amplitude and duration. Mol. Cell Biol. 24:144-153.

374. Eferl, R. and E. F. Wagner. 2003. AP-1: a double-edged sword in tumorigenesis. Nat. Rev. Cancer 3:859-868.

375. Gille, H., A. D. Sharrocks, and P. E. Shaw. 1992. Phosphorylation of transcription factor p62TCF by MAP kinase stimulates ternary complex formation at c-fos promoter. Nature 358:414-417.

376. Kato, S., H. Endoh, Y. Masuhiro, T. Kitamoto, S. Uchiyama, H. Sasaki, S. Masushige, Y. Gotoh, E. Nishida, H. Kawashima, D. Metzger, and P. Chambon. 1995. Activation of the estrogen receptor through phosphorylation by mitogen-activated protein kinase. Science 270:1491-1494.

377. Hu, E., J. B. Kim, P. Sarraf, and B. M. Spiegelman. 1996. Inhibition of adipogenesis through MAP kinase-mediated phosphorylation of PPARgamma. Science 274:21002103.

378. Solomon, C., J. H. White, and R. Kremer. 1999. Mitogen-activated protein kinase inhibits 1,25-dihydroxyvitamin D3-dependent signal transduction by phosphorylating human retinoid X receptor alpha. J. Clin. Invest 103:1729-1735. 
379. Krstic, M. D., I. Rogatsky, K. R. Yamamoto, and M. J. Garabedian. 1997. Mitogenactivated and cyclin-dependent protein kinases selectively and differentially modulate transcriptional enhancement by the glucocorticoid receptor. Mol. Cell Biol. 17:39473954.

380. Treisman, R. 1996. Regulation of transcription by MAP kinase cascades. Curr. Opin. Cell Biol. 8:205-215.

381. Anjum, R. and J. Blenis. 2008. The RSK family of kinases: emerging roles in cellular signalling. Nat. Rev. Mol. Cell Biol. 9:747-758.

382. Rossomando, A. J., P. Dent, T. W. Sturgill, and D. R. Marshak. 1994. Mitogenactivated protein kinase kinase 1 (MKK1) is negatively regulated by threonine phosphorylation. Mol. Cell Biol. 14:1594-1602.

383. Buday, L., P. H. Warne, and J. Downward. 1995. Downregulation of the Ras activation pathway by MAP kinase phosphorylation of Sos. Oncogene 11:1327-1331.

384. Ueki, K., S. Matsuda, K. Tobe, Y. Gotoh, H. Tamemoto, M. Yachi, Y. Akanuma, Y. Yazaki, E. Nishida, and T. Kadowaki. 1994. Feedback regulation of mitogen-activated protein kinase kinase kinase activity of c-Raf-1 by insulin and phorbol ester stimulation. J Biol. Chem. 269:15756-15761.

385. McKay, M. M. and D. K. Morrison. 2007. Integrating signals from RTKs to ERK/MAPK. Oncogene 26:3113-3121.

386. Peraldi, P., J. C. Scimeca, C. Filloux, and O. E. Van. 1993. Regulation of extracellular signal-regulated protein kinase-1 (ERK-1; pp44/mitogen-activated protein kinase) by epidermal growth factor and nerve growth factor in PC12 cells: implication of ERK1 inhibitory activities. Endocrinology 132:2578-2585.

387. Vaudry, D., P. J. Stork, P. Lazarovici, and L. E. Eiden. 2002. Signaling pathways for PC12 cell differentiation: making the right connections. Science 296:1648-1649.

388. Vigil, D., J. Cherfils, K. L. Rossman, and C. J. Der. 2010. Ras superfamily GEFs and GAPs: validated and tractable targets for cancer therapy? Nat. Rev. Cancer 10:842-857.

389. Yoshimura, A. 2009. Regulation of cytokine signaling by the SOCS and Spred family proteins. Keio J. Med. 58:73-83.

390. Boguski, M. S. and F. Mccormick. 1993. Proteins regulating Ras and its relatives. Nature 366:643-654.

391. Trahey, M. and F. Mccormick. 1987. A cytoplasmic protein stimulates normal N-ras p21 GTPase, but does not affect oncogenic mutants. Science 238:542-545. 
392. Manne, V., E. Bekesi, and H. F. Kung. 1985. Ha-ras proteins exhibit GTPase activity: point mutations that activate Ha-ras gene products result in decreased GTPase activity. Proc. Natl. Acad. Sci. U. S. A 82:376-380.

393. Sweet, R. W., S. Yokoyama, T. Kamata, J. R. Feramisco, M. Rosenberg, and M. Gross. 1984. The product of ras is a GTPase and the T24 oncogenic mutant is deficient in this activity. Nature 311:273-275.

394. Gibbs, J. B., I. S. Sigal, M. Poe, and E. M. Scolnick. 1984. Intrinsic GTPase activity distinguishes normal and oncogenic ras p21 molecules. Proc. Natl. Acad. Sci. U. S. A 81:5704-5708.

395. McGrath, J. P., D. J. Capon, D. V. Goeddel, and A. D. Levinson. 1984. Comparative biochemical properties of normal and activated human ras p21 protein. Nature 310:644649 .

396. Trahey, M., G. Wong, R. Halenbeck, B. Rubinfeld, G. A. Martin, M. Ladner, C. M. Long, W. J. Crosier, K. Watt, K. Koths, and . 1988. Molecular cloning of two types of GAP complementary DNA from human placenta. Science 242:1697-1700.

397. Vogel, U. S., R. A. Dixon, M. D. Schaber, R. E. Diehl, M. S. Marshall, E. M. Scolnick, I. S. Sigal, and J. B. Gibbs. 1988. Cloning of bovine GAP and its interaction with oncogenic ras p21. Nature 335:90-93.

398. Gibbs, J. B., M. D. Schaber, W. J. Allard, I. S. Sigal, and E. M. Scolnick. 1988. Purification of ras GTPase activating protein from bovine brain. Proc. Natl. Acad. Sci. U. S. A 85:5026-5030.

399. Ballester, R., D. Marchuk, M. Boguski, A. Saulino, R. Letcher, M. Wigler, and F. Collins. 1990. The NF1 locus encodes a protein functionally related to mammalian GAP and yeast IRA proteins. Cell 63:851-859.

400. Martin, G. A., D. Viskochil, G. Bollag, P. C. McCabe, W. J. Crosier, H. Haubruck, L. Conroy, R. Clark, P. O'Connell, R. M. Cawthon, and . 1990. The GAP-related domain of the neurofibromatosis type 1 gene product interacts with ras p21. Cell 63:843-849.

401. Xu, G. F., B. Lin, K. Tanaka, D. Dunn, D. Wood, R. Gesteland, R. White, R. Weiss, and F. Tamanoi. 1990. The catalytic domain of the neurofibromatosis type 1 gene product stimulates ras GTPase and complements ira mutants of S. cerevisiae. Cell 63:835-841.

402. Mitin, N., K. L. Rossman, and C. J. Der. 2005. Signaling interplay in Ras superfamily function. Curr. Biol. 15:R563-R574.

403. Mccormick, F. 1992. Coupling of ras p21 signalling and GTP hydrolysis by GTPase activating proteins. Philos. Trans. R. Soc. Lond B Biol. Sci. 336:43-47. 
404. Bollag, G. and F. Mccormick. 1991. Differential regulation of rasGAP and neurofibromatosis gene product activities. Nature 351:576-579.

405. Mccormick, F., G. A. Martin, R. Clark, G. Bollag, and P. Polakis. 1991. Regulation of ras p21 by GTPase activating proteins. Cold Spring Harb. Symp. Quant. Biol. 56:237241.

406. Berry, D. M., P. Nash, S. K. Liu, T. Pawson, and C. J. McGlade. 2002. A high-affinity Arg-X-X-Lys SH3 binding motif confers specificity for the interaction between Gads and SLP-76 in T cell signaling. Curr. Biol. 12:1336-1341.

407. Lowenstein, E. J., R. J. Daly, A. G. Batzer, W. Li, B. Margolis, R. Lammers, A. Ullrich, E. Y. Skolnik, D. Bar-Sagi, and J. Schlessinger. 1992. The SH2 and SH3 domain-containing protein GRB2 links receptor tyrosine kinases to ras signaling. Cell 70:431-442.

408. Matheny, S. A., C. Chen, R. L. Kortum, G. L. Razidlo, R. E. Lewis, and M. A. White. 2004. Ras regulates assembly of mitogenic signalling complexes through the effector protein IMP. Nature 427:256-260.

409. Muller, J., S. Ory, T. Copeland, H. Piwnica-Worms, and D. K. Morrison. 2001. CTAK1 regulates Ras signaling by phosphorylating the MAPK scaffold, KSR1. Mol. Cell 8:983-993.

410. Denouel-Galy, A., E. M. Douville, P. H. Warne, C. Papin, D. Laugier, G. Calothy, J. Downward, and A. Eychene. 1998. Murine Ksr interacts with MEK and inhibits Rasinduced transformation. Curr. Biol. 8:46-55.

411. Ory, S., M. Zhou, T. P. Conrads, T. D. Veenstra, and D. K. Morrison. 2003. Protein phosphatase $2 \mathrm{~A}$ positively regulates Ras signaling by dephosphorylating KSR1 and Raf-1 on critical 14-3-3 binding sites. Curr. Biol. 13:1356-1364.

412. Ritt, D. A., M. Zhou, T. P. Conrads, T. D. Veenstra, T. D. Copeland, and D. K. Morrison. 2007. CK2 Is a component of the KSR1 scaffold complex that contributes to Raf kinase activation. Curr. Biol. 17:179-184.

413. Teis, D., N. Taub, R. Kurzbauer, D. Hilber, M. E. de Araujo, M. Erlacher, M. Offterdinger, A. Villunger, S. Geley, G. Bohn, C. Klein, M. W. Hess, and L. A. Huber. 2006. p14-MP1-MEK1 signaling regulates endosomal traffic and cellular proliferation during tissue homeostasis. J. Cell Biol. 175:861-868.

414. Teis, D., W. Wunderlich, and L. A. Huber. 2002. Localization of the MP1-MAPK scaffold complex to endosomes is mediated by p14 and required for signal transduction. Dev. Cell 3:803-814.

415. Torii, S., M. Kusakabe, T. Yamamoto, M. Maekawa, and E. Nishida. 2004. Sef is a spatial regulator for Ras/MAP kinase signaling. Dev. Cell 7:33-44. 
416. Ishibe, S., D. Joly, Z. X. Liu, and L. G. Cantley. 2004. Paxillin serves as an ERKregulated scaffold for coordinating FAK and Rac activation in epithelial morphogenesis. Mol. Cell 16:257-267.

417. Huang, Y. Z., M. Zang, W. C. Xiong, Z. Luo, and L. Mei. 2003. Erbin suppresses the MAP kinase pathway. J. Biol. Chem. 278:1108-1114.

418. Yeung, K., P. Janosch, B. McFerran, D. W. Rose, H. Mischak, J. M. Sedivy, and W. Kolch. 2000. Mechanism of suppression of the Raf/MEK/extracellular signal-regulated kinase pathway by the raf kinase inhibitor protein. Mol. Cell Biol. 20:3079-3085.

419. Hacohen, N., S. Kramer, D. Sutherland, Y. Hiromi, and M. A. Krasnow. 1998. sprouty encodes a novel antagonist of FGF signaling that patterns apical branching of the Drosophila airways. Cell 92:253-263.

420. Casci, T., J. Vinos, and M. Freeman. 1999. Sprouty, an intracellular inhibitor of Ras signaling. Cell 96:655-665.

421. Hanafusa, H., S. Torii, T. Yasunaga, K. Matsumoto, and E. Nishida. 2004. Shp2, an $\mathrm{SH} 2$-containing protein-tyrosine phosphatase, positively regulates receptor tyrosine kinase signaling by dephosphorylating and inactivating the inhibitor Sprouty. J. Biol. Chem. 279:22992-22995.

422. Bundschu, K., U. Walter, and K. Schuh. 2006. The VASP-Spred-Sprouty domain puzzle. J Biol. Chem. 281:36477-36481.

423. Kim, H. J. and D. Bar-Sagi. 2004. Modulation of signalling by Sprouty: a developing story. Nat. Rev. Mol. Cell Biol. 5:441-450.

424. Edwin, F., K. Anderson, C. Ying, and T. B. Patel. 2009. Intermolecular interactions of Sprouty proteins and their implications in development and disease. Mol. Pharmacol. 76:679-691.

425. Guy, G. R., R. A. Jackson, P. Yusoff, and S. Y. Chow. 2009. Sprouty proteins: modified modulators, matchmakers or missing links? J. Endocrinol. 203:191-202.

426. Wakioka, T., A. Sasaki, R. Kato, T. Shouda, A. Matsumoto, K. Miyoshi, M. Tsuneoka, S. Komiya, R. Baron, and A. Yoshimura. 2001. Spred is a Sprouty-related suppressor of Ras signalling. Nature 412:647-651.

427. Nonami, A., T. Taketomi, A. Kimura, K. Saeki, H. Takaki, T. Sanada, K. Taniguchi, M. Harada, R. Kato, and A. Yoshimura. 2005. The Sprouty-related protein, Spred-1, localizes in a lipid raft/caveola and inhibits ERK activation in collaboration with caveolin-1. Genes Cells 10:887-895.

428. Inoue, H., R. Kato, S. Fukuyama, A. Nonami, K. Taniguchi, K. Matsumoto, T. Nakano, M. Tsuda, M. Matsumura, M. Kubo, F. Ishikawa, B. G. Moon, K. Takatsu, Y. 
Nakanishi, and A. Yoshimura. 2005. Spred-1 negatively regulates allergen-induced airway eosinophilia and hyperresponsiveness. J. Exp. Med. 201:73-82.

429. Miyoshi, K., T. Wakioka, H. Nishinakamura, M. Kamio, L. Yang, M. Inoue, M. Hasegawa, Y. Yonemitsu, S. Komiya, and A. Yoshimura. 2004. The Sprouty-related protein, Spred, inhibits cell motility, metastasis, and Rho-mediated actin reorganization. Oncogene 23:5567-5576.

430. Nonami, A., R. Kato, K. Taniguchi, D. Yoshiga, T. Taketomi, S. Fukuyama, M. Harada, A. Sasaki, and A. Yoshimura. 2004. Spred-1 negatively regulates interleukin3-mediated ERK/mitogen-activated protein (MAP) kinase activation in hematopoietic cells. J. Biol. Chem. 279:52543-52551.

431. Stowe, I. B., E. L. Mercado, T. R. Stowe, E. L. Bell, J. A. Oses-Prieto, H. Hernandez, A. L. Burlingame, and F. Mccormick. 2012. A shared molecular mechanism underlies the human rasopathies Legius syndrome and Neurofibromatosis-1. Genes Dev. 26:1421-1426.

432. Owens, D. M. and S. M. Keyse. 2007. Differential regulation of MAP kinase signalling by dual-specificity protein phosphatases. Oncogene 26:3203-3213.

433. Anderson, N. G., J. L. Maller, N. K. Tonks, and T. W. Sturgill. 1990. Requirement for integration of signals from two distinct phosphorylation pathways for activation of MAP kinase. Nature 343:651-653.

434. Ritt, D. A., D. M. Monson, S. I. Specht, and D. K. Morrison. 2010. Impact of feedback phosphorylation and Raf heterodimerization on normal and mutant B-Raf signaling. Mol. Cell Biol. 30:806-819.

435. Gilmartin, A. G., M. R. Bleam, A. Groy, K. G. Moss, E. A. Minthorn, S. G. Kulkarni, C. M. Rominger, S. Erskine, K. E. Fisher, J. Yang, F. Zappacosta, R. Annan, D. Sutton, and S. G. Laquerre. 2011. GSK1120212 (JTP-74057) is an inhibitor of MEK activity and activation with favorable pharmacokinetic properties for sustained in vivo pathway inhibition. Clin Cancer Res 17:989-1000.

436. Duncan, J. S., M. C. Whittle, K. Nakamura, A. N. Abell, A. A. Midland, J. S. Zawistowski, N. L. Johnson, D. A. Granger, N. V. Jordan, D. B. Darr, J. Usary, P. F. Kuan, D. M. Smalley, B. Major, X. He, K. A. Hoadley, B. Zhou, N. E. Sharpless, C. M. Perou, W. Y. Kim, S. M. Gomez, X. Chen, J. Jin, S. V. Frye, H. S. Earp, L. M. Graves, and G. L. Johnson. 2012. Dynamic reprogramming of the kinome in response to targeted MEK inhibition in triple-negative breast cancer. Cell 149:307-321.

437. Gual, P., S. Giordano, S. Anguissola, P. J. Parker, and P. M. Comoglio. 2001. Gab1 phosphorylation: a novel mechanism for negative regulation of HGF receptor signaling. Oncogene 20:156-166. 
438. Yu, C. F., Z. X. Liu, and L. G. Cantley. 2002. ERK negatively regulates the epidermal growth factor-mediated interaction of Gab1 and the phosphatidylinositol 3-kinase. J Biol. Chem. 277:19382-19388.

439. Lax, I., A. Wong, B. Lamothe, A. Lee, A. Frost, J. Hawes, and J. Schlessinger. 2002. The docking protein FRS2alpha controls a MAP kinase-mediated negative feedback mechanism for signaling by FGF receptors. Mol. Cell 10:709-719.

440. Mothe, I. and O. E. Van. 1996. Phosphorylation of insulin receptor substrate-1 on multiple serine residues, 612, 632, 662, and 731, modulates insulin action. J Biol. Chem. 271:11222-11227.

441. Li, X., Y. Huang, J. Jiang, and S. J. Frank. 2008. ERK-dependent threonine phosphorylation of EGF receptor modulates receptor downregulation and signaling. Cell Signal. 20:2145-2155.

442. Sorkin, A. and L. K. Goh. 2009. Endocytosis and intracellular trafficking of ErbBs. Exp. Cell Res 315:683-696.

443. Zwang, Y. and Y. Yarden. 2009. Systems biology of growth factor-induced receptor endocytosis. Traffic. 10:349-363.

444. Amit, I., A. Citri, T. Shay, Y. Lu, M. Katz, F. Zhang, G. Tarcic, D. Siwak, J. Lahad, J. Jacob-Hirsch, N. Amariglio, N. Vaisman, E. Segal, G. Rechavi, U. Alon, G. B. Mills, E. Domany, and Y. Yarden. 2007. A module of negative feedback regulators defines growth factor signaling. Nat. Genet. 39:503-512.

445. Sears, R., F. Nuckolls, E. Haura, Y. Taya, K. Tamai, and J. R. Nevins. 2000. Multiple Ras-dependent phosphorylation pathways regulate Myc protein stability. Genes Dev $14: 2501-2514$.

446. Fernandez-Medarde, A. and E. Santos. 2011. Ras in cancer and developmental diseases. Genes Cancer 2:344-358.

447. Cawthon, R. M., P. O'Connell, A. M. Buchberg, D. Viskochil, R. B. Weiss, M. Culver, J. Stevens, N. A. Jenkins, N. G. Copeland, and R. White. 1990. Identification and characterization of transcripts from the neurofibromatosis 1 region: the sequence and genomic structure of EVI2 and mapping of other transcripts. Genomics 7:555-565.

448. Jett, K. and J. M. Friedman. 2010. Clinical and genetic aspects of neurofibromatosis 1. Genet. Med. 12:1-11.

449. Korf, B. R. 2000. Malignancy in neurofibromatosis type 1. Oncologist. 5:477-485.

450. Revencu, N., L. M. Boon, J. B. Mulliken, O. Enjolras, M. R. Cordisco, P. E. Burrows, P. Clapuyt, F. Hammer, J. Dubois, E. Baselga, F. Brancati, R. Carder, J. M. Quintal, B. Dallapiccola, G. Fischer, I. J. Frieden, M. Garzon, J. Harper, J. Johnson-Patel, C. Labreze, L. Martorell, H. J. Paltiel, A. Pohl, J. Prendiville, I. Quere, D. H. Siegel, E. M. 
Valente, H. A. Van, H. L. Van, K. K. Vaux, A. Vicente, L. Weibel, D. Chitayat, and M. Vikkula. 2008. Parkes Weber syndrome, vein of Galen aneurysmal malformation, and other fast-flow vascular anomalies are caused by RASA1 mutations. Hum. Mutat. 29:959-965.

451. Eerola, I., L. M. Boon, J. B. Mulliken, P. E. Burrows, A. Dompmartin, S. Watanabe, R. Vanwijck, and M. Vikkula. 2003. Capillary malformation-arteriovenous malformation, a new clinical and genetic disorder caused by RASA1 mutations. Am. J. Hum. Genet. 73:1240-1249.

452. Sarkozy, A., M. C. Digilio, and B. Dallapiccola. 2008. Leopard syndrome. Orphanet. J. Rare. Dis. 3:13.

453. Tartaglia, M., E. L. Mehler, R. Goldberg, G. Zampino, H. G. Brunner, H. Kremer, d. B. van, I, A. H. Crosby, A. Ion, S. Jeffery, K. Kalidas, M. A. Patton, R. S. Kucherlapati, and B. D. Gelb. 2001. Mutations in PTPN11, encoding the protein tyrosine phosphatase SHP-2, cause Noonan syndrome. Nat. Genet. 29:465-468.

454. Aoki, Y., T. Niihori, H. Kawame, K. Kurosawa, H. Ohashi, Y. Tanaka, M. Filocamo, K. Kato, Y. Suzuki, S. Kure, and Y. Matsubara. 2005. Germline mutations in HRAS proto-oncogene cause Costello syndrome. Nat. Genet. 37:1038-1040.

455. Niihori, T., Y. Aoki, Y. Narumi, G. Neri, H. Cave, A. Verloes, N. Okamoto, R. C. Hennekam, G. Gillessen-Kaesbach, D. Wieczorek, M. I. Kavamura, K. Kurosawa, H. Ohashi, L. Wilson, D. Heron, D. Bonneau, G. Corona, T. Kaname, K. Naritomi, C. Baumann, N. Matsumoto, K. Kato, S. Kure, and Y. Matsubara. 2006. Germline KRAS and BRAF mutations in cardio-facio-cutaneous syndrome. Nat. Genet. 38:294-296.

456. Rodriguez-Viciana, P., O. Tetsu, W. E. Tidyman, A. L. Estep, B. A. Conger, M. S. Cruz, F. Mccormick, and K. A. Rauen. 2006. Germline mutations in genes within the MAPK pathway cause cardio-facio-cutaneous syndrome. Science 311:1287-1290.

457. McCubrey, J. A., L. S. Steelman, S. L. Abrams, J. T. Lee, F. Chang, F. E. Bertrand, P. M. Navolanic, D. M. Terrian, R. A. Franklin, A. B. D'Assoro, J. L. Salisbury, M. C. Mazzarino, F. Stivala, and M. Libra. 2006. Roles of the RAF/MEK/ERK and $\mathrm{PI} 3 \mathrm{~K} / \mathrm{PTEN} / \mathrm{AKT}$ pathways in malignant transformation and drug resistance. Adv. Enzyme Regul. 46:249-279.

458. Roberts, P. J. and C. J. Der. 2007. Targeting the Raf-MEK-ERK mitogen-activated protein kinase cascade for the treatment of cancer. Oncogene 26:3291-3310.

459. Davies, H., G. R. Bignell, C. Cox, P. Stephens, S. Edkins, S. Clegg, J. Teague, H. Woffendin, M. J. Garnett, W. Bottomley, N. Davis, E. Dicks, R. Ewing, Y. Floyd, K. Gray, S. Hall, R. Hawes, J. Hughes, V. Kosmidou, A. Menzies, C. Mould, A. Parker, C. Stevens, S. Watt, S. Hooper, R. Wilson, H. Jayatilake, B. A. Gusterson, C. Cooper, J. Shipley, D. Hargrave, K. Pritchard-Jones, N. Maitland, G. Chenevix-Trench, G. J. Riggins, D. D. Bigner, G. Palmieri, A. Cossu, A. Flanagan, A. Nicholson, J. W. Ho, S. Y. Leung, S. T. Yuen, B. L. Weber, H. F. Seigler, T. L. Darrow, H. Paterson, R. 
Marais, C. J. Marshall, R. Wooster, M. R. Stratton, and P. A. Futreal. 2002. Mutations of the BRAF gene in human cancer. Nature 417:949-954.

460. Gschwind, A., O. M. Fischer, and A. Ullrich. 2004. The discovery of receptor tyrosine kinases: targets for cancer therapy. Nat. Rev. Cancer 4:361-370.

461. Bromberg-White, J. L., N. J. Andersen, and N. S. Duesbery. 2012. MEK genomics in development and disease. Brief. Funct. Genomics 11:300-310.

462. Calvisi, D. F., S. Ladu, E. A. Conner, D. Seo, J. T. Hsieh, V. M. Factor, and S. S. Thorgeirsson. 2011. Inactivation of Ras GTPase-activating proteins promotes unrestrained activity of wild-type Ras in human liver cancer. J. Hepatol. 54:311-319.

463. Santen, R. J., R. X. Song, R. McPherson, R. Kumar, L. Adam, M. H. Jeng, and W. Yue. 2002. The role of mitogen-activated protein (MAP) kinase in breast cancer. J. Steroid Biochem. Mol. Biol. 80:239-256.

464. Whyte, J., O. Bergin, A. Bianchi, S. McNally, and F. Martin. 2009. Key signalling nodes in mammary gland development and cancer. Mitogen-activated protein kinase signalling in experimental models of breast cancer progression and in mammary gland development. Breast Cancer Res. 11:209.

465. Eckert, L. B., G. A. Repasky, A. S. Ulku, A. McFall, H. Zhou, C. I. Sartor, and C. J. Der. 2004. Involvement of Ras activation in human breast cancer cell signaling, invasion, and anoikis. Cancer Res 64:4585-4592.

466. Mattingly, R. R. 2013. Activated Ras as a Therapeutic Target: Constraints on Directly Targeting Ras Isoforms and Wild-Type versus Mutated Proteins. ISRN. Oncol. 2013:536529.

467. Johnson, G. L., T. J. Stuhlmiller, S. P. Angus, J. S. Zawistowski, and L. M. Graves. 2014. Molecular pathways: adaptive kinome reprogramming in response to targeted inhibition of the BRAF-MEK-ERK pathway in cancer. Clin Cancer Res 20:2516-2522.

468. Liu, Q., Y. Sabnis, Z. Zhao, T. Zhang, S. J. Buhrlage, L. H. Jones, and N. S. Gray. 2013. Developing irreversible inhibitors of the protein kinase cysteinome. Chem. Biol. 20:146-159.

469. Klink, B. U. and A. J. Scheidig. 2010. New insight into the dynamic properties and the active site architecture of H-Ras p21 revealed by X-ray crystallography at very high resolution. BMC Struct. Biol. 10:38.

470. Adjei, A. A. 2001. Blocking oncogenic Ras signaling for cancer therapy. J Natl. Cancer Inst. 93:1062-1074.

471. Kohl, N. E., C. A. Omer, M. W. Conner, N. J. Anthony, J. P. Davide, S. J. deSolms, E. A. Giuliani, R. P. Gomez, S. L. Graham, K. Hamilton, and . 1995. Inhibition of 
farnesyltransferase induces regression of mammary and salivary carcinomas in ras transgenic mice. Nat. Med 1:792-797.

472. Adjei, A. A., C. Erlichman, J. N. Davis, D. L. Cutler, J. A. Sloan, R. S. Marks, L. J. Hanson, P. A. Svingen, P. Atherton, W. R. Bishop, P. Kirschmeier, and S. H.

Kaufmann. 2000. A Phase I trial of the farnesyl transferase inhibitor SCH66336:

evidence for biological and clinical activity. Cancer Res 60:1871-1877.

473. Zujewski, J., I. D. Horak, C. J. Bol, R. Woestenborghs, C. Bowden, D. W. End, V. K. Piotrovsky, J. Chiao, R. T. Belly, A. Todd, W. C. Kopp, D. R. Kohler, C. Chow, M. Noone, F. T. Hakim, G. Larkin, R. E. Gress, R. B. Nussenblatt, A. B. Kremer, and K. H. Cowan. 2000. Phase I and pharmacokinetic study of farnesyl protein transferase inhibitor R115777 in advanced cancer. J Clin Oncol. 18:927-941.

474. Sepp-Lorenzino, L., Z. Ma, E. Rands, N. E. Kohl, J. B. Gibbs, A. Oliff, and N. Rosen. 1995. A peptidomimetic inhibitor of farnesyl:protein transferase blocks the anchoragedependent and -independent growth of human tumor cell lines. Cancer Res 55:53025309.

475. Mangues, R., T. Corral, N. E. Kohl, W. F. Symmans, S. Lu, M. Malumbres, J. B. Gibbs, A. Oliff, and A. Pellicer. 1998. Antitumor effect of a farnesyl protein transferase inhibitor in mammary and lymphoid tumors overexpressing N-ras in transgenic mice. Cancer Res 58:1253-1259.

476. Omer, C. A., Z. Chen, R. E. Diehl, M. W. Conner, H. Y. Chen, M. E. Trumbauer, S. Gopal-Truter, G. Seeburger, H. Bhimnathwala, M. T. Abrams, J. P. Davide, M. S. Ellis, J. B. Gibbs, I. Greenberg, K. S. Koblan, A. M. Kral, D. Liu, R. B. Lobell, P. J. Miller, S. D. Mosser, T. J. O'Neill, E. Rands, M. D. Schaber, E. T. Senderak, A. Oliff, and N. E. Kohl. 2000. Mouse mammary tumor virus-Ki-rasB transgenic mice develop mammary carcinomas that can be growth-inhibited by a farnesyl:protein transferase inhibitor. Cancer Res 60:2680-2688.

477. Zhang, F. L., P. Kirschmeier, D. Carr, L. James, R. W. Bond, L. Wang, R. Patton, W. T. Windsor, R. Syto, R. Zhang, and W. R. Bishop. 1997. Characterization of Ha-ras, Nras, $\mathrm{Ki}-\mathrm{Ras} 4 \mathrm{~A}$, and $\mathrm{Ki}-\mathrm{Ras} 4 \mathrm{~B}$ as in vitro substrates for farnesyl protein transferase and geranylgeranyl protein transferase type I. J Biol. Chem. 272:10232-10239.

478. Whyte, D. B., P. Kirschmeier, T. N. Hockenberry, I. Nunez-Oliva, L. James, J. J. Catino, W. R. Bishop, and J. K. Pai. 1997. K- and N-Ras are geranylgeranylated in cells treated with farnesyl protein transferase inhibitors. J Biol. Chem. 272:14459-14464.

479. Rowell, C. A., J. J. Kowalczyk, M. D. Lewis, and A. M. Garcia. 1997. Direct demonstration of geranylgeranylation and farnesylation of Ki-Ras in vivo. J Biol. Chem. 272:14093-14097.

480. Gibbs, B. S., T. J. Zahn, Y. Mu, J. S. Sebolt-Leopold, and R. A. Gibbs. 1999. Novel farnesol and geranylgeraniol analogues: A potential new class of anticancer agents directed against protein prenylation. J Med Chem. 42:3800-3808. 
481. Mattingly, R. R., R. A. Gibbs, R. E. Menard, and J. J. Reiners, Jr. 2002. Potent suppression of proliferation of a10 vascular smooth muscle cells by combined treatment with lovastatin and 3-allylfarnesol, an inhibitor of protein farnesyltransferase. J Pharmacol. Exp. Ther 303:74-81.

482. Wojtkowiak, J. W., K. M. Sane, M. Kleinman, B. F. Sloane, J. J. Reiners, Jr., and R. R. Mattingly. 2011. Aborted autophagy and nonapoptotic death induced by farnesyl transferase inhibitor and lovastatin. J Pharmacol. Exp. Ther 337:65-74.

483. Clark, M. K., S. A. Scott, J. Wojtkowiak, R. Chirco, P. Mathieu, J. J. Reiners, Jr., R. R. Mattingly, R. F. Borch, and R. A. Gibbs. 2007. Synthesis, biochemical, and cellular evaluation of farnesyl monophosphate prodrugs as farnesyltransferase inhibitors. J Med Chem. 50:3274-3282.

484. Wojtkowiak, J. W., F. Fouad, D. T. Lalonde, M. D. Kleinman, R. A. Gibbs, J. J. Reiners, Jr., R. F. Borch, and R. R. Mattingly. 2008. Induction of apoptosis in neurofibromatosis type 1 malignant peripheral nerve sheath tumor cell lines by a combination of novel farnesyl transferase inhibitors and lovastatin. J Pharmacol. Exp. Ther 326:1-11.

485. Wojtkowiak, J. W., R. A. Gibbs, and R. R. Mattingly. 2009. Working together: Farnesyl transferase inhibitors and statins block protein prenylation. Mol. Cell Pharmacol. 1:1-6.

486. Sane, K. M., M. Mynderse, D. T. Lalonde, I. S. Dean, J. W. Wojtkowiak, F. Fouad, R. F. Borch, J. J. Reiners, Jr., R. A. Gibbs, and R. R. Mattingly. 2010. A novel geranylgeranyl transferase inhibitor in combination with lovastatin inhibits proliferation and induces autophagy in STS-26T MPNST cells. J Pharmacol. Exp. Ther 333:23-33.

487. Tanaka, T. and T. H. Rabbitts. 2010. Interfering with RAS-effector protein interactions prevent RAS-dependent tumour initiation and causes stop-start control of cancer growth. Oncogene 29:6064-6070.

488. Kato-Stankiewicz, J., I. Hakimi, G. Zhi, J. Zhang, I. Serebriiskii, L. Guo, H. Edamatsu, H. Koide, S. Menon, R. Eckl, S. Sakamuri, Y. Lu, Q. Z. Chen, S. Agarwal, W. R. Baumbach, E. A. Golemis, F. Tamanoi, and V. Khazak. 2002. Inhibitors of Ras/Raf-1 interaction identified by two-hybrid screening revert Ras-dependent transformation phenotypes in human cancer cells. Proc. Natl. Acad. Sci. U. S. A 99:14398-14403.

489. Maurer, T., L. S. Garrenton, A. Oh, K. Pitts, D. J. Anderson, N. J. Skelton, B. P. Fauber, B. Pan, S. Malek, D. Stokoe, M. J. Ludlam, K. K. Bowman, J. Wu, A. M. Giannetti, M. A. Starovasnik, I. Mellman, P. K. Jackson, J. Rudolph, W. Wang, and G. Fang. 2012. Small-molecule ligands bind to a distinct pocket in Ras and inhibit SOSmediated nucleotide exchange activity. Proc. Natl. Acad. Sci. U. S. A 109:5299-5304.

490. Sun, Q., J. P. Burke, J. Phan, M. C. Burns, E. T. Olejniczak, A. G. Waterson, T. Lee, O. W. Rossanese, and S. W. Fesik. 2012. Discovery of small molecules that bind to K-Ras and inhibit Sos-mediated activation. Angew. Chem. Int Ed Engl. 51:6140-6143. 
491. Patgiri, A., K. K. Yadav, P. S. Arora, and D. Bar-Sagi. 2011. An orthosteric inhibitor of the Ras-Sos interaction. Nat. Chem. Biol. 7:585-587.

492. Jeng, H. H., L. J. Taylor, and D. Bar-Sagi. 2012. Sos-mediated cross-activation of wildtype Ras by oncogenic Ras is essential for tumorigenesis. Nat. Commun. 3:1168.

493. Young, A., D. Lou, and F. Mccormick. 2013. Oncogenic and wild-type Ras play divergent roles in the regulation of mitogen-activated protein kinase signaling. Cancer Discov. 3:112-123.

494. Grabocka, E., Y. Pylayeva-Gupta, M. J. Jones, V. Lubkov, E. Yemanaberhan, L. Taylor, H. H. Jeng, and D. Bar-Sagi. 2014. Wild-type H- and N-Ras promote mutant KRas-driven tumorigenesis by modulating the DNA damage response. Cancer Cell 25:243-256.

495. Gibney, G. T. and J. S. Zager. 2013. Clinical development of dabrafenib in BRAF mutant melanoma and other malignancies. Expert Opin. Drug Metab Toxicol. 9:893899.

496. Bollag, G., P. Hirth, J. Tsai, J. Zhang, P. N. Ibrahim, H. Cho, W. Spevak, C. Zhang, Y. Zhang, G. Habets, E. A. Burton, B. Wong, G. Tsang, B. L. West, B. Powell, R. Shellooe, A. Marimuthu, H. Nguyen, K. Y. Zhang, D. R. Artis, J. Schlessinger, F. Su, B. Higgins, R. Iyer, K. D'Andrea, A. Koehler, M. Stumm, P. S. Lin, R. J. Lee, J. Grippo, I. Puzanov, K. B. Kim, A. Ribas, G. A. McArthur, J. A. Sosman, P. B. Chapman, K. T. Flaherty, X. Xu, K. L. Nathanson, and K. Nolop. 2010. Clinical efficacy of a RAF inhibitor needs broad target blockade in BRAF-mutant melanoma. Nature 467:596-599.

497. Flaherty, K. T., J. R. Infante, A. Daud, R. Gonzalez, R. F. Kefford, J. Sosman, O. Hamid, L. Schuchter, J. Cebon, N. Ibrahim, R. Kudchadkar, H. A. Burris, III, G. Falchook, A. Algazi, K. Lewis, G. V. Long, I. Puzanov, P. Lebowitz, A. Singh, S. Little, P. Sun, A. Allred, D. Ouellet, K. B. Kim, K. Patel, and J. Weber. 2012. Combined BRAF and MEK inhibition in melanoma with BRAF V600 mutations. N. Engl. J Med 367:1694-1703.

498. Davies, B. R., A. Logie, J. S. McKay, P. Martin, S. Steele, R. Jenkins, M. Cockerill, S. Cartlidge, and P. D. Smith. 2007. AZD6244 (ARRY-142886), a potent inhibitor of mitogen-activated protein kinase/extracellular signal-regulated kinase kinase $1 / 2$ kinases: mechanism of action in vivo, pharmacokinetic/pharmacodynamic relationship, and potential for combination in preclinical models. Mol. Cancer Ther 6:2209-2219.

499. Yeh, T. C., V. Marsh, B. A. Bernat, J. Ballard, H. Colwell, R. J. Evans, J. Parry, D. Smith, B. J. Brandhuber, S. Gross, A. Marlow, B. Hurley, J. Lyssikatos, P. A. Lee, J. D. Winkler, K. Koch, and E. Wallace. 2007. Biological characterization of ARRY-142886 (AZD6244), a potent, highly selective mitogen-activated protein kinase kinase 1/2 inhibitor. Clin Cancer Res 13:1576-1583. 
500. Ohori, M., T. Kinoshita, M. Okubo, K. Sato, A. Yamazaki, H. Arakawa, S. Nishimura, N. Inamura, H. Nakajima, M. Neya, H. Miyake, and T. Fujii. 2005. Identification of a selective ERK inhibitor and structural determination of the inhibitor-ERK2 complex. Biochem. Biophys. Res Commun. 336:357-363.

501. Rodrik-Outmezguine, V. S., S. Chandarlapaty, N. C. Pagano, P. I. Poulikakos, M. Scaltriti, E. Moskatel, J. Baselga, S. Guichard, and N. Rosen. 2011. mTOR kinase inhibition causes feedback-dependent biphasic regulation of AKT signaling. Cancer Discov. 1:248-259.

502. Garrett, J. T., M. G. Olivares, C. Rinehart, N. D. Granja-Ingram, V. Sanchez, A. Chakrabarty, B. Dave, R. S. Cook, W. Pao, E. McKinely, H. C. Manning, J. Chang, and C. L. Arteaga. 2011. Transcriptional and posttranslational up-regulation of HER3 (ErbB3) compensates for inhibition of the HER2 tyrosine kinase. Proc. Natl. Acad. Sci. U. S. A 108:5021-5026.

503. Chandarlapaty, S., A. Sawai, M. Scaltriti, V. Rodrik-Outmezguine, O. Grbovic-Huezo, V. Serra, P. K. Majumder, J. Baselga, and N. Rosen. 2011. AKT inhibition relieves feedback suppression of receptor tyrosine kinase expression and activity. Cancer Cell 19:58-71.

504. Engelman, J. A., K. Zejnullahu, T. Mitsudomi, Y. Song, C. Hyland, J. O. Park, N. Lindeman, C. M. Gale, X. Zhao, J. Christensen, T. Kosaka, A. J. Holmes, A. M. Rogers, F. Cappuzzo, T. Mok, C. Lee, B. E. Johnson, L. C. Cantley, and P. A. Janne. 2007. MET amplification leads to gefitinib resistance in lung cancer by activating ERBB3 signaling. Science 316:1039-1043.

505. Villanueva, J., J. R. Infante, C. Krepler, P. Reyes-Uribe, M. Samanta, H. Y. Chen, B. Li, R. K. Swoboda, M. Wilson, A. Vultur, M. Fukunaba-Kalabis, B. Wubbenhorst, T. Y. Chen, Q. Liu, K. Sproesser, D. J. DeMarini, T. M. Gilmer, A. M. Martin, R. Marmorstein, D. C. Schultz, D. W. Speicher, G. C. Karakousis, W. Xu, R. K. Amaravadi, X. Xu, L. M. Schuchter, M. Herlyn, and K. L. Nathanson. 2013. Concurrent MEK2 mutation and BRAF amplification confer resistance to BRAF and MEK inhibitors in melanoma. Cell Rep. 4:1090-1099.

506. Shi, H., A. Hong, X. Kong, R. C. Koya, C. Song, G. Moriceau, W. Hugo, C. C. Yu, C. Ng, T. Chodon, R. A. Scolyer, R. F. Kefford, A. Ribas, G. V. Long, and R. S. Lo. 2014. A novel AKT1 mutant amplifies an adaptive melanoma response to BRAF inhibition. Cancer Discov. 4:69-79.

507. Nazarian, R., H. Shi, Q. Wang, X. Kong, R. C. Koya, H. Lee, Z. Chen, M. K. Lee, N. Attar, H. Sazegar, T. Chodon, S. F. Nelson, G. McArthur, J. A. Sosman, A. Ribas, and R. S. Lo. 2010. Melanomas acquire resistance to B-RAF(V600E) inhibition by RTK or N-RAS upregulation. Nature 468:973-977.

508. Sos, M. L., S. Fischer, R. Ullrich, M. Peifer, J. M. Heuckmann, M. Koker, S. Heynck, I. Stuckrath, J. Weiss, F. Fischer, K. Michel, A. Goel, L. Regales, K. A. Politi, S. Perera, M. Getlik, L. C. Heukamp, S. Ansen, T. Zander, R. Beroukhim, H. Kashkar, K. M. 
Shokat, W. R. Sellers, D. Rauh, C. Orr, K. P. Hoeflich, L. Friedman, K. K. Wong, W. Pao, and R. K. Thomas. 2009. Identifying genotype-dependent efficacy of single and combined. Proc. Natl. Acad. Sci. U. S. A 106:18351-18356.

509. Hoeflich, K. P., C. O'Brien, Z. Boyd, G. Cavet, S. Guerrero, K. Jung, T. Januario, H. Savage, E. Punnoose, T. Truong, W. Zhou, L. Berry, L. Murray, L. Amler, M. Belvin, L. S. Friedman, and M. R. Lackner. 2009. In vivo antitumor activity of MEK and phosphatidylinositol 3-kinase inhibitors in basal-like breast cancer models. Clin Cancer Res 15:4649-4664.

510. Sun, C., S. Hobor, A. Bertotti, D. Zecchin, S. Huang, F. Galimi, F. Cottino, A. Prahallad, W. Grernrum, A. Tzani, A. Schlicker, L. F. Wessels, E. F. Smit, E. Thunnissen, P. Halonen, C. Lieftink, R. L. Beijersbergen, N. F. Di, A. Bardelli, L. Trusolino, and R. Bernards. 2014. Intrinsic resistance to MEK inhibition in KRAS mutant lung and colon cancer through transcriptional induction of ERBB3. Cell Rep. 7:86-93.

511. Heidorn, S. J., C. Milagre, S. Whittaker, A. Nourry, I. Niculescu-Duvas, N. Dhomen, J. Hussain, J. S. Reis-Filho, C. J. Springer, C. Pritchard, and R. Marais. 2010. Kinasedead BRAF and oncogenic RAS cooperate to drive tumor progression through CRAF. Cell 140:209-221.

512. Poulikakos, P. I., C. Zhang, G. Bollag, K. M. Shokat, and N. Rosen. 2010. RAF inhibitors transactivate RAF dimers and ERK signalling in cells with wild-type BRAF. Nature 464:427-430.

513. Hatzivassiliou, G., K. Song, I. Yen, B. J. Brandhuber, D. J. Anderson, R. Alvarado, M. J. Ludlam, D. Stokoe, S. L. Gloor, G. Vigers, T. Morales, I. Aliagas, B. Liu, S. Sideris, K. P. Hoeflich, B. S. Jaiswal, S. Seshagiri, H. Koeppen, M. Belvin, L. S. Friedman, and S. Malek. 2010. RAF inhibitors prime wild-type RAF to activate the MAPK pathway and enhance growth. Nature 464:431-435.

514. Perou, C. M., T. Sorlie, M. B. Eisen, R. M. van de, S. S. Jeffrey, C. A. Rees, J. R. Pollack, D. T. Ross, H. Johnsen, L. A. Akslen, O. Fluge, A. Pergamenschikov, C. Williams, S. X. Zhu, P. E. Lonning, A. L. Borresen-Dale, P. O. Brown, and D. Botstein. 2000. Molecular portraits of human breast tumours. Nature 406:747-752.

515. Herschkowitz, J. I., K. Simin, V. J. Weigman, I. Mikaelian, J. Usary, Z. Hu, K. E. Rasmussen, L. P. Jones, S. Assefnia, S. Chandrasekharan, M. G. Backlund, Y. Yin, A. I. Khramtsov, R. Bastein, J. Quackenbush, R. I. Glazer, P. H. Brown, J. E. Green, L. Kopelovich, P. A. Furth, J. P. Palazzo, O. I. Olopade, P. S. Bernard, G. A. Churchill, D. T. Van, and C. M. Perou. 2007. Identification of conserved gene expression features between murine mammary carcinoma models and human breast tumors. Genome Biol. 8:R76.

516. Prat, A. and C. M. Perou. 2011. Deconstructing the molecular portraits of breast cancer. Mol. Oncol. 5:5-23. 
517. Perou, C. M. 2011. Molecular stratification of triple-negative breast cancers. Oncologist. 16 Suppl 1:61-70.

518. Prat, A., J. S. Parker, O. Karginova, C. Fan, C. Livasy, J. I. Herschkowitz, X. He, and C. M. Perou. 2010. Phenotypic and molecular characterization of the claudin-low intrinsic subtype of breast cancer. Breast Cancer Res 12:R68.

519. Rakha, E. A., J. S. Reis-Filho, and I. O. Ellis. 2008. Basal-like breast cancer: a critical review. J. Clin. Oncol. 26:2568-2581.

520. Nielsen, T. O., F. D. Hsu, K. Jensen, M. Cheang, G. Karaca, Z. Hu, T. HernandezBoussard, C. Livasy, D. Cowan, L. Dressler, L. A. Akslen, J. Ragaz, A. M. Gown, C. B. Gilks, R. M. van de, and C. M. Perou. 2004. Immunohistochemical and clinical characterization of the basal-like subtype of invasive breast carcinoma. Clin Cancer Res 10:5367-5374.

521. Prat, A., B. Adamo, M. C. Cheang, C. K. Anders, L. A. Carey, and C. M. Perou. 2013. Molecular characterization of basal-like and non-basal-like triple-negative breast cancer. Oncologist. 18:123-133.

522. Mueller, H., N. Flury, S. Eppenberger-Castori, W. Kueng, F. David, and U. Eppenberger. 2000. Potential prognostic value of mitogen-activated protein kinase activity for disease-free survival of primary breast cancer patients. Int. J. Cancer 89:384-388.

523. Sivaraman, V. S., H. Wang, G. J. Nuovo, and C. C. Malbon. 1997. Hyperexpression of mitogen-activated protein kinase in human breast cancer. J. Clin. Invest 99:1478-1483.

524. McGlynn, L. M., T. Kirkegaard, J. Edwards, S. Tovey, D. Cameron, C. Twelves, J. M. Bartlett, and T. G. Cooke. 2009. Ras/Raf-1/MAPK pathway mediates response to tamoxifen but not chemotherapy in breast cancer patients. Clin. Cancer Res. 15:14871495.

525. Bergqvist, J., G. Elmberger, J. Ohd, B. Linderholm, J. Bjohle, H. Hellborg, H. Nordgren, A. L. Borg, L. Skoog, and J. Bergh. 2006. Activated ERK1/2 and phosphorylated oestrogen receptor alpha are associated with improved breast cancer survival in women treated with tamoxifen. Eur. J. Cancer 42:1104-1112.

526. Svensson, S., K. Jirstrom, L. Ryden, G. Roos, S. Emdin, M. C. Ostrowski, and G. Landberg. 2005. ERK phosphorylation is linked to VEGFR2 expression and Ets-2 phosphorylation in breast cancer and is associated with tamoxifen treatment resistance and small tumours with good prognosis. Oncogene 24:4370-4379.

527. Cancer Genome, A. N. 2012. Comprehensive molecular portraits of human breast tumours. Nature 490:61-70.

528. Hoeflich, K. P., C. O'Brien, Z. Boyd, G. Cavet, S. Guerrero, K. Jung, T. Januario, H. Savage, E. Punnoose, T. Truong, W. Zhou, L. Berry, L. Murray, L. Amler, M. Belvin, 
L. S. Friedman, and M. R. Lackner. 2009. In vivo antitumor activity of MEK and phosphatidylinositol 3-kinase inhibitors in basal-like breast cancer models. Clin. Cancer Res. 15:4649-4664.

529. Mirzoeva, O. K., D. Das, L. M. Heiser, S. Bhattacharya, D. Siwak, R. Gendelman, N. Bayani, N. J. Wang, R. M. Neve, Y. Guan, Z. Hu, Z. Knight, H. S. Feiler, P. Gascard, B. Parvin, P. T. Spellman, K. M. Shokat, A. J. Wyrobek, M. J. Bissell, F. Mccormick, W. L. Kuo, G. B. Mills, J. W. Gray, and W. M. Korn. 2009. Basal subtype and MAPK/ERK kinase (MEK)-phosphoinositide 3-kinase feedback signaling determine susceptibility of breast cancer cells to MEK inhibition. Cancer Res 69:565-572.

530. van Beers, E. H., W. T. van, L. F. Wessels, Y. Li, R. A. Oldenburg, P. Devilee, C. J. Cornelisse, S. Verhoef, F. B. Hogervorst, L. J. van't Veer, and P. M. Nederlof. 2005. Comparative genomic hybridization profiles in human BRCA1 and BRCA 2 breast tumors highlight differential sets of genomic aberrations. Cancer Res 65:822-827.

531. Hu, X., H. M. Stern, L. Ge, C. O'Brien, L. Haydu, C. D. Honchell, P. M. Haverty, B. A. Peters, T. D. Wu, L. C. Amler, J. Chant, D. Stokoe, M. R. Lackner, and G. Cavet. 2009. Genetic alterations and oncogenic pathways associated with breast cancer subtypes. Mol. Cancer Res. 7:511-522.

532. Balko, J. M., L. J. Schwarz, N. E. Bhola, R. Kurupi, P. Owens, T. W. Miller, H. Gomez, R. S. Cook, and C. L. Arteaga. 2013. Activation of MAPK pathways due to DUSP4 loss promotes cancer stem cell-like phenotypes in basal-like breast cancer. Cancer Res. 73:6346-6358.

533. Balko, J. M., R. S. Cook, D. B. Vaught, M. G. Kuba, T. W. Miller, N. E. Bhola, M. E. Sanders, N. M. Granja-Ingram, J. J. Smith, I. M. Meszoely, J. Salter, M. Dowsett, K. Stemke-Hale, A. M. Gonzalez-Angulo, G. B. Mills, J. A. Pinto, H. L. Gomez, and C. L. Arteaga. 2012. Profiling of residual breast cancers after neoadjuvant chemotherapy identifies DUSP4 deficiency as a mechanism of drug resistance. Nat. Med. 18:10521059.

534. Infante, J. R., K. P. Papadopoulos, J. C. Bendell, A. Patnaik, H. A. Burris, III, D. Rasco, S. F. Jones, L. Smith, D. S. Cox, M. Durante, K. M. Bellew, J. J. Park, N. T. Le, and A. W. Tolcher. 2013. A phase 1b study of trametinib, an oral Mitogen-activated protein kinase kinase (MEK) inhibitor, in combination with gemcitabine in advanced solid tumours. Eur. J. Cancer 49:2077-2085.

535. Britten, C. D. 2013. PI3K and MEK inhibitor combinations: examining the evidence in selected tumor types. Cancer Chemother. Pharmacol. 71:1395-1409.

536. Bartholomeusz, C., A. M. Gonzalez-Angulo, P. Liu, N. Hayashi, A. Lluch, J. FerrerLozano, and G. N. Hortobagyi. 2012. High ERK protein expression levels correlate with shorter survival in triple-negative breast cancer patients. Oncologist. 17:766-774.

537. Giltnane, J. M. and J. M. Balko. 2014. Rationale for targeting the Ras/MAPK pathway in triple-negative breast cancer. Discov. Med. 17:275-283. 
538. Carey, L., E. Winer, G. Viale, D. Cameron, and L. Gianni. 2010. Triple-negative breast cancer: disease entity or title of convenience? Nat. Rev. Clin. Oncol. 7:683-692.

539. Pearson, R., J. Fleetwood, S. Eaton, M. Crossley, and S. Bao. 2008. Krüppel-like transcription factors: a functional family. Int J Biochem. Cell Biol. 40:1996-2001.

540. Wei, D., M. Kanai, S. Huang, and K. Xie. 2006. Emerging role of KLF4 in human gastrointestinal cancer. Carcinogenesis 27:23-31. 


\section{CHAPTER 2}

microRNAs-206 and -21 cooperate to promote RAS-Extracellular Signal Regulated Kinase (ERK) signaling by suppressing the translation of RASA1 and SPREDI

Sriganesh B. Sharma ${ }^{1,2 \dagger}$, Chen-Chung Lin ${ }^{1,3 \dagger}$, Mark K. Farrugia ${ }^{1,2}$, Sarah L. McLaughlin $^{3}$, Emily J. Ellis ${ }^{3}$, Kathleen M. Brundage ${ }^{3}$, Mohamad A. Salkeni ${ }^{3,4}$, and J. Michael Ruppert ${ }^{1,3, \#}$

${ }^{1}$ Department of Biochemistry, ${ }^{2}$ Program in Cancer Cell Biology, ${ }^{3}$ The Mary Babb Randolph Cancer Center, and ${ }^{4}$ Department of Medicine, West Virginia University, Morgantown, West Virginia, 26506

\section{${ }^{\dagger}$ Equal contributions}

\#To whom correspondence should be addressed: E-mail, mruppert@hsc.wvu.edu; Phone: 304293-5246.

Running title: miR-206 and miR-21 cooperate to promote RAS signaling

Note: This manuscript has been published in Molecular and Cellular Biology, 2014 November; 34 (22): 4143-4164 


\section{ABSTRACT}

Despite the low prevalence of activating point mutation of RAS or RAF genes, the RAS-ERK pathway is implicated in breast cancer pathogenesis. Indeed, in triple-negative breast cancer (TNBC) there is recurrent genetic alteration of pathway components. Using shRNA methods, we observed that the zinc finger transcription factor Krüppel-like factor 4 (KLF4) can promote RAS-ERK signaling in TNBC cells. Endogenous KLF4 bound to the promoter regions and promoted the expression of two microRNAs (miRs), miR-206 and miR-21 (miR-206/21). Antisense-mediated knockdown (anti-miR) revealed that miR-206/21 coordinately promote RAS-ERK signaling and the corresponding cell phenotypes by inhibiting translation of the pathway suppressors $R A S A 1$ and SPRED1. In TNBC cells, including cells with mutation of $R A S$, the suppression of either RASA1 or SPRED1 increased the levels of GTP-bound, wild-type RAS and activated ERK 1/2. Unlike the control cells, treatment of RASA1- or SPRED1-suppressed cells with anti-miR-206/21 had little or no impact on the level of activated ERK 1/2 or on cell proliferation, and failed to suppress tumor initiation. These results identify RASA1 and SPREDI mRNAs as latent RAS-ERK pathway suppressors that can be upregulated in tumor cells by antimiR treatment. Consequently, KLF4-regulated miRs are important for the maintenance of RASERK pathway activity in TNBC cells. 


\section{INTRODUCTION}

In comparison to simpler organisms, the evolution of metazoans required adaptations for the proper regulation of cell fate (1). One such adaptation is the mitogen-activated protein kinase (MAPK) pathway composed of RAS, RAF, MEK, and ERK, which regulates a variety of cell physiologic processes (2-9). Diverse stimuli including growth factors, interaction with extracellular components, and cell stress can signal through receptor tyrosine kinases (RTKs), integrins, or ion channels to regulate signaling through the RAS GTPases. GTP-bound RAS (RAS-GTP) can activate MAP3Ks (i.e., the RAF family of protein kinases), leading to sequential phosphorylation and activation of MAP2Ks (i.e., MEK 1/2) and the extracellular signal-regulated kinases (ERK 1/2).

Inhibitory proteins play important roles in RAS-ERK pathway regulation. These include the RAS p21 protein activator (GTPase activating protein [GAP]) 1 (RASA1), the GAP neurofibromin 1 (NF1), the sprouty homologs SPRY1 and SPRY2, and the sprouty-related, EVH1 domain containing (SPRED) proteins, SPRED1 and SPRED2 (10-12). SPRED1 associates with NF1 to mediate its membrane localization, implicating GAP activity as a shared molecular mechanism among pathway inhibitory proteins (13). Congenital disorders that feature deregulation of this kinase cascade include Neurofibromatosis type I, Legius syndrome, Noonan syndrome, Costello syndrome, and cardiofaciocutaneous syndrome (8,9,14-16).

In addition, somatic alteration of this pathway is critical for the initiation and progression

of a variety of cancers. Activating point mutation of $R A S$ genes or $B R A F$ occur in approximately $15-30 \%$ and $7 \%$ of all human cancers, respectively $(3,17-20)$. In human breast cancer, point mutation of these genes is rare, but activated ERK 1/2 levels are frequently elevated and contribute to the aggressive behavior of cancer cells $(21,22)$. 
RAS-ERK pathway activity appears particularly critical in triple-negative breast cancers (TNBCs), tumors that are deficient in estrogen receptor (ER) $\alpha$, HER2, and progesterone receptor $(23,24)$. This group of clinically aggressive tumors overlaps extensively with the basallike and claudin-low molecular subtypes (25). Genomic analysis of human basal-like breast tumors indicates frequent copy number gain of $K R A S(32 \%)$ and $B R A F(30 \%)$, and reduced gene copy number for pathway inhibitors such as RASAl and DUSP4 (26-31). For RASAl, the correlation of mRNA levels, genomic copy number and clinical outcome supports a functional role in TNBC (29). Consistent with these results, basal-like breast tumors have a high RAS-ERK pathway activity signature $(24,30)$. Despite this insight, therapeutic targeting of the pathway is hindered by cellular mechanisms of escape, including dynamic reprogramming of the kinome and PI 3-kinase activation, and improved strategies for inhibiting the pathway are needed $(32,33)$.

The zinc-finger transcription factor Krüppel-like factor 4 (KLF4) is a pluripotency factor that functions in tumors in a context-dependent fashion, capable of exerting both protumorigenic and anti-tumorigenic effects (34-36). Supporting a tumor suppressor role, its expression is reduced during development of tumors such as colorectal cancer, and endogenous Klf4 suppresses tumorigenesis in the $\mathrm{Apc}^{\mathrm{Min}}$ mouse model (37). In normal cells, KLF4 is often induced in response to cell stress or wounding, and protumorigenic influences may reflect its role in the stress response (38-46). Loss- or gain-of-function studies show that KLF4 can promote malignant properties, including epithelial transformation in vitro, escape from RAS-induced senescence, enhanced cell survival following $\gamma$-radiation-induced DNA damage, increased tumorigenicity of colorectal cancer stem cell-enriched spheroid cells, and skin tumor initiation in transgenic mice (43,47-50). 
In human breast cancers, there is typically higher expression of KLF4 in tumor cells compared with the adjacent, uninvolved epithelium. This elevated protein level, or else demethylation of the KLF4 promoter, portends a poor prognosis (51-54). We previously identified microRNA (miR)-206 as a potential downstream effector of KLF4 that, in turn, directly regulates KLF4 translation, constituting a feedback loop (55). miRs associate with the RNA-induced silencing complex (RISC) to regulate the translation of cognate mRNAs. miR deregulation occurs in multiple cancer types and can promote or inhibit tumorigenesis (56-58).

In the current study, we suppressed KLF4 in TNBC cells and observed a decrease in miR-206 levels, attributed to the reduced association of KLF4 with a MIR206 promoter-proximal consensus site. Pathway analysis of putative miR-206 regulated genes identified this miR as a likely regulator of MAPK signaling, and in KLF4-deficient cells we observed marked downregulation of activated ERK 1/2 regardless of the RAS mutational status. As miRs can function in a combinatorial fashion, we sought additional miR effectors of KLF4 signaling to RAS-ERK. The protumorigenic miR-21 is upregulated in breast cancer and was previously validated to target RAS-ERK pathway inhibitory proteins (59-65). Furthermore, pathway enrichment identified MAPK signaling as likely to be co-targeted by miR-206 and miR-21 (miR206/21). We subsequently observed reduced levels of miR-21 in KLF4-deficient cells, attributed to a direct interaction of KLF4 with the MIR21 promoter. These results identified a pathway by which a pluripotency factor can signal through two distinct miRs to impact RAS-ERK signaling.

The loss of activated ERK 1/2 upon KLF4 depletion corresponded to a decrease in the level of GTP-bound wild-type (WT) RAS, and we found that miR-206 and miR-21 co-target both RASA1 and SPREDI to repress their translation. Although each miR alone had only modest effects on the level of activated ERK 1/2, simultaneous inhibition of both miRs led to marked 
downregulation of activated ERK 1/2, similarly as observed for KLF4-deficient cells. In RASWT and RAS-mutant cells alike, depletion of either RASA1 or SPRED1 promoted RAS-ERK pathway activity by modulating the levels of WT RAS-GTP. Knockdown of either RASA1 or SPRED1 conferred resistance to antisense miR (anti-miR) mediated inhibition of RAS-ERK signaling and promoted in vivo tumor initiation. These studies identify miR-206/21 as protumorigenic outputs of KLF4 signaling in TNBC cells, identify RASA1 and SPRED1 transcripts as latent RAS-ERK suppressors, and point to antagonists of KLF4-dependent miRs as potential agents for the therapeutic re-expression of RAS-ERK pathway inhibitory proteins.

\section{MATERIALS AND METHODS}

Cell lines, cell culture, and drug treatments. MDA-MB-231, HCC1143, HCC1937, MDAMB-468, and Hs578t breast cancer cell lines were obtained from ATCC. MCF10A and MCF10AT cells were provided by Steven M. Frisch (West Virginia University). SUM159PT cells were provided by Gary L. Johnson (University of North Carolina at Chapel Hill) and M6 cells were provided by Jeffrey E. Green (National Cancer Institute). MDA-MB-231 and M6 cells were cultured in DMEM supplemented with 10\% (v/v) FBS. HCC1143 cells were cultured in RPMI-1640 supplemented with 10\% FBS. Hs578t cells were cultured in DMEM supplemented with $5 \mu \mathrm{g} / \mathrm{ml}$ insulin and 10\% FBS. HCC1937 and MDA-MB-468 cells were cultured in RPMI1640 supplemented with $5 \mu \mathrm{g} / \mathrm{ml}$ insulin and 10\% FBS. SUM159PT cells were cultured in 50:50 DMEM/F12 supplemented with $5 \mu \mathrm{g} / \mathrm{ml}$ insulin, $1 \mu \mathrm{g} / \mathrm{ml}$ hydrocortisone, and $5 \%$ horse serum. MCF10A and MCF10AT cells were cultured in 50:50 DMEM/F12 supplemented with $10 \mu \mathrm{g} / \mathrm{ml}$ insulin, $0.5 \mu \mathrm{g} / \mathrm{ml}$ hydrocortisone, $20 \mathrm{ng} / \mathrm{ml} \mathrm{EGF,} 100 \mathrm{ng} / \mathrm{ml}$ cholera toxin, and 5\% horse serum. Cell culture media was also supplemented with penicillin and streptomycin. 4-hydroxytamoxifen 
(4-OHT) was dissolved in ethanol and used at $0.3 \mu \mathrm{M}$. U0126 (Sigma) was dissolved in DMSO and used at $20 \mu \mathrm{M}$. 5-aza-2'-deoxycytidine/decitabine (AZA; Selleck Chemicals) and trichostatin A (TSA; Selleck Chemicals) were dissolved in DMSO and used at $10 \mu \mathrm{M}$ and 400 nM respectively. Subconfluent cell cultures were treated with AZA for 96 hours or TSA for 12 hours. For the AZA treatment, the drug containing media was replenished every 24 hours.

Retroviral transduction. Suppression studies utilized pGIPZ lentiviral shRNAmir plasmids (V2LHS_28277 - shKLF4-1, V3LHS_410934 - shKLF4-2, V2LHS_28349 - shKLF4-3, V3LHS_376638 - shKLF4-4, V3LHS_410935 - shKLF4-5, V2LHS_149857 - shRASA1-1, V2LHS_203287 - shRASA1-2, V2LHS_49151 - shSPRED1-1, or V3LHS_634744 shSPRED1-2; Open Biosystems). The retroviral vector pLJD-HA-KLF4 was previously described (51). pLJD-KLF4-ER was generated by transfer of the insert from pBpuro-KLF4-ER to pLJD (48). Viral transduction was performed as described previously, and pGIPZ and pLJDtransduced cells were selected using puromycin $(0.5 \mu \mathrm{g} / \mathrm{ml}$ : HCC1937, MDA-MB-468; $1 \mu \mathrm{g} / \mathrm{ml}$ : MCF10A, MCF10AT, MDA-MB-231, Hs578t, SUM159PT, HCC1143; $5 \mu \mathrm{g} / \mathrm{ml}$ : M6) or Geneticin $(200 \mu \mathrm{g} / \mathrm{ml})$, respectively (55).

Plasmid construction. pMIR-REPORT firefly luciferase (luc) vector was purchased from Ambion/Invitrogen. pRL-TK Renilla luc reporter was obtained from Promega (Madison, WI). cDNA clones containing full length RASAl (clone ID BC033015) and a $1.2 \mathrm{~kb}$ fragment of the 3 ' untranslated region (3' UTR) of SPREDI (clone ID BG167687) were purchased from Open Biosystems. To construct a WT RASA1 translational reporter, a 926 bp fragment of the RASAl 3' UTR region was generated by sequential treatment with BamHI, Klenow fragment, and XbaI. This fragment was inserted into pMIR-REPORT vector that was prepared by sequential treatment with HindIII, Klenow fragment, and SpeI. To construct a WT SPREDI reporter 
containing the putative miR-206 binding site, the $1.2 \mathrm{~kb}$ SPRED1 3' UTR from clone BG167687 was excised by treatment with MluI and inserted into the same site of pMIR-REPORT. Finally, a 744 bp fragment of the SPRED1 3' UTR containing two putative miR-21 binding sites was amplified by PCR from MDA-MB-231 cDNA using the oligonucleotides 5'cccacgcgtTGAAAAACTGTTTAACTCATGT-3' and 5'cccacgcgtTGAAAAACCTGTAAATAAGCAC-3' (SPRED1 sequence indicated in uppercase). Following MluI digestion, the product was cloned into the same site of pMIR-REPORT vector. To generate RASAl and SPREDI reporters with mutation of miR seed complementary regions, the WT reporter sequence was altered so as to conserve the predicted secondary structure of the WT 3' UTR, as indicated by the mfold Web Server (66). Oligonucleotides (forward, reverse; mutated bases in lowercase) for PCR mutagenesis included RASA1 - miR-206 site: 5'-AAAAATAGCACACTTTTCCAacTctCAGTGATGTGTGAGCTATGC-3', 5'GCATAGCTCACACATCACTGagAgtTGGAAAAGTGTGCTATTTTT-3'; SPREDI - miR206 site: 5'- ATATATATATCTACTGTCAacTctCATATATTTTGAATATTTAAC-3'; 5'GTTAAATATTCAAAATATATGagAgtTGACAGTAGATATATATAT-3'; SPREDI - miR-21 Site 1: 5'-GTATTCAGATTTTTTTTTAAGTCTCTAgatcAATAATGTTATATTTATTG-3', 5'CAATAAATATAACATTATTgatcTAGAGACTTAAAAAAAAATCTGAATAC-3’; and SPRED1 - miR-21 Site 2: 5'TTGGTAACATGTTGCAGCTAccCTAATGACCTTAAGTGGCAATTG-3', 5'CAATTGCCACTTAAGGTCATTAGggTAGCTGCAACATGTTACCAA-3'. Cloned PCR products were confirmed by sequence analysis.

Transient transfection and translational reporter assays. Anti-miR inhibitors (AM) and miRmimics (PM) were obtained from Ambion/Invitrogen including, hsa-miR-206 (AM10409, 
PM10409), hsa-miR-21-5p (AM17000, PM17100), AM Negative Control \#1 (AM17010), and PM Negative Control \#1 (AM17110). Inhibitors and mimics were diluted to $20 \mu \mathrm{M}$ in nuclease free water and where indicated, transfected either singly or in combination into cells at a final total concentration of $25 \mathrm{nM}$. Where two miR reagents were cotransfected, the final concentration was $12.5 \mathrm{nM}$ each. For analysis of endogenous protein or miR levels, two sequential transfections were performed. Cells were subjected to reverse transfection and, 24 hours later, forward transfection was performed as described (55). At 24 hours after the start of the forward transfection, cell extracts were prepared for expression studies, or cells were used for phenotypic studies. Translational reporter assays were performed after only a single transfection, and extracts were prepared at 24 hours after the start of the reverse transfection. Inhibitors or mimics were cotransfected with reporter plasmids, including the pRL-TK control, and DualLuciferase ${ }^{\circledR}$ Reporter Assays (DLR Assay, Promega) were performed as described (55).

In vitro cell proliferation, transwell migration, and anoikis assays. Cells were plated for the respective assay at 24 hours following the final transfection with AM or PM. To measure cell proliferation, $1 \times 10^{3}$ cells/well were transferred to $96-$ well plates and cultured for the indicated interval. Cell proliferation was determined using the ATPlite Luminescence ATP Detection Assay System (PerkinElmer). Cell number was calculated by constructing a standard curve and correlating cell number with the luminescence signal. In parallel, 2D cell viability was measured by trypan blue exclusion.

For transwell migration assays, $1 \times 10^{4}$ cells were plated in the top chamber using growth medium containing $0.5 \%$ FBS ( 24 well plates, pore size, $8 \mu \mathrm{m}$; BD Biosciences). Growth medium containing 10\% FBS was used as chemoattractant in the lower chamber. After 24 hours, 
cells on the lower surface of the membrane were stained using the Diff-Quik ${ }^{\mathrm{TM}}$ Stain Set (Siemens) and counted.

To quantitate cell death (anoikis), $1 \times 10^{5}$ cells/well in DMEM complete growth medium containing $1 \%(w / v)$ methylcellulose were added to a 6-well Ultra-Low Attachment Cluster Dish (Costar). After 24 hours in suspension, the cells were washed twice with PBS and suspended in $200 \mu \mathrm{L}$ of AccuMax (Innovative Cell Tech). Cell death was measured by trypan blue exclusion. Alternatively, suspended cells were analyzed for cell death by flow cytometry.

Flow cytometry. Flow cytometric analysis of apoptosis was performed using Alexa Flour® 488 Annexin V/Dead Cell Apoptosis Kit (Invitrogen). Samples were analyzed on a BD Fortessa flow cytometer using BD FACSDiva 7.0 software (BD Biosciences). 10,000 events were collected per sample. Data analysis was performed using FCS Express 4 Research Edition software (De Novo Software).

Immunoblot analysis and antibodies. Cells were maintained as subconfluent monolayers in complete growth medium. For analysis of protein levels, including the levels of phosphorylated MEK 1/2 and ERK 1/2, cells were fed with complete growth medium 18-24 hours prior to extraction. Cells were washed twice in PBS and then lysed in ice-cold RIPA buffer (150 mM $\mathrm{NaCl}, 1 \%[\mathrm{w} / \mathrm{v}]$ sodium deoxycholate, $1 \%$ [v/v] Triton X-100, 5 mM EDTA, $50 \mathrm{mM}$ Tris-HCl, pH 7.5, $0.25 \mathrm{mM}$ PMSF, $1 \mathrm{mM}$ benzamidine, $1 \mathrm{mM}$ pepstatin, $1 \mu \mathrm{g} / \mathrm{mL}$ leupeptin, $1 \mu \mathrm{g} / \mathrm{mL}$ aprotinin, $0.4 \mathrm{mM}$ sodium orthovanadate, $40 \mathrm{mM} \beta$-glycerophosphate, and $20 \mathrm{mM} \mathrm{NaF}$ ). Extracts were centrifuged at $15,000 \times \mathrm{g}$ and protein concentration was determined using the Bradford Assay (Bio-Rad). Following electrophoresis, proteins were transferred onto nitrocellulose membranes and probed with the indicated antibody. Antibodies included phospho-ERK 1/2 (pERK 1/2; Thr202/Tyr204; Cell Signaling, 9101), ERK2 (Santa Cruz, C-14), 
phospho-MEK 1/2 (pMEK 1/2; Cell Signaling, 9154), total MEK 1/2 (tMEK 1/2; Cell Signaling, 9126), anti-hemagglutinin (HA) (Roche, 3F10), KLF4 (Santa Cruz, H-180), $\beta$-actin (Santa Cruz, C-4), p21 ${ }^{\text {Cip1/Waf1 }}$ (Santa Cruz, C-19), RASA1 (Santa Cruz, B4F8), SPRED1 (Abcam, 77079), PDCD4 (Rockland Immunochemicals, 600-401-965), HRAS (Santa Cruz, C-20), NRAS (Santa Cruz, F155), and KRAS (Santa Cruz, F234). Bound antibodies were detected using Pierce ECL Western Blotting Substrate (Thermo Scientific). Scanned images were quantitated using ImageJ software, with normalization to the loading control. Column data indicates the average of three independent experiments.

RAS-GTP affinity precipitation. Affinity precipitation of active RAS (RAS-GTP) utilized the RAS Assay Reagent, a GST-fusion protein corresponding to the RBD (residues 1-149) of Raf-1 (Millipore). For analysis of RAS-GTP levels, cells were fed with complete growth medium 1824 hours prior to protein extraction. Cells were washed twice in PBS and then lysed in ice-cold magnesium-containing lysis buffer (MLB; $25 \mathrm{mM}$ HEPES, pH 7.5, $150 \mathrm{mM} \mathrm{NaCl}, 1 \%$ Igepal CA-630, 0.25\% sodium deoxycholate, $10 \%$ glycerol, $10 \mathrm{mM} \mathrm{MgCl} 2,1 \mathrm{mM}$ EDTA, $25 \mathrm{mM} \mathrm{NaF}$, $1 \mathrm{mM}$ sodium orthovanadate, $10 \mu \mathrm{g} / \mathrm{ml}$ leupeptin, and $10 \mu \mathrm{g} / \mathrm{ml}$ aprotinin). Whole cell lysates (WCL) were centrifuged at $15,000 \times \mathrm{g}$ for 15 minutes and the protein concentration was determined using the Bradford Assay (Bio-Rad). WCLs were diluted to $1 \mathrm{mg} / \mathrm{ml}$ and $1 \mathrm{ml}$ of the lysate was precleared with glutathione agarose and used for affinity precipitation with $10 \mu \mathrm{g}$ of the Raf-1 RBD agarose conjugate. The agarose beads were collected by centrifugation at 15,000 $\times \mathrm{g}$ and washed three times with MLB. Beads were resuspended in 2X Laemmli sample buffer and boiled at $95^{\circ} \mathrm{C}$ for 5 minutes. Samples were diluted to $1 \mathrm{X}$ Laemmli buffer and subjected to SDS-PAGE and immunoblot analysis. 
Reverse Transcription and real-time PCR detection of miRs. Total RNA was extracted using mirVana $^{\mathrm{TM}}$ miRNA Isolation Kit (Ambion/Invitrogen). For mRNA analysis, total RNA was reverse transcribed using SuperScript II reverse transcriptase (Invitrogen). ESRI transcripts were analyzed using the Brilliant II SYBR ${ }^{\circledR}$ Green QPCR Master Mix (Agilent) with the following primers: 5'-AGGTGGACCTGATCATGGAG-3'， 5'-AAGCTTCGATGATGGGCTTA-3'. Reactions were normalized to B2M: 5'-TCTCTGCTGGATGACGTGAG-3', 5'TAGCTGTGCTCGCGCTACT-3'. Individual miRs were analyzed by stem-loop reverse transcription followed by quantitative real-time PCR (qPCR) detection using TaqMan MicroRNA Assays (Applied Biosystems) and normalized to U6 snRNA: hsa-miR-206 (\#000510), hsa-miR-21-5p (\#000397), U6 snRNA (\#001973). PCR reactions were performed on a Mx3005PTM Real-Time PCR System (Stratagene). mRNA and miR levels were determined by the $\Delta \Delta \mathrm{C}_{\mathrm{T}}$ method. Three independent experiments were performed in duplicate fashion.

Chromatin immunoprecipitation (ChIP). Potential KLF4 binding sites were identified using JASPAR (67). Chromatin from $4 \times 10^{7}$ cells was prepared as described (68) and used as input for each immunoprecipitation (IP). Chromatin was sonicated in ice water using a Bioruptor (Diagenode) set at high energy, cycling on/off at 30 second intervals for 6 cycles of 10 minutes each. IP was performed using $1 \mu \mathrm{g}$ of the indicated antibody. Following IP, elution, reversal of crosslinks, and proteinase K digestion, DNA was purified using Qiaquick spin columns (Qiagen) and then eluted in $50 \mu \mathrm{l}$ of $10 \mathrm{mM}$ Tris- $\mathrm{HCl}(\mathrm{pH} 8.0) .2 \%$ of the ChIP yield was used as input for each PCR reaction. ChIP Intensity levels were determined by use of the $\Delta \mathrm{C}_{\mathrm{T}}$ method to compare the yield obtained using anti-KLF4 or normal IgG. The sequences of oligonucleotides are: miR21 site 1, 5'-CTTAGATTGAGAAAGACCGC-3' and 5'-ACTTATGCTTGTGTCATCCC-3'; miR-21 site 2, 5'-GCAACCTCCACTTCCTGGGT-3' and 5'-CCAACACAGTGAAACCCTGT- 
3'; miR-206 site 1, 5'-CATCAACAACACCCCAAGCG-3' and 5'-

GGCACAGTTTTGGATCAACCC-3'; miR-206 site 2, 5'-TGCAAAGCACAGAGAAACGTG3' and 5'-ACCTTCTTCCCATTTTCCTGGAC-3'.

Animal Studies. Female Athymic Nude mice (Crl:NU(NCr)-Foxn1 ${ }^{\text {nu }}$, Charles River) were obtained at 6-8 weeks of age. $2 \times 10^{6}$ cells in in $100 \mu 1$ of DMEM were injected into the 4th mammary fat pad. Caliper measurements were performed twice a week and tumor initiation was defined as $\geq 4 \mathrm{~mm}$ for both $\mathrm{L}_{1}$ and $\mathrm{L}_{2}\left(\mathrm{~L}_{1}\right.$, long axis; $\mathrm{L}_{2}$, short axis). All animal procedures were performed under an approved protocol.

Statistical Analysis. Data were analyzed using the unpaired t-test (two-tailed), or else one-way analysis of variance (ANOVA) followed by Tukey's multiple comparison ad hoc post-test. Growth curves were analyzed using non-linear regression curve fitting. Tumor initiation was analyzed using a $2 \times 2$ contingency table with Fisher's exact test. Statistical analyses were performed in GraphPad Prism 5 (GraphPad Software). Except where noted, assays were performed three times in duplicate fashion. Cell proliferation assays were performed in three independent experiments, each containing 5 replicates. Differences were considered significant when the analysis yielded $\mathrm{P}<0.05$.

\section{RESULTS}

KLF4 regulates miR-206 and ERK signaling in TNBC cells. Consistent with our previous study, miR-206 levels were markedly repressed in KLF4-depleted cells (Fig. 1A) (55). Similar results were obtained in the RAS-mutant $\left(K R A S^{G 13 D}\right)$ and claudin-low tumor line MDAMB-231, and the RAS-WT and basal-like HCC1143 cells. ChIP analysis of KLF4 at consensus sites in the MIR206 locus identified enrichment of site 1, located within the promoter-proximal 
region (Fig. 1B). Supporting specificity, this enrichment was reduced in KLF4-deficient cells and increased in cells with exogenous KLF4 (Fig. 1C).

As protumorigenic mechanisms of KLF4 signaling remain poorly understood, we sought potential effectors of miR-206. In silico enrichment analysis identified MAPK signaling as likely to be regulated by miR-206, which has the potential to target 17 genes in this pathway $(\mathrm{p}=1.24$ $\times 10^{-2}$, Table 1). Because of its ability to regulate miR-206, we first determined whether endogenous KLF4 can regulate steady state RAS-ERK activity in TNBC cells by analyzing ERK 1/2 activation loop phosphorylation (i.e., activated ERK $1 / 2$ or pERK 1/2). In KLF4-deficient cells the pERK 1/2 levels were suppressed (Fig. 2A). In these cell lines, the reduction of pERK $1 / 2$ reflected lower levels of WT RAS-GTP, whereas mutant RAS-GTP levels were unaffected by KLF4 knockdown (Fig. 2B). In KLF4-deficient MDA-MB-231 cells, introduction of HAtagged KLF4 rescued the levels of miR-206 and pERK 1/2 (Fig. 2C).

To temporally correlate the induction of miR-206 and pERK $1 / 2$ by KLF4, we transduced KLF4-depleted MDA-MB-231 cells with KLF4-ER or empty vector (Fig. 2D). The KLF4-ER fusion protein is constitutively expressed but functionally inactive until treatment of cells with 4hydroxytamoxifen (4-OHT) $(48,69)$. As previously reported, addition of 4-OHT to KLF4-ER cells resulted in upregulation of miR-206 between 0.5 and 2 hours post-treatment (Fig. 2E, left panel) (55). In this experiment the induction of pERK 1/2 was apparent by 2 hours (Fig. 2E, right panel). KLF4-ER activity was supported by the induction of cyclin-dependent kinase inhibitor 1A (p21 $\left.{ }^{\text {Cip1/Wafl }}\right)$ in these cells (70) (Fig. 2E, right panel). The modest induction of activated ERK $1 / 2$ by exogenous KLF4 (Figs. $2 \mathrm{C}$ and 2E) was in contrast to the larger fold effect of endogenous KLF4 (Fig. 2A), identifying a discordance between the two approaches. 


\section{miR-206 suppresses the translation of the RAS-ERK pathway inhibitors RASA1 and}

SPRED1. Consistent with the mutual dependence of both miR-206 and RAS-ERK activity upon KLF4, we sought to identify specific components of the RAS-ERK pathway that are regulated by this miR. The two RAS-ERK pathway suppressors RASAl and SPREDI were consistently identified as likely miR-206 targets across multiple miR algorithms (Table 2). Consistent with regulation by miR-206, KLF4 depletion was associated with higher levels of RASA1 and SPRED1 (Fig. 3A). To examine a role for endogenous miR-206 we utilized antisense inhibitor specific to the mature miR (anti-miR). As compared to the control, anti-miR-206 depleted the miR levels in TNBC cells (Fig. 3B). This suppression of miR-206 activity was sufficient to increase the levels of the two pathway inhibitors (Fig. 3C, left panels). Conversely, transfection of exogenous miR-206 (miR-206-mimic) decreased the level of each protein (Fig. 3C, right panels). These results identified miR-206 as a potential link between KLF4 and RAS-ERK signaling.

To analyze the regulation of protein translation by miR-206, we utilized translational reporter assays. Fragments of the 3' UTRs containing putative miR-206 binding sites were cloned downstream of the open reading frame of firefly luc (Figs. 4A-4B). Relative to the control anti-miR, in MDA-MB-231 cells transfected with anti-miR-206, the luc activity was 3.9 fold induced for the WT RASAI reporter and 2.4 fold induced for the WT SPREDI reporter (Figs. 4C-4D, upper and middle panels). Conversely, transfection of each reporter with miR-206-mimic decreased luc activity by $32 \%$ for the RASAl reporter and by $64 \%$ for the SPREDI reporter (Figs. 4C-4D, lower panels). Reporter regulation by miR-206 was abolished by mutation of RASA1 or SPRED1 sequences important for miR-206 binding (Figs. 4C-4D). These results identify miR-206 as a direct regulator of these transcripts, supporting a role for KLF4 in 
promotion of RAS-ERK signaling through miR-206 mediated suppression of RASAl and SPREDI.

miR-21 is a KLF4-dependent miR that represses the translation of both RASA1 and

SPRED1. As modulation of miR-206 alone was not sufficient to recapitulate the effects of KLF4 on pERK 1/2 levels, we therefore sought additional downstream effectors. Similarly to miR-206, miR-21 is upregulated in breast cancer and is predicted by pathway enrichment analysis to regulate MAPK signaling ( $\mathrm{p}=2.09 \times 10^{-3}$; Table 3 ) (59). Furthermore, miR-21 has been validated to directly regulate the translation of several RAS-ERK-activator protein 1 (AP-1) inhibitory components, including RASA1, SPRY1, SPRY2, and PDCD4 (56-58). These common features suggested the possibility of shared signaling by these two miRs. Providing compelling support for this idea, MAPK signaling was ranked first among the pathways likely to be coregulated by miR-206/21 ( $\mathrm{p}=3.00 \times 10^{-4}$; Table 4$)$.

The ability of KLF4 to regulate RAS-ERK signaling, and the established role of miR-21 in regulation of this pathway identified KLF4 as a potential regulator of miR-21. To determine whether KLF4 might signal through miR-21, we assayed KLF4-deficient TNBC cells for miR-21 levels, observing marked suppression (Fig. 5A). ChIP analysis of KLF4 at consensus sites in the MIR21 locus of MDA-MB-231 cells identified enrichment of site 1, located within the promoter region (Fig. 5B). This enrichment was reduced in KLF4-deficient cells and increased in cells with exogenous KLF4 (Fig. 5C). In contrast to the enhanced ChIP intensity signal in cells with exogenous KLF4, miR-21 levels were not enhanced (Fig. 5D). Restoration of KLF4 in MDAMB-231/shKLF4 cells was likewise insufficient to increase miR-21 (Fig. 5E). As a control, miR206 levels were induced by exogenous KLF4 in these experiments (Fig. 5D), suggesting different modes of miR regulation by KLF4. 
To address possible off-targeting by KLF4 shRNAs (shKLF4), we analyzed additional shKLF4 constructs. We observed only weak activity of shKLF4-3, but more efficient KLF4 suppression by shKLF4-4 and shKLF4-5 (Fig. 5F, left panels). Compared to shCtl and shKLF43, cells transduced with the active constructs had consistently reduced levels of miR-21 (Fig. 5F, right panels). Consequently, the regulation of miR-21 by endogenous KLF4 was supported by a total of four active shRNAs (Figs. 5A and 5F).

The failure of exogenous KLF4 to restore miR-21 levels appeared consistent with a stable alteration of MIR21 chromatin in KLF4-deficient cells $(71,72)$. To examine this possibility we treated shCtl cells or shKLF4-1 cells with the DNA methyltransferase inhibitor AZA or the histone deacetylase inhibitor TSA (Fig. 5G). Unlike TSA, AZA largely restored miR-21 levels (Fig. 5G, left panel). Neither AZA nor TSA significantly altered miR-206 levels, whereas ESR1 served as a positive control and was induced by both agents (Fig. 5G, middle and right panels respectively) $(73,74)$. These results show that endogenous KLF4 is permissive for the expression of miR-21 in TNBC cells, and support a role for chromatin modification in the suppression of miR-21 following KLF4 depletion.

The regulation of RASA1 by miR-21 is well established (64). Our results above indicate that RASA1 is co-targeted by miR-206/21, suggesting a broader role for this pair as coregulators of RAS-ERK pathway components (Fig. 4). Although miR-206 was predicted to regulate SPRED1 and this was subsequently validated, whether miR-21 can likewise regulate this factor was unknown. Nevertheless, transfection of anti-miR-21 into MDA-MB-231 cells increased the protein levels of both RASA1 and SPRED1, whereas exogenous miR-21 was suppressive (Fig. 6A). Unlike the well-conserved miR-21 sites in RASA1, analysis in TargetScan revealed that SPRED1 contains two candidate binding sites for miR-21 with only limited species 
conservation (Figs. 6B-6C) (75). Translational reporter assays identified only one of these sites as functional, and supported the direct regulation of both RASAl and SPRED1 transcripts by miR-21 (Figs. 6D-6E). As a control, transfection of anti-miR-21 led to reduced miR-21 activity, as indicated by immunoblot analysis of PDCD4, a well established target of this miR (Fig. 6A) (63). These results validate SPREDI as a miR-21 targeted transcript. Therefore, the miR-206/21 pair can indeed co-target distinct RAS-ERK pathway components, validating the idea that MAPK signaling represents an important signaling intersection for these two miRs (Table 4).

\section{Consistent KLF4 regulation of miR-206/21 levels and RAS-ERK signaling in RAS-}

WT and RAS-mutant tumor cells. The results observed for MDA-MD-231 and HCC1143 cells suggested a functional relationship between the KLF4-miR-206/21 axis and the RAS-ERK pathway. We analyzed this signaling in a broader panel of mammary epithelial cells (MECs) and cancer cells (Fig. 7A). KLF4 depletion in nontumorigenic MCF10A cells, in HRAS-mutant MCF10AT cells, or in a variety of human or mouse TNBC lines led to consistently reduced levels of activated ERK 1/2 and to increased levels of RASA1 and/or SPRED1. In the KLF4deficient TNBC cells, RASA1 and SPRED1 were concordantly upregulated. However, in MECs, the RASA1 levels were not appreciably altered, and reduced pERK 1/2 levels were associated with increased SPRED1 alone. KLF4 depletion was likewise associated with reduced miR206/21 levels, except in MDA-MB-468 cells where miR-206 was undetected (Fig. 7B). In this miR-206-deficient cell line, the overall effect of KLF4 on pERK 1/2 appeared more modest. In summary, KLF4-miR-206/21 signaling appears to generally regulate the RAS-ERK pathway in TNBC cells, with similar effects in RAS-WT (HCC1143, HCC1937, and MDA-MB-468) and RAS-mutant cells (MDA-MB-231, Hs578t, and SUM159PT). 


\section{miR-206 and miR-21 cooperate to promote RAS-ERK signaling and ERK 1/2}

dependent phenotypes. Transfection of anti-miR-206 or anti-miR-21 alone did not have prominent effects on pERK 1/2 levels (Fig. 8, lanes 2, 3, 6, and 7). To test for cooperativity we inhibited both miRs in TNBC cells (i.e., anti-miR-206/21), and then assayed for RASA1 and SPRED1 levels (Fig. 8, lanes 4 and 8). Compared to the individual anti-miRs, SPRED1 was consistently induced to a greater extent by anti-miR-206/21. In contrast, RASA1 levels responded to each of the anti-miRs (lanes 2 and 6), but anti-miR-206/21 cooperativity was only apparent in HCC1143 cells (lanes 4 and 8). Nevertheless, anti-miR-206/21 transfection of TNBC cells reduced the levels of pMEK $1 / 2$ and pERK $1 / 2$ to a greater extent than did either anti-miR when used alone. These findings identify two KLF4-dependent miRs as maintenance factors for RAS-ERK signaling in TNBC cells, potentially through cooperativity for regulation of SPRED1 and/or other pathway regulators.

In breast cancer cells the RAS-ERK pathway promotes cell proliferation, migration, and resistance to cell death $(21,22)$. TNBC cells transfected with both anti-miRs displayed slower growth over a three day time course than did control or single anti-miR transfected cells (Fig. 9A, upper panels). This marked attenuation of proliferation was not explained by changes in cell viability as measured by trypan blue exclusion (Fig. 9A, lower panels).

Similarly to cell proliferation, inhibition of both miRs abrogated TNBC cell migration in transwell chambers to a greater extent than the inhibition of either miR alone (Fig. 9B). Finally, anti-miR-206/21 rendered TNBC cells more susceptible to cell death in anoikis assays (Fig. 9C9D). As a control, treatment of matrix-deprived MDA-MB-231 cells with the MEK 1/2 inhibitor U0126 yielded a higher rate of cell death (76). These results indicate that miR-206/21 can cooperate to promote RAS-ERK pathway signaling as well as pathway dependent phenotypes. 


\section{miR-206 and miR-21 promote RAS-ERK signaling by repression of RASA1 and}

SPRED1. To determine whether miR-206/21 impact RAS-ERK signaling through their coregulation of RASA1 and/or SPRED1, we depleted either RASA1 (Fig. 10A) or SPRED1 (Fig. 10B). Knockdown of either factor had little effect upon the other protein. Regardless of the RAS mutational status, knockdown of RASA1 or SPRED1 led to increased steady-state levels of pERK 1/2 relative to control cells. This increase in pathway activity was associated with elevated levels of WT RAS-GTP (Fig. 10C). In contrast to the results obtained for WT RAS proteins, the KRAS-GTP levels in MDA-MB-231 cells $\left(K R A S^{G 13 D}\right)$ were not appreciably altered by suppression of RASA1 or SPRED1. Similarly, the HRAS-GTP levels in SUM159PT cells $\left(H R A S^{G I 2 D}\right)$ were unchanged by suppression of either RASA1 or SPRED1, even though pERK 1/2 levels were increased (Fig. 10D). These results suggest that WT RAS proteins mediate the enhanced RAS-ERK pathway activity in RASA1- or SPRED1-deficient TNBC cells.

To test for a function of RASA1 and SPRED1 as mediators of miR-206/21 effects on RAS-ERK signaling, we delivered anti-miR-206/21 to control cells or cells deficient in either protein (Fig. 11A). As an indicator of successful miR suppression we assayed the protein levels of PDCD4, which is regulated by miR-21. As expected, combined miR-206/21 inhibition in control cells reduced the levels of pERK 1/2 (Fig. 11A; lanes 1-2 in each panel). Suppression of RASA1 rendered cells largely independent of miR-206/21, as pERK 1/2 showed little or no attenuation by anti-miR (lanes 3-4). In SPRED1-suppressed cells, RASA1 and/or some other miR-206/21-dependent component appeared to be limiting for pathway activity, as anti-miRs induced RASA1 and also suppressed the levels of pERK 1/2 (lanes 5-6). In TNBC cells depleted of RASA1 or SPRED1 and then treated with anti-miR-206/21, the residual activated ERK 1/2 was increased relative to control cells (Fig. 11A, lanes 2, 4, and 6). This anti-miR-resistant 
signaling supports functional roles for both RASA1 and SPRED1 as mediators of the KLF4dependent miRs.

Phenotypic data consistent with these immunoblot results were obtained by analysis of cell proliferation (Fig. 11B). Compared to parental (untransduced and untransfected) TNBC cells or control (shCtl) cells, shRASA1 and shSPRED1 cells proliferated at a rate that was only slightly faster. For each cell line we next measured cell proliferation following treatment with either anti-miR-Ctl or anti-miR-206/21. Whereas shCtl cells transfected with anti-miR-206/21 proliferated much more slowly ( $\mathrm{p}<0.001$ ), shRASA1 cells displayed anti-miR-206/21-resistant cell proliferation ( $\mathrm{p}>0.05$; Fig. 11B). shSPRED1 cells had an intermediate phenotype, with a smaller size effect than observed for shCtl cells that were treated with anti-miR-206/21 ( $p<0.05$; Fig. 11B). The effects of anti-miR-206/21 on cell proliferation appeared consistent with the residual levels of activated ERK 1/2 (see Fig. 11A).

Consistent results were obtained when anti-miR-treated TNBC cells were orthotopically injected into the mammary gland of immunodeficient mice (Fig. 11C). Relative to anti-miR-Ctl, anti-miR-206/21 suppressed tumor initiation by the control cells (shCtl), attributed to their decreased proliferation and/or increased cell death following implantation into the mammary gland. In contrast, cells deficient in RASA1 or SPRED1 were competent for tumor initiation. These results support functional roles for both RASA1 and SPRED1 in miR-206/21 signaling.

\section{Restoration of RAS-ERK signaling by exogenous miR-206/21 in KLF4-depleted cells}

promotes resistance to cell death. To complement the anti-miR data, we delivered exogenous miRs into KLF4-deficient TNBC cells (Fig. 12A). As compared to the individual miR-mimics (Fig. 12A, lanes 2, 3, 6, and 7), more pronounced signaling effects were obtained using the miR- 
206/21-mimic (lanes 4 and 8). These effects included suppression of RASA1 and SPRED1 and the induction of activated MEK 1/2 and ERK 1/2.

Relative to the control, transfection of either miR-206- or miR-21-mimic into KLF4depleted cells reduced the cell death following matrix deprivation (Fig. 12B, lanes 2, 3, 8, and 9). Consistent with the cooperative regulation of pERK 1/2 levels (Fig. 12A, lanes 4 and 8), a greater fold effect on cell death was observed for cells treated with both mimics (Fig. 12B, lanes 4 and 10). In these studies, exogenous KLF4 effects were similar to that of the miR-mimics, and suppressed cell death (Fig. 12B, left panel, lanes 5 and 6). These gain-of-function studies provide independent support for the cooperative regulation of RAS-ERK signaling by KLF4-dependent miRs-206/21. In the light of previous studies by others, our results support a model for miR206/21 co-targeting and co-regulation of RAS-ERK signaling (Fig. 13).

\section{DISCUSSION}

Compared to other breast cancer subtypes, TNBCs express elevated levels of RTKs such as EGFR and FGFRs that represent major regulators of RAS-ERK signaling $(30,77)$. Although genetic changes in these receptors or the mutational activation of RAS or RAF are rare, these tumors often harbor other genetic alterations that promote RAS-ERK pathway activity (26-31). Supporting the importance of this signaling, TNBC cells are particularly sensitive to drugs such as MEK or PI3K inhibitors, and combination therapies have been analyzed in clinical trials $(23,24,78,79)$.

KLF4 is a major regulator of pluripotency with the potential to either promote or suppress malignant properties, and dissecting the relevant mechanisms has the potential to identify new therapeutic approaches. We previously implicated miR-206 as a potential downstream effector of 
KLF4 (55). Evidence from the current study supported a direct role of KLF4 in regulation of miR-206. Consistent with a role for this signaling in TNBCs, miR-206 is upregulated in breast cancer and ER-negative tumors express higher levels relative to ER-positive tumors $(59,80)$.

miR-206 has been well characterized in muscle cells, where it promotes skeletal muscle regeneration in response to injury (81-83). An in silico search for cancer-relevant influences of miR-206 identified regulation of MAPK signaling as a likely effector pathway, with potential effects on RASA1 and SPRED1 (Tables 1-2). Subsequently, analysis of KLF4-deficient cells revealed upregulation of these two pathway inhibitors in conjunction with markedly reduced levels of pERK 1/2, and protein translation reporter studies identified direct roles for miR-206 in regulation of RASA1 and SPRED1.

As compared to the pronounced effect of endogenous KLF4 on pERK 1/2 levels, modulation of miR-206 alone revealed only modest effects (Fig. 12A), and we therefore sought additional effectors downstream of KLF4. We evaluated miR-21 as a candidate because of its upregulation in breast cancer and its known role in regulation of RAS-ERK pathway components, including RASA1 (Table 3) (58-65). Strikingly, an intersection approach identified MAPK signaling as the pathway most likely to be co-regulated by miR-206/21 (Table 4). We subsequently observed a critical role for endogenous KLF4 in maintenance of miR-21 levels, and found that both RASA1 and SPRED1 contain binding sites for miR-206/21. These results identified a recurrent regulatory strategy in which two KLF4-regulated miRs can impact the same transcript. As shown by suppression of KLF4 or by the introduction of anti-miR-206/21, this regulation results in pronounced alteration of RAS-ERK signaling in the multiple TNBC models examined. 
The protumorigenic miR-21 is abundant in TNBC cells and inhibits the translation of multiple negative regulators of RAS-ERK-AP1 signaling (Fig. 13). Despite extensive interactions with the RAS-ERK pathway, we and others have observed that antisense-mediated inhibition of endogenous miR-21 gives relatively modest effects on overall pathway activity, as indicated by analysis of activated ERK 1/2 (Fig. 8). In the current study, we observed that miR206/21 function as a pair to co-target RAS-ERK pathway inhibitory proteins, with profound consequences on RAS-ERK signaling. Such co-targeting is not without precedent. For example, miR-27a, miR-96, and miR-182 co-target the tumor suppressor FOXO1 in breast cancer cells (84).

Our analysis of KLF4-depleted TNBC cells indicated that endogenous KLF4 could influence the levels of both miR-206 and miR-21, a response obtained using each of four distinct KLF4 shRNAs (Fig. 5A and 5F). We also characterized the temporal KLF4 regulation of miR levels using a 4OHT-conditional KLF4-ER fusion protein. In combination with ChIP data that strongly supported direct interaction of KLF4 with the promoter-proximal regions of MIR206 and MIR21, these studies implicated MIR206 and MIR21 as direct transcriptional targets of KLF4, but with distinct modes of regulation.

Unlike for the regulation of MIR206, we observed that endogenous and exogenous KLF4 function discordantly for regulation of MIR21. Previous studies have shown that MIR21 is transcribed as an independent unit located in the intron of the TMEM49 gene (85). Relative to the control cells, in KLF4-deficient cells we observed a decrease of TMEM49 of similar to 35\% (not shown). Unlike for miR-206, exogenous KLF4 did not alter miR-21 levels in TNBC cells. Similarly, restoration of KLF4 activity in KLF4-depleted tumor cells induced miR-206 but did not significantly alter miR-21 levels. The insufficiency of exogenous KLF4 for induction of 
miR-21 suggests an "on-off" mode of regulation, and identifies KLF4 suppression as a potential hit-and-run strategy for the therapeutic silencing of miR-21 in tumors. Also, this lack of regulation of miR-21 by exogenous KLF4 is quite consistent with the more limited effect of exogenous KLF4 on activated ERK 1/2 levels relative to the endogenous transcription factor (Figs. 2A and 2C).

These results suggested a working model in which endogenous KLF4 maintains an open chromatin structure at MIR21. Initial support for this model was obtained by analyzing a role for DNA methylation. In KLF4-deficient cells, but not in control cells, treatment with the DNA methyltransferase inhibitor AZA was sufficient to upregulate miR-21 levels. Krüppel-like factors such as Erythroid Krüppel-like factor (EKLF or KLF1) can regulate chromatin structure by interacting with chromatin modifying proteins or chromatin remodelers (86-88). The observed regulation of MIR21 by KLF4 is especially interesting given the relationship between open chromatin and pluripotency (89).

KLF4-miR-21 signaling highlights the potential for distinct effects of KLF4 in loss- and gain-of-function experimental settings. As the oncogenic miR-21 is expressed independently of exogenous KLF4, KLF4 gain-of-function phenotypic studies may potentially underestimate protumorigenic signaling by the endogenous transcription factor. It is currently unclear whether KLF4 can regulate other loci in a similar fashion as for MIR21, or whether its regulation of MIR21 extends to other cell types.

Importantly, shRNA studies revealed both RASA1 and SPRED1 to be limiting endogenous factors for steady state RAS-ERK signaling through modulation of WT RAS-GTP levels, identifying these components as potential mediators of miR-206/21 effects (Fig. 10). miR loss- and gain-of-function studies using single anti-miRs or miR-mimics indicated that either 
miR-21 or miR-206 could individually regulate the level of these pathway inhibitors, but with only subtle effects on pERK 1/2 levels. Indicating cooperativity, larger fold effects were observed when anti-miRs or miR-mimics were combined to modulate both miR-206 and -21 . Cooperativity was observed for the expression level of SPRED1, for the levels of pERK 1/2, and for relevant phenotypic parameters including tumor cell proliferation, migration, and survival.

As observed for SPRED1, RASA1 protein levels were dependent upon both miR-206 and miR-21. However, RASA1 did not consistently show cooperative regulation. Strikingly, RASA1 expression was nevertheless critical for miR-206/21 signaling, as anti-miR-206/21 had little or no discernible effect on pERK 1/2 levels in RASA1-deficient cells (Fig. 11). Likewise, cell proliferation and tumor initiation were concordantly anti-miR resistant in RASA1-deficient cells.

Supporting a functional role for SPRED1 in KLF4-miR signaling to RAS-ERK, SPRED1-suppressed cells not only displayed elevated pERK 1/2 levels, but also had resistance to anti-miR-206/21 that was intermediate as compared to shCtl cells and shRASA1 cells. This was shown by analysis of pERK $1 / 2$ and cell proliferation, and tumor initiation in mice was antimiR-resistant. The failure of anti-miR-206/21 to regulate pERK 1/2 levels in RASA1-deficient cells, despite upregulation of SPRED1, suggests that SPRED1 activity may be somehow limited in this context (Fig. 11). For example, this data would appear consistent with a critical role for RASA1 in signaling by SPRED1 or SPRED1-NF1 (13). On the other hand, in the context of MDA-MB-231 cells where SPRED1 but not RASA1 was cooperatively induced by anti-miR206/21, the cooperative suppression of pERK 1/2 levels may be largely attributed to SPRED1, with RASA1 serving a more permissive role (Fig. 8).

Of interest in the current study was the similar effect of anti-miR-206/21 on RAS-ERK signaling in RAS-WT and RAS-mutant breast cancer cells alike. In RAS-mutant cells that were 
analyzed for RAS-GTP levels, only the WT RAS GTP was increased following depletion of either RASA1 or SPRED1. Consistent with our results, previous studies support the potential for these pathway inhibitors to antagonize signaling in cells harboring activated RAS (90-92). The effects of RASA1 and SPRED1 that we observed appear consistent with an important role of WT RAS proteins (i.e., KRAS, NRAS, and/or HRAS) for pathway activation in RAS-mutant TNBC cells. This model is supported by several previous analyses in non-mammary contexts (93-96). For example, suppression of the guanine nucleotide exchange factor SOS1 in a RAS-mutant context results in attenuation of WT RAS-GTP levels and pERK 1/2 levels, and suppresses tumorigenesis (94). In addition to effects on WT RAS proteins, the possibility that RASA1 and/or SPRED1 could suppress signaling by impacting other steps in the pathway is not excluded. Although additional studies are needed, these results support the targeting of RASA1 and SPRED1 for therapy of RAS-mutant as well as RAS-WT cancers.

We have identified a facet of KLF4 signaling that promotes malignant properties in TNBC cells, through miR-mediated activation of RAS-ERK signaling. The results highlight RASAl and SPREDI transcripts as latent tumor suppressors in TNBC cells, held at bay through KLF4-dependent miRs. The pronounced inhibitory effect of anti-miR-206/21 on the level of activated ERK 1/2 identifies the enhanced translation of RASA1 and SPRED1 as an attractive therapeutic strategy. In TNBCs the use of MEK 1/2 inhibitors typically induces a rapid compensatory reprogramming of the kinome, leading to drug resistance (33). Suppression of KLF4 or else the anti-sense mediated silencing of miR-206 and/or miR-21 might be used in combination with MEK inhibitors or other pathway antagonists to attenuate this drug resistance. 


\section{ACKNOWLEDGMENTS}

This work was supported by grant NCI RO1 CA127405 and the Jo and Ben Statler Chair in Breast Cancer Research. We thank Steven M. Frisch (West Virginia University), Gary L. Johnson (University of North Carolina at Chapel Hill) and Jeffrey E. Green (NIH) for providing cell lines. Flow cytometry experiments were performed in the West Virginia University Flow Cytometry Core Facility, which is supported by the NIH equipment grant RR020866 and the Institutional Development Award (IDeA) from the National Institute of General Medical Sciences under grants P30GM103488 (CoBRE) and P20GM103434 (INBRE). Orthotopic tumor cell injections were performed in the West Virginia University Animal Models \& Imaging Facility, which has been supported by the Mary Babb Randolph Cancer Center and NIH grants P20 RR016440, P30 RR032138/GM103488 and S10 RR026378. 


\section{REFERENCES}

1. Weinberg RA. 2007. Growth Factors, Receptors, and Cancer, p. 119-158. In: Weinberg RA (ed.), The Biology of Cancer. Garland Science, Taylor and Francis Group, LLC, New York.

2. Johnson GL and Lapadat R. 2002. Mitogen-activated protein kinase pathways mediated by ERK, JNK, and p38 protein kinases. Science 298:1911-1912.

3. Downward J. 2003. Targeting RAS signalling pathways in cancer therapy. Nat Rev Cancer 3:11-22.

4. Kolch W. 2005. Coordinating ERK/MAPK signalling through scaffolds and inhibitors. Nat Rev Mol Cell Biol 6:827-837.

5. McCubrey JA, Steelman LS, Abrams SL, Lee JT, Chang F, Bertrand FE, Navolanic PM, Terrian DM, Franklin RA, D'Assoro AB, Salisbury JL, Mazzarino MC, Stivala F, and Libra M. 2006. Roles of the RAF/MEK/ERK and PI3K/PTEN/AKT pathways in malignant transformation and drug resistance. Adv Enzyme Regul 46:249-279.

6. Roberts PJ and Der CJ. 2007. Targeting the Raf-MEK-ERK mitogen-activated protein kinase cascade for the treatment of cancer. Oncogene 26:3291-3310.

7. Mebratu Y and Tesfaigzi Y. 2009. How ERK1/2 activation controls cell proliferation and cell death: Is subcellular localization the answer? Cell Cycle 8:1168-1175.

8. Young A, Lyons J, Miller AL, Phan VT, Alarcon IR, and McCormick F. 2009. Ras signaling and therapies. Adv Cancer Res 102:1-17.

9. Pylayeva-Gupta Y, Grabocka E, and Bar-Sagi D. 2011. RAS oncogenes: weaving a tumorigenic web. Nat Rev Cancer 11:761-774.

10. Boguski MS and McCormick F. 1993. Proteins regulating Ras and its relatives. Nature 366:643-654.

11. Yoshimura A. 2009. Regulation of cytokine signaling by the SOCS and Spred family proteins. Keio J Med 58:73-83.

12. Vigil D, Cherfils J, Rossman KL, and Der CJ. 2010. Ras superfamily GEFs and GAPs: validated and tractable targets for cancer therapy? Nat Rev Cancer 10:842-857.

13. Stowe IB, Mercado EL, Stowe TR, Bell EL, Oses-Prieto JA, Hernandez H, Burlingame AL, and McCormick F. 2012. A shared molecular mechanism underlies the human rasopathies Legius syndrome and Neurofibromatosis-1. Genes Dev 26:1421-1426. 
14. Brems H, Chmara M, Sahbatou M, Denayer E, Taniguchi K, Kato R, Somers R, Messiaen L, De Schepper S, Fryns JP, Cools J, Marynen P, Thomas G, Yoshimura A, and Legius E. 2007. Germline loss-of-function mutations in SPRED1 cause a neurofibromatosis 1-like phenotype. Nat Genet 39:1120-1126.

15. Schubbert S, Shannon K, and Bollag G. 2007. Hyperactive Ras in developmental disorders and cancer. Nat Rev Cancer 7:295-308.

16. Tidyman WE and Rauen KA. 2009. The RASopathies: developmental syndromes of Ras/MAPK pathway dysregulation. Curr Opin Genet Dev 19:230-236.

17. Bos JL. 1989. RAS oncogenes in human cancer: a review. Cancer Res 49:4682-4689.

18. Davies H, Bignell GR, Cox C, Stephens P, Edkins S, Clegg S, Teague J, Woffendin H, Garnett MJ, Bottomley W, Davis N, Dicks E, Ewing R, Floyd Y, Gray K, Hall S, Hawes R, Hughes J, Kosmidou V, Menzies A, Mould C, Parker A, Stevens C, Watt S, Hooper S, Wilson R, Jayatilake H, Gusterson BA, Cooper C, Shipley J, Hargrave D, Pritchard-Jones K, Maitland N, Chenevix-Trench G, Riggins GJ, Bigner DD, Palmieri G, Cossu A, Flanagan A, Nicholson A, Ho JW, Leung SY, Yuen ST, Weber BL, Seigler HF, Darrow TL, Paterson H, Marais R, Marshall CJ, Wooster R, Stratton MR, and Futreal PA. 2002. Mutations of the BRAF gene in human cancer. Nature 417:949-954.

19. Prior IA, Lewis PD, and Mattos C. 2012. A comprehensive survey of Ras mutations in cancer. Cancer Res 72:2457-2467.

20. Garnett MJ and Marais R. 2004. Guilty as charged: B-RAF is a human oncogene. Cancer Cell 6:313-319.

21. Santen RJ, Song RX, McPherson R, Kumar R, Adam L, Jeng MH, and Yue W. 2002. The role of mitogen-activated protein (MAP) kinase in breast cancer. J Steroid Biochem Mol Biol 80:239-256.

22. Whyte J, Bergin O, Bianchi A, McNally S, and Martin F. 2009. Key signalling nodes in mammary gland development and cancer. Mitogen-activated protein kinase signalling in experimental models of breast cancer progression and in mammary gland development. Breast Cancer Res 11:209.

23. Mirzoeva OK, Das D, Heiser LM, Bhattacharya S, Siwak D, Gendelman R, Bayani N, Wang NJ, Neve RM, Guan Y, Hu Z, Knight Z, Feiler HS, Gascard P, Parvin B, Spellman PT, Shokat KM, Wyrobek AJ, Bissell MJ, McCormick F, Kuo WL, Mills GB, Gray JW, and Korn WM. 2009. Basal subtype and MAPK/ERK kinase (MEK)-phosphoinositide 3kinase feedback signaling determine susceptibility of breast cancer cells to MEK inhibition. Cancer Res 69:565-572.

24. Hoeflich KP, O'Brien C, Boyd Z, Cavet G, Guerrero S, Jung K, Januario T, Savage H, Punnoose E, Truong T, Zhou W, Berry L, Murray L, Amler L, Belvin M, Friedman LS, 
and Lackner MR. 2009. In vivo antitumor activity of MEK and phosphatidylinositol 3kinase inhibitors in basal-like breast cancer models. Clin Cancer Res 15:4649-4664.

25. Prat A and Perou CM. 2011. Deconstructing the molecular portraits of breast cancer. Mol Oncol 5:5-23.

26. van Beers EH, van Welsem T, Wessels LF, Li Y, Oldenburg RA, Devilee P, Cornelis se CJ, Verhoef S, Hogervorst FB, van't Veer LJ, and Nederlof PM. 2005. Comparative genomic hybridization profiles in human BRCA1 and BRCA2 breast tumors highlight differential sets of genomic aberrations. Cancer Res 65:822-827.

27. Herschkowitz JI, Simin K, Weigman VJ, Mikaelian I, Usary J, Hu Z, Rasmussen KE, Jones LP, Assefnia S, Chandrasekharan S, Backlund MG, Yin Y, Khramtsov AI, Bastein R, Quackenbush J, Glazer RI, Brown PH, Green JE, Kopelovich L, Furth PA, Palazzo JP, Olopade OI, Bernard PS, Churchill GA, Van Dyke T, and Perou CM. 2007. Identification of conserved gene expression features between murine mammary carcinoma models and human breast tumors. Genome Biol 8:R76.

28. Rakha EA, Reis-Filho JS, and Ellis IO. 2008. Basal-like breast cancer: a critical review. J Clin Oncol 26:2568-2581.

29. Hu X, Stern HM, Ge L, O'Brien C, Haydu L, Honchell CD, Haverty PM, Peters BA, Wu TD, Amler LC, Chant J, Stokoe D, Lackner MR, and Cavet G. 2009. Genetic alterations and oncogenic pathways associated with breast cancer subtypes. Mol Cancer Res 7:511522.

30. Cancer Genome Atlas Network. 2012. Comprehensive molecular portraits of human breast tumours. Nature 490:61-70.

31. Balko JM, Cook RS, Vaught DB, Kuba MG, Miller TW, Bhola NE, Sanders ME, GranjaIngram NM, Smith JJ, Meszoely IM, Salter J, Dowsett M, Stemke-Hale K, GonzalezAngulo AM, Mills GB, Pinto JA, Gomez HL, and Arteaga CL. 2012. Profiling of residual breast cancers after neoadjuvant chemotherapy identifies DUSP4 deficiency as a mechanism of drug resistance. Nat Med 18:1052-1059.

32. Gysin S, Salt M, Young A, and McCormick F. 2011. Therapeutic strategies for targeting ras proteins. Genes Cancer 2:359-372.

33. Duncan JS, Whittle MC, Nakamura K, Abell AN, Midland AA, Zawistowski JS, Johnson NL, Granger DA, Jordan NV, Darr DB, Usary J, Kuan PF, Smalley DM, Major B, He X, Hoadley KA, Zhou B, Sharpless NE, Perou CM, Kim WY, Gomez SM, Chen X, Jin J, Frye SV, Earp HS, Graves LM, and Johnson GL. 2012. Dynamic reprogramming of the kinome in response to targeted MEK inhibition in triple-negative breast cancer. Cell 149:307-321. 
34. Takahashi K and Yamanaka S. 2006. Induction of pluripotent stem cells from mouse embryonic and adult fibroblast cultures by defined factors. Cell 126:663-676.

35. Rowland BD and Peeper DS. 2006. KLF4, p21 and context-dependent opposing forces in cancer. Nat Rev Cancer 6:11-23.

36. McConnell BB and Yang VW. 2010. Mammalian Krüppel-like factors in health and diseases. Physiol Rev 90:1337-1381.

37. Ghaleb AM, McConnell BB, Nandan MO, Katz JP, Kaestner KH, and Yang VW. 2007. Haploinsufficiency of Krüppel-like factor 4 promotes adenomatous polyposis coli dependent intestinal tumorigenesis. Cancer Res 67:7147-7154.

38. McCormick SM, Eskin SG, McIntire LV, Teng CL, Lu CM, Russell CG, and Chittur KK. 2001. DNA microarray reveals changes in gene expression of shear stressed human umbilical vein endothelial cells. Proc Natl Acad Sci U S A 98:8955-8960.

39. Yoon HS, Chen X, and Yang VW. 2003. Krüppel-like factor 4 mediates p53-dependent G1/S cell cycle arrest in response to DNA damage. J Biol Chem 278:2101-2105.

40. Pedersen TX, Leethanakul C, Patel V, Mitola D, Lund LR, Dano K, Johnsen M, Gutkind JS, and Bugge TH. 2003. Laser capture microdissection-based in vivo genomic profiling of wound keratinocytes identifies similarities and differences to squamous cell carcinoma. Oncogene 22:3964-3976.

41. Liu Y, Sinha S, McDonald OG, Shang Y, Hoofnagle MH, and Owens GK. 2005. Krüppel-like factor 4 abrogates myocardin-induced activation of smooth muscle gene expression. J Biol Chem 280:9719-9727.

42. Liu Y, Wang J, Yi Y, Zhang H, Liu J, Liu M, Yuan C, Tang D, Benjamin IJ, and Xiao X. 2006. Induction of KLF4 in response to heat stress. Cell Stress Chaperones 11:379-389.

43. Ghaleb AM, Katz JP, Kaestner KH, Du JX, and Yang VW. 2007. Krüppel-like factor 4 exhibits antiapoptotic activity following gamma-radiation-induced DNA damage. Oncogene 26:2365-2373.

44. Hamik A, Lin Z, Kumar A, Balcells M, Sinha S, Katz J, Feinberg MW, Gerzsten RE, Edelman ER, and Jain MK. 2007. Krüppel-like factor 4 regulates endothelial inflammation. J Biol Chem 282:13769-13779.

45. Liao X, Haldar SM, Lu Y, Jeyaraj D, Paruchuri K, Nahori M, Cui Y, Kaestner KH, and Jain MK. 2010. Krüppel-like factor 4 regulates pressure-induced cardiac hypertrophy. J Mol Cell Cardiol 49:334-338. 
46. Lai JK, Wu HC, Shen YC, Hsieh HY, Yang SY, and Chang CC. 2012. Krüppel-like factor 4 is involved in cell scattering induced by hepatocyte growth factor. J Cell Sci $125: 4853-4864$.

47. Foster KW, Ren S, Louro ID, Lobo-Ruppert SM, McKie-Bell P, Grizzle W, Hayes MR, Broker TR, Chow LT, and Ruppert JM. 1999. Oncogene expression cloning by retroviral transduction of adenovirus E1A-immortalized rat kidney RK3E cells: transformation of a host with epithelial features by c-MYC and the zinc finger protein GKLF. Cell Growth Differ 10:423-434.

48. Foster KW, Liu Z, Nail CD, Li X, Fitzgerald TJ, Bailey SK, Frost AR, Louro ID, Townes TM, Paterson AJ, Kudlow JE, Lobo-Ruppert SM, and Ruppert JM. 2005. Induction of KLF4 in basal keratinocytes blocks the proliferation-differentiation switch and initiates squamous epithelial dysplasia. Oncogene 24:1491-1500.

49. Rowland BD, Bernards R, and Peeper DS. 2005. The KLF4 tumour suppressor is a transcriptional repressor of p53 that acts as a context-dependent oncogene. Nat Cell Biol 7:1074-1082.

50. Leng Z, Tao K, Xia Q, Tan J, Yue Z, Chen J, Xi H, Li J, and Zheng H. 2013. Krüppellike factor 4 acts as an oncogene in colon cancer stem cell-enriched spheroid cells. PLoS One 8:e56082.

51. Pandya AY, Talley LI, Frost AR, Fitzgerald TJ, Trivedi V, Chakravarthy M, Chhieng DC, Grizzle WE, Engler JA, Krontiras H, Bland KI, Lobuglio AF, Lobo-Ruppert SM, and Ruppert JM. 2004. Nuclear localization of KLF4 is associated with an aggressive phenotype in early-stage breast cancer. Clin Cancer Res 10:2709-2719.

52. Chu PY, Hsu NC, Liao AT, Yeh KT, Hou MF, and Liu CH. 2011. Elevated Krüppel-like factor 4 transcription factor in canine mammary carcinoma. BMC Vet Res 7:58.

53. Kamalakaran S, Varadan V, Giercksky Russnes HE, Levy D, Kendall J, Janevski A, Riggs M, Banerjee N, Synnestvedt M, Schlichting E, Karesen R, Shama PK, Rotti H, Rao R, Rao L, Eric Tang MH, Satyamoorthy K, Lucito R, Wigler M, Dimitrova N, Naume B, Borresen-Dale AL, and Hicks JB. 2011. DNA methylation patterns in luminal breast cancers differ from non-luminal subtypes and can identify relapse risk independent of other clinical variables. Mol Oncol 5:77-92.

54. Chen CJ, Lin SE, Lin YM, Lin SH, Chen DR, and Chen CL. 2012. Association of expression of Krüppel-like Factor 4 and Krüppel-like Factor 5 with the clinical manifestations of breast cancer. Pathol Oncol Res 18:161-168.

55. Lin CC, Liu LZ, Addison JB, Ivanov AV, and Ruppert JM. 2011. A KLF4-miRNA-206 autoregulatory feedback loop can promote or inhibit protein translation depending upon cell context. Mol Cell Biol 31:2513-2527. 
56. Lee YS and Dutta A. 2009. MicroRNAs in cancer. Annu Rev Pathol 4:199-227.

57. Kasinski AL and Slack FJ. 2011. Epigenetics and genetics. MicroRNAs en route to the clinic: progress in validating and targeting microRNAs for cancer therapy. Nat Rev Cancer 11:849-864.

58. Iorio MV and Croce CM. 2012. MicroRNA dysregulation in cancer: diagnostics, monitoring and therapeutics. A comprehensive review. EMBO Mol Med 4:143-159.

59. Iorio MV, Ferracin M, Liu CG, Veronese A, Spizzo R, Sabbioni S, Magri E, Pedriali M, Fabbri M, Campiglio M, Menard S, Palazzo JP, Rosenberg A, Musiani P, Volinia S, Nenci I, Calin GA, Querzoli P, Negrini M, and Croce CM. 2005. MicroRNA gene expression deregulation in human breast cancer. Cancer Res 65:7065-7070.

60. Meng F, Henson R, Wehbe-Janek H, Ghoshal K, Jacob ST, and Patel T. 2007. MicroRNA-21 regulates expression of the PTEN tumor suppressor gene in human hepatocellular cancer. Gastroenterology 133:647-658.

61. Thum T, Gross C, Fiedler J, Fischer T, Kissler S, Bussen M, Galuppo P, Just S, Rottbauer W, Frantz S, Castoldi M, Soutschek J, Koteliansky V, Rosenwald A, Basson MA, Licht JD, Pena JT, Rouhanifard SH, Muckenthaler MU, Tuschl T, Martin GR, Bauersachs J, and Engelhardt S. 2008. MicroRNA-21 contributes to myocardial disease by stimulating MAP kinase signalling in fibroblasts. Nature 456:980-984.

62. Sayed D, Rane S, Lypowy J, He M, Chen IY, Vashistha H, Yan L, Malhotra A, Vatner D, and Abdellatif M. 2008. MicroRNA-21 targets Sprouty2 and promotes cellular outgrowths. Mol Biol Cell 19:3272-3282.

63. Asangani IA, Rasheed SA, Nikolova DA, Leupold JH, Colburn NH, Post S, and Allgayer H. 2008. MicroRNA-21 (miR-21) post-transcriptionally downregulates tumor suppressor Pdcd4 and stimulates invasion, intravasation and metastasis in colorectal cancer. Oncogene 27:2128-2136.

64. Wickramasinghe NS, Manavalan TT, Dougherty SM, Riggs KA, Li Y, and Klinge CM. 2009. Estradiol downregulates miR-21 expression and increases miR-21 target gene expression in MCF-7 breast cancer cells. Nucleic Acids Res 37:2584-2595.

65. Hatley ME, Patrick DM, Garcia MR, Richardson JA, Bassel-Duby R, van Rooij E, and Olson EN. 2010. Modulation of K-Ras-dependent lung tumorigenesis by MicroRNA-21. Cancer Cell 18:282-293.

66. Zuker M. 2003. Mfold web server for nucleic acid folding and hybridization prediction. Nucleic Acids Res 31:3406-3415.

67. Mathelier A, Zhao X, Zhang AW, Parcy F, Worsley-Hunt R, Arenillas DJ, Buchman S, Chen CY, Chou A, Ienasescu H, Lim J, Shyr C, Tan G, Zhou M, Lenhard B, Sandelin A, 
and Wasserman WW. 2014. JASPAR 2014: an extensively expanded and updated openaccess database of transcription factor binding profiles. Nucleic Acids Res 42:D142D147.

68. Boyd KE and Farnham PJ. 1999. Coexamination of site-specific transcription factor binding and promoter activity in living cells. Mol Cell Biol 19:8393-8399.

69. Littlewood TD, Hancock DC, Danielian PS, Parker MG, and Evan GI. 1995. A modified oestrogen receptor ligand-binding domain as an improved switch for the regulation of heterologous proteins. Nucleic Acids Res 23:1686-1690.

70. Zhang W, Geiman DE, Shields JM, Dang DT, Mahatan CS, Kaestner KH, Biggs JR, Kraft AS, and Yang VW. 2000. The gut-enriched Krüppel-like factor (Krüppel-like factor 4) mediates the transactivating effect of p53 on the p21WAF1/Cip1 promoter. J Biol Chem 275:18391-18398.

71. Geiman DE, Han TT, Johnson JM, and Yang VW. 2000. Transactivation and growth suppression by the gut-enriched Krüppel-like factor (Krüppel-like factor 4) are dependent on acidic amino acid residues and protein-protein interaction. Nucleic Acids Res 28:1106-1113.

72. Evans PM, Zhang W, Chen X, Yang J, Bhakat KK, and Liu C. 2007. Krüppel-like factor 4 is acetylated by p300 and regulates gene transcription via modulation of histone acetylation. J Biol Chem 282:33994-34002.

73. Yang X, Ferguson AT, Nass SJ, Phillips DL, Butash KA, Wang SM, Herman JG, and Davidson NE. 2000. Transcriptional activation of estrogen receptor alpha in human breast cancer cells by histone deacetylase inhibition. Cancer Res 60:6890-6894.

74. Sharma D, Blum J, Yang X, Beaulieu N, Macleod AR, and Davidson NE. 2005. Release of methyl $\mathrm{CpG}$ binding proteins and histone deacetylase 1 from the Estrogen receptor alpha (ER) promoter upon reactivation in ER-negative human breast cancer cells. Mol Endocrinol 19:1740-1751.

75. Lewis BP, Burge CB, and Bartel DP. 2005. Conserved seed pairing, often flanked by adenosines, indicates that thousands of human genes are microRNA targets. Cell 120:1520.

76. Fukazawa H, Noguchi K, Murakami Y, and Uehara Y. 2002. Mitogen-activated protein/extracellular signal-regulated kinase kinase (MEK) inhibitors restore anoikis sensitivity in human breast cancer cell lines with a constitutively activated extracellularregulated kinase (ERK) pathway. Mol Cancer Ther 1:303-309.

77. Turner N, Lambros MB, Horlings HM, Pearson A, Sharpe R, Natrajan R, Geyer FC, van Kouwenhove M, Kreike B, Mackay A, Ashworth A, Van de Vijver MJ, and Reis-Filho 
JS. 2010. Integrative molecular profiling of triple negative breast cancers identifies amplicon drivers and potential therapeutic targets. Oncogene 29:2013-2023.

78. Infante JR, Papadopoulos KP, Bendell JC, Patnaik A, Burris HA, III, Rasco D, Jones SF, Smith L, Cox DS, Durante M, Bellew KM, Park JJ, Le NT, and Tolcher AW. 2013. A phase $1 \mathrm{~b}$ study of trametinib, an oral Mitogen-activated protein kinase kinase (MEK) inhibitor, in combination with gemcitabine in advanced solid tumours. Eur J Cancer 49:2077-2085.

79. Britten CD. 2013. PI3K and MEK inhibitor combinations: examining the evidence in selected tumor types. Cancer Chemother Pharmacol 71:1395-1409.

80. Kondo N, Toyama T, Sugiura H, Fujii Y, and Yamashita H. 2008. miR-206 Expression is down-regulated in estrogen receptor $\alpha$-positive human breast cancer. Cancer Res 68:5004-5008.

81. Williams AH, Valdez G, Moresi V, Qi X, McAnally J, Elliott JL, Bassel-Duby R, Sanes JR, and Olson EN. 2009. MicroRNA-206 delays ALS progression and promotes regeneration of neuromuscular synapses in mice. Science 326:1549-1554.

82. Dey BK, Gagan J, and Dutta A. 2011. miR-206 and -486 induce myoblast differentiation by downregulating Pax7. Mol Cell Biol 31:203-214.

83. Liu N, Williams AH, Maxeiner JM, Bezprozvannaya S, Shelton JM, Richardson JA, Bassel-Duby R, and Olson EN. 2012. microRNA-206 promotes skeletal muscle regeneration and delays progression of Duchenne muscular dystrophy in mice. J Clin Invest 122:2054-2065.

84. Guttilla IK and White BA. 2009. Coordinate regulation of FOXO1 by miR-27a, miR-96, and miR-182 in breast cancer cells. J Biol Chem 284:23204-23216.

85. Fujita S, Ito T, Mizutani T, Minoguchi S, Yamamichi N, Sakurai K, and Iba H. 2008. miR-21 Gene expression triggered by AP-1 is sustained through a double-negative feedback mechanism. J Mol Biol 378:492-504.

86. Armstrong JA, Bieker JJ, and Emerson BM. 1998. A SWI/SNF-related chromatin remodeling complex, $\mathrm{E}-\mathrm{RC} 1$, is required for tissue-specific transcriptional regulation by EKLF in vitro. Cell 95:93-104.

87. Kadam S and Emerson BM. 2003. Transcriptional specificity of human SWI/SNF BRG1 and BRM chromatin remodeling complexes. Mol Cell 11:377-389.

88. Wu JI, Lessard J, and Crabtree GR. 2009. Understanding the words of chromatin regulation. Cell 136:200-206. 
89. Gaspar-Maia A, Alajem A, Meshorer E, and Ramalho-Santos M. 2011. Open chromatin in pluripotency and reprogramming. Nat Rev Mol Cell Biol 12:36-47.

90. Clark GJ, Quilliam LA, Hisaka MM, and Der CJ. 1993. Differential antagonism of Ras biological activity by catalytic and Src homology domains of Ras GTPase activation protein. Proc Natl Acad Sci U S A 90:4887-4891.

91. Huang DC, Marshall CJ, and Hancock JF. 1993. Plasma membrane-targeted ras GTPaseactivating protein is a potent suppressor of p21ras function. Mol Cell Biol 13:2420-2431.

92. Wakioka T, Sasaki A, Kato R, Shouda T, Matsumoto A, Miyoshi K, Tsuneoka M, Komiya S, Baron R, and Yoshimura A. 2001. Spred is a Sprouty-related suppressor of Ras signalling. Nature 412:647-651.

93. Fotiadou PP, Takahashi C, Rajabi HN, and Ewen ME. 2007. Wild-type NRas and KRas perform distinct functions during transformation. Mol Cell Biol 27:6742-6755.

94. Jeng HH, Taylor LJ, and Bar-Sagi D. 2012. Sos-mediated cross-activation of wild-type Ras by oncogenic Ras is essential for tumorigenesis. Nat Commun 3:1168.

95. Young A, Lou D, and McCormick F. 2013. Oncogenic and wild-type Ras play divergent roles in the regulation of mitogen-activated protein kinase signaling. Cancer Discov 3:112-123.

96. Grabocka E, Pylayeva-Gupta Y, Jones MJ, Lubkov V, Yemanaberhan E, Taylor L, Jeng $\mathrm{HH}$, and Bar-Sagi D. 2014. Wild-type H- and N-ras promote mutant K-ras-driven tumorigenesis by modulating the DNA damage response. Cancer Cell 25:243-256.

97. Vlachos IS, Kostoulas N, Vergoulis T, Georgakilas G, Reczko M, Maragkakis M, Paraskevopoulou MD, Prionidis K, Dalamagas T, and Hatzigeorgiou AG. 2012. DIANA miRPath v.2.0: investigating the combinatorial effect of microRNAs in pathways. Nucleic Acids Res 40:W498-W504.

98. Lu TP, Lee CY, Tsai MH, Chiu YC, Hsiao CK, Lai LC, and Chuang EY. 2012. miRSystem: an integrated system for characterizing enriched functions and pathways of microRNA targets. PLoS One 7:e42390. 


\section{FIGURE LEGENDS}

Figure 1. KLF4 is bound to the MIR206 promoter region and induces miR-206 expression.

(A) TNBC cells were transduced with lentiviral vectors expressing KLF4 shRNA or a nontargeting control (Ctl). Endogenous miR-206 levels in stably selected cells were measured by stem-loop reverse transcriptase real time quantitative PCR. miR-206 levels were measured relative to U6 snRNA (columns, mean; bars, SE; *, $\mathrm{P}<0.05$; **, $\mathrm{P}<0.01$; ***, $\mathrm{P}<0.001$ ). (B) Schematic of the MIR206 locus (left panel) indicating KLF4 consensus binding sites that were analyzed by ChIP analysis of MDA-MB-231 cells (right panel).

(C) KLF4 protein levels (upper panels), and the KLF4-MIR206 ChIP Intensity levels (lower panels) were analyzed in MDA-MB-231 cells expressing shKLF4, a KLF4 transgene, or controls. $\beta$-actin served as a loading control for immunoblot analysis. 


\section{Figure 2. KLF4 rapidly induces miR-206 and RAS-ERK signaling.}

(A) The indicated proteins, including phospho-ERK 1/2 (pERK 1/2) and ERK2, were analyzed by immunoblot analysis of TNBC cells expressing shKLF4 or shCtl. Column data indicates the average of three independent experiments (bars, SD).

(B) The levels of GTP-bound (active) HRAS, NRAS, and KRAS in cell extracts were analyzed by affinity precipitation using the RAS binding domain of Raf-1 (RBD). RAS proteins were analyzed by immunoblot. Levels of each RAS isoform in the whole cell lysate (WCL) served as the loading control.

(C) KLF4-deficient MDA-MB-231 cells were transduced with retroviral vector expressing hemagglutinin (HA) epitope-tagged KLF4 or empty vector (Ctl). miR-206 levels (left panel) and levels of the indicated proteins (right panel) were analyzed.

(D) KLF4-deficient MDA-MB-231 cells were transduced with a 4-hydroxytamoxifen (4-OHT) dependent transgene, KLF4-ER, or else empty vector. KLF4 and KLF4-ER levels were analyzed by immunoblot.

(E) miR-206 levels (left panel) and levels of the indicated proteins (right panel) were analyzed. CDKN1A is regulated by KLF4 and $\mathrm{p} 21^{\mathrm{Cip} 1 / \mathrm{Waf} 1}$ therefore served as an indicator of KLF4 activity. 
Figure 3: KLF4 and miR-206 regulate the levels of two RAS-ERK pathway suppressors, RASA1 and SPRED1.

(A) RASA1 and SPRED1 levels were determined by immunoblot analysis of KLF4-deficient cells and control cells.

(B) TNBC cells were transfected with either anti-miR-206 or a non-targeting anti-miR (Ctl) and miR-206 levels were determined.

(C) TNBC cells were transfected with the indicated anti-miR or miR-mimic. RASA1 and SPRED1 levels were analyzed by immunoblot. 
Figure 4: miR-206 represses the translation of $R A S A 1$ and $S P R E D 1$ by directly targeting the 3' UTRs.

(A-B) Schematic of the RASA1 3' UTR (A) and the SPRED1 3' UTR (B) indicating potential miR-206 binding sites. The portion of each 3' UTR that was cloned into the translational reporter is indicated relative to the stop codon and poly-adenylation signal. The sequence of the miR-206 candidate binding site is indicated below each schematic for several mammals (underline, seed sequence complement).

(C-D) For analysis of protein translation, WT and mutant versions of the RASA1 3' UTR (C) or

SPRED1 3' UTR (D) were inserted into the 3' UTR of firefly luc (upper panels, 3' UTR WT and 3' UTR Mut). MDA-MB-231 cells were co-transfected with reporters in combination with either anti-miR (middle panel) or miR-mimic (lower panel). The normalized activity of the reporters relative to empty luc vector was analyzed at 24 hours post-transfection. 
Figure 5: Endogenous KLF4 is bound to the MIR21 promoter region and maintains miR-21 expression in TNBC cells.

(A) miR-21 levels were analyzed in KLF4-deficient or control TNBC cells.

(B) Schematic of the MIR21 locus (left panel) indicating a KLF4 consensus binding site that was analyzed in MDA-MB-231 cells by ChIP (right panel).

(C) Similarly as shown in Fig. 1C, the KLF4-MIR21 ChIP Intensity levels were analyzed in MDA-MB-231 cells expressing shKLF4, a KLF4 transgene, or controls.

(D) TNBC cells were transduced with empty vector or with vector encoding KLF4 or KLF4-ER. Where indicated, cells were treated with 4-OHT, and the levels of the indicated miRs were analyzed.

(E) Similarly as shown in Fig. 2C-2E, miR-21 levels were analyzed in KLF4-deficient TNBC cells following rescue with exogenous KLF4 (left panel) or KLF4-ER (right panel).

(F) TNBC cells were transduced with the indicated shRNA vector. Proteins were analyzed by immunoblot (left panels) and miR levels were determined (right panels).

(G) Cells were treated with 5-aza-2'-deoxycytidine (AZA; 96 hours) or trichostatin A (TSA; 12 hours). ESRI mRNA levels were analyzed as a positive control for drug activity (right panel). 
Figure 6: miR-21 directly represses the translation of RASA1 and SPRED1.

(A) MDA-MB-231 cells were transfected with the indicated anti-miR or miR-mimic, and RASA1 and SPRED1 levels were determined by immunoblot. PDCD4 is encoded by a miR-21 targeted transcript and was analyzed in parallel.

(B) To construct a WT translational reporter, a portion of the RASA1 3' UTR (B) containing an established miR-21 binding site was inserted into the 3' UTR of firefly luc (64). The sequence of the miR-21 binding site is indicated for several vertebrates (underline, seed sequence complement).

(C) Schematic of the SPRED1 3' UTR indicating potential miR-21 binding sites. (D-E) For analysis of protein translation, WT or mutant versions of the indicated 3' UTR were inserted into the 3' UTR of firefly luc (upper panels). MDA-MB-231 cells were co-transfected with reporters in combination with either anti-miR or miR-mimic. The normalized activity of the reporters relative to empty luc vector was analyzed at 24 hours post-transfection. 
Figure 7: KLF4 promotes activated ERK 1/2 levels, miR-206, and miR-21 expression in a panel composed of human mammary epithelial cells and TNBC cell lines.

(A) Cells were transduced with the indicated shRNA vector. Stably selected cells were analyzed for the indicated proteins by immunoblot.

(B) miR levels were analyzed in the indicated cells. For MDA-MB-468 cells, the miR-206 cycle threshold exceeded 40 and the expression level was therefore designated as not detected (n.d.). 
Figure 8: Endogenous miR-206 and miR-21 cooperate to promote RAS-ERK signaling. TNBC cells were transfected with the indicated anti-miR and proteins levels were analyzed by immunoblot (pMEK 1/2: phospho-MEK 1/2; tMEK 1/2: total MEK 1/2). Transfections were performed using $12.5 \mathrm{nM}$ of the indicated anti-miR, with $25 \mathrm{nM}$ as the final concentration of all species combined, using anti-miR-Ctl where indicated (-). 
Figure 9: Inhibition of miR-206 and miR-21 cooperatively suppresses ERK 1/2 dependent phenotypes in TNBC cells.

(A) TNBC cells were transfected with the indicated anti-miR and plated 24 hours post transfection (i.e., Day 0). Cell proliferation was measured using an ATP based luminescence assay $(\mathrm{N}=3$; bars, $\mathrm{SE})$.

(B) Migration of anti-miR transfected TNBC cells was measured in a Boyden chamber transwell assay $(\mathrm{N}=3$; bars, $\mathrm{SE})$.

(C) Anti-miR transfected cells were analyzed in an anoikis assay. After 24 hours in suspension, cell death was analyzed by trypan blue staining ( $\mathrm{N}=3$; bars, $\mathrm{SE}$ ). In parallel, cells were treated with MEK inhibitor U0126.

(D) Three experiments were performed independently of those in (C), and anoikis was assessed by Annexin V-PI staining. The percent of Annexin V+ cells, representing both early and late apoptotic cells, is depicted in the right panel for each treatment group (N=3; bars, SD). 
Figure 10: RASA1 and SPRED1 are limiting factors for RAS-ERK signaling in TNBC cells. (A) TNBC cells were stably transduced with shRNA vector targeting RASA1 (R1 or R2) or with a nontargeting Ctl. The indicated proteins were analyzed by immunoblot.

(B) Cells were stably transduced with shRNA vector targeting SPRED1 (S1 or S2) or with a nontargeting $\mathrm{Ctl}$. The indicated proteins were analyzed by immunoblot.

(C) RAS-GTP levels were analyzed by the RBD pulldown assay in RASA1 and SPRED1 knockdown cells and control cells.

(D) SUM159PT TNBC cells were stably transduced with the indicated shRNA expression vector and the indicated proteins and RAS-GTP levels were analyzed. 
Figure 11: RASA1 and SPRED1 mediate the regulation of RAS-ERK pathway signaling by miR-206 and miR-21.

(A) TNBC cells expressing the indicated shRNAs were treated with anti-miR-206 and anti-miR21 in combination (anti-miR-206/21), or with anti-miR-Ctl. Whole cell extracts were prepared and the indicated proteins were analyzed by immunoblot. Two distinct cell culture models were analyzed (MDA-MB-231 vs. HCC1143) using independent shRNAs (R1, S1, R2 and S2). For pERK 1/2 both short (S) and long (L) exposures are indicated.

(B) Absent any anti-miR transfection (Untransfected), baseline cell proliferation was analyzed for cells expressing the indicated shRNA and for the untransduced, parental cells (P). Following anti-miR transfection, cell proliferation was measured for control TNBC cells (shCtl) or cells deficient in RASA1 (shR1, shR2) or SPRED1 (shS1, shS2). Assays were performed following transfection of anti-miR-Ctl (AC) or anti-miR-206/21 (A+).

(C) MDA-MB-231 cells expressing the indicated shRNAs were transfected with either anti-miR$206 / 21$ or anti-miR-Ctl. $2 \times 10^{6}$ cells were orthotopically injected into the left fourth mammary gland of athymic mice. Bi-weekly tumor measurements were made by using calipers. For each treatment group, the number of mice in which tumor initiation occurred by day 28 is indicated (Initiated/Total). 
Figure 12: Exogenous miR-206 and miR-21 cooperate to promote RAS-ERK signaling and cell survival in KLF4-depleted cells.

(A) KLF4-depleted TNBC cells were transfected with the indicated miR-mimic, and protein levels were analyzed by immunoblot.

(B) Anoikis assays were performed following transfection of the indicated miR-mimic into KLF4-depleted TNBC cells. For MDA-MB-231, cells transduced with KLF4 vector or empty vector were analyzed in parallel. 
Figure 13: KLF4-dependent miRs cooperatively promote RAS-ERK pathway activity by co-targeting of pathway inhibitors. The schematic shows the organization of the RAS-ERK pathway. miR-206/21 co-targeted repressors of RAS-ERK signaling are shown in blue ovals. The GAP protein NF1 is indicated as a likely catalytic partner of SPRED1 (13). 


\section{TABLES}

Table 1: Pathway enrichment analysis of putative miR-206-regulated genes.

\begin{tabular}{|c|c|c|}
\hline KEGG pathway & p-value & Predicted targets in pathway \\
\hline Dorso-ventral axis formation & $2.58 \mathrm{E}-06$ & 6 \\
\hline Gap junction & $2.58 \mathrm{E}-06$ & 9 \\
\hline Glioma & $1.39 \mathrm{E}-05$ & 9 \\
\hline Long-term potentiation & $5.16 \mathrm{E}-05$ & 9 \\
\hline Transcriptional misregulation in cancer & $8.44 \mathrm{E}-05$ & 16 \\
\hline Thyroid cancer & $2.86 \mathrm{E}-04$ & 4 \\
\hline Chronic myeloid leukemia & $1.06 \mathrm{E}-03$ & 8 \\
\hline Prostate cancer & $1.48 \mathrm{E}-03$ & 9 \\
\hline Pentose phosphate pathway & $2.14 \mathrm{E}-03$ & 4 \\
\hline Neurotrophin signaling pathway & $4.13 \mathrm{E}-03$ & 10 \\
\hline Insulin signaling pathway & $4.13 \mathrm{E}-03$ & 11 \\
\hline Melanoma & $4.13 \mathrm{E}-03$ & 7 \\
\hline Spliceosome & $5.11 \mathrm{E}-03$ & 11 \\
\hline Bacterial invasion of epithelial cells & $5.11 \mathrm{E}-03$ & 7 \\
\hline mRNA surveillance pathway & $6.73 \mathrm{E}-03$ & 7 \\
\hline Alcoholism & $6.73 \mathrm{E}-03$ & 12 \\
\hline Non-small cell lung cancer & $8.82 \mathrm{E}-03$ & 6 \\
\hline MAPK signaling pathway & $1.24 E-02$ & 17 \\
\hline Regulation of actin cytoskeleton & $1.38 \mathrm{E}-02$ & 13 \\
\hline Endometrial cancer & $1.47 \mathrm{E}-02$ & 5 \\
\hline Lysine degradation & $1.73 \mathrm{E}-02$ & 4 \\
\hline GnRH signaling pathway & $1.73 \mathrm{E}-02$ & 7 \\
\hline Dilated cardiomyopathy & $1.85 \mathrm{E}-02$ & 7 \\
\hline Progesterone-mediated oocyte maturation & $2.25 \mathrm{E}-02$ & 7 \\
\hline Glycosphingolipid biosynthesis - lacto and neolacto series & $2.75 \mathrm{E}-02$ & 1 \\
\hline
\end{tabular}

Genes containing potential miR-206 binding sites were analyzed using DIANA miRPath (97). 
Table 2: Putative miR-206 target genes related to the MAPK/ERK signaling pathway.

\begin{tabular}{|l|c|c|c|c|c|c|c|c|}
\hline $\begin{array}{c}\text { TARGET } \\
\text { GENE }\end{array}$ & DIANA & MIRANDA & MIRBRIDGE & PICTAR & PITA & RNA22 & TARGETSCAN & $\begin{array}{c}\text { TOTAL } \\
\text { HIT }\end{array}$ \\
\hline RASA1 & $\mathrm{V}$ & $\mathrm{V}$ & $\mathrm{V}$ & $\mathrm{V}$ & $\mathrm{V}$ & $\mathrm{V}$ & $\mathrm{V}$ & 7 \\
\hline SPRED1 & $\mathrm{V}$ & $\mathrm{V}$ & & $\mathrm{V}$ & $\mathrm{V}$ & $\mathrm{V}$ & $\mathrm{V}$ & 6 \\
\hline RIT2 & $\mathrm{V}$ & $\mathrm{V}$ & $\mathrm{V}$ & $\mathrm{V}$ & $\mathrm{V}$ & & $\mathrm{V}$ & 6 \\
\hline MAP4K3 & $\mathrm{V}$ & $\mathrm{V}$ & $\mathrm{V}$ & $\mathrm{V}$ & $\mathrm{V}$ & & $\mathrm{V}$ & 6 \\
\hline BDNF & $\mathrm{V}$ & $\mathrm{V}$ & $\mathrm{V}$ & $\mathrm{V}$ & $\mathrm{V}$ & & $\mathrm{V}$ & 6 \\
\hline NGFR & $\mathrm{V}$ & $\mathrm{V}$ & $\mathrm{V}$ & $\mathrm{V}$ & & & $\mathrm{V}$ & 5 \\
\hline PDGFA & $\mathrm{V}$ & $\mathrm{V}$ & $\mathrm{V}$ & & $\mathrm{V}$ & & $\mathrm{V}$ & 5 \\
\hline PDCD4 & $\mathrm{V}$ & & & $\mathrm{V}$ & & $\mathrm{V}$ & $\mathrm{V}$ & 4 \\
\hline MAPK1 & $\mathrm{V}$ & & $\mathrm{V}$ & & $\mathrm{V}$ & & $\mathrm{V}$ & 4 \\
\hline CRK & $\mathrm{V}$ & $\mathrm{V}$ & $\mathrm{V}$ & $\mathrm{V}$ & & & & 4 \\
\hline MAP3K1 & $\mathrm{V}$ & $\mathrm{V}$ & & & $\mathrm{V}$ & & $\mathrm{V}$ & 4 \\
\hline SRC & $\mathrm{V}$ & $\mathrm{V}$ & & & & $\mathrm{V}$ & & 3 \\
\hline RPS6KA5 & $\mathrm{V}$ & $\mathrm{V}$ & $\mathrm{V}$ & & & & & 3 \\
\hline RAP1A & $\mathrm{V}$ & & & $\mathrm{V}$ & & & $\mathrm{V}$ & 3 \\
\hline RALA & $\mathrm{V}$ & & $\mathrm{V}$ & $\mathrm{V}$ & & & & 3 \\
\hline MAPK3 & & & $\mathrm{V}$ & & $\mathrm{V}$ & & $\mathrm{V}$ & 3 \\
\hline KRAS & $\mathrm{V}$ & & $\mathrm{V}$ & & & & $\mathrm{V}$ & 3 \\
\hline GNB1 & $\mathrm{V}$ & & $\mathrm{V}$ & $\mathrm{V}$ & & & & 3 \\
\hline IKBKB & $\mathrm{V}$ & & $\mathrm{V}$ & & & $\mathrm{V}$ & & 3 \\
\hline RAPGEF2 & $\mathrm{V}$ & & $\mathrm{V}$ & & & $\mathrm{V}$ & $\mathrm{V}$ & 3 \\
\hline PRKACB & $\mathrm{V}$ & & $\mathrm{V}$ & $\mathrm{V}$ & & & & 3 \\
\hline
\end{tabular}

Candidate miR-206 target genes relevant to MAPK/ERK signaling were identified using KEGG, BIOCARTA, or REACTOME pathway analysis tools. Ranking (Total Hit) was performed using miRSystem (98). 
Table 3: Pathway enrichment analysis of putative miR-21-regulated genes.

\begin{tabular}{|c|c|c|}
\hline KEGG pathway & p-value & Predicted targets in pathway \\
\hline Cytokine-cytokine receptor interaction & $6.55 \mathrm{E}-07$ & 9 \\
\hline Steroid biosynthesis & $1.26 \mathrm{E}-03$ & 2 \\
\hline Jak-STAT signaling pathway & $1.31 \mathrm{E}-03$ & 7 \\
\hline MAPK signaling pathway & $2.09 E-03$ & 9 \\
\hline TGF- $\beta$ signaling pathway & $5.88 \mathrm{E}-03$ & 4 \\
\hline Pancreatic cancer & $6.23 \mathrm{E}-03$ & 4 \\
\hline N-Glycan biosynthesis & $7.71 \mathrm{E}-03$ & 2 \\
\hline Hepatitis B & $1.06 \mathrm{E}-02$ & 5 \\
\hline Neurotrophin signaling pathway & $1.49 \mathrm{E}-02$ & 5 \\
\hline Viral carcinogenesis & $1.49 \mathrm{E}-02$ & 6 \\
\hline Small cell lung cancer & $1.49 \mathrm{E}-02$ & 4 \\
\hline Regulation of actin cytoskeleton & $2.60 \mathrm{E}-02$ & 6 \\
\hline Cell cycle & $2.76 \mathrm{E}-02$ & 5 \\
\hline Pathways in cancer & $3.06 \mathrm{E}-02$ & 9 \\
\hline
\end{tabular}

Genes containing potential miR-21 binding sites were analyzed using DIANA miRPath (97). 
Table 4: Intersection of the pathways targeted by miR-206 and miR-21.

\begin{tabular}{|l|c|c|}
\hline \multicolumn{1}{|c|}{ KEGG pathway } & p-value & Predicted targets in pathway \\
\hline MAPK signaling pathway & $3.00 \mathrm{E}-04$ & 26 \\
\hline Neurotrophin signaling pathway & $6.59 \mathrm{E}-04$ & 14 \\
\hline Regulation of actin cytoskeleton & $3.19 \mathrm{E}-03$ & 19 \\
\hline
\end{tabular}

Analysis was performed using DIANA miRPath (97). 


\section{FIGURES}

A

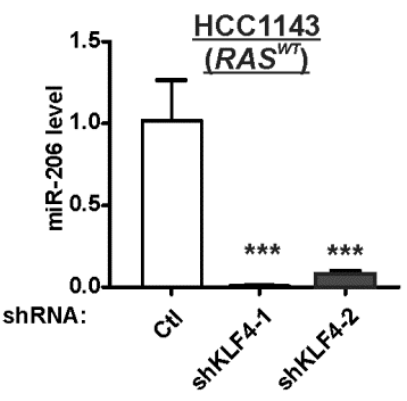

shRNA:

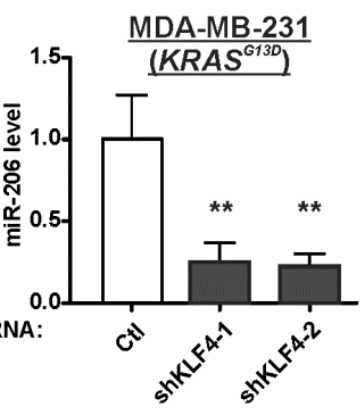

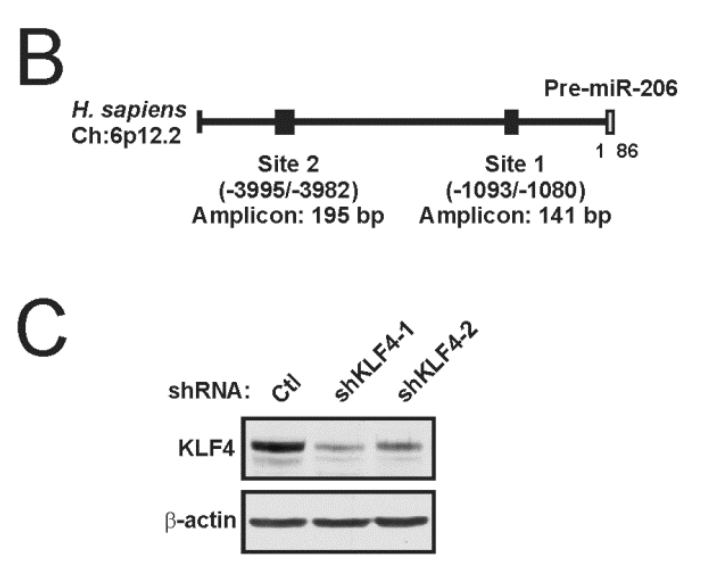
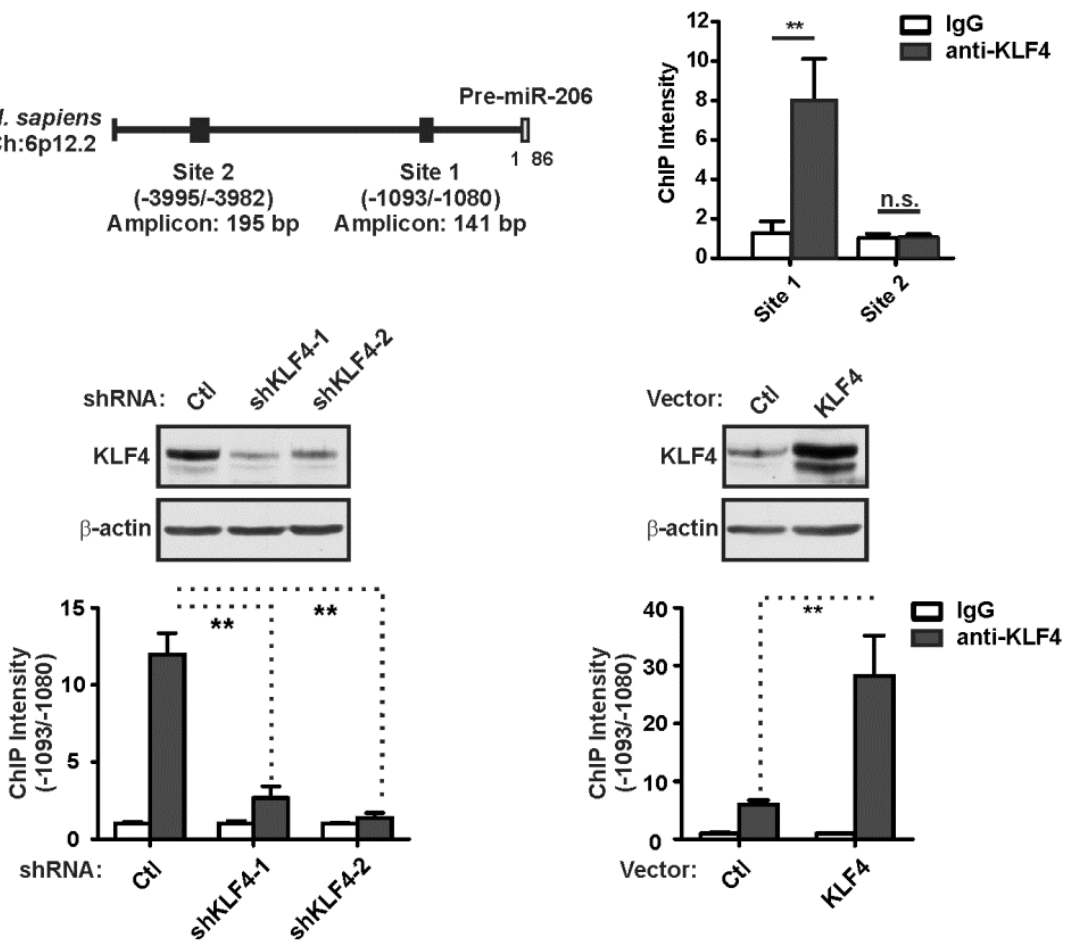

Figure 1 


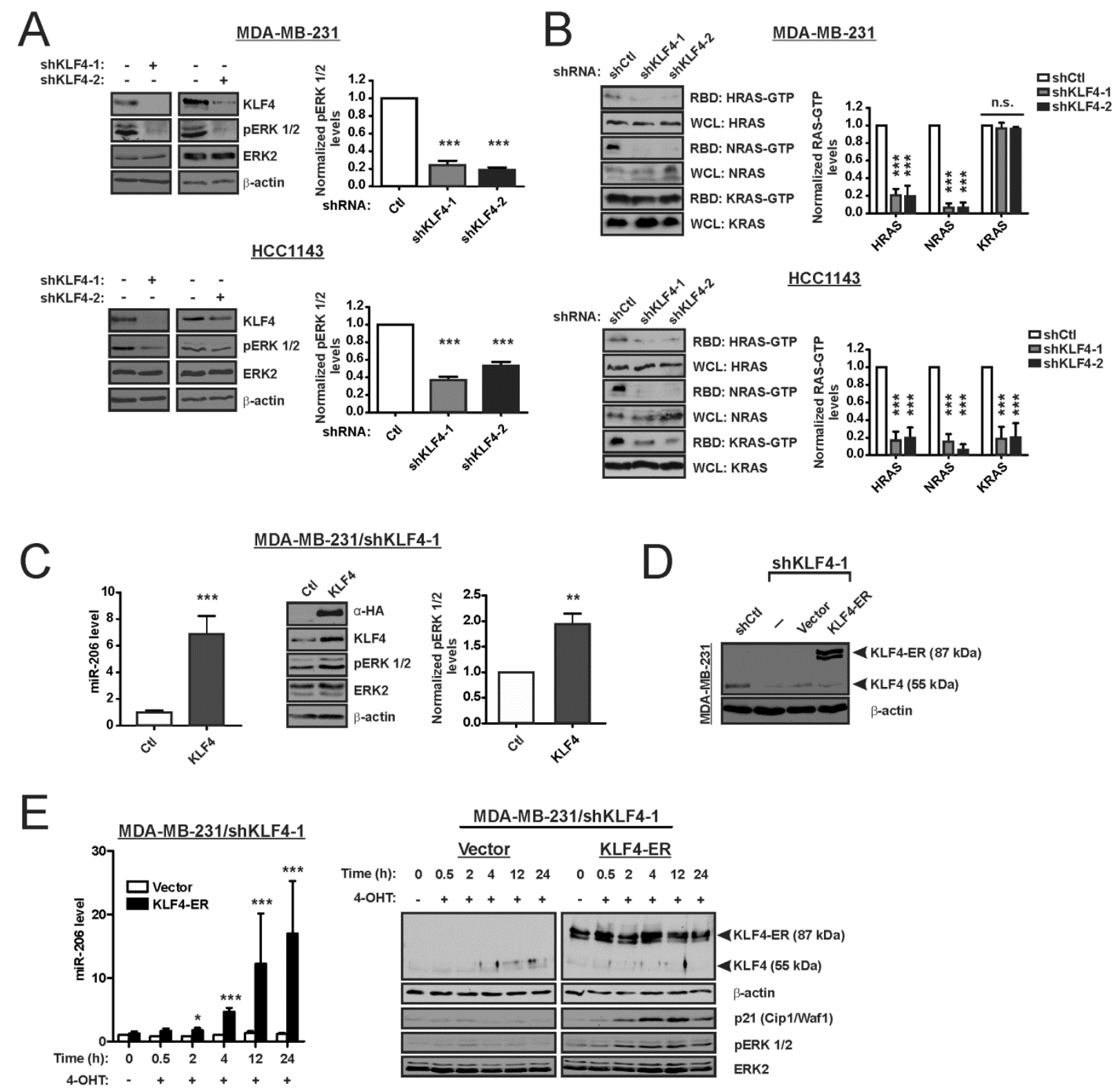

Figure 2 

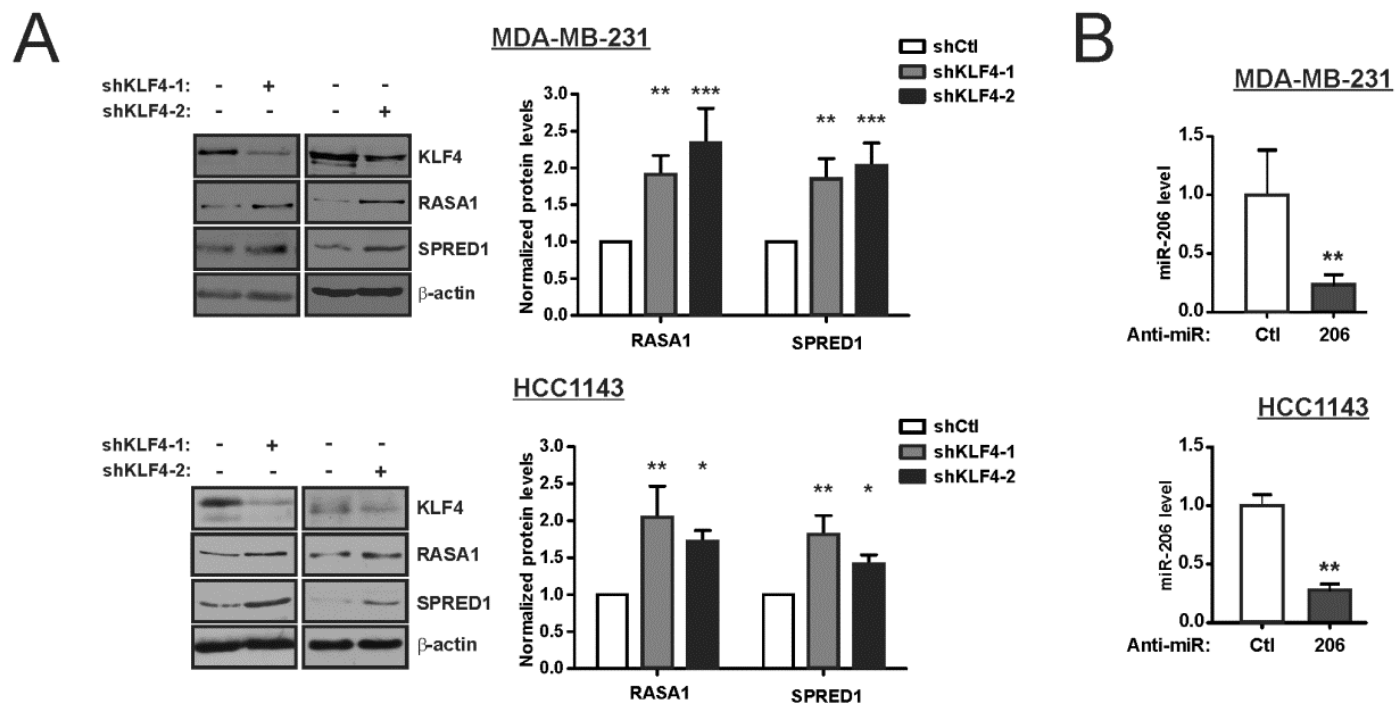

C

MDA-MB-231

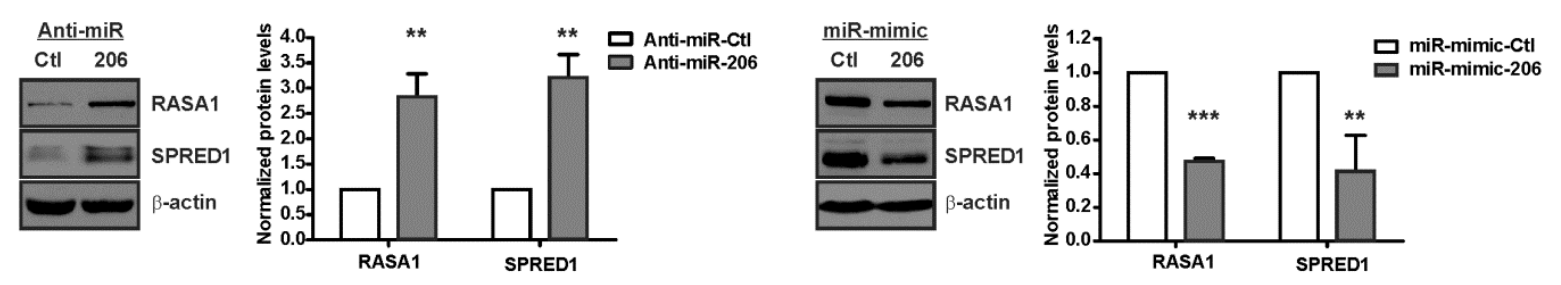

HCC1143

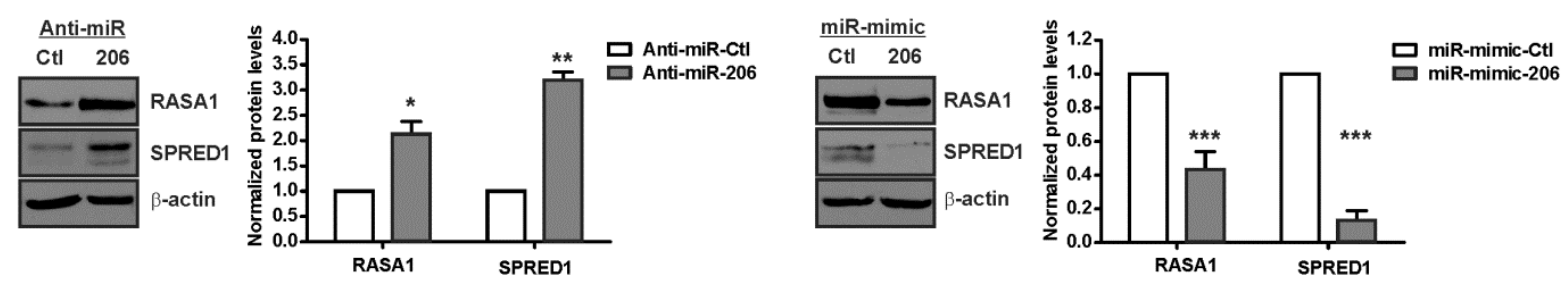

Figure 3 
A

Hsa RASA1 3' UTR (1033 nt)

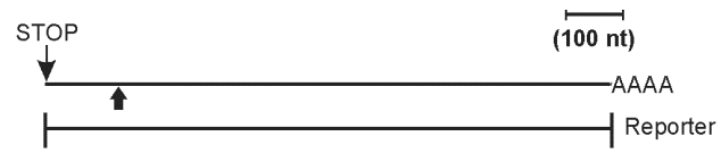

Species Accession (bp) miR-206 binding site

Hsa NM_002890 (3434-55) ^ UAGCACACUUUUCCACAUUCCA

Ptr XM_003310643 (2939-60) UAGCACACUUUUCCACAUUCCA

Mmu NM 145452 (3800-21) UAGCACACUUUCCCACAUUCCA

Bta NM_174449 (3824-45) UAGCACACCUUUCCACAUUCCA

0
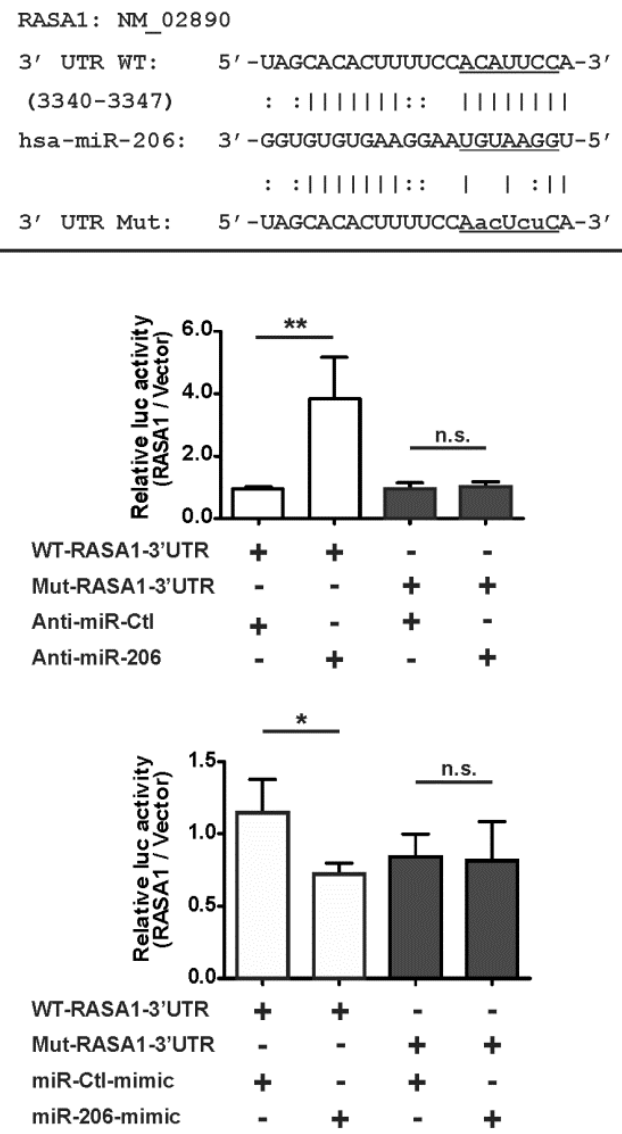

B

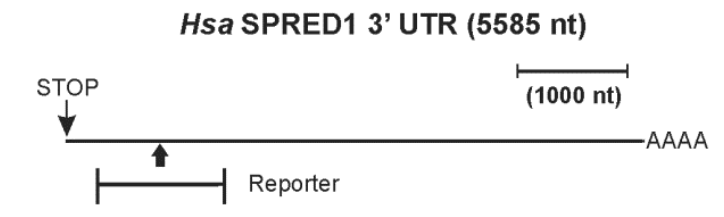

Species Accession (bp) miR-206 binding site Hsa NM 152594 (2262-81) AUAUCUACUGUCACAUUCCA Ptx XM_001138171 (2785-804) AUAUCUACUGUCACAUUCCA Mma NM_033524 (2180-201) AUAUCUGCCACCACAUUCCA Bta NM_001192516.1 (2066-85) AUAUCUACUGUCACAUUCCA

D

\begin{tabular}{|c|c|}
\hline \multicolumn{2}{|c|}{ SPRED1: NM_152594 } \\
\hline $\begin{array}{l}3^{\prime} \text { UTR WT: } \\
(2274-2281)\end{array}$ & $\begin{array}{c}5^{\prime} \text {-AUAUAUCUACUGUCACAUUCCA-3' } \\
:|:|::: \quad|||1| 1|1| 1\end{array}$ \\
\hline hsa-miR-206: & 3' -GGUGUGUGAAGGAAUGUAAGGU-5' \\
\hline & $:|:|:: \quad:|||||:| \mid$ \\
\hline 3' UTR Mut: & $5^{\prime}$-AUAUAUCUACUGUCAacUcuCA-3' \\
\hline
\end{tabular}
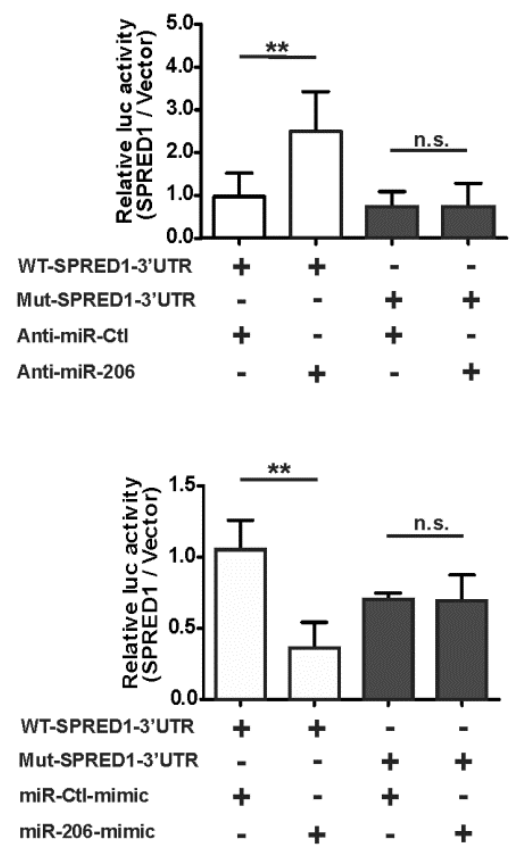

Figure 4 


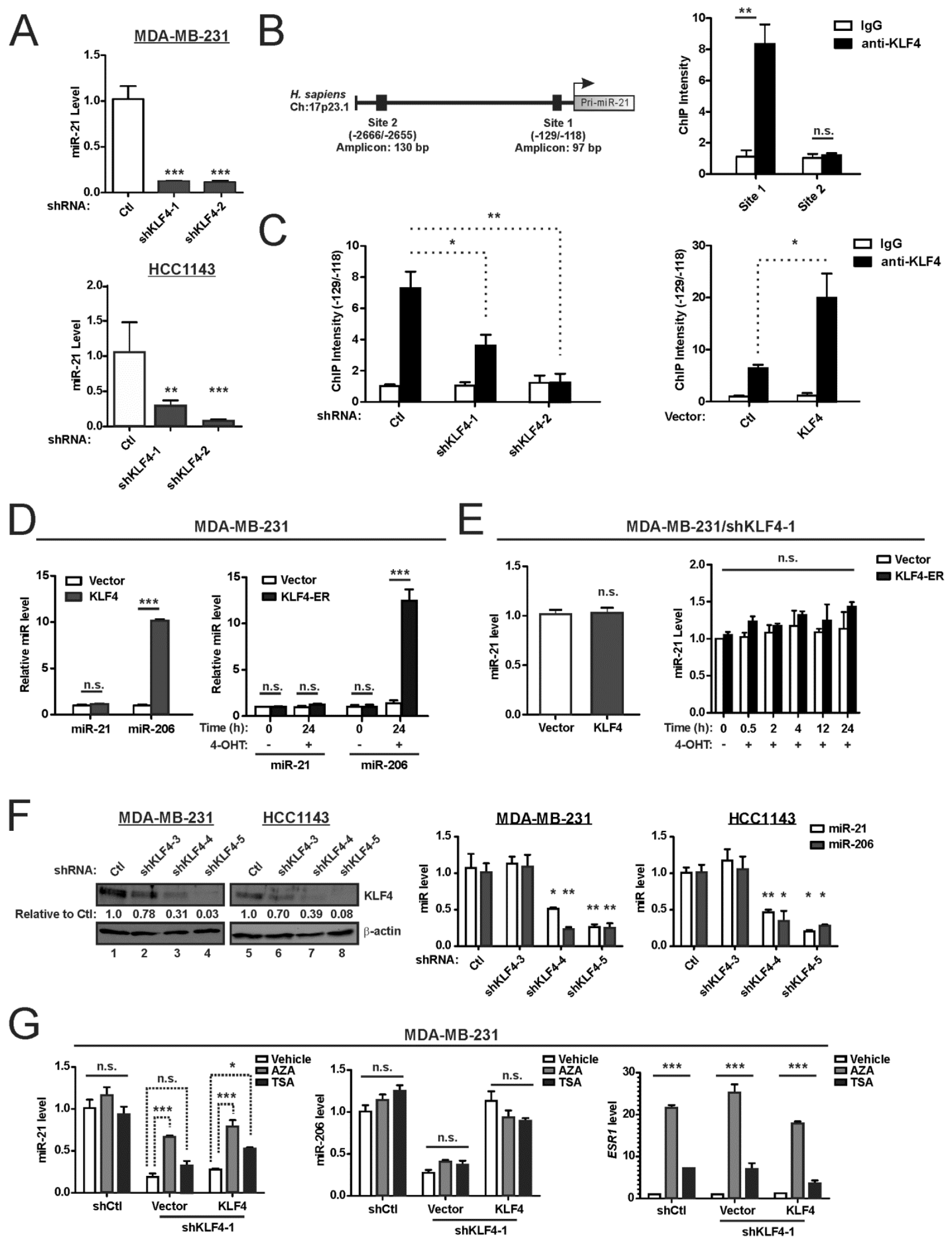

Figure 5 


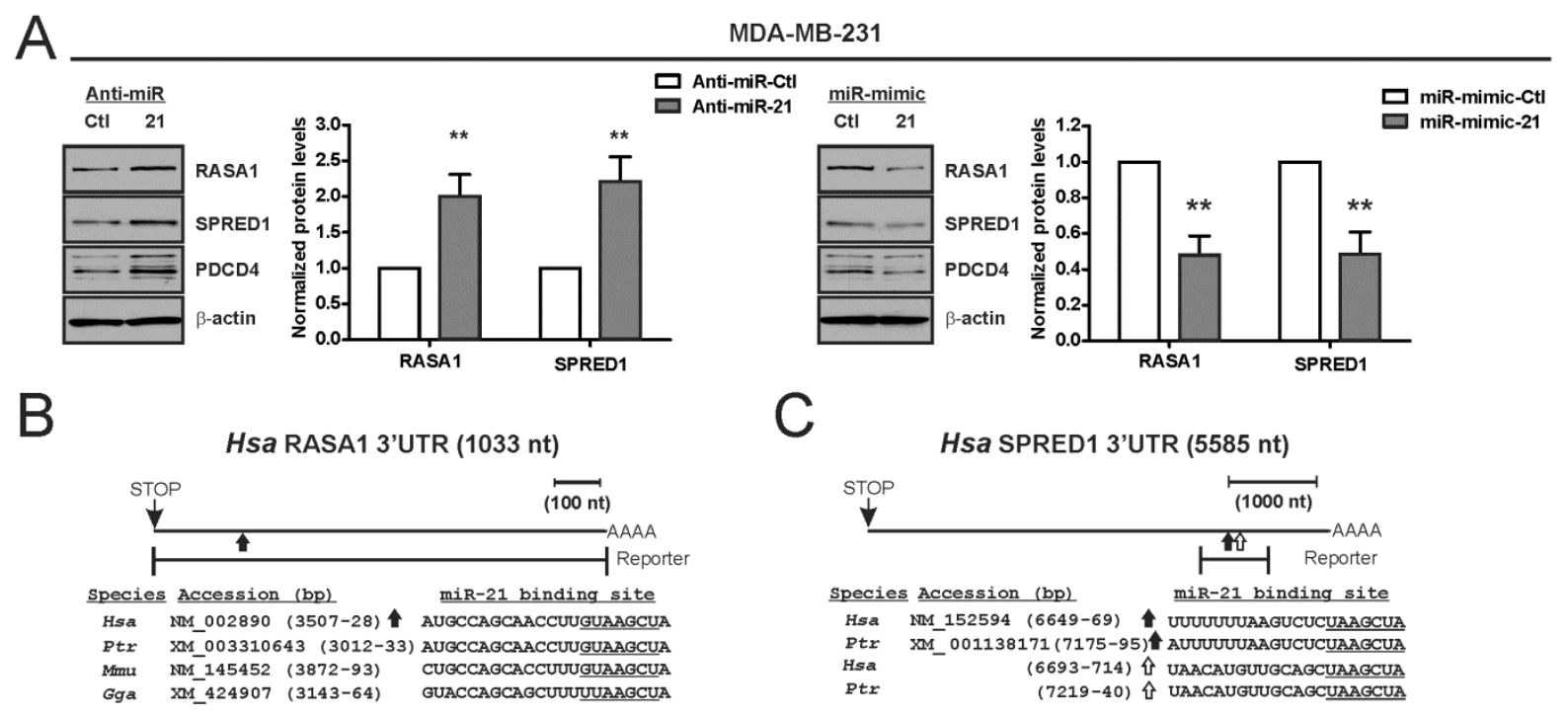

D

\begin{tabular}{|c|c|}
\hline \multicolumn{2}{|c|}{ RASA1: NM_02890 } \\
\hline 3' UTR: & $5^{\prime}$-AACCUUGUAAGCUA-3' \\
\hline (3522-3528) & $1|1|: \mid 11111$ \\
\hline $\min$ & JAUUC \\
\hline
\end{tabular}
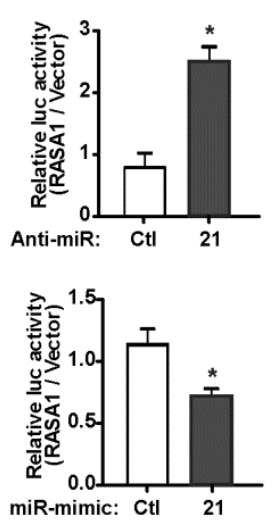
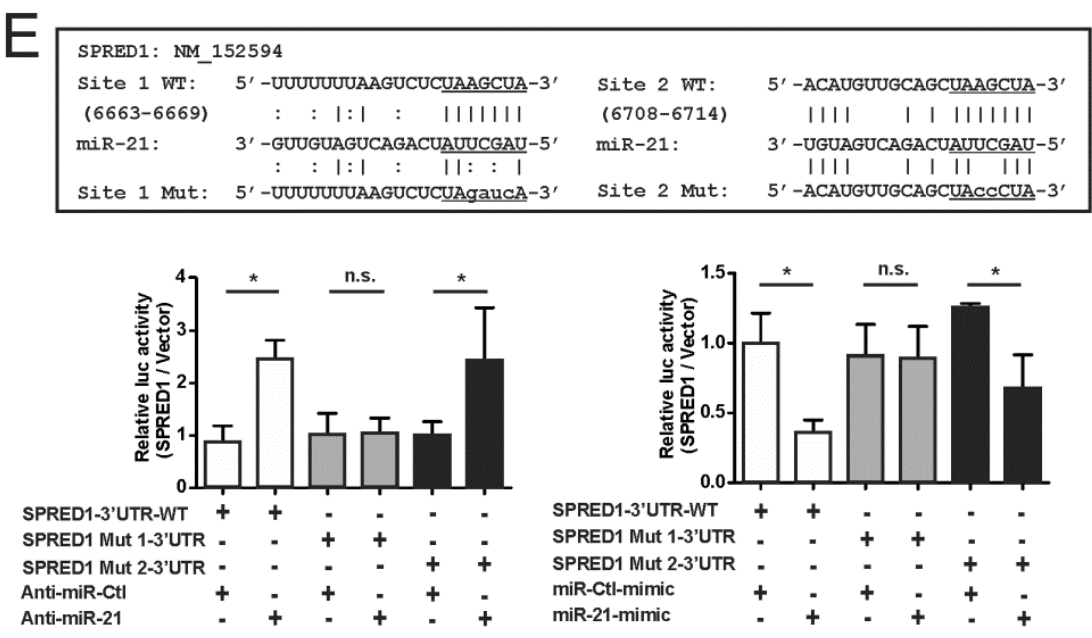

Figure 6 


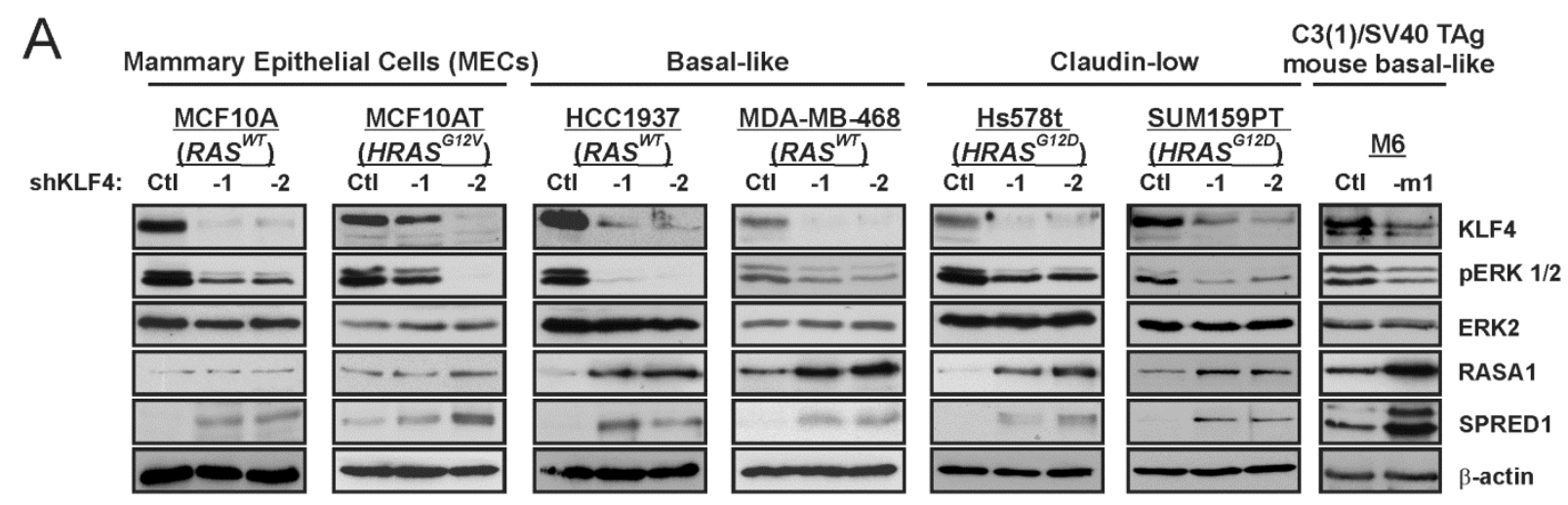

B
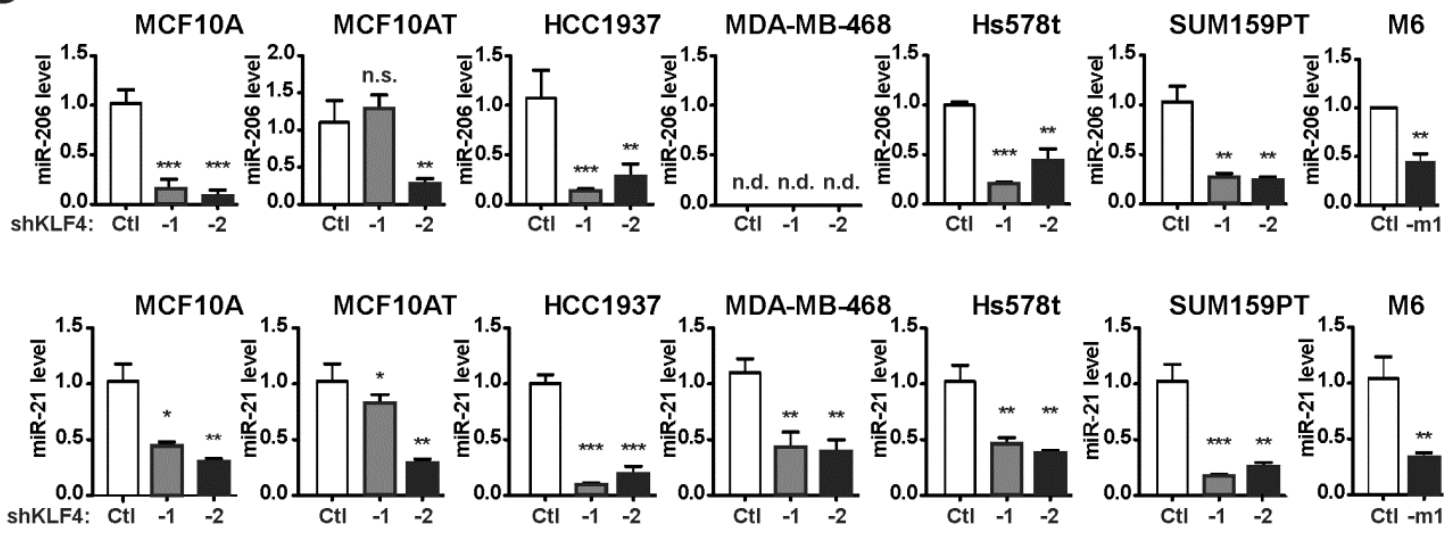

Figure 7 
MDA-MB-231

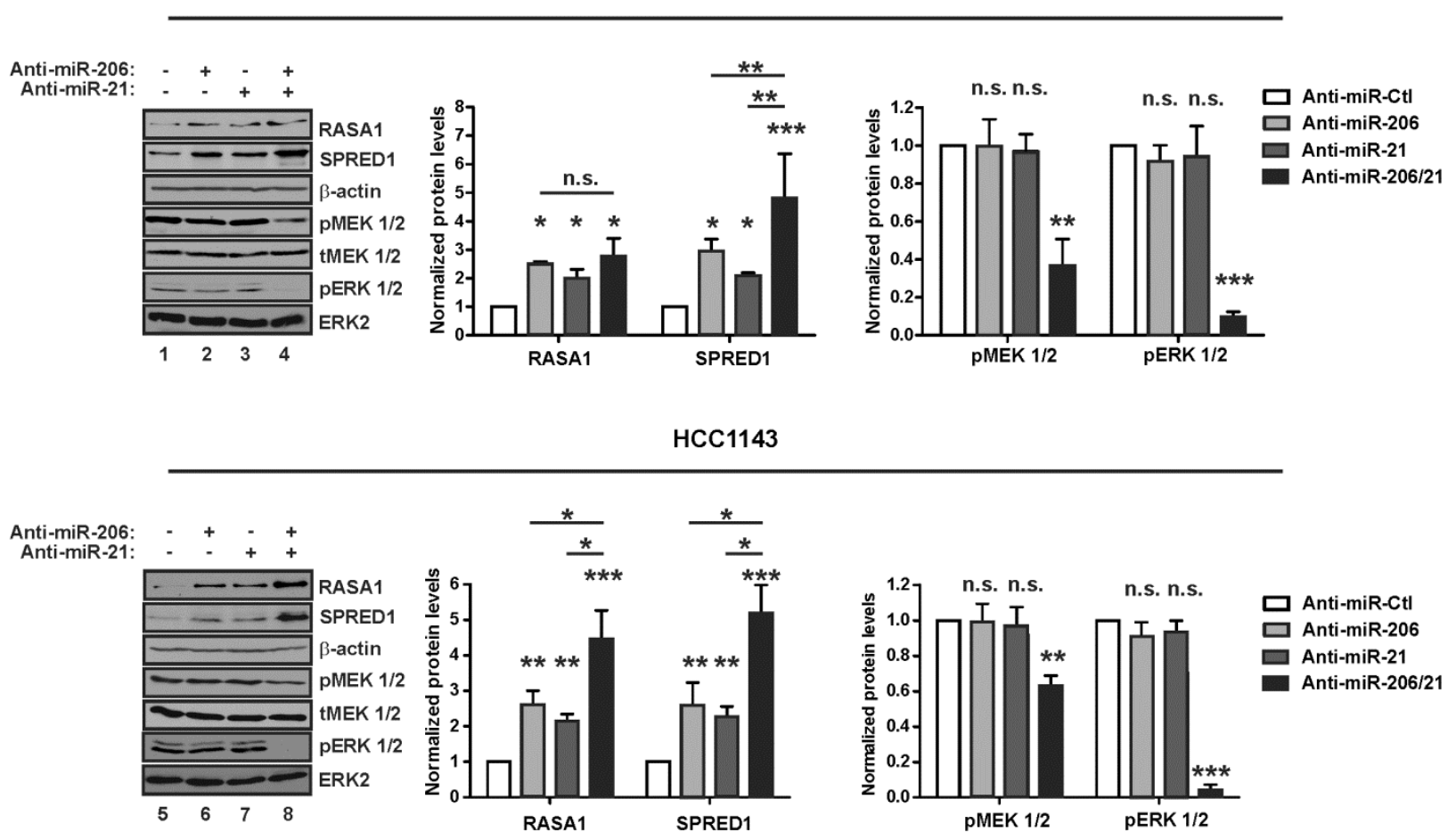

Figure 8 
A

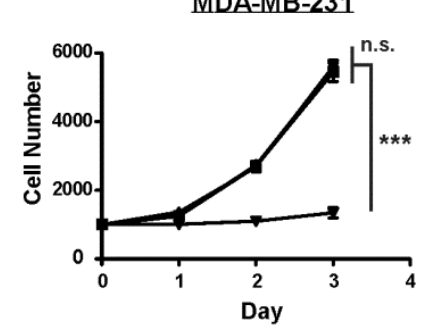

- Anti-miR-CtI

- Anti-miR-206

$\rightarrow$ Anti-miR-206/21

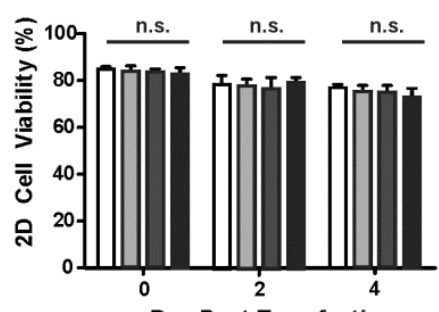

Day Post Transfection
HCC1143

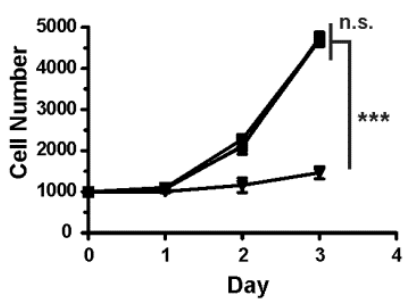

- Anti-miR-Ctl

- Anti-miR-206

- Anti-miR-206/21

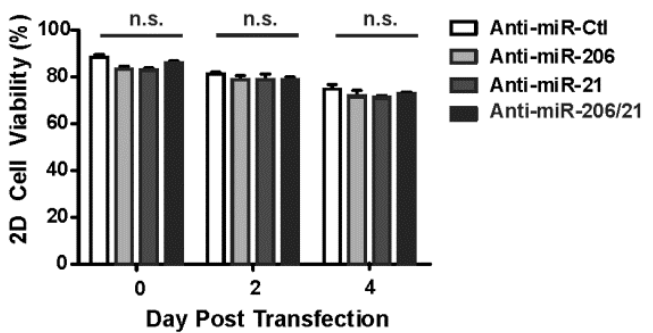

B

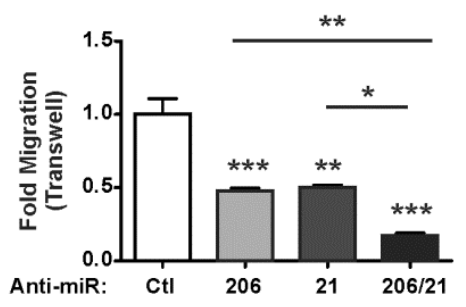

HCC1143

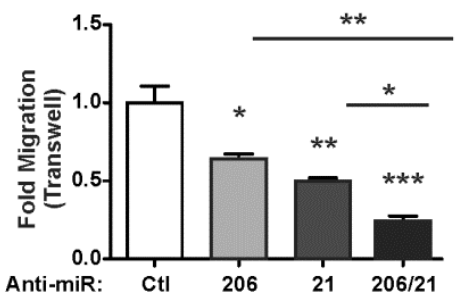

C
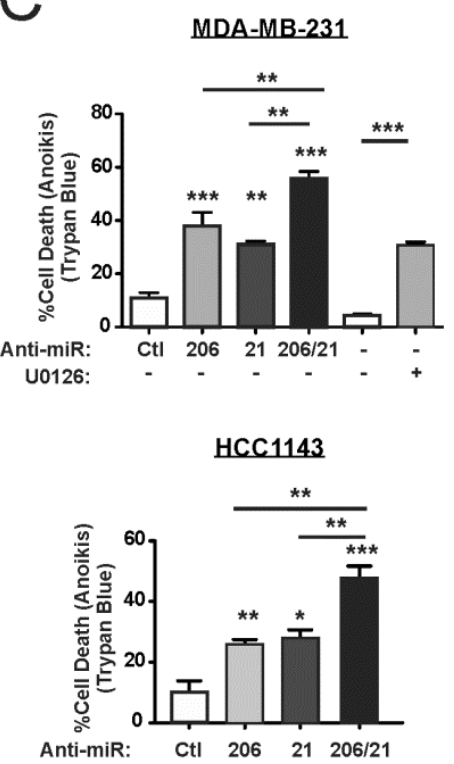

D
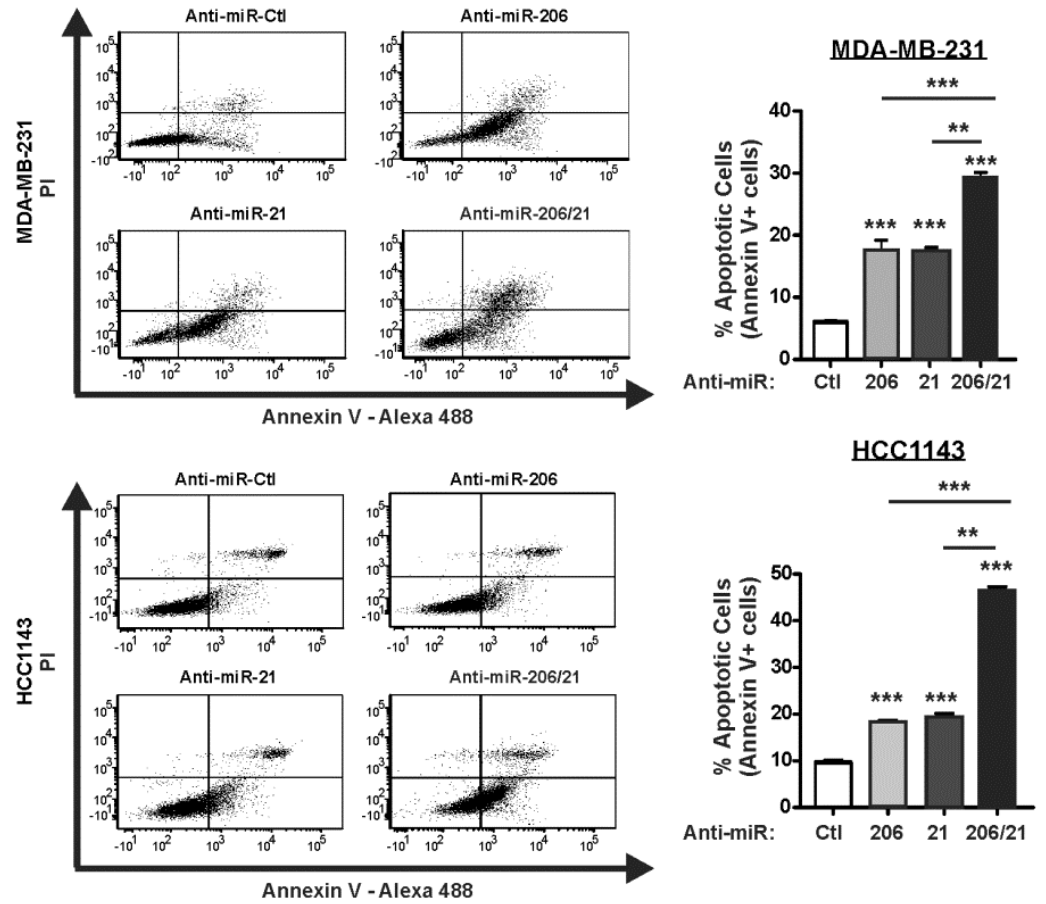

Figure 9 


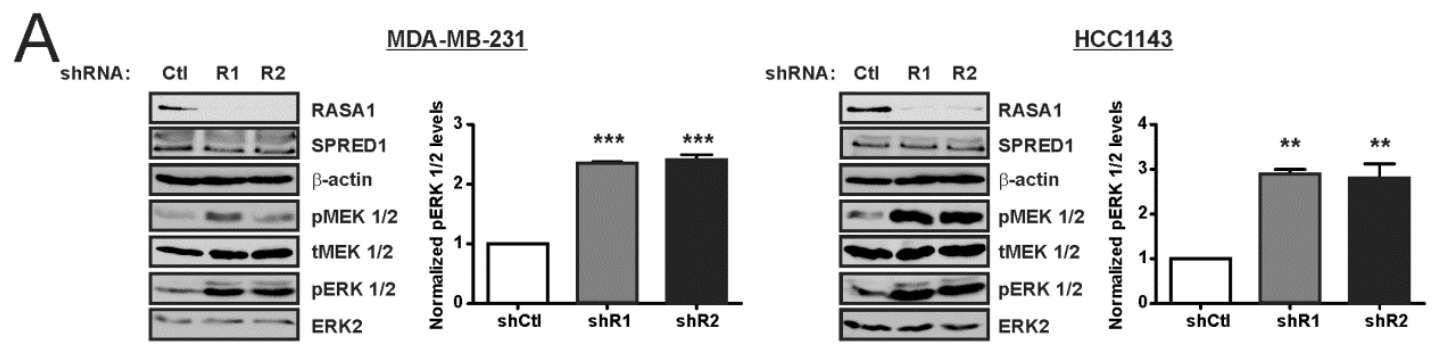

MDA-MB-231

HCC1143
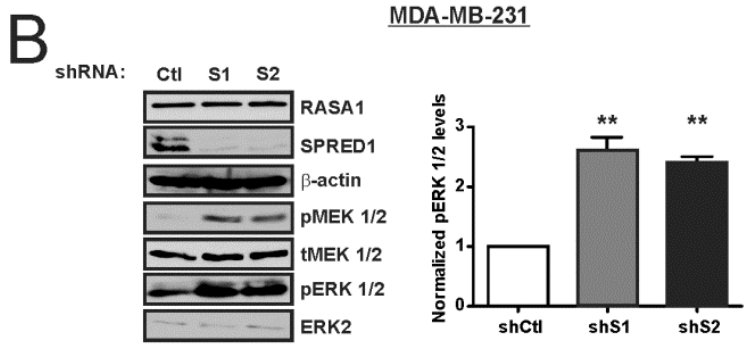

ShRNA: Ctl S1 S2
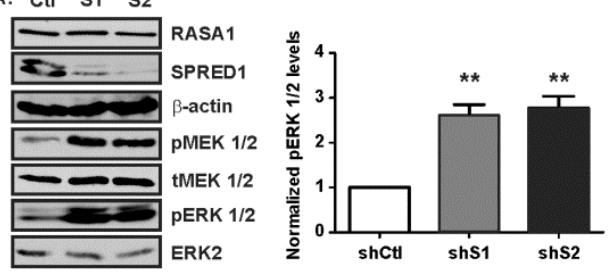
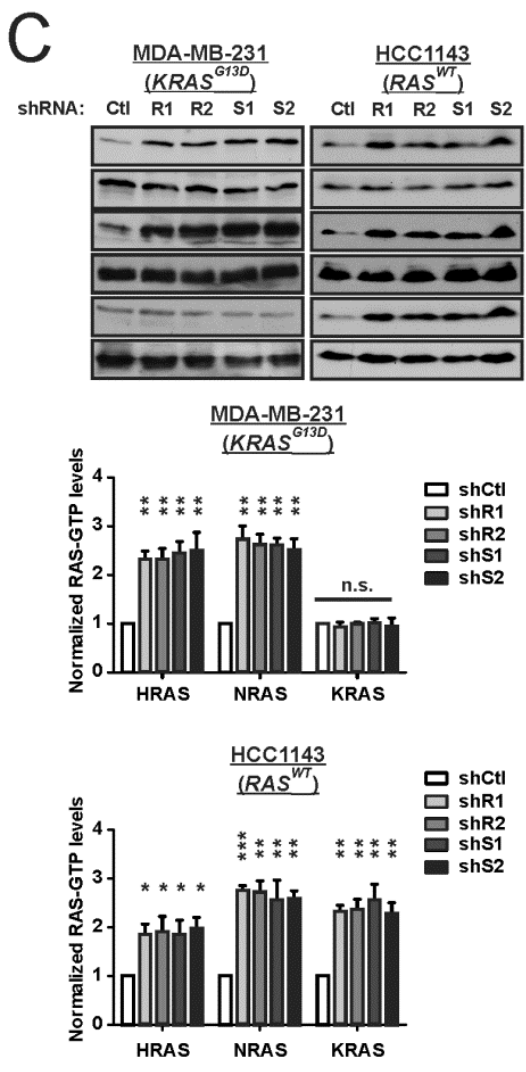

\section{RBD: HRAS-GTP SUM159PT \\ (HRAS $^{\text {G12D }}$}

WCL: HRAS ShRNA: CtI R1 R2 S1 S2

RBD: NRAS-GTP

WCL: NRAS

RBD: KRAS-GTP

WCL: KRAS
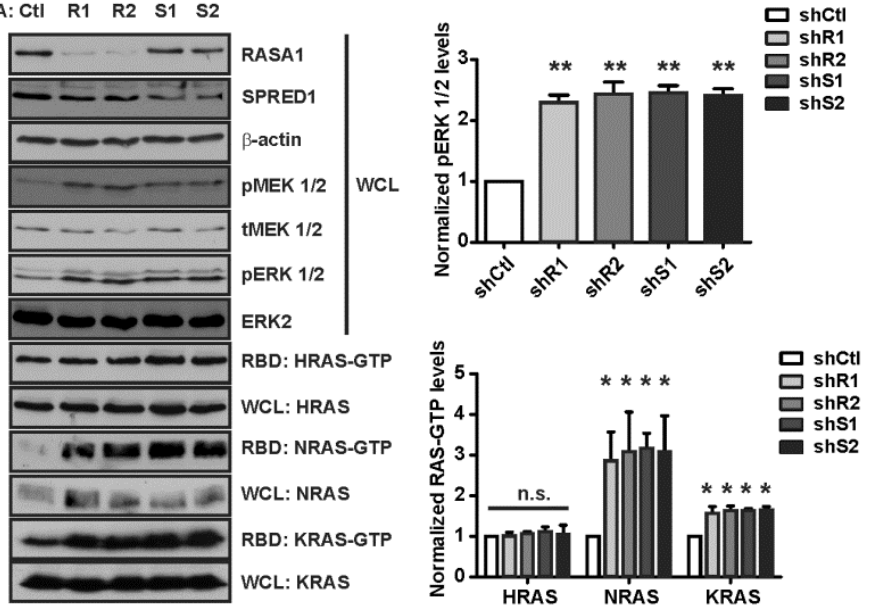

Figure 10 
A

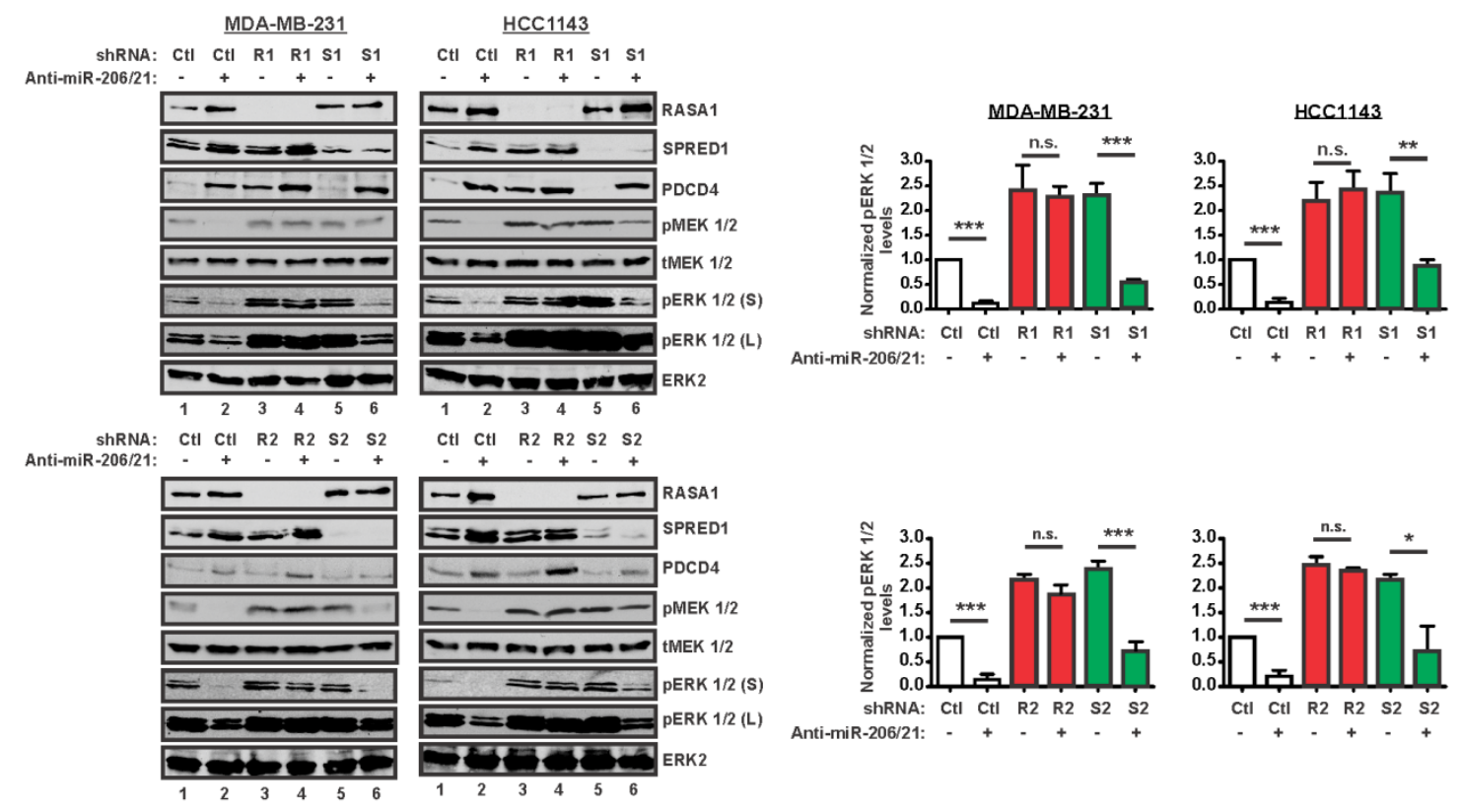

B

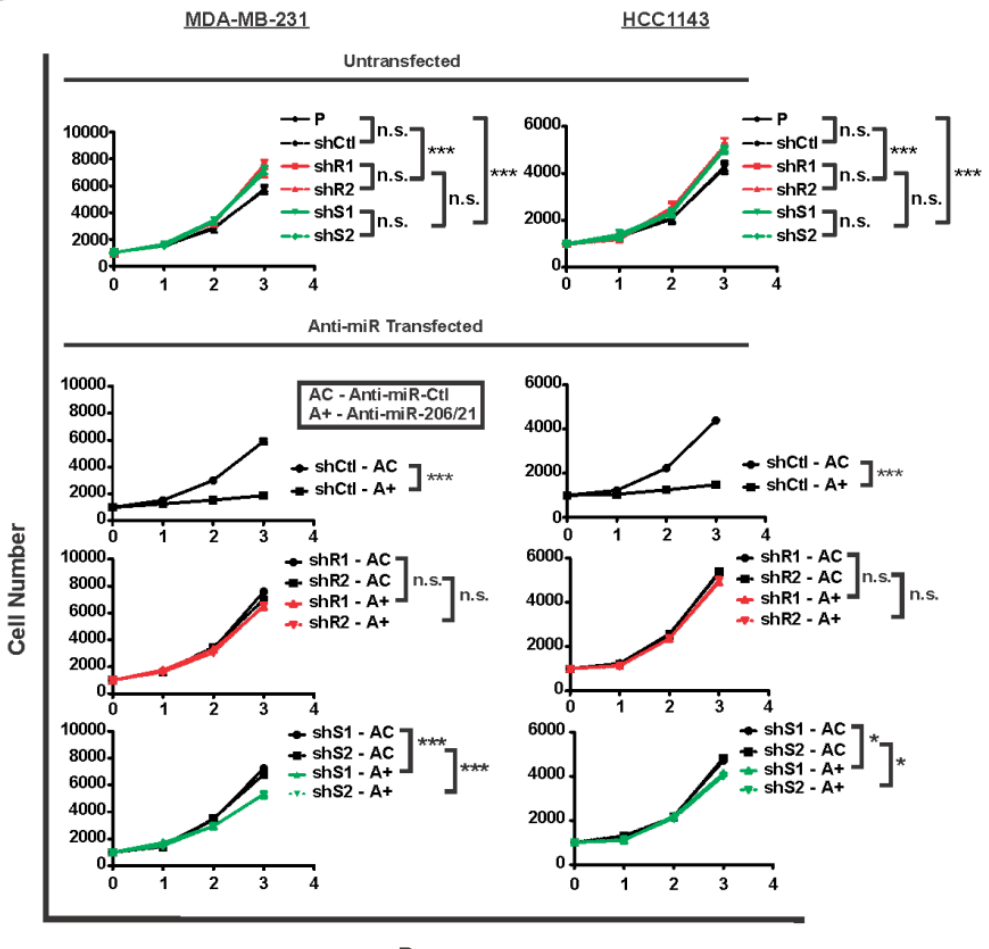

Day
C MDA-MB-231

\begin{tabular}{|c|c|}
\hline shRNA/Anti-miR & Initiated/Total (Day 28) \\
\hline shCt//Anti-miR-Ctl & \\
\hline shCtVAnti-miR-206/21 & \\
\hline shR1/Anti-miR-Ctl & $5 / 5$ \\
\hline shR1/Anti-miR-206/21 & $5 / 5$ \\
\hline shS1/Anti-miR-Ctl & $5 / 5$ \\
\hline shS1/Anti-miR-206/21 & 4/4 \\
\hline
\end{tabular}

Figure 11 

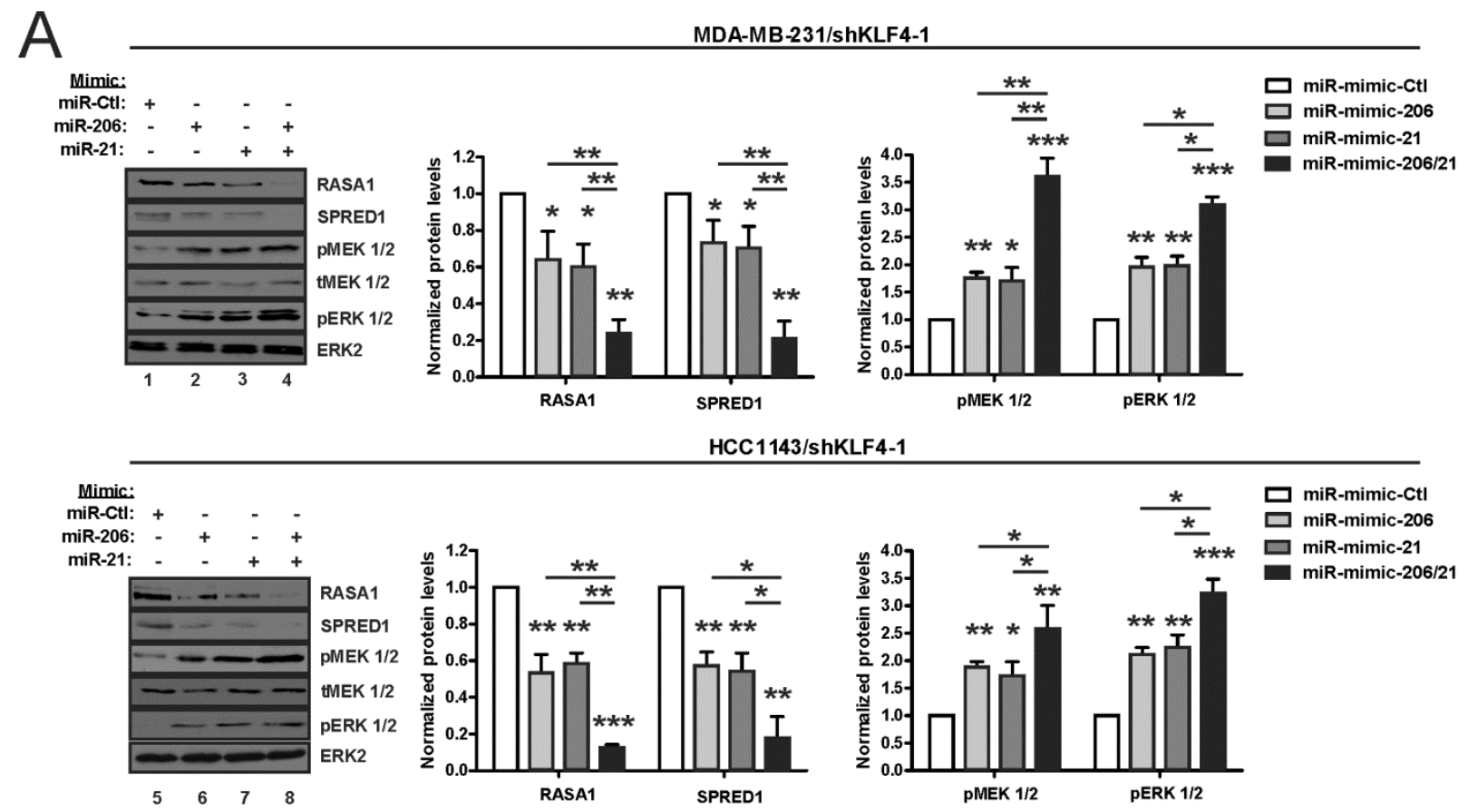

B

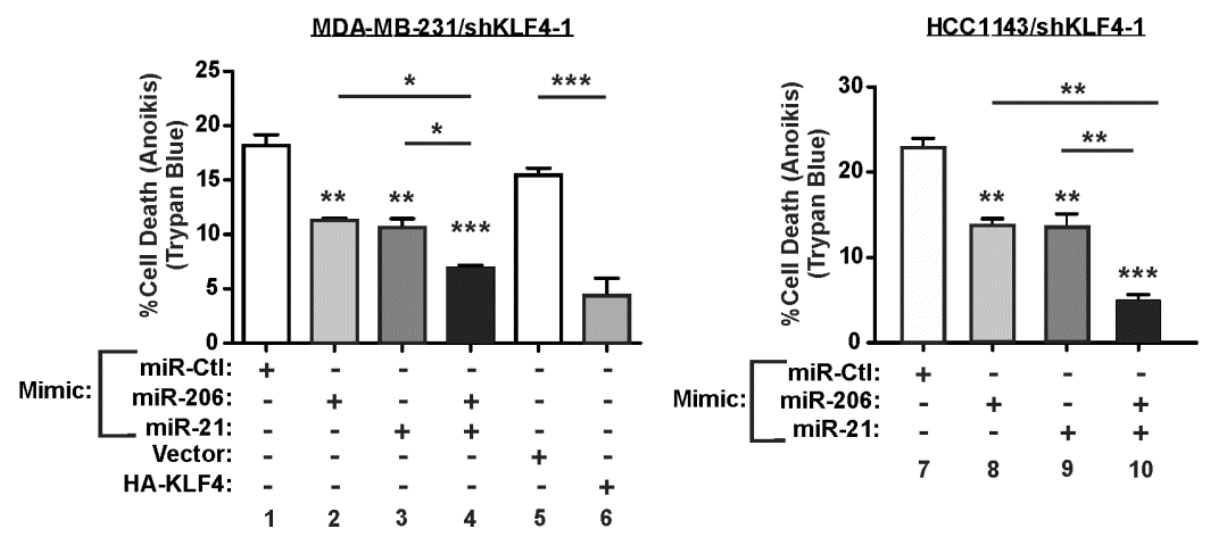

Figure 12 


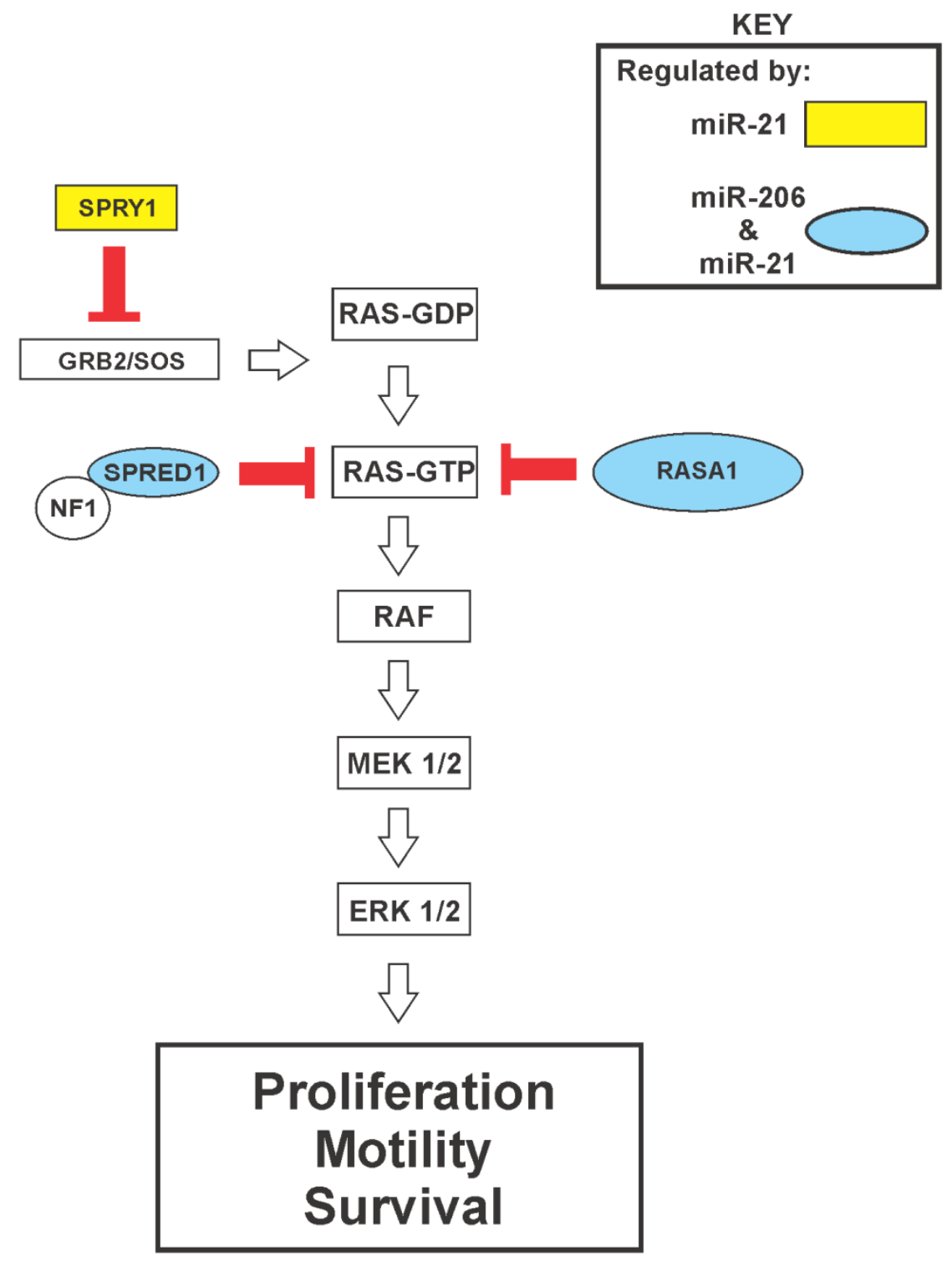

Figure 13 
This page is intentionally left blank 


\section{CHAPTER 3}

\section{Krüppel-like factor 4 signals through microRNA-206 to promote tumor initiation and cell survival}

Chen-Chung $\operatorname{Lin}^{1,3,5}$, Sriganesh B. Sharma ${ }^{\mathbf{1}, \mathbf{5}}$, Mark K. Farrugia ${ }^{1,2}$, Sarah L. McLaughlin $^{3}$, Ryan J. Ice ${ }^{1,2}$, Yuriy V. Loskutov ${ }^{1,2}$,Elena N. Pugacheva ${ }^{1,3}$, Kathleen M. Brundage ${ }^{3}$, Dongquan $\mathrm{Chen}^{4}$ and J. Michael Ruppert ${ }^{1,3, *}$

${ }^{1}$ Department of Biochemistry, ${ }^{2}$ Program in Cancer Cell Biology, and ${ }^{3}$ The Mary Babb Randolph Cancer Center, West Virginia University, Morgantown, West Virginia 26506; ${ }^{4}$ Division of Preventive Medicine, University of Alabama at Birmingham, Birmingham, Alabama 35205

${ }^{5}$ These authors contributed equally to this work.

*Corresponding author. Mailing address: Department of Biochemistry, PO Box 9142,

1 Medical Center Dr, West Virginia University, School of Medicine, Morgantown, WV, 26506. Phone: (304) 293-5246; Fax: (304) 293-4667; Email: mruppert@hsc.wvu.edu.

Running title: Enhanced KLF4-miR-206 signaling in MaCSCs

Key words: Breast cancer, Krüppel-like factor 4/KLF4, microRNA-206/miR-206, PDCD4, CX43/GJA1

Note: This manuscript has been published in Oncogenesis, 2015, doi: 10.1038/oncsis.2015.8 


\section{ABSTRACT}

Tumor cell heterogeneity poses a major hurdle in the treatment of cancer. Mammary cancer stem-like cells (MaCSCs), or tumor initiating cells, are highly tumorigenic subpopulations that have the potential to self-renew and to differentiate. These cells are clinically important, as they display therapeutic resistance and may contribute to treatment failure and recurrence, but the signaling axes relevant to the tumorigenic phenotype are poorly defined. The zinc finger transcription factor Krüppel-like factor 4 (KLF4) is a pluripotency mediator that is enriched in MaCSCs. KLF4 promotes RAS-ERK pathway activity and tumor cell survival in triple-negative breast cancer (TNBC) cells. In the present study we found that both $K L F 4$ and a downstream effector, microRNA-206 (miR-206), are selectively enriched in the MaCSC fractions of cultured human TNBC cell lines, as well as in the aldehyde dehydrogenase-high MaCSC subpopulation of cells derived from xenografted human mammary carcinomas. The suppression of endogenous KLF4 or miR-206 activities abrogated cell survival and in vivo tumor initiation, despite having only subtle effects on MaCSC abundance. Using a combinatorial approach that included in silico as well as loss- and gain-of-function in vitro assays, we identified miR-206 mediated repression of the pro-apoptotic molecules Programmed Cell Death 4 (PDCD4) and Connexin 43 (CX43/GJA1). Depletion of either of these two miR-206-regulated transcripts promoted resistance to anoikis, a prominent feature of CSCs, but did not consistently alter MaCSC abundance. Consistent with increased levels of miR-206 in MaCSCs, the expression of both PDCD4 and CX43 was suppressed in these cells relative to control cells. These results identifiy miR-206 as an effector of KLF4-mediated prosurvival signaling in MaCSCs through repression of PDCD4 and CX43. Consequently, our study suggests that a pluripotency factor exerts prosurvival signaling in MaCSCs, and that antagonism of KLF4-miR206 signaling may selectively target the MaCSC niche in TNBC. 


\section{INTRODUCTION}

Compelling experimental evidence supports the hierarchical organization of certain human tumor types, including breast cancer (1-6). These tumors are comprised of heterogenous mixtures of tumor cell populations that include cancer stem-like cells (CSCs), typically defined by their ability to initiate tumors in limiting dilution assays (i.e., tumor initiating cells, TICs). Furthermore, CSCs can survive and form tumorspheres in suspension culture, self-renew and differentiate $(7,8)$. These cells display resistance to chemotherapy, radiation therapy, and other triggers of cell death, and are thought to contribute to cancer recurrence. Therefore CSCs represent an important subpopulation for therapeutic targeting $(3,5)$.

In mammary carcinoma, functionally validated cancer stem-like cells (termed MaCSC) have been identified by profiling the expression of cell surface markers such as PROCR (P) and ESA (E) and/or by assaying aldehyde dehydrogenase (ALDH) activity (9-12). Despite this insight, the underlying mechanisms that mediate the MaCSC phenotype are unclear. For regulation of their abundance and/or their intrinsic properties such as resistance to cell death, several cellular signaling axes have been implicated including the WNT, NOTCH, TGF $\beta$ and SHH pathways (13-15).

A potential mediator of the MaCSC phenotype is the pluripotency factor Krüppel-like factor 4 (KLF4). This zinc finger transcription factor promotes the formation of induced pluripotent stem (iPS) cells from adult somatic cells and can play both anti-tumorigenic and protumorigenic roles in a context-dependent fashion (16-20). The capability of KLF4 to exert protumorigenic influences may reflect its role as a prosurvival stress response factor (21-28). In support of a protumorigenic role, KLF4 promotes epithelial transformation in vitro, escape from RAS-induced senescence, and skin tumor initiation in transgenic mice $(16,29,30)$. Furthermore, 
loss-of-function studies reveal that KLF4 promotes cell survival following radiation-induced DNA damage, and promotes the tumorigenicity of colorectal CSCs-enriched spheroid cells $(26,31)$.

In human breast cancer, KLF4 promoter demethylation and KLF4 protein expression indicate an unfavorable prognosis (32-34). KLF4 expression is positively correlated with tumor size, advanced grade and stage (35). We previously identified microRNAs, including miR-206 and miR-21, as direct transcriptional targets of KLF4 that promote RAS-Extracellular Signal Regulated-Kinase (ERK) signaling in triple-negative breast cancer (TNBC) cells $(36,37)$. Although on its own each miR exerts only subtle influences on RAS-ERK pathway activity, the coexpression of miR-206 and miR-21 potently represses the expression of pathway inhibitors including RASA1 and SPRED1. Furthermore, miR-206 directly represses KLF4 translation, constituting a feedback loop (36).

In the present study, we observed elevation of KLF4 and miR-206 in the $\mathrm{P}^{+} / \mathrm{E}^{+}$and ALDH $^{\text {High }}$ MaCSC fractions. In TNBC cells, both KLF4 and miR-206 were critical for cell survival and in vivo tumor initiation. We identified the tumor suppressor Programmed Cell Death 4 (PDCD4) as a potential mediator of cell survival by miR-206. Furthermore, in TNBC cells we demonstrated the miR-206 regulation of a previously validated transcript, the gap junction protein Connexin 43 (CX43/GJAl) (38).

Consistent with the elevated levels of miR-206 in MaCSCs, PDCD4 and CX43 levels were decreased. Supporting functional roles downstream of KLF4 and miR-206, suppression of either PDCD4 or CX43 led to anoikis resistance, an intrinsic property of CSCs (7,39-43). Finally, further documenting a prosurvival role, miR-206 promoted chemoresistance of TNBC cells against paclitaxel or doxorubicin. Our studies identify KLF4 and miR-206 as functional 
MaCSC markers that mediate cell survival. Consequently KLF4 and/or miR-206 may be therapeutically targeted to selectively cripple MaCSCs in TNBCs.

\section{MATERIALS AND METHODS}

Cell lines, cell culture, and drug treatments. MDA-MB-231 cells were provided by Katri S. Selander (University of Alabama at Birmingham), SUM159PT cells were provided by Gary L. Johnson (University of North Carolina at Chapel Hill), and M6 mammary carcinoma cells derived from the C3(1)/SV40 TAg mouse model were provided by Jeffrey E. Green (NIH). HCC1143 cells were from ATCC (Manassas, VA). Cells were maintained as subconfluent monolayers as previously described $(36,37)$.

For chemoresistance experiments, cells were treated with the indicated doses of paclitaxel (Sigma, St. Louis, MO) or doxorubicin (Merck, Billerica, MA) for 72 hours. Cells were treated with cycloheximide (CHX; Sigma) at $20 \mu \mathrm{g} / \mathrm{ml}$ for 24 hours. Cell proliferation was determined using the ATPlite ${ }^{\mathrm{TM}}$ Luminescence Assay System (PerkinElmer, Waltham, MA).

Retroviral transduction. Suppression studies utilized the following pGIPZ lentiviral shRNAmir plasmids (V2LHS_28277 - shKLF4-1, V3LHS_410934 - shKLF4-2, V3LHS_411731 shCX43-1, V3LHS_411733 - shCX43-2, V3LHS_366084 - shPDCD4-1, V3LHS_366087 shPDCD4-2; GE Dharmacon/Open Biosystems, Lafayette, CO). The retroviral vector pBABEpuro-HA-KLF4 and viral transduction was previously described (36). Cells were selected using puromycin $(1 \mu \mathrm{g} / \mathrm{ml})$.

Plasmid Construction. pMIR-REPORT firefly luciferase vector was purchased from Ambion (Austin, TX). pRL-TK Renilla luc reporter was obtained from Promega (Madison, WI). 
cDNA clones containing fragments of the 3' UTR of PDCD4 (Clone ID: NM_014456) and CX43/GJA1 were purchased from Open Biosystems and OriGene (Rockville, MD) respectively.

To construct a WT PDCD4 translational reporter, a $1.7 \mathrm{~kb}$ fragment representing the 3' UTR was excised using MluI and inserted into MluI-digested pMIR-REPORT. To construct a WT $C X 43 / G J A 1$ translational reporter, a $1.7 \mathrm{~kb}$ fragment representing the $C X 43$ 3' UTR was generated by sequential treatment with EcoRI, Klenow fragment, and MluI. This fragment was inserted into pMIR-REPORT vector that was prepared by sequential treatment with SacI, Klenow fragment, and MluI.

PDCD4 and $C X 43$ reporters with mutation in the miR-seed complementary regions were generated by PCR-mutagenesis. Oligonucleotides are listed in Table S1. WT reporters were mutated so as to conserve the predicted secondary structure of the 3' UTR (44). Cloned PCR products were confirmed by sequence analysis.

Transient transfection and translation reporter assays. The following Anti-miR inhibitors (AM) and miR-mimics (PM) were obtained from Ambion and diluted to $20 \mu \mathrm{M}$ in nuclease-free water: hsa-miR-206 (AM10409, PM10409), AM Negative Control (AM17010), and PM Negative Control (AM17110). Cells were subjected to reverse transfection and, 24 hours later, forward transfection was performed as described (36). At 24 hours after the start of the forward transfection, cell extracts were prepared for expression studies, or cells were used for phenotypic studies. Translational reporter assays were performed following just one transfection, at 24 hours after the start of the reverse transfection. Inhibitors/mimics were cotransfected with reporter plasmids, and Dual-Luciferase ${ }^{\circledR}$ Reporter Assays (Promega) were performed as described (36). 
Immunoblot analysis and antibodies. Cell extracts for immunoblot analysis were prepared as previously described (36). PARP cleavage assays were performed as recommended (Roche, Indianapolis, IN). Following electrophoresis, proteins were transferred onto nitrocellulose membranes and probed with the indicated antibody: KLF4 (Santa Cruz Biotechnology, Dallas, TX), PDCD4 (Rockland Immunochemicals, Philadelphia, PA), CX43 (Sigma), PARP (Roche), or $\beta$-actin (Santa Cruz). Bound antibodies were detected using Pierce ECL Western Blotting Substrate (ThermoFisher Scientific, Waltham, MA).

Animal studies. Female Athymic Nude mice (Crl:NU(NCr)-Foxn1 ${ }^{\text {nu }}$, Charles River) were obtained at 6-8 weeks of age. $2 \times 10^{6}$ cells were suspended in DMEM and injected into the $4^{\text {th }}$ mammary fat pad. For tumor initiation/limiting dilution assays, NOD/SCID-Gamma mice (NSG; NOD.Cg-Prkdcscid I12rgtm1Wj1/SzJ; Jackson lab, Bar Harbor, ME) were obtained at 6-8 weeks of age. Tumor cells were suspended in DMEM containing matrigel (50\% [vol/vol]) and injected into the $4^{\text {th }}$ mammary fat pad. Tumors were measured semiweekly using digital calipers. Tumor volume was determined by $\pi\left(\mathrm{L} 1 \times \mathrm{L} 2^{2}\right) / 6$ (L1, long axis; L2, short axis), and tumor initiation was defined as $\geq 2 \mathrm{~mm}$ for both $\mathrm{L}_{1}$ and $\mathrm{L}_{2}$. Animal procedures were performed under an approved protocol.

Isolation of mammary carcinoma cells from tumors. Human mammary cancer tissue was passaged as PDXs in NSG mice. HCI-001 and HCI-002 were obtained from Alana L. Welm, University of Utah, and PEN-025 and PEN-027 were obtained from the West Virginia University Tissue Bank. PDX tumors and tumors arising in female C3(1)/TAg mice were harvested upon reaching a size of $1-2 \mathrm{~cm}^{3}$. To isolate mammary carcinoma cells, tumors were minced and suspended in DMEM/F12 containing Gentle Collagenase/Hyaluronidase (STEMCELL Technologies, Vancouver, BC, Canada) and then processed as recommended by the 
manufacturer. Briefly, tumor cell suspensions were incubated with mild agitation at $37^{\circ} \mathrm{C}$ for 15 hours. Red blood cells (RBCs) were lysed by the addition of $0.16 \mathrm{M}$ Tris- $\mathrm{NH}_{4} \mathrm{Cl}(\mathrm{pH} 7.6)$ and incubation at $25^{\circ} \mathrm{C}$ for 3 minutes. $\mathrm{RBC}$ lysis was stopped by the addition of DMEM/F12 containing $10 \%$ FBS. The suspension was centrifuged and the resulting cell pellet was washed twice with DMEM/F12 containing 10\% FBS, resuspended in Trypsin-EDTA (0.25\%; Media Tech, Corning, NY) for 3 minutes with disaggregation by pipette, and then washed once again. Cells were resuspended in dispase and DNAse I (STEMCELL Technologies) at final concentrations of $4.2 \mathrm{mg} / \mathrm{ml}$ and $192 \mu \mathrm{g} / \mathrm{ml}$, respectively. The cells were centrifuged and the cell pellet was resuspended in HBSS containing $10 \mathrm{mM}$ HEPES-KOH, $\mathrm{pH} 7.2$, and $2 \% \mathrm{FBS}$.

Depletion of lineage-positive $\left(\mathrm{Lin}^{+}\right)$cells from prepared tumor cell suspensions was performed using an AutoMACS sorter (Miltenyi Biotec, San Diego, CA). Briefly, cells were suspended in ice cold staining buffer (PBS supplemented with 0.5\% [wt/vol] BSA) and blocked with $10 \mu \mathrm{g} / \mathrm{ml}$ mouse IgG (Sigma) for 15 minutes. Cells were stained with the following biotinconjugated antibodies (BD-Bioscience, San Jose, CA): anti-mouse-CD31 (clone 390), anti-CD45 (clone 30-F11), anti-TER-119 (clone TER-119). Anti-CD140b was from eBioscience, San Diego, CA (clone APB5). Cells were washed with labeling buffer (PBS, pH 7.2 containing 0.5\% BSA and 2 mM EDTA) and incubated with streptavidin microbeads (Miltenyi) prior to magnetic cell sorting as recommend by manufacturer.

Analysis and purification of MaCSCs. For analysis of PROCR/ESA expression, cells were blocked with $10 \mu \mathrm{g} / \mathrm{ml}$ normal human IgG (R\&D system, Minneapolis, MN) in ice cold staining buffer (PBS supplemented with 1\% [vol/vol] FBS) for 15 min. Cells were stained with antihuman PROCR-APC (clone RCR-227; eBioscience) and anti-human ESA-PerCP-Cy5.5 (clone 
EBA-1; BD Bioscience). Cells were centrifuged at $300 \times \mathrm{g}$ for $5 \mathrm{~min}$ at $4^{\circ} \mathrm{C}$, and washed twice with staining buffer before analysis.

ALDH activity was evaluated by flow cytometry using the ALDEFLUOR assay (STEMCELL Technologies). Cell sorting or flow cytometry was performed on a BD FACSAria using BDFACSDiva software version 6.1, or on a BD Fortessa using BDFACSDiva software version 7.0. For analysis, a minimum of 10,000 events were collected for each sample. The data were analyzed by using FCS Express 4 Research Edition software (De Novo software, Glendale, CA).

Tumorsphere formation and anoikis assays. To grow tumorspheres, $2 \times 10^{4} \mathrm{Lin}^{-}$cells were placed in suspension cultures in low attachment plates (Costar; Corning, NY) using DMEM/F-12 supplemented with B27, $4 \mu \mathrm{g} / \mathrm{ml}$ heparin, $20 \mathrm{ng} / \mathrm{ml}$ epidermal growth factor, $20 \mathrm{ng} / \mathrm{ml}$ fibroblast growth factor and 1\% (wt/vol) methylcellulose. For analysis of anoikis, cells were suspended in culture as previously described (37). Cell death was analyzed by propidium iodide (PI) staining and flow cytometry (Invitrogen; Carlsbad, CA), by trypan blue exclusion, or by analysis of cleaved PARP.

Expression analyses. Microarray data was extracted from GEO accessions GSE45666 and GSE23978 and then normalized to the geometric median $(45,46)$.

For qRT-PCR, total RNA was extracted and mRNA and miR levels were analyzed as previously described (36). Reactions were normalized to $B 2 M$ or $R P L P O$ for mRNA analysis, or to U6 snRNA for miR analysis. Primer sequences are listed in Table S2. PCR reactions were performed on a Mx3005PTM Real-Time PCR System (Stratagene, La Jolla, CA). mRNA and miR 
levels were determined by the $\Delta \Delta \mathrm{C}_{\mathrm{T}}$ method (47). For all RNA measurements, three independent experiments were performed in duplicate fashion.

Statistical analysis. Data were analyzed using either the unpaired t-test (two-tailed), or else oneway analysis of variance (ANOVA) followed by Tukey's multiple comparison ad hoc post-test. Tumor volumes were analyzed using two-way ANOVA with a Bonferroni post-test. Tumor initiation was analyzed using a $2 \times 2$ contingency table with a Fisher's exact test. Statistical analyses were performed in GraphPad Prism 5 (GraphPad Software, La Jolla, CA). Differences were considered significant when the analysis yielded $\mathrm{P}<0.05$.

\section{RESULTS}

\section{miR-206 is highly expressed in basal-like breast cancers and MaCSCs}

KLF4 protein levels correlate with an aggressive phenotype in breast tumors $(32,33,35)$. Similar to KLF4, miR-206 was increased in human tumors of advanced histological grade (Fig. 1A, left panel). Consistent with studies that identified upregulation of miR-206 in ER ${ }^{-}$breast tumors, miR-206 levels were elevated in TNBCs compared to both $\mathrm{ER}^{+}$and $\mathrm{HER} 2^{+}$human subgroups (Fig. 1A, right panel) $(48,49)$. Enrichment of miR-206 was likewise observed in murine basal-like mammary tumors (Fig. 1B). Compared to normal mammary tissues or tumors arising in the luminal MMTV_Neu model (50), we observed upregulation of both KLF4 and miR-206 in basal-like tumors derived from the C3(1)/SV40 large $T$ antigen (C3(1)/TAg) genetically engineered mouse model (GEMM) (Fig. 1C). These results are consistent with the direct regulation of miR-206 by KLF4 as previously reported (37).

MaCSCs are enriched in the triple-negative subgroup of breast cancer and are thought to contribute to the aggressive behavior of these cancers (51-53). Similar to human and murine 
mammary carcinoma cells displaying high ALDH activity (11,54-56), MDA-MB-231 TNBC cells displaying the $\mathrm{P}^{+} / \mathrm{E}^{+}$surface marker profile represent TICs (12). For SUM159PT cells, $\mathrm{CD}^{+} 4^{+} / \mathrm{CD} 24 / \mathrm{ESA}^{+}$subset was previously identified as TICs (57). As the $\mathrm{P}^{+}$phenotype is a surrogate for the $\mathrm{CD} 44^{+} / \mathrm{CD} 24^{-}$profile, the $\mathrm{P}^{+} / \mathrm{E}^{+}$SUM159PT cells are likely to represent $\operatorname{MaCSCs}(10)$.

We analyzed KLF4 and miR-206 levels in flow-sorted subpopulations of MDA-MB-231 cells (Fig.1D, left panel). Compared to non-MaCSCs (i.e., $\mathrm{P}^{-} / \mathrm{E}^{-}$), miR-206 and KLF4 were increased in the $\mathrm{P}^{+} / \mathrm{E}^{+}$subpopulation (Fig.1D, middle panels). Using $\mathrm{P}^{+} / \mathrm{E}^{+}$cells we profiled the expression of other genes associated with stem-like cell phenotypes $(9,14,18,19)$. Compared to $\mathrm{P}^{-}$ /E ${ }^{-}$cells, the expression of $C D 44, M Y C$, SOX2, NANOG, ZEB1, and SNAI2 was upregulated in $\mathrm{P}^{+} / \mathrm{E}^{+}$cells, whereas CD24 and POU5F1 (OCT3/4) expression were decreased (Fig.1D, right panel). Likewise, the $\mathrm{P}^{+} / \mathrm{E}^{+}$fraction of SUM159PT cells displayed elevated levels of KLF4 and miR-206, and showed a similar stem cell marker profile as the MDA-MB-231 cells (Fig. 1E). These results associate KLF4 and miR-206 with the MaCSC phenotype in human breast cancer models.

\section{KLF4 and miR-206 are enriched in MaCSCs derived from human patient-derived xenografts (PDXs) and the C3(1)/TAg GEMM}

KLF4 was likewise consistently elevated in Lineage-negative ( $\left.\operatorname{Lin}^{-}\right) / \mathrm{ALDH}^{\mathrm{High}} \mathrm{MaCSC}$ isolated from human mammary tumor tissues that were passaged as PDXs (Fig. 2A). miR-206 was upregulated in three of these four cases. Notably, none of these tumors displayed an appreciable $\mathrm{CD}_{4} 4^{+} \mathrm{CD} 24^{-}$MaCSC population (data not shown), consistent with the variable expression of these markers in patient samples $(10,58,59)$. 
Tumorspheres are enriched for MaCSCs $(7,8)$. Compared to cells grown in adherent (2D) monolayers, tumorspheres formed from the Lin ${ }^{-}$cells of C3(1)/TAg mammary tumors showed elevated levels of Klf4 and miR-206 (Fig. 2B). ALDH ${ }^{\text {High }}$ cells from other mammary cancer GEMMs were previously shown to have properties of MaCSCs $(54,56)$. Similar to the human

tumors, Lin/ALDH ${ }^{\text {High }}$ cells of C3(1)/TAg mammary tumors also had increased Klf4 and miR206 relative to $\mathrm{ALDH}^{\mathrm{Low}}$ cells (Fig. 2C). These results identify KLF4 and miR-206 as MaCSC markers and potential mediators of MaCSC malignant properties.

\section{KLF4 and miR-206 can promote MaCSC abundance}

To determine the effect of KLF4-miR-206 signaling on MaCSC abundance we depleted KLF4 in MDA-MB-231 cells using two distinct lentiviral shRNA constructs (Fig. 3A, left upper panel). Consistent with previous studies, miR-206 was suppressed following KLF4 knockdown (Fig. 3A, left lower panel). In addition, $\mathrm{P}^{+} / \mathrm{E}^{+}$cell abundance was modestly decreased upon KLF4 depletion (Fig. 3A, middle and right panels). Conversely, gain-of-function experiments showed that exogenous KLF4 promoted both miR-206 levels and the abundance of $\mathrm{P}^{+} / \mathrm{E}^{+}$cells (Fig. 3B).

We next sought to determine whether miR-206 could play a causal role downstream of KLF4 to regulate MaCSC abundance. As expected, transfection of miR-206-mimic into MDAMB-231 cells elevated the miR-206 level as detected by quantitative reverse transcription and PCR (qRT-PCR; Fig. 3C, left upper panel). In addition, the level of KLF4 was suppressed, attributed to direct regulation of KLF4 protein translation by miR-206 (Fig. 3C, left lower panel) (36). Despite the reduced levels of KLF4, miR-206-transfected cells displayed higher $\mathrm{P}^{+} / \mathrm{E}^{+}$cell abundance relative to the control cells (Fig. 3C, right panel). Similar regulation of $\mathrm{P}^{+} / \mathrm{E}^{+}$cell 
abundance by miR-206 was observed for SUM159PT cells (Fig. 3D). These results establish miR-206 as a potential effector of KLF4 for regulation of MaCSC abundance.

To determine whether miR-206 can promote the MaCSC phenotype, we assayed by limiting dilution the capability of miR-206-transfected MDA-MB-231 cells to initiate tumors in vivo. Consistent with an increased number of $\mathrm{P}^{+} / \mathrm{E}^{+}$cells, miR-206-transfected cells formed tumors more efficiently in NOD/SCID-gamma (NSG) mice compared to control cells (Fig. 3E; 2 $\times 10^{3}$ cells, $\mathrm{p}=0.0022$ ). These results implicate miR-206 as an effector of KLF4 that promotes tumor initiation.

\section{Endogenous KLF4 and miR-206 promote tumor cell survival and in vivo tumorigenesis}

We next examined the impact of endogenous KLF4-miR-206 signaling on tumor initiation. Depletion of KLF4 reduced the tumor initiation rate of MDA-MB-231 cells in athymic nude mice (Fig. 4A, left panels). This decrease in tumor incidence was reflected by the reduced mean tumor volume for all animals combined (Fig. 4A, middle panels). Indicating that the major effect of KLF4 in this setting is restricted to tumor initiation, analysis of the tumor-positive subset revealed little difference in the tumor growth rate between KLF4 depleted cells and the control (Fig. 4A, right panels).

To study the role of endogenous miR-206 during in vivo tumorigenesis, we analyzed the tumorigenicity of MDA-MB-231 cells treated by in vitro transfection of anti-sense oligonucleotides (anti-miR-206). Compared to cells transfected with the control, anti-miR-206 treatment reduced both tumor incidence and tumor growth (Fig. 4B). As an indicator of successful transfection, KLF4 expression was increased (Fig. 4C, left panel). Effects on tumor growth were not likely attributed to differences in cell proliferation rates, as anti-miR-206 had little effect (Fig. 4C, right panel). 
The critical role of endogenous miR-206 for tumor initiation following orthotopic injection, despite its minimal effects on cell proliferation or MaCSC abundance, pointed to a potential role in regulating cell survival. We therefore assayed for resistance to cell death following matrix deprivation (anoikis), an intrinsic property of CSCs (7,39-43). Indeed, consistent with our previous report that analyzed two human TNBC cell lines (37), anti-miR-206 transfection sensitized several human TNBC models and a murine basal-like mammary cancer model (i.e., M6 cells) to anoikis (Fig. 4D, left panel). Consistent results were obtained when anoikis was analyzed by poly ADP ribose polymerase (PARP) cleavage (Fig. 4D, right panel). In support of a prosurvival role for endogenous miR-206, depletion of KLF4 sensitized TNBC cells to anoikis (Fig. 4E). These results suggest that endogenous KLF4 exerts a prosurvival effect by induction of miR-206.

miR-206 suppresses the translation of the tumor suppressor PDCD4 and promotes tumor cell survival

We previously reported that RAS-ERK signaling, a prosurvival pathway, is maintained in TNBC cells by KLF4, in part through its regulation of miR-206 (37). In contrast to the prominent effect of miR-206 on tumor initiation and cell survival, on its own this miR has only limited effects on ERK activity (37). We therefore sought to better understand how endogenous miR-206 can promote anoikis resistance.

The tumor suppressor PDCD4 was identified as a potential miR-206 targeted transcript by multiple miR-target prediction tools $(37,60)$. PDCD4 is a negative regulator of RAS-ERKAP1 signaling and protein translation, and promotes breast cancer cell apoptosis (61-63). We therefore analyzed PDCD4 as a miR-206-regulated transcript. 
Consistent with regulation of PDCD4 by miR-206, KLF4 depletion in MDA-MB-231 cells increased PDCD4 expression (Fig. 5A, left panel). Similarly, while anti-miR-206 treatment elevated PDCD4, transfection of miR-206-mimic was suppressive (Fig. 5A, middle and right panels). Direct regulation of PDCD4 by miR-206 was determined using translational reporter assays. Fragments of the PDCD4 3' UTR containing two putative miR-206 binding sites (denoted WT-A and WT-B; Fig. 5B) were cloned downstream of the open reading frame of firefly luciferase (luc). Relative to the controls, in MDA-MB-231 cells miR-206 mimic repressed WT-reporter luc activity by 72\%, and anti-miR-206 induced the reporter by 1.9 -fold (Fig. 5C). Reporter regulation by miR-206 was abolished by mutation of site WT-A, but not by mutation of site WT-B, thus identifying site WT-A as a functional miR-206 binding site (Fig. 5B and 5C). In agreement with previous studies, PDCD4 depletion in TNBC cells promoted resistance to anoikis, with little or no effect on 2D proliferation (Fig. 5D).

Consistent with miR-206 regulation of $P D C D 4$ in MaCSCs, the $\mathrm{P}^{+} / \mathrm{E}^{+}$subpopulation of MDA-MB-231 cells exhibited decreased levels of PDCD4 mRNA and protein compared to nonMaCSCs (Fig. 5E). In TNBC cells the depletion of PDCD4 was not sufficient to alter the abundance of the $\mathrm{P}^{+} / \mathrm{E}^{+}$fraction (Fig. 5F). These results appear to support a selective role of PDCD4 for suppression of tumor cell survival.

\section{miR-206 promotes cell survival by suppressing $C X 43$ in MaCSCs}

Our identification of miR-206 regulation of PDCD4 led us to seek additional targets of this miR that may be important for promoting cell survival. DIANA-miRPath analysis identifies gap junction signaling as the top-ranked miR-206-regulated pathway $\left(\mathrm{P}=2.58 \times 10^{-6}\right)(64)$. Among the targeted gap junction proteins, CX43 is a validated miR-206-regulated transcript, as 
previously shown in muscle cells $(38,65)$. CX43 is deficient in human breast tumor cells and MaCSCs, and may exert a tumor suppressor role (66-72).

Consistent with its regulation by miR-206 in breast cancer cells, CX43 was increased in KLF4-depleted MDA-MB-231 cells (Fig. 6A, left panel). Similarly, inhibition of miR-206 led to elevated CX43 levels, and transfection of miR-206-mimic was suppressive (Fig. 6A, middle and right panels). In TNBC cells the activity of a translational reporter containing the CX43 3' UTR was induced by 1.5 -fold following anti-miR-206 treatment, and suppressed by $53 \%$ following transfection of miR-206-mimic (Fig. 6B and 6C). Supporting the direct regulation of CX43 by miR-206 in breast tumor cells, mutation of site A (mut206-A) abolished regulation by miR-206 (Fig. 6C). Similar to PDCD4 depletion, suppression of CX43 in TNBC cells promoted resistance to anoikis, with only subtle effects on cell proliferation (Fig. 6D).

Compared to the non-MaCSC fraction, $\mathrm{P}^{+} / \mathrm{E}^{+} \mathrm{MDA}-\mathrm{MB}-231$ cells displayed lower $\mathrm{CX} 43$ mRNA and protein (Fig. 6E). These results support a previous study that reported low CX43 expression in mammary TICs (68). Similarly to PDCD4, knockdown of CX43 did not consistently alter the $\mathrm{P}^{+} / \mathrm{E}^{+}$cell abundance in TNBC cells, suggesting a selective role in tumor cell survival (data not shown).

\section{miR-206 confers chemoresistance in TNBC cells}

Consistent with the promotion of cell survival by miR-206 as determined by anoikis assays, TNBC cells transfected with miR-206-mimic were more resistant to paclitaxel or doxorubicin (Fig. 7A). Furthermore, inhibition of the endogenous miR-206 moderately sensitized TNBC cells to either agent (Fig. 7B). Collectively, these results link pluripotency factor signaling and the enhanced cell survival of MaCSCs, supporting roles of KLF4-miR-206 
signaling for breast tumor cell survival, chemoresistance, and tumor initiation through the repression of PDCD4 and $C X 43$ (Fig. 7C).

\section{DISCUSSION}

Cancer stem-like cells (CSCs) were first identified in hematopoietic malignancies and subsequently in solid tumors such as breast cancer $(1,3,5,73)$. Despite substantial progress, questions remain regarding the relationship of CSCs to the adult stem cells of normal tissue, and the nature of the signaling pathways that regulate CSC properties (6). Despite this uncertainty, it is clear that CSCs represent a highly malignant subpopulation of tumor cells with the capability to resist therapy $(3,5)$.

In TNBC cells KLF4 directly regulates miR-206 transcription, and depletion of KLF4 consistently results in loss of the vast majority of miR-206 $(36,37)$. In the present study we identified KLF4 and miR-206 as critical promoters of breast tumor cell survival. Both factors were preferentially expressed in the MaCSCs purified from 2D cell culture models of TNBC, from tumorspheres cultured in 3D, from human PDXs, and from primary mouse mammary cancers. As shown by anti-miR treatment of TNBC cells, endogenous miR-206 directly repressed the translation of the tumor suppressors PDCD4 and CX43 and promoted tumor cell survival, chemoresistance and in vivo tumor initiation. Immunoblot analysis of MaCSCs revealed suppressed levels of both PDCD4 and CX43. Mirroring the role of endogenous miR-206, depletion of each tumor suppressor did not alter the abundance of CSCs, but instead enhanced tumor cell survival consistent with previous reports $(61,74)$.

miRs can act as critical factors for regulating the abundance and/or survival of MaCSCs

(75-78). In skeletal muscle miR-206 is important for repression of PAX7 during stem cell 
differentiation, and for muscle regeneration following injury (79-83). In a mammary cancer context, miR-206 expression is elevated in $\mathrm{ER}^{-}$tumors, which are enriched for MaCSCs $(48,49,51-53)$. In agreement with previous studies, we observed that miR-206 is upregulated in human breast cancers that display a higher grade, in human triple-negative breast cancers, and in basal-like mammary tumors derived from the C3(1)/TAg GEMM (Fig. 1A-C).

Multiple previous studies have reported that enforced expression of miR-206 can suppress tumor cell proliferation, invasion or metastasis $(49,84)$. These tumor suppressor-like effects of miR-206 may result from higher level enforced expression of the exogenous miR. In the current study, suppression of endogenous miR-206 blocked tumor initiation, and moderate (5-fold) overexpression of exogenous miR-206 promoted initiation in a limiting dilution assay. In addition, we observed that either exogenous or endogenous miR-206 could promote malignant properties including tumor cell survival and drug resistance.

Depletion of endogenous KLF4 suppressed in vivo tumor initiation by MDA-MB-231 cells in athymic nude mice, yet had little effect on the growth rate of established tumors. Similarly as observed for KLF4, transient inhibition of endogenous miR-206 by anti-miR-206 transfection suppressed tumor initiation in vivo but did not alter the in vitro proliferation or the MaCSC abundance. These results suggest that endogenous KLF4 can signal through miR-206 to promote tumor initiation, probably by impacting cell survival rather than MaCSC abundance. In contrast, exogenous KLF4 or miR-206 promoted MaCSC abundance, mirroring the role of exogenous KLF4 for generation of iPS cells $(18,19)$. It will be interesting to determine whether miR-206 likewise influences the generation of iPS cells.

In the current study, we have identified endogenous KLF4 and a downstream effector, miR-206, as functional markers and prosurvival factors that are enriched in MaCSCs. 
Prosurvival signaling by miR-206 was attributed to direct regulation of PDCD4 and $C X 43$, and miR-206 enhanced the chemoresistance of TNBC cells. Our study therefore provides a rationale for miR-206-directed antago-miR therapy for the sensitization of the MaCSCs (78,85-89).

\section{ACKNOWLEDGMENTS}

We thank Katri Selander, Gary Johnson and Jeffrey Green for providing cell lines. We thank Alana Welm for providing human mammary carcinoma xenografts. We thank Max Wicha for helpful discussions. This work was supported by NCI grant R01CA127405 (J.M.R.), the Jo and Ben Statler Chair in Breast Cancer Research (J.M.R.) and by grant R01CA148671 (E.N.P). Flow cytometry experiments were performed in the West Virginia University Flow Cytometry Core Facility, which is supported by NIH equipment grant RR020866/OD016165 and the Institutional Development Award (IDeA) from the National Institute of General Medical Sciences under grants P30GM103488 (CoBRE) and P20GM103434 (INBRE). Orthotopic tumor cell injections were performed in the West Virginia University Animal Models \& Imaging Facility, which has been supported by the Mary Babb Randolph Cancer Center and NIH grants P20RR016440, P30RR032138/GM103488, and S10RR026378. 


\section{REFERENCES}

1. Reya, T., S. J. Morrison, M. F. Clarke, and I. L. Weissman. 2001. Stem cells, cancer, and cancer stem cells. Nature 414:105-111.

2. Hwang-Verslues, W. W., K. J. Chang, E. Y. Lee, and W. H. Lee. 2008. Breast cancer stem cells and tumor suppressor genes. J Formos. Med Assoc. 107:751-766.

3. Visvader, J. E. and G. J. Lindeman. 2008. Cancer stem cells in solid tumours: accumulating evidence and unresolved questions. Nat. Rev. Cancer 8:755-768.

4. Shackleton, M., E. Quintana, E. R. Fearon, and S. J. Morrison. 2009. Heterogeneity in cancer: cancer stem cells versus clonal evolution. Cell 138:822-829.

5. Clevers, H. 2011. The cancer stem cell: premises, promises and challenges. Nat Med 17:313-319.

6. Beck, B. and C. Blanpain. 2013. Unravelling cancer stem cell potential. Nat Rev Cancer 13:727-738.

7. Dontu, G., W. M. Abdallah, J. M. Foley, K. W. Jackson, M. F. Clarke, M. J. Kawamura, and M. S. Wicha. 2003. In vitro propagation and transcriptional profiling of human mammary stem/progenitor cells. Genes Dev 17:1253-1270.

8. Dontu, G., M. Al-Hajj, W. M. Abdallah, M. F. Clarke, and M. S. Wicha. 2003. Stem cells in normal breast development and breast cancer. Cell Prolif. 36 Suppl 1:59-72.

9. Al-Hajj, M., M. S. Wicha, A. ito-Hernandez, S. J. Morrison, and M. F. Clarke. 2003. Prospective identification of tumorigenic breast cancer cells. Proc Natl Acad Sci U. S. A 100:3983-3988.

10. Shipitsin, M., L. L. Campbell, P. Argani, S. Weremowicz, N. Bloushtain-Qimron, J. Yao, T. Nikolskaya, T. Serebryiskaya, R. Beroukhim, M. Hu, M. K. Halushka, S. Sukumar, L. M. Parker, K. S. Anderson, L. N. Harris, J. E. Garber, A. L. Richardson, S. J. Schnitt, Y. Nikolsky, R. S. Gelman, and K. Polyak. 2007. Molecular definition of breast tumor heterogeneity. Cancer Cell 11:259-273.

11. Ginestier, C., M. H. Hur, E. Charafe-Jauffret, F. Monville, J. Dutcher, M. Brown, J. Jacquemier, P. Viens, C. G. Kleer, S. Liu, A. Schott, D. Hayes, D. Birnbaum, M. S. Wicha, and G. Dontu. 2007. ALDH1 is a marker of normal and malignant human mammary stem cells and a predictor of poor clinical outcome. Cell Stem Cell 1:555-567. 
12. Hwang-Verslues, W. W., W. H. Kuo, P. H. Chang, C. C. Pan, H. H. Wang, S. T. Tsai, Y. M. Jeng, J. Y. Shew, J. T. Kung, C. H. Chen, E. Y. Lee, K. J. Chang, and W. H. Lee. 2009. Multiple lineages of human breast cancer stem/progenitor cells identified by profiling with stem cell markers. PLoS One 4:e8377.

13. Reya, T. and H. Clevers. 2005. Wnt signalling in stem cells and cancer. Nature 434:843850.

14. Mani, S. A., W. Guo, M. J. Liao, E. N. Eaton, A. Ayyanan, A. Y. Zhou, M. Brooks, F. Reinhard, C. C. Zhang, M. Shipitsin, L. L. Campbell, K. Polyak, C. Brisken, J. Yang, and R. A. Weinberg. 2008. The epithelial-mesenchymal transition generates cells with properties of stem cells. Cell 133:704-715.

15. Karamboulas, C. and L. Ailles. 2013. Developmental signaling pathways in cancer stem cells of solid tumors. Biochim Biophys Acta 1830:2481-2495.

16. Rowland, B. D., R. Bernards, and D. S. Peeper. 2005. The KLF4 tumour suppressor is a transcriptional repressor of p53 that acts as a context-dependent oncogene. Nat Cell Biol 7:1074-1082.

17. Rowland, B. D. and D. S. Peeper. 2006. KLF4, p21 and context-dependent opposing forces in cancer. Nat. Rev. Cancer 6:11-23.

18. Takahashi, K. and S. Yamanaka. 2006. Induction of pluripotent stem cells from mouse embryonic and adult fibroblast cultures by defined factors. Cell 126:663-676.

19. Takahashi, K., K. Tanabe, M. Ohnuki, M. Narita, T. Ichisaka, K. Tomoda, and S. Yamanaka. 2007. Induction of pluripotent stem cells from adult human fibroblasts by defined factors. Cell 131:861-872.

20. McConnell, B. B. and V. W. Yang. 2010. Mammalian krüppel-like factors in health and diseases. Physiol Rev. 90:1337-1381.

21. McCormick, S. M., S. G. Eskin, L. V. McIntire, C. L. Teng, C. M. Lu, C. G. Russell, and K. K. Chittur. 2001. DNA microarray reveals changes in gene expression of shear stressed human umbilical vein endothelial cells. Proc Natl Acad Sci U S A 98:8955-8960.

22. Pedersen, T. X., C. Leethanakul, V. Patel, D. Mitola, L. R. Lund, K. Dano, M. Johnsen, J. S. Gutkind, and T. H. Bugge. 2003. Laser capture microdissection-based in vivo genomic profiling of wound keratinocytes identifies similarities and differences to squamous cell carcinoma. Oncogene 22:3964-3976. 
23. Yoon, H. S., X. Chen, and V. W. Yang. 2003. Krüppel-like factor 4 mediates p53dependent G1/S cell cycle arrest in response to DNA damage. J Biol. Chem. 278:21012105.

24. Liu, Y., S. Sinha, O. G. McDonald, Y. Shang, M. H. Hoofnagle, and G. K. Owens. 2005. Krüppel-like factor 4 abrogates myocardin-induced activation of smooth muscle gene expression. J Biol. Chem. 280:9719-9727.

25. Liu, Y., J. Wang, Y. Yi, H. Zhang, J. Liu, M. Liu, C. Yuan, D. Tang, I. J. Benjamin, and X. Xiao. 2006. Induction of KLF4 in response to heat stress. Cell Stress Chaperones 11:379-389.

26. Ghaleb, A. M., J. P. Katz, K. H. Kaestner, J. X. Du, and V. W. Yang. 2007. Krüppel-like factor 4 exhibits antiapoptotic activity following gamma-radiation-induced DNA damage. Oncogene 26:2365-2373.

27. Hamik, A., Z. Lin, A. Kumar, M. Balcells, S. Sinha, J. Katz, M. W. Feinberg, R. E. Gerzsten, E. R. Edelman, and M. K. Jain. 2007. Krüppel-like factor 4 regulates endothelial inflammation. J Biol Chem 282:13769-13779.

28. Liao, X., S. M. Haldar, Y. Lu, D. Jeyaraj, K. Paruchuri, M. Nahori, Y. Cui, K. H. Kaestner, and M. K. Jain. 2010. Krüppel-like factor 4 regulates pressure-induced cardiac hypertrophy. J Mol Cell Cardiol. 49:334-338.

29. Foster, K. W., S. Ren, I. D. Louro, S. M. Lobo-Ruppert, P. Kie-Bell, W. Grizzle, M. R. Hayes, T. R. Broker, L. T. Chow, and J. M. Ruppert. 1999. Oncogene expression cloning by retroviral transduction of adenovirus E1A-immortalized rat kidney RK3E cells: transformation of a host with epithelial features by c-MYC and the zinc finger protein GKLF. Cell Growth Differ 10:423-434.

30. Foster, K. W., Z. Liu, C. D. Nail, X. Li, T. J. Fitzgerald, S. K. Bailey, A. R. Frost, I. D. Louro, T. M. Townes, A. J. Paterson, J. E. Kudlow, S. M. Lobo-Ruppert, and J. M. Ruppert. 2005. Induction of KLF4 in basal keratinocytes blocks the proliferationdifferentiation switch and initiates squamous epithelial dysplasia. Oncogene 24:14911500 .

31. Leng, Z., K. Tao, Q. Xia, J. Tan, Z. Yue, J. Chen, H. Xi, J. Li, and H. Zheng. 2013. Krüppel-Like Factor 4 Acts as an Oncogene in Colon Cancer Stem Cell-Enriched Spheroid Cells. PLoS One 8:e56082.

32. Pandya, A. Y., L. I. Talley, A. R. Frost, T. J. Fitzgerald, V. Trivedi, M. Chakravarthy, D. C. Chhieng, W. E. Grizzle, J. A. Engler, H. Krontiras, K. I. Bland, A. F. LoBuglio, S. M. 
Lobo-Ruppert, and J. M. Ruppert. 2004. Nuclear localization of KLF4 is associated with an aggressive phenotype in early-stage breast cancer. Clin Cancer Res 10:2709-2719.

33. Chu, P. Y., N. C. Hsu, A. T. Liao, K. T. Yeh, M. F. Hou, and C. H. Liu. 2011. Elevated Krüppel-like factor 4 transcription factor in canine mammary carcinoma. BMC Vet. Res 7:58.

34. Kamalakaran, S., V. Varadan, H. E. Giercksky Russnes, D. Levy, J. Kendall, A. Janevski, M. Riggs, N. Banerjee, M. Synnestvedt, E. Schlichting, R. Karesen, P. K. Shama, H. Rotti, R. Rao, L. Rao, M. H. Eric Tang, K. Satyamoorthy, R. Lucito, M. Wigler, N. Dimitrova, B. Naume, A. L. Borresen-Dale, and J. B. Hicks. 2011. DNA methylation patterns in luminal breast cancers differ from non-luminal subtypes and can identify relapse risk independent of other clinical variables. Mol Oncol 5:77-92.

35. Chen, C. J., S. E. Lin, Y. M. Lin, S. H. Lin, D. R. Chen, and C. L. Chen. 2012. Association of expression of krüppel-like factor 4 and krüppel-like factor 5 with the clinical manifestations of breast cancer. Pathol. Oncol. Res 18:161-168.

36. Lin, C. C., L. Z. Liu, J. B. Addison, A. V. Ivanov, and J. M. Ruppert. 2011. A KLF4miRNA-206 Autoregulatory Feedback Loop can Promote or Inhibit Protein Translation Depending upon Cell Context. Mol. Cell Biol. 31:2513-2527.

37. Sharma, S. B., C. C. Lin, M. K. Farrugia, S. L. McLaughlin, E. J. Ellis, K. M. Brundage, M. A. Salkeni, and J. M. Ruppert. 2014. microRNAs-206 and -21 cooperate to promote RAS-ERK signaling by suppressing the translation of RASA1 and SPRED1. Mol Cell Biol 34:4143-4164.

38. Anderson, C., H. Catoe, and R. Werner. 2006. MIR-206 regulates connexin43 expression during skeletal muscle development. Nucleic Acids Res 34:5863-5871.

39. Reynolds, B. A. and S. Weiss. 1996. Clonal and population analyses demonstrate that an EGF-responsive mammalian embryonic CNS precursor is a stem cell. Dev Biol 175:1-13.

40. Uchida, N., D. W. Buck, D. He, M. J. Reitsma, M. Masek, T. V. Phan, A. S. Tsukamoto, F. H. Gage, and I. L. Weissman. 2000. Direct isolation of human central nervous system stem cells. Proc Natl Acad Sci U S A 97:14720-14725.

41. Dontu, G. and M. S. Wicha. 2005. Survival of mammary stem cells in suspension culture: implications for stem cell biology and neoplasia. J Mammary Gland Biol Neoplasia 10:75-86. 
42. Harrison, H., G. Farnie, S. J. Howell, R. E. Rock, S. Stylianou, K. R. Brennan, N. J. Bundred, and R. B. Clarke. 2010. Regulation of breast cancer stem cell activity by signaling through the Notch4 receptor. Cancer Res 70:709-718.

43. Chaffer, C. L., I. Brueckmann, C. Scheel, A. J. Kaestli, P. A. Wiggins, L. O. Rodrigues, M. Brooks, F. Reinhardt, Y. Su, K. Polyak, L. M. Arendt, C. Kuperwasser, B. Bierie, and R. A. Weinberg. 2011. Normal and neoplastic nonstem cells can spontaneously convert to a stem-like state. Proc Natl Acad Sci U S A 108:7950-7955.

44. Zuker, M. 2003. Mfold web server for nucleic acid folding and hybridization prediction. Nucleic Acids Res 31:3406-3415.

45. Zhu, M., M. Yi, C. H. Kim, C. Deng, Y. Li, D. Medina, R. M. Stephens, and J. E. Green. 2011. Integrated miRNA and mRNA expression profiling of mouse mammary tumor models identifies miRNA signatures associated with mammary tumor lineage. Genome Biol 12:R77.

46. Lee, C. H., W. H. Kuo, C. C. Lin, Y. J. Oyang, H. C. Huang, and H. F. Juan. 2013. MicroRNA-Regulated Protein-Protein Interaction Networks and Their Functions in Breast Cancer. Int J Mol Sci 14:11560-11606.

47. Chen, C., D. A. Ridzon, A. J. Broomer, Z. Zhou, D. H. Lee, J. T. Nguyen, M. Barbisin, N. L. Xu, V. R. Mahuvakar, M. R. Andersen, K. Q. Lao, K. J. Livak, and K. J. Guegler. 2005. Real-time quantification of microRNAs by stem-loop RT-PCR. Nucleic Acids Res 33:e179.

48. Iorio, M. V., M. Ferracin, C. G. Liu, A. Veronese, R. Spizzo, S. Sabbioni, E. Magri, M. Pedriali, M. Fabbri, M. Campiglio, S. Menard, J. P. Palazzo, A. Rosenberg, P. Musiani, S. Volinia, I. Nenci, G. A. Calin, P. Querzoli, M. Negrini, and C. M. Croce. 2005. MicroRNA gene expression deregulation in human breast cancer. Cancer Res 65:70657070 .

49. Kondo, N., T. Toyama, H. Sugiura, Y. Fujii, and H. Yamashita. 2008. miR-206 Expression is down-regulated in estrogen receptor alpha-positive human breast cancer. Cancer Res 68:5004-5008.

50. Herschkowitz, J. I., K. Simin, V. J. Weigman, I. Mikaelian, J. Usary, Z. Hu, K. E. Rasmussen, L. P. Jones, S. Assefnia, S. Chandrasekharan, M. G. Backlund, Y. Yin, A. I. Khramtsov, R. Bastein, J. Quackenbush, R. I. Glazer, P. H. Brown, J. E. Green, L. Kopelovich, P. A. Furth, J. P. Palazzo, O. I. Olopade, P. S. Bernard, G. A. Churchill, D. T. Van, and C. M. Perou. 2007. Identification of conserved gene expression features 
between murine mammary carcinoma models and human breast tumors. Genome Biol. 8:R76.

51. Honeth, G., P. O. Bendahl, M. Ringner, L. H. Saal, S. K. Gruvberger-Saal, K. Lovgren, D. Grabau, M. Ferno, A. Borg, and C. Hegardt. 2008. The CD44+/CD24- phenotype is enriched in basal-like breast tumors. Breast Cancer Res 10:R53.

52. Creighton, C. J., X. Li, M. Landis, J. M. Dixon, V. M. Neumeister, A. Sjolund, D. L. Rimm, H. Wong, A. Rodriguez, J. I. Herschkowitz, C. Fan, X. Zhang, X. He, A. Pavlick, M. C. Gutierrez, L. Renshaw, A. A. Larionov, D. Faratian, S. G. Hilsenbeck, C. M. Perou, M. T. Lewis, J. M. Rosen, and J. C. Chang. 2009. Residual breast cancers after conventional therapy display mesenchymal as well as tumor-initiating features. Proc Natl Acad Sci U S A 106:13820-13825.

53. Perou, C. M. 2010. Molecular stratification of triple-negative breast cancers. Oncologist. 15 Suppl 5:39-48.

54. Ibarra, I., Y. Erlich, S. K. Muthuswamy, R. Sachidanandam, and G. J. Hannon. 2007. A role for microRNAs in maintenance of mouse mammary epithelial progenitor cells. Genes Dev 21:3238-3243.

55. Charafe-Jauffret, E., C. Ginestier, F. Iovino, J. Wicinski, N. Cervera, P. Finetti, M. H. Hur, M. E. Diebel, F. Monville, J. Dutcher, M. Brown, P. Viens, L. Xerri, F. Bertucci, G. Stassi, G. Dontu, D. Birnbaum, and M. S. Wicha. 2009. Breast cancer cell lines contain functional cancer stem cells with metastatic capacity and a distinct molecular signature. Cancer Res 69:1302-1313.

56. Luo, M., H. Fan, T. Nagy, H. Wei, C. Wang, S. Liu, M. S. Wicha, and J. L. Guan. 2009. Mammary epithelial-specific ablation of the focal adhesion kinase suppresses mammary tumorigenesis by affecting mammary cancer stem/progenitor cells. Cancer Res 69:466474.

57. Fillmore, C. M. and C. Kuperwasser. 2008. Human breast cancer cell lines contain stemlike cells that self-renew, give rise to phenotypically diverse progeny and survive chemotherapy. Breast Cancer Res 10:R25.

58. Park, S. Y., H. E. Lee, H. Li, M. Shipitsin, R. Gelman, and K. Polyak. 2010. Heterogeneity for stem cell-related markers according to tumor subtype and histologic stage in breast cancer. Clin Cancer Res 16:876-887.

59. Giatromanolaki, A., E. Sivridis, A. Fiska, and M. I. Koukourakis. 2011. The CD44+/CD24- phenotype relates to 'triple-negative' state and unfavorable prognosis in breast cancer patients. Med Oncol 28:745-752. 
60. Lu, T. P., C. Y. Lee, M. H. Tsai, Y. C. Chiu, C. K. Hsiao, L. C. Lai, and E. Y. Chuang. 2012. miRSystem: an integrated system for characterizing enriched functions and pathways of microRNA targets. PLoS One 7:e42390.

61. Afonja, O., D. Juste, S. Das, S. Matsuhashi, and H. H. Samuels. 2004. Induction of PDCD4 tumor suppressor gene expression by RAR agonists, antiestrogen and HER-2/neu antagonist in breast cancer cells. Evidence for a role in apoptosis. Oncogene 23:81358145 .

62. Lankat-Buttgereit, B. and R. Goke. 2009. The tumour suppressor Pdcd4: recent advances in the elucidation of function and regulation. Biol Cell 101:309-317.

63. Santhanam, A. N., A. R. Baker, G. Hegamyer, D. A. Kirschmann, and N. H. Colburn. 2010. Pdcd4 repression of lysyl oxidase inhibits hypoxia-induced breast cancer cell invasion. Oncogene 29:3921-3932.

64. Vlachos, I. S., N. Kostoulas, T. Vergoulis, G. Georgakilas, M. Reczko, M. Maragkakis, M. D. Paraskevopoulou, K. Prionidis, T. Dalamagas, and A. G. Hatzigeorgiou. 2012. DIANA miRPath v.2.0: investigating the combinatorial effect of microRNAs in pathways. Nucleic Acids Res 40:W498-W504.

65. Kim, H. K., Y. S. Lee, U. Sivaprasad, A. Malhotra, and A. Dutta. 2006. Muscle-specific microRNA miR-206 promotes muscle differentiation. J Cell Biol. 174:677-687.

66. Laird, D. W., P. Fistouris, G. Batist, L. Alpert, H. T. Huynh, G. D. Carystinos, and M. A. aoui-Jamali. 1999. Deficiency of connexin43 gap junctions is an independent marker for breast tumors. Cancer Res 59:4104-4110.

67. Qin, H., Q. Shao, H. Curtis, J. Galipeau, D. J. Belliveau, T. Wang, M. A. aoui-Jamali, and D. W. Laird. 2002. Retroviral delivery of connexin genes to human breast tumor cells inhibits in vivo tumor growth by a mechanism that is independent of significant gap junctional intercellular communication. J Biol. Chem. 277:29132-29138.

68. Ponti, D., A. Costa, N. Zaffaroni, G. Pratesi, G. Petrangolini, D. Coradini, S. Pilotti, M. A. Pierotti, and M. G. Daidone. 2005. Isolation and in vitro propagation of tumorigenic breast cancer cells with stem/progenitor cell properties. Cancer Res 65:5506-5511.

69. Shao, Q., H. Wang, E. McLachlan, G. I. Veitch, and D. W. Laird. 2005. Down-regulation of Cx43 by retroviral delivery of small interfering RNA promotes an aggressive breast cancer cell phenotype. Cancer Res 65:2705-2711. 
70. McLachlan, E., Q. Shao, and D. W. Laird. 2007. Connexins and gap junctions in mammary gland development and breast cancer progression. J Membr. Biol. 218:107121.

71. Li, Z., Z. Zhou, D. R. Welch, and H. J. Donahue. 2008. Expressing connexin 43 in breast cancer cells reduces their metastasis to lungs. Clin Exp. Metastasis 25:893-901.

72. Plante, I., M. K. Stewart, K. Barr, A. L. Allan, and D. W. Laird. 2011. Cx43 suppresses mammary tumor metastasis to the lung in a $\mathrm{Cx} 43$ mutant mouse model of human disease. Oncogene 30:1681-1692.

73. Dick, J. E. 2008. Stem cell concepts renew cancer research. Blood 112:4793-4807.

74. Sirnes, S., J. Bruun, M. Kolberg, A. Kjenseth, G. E. Lind, A. Svindland, A. Brech, A. Nesbakken, R. A. Lothe, E. Leithe, and E. Rivedal. 2012. Connexin43 acts as a colorectal cancer tumor suppressor and predicts disease outcome. Int J Cancer 131:570-581.

75. Yu, F., H. Yao, P. Zhu, X. Zhang, Q. Pan, C. Gong, Y. Huang, X. Hu, F. Su, J. Lieberman, and E. Song. 2007. let-7 regulates self renewal and tumorigenicity of breast cancer cells. Cell 131:1109-1123.

76. Shimono, Y., M. Zabala, R. W. Cho, N. Lobo, P. Dalerba, D. Qian, M. Diehn, H. Liu, S. P. Panula, E. Chiao, F. M. Dirbas, G. Somlo, R. A. Pera, K. Lao, and M. F. Clarke. 2009. Downregulation of miRNA-200c links breast cancer stem cells with normal stem cells. Cell 138:592-603.

77. Hwang-Verslues, W. W., P. H. Chang, P. C. Wei, C. Y. Yang, C. K. Huang, W. H. Kuo, J. Y. Shew, K. J. Chang, E. Y. Lee, and W. H. Lee. 2011. miR-495 is upregulated by E12/E47 in breast cancer stem cells, and promotes oncogenesis and hypoxia resistance via downregulation of E-cadherin and REDD1. Oncogene 30:2463-2474.

78. Deng, L., L. Shang, S. Bai, J. Chen, X. He, R. Martin-Trevino, S. Chen, X. Y. Li, X. Meng, B. Yu, X. Wang, Y. Liu, S. P. McDermott, A. E. Ariazi, C. Ginestier, I. Ibarra, J. Ke, T. Luther, S. G. Clouthier, L. Xu, G. Shan, E. Song, H. Yao, G. J. Hannon, S. J. Weiss, M. S. Wicha, and S. Liu. 2014. MicroRNA100 inhibits self-renewal of breast cancer stem-like cells and breast tumor development. Cancer Res 74:6648-6660.

79. McCarthy, J. J. 2008. MicroRNA-206: the skeletal muscle-specific myomiR. Biochim. Biophys. Acta 1779:682-691.

80. Williams, A. H., G. Valdez, V. Moresi, X. Qi, J. McAnally, J. L. Elliott, R. Bassel-Duby, J. R. Sanes, and E. N. Olson. 2009. MicroRNA-206 delays ALS progression and promotes regeneration of neuromuscular synapses in mice. Science 326:1549-1554. 
81. Cacchiarelli, D., J. Martone, E. Girardi, M. Cesana, T. Incitti, M. Morlando, C. Nicoletti, T. Santini, O. Sthandier, L. Barberi, A. Auricchio, A. Musaro, and I. Bozzoni. 2010. MicroRNAs involved in molecular circuitries relevant for the Duchenne muscular dystrophy pathogenesis are controlled by the dystrophin/nNOS pathway. Cell Metab 12:341-351.

82. Dey, B. K., J. Gagan, and A. Dutta. 2011. miR-206 and -486 induce myoblast differentiation by downregulating Pax7. Mol Cell Biol 31:203-214.

83. Liu, N., A. H. Williams, J. M. Maxeiner, S. Bezprozvannaya, J. M. Shelton, J. A. Richardson, R. Bassel-Duby, and E. N. Olson. 2012. microRNA-206 promotes skeletal muscle regeneration and delays progression of Duchenne muscular dystrophy in mice. $\mathbf{J}$ Clin Invest 122:2054-2065.

84. Tavazoie, S. F., C. Alarcon, T. Oskarsson, D. Padua, Q. Wang, P. D. Bos, W. L. Gerald, and J. Massague. 2008. Endogenous human microRNAs that suppress breast cancer metastasis. Nature 451:147-152.

85. Ma, L., F. Reinhardt, E. Pan, J. Soutschek, B. Bhat, E. G. Marcusson, J. TeruyaFeldstein, G. W. Bell, and R. A. Weinberg. 2010. Therapeutic silencing of miR-10b inhibits metastasis in a mouse mammary tumor model. Nat. Biotechnol. 28:341-347.

86. Kasinski, A. L. and F. J. Slack. 2011. Epigenetics and genetics. MicroRNAs en route to the clinic: progress in validating and targeting microRNAs for cancer therapy. Nat Rev Cancer 11:849-864.

87. Kasinski, A. L. and F. J. Slack. 2012. Arresting the Culprit: Targeted Antagomir Delivery to Sequester Oncogenic miR-221 in HCC. Mol Ther Nucleic Acids 1:e12.

88. Babar, I. A., C. J. Cheng, C. J. Booth, X. Liang, J. B. Weidhaas, W. M. Saltzman, and F. J. Slack. 2012. Nanoparticle-based therapy in an in vivo microRNA-155 (miR-155)dependent mouse model of lymphoma. Proc Natl Acad Sci U S A 109:E1695-E1704.

89. Janssen, H. L., H. W. Reesink, E. J. Lawitz, S. Zeuzem, M. Rodriguez-Torres, K. Patel, A. J. van der Meer, A. K. Patick, A. Chen, Y. Zhou, R. Persson, B. D. King, S. Kauppinen, A. A. Levin, and M. R. Hodges. 2013. Treatment of HCV infection by targeting microRNA. N Engl J Med 368:1685-1694. 


\section{FIGURE LEGENDS}

Figure 1. KLF4 and miR-206 are selectively expressed in basal-like mammary cancers and in the MaCSC population.

(A) miR-206 levels were analyzed by microarray in 98 primary human breast tumors (46). The Gene Expression Omnibus (GEO) accession number is indicated. (Columns, mean; bars, SEM; Hist. grade, histologic grade.)

(B) miR-206 levels were analyzed by microarray in 42 mammary tumors from geneticallyengineered mouse models (GEMMs) (45). The GEO accession number is indicated.

(C) Klf4 and miR-206 expression was evaluated in normal mammary tissues from FVB/N mice and in primary tumors arising in the MMTV-Neu and C3(1)/TAg GEMMs. RNA levels were determined by qRT-PCR.

(D) MaCSCs were isolated from MDA-MB-231 cells by sorting using PROCR (P) and ESA (E) as described (12). Transcript levels were analyzed in $\mathrm{P}^{+} / \mathrm{E}^{+}$and $\mathrm{P}^{-} / \mathrm{E}^{-}$cells.

(E) MaCSCs were isolated from SUM159PT cells and analyzed similarly as described above for MDA-MB-231 cells (57). For these cells the $\mathrm{P}^{+}$profile was used as a surrogate for $\mathrm{CD}^{+} 4^{+} / \mathrm{CD} 24^{-}$ (10). $(*, \mathrm{P}<0.05 ; * *, \mathrm{P}<0.01 ; * * *, \mathrm{P}<0.001)$ 
Figure 2. KLF4 and miR-206 are enriched in $\mathrm{ALDH}^{\mathrm{High}}$ MaCSCs derived from human patient-derived xenografts (PDXs) and the C3(1)/TAg GEMM.

(A) KLF4 and miR-206 levels were measured in MaCSCs purified in replicate fashion from four cases of human mammary carcinoma passaged as xenografts in mice (PDXs). Purified lineagenegative ( Lin $^{-}$) cells were sorted based on aldehyde dehydrogenase (ALDH) activity. Fluorescence was analyzed in the presence of the ALDH substrate BAAA and in presence/absence of the ALDH inhibitor DEAB. The number of xenografted tumors that were analyzed for each case is indicated below the column data (bars, SEM).

(B) Lin $^{-}$cells were isolated from the spontaneous mammary tumors arising in C3(1)/TAg females $(\mathrm{N}=3)$. Cells from each animal were grown as either adherent monolayers (2D) or in suspension (3D) for 7-10 days. Photomicrographs (left) depict the morphology of cultured cells. RNA was extracted from 2D or 3D cell cultures and Klf4 and miR-206 levels were determined.

(C) Klf4 and miR-206 levels were measured in the ALDH ${ }^{\mathrm{High}}$ tumor cells isolated from C3(1)/TAg animals $\left(\mathrm{N}=3\right.$, see panel B). ALDH ${ }^{\mathrm{High}}$ tumor cells derived from other GEMMs of mammary cancer have been demonstrated to be enriched for TICs $(54,56)$. 


\section{Figure 3. KLF4 and miR-206 promote MaCSC abundance}

(A) MDA-MB-231 cells were transduced with lentiviral vectors expressing KLF4 shRNAs or a non-targeting control $(\mathrm{Ctl})$. KLF4 protein expression was analyzed by immunoblot (left upper panel). $\beta$-actin served as a loading control. miR-206 levels were measured by stem loop qRTPCR (left lower panel). The cell surface marker profile of the transduced cells was analyzed by flow cytometry (representative scatter plot, middle panel; column data, right panel) $(\mathrm{N}=3$; bars, SEM).

(B) MDA-MB-231 cells were transduced with a retroviral vector encoding KLF4 or empty vector (Ctl). KLF4 and miR-206 levels were analyzed in these cells (left panels) and the MaCSC abundance was determined by flow cytometry.

(C) MDA-MB-231 cells were transfected with either miR-206-mimic or control oligonucleotides (Ctl) and then analyzed as in the previous panels.

(D) SUM159PT cells were transfected with the indicated miR-mimics and then analyzed as in the previous panels.

(E) MDA-MB-231 cells were transfected with miR-206-mimic or control. The indicated number of cells were mixed with matrigel (50\% [vol/vol] in DMEM) and injected into NOD/SCIDgamma mice. Tumor initiation was measured at 4 weeks post-injection. 
Figure 4. Endogenous KLF4-miR-206 signaling promotes in vivo tumorigenesis and cell survival.

(A) KLF4-depleted and control MDA-MB-231 cells were orthotopically injected into athymic nude mice. Tumor initiation and tumor size were determined twice per week using digital calipers (right panels; bars, SEM).

(B) MDA-MB-231 cells were transfected with the indicated anti-miRs. Briefly, cells were subjected to sequential transfections in vitro. At 2 days post-transfection the cells were injected into athymic nude mice. Tumor incidence and growth were measured as described above.

(C) Residual transfected cells (see panel B) were directly lysed for immunobot analysis (left panel) or else placed in culture for 2D cell proliferation analysis (right panel, ATPlite; $\mathrm{N}=6$, bars, SD). Post-tf, post-transfection.

(D) TNBC cells were transfected with either anti-miR-206 or anti-miR-Ctl and then deprived of matrix for 24 hours. Anoikis was measured by trypan blue exclusion (left panel, N=3, bars, SD). In parallel, cells were assayed by immunoblot analysis of cleaved poly ADP ribose polymerase (PARP). Cyclohexamide (CHX) treatment served as a positive control for induction of cell death.

(E) Anoikis was measured in KLF4-depleted MDA-MB-231 cells or control cells by trypan blue exclusion $(\mathrm{N}=3$, bars, $\mathrm{SD})$, by flow cytometric analysis of propidium iodide (PI)-stained cells $(\mathrm{N}=3$, bars, $\mathrm{SD})$, and by analysis of cleaved PARP. 


\section{Figure 5. miR-206 suppresses the translation of the tumor suppressor PDCD4.}

(A) PDCD4 levels were determined by immunoblot analysis of the indicated cells.

(B) Alignment of the PDCD4 3'UTR region indicating two potential miR-206 binding sites, WT-A and WT-B. The miR-206 seed sequence is underlined. Mutated miR-206 binding sites in the PDCD4 3' UTR that were utilized in translational reporter assays are indicated (mt206-A and mt206-B).

(C) For analysis of PDCD4 protein translation, MDA-MB-231 cells were co-transfected with reporters in combination with either miR-mimic (left panel) or anti-miR (right panel). The normalized activity of the reporters relative to empty luc vector was analyzed 24 hours posttransfection ( $\mathrm{N}=3$; bars, $\mathrm{SEM})$.

(D) PDCD4 was depleted in the indicated TNBC cells and PDCD4 levels were determined by immunoblot (upper panels). Cells were suspended in 3D culture for 24 hours, and anoikis was measured by flow cytometric analysis of PI stained cells (middle panels; N=3; bars, SEM). Following 4 days of 2D culture, the relative cell number of PDCD4-depleted cells and control cells was determined by the ATPlite assay (N=6; bars, SD).

(E) PDCD4 mRNA and protein expression was analyzed in the indicated subpopulations of TNBC cells. Non-MaCSCs were comprised of the $\mathrm{P}^{+} / \mathrm{E}^{-}$and $\mathrm{P}^{-} / \mathrm{E}^{-}$subgroups. The immunoblot data corresponds to one of the three independent experiments that analyzed mRNA levels $(\mathrm{N}=3$, bars, SEM).

(F) MaCSC abundance was analyzed in PDCD4-depleted TNBC cells and control cells ( $\mathrm{N}=3$; bars, SD). 


\section{Figure 6. KLF4-miR-206 signaling suppresses CX43 in MaCSCs.}

(A) CX43 expression was analyzed in shKLF4 cells and control cells by immunoblot. Similarly, CX43 expression was analyzed in cells transfected with the indicated miR mimic or anti-miR.

(B) Alignment of CX43 3'UTR region, indicating two previously-validated miR-206 binding sites, WT-A and WT-B (38). The miR-206 seed sequence is underlined. The mutation generated in miR-206 binding site $\mathrm{A}$ is indicated (mt206-A).

(C) For analysis of CX43 protein translation, MDA-MB-231 cells were co-transfected with reporters in combination with anti-miR (left panel) or miR-mimic (right panel). The normalized activity of the reporters relative to empty luc vector was analyzed at 24 hours post-transfection.

(D) CX43 expression was assayed in CX43-depleted TNBC cells and control cells (upper panels). Cells were suspended in 3D culture for 24 hours, and anoikis was measured by flow cytometric analysis of PI-stained cells (middle panels, N=3; bars, SEM). Following 4 days of 2D culture, the relative cell number of CX43-depleted cells and control cells was determined by the ATPlite assay (lower panels, $\mathrm{N}=6$; bars, SD).

(E) CX43 mRNA and protein expression was analyzed in the indicated subpopulations of MDAMB-231 cells ( $\mathrm{N}=3$; bars, SEM). Non-MaCSCs were composed of the $\mathrm{P}^{+} / \mathrm{E}^{-}$and $\mathrm{P}^{-} / \mathrm{E}^{-}$subgroups. 


\section{Figure 7. miR-206 promotes chemoresistance in TNBC cells.}

(A and B) TNBC cells were transfected as indicated. At 48 hours post-transfection, cells were treated with the indicated concentrations of paclitaxel or doxorubicin for a duration of 72 hours. Cell viability was determined by the ATPlite assay (N=6; bars, SD).

(C) Schematic of KLF4-miR-206 regulation of PDCD4 and CX43. 


\section{FIGURES}

A

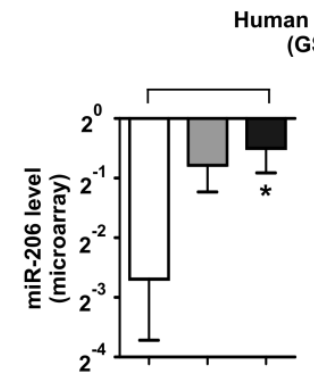

Hist. grade: I II III

(N) (10) (41) (47) n breast tumor

SE45666)

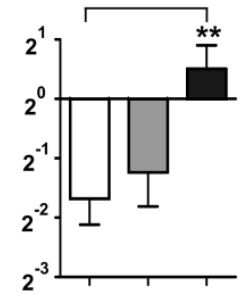

Subtype: $\mathrm{ER}^{+} \mathrm{HER}^{+}{ }^{+} \mathrm{TN}$

(N) (44) (18) (36)
B

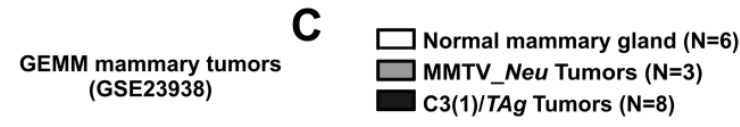

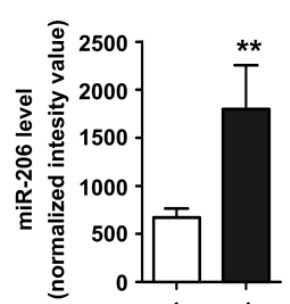

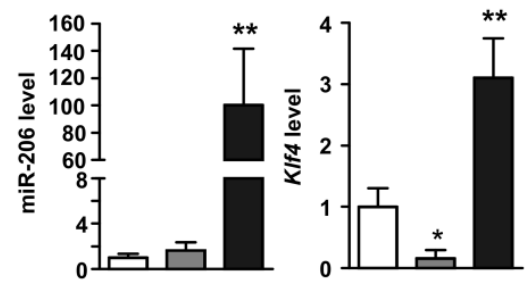

MDA-MB-231

D

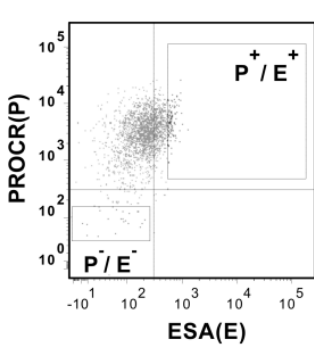

E

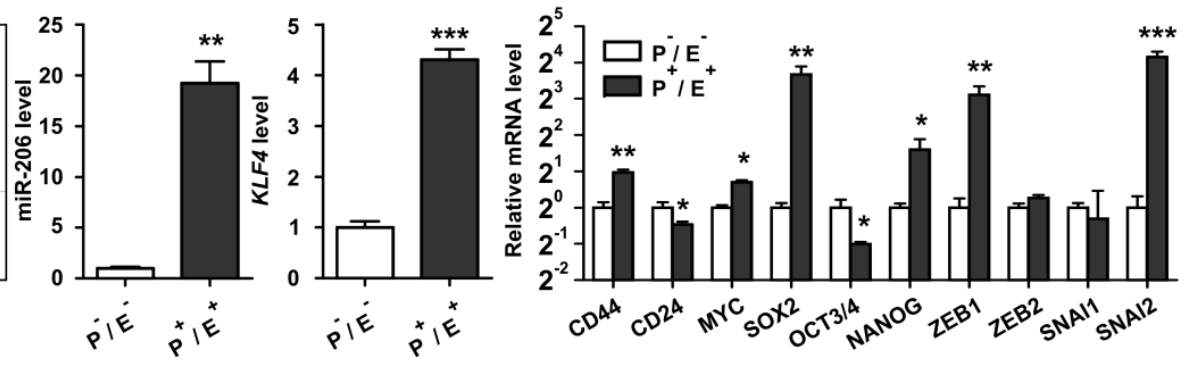

SUM159PT

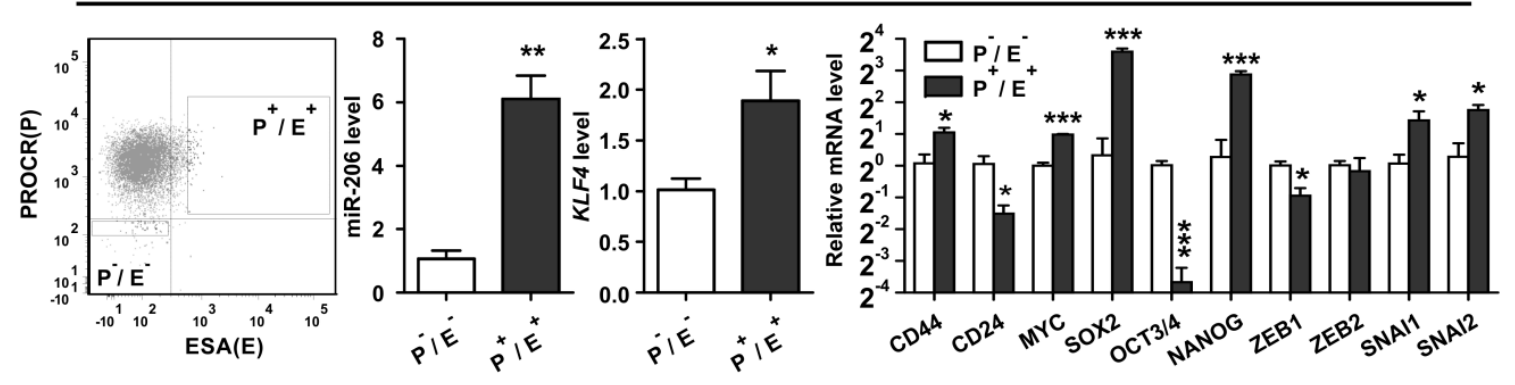

Figure 1 


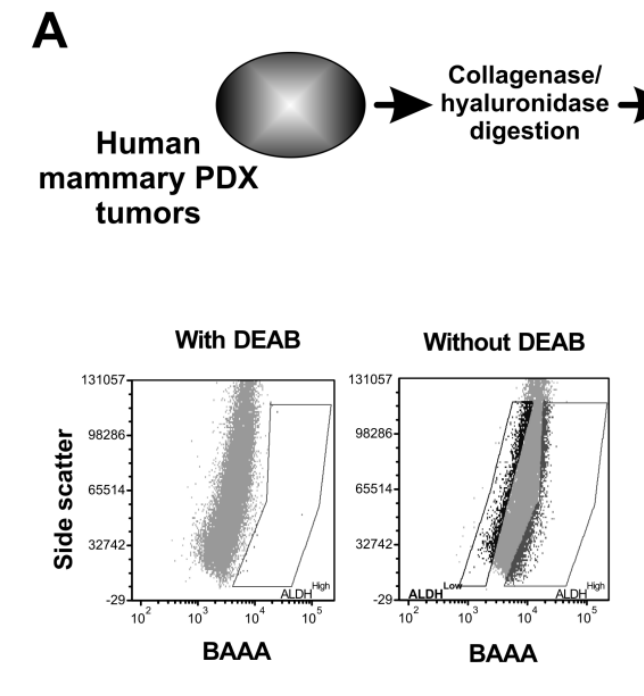

B

Human mammary PDX tumor cells

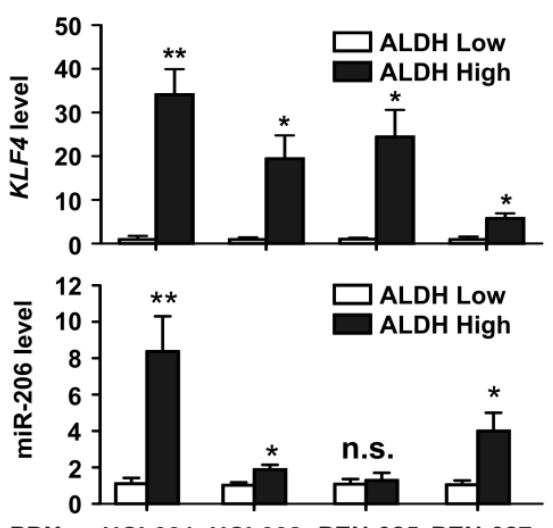

PDXs: HCl-001 HCl-002 PEN-025 PEN-027

$\begin{array}{rllll}\text { N: } & 4 & 3 & 3 & 3 \\ \text { ER: } & - & - & - & + \\ \text { PR: } & - & - & - & + \\ \text { HER2: } & - & - & - & -\end{array}$

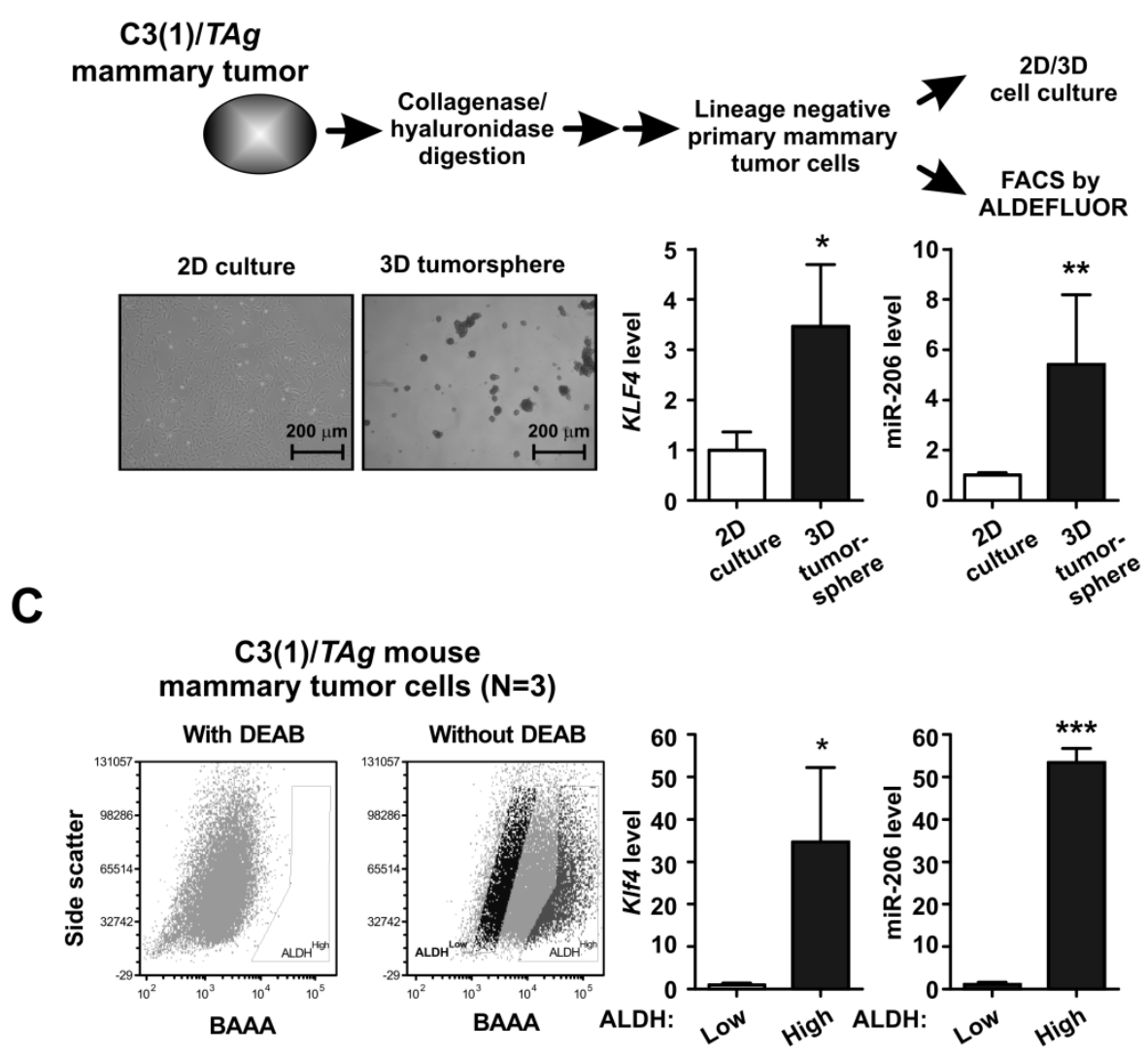

Figure 2 

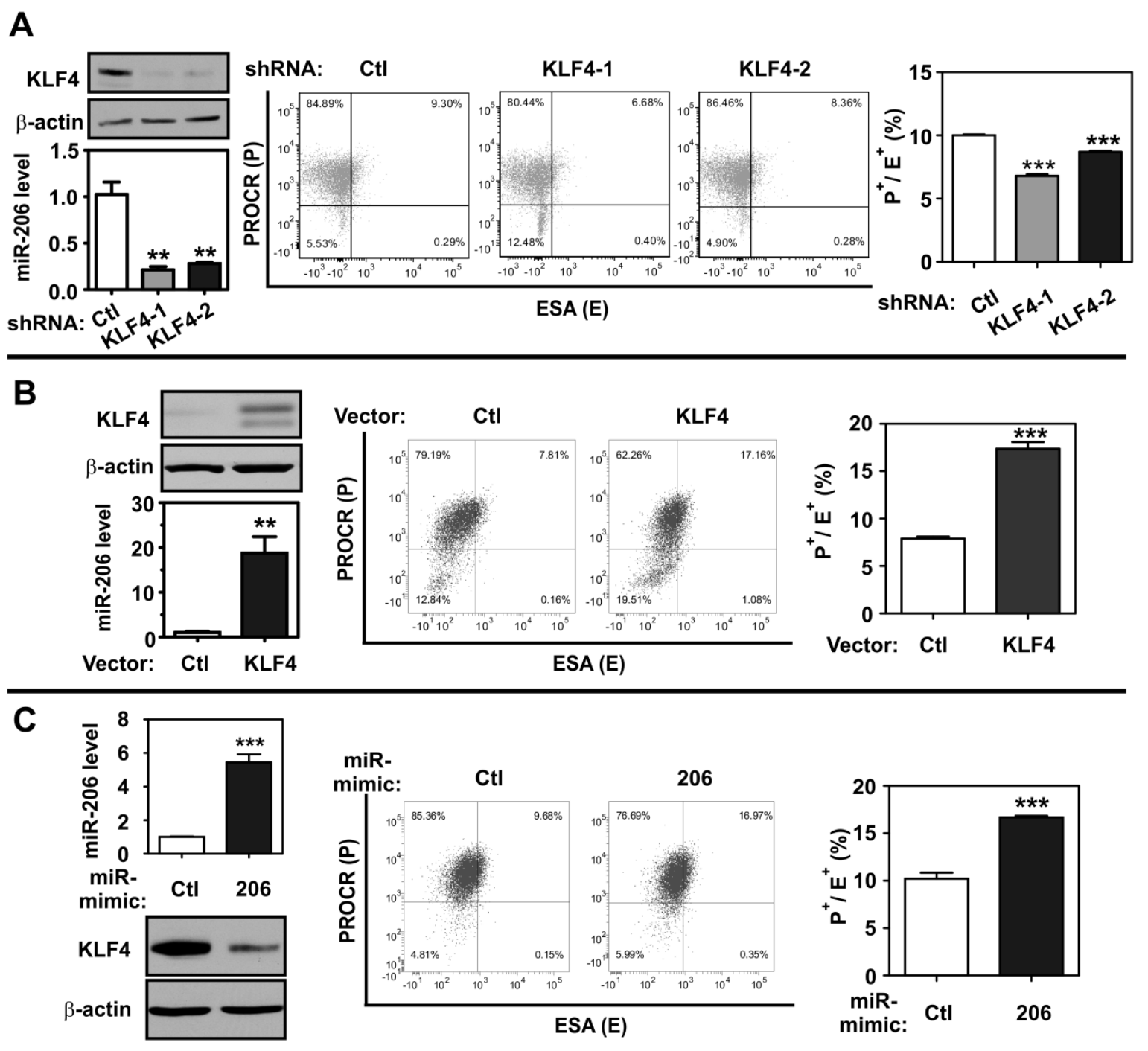

ESA (E)

miR-

mimic: Ctl 206

D

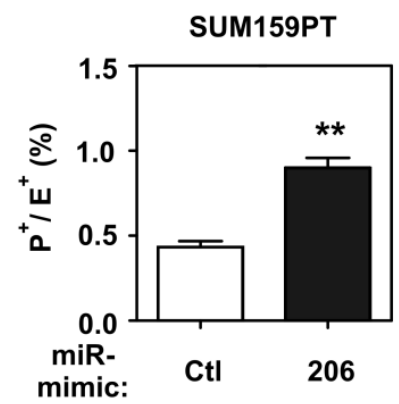

E

\begin{tabular}{ccccc}
\multicolumn{4}{c}{$\begin{array}{c}\text { Tumor incidence of miR-mimic transfected } \\
\text { MDA-MB-231 cells in mice }\end{array}$} \\
\hline & \multicolumn{4}{c}{ Tumor incidence / Cell number } \\
\cline { 2 - 5 } Cell injected & $2 \times 10^{6}$ & $2 \times 10^{5}$ & $2 \times 10^{4}$ & $2 \times 10^{3}$ \\
\hline miR-Ctl-mimic & $1 / 1$ & $6 / 6$ & $4 / 6$ & $0 / 6$ \\
\hline miR-206-mimic & $1 / 1$ & $6 / 6$ & $5 / 6$ & $6 / 6$ \\
\hline
\end{tabular}

Figure 3 
A

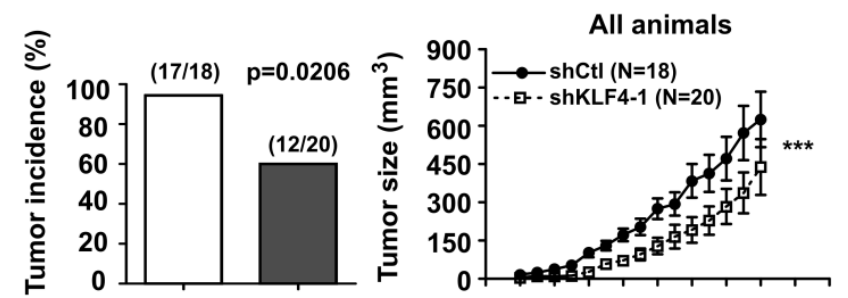

shCtl shKLF4-1 Weeks: $\begin{array}{lllllllllll}0 & 1 & 2 & 3 & 4 & 5 & 6 & 7 & 8 & 9 & 10\end{array}$

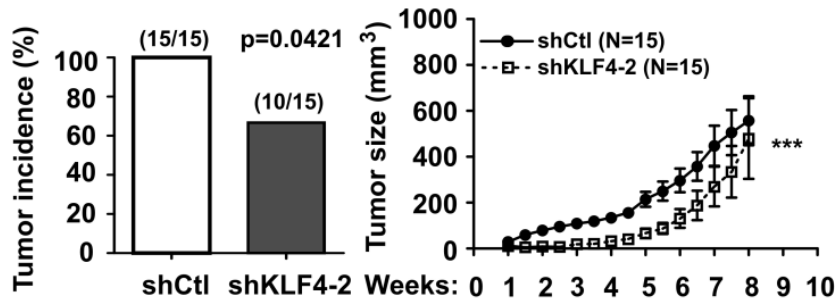

shCtl shKLF4-2 Weeks: $\begin{array}{llllllllllllllllllllll}0 & 1 & 2 & 3 & 4 & 5 & 6 & 7 & 8 & 9 & 10 & \text { Weeks: } 0 & 1 & 2 & 3 & 4 & 5 & 6 & 7 & 8 & 9 & 10\end{array}$

\section{B}

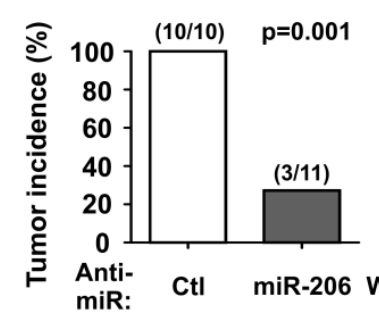

All animals

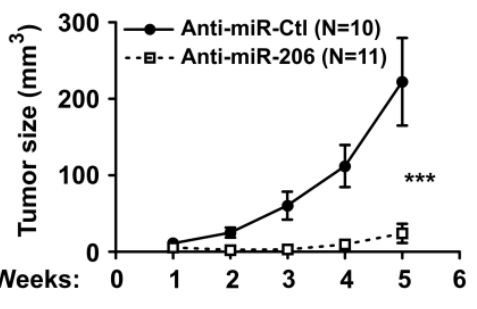

Tumor-positive $(>2.0 \mathrm{~mm})$

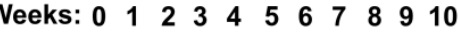

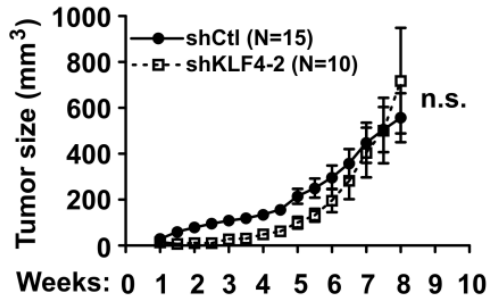

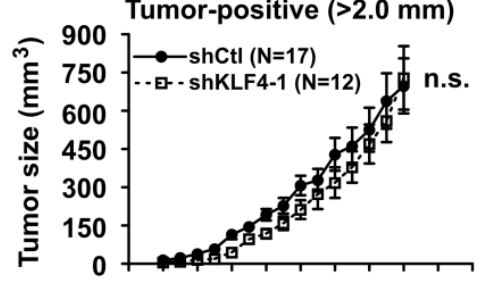

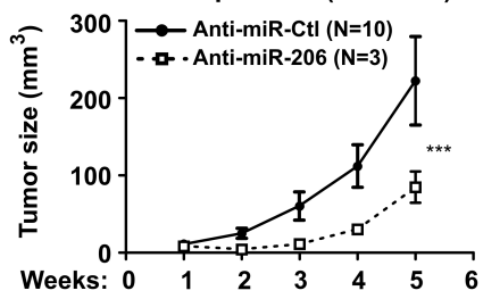

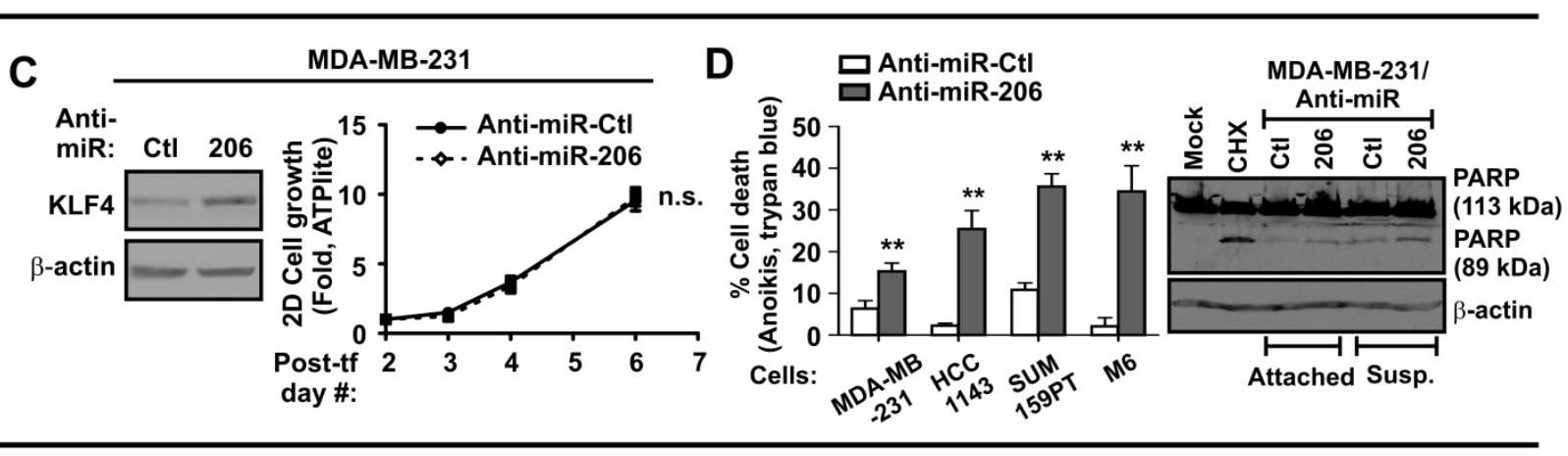

E

MDA-MB-231

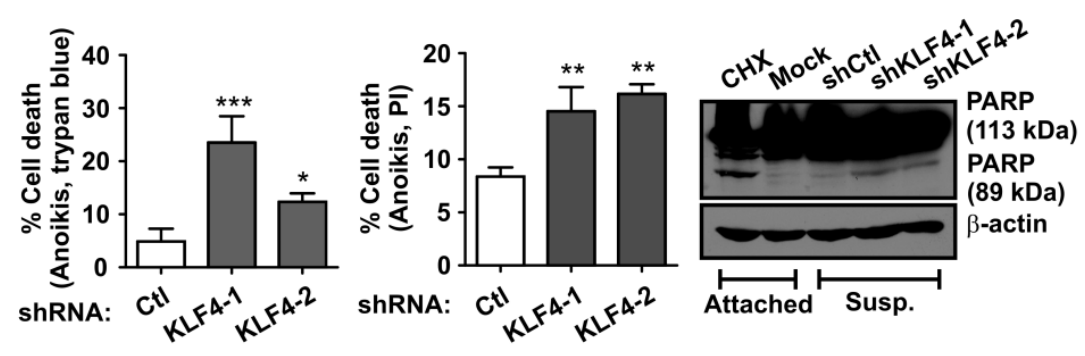

Figure 4 
A

MDA-MB-231

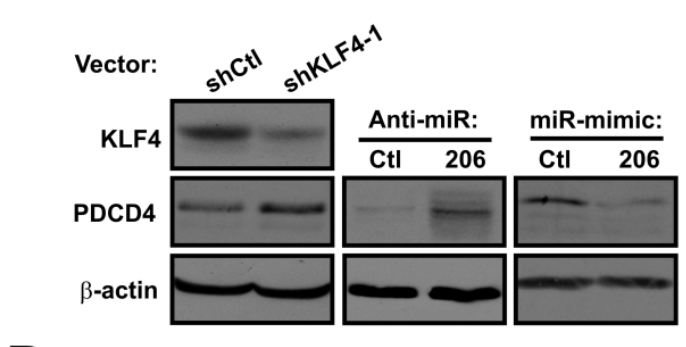

B

Luc-PDCD4 Reporters

PDCD4 : NM_014456

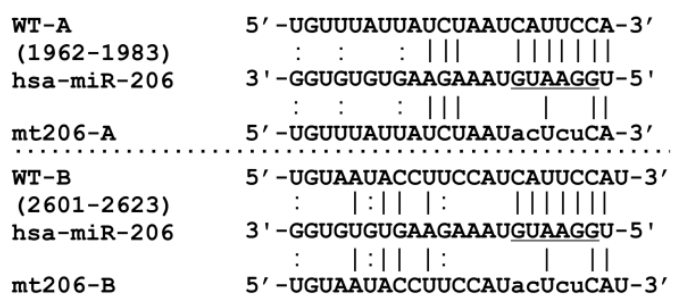

C

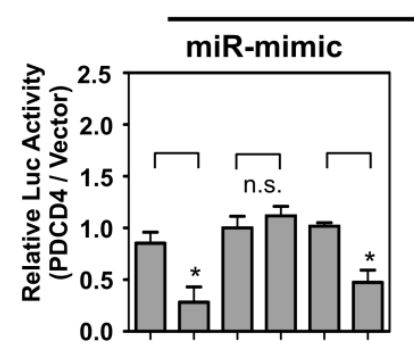

Reporters

WT:

miR mimic

Ctl:
R-206:
MDA-MB-231

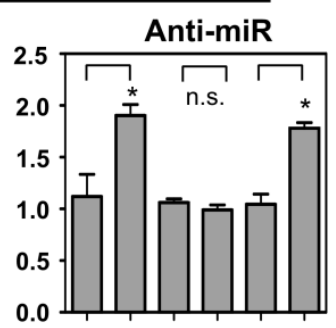

WT: + + - - -

mt206-A: - \pm++-

$\mathrm{mt206-B:}$

Anti-miR

Ctl: +-+-+
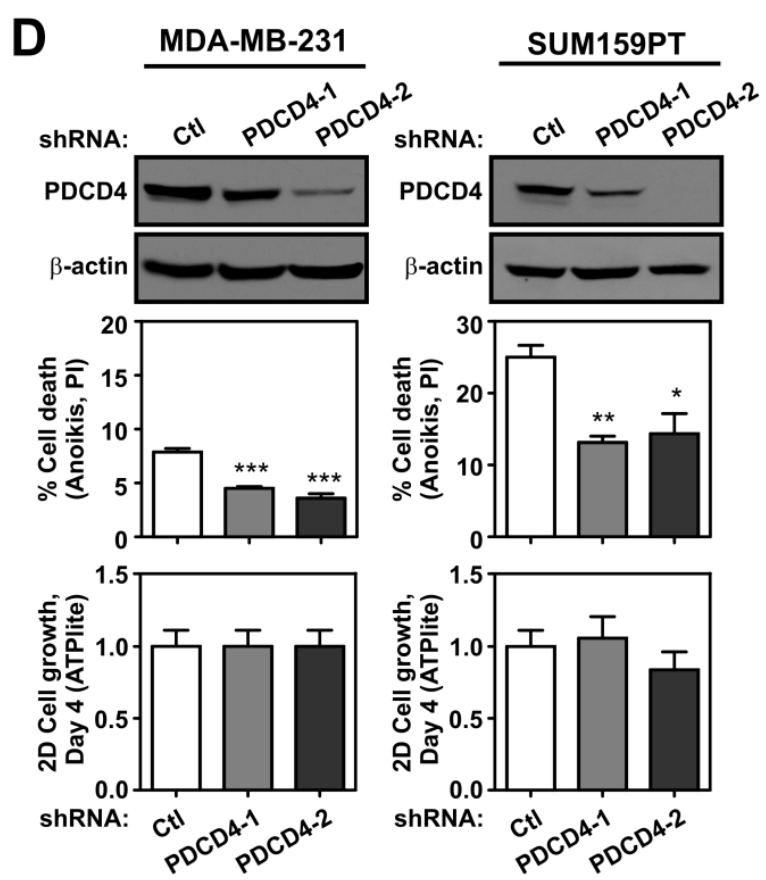

E

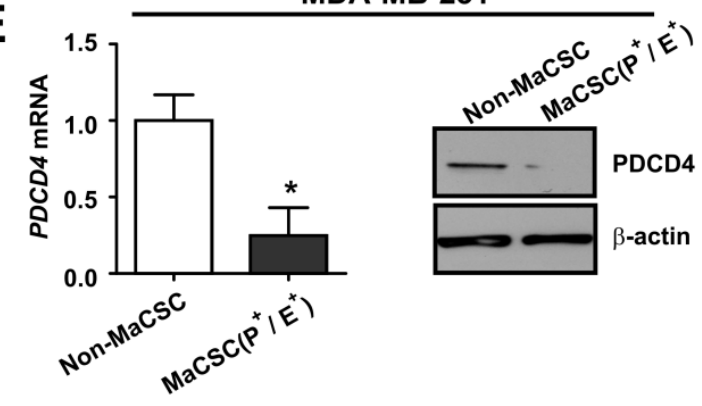

$\mathbf{F}$

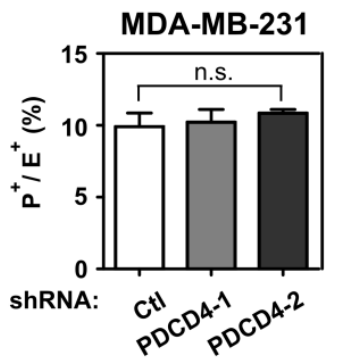

Figure 5 
A

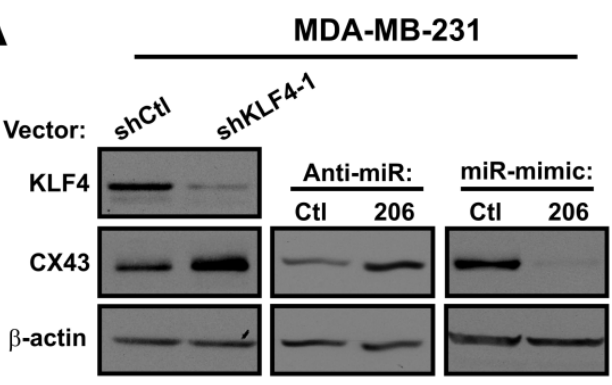

B

Luc-CX43 Reporters

Cx43: NM_000165

WT-A 5'-AAGUCCCUGCUAAAACAUUCCAU-3' (1863-1885) $\quad:: \mid::$ : ||||||||

hsa-miR-206 3'-GGUGUGUGAAGAAAUGUAAGGU-5 ' $::|: \quad: \quad| \quad|| \mid$ mt206-A 5' -AAGUCCCUGCUAAAAacUCuCAU-3' WT-B 5'-UACUAAUUUGUUUGACAUUCCAU-3' (2994-3016) : : | : | : | | | | | || ||| hsa-miR-206 3' -GGUGUGUGAAGAAAUGUAAGGU-5

C

MDA-MB-231

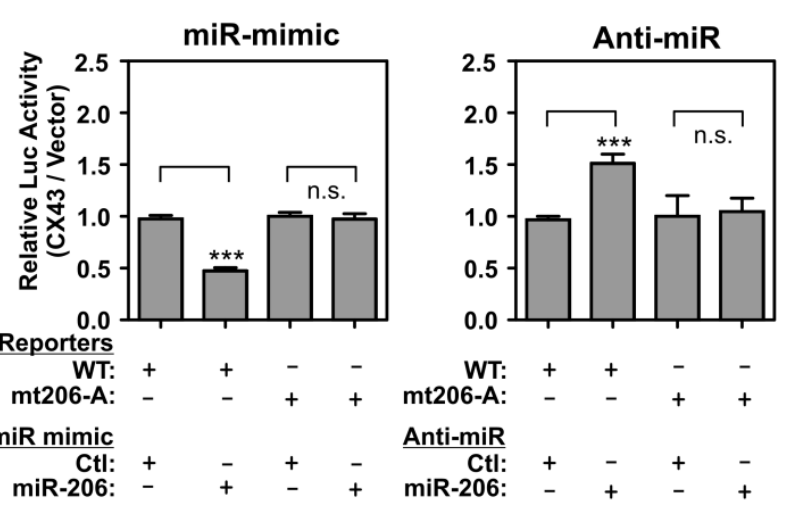

D
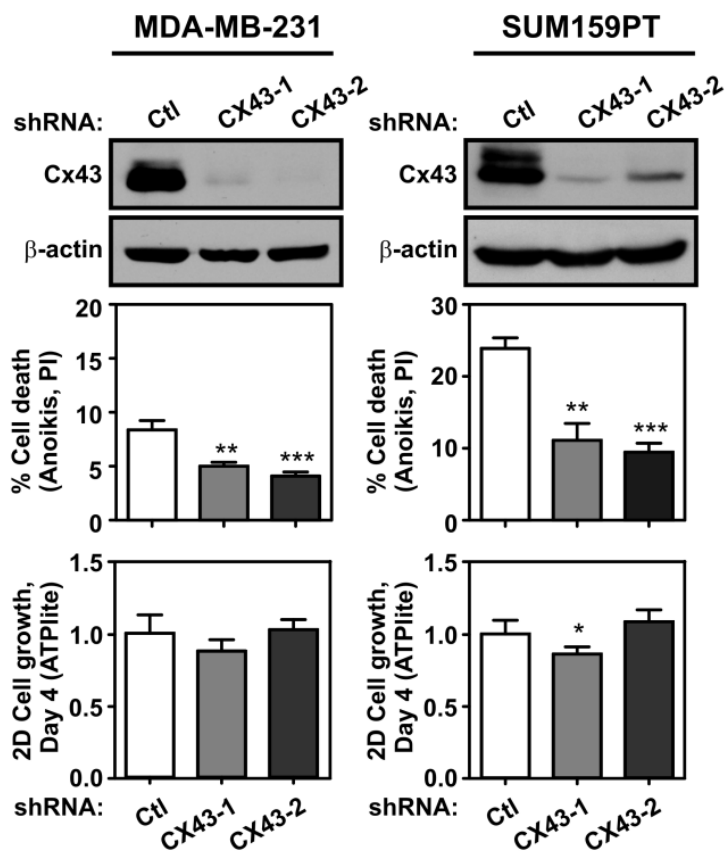

E

MDA-MB-231

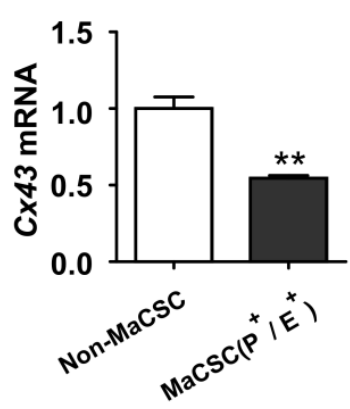

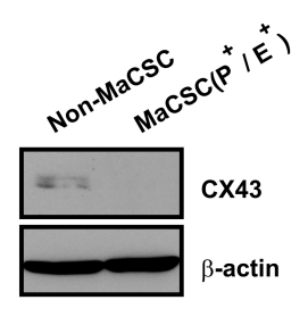

$\times 43$

Figure 6 
A

MDA-MB-231

SUM159PT
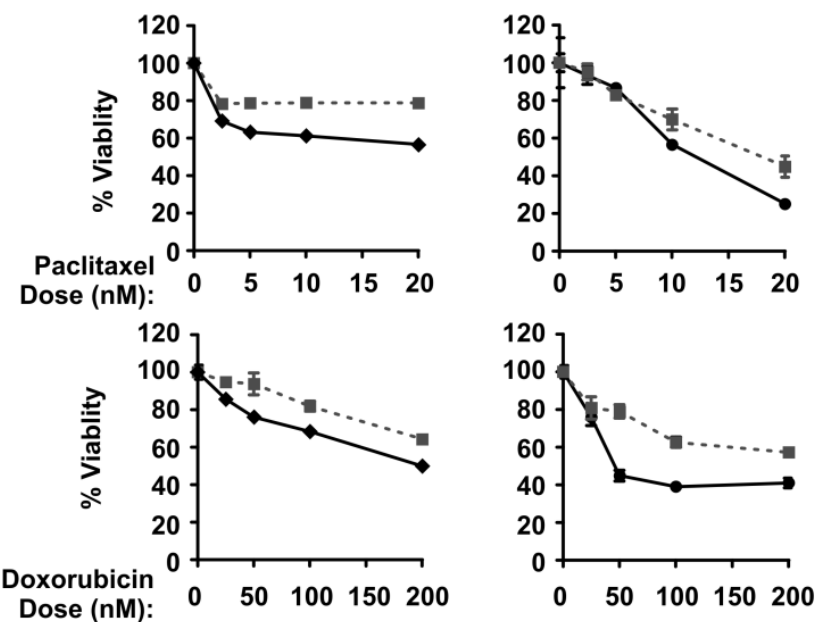

B

\section{$\square$ Anti-miR-Ctl}

Anti-miR-206

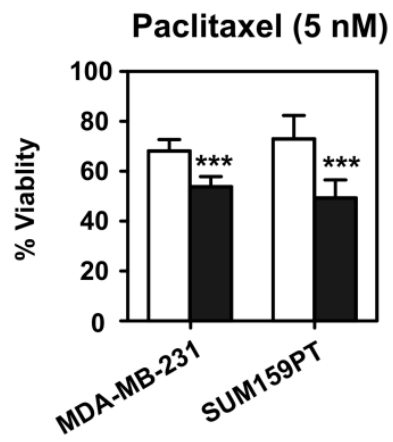

Doxorubicin (50 nM)

C

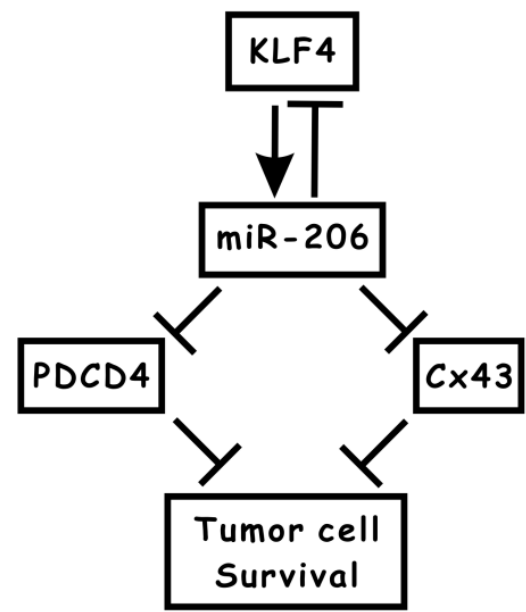

Figure 7 


\section{TABLES (SUPPLEMENT)}

Table 1: Oligonucleotides for PCR mutagenesis

\begin{tabular}{|l|l|l|}
\hline \multicolumn{1}{|c|}{ Gene } & \multicolumn{1}{|c|}{ Sense Primer (5'-3')* } & \multicolumn{1}{c|}{ Antisense Primer (5'-3')* } \\
\hline PDCD4-mt206-A & $\begin{array}{l}\text { GCCATGTTTATTATCTAATacTctCAAGTTT } \\
\text { TGCATTGATGTCTG }\end{array}$ & $\begin{array}{l}\text { CAGACATCAATGCAAAACTTGagAgtATTA } \\
\text { GATAATAAACATGGC }\end{array}$ \\
\hline PDCD4-mt206-B & $\begin{array}{l}\text { AATATGTAATACCTTCCATacTctCATCATC } \\
\text { CTTAAATTCTGTTA }\end{array}$ & $\begin{array}{l}\text { TAACAGAATTTAAGGATGATGagAgtATGG } \\
\text { AAGGTATTACATATT }\end{array}$ \\
\hline CX43-mt206-A & $\begin{array}{l}\text { CCTTAAGTCCCTGCTAAAAacTctCATTGT } \\
\text { TAAAATTTGCACTTT }\end{array}$ & $\begin{array}{l}\text { AAAGTGCAAATTTTAACAATGagAgtTTTT } \\
\text { AGCAGGGACTTAAGG }\end{array}$ \\
\hline
\end{tabular}

*Lower case showed mutated-nucleotide. 
Table 2: Oligonucleotides for real-time quantitative PCR analysis

\begin{tabular}{|l|l|l|}
\hline \multicolumn{1}{|c|}{ Gene } & \multicolumn{1}{c|}{ Sense Primer (5'-3') } & \multicolumn{1}{c|}{ Antisense Primer (5'-3') } \\
\hline KLF4 & AGAGTTCCCATCTCAAGGCA & GTCAGTTCATCTGAGCGGG \\
\hline CD44 & TGCCGCTTTGCAGGTGTAT & GGCCTCCGTCCGAGAGA \\
\hline CD24 & AAACAACAACTGGAACTTCAAGTAACT & GGTGGTGGCATTAGTTGGATTT \\
\hline c-MYC & CGACGAGACCTTCATCAAAA & TGCTGTCGTTGAGAGGGTAG \\
\hline SOX2 & AACCCCAAGATGCACAACTC & GCTTAGCCTCGTCGATGAAC \\
\hline OCT3/4 & GAAGCAGAAGAGGATCACCTTG & TTCTTAAGGCTGAGCTGCAAG \\
\hline NANOG & AATACCTCAGCCTCCAGCAGATG & TGCGTCACACCATTGCTATTCTTC \\
\hline ZEB1 & GCACAACCAAGTGCAGAAGA & CATTTGCAGATTGAGGCTGA \\
\hline ZEB2 & GCCATCTGATCCGCTCTTATC & ACCTGTGTCCACTACATTGTC \\
\hline SNAI1 & TCTGAGTGGGTCTGGAGGTG & CTCTAGGCCCTGGCTGCTAC \\
\hline SNAI2 & AGCATTTCAACGCCTCCA & GGATCTCTGGTTGTGGTATGAC \\
\hline CX43 & AGGAGTTCAATCACTTGGCG & GAGTTTGCCTAAGGCGCTC \\
\hline PDCD4 & TGCAAGCGAAATTAAGGGAA & TCCTCAGTCCCAGCATTTTC \\
\hline B2M & TGCTCGCGCTACTCTCTCTTT & TCTGCTGGATGACGTGAGTAAAC \\
\hline RPLP0 & GGACCCGAGAAGACCTCCTT & GCACATCACTCAGAATTTCAATGG \\
\hline
\end{tabular}




\title{
CHAPTER 4
}

\section{Krüppel-like Factor 4 (KLF4) promotes the expression of platelet-} derived growth factor receptor beta polypeptide (PDGFRß) and resistance to MEK 1/2 inhibition in triple-negative breast cancers (TNBCs)

\author{
Sriganesh B. Sharma ${ }^{\mathbf{1 , 2}}$, Mark K. Farrugia ${ }^{1,2}$, Phillip M. Pifer ${ }^{1,2}$, Chen-Chung \\ $\operatorname{Lin}^{1,2}$, J. Michael Ruppert ${ }^{1,3, \#}$
}

${ }^{1}$ Department of Biochemistry, ${ }^{2}$ Program in Cancer Cell Biology, and ${ }^{3}$ The Mary Babb Randolph Cancer Center, West Virginia University, Morgantown, WV, 26506

${ }^{\#}$ To whom correspondence should be addressed: J. Michael Ruppert, Department of Biochemistry and The Mary Babb Randolph Cancer Center, Morgantown, West Virginia, 26506. Email: mruppert@hsc.wvu.edu

Running Title: KLF4-PDGFR $\beta$ signaling promotes MEK 1/2 inhibitor resistance

Keywords: KLF4, PDGFR $\beta$, MEK 1/2 inhibitor, Breast Cancer, RAS, ERK, Drug Resistance

Note: This manuscript is in the process of submission to The Journal of Biological Chemistry 


\section{CAPSUle}

Background: RAS-ERK signaling is critical in triple-negative breast cancers (TNBCs) but therapeutic targeting of this pathway is hindered by cellular resistance.

Results: KLF4 directly promotes PDGFR $\beta$ expression and TNBC resistance to RAS-ERK pathway inhibition.

Conclusion: KLF4 is a critical MEK 1/2 inhibitor resistance factor.

Significance: Coordinate targeting of KLF4 and RAS-ERK signaling may be an effective therapeutic strategy for TNBCs. 


\section{ABSTRACT}

RAS-Extracellular signal regulated kinase (ERK) signaling is critical for development and progression of many cancers, including triple-negative breast cancers (TNBCs). However, therapeutic targeting of this pathway using MEK 1/2 inhibitors has been largely unsuccessful. Pathway inhibition results in dynamic reprogramming of cell signaling and induction of multiple receptor tyrosine kinases (RTKs), notably platelet-derived growth factor receptor beta polypeptide (PDGFR $\beta$ ). These processes enable cellular escape from MEK 1/2 inhibition by circumventing blocked signaling, restoring cell proliferation, and contribute to therapeutic failure. We previously identified the zinc finger pluripotency factor, Krüppel-like factor 4 (KLF4), as a potent positive regulator of steady-state RAS-ERK signaling and cellular resistance to chemotherapy and anti-HER2 therapies. In the present study, we observed that KLF4 directly promotes the expression of PDGFR $\beta$ in human and murine models of TNBC. KLF4, however, did not appear to regulate the levels of cMYC protein, which represses PDGFRB transcription and is stabilized by RAS-ERK signaling. Instead, KLF4 opposed cMYC mediated repression of PDGFRB and was required for the induction of PDGFR $\beta$ following MEK 1/2 inhibition and cMYC knockdown. Consequently, depletion of KLF4 sensitized TNBC cells to MEK 1/2 inhibition and enforced expression of PDGFR $\beta$ in KLF4 deficient cells restored cellular resistance to pathway inhibition. Interestingly, both KLF4 and PDGFR $\beta$ were dispensable for the reemergence of RAS-ERK signaling following pathway inhibition. These results identify KLF4PDGFR $\beta$ signaling as a critical participant in promoting MEK 1/2 inhibitor resistance and provide rationale for concurrent anti-KLF4 therapies with pharmacological MEK 1/2 inhibition in TNBC. 


\section{INTRODUCTION}

Signaling through the RAS-Extracellular signal regulated kinase (ERK) pathway is crucial for the initiation and progression of many cancers, and therapeutic inhibition of this signaling is of clinical significance (1-6). Despite the successful development of potent pharmacological inhibitors targeting major signaling pathways, including the RAS-ERK pathway (e.g., BRAF and MEK 1/2 inhibitors), the effective use of these agents in the treatment of cancers is hampered by the development of therapeutic resistance by different mechanisms (611). Acute loss of RAS-ERK pathway activity results in adaptive changes in the expression and activation of the kinome $(11,12)$. MEK 1/2 inhibition disrupts ERK dependent negative feedback regulation of the activity of positive pathway regulators including MEK1 and BRAF $(11,13)$. Furthermore, tumor cells can acquire of somatic mutations in $N R A S, M E K 2$, or $A K T 1$ and counteract sustained inhibited RAS-ERK signaling (14-16). Finally, the induction of multiple receptor tyrosine kinases (RTKs) and the activation of alternative signaling pathways (e.g., phosphoinositide 3-kinase (PI3-K-AKT) can compensate for the inhibited RAS-ERK signaling (11,14,17-21). Either singly or in combination, these adaptive changes ultimately circumvent blocked signaling and restore cell proliferation.

Triple-negative breast cancers (TNBCs), tumors that are deficient in estrogen receptor $(\mathrm{ER} \alpha)$, progesterone receptor (PR), and human epidermal growth factor receptor 2 (HER2) gene amplification, display a particular reliance on RAS-ERK signaling $(19,20)$. These tumors, which overlap with the basal-like and claudin-low molecular subtypes of breast cancer, display consistent genetic alterations in RTK and RAS-ERK pathway component genes (22-25). Consistent with these genetic changes, basal-like breast cancers display a high RAS-ERK pathway signature and TNBC cells are sensitive to MEK 1/2 inhibition in experimental models 
$(19,20,25)$. However, similar to other contexts, resistance to pathway inhibition emerges rapidly and involves the restoration of RAS-ERK signaling and induction of multiple RTKs (11).

A prominent feature of kinome reprogramming in response to MEK 1/2 inhibition in TNBC cells is the robust induction of platelet-derived growth factor beta polypeptide (PDGFR $\beta$ ) $(11,12)$. Upregulation of PDGFR $\beta$ occurs in response to the loss of ERK signaling and consequently the destabilization of cMYC protein, which represses PDGFRB transcription (11,26-28). A similar induction of PDGFR $\beta$ in response to RAS-ERK inhibition occurs in other cancers including malignant melanoma and glioblastoma multiforme, and has been functionally implicated in mediating cellular resistance to pathway inhibition $(14,28)$.

We previously identified the zinc finger transcription factor, Krüppel-like factor 4 (KLF4), as a critical positive regulator of steady state RAS-ERK activity in TNBC cells through microRNA (miR) - 206 and miR-21 mediated suppression of RAS GTPase activating protein (GAP) activity (29). KLF4 is a mediator of pluripotency in adult somatic cells and a stress response factor that plays prosurvival roles in many diverse contexts (30-38). In breast cancer, KLF4 protein expression is often increased, and this increased expression and the demethylation of the KLF4 promoter are poor prognostic factors (39-41). KLF4 and its effector miR-206 are functional markers of mammary cancer stem-like cells that represses the translation of proapoptotic proteins to promote chemoresistance in TNBC cells (42) and (manuscript in press) ${ }^{2}$. Furthermore, KLF4 collaborates with Krüppel-like factor 5 to promote the expression of antiapoptotic proteins and resistance to HER2 targeted therapies (43). These observations suggest that KLF4 has the potential to be a resistance factor against other targeted therapies, including MEK 1/2 inhibitors. 
In the present study, we observed that KLF4 mRNA expression was positively correlated with PDGFRB mRNA expression in human breast tumor samples. Consistent with this relationship in vivo, KLF4 loss- and gain-of-function studies as well as chromatin immunoprecipitation (ChIP) analysis showed direct regulation of PDGFR $\beta$ in both human TNBC cells and in cells derived from spontaneously arising tumors in the C3(1)/TAg (SV40 large T antigen transgenic mice under control of the C3(1)/prostatein promoter) genetically engineered mouse model (GEMM) of TNBC. In addition to regulating the steady state levels of this RTK, KLF4 was required for the PDGFR $\beta$ induction in response to MEK $1 / 2$ inhibition. Similarly, KLF4 knockdown blunted the upregulation of PDGFR $\beta$ upon siRNA mediated depletion of cMYC, suggesting functional antagonism of KLF4 on cMYC mediated repression of PDGFRB transcription. KLF4-PDGFR $\beta$ signaling promoted cellular resistance against MEK 1/2 inhibition and ectopic expression of PDGFR $\beta$ in KLF4-deficient cells was sufficient to rescue the sensitivity of KLF4 knockdown cells to pathway inhibition. Despite profound effects on drug resistance, neither KLF4 nor PDGFR $\beta$ was required for the restoration of RAS-ERK pathway activity following MEK $1 / 2$ inhibition. These results identify KLF4 as a critical MEK 1/2 inhibitor resistance factor and suggest that coordinate inhibition of KLF4 and RAS-ERK signaling in the treatment of TNBCs.

\section{MATERIALS AND METHODS}

Cell lines, cell culture, drug treatments, and drug sensitivity assays. MDA-MB-231, MDAMB-468 and Hs578t cells were obtained from American Type Culture Collection (ATCC). SUM159PT cells were provided by Gary L. Johnson (University of North Carolina at Chapel 
Hill). MDA-MB-231 cells were cultured in DMEM supplemented with $10 \%$ (v/v) fetal bovine serum (FBS). SUM159PT cells were cultured in 50:50 DMEM/F12 supplemented with $5 \mu \mathrm{g} / \mathrm{ml}$ insulin, $1 \mu \mathrm{g} / \mathrm{ml}$ hydrocortisone, and 5\% horse serum. Hs578t cells were cultured in DMEM supplemented with $5 \mu \mathrm{g} / \mathrm{ml}$ insulin and 10\% FBS. MDA-MB-468 cells were cultured in RPMI1640 supplemented with $5 \mu \mathrm{g} / \mathrm{ml}$ insulin and 10\% FBS. Cells were maintained as subconfluent monolayers.

Cells were treated with the indicated doses of the following MEK 1/2 inhibitors: AZD6244 (Selleck Chemicals) and U0126 (Sigma) (both dissolved in DMSO). Media containing inhibitors was replenished every 24 hours. Cell proliferation/viability was determined using the ATPlite Luminescence Assay System (Perkin Elmer), or alternatively by trypan blue exclusion followed by cell counting. For analysis of drug sensitivity, cells were plated at a density of 1000 cells per well in 96 well plates, and cell viability was measured after treatment with the indicated drug or vehicle for 72 hours.

Plasmid Construction and Retroviral transduction. The lentiviral vector encoding PDGFR $\beta$ (PLX303-PDGFR $\beta$ ) was constructed by recombination cloning using LR clonase II enzyme mix (Life Technologies) according to the enzyme manufacturer's recommendation. The entry vector (pDONR223-PDGFRB; Addgene plasmid \# 23893), and the destination lentiviral vector (pLX303; Addgene plasmid \# 25897) were gifts from William Hahn and David Root $(44,45)$. The corresponding control lentiviral vector in PDGFRB overexpression studies was similarly constructed by recombination of the entry vector pENTR-GUS (Life Technologies) with pLX303 (PLX303-GUS). 
KLF4 knockdown studies utilized the following pGIPZ lentiviral shRNAmir plasmids, which were obtained from Open Biosystems: human (hs): V2LHS_28277 - shKLF4-1, V3LHS_410934 - shKLF4-2, V3LHS_411731. The retroviral vector pBABEpuro-HA-KLF4 was previously described (46). Viral transduction was performed as described previously (42). Transduced cells were selected using puromycin $(1 \mu \mathrm{g} / \mathrm{ml})$ or blasticidin $(5 \mu \mathrm{g} / \mathrm{ml})$.

Immunoblot analysis and antibodies. Cells extracts for immunoblot analysis were prepared as previously described (29). Following electrophoresis, proteins were transferred onto nitrocellulose membranes and probed with the indicated antibody, including: KLF4 (Santa Cruz), $\beta$-actin (Santa Cruz), phospho-ERK 1/2 (pERK 1/2; Cell Signaling), ERK2 (Santa Cruz), phospho-MEK 1/2 (pMEK 1/2; Cell Signaling), total-MEK 1/2 (tMEK 1/2; Cell Signaling), cMYC (BD biosciences), and PDGFR $\beta$ (Cell Signaling). Bound antibodies were detected using Pierce ECL Western Blotting Substrate (Thermo Scientific). Scanned images were quantitated using ImageJ software, with normalization to loading control. Column data indicates the average of three independent experiments.

Transient transfections and AP-1 responsive promoter luciferase assays. The following siRNAs were obtained from GE Dharmacon: siMYC\#1 (D-003282-14), siMYC\#2 (D-00328216), and siCtl (siGENOME control pool non-targeting \#2 [D-001206-14-05]). siRNAs were diluted to $20 \mu \mathrm{M}$ in nuclease free water and transfected at a final concentration of $25 \mathrm{nM}$. Cells were subjected to forward transfection as previously described (42). Extracts of transfected cells were prepared for analysis at 48 hours post-transfection.

Analysis of activator protein 1 (AP-1) transcriptional activity, a downstream effector of RAS-ERK the pathway, was performed using AP-1 responsive luciferase (luc) construct. AP-1 
luc (pGL4.44[luc2P/AP1 RE/Hygro]) and vector control (pGL4.27[luc2P/minP/Hygro]) firefly luc reporter plasmids were obtained from Promega. pRLTK Renilla luc reporter plasmid was obtained from Promega and served as the internal control for luciferase experiments. Cells were plated in 12 well plates and subjected to forward transfection with luc plasmids (1.0 $\mu \mathrm{g}$ of AP-1 luc or vector and $100 \mathrm{ng}$ of pRLTK per well) as previously described (42). 24 hours posttransfection, MEK 1/2 inhibitors were added to transfected cells in a reverse time course fashion, i.e., with cells treated with drug for longer time intervals received the drug earlier in the time course than the cells treated for shorter time intervals. At the conclusion of the time course, cell extracts for all experimental conditions were prepared and Dual-Luciferase ${ }^{\circledR}$ Reporter Assays (DLR Assay, Promega) were performed as described previously (42).

Reverse Transcription and real-time PCR detection of mRNA. For quantitative real-time PCR (qRT-PCR), total RNA was extracted and mRNA levels were analyzed as previously described (42). Reactions were normalized to B2M or Rplo for analysis of human (hs) or murine (mmu) mRNA respectively. Primer sequences are as follow: hs PDGFRB: 5'CAGGAGAGACAGCAACAGCA-3', 5'-AACTGTGCCCACACCAGAAG-3'; hs B2M: 5'TCTCTGCTGGATGACGTGAG-3', 5'-TAGCTGTGCTCGCGCTACT-3'; mmu Pdgfrb: 5'TGGTATCACTCCTGGAAGCC-3', 5'-AACAGAAGACAGCGAGGTGG-3'; mmu Klf4: 5'GGAAAAGAACAGCCACCCAC-3', 5'-GTGGTAAGGTTTCTCGCCTGT-3'; mmu Rplpo: 5'-GGACCCGAGAAGACCTCCTT-3', 5'-GCACATCACTCAGAATTTCAATGG-3'． PCR reactions were performed on an ABI 7500 Real Time PCR system (Applied Biosystems). mRNA levels were determined by the $\Delta \Delta \mathrm{C}_{\mathrm{T}}$ method.

Chromatin Immunoprecipitation (ChIP). Potential KLF4 binding sites were identified using JASPAR (47). Chromatin preparation and immunoprecipitation (IP) was performed as 
previously described (48). Where indicated, cells were treated with AZD6244 or vehicle (DMSO) for 48 hours prior to chromatin preparation. IP was performed using $5 \mu \mathrm{g}$ of the following antibodies: anti-KLF4 (Santa Cruz), anti-cMYC (Santa Cruz), normal rabbit IgG, or normal mouse IgG. The sequences of primers used for PCR reactions are as follow; KLF4 Site 1: 5'-GCCACTCTTTGACAAAAGTGAC-3', 5'-GACGCGTGCGTCTGTTTTCA-3'; KLF4 Site 2: 5'-GCACCTGCTTGCCCAATGAA-3', 5'- GGTTGATTCAGTCAACCTTCATTC-3'; cMYC: 5'-GGCTTTGAGACGTGAAAAGGA-3', 5'-GGTCATCCAGCACAGATTGG-3'. ChIP intensity levels were determined by using the $\Delta \mathrm{C}_{\mathrm{T}}$ method to compare the yield obtained by using anti-KLF4 or anti-cMYC to normal IgG.

Animal Studies. Tumors arising in female $K l f 4^{+/+}-\mathrm{C} 3(1) / \mathrm{TAg}$ and $K l f 4^{+/-}-\mathrm{C} 3(1) / \mathrm{TAg}$ mice were harvested upon reaching a size of $1-2 \mathrm{~cm}^{3}$ and snap frozen in liquid nitrogen. Total RNA was extracted from tumors using the mirVana ${ }^{\mathrm{TM}}$ miRNA Isolation Kit (Ambion/Invitrogen) and used for analysis of Pdgfrb and Klf4 mRNA expression. All animal studies were performed under an approved protocol.

Statistical analysis. Unless otherwise stated, three independent experiments were performed in duplicate fashion. Data were analyzed using either the unpaired t-test (two-tailed), or else oneway analysis of variance (ANOVA) followed by Tukey's multiple comparison ad hoc post-test. Statistical analyses were performed in GraphPad Prism 5 (GraphPad software). Differences were considered significant when the analysis yielded $\mathrm{P}<0.05$. 


\section{RESULTS}

\section{KLF4 directly regulates PDGFRß expression in human and murine models of TNBC.}

We previously characterized the role of KLF4 as a critical regulator of steady state RASERK signaling and drug resistance in TNBC and HER2+ breast cancer cells $(29,43)$ and (manuscript in press) $^{2}$. We sought to identify whether KLF4 exerted a similar prosurvival role in response to RAS-ERK pathway inhibition in TNBC cells. To identify putative KLF4 effectors in mediating this phenotype, we analyzed whole transcriptome sequencing (RNAseq) data from 1099 breast tumor samples representing virtually all molecular and histological breast cancer subtypes $(25,49,50)$. In this analysis, we observed statistically significant positive correlations between the mRNA expression of KLF4 and the mRNA levels of RTKs that are dynamically regulated by MEK 1/2 inhibition and functionally implicated in drug resistance in TNBC cells (11). KLF4 mRNA expression was positively correlated with that of $P D G F R B$ (Spearman $\mathrm{R}=$ 0.2999, $\mathrm{P}<0.0001), A X L(\mathrm{R}=0.2853, \mathrm{P}<0.0001)$, and KDR/VEGFR2 $(\mathrm{R}=0.2543, \mathrm{P}<$ 0.0001), suggesting that KLF4 could promote RTK expression and consequently MEK $1 / 2$ inhibitor resistance in TNBC cells.

To further investigate the relationship between KLF4 and these RTKs, we assayed the mRNA expression of PDGFRB, $A X L$, and VEGFR2 in KLF4-depleted claudin-low MDA-MB231 and SUM159PT cells and control cells (Fig. 1A and data not shown). KLF4 knockdown cells displayed reduced PDGFRB mRNA and protein levels (Fig. 1A-1C). KLF4 knockdown, however, did not consistently alter the expression of $A X L$ and VEGFR2 (data not shown). Complementing KLF4 loss-of-function studies, ectopic expression of KLF4 in TNBC cells promoted PDGFR $\beta$ mRNA and protein expression (Figs. 1D-1F). Interestingly, KLF4 did not 
appear to regulate the expression of cMYC protein, which is a repressor of PDGFRB transcription (Figs. 1B and 1E) (27). To determine direct regulation of PDGFR $\beta$ by KLF4, we performed ChIP analysis and observed enrichment of KLF4 at a promoter proximal site (Site 1) (Fig. 1G and Fig. 1H, left panel). Supporting specificity, this enrichment was reduced in KLF4deficient cells (Fig. 1H, right panel).

We next analyzed KLF4 regulation of PDGFR $\beta$ in a broader context. Consistent with our previous observations, PDGFR $\beta$, but not cMYC protein levels, were dependent on KLF4 in two additional TNBC cell lines (Figs. 1I and 1J). Suggesting conservation of KLF4-PDGFR $\beta$ regulation across species, we observed a positive correlation between Pdgfrb and Klf4 mRNA levels through the analysis of RNAseq data of tumors derived from genetically engineered mouse models (GEMMs) of mammary cancer representing a wide variety of histological and molecular subtypes (Spearman $\mathrm{R}=0.3673, \mathrm{P}<0.01)(22)$. We also observed PDGFR $\beta$ dependence on KLF4 first-hand in the C3(1)/TAg GEMM of basal-like breast cancer $(22,51)$. In

this model, female mice with a hemizygous loss of $\mathrm{Klf}\left(\mathrm{Klf}^{+{ }^{+}}\right)$displayed reduced Pdgfrb mRNA in spontaneously arising tumors compared to tumors from $K l f 4^{+/+}-\mathrm{C} 3(1) / \mathrm{TAg}$ mice (Fig. 1K). These results suggest KLF4 regulates the steady state expression of PDGFR $\beta$ in human and murine models of TNBC.

KLF4 is required for the time-dependent induction of PDGFR in response to MEK 1/2 inhibition.

TNBC cells display dynamic reprogramming of the kinome and induction of RTK signaling in response to MEK 1/2 inhibition. Collectively, these compensatory changes restore blocked RAS-ERK signaling and activate alternative prosurvival signaling pathways. A 
prominent feature of this cellular response to RAS-ERK pathway inhibition is the induction of PDGFR $\beta$, which has been functionally implicated in promoting cellular escape from RAS-ERK pathway inhibition in numerous contexts $(11,12,14,28)$. Upon MEK $1 / 2$ inhibition, PDGFR $\beta$ levels are increased due to transcriptional derepression resulting from the loss of cMYC protein stability $(11,26,27)$. Indeed, TNBC cells displayed time-dependent loss of cMYC protein and upregulation of PDGFR $\beta$ protein and mRNA levels when treated with the allosteric ATPuncompetitive MEK 1/2 inhibitor AZD6244 (Figs. 2A and 2B) (52,53). Furthermore, reemergence of RAS-ERK signaling was observed as early as 12 hours following drug treatment by analysis of phospho-MEK 1/2 (pMEK 1/2) and phospho-ERK 1/2 (pERK 1/2) protein levels. However, KLF4 protein levels did not appear to be dynamically regulated in response to MEK 1/2 inhibition (Fig. 2A).

We next chose to investigate whether the induction of PDGFR $\beta$ and/or the reemergence of RAS-ERK signaling following MEK 1/2 inhibition were dependent on KLF4. Compared to AZD6244 treated control ( $\mathrm{shCtl}$ ) cells, drug treated KLF4 knockdown TNBC cells showed blunted PDGFR $\beta$ protein upregulation (Figs. 2C-2F). Likewise, attenuation of PDGFRB mRNA induction was observed in KLF4 knockdown cells (Fig. 2G). However, unlike the profound effect on PDGFR $\beta$ induction, KLF4 knockdown did not appear to impact the reemergence of RAS-ERK signaling after MEK 1/2 inhibition (Fig. 2C, lanes 4, 8, and 12; Fig. 2D, lanes 2, 4, and 6). Instead, KLF4 promoted steady state levels of RAS-ERK signaling (Fig. 2C, lanes 1, 5, 9; Fig. 2D, lanes 1, 3, 5), and this effect of KLF4 was attributed to the regulation of RASGAP activity (29). Analysis of the activator protein 1 (AP-1) mediated transcriptional activity, a correlate of active RAS-ERK signaling, using an AP-1 responsive firefly luciferase (luc) construct supported the differential regulation of steady state and MEK 1/2 inhibitor responsive 
RAS-ERK signaling by KLF4 (Figs $2 \mathrm{H}$ and 2I) $(54,55)$. These results highlight a critical role of KLF4 in the induction of PDGFR $\beta$ in response to MEK $1 / 2$ inhibition, and suggest that KLF4 could promote resistance to RAS-ERK pathway inhibition.

\section{KLF4 opposes cMYC mediated repression of PDGFRß.}

We next chose to investigate the relationship between KLF4 regulation of PDGFR $\beta$ and cMYC mediated repression of this RTK. Consistent with previous reports, we observed cMYC ChIP enrichment on the PDGFRB promoter, and a reduction of this interaction when MDA-MB231/shCtl cells were treated with AZD6244 (Figs. 3A, and 3B, left panel) (11). A similar reduction in cMYC enrichment was observed in KLF4-depleted MDA-MB-231 cells (Fig. 3B, left panel). Unlike cMYC, KLF4 ChIP intensity levels on the PDGFRB promoter did not appear to be dependent on RAS-ERK signaling in either KLF4 knockdown cells or control cells, as AZD6244 treatment did not alter KLF4 ChIP intensity relative to the vehicle control (Fig. 3B, right panel). These results seem to indicate that KLF4 binding to PDGFRB may not be dependent on cMYC.

To further characterize the relationship between these two factors, we employed cMYC loss-of-function studies. As previously reported, siRNA mediated knockdown of cMYC induced PDGFR $\beta$ mRNA and protein levels (Figs. 3C and 3D) (11). Furthermore, consistent with a previous study, pERK 1/2 levels were not altered in cMYC depleted cells (Fig. 3D). Indicating KLF4 protein levels were likely not dependent on cMYC, KLF4 expression was not altered by cMYC knockdown either (Fig. 3D). This result and our previous observations on modulating KLF4 levels in TNBC cells (Figs. 1B and 1E) appear to exclude reciprocal regulation of KLF4 and cMYC in the present context. Finally, we sought to determine whether KLF4 was required 
for PDGFR $\beta$ induction upon loss of cMYC protein. Similar to the KLF4 dependence of PDGFR $\beta$ upregulation in response to MEK 1/2 inhibition, KLF4 was critical for PDGFR $\beta$ induction upon cMYC knockdown (Figs. 3F and 3G). These results are consistent with the role for KLF4 as a constitutive positive regulator of PDGFR $\beta$ expression which opposes cMYC mediated repression of this RTK.

\section{KLF4 promotes resistance to MEK $1 / 2$ inhibition in TNBC cells.}

We next wanted to investigate whether KLF4 could mediate resistance to MEK 1/2 inhibition. We had previously identified endogenous KLF4 as a resistance factor that protected cells from cytotoxic chemotherapy as well as anti-HER2 therapies (43) and (manuscript in press) $)^{2}$. The KLF4 dependence of PDGFR $\beta$ for upregulation in response to MEK 1/2 inhibition, suggested that KLF4 could mediate resistance against RAS-ERK pathway inhibition in TNBC cells. Indeed, compared to the control, we observed a reduction in viable cell number of KLF4 knockdown cells that were treated with a range of doses of AZD6244 (Fig. 4A, top panels). This left-shift in the cell viability dose response curve was likewise observed in KLF4-depleted cells treated with a second allosteric MEK 1/2 inhibitor, U0126 (Fig. 4A, bottom panels) (56). Furthermore, KLF4 knockdown cells displayed reduced MEK 1/2 inhibitor resistant cell proliferation compared to shCtl cells (Fig. 4B). The effects on cell number were partly attributed to the increased cell death observed in KLF4 knockdown cells treated with AZD6244 (Fig. 4C). These results implicate KLF4 as a MEK 1/2 inhibitor resistance factor in TNBC cells.

\section{KLF4-PDGFRß regulation is critical for MEK 1/2 inhibitor resistance.}

To assess the significance of PDGFR $\beta$ in KLF4 mediated MEK 1/2 inhibitor resistance, we employed PDGFR $\beta$ gain-of-function studies. Previous studies have shown that both MDA- 
MB-231 and SUM159PT cells display PDGFB and PDGFD ligand positivity and autocrine/paracrine PDGFR $\beta$ signaling $(11,57,58)$. Despite this insight, how this signaling impacts RAS-ERK pathway activity is not well understood. Surprisingly, ectopic expression of this RTK did not alter steady-state levels of pERK 1/2 in either KLF4 knockdown cells or control cells (Fig. 5A, compare lanes 1 and 2, 5 and 6, 9 and 10). Furthermore, regardless of KLF4 knockdown, PDGFR $\beta$ expression did not affect ERK 1/2 activation after treatment with AZD6244 (Fig. 5A, compare lanes 3 and 4, 7 and 8, 11 and 12). These observations suggest that in these cell lines, PDGFR $\beta$ signaling may be dispensable for both steady state RAS-ERK signaling and the reactivation of this pathway activity in response to MEK 1/2 inhibition.

Nonetheless, PDGFR $\beta$ exerted a pronounced effect on restoring MEK 1/2 inhibitor resistance in KLF4 knockdown cells, as an increase in viable cell number was observed in AZD6244 treated KLF4-depleted cells expressing PDGFR $\beta$ compared to the respective vector control cells (Fig. 5B, left panels). PDGFR $\beta$ overexpression yielded more subtle effects in SUM159PT/shCtl cells (Fig. 5B, top right panel) and virtually no increase in cell viability was observed in MDA-MB-231/shCtl cells upon ectopic expression of this RTK (Fig. 5B, bottom right panel). Furthermore, analysis of cell death by trypan blue exclusion showed that PDGFR $\beta$ overexpression promoted survival in KLF4 knockdown cells treated with AZD6244 compared to shCtl cells (Fig. 5C). These results identify PDGFR $\beta$ as a KLF4 regulated prosurvival factor in promoting resistance to MEK 1/2 inhibition in TNBC cells, and suggest that alternative pathways may be activated by this receptor in mediating cellular escape in response to RAS-ERK pathway inhibition (Fig. 6). 


\section{DISCUSSION}

Targeted therapies have significantly impacted the treatment of ER $\alpha+$ and HER2+ breast cancers and hold great promise for TNBCs. Blockade of the RAS-ERK signaling pathway using MEK 1/2 inhibitors is an attractive therapeutic strategy for the treatment of these cancers. However, dynamic reprogramming of cell signaling and compensatory genetic events occur in response to the inhibition of major signaling pathways, including the RAS-ERK pathway, contribute to therapeutic resistance $(6-12,14,16)$. Thus, the successful use of MEK 1/2 inhibitors would require concurrent blockade of the adaptive programming that drives cellular escape.

In the present study, we identified KLF4 as a major regulator of PDGFR $\beta$ in TNBC cells and a critical resistance factor that protects cells from MEK 1/2 inhibition. These observations are consistent with our previous studies that characterized a prosurvival role for endogenous KLF4 in breast cancer cells (43) and (manuscript in press) ${ }^{2}$. PDGFR $\beta$ (and PDGFR $\alpha$ ) are prominent mesenchymal mitogenic signaling molecules and the expression of these proteins is normally low in epithelial cells (59-61). However, cancerous epithelial cells in many tumors often upregulate this RTK and exploit PDGF signaling. In breast cancer cells, the levels of PDGFR $\beta$ and its ligand PDGF-B are increased compared to normal epithelia (62). Furthermore, the protein expression of PDGFR $\alpha$, PDGFR $\beta$, and the PDGF ligands are adverse prognostic factors which signify aggressive disease $(63,64)$. PDGFR $\beta$ is prominently upregulated in breast epithelial cells that undergo epithelial-mesenchymal transition and autocrine PDGFR signaling is required for the maintenance of this phenotype and cell survival during breast cancer cell metastasis $(57,65)$. In addition to promoting tumor cell aggressiveness, PDGFR $\beta$ is an important mediator of resistance to chemotherapy and radiotherapy in breast cancer. For 
example, PDGFR $\beta$ signaling promotes tumor cell survival in ER $\alpha+$ breast cancers upon estradiol deprivation and the expression of this RTK is correlated with a poor response to aromatase

In broad contexts including breast cancer, PDGFR $\beta$ upregulation is a prominent adaptive response to RAS-ERK pathway inhibition and induction of this RTK has been functionally implicated in promoting therapeutic resistance $(11,14,28,66)$. In human TNBC cells, PDGFR $\beta$ knockdown synergizes with MEK 1/2 inhibition and attenuates drug resistant cell proliferation (11). In glioblastoma multiforme, PDGFR $\beta$ signaling is induced upon epithelial growth factor inhibition with erlotinib, and promotes drug resistant tumor cell survival and proliferation (28). Finally, PDGFR $\beta$ induction and NRAS mutations are mutually exclusive but functionally equivalent events that occur in malignant melanoma is response treatment with BRAF-inhibitors $(14,66)$. Consistent with these previous studies, we observed that KLF4, by way of regulating PDGFR $\beta$ expression, promoted MEK 1/2 inhibitor resistance.

However, the precise mechanism of how PDGFR $\beta$ mediates MEK 1/2 resistant RASERK signaling is unclear. Activation of PDGFR $\beta$ can result in signaling through a multitude of prosurvival pathways including through RAS-ERK, PI3-K-AKT, phospholipase C- $\gamma$, and Src family kinases (61,67-69). It is possible that differential activation of these PDGFR $\beta$ effector pathways can occur in a context dependent fashion to promote MEK 1/2 inhibitor resistance. Indeed, in melanoma, PDGFR $\beta$ knockdown yields a reduction in drug resistant RAS-ERK signaling suggesting a major contribution to the restoration of this pathway (14). However, the effect of PDGFR $\beta$ in promoting RAS-ERK signaling in TNBC may be more limited as overexpression of this RTK in cells does not greatly alter RAS-ERK pathway activity (Fig. 5A). Furthermore, the reduction in steady-state RAS-ERK signaling was not restored by ectopic PDGFR $\beta$ expression (Fig. 5A). Finally, despite severely blunted PDGFR $\beta$ induction in KLF4 
knockdown cells, reemergence of RAS-ERK signaling occurred in a similar fashion to control cells (Fig. 2). Nonetheless, PDGFR $\beta$ was critical in promoting MEK 1/2 resistant cell proliferation and cell survival in KLF4 knockdown cells (Figs. 5B and 5C). These results suggest that PDGFR $\beta$ may activate alternate signaling pathways to promote MEK $1 / 2$ resistance in TNBC cells.

Interestingly, our results suggest that restoration of RAS-ERK signaling in response to MEK 1/2 inhibition may not be limited by the action of RAS GAPs as KLF4 knockdown cells, which display increased GAP activity, featured similar reactivation of the pathway compared to control cells (Fig. 2). Our results are consistent with the idea that in the absence of oncogenic mutations in RAS, the activation of RAS molecules is governed by guanine nucleotide exchange factors (GEFs) (70,71). With the induction of RTK signaling upon MEK 1/2 inhibition, RAS activation by GEFs may override the inhibitory action of GAPs. Another explanation involves the feedback repression of BRAF and MEK1 activity by activated ERK 1/2 at inhibitory sites $(11,13)$. Derepression of this feedback occurs upon MEK 1/2 inhibition and results in reflexive activation of MEK2, which does not possess an ERK-responsive inhibitory site, and not MEK1. Upstream activation of MEK2, which is not discernible from MEK1 from our western blotting results, causes a reduction in the affinity for the enzyme with allosteric MEK 1/2 inhibitors (such as AZD6244), and thus MEK2 is able to mediate downstream signaling (Fig. 2).

This study identifies KLF4 as a critical resistance factor against RAS-ERK pathway inhibition in TNBC cells through the regulation of PDGFR $\beta$. In response to MEK 1/2 inhibition, TNBC cells rely upon KLF4 mediated induction of PDGFR $\beta$ for tumor cell proliferation and survival. Given the profound prosurvival role of KLF4 in breast cancer cells, our current study 
provides the rationale for concurrent inhibition of KLF4 signaling along with MEK 1/2 inhibition as a potentially efficacious therapeutic combination in combating TNBC.

\section{ACKNOWLEDGEMENTS}

This work was supported by grants NCI RO1 CA127405 (to JMR), the Jo and Ben Statler Chair in Breast Cancer Research, and the Wilmer V. and Helen B. Morley Memorial Fund at the Mary Babb Randolph Cancer Center (MBRCC). Animal work and imaging experiments were performed in the West Virginia University Microscope Imaging Facility and West Virginia University Animal Models and Imaging Facility, which was supported by the MBRCC and NIH grants P20 RR016440, P30 RR032138/GM103488, P20 RR016477 and S10 RR026378. We thank Gary Johnson (University of North Carolina) and Jeffrey Green (National Cancer Institute) for providing cell lines. We thank Laura Gibson (West Virginia University) and Steven Frisch (West Virginia University) for sharing reagents.

\section{CONFLICTS OF INTEREST}

The authors declare no conflicts of interest.

\section{FOOTNOTES}

${ }^{1}$ Abbreviations: KLF4 - Krüppel like factor 4; PDGFR $\beta$ - Platelet derived growth factor receptor beta polypeptide; TNBC - Triple-negative breast cancer; ERK - Extracellular signal regulated 
kinase; RTK - Receptor tyrosine kinase; PI3-K - phosphoinositide 3-kinase; ER $\alpha$ - Estrogen receptor $\alpha$; PR - Progesterone receptor; HER2 - human epidermal growth factor receptor 2; GAP - GTPase activating protein; GEF - Guanine nucleotide exchange factor; C3(1)/TAg SV40 large T antigen transgenic mice under control of the C3(1)/prostatein promoter; GEMM Genetically engineered mouse model; AP-1 - activator protein 1

${ }^{2}$ Manuscript in press 


\section{REFERENCES}

1. Johnson, G. L. and R. Lapadat. 2002. Mitogen-activated protein kinase pathways mediated by ERK, JNK, and p38 protein kinases. Science 298:1911-1912.

2. Downward, J. 2003. Targeting RAS signalling pathways in cancer therapy. Nat. Rev. Cancer 3:11-22.

3. Roberts, P. J. and C. J. Der. 2007. Targeting the Raf-MEK-ERK mitogen-activated protein kinase cascade for the treatment of cancer. Oncogene 26:3291-3310.

4. Young, A., J. Lyons, A. L. Miller, V. T. Phan, I. R. Alarcon, and F. McCormick. 2009. Ras signaling and therapies. Adv. Cancer Res 102:1-17.

5. De Luca A., M. R. Maiello, A. D'Alessio, M. Pergameno, and N. Normanno. 2012. The RAS/RAF/MEK/ERK and the PI3K/AKT signalling pathways: role in cancer pathogenesis and implications for therapeutic approaches. Expert Opin. Ther Targets. 16 Suppl 2:S17-S27.

6. Johnson, G. L., T. J. Stuhlmiller, S. P. Angus, J. S. Zawistowski, and L. M. Graves. 2014. Molecular pathways: adaptive kinome reprogramming in response to targeted inhibition of the BRAF-MEK-ERK pathway in cancer. Clin Cancer Res 20:2516-2522.

7. Engelman, J. A., K. Zejnullahu, T. Mitsudomi, Y. Song, C. Hyland, J. O. Park, N. Lindeman, C. M. Gale, X. Zhao, J. Christensen, T. Kosaka, A. J. Holmes, A. M. Rogers, F. Cappuzzo, T. Mok, C. Lee, B. E. Johnson, L. C. Cantley, and P. A. Janne. 2007. MET amplification leads to gefitinib resistance in lung cancer by activating ERBB3 signaling. Science 316:1039-1043.

8. Rodrik-Outmezguine, V. S., S. Chandarlapaty, N. C. Pagano, P. I. Poulikakos, M. Scaltriti, E. Moskatel, J. Baselga, S. Guichard, and N. Rosen. 2011. mTOR kinase inhibition causes feedback-dependent biphasic regulation of AKT signaling. Cancer Discov. 1:248-259.

9. Garrett, J. T., M. G. Olivares, C. Rinehart, N. D. Granja-Ingram, V. Sanchez, A. Chakrabarty, B. Dave, R. S. Cook, W. Pao, E. McKinely, H. C. Manning, J. Chang, and C. L. Arteaga. 2011. Transcriptional and posttranslational up-regulation of HER3 (ErbB3) compensates for inhibition of the HER2 tyrosine kinase. Proc. Natl. Acad. Sci. U. S. A 108:5021-5026.

10. Chandarlapaty, S., A. Sawai, M. Scaltriti, V. Rodrik-Outmezguine, O. Grbovic-Huezo, V. Serra, P. K. Majumder, J. Baselga, and N. Rosen. 2011. AKT inhibition relieves feedback suppression of receptor tyrosine kinase expression and activity. Cancer Cell 19:58-71.

11. Duncan, J. S., M. C. Whittle, K. Nakamura, A. N. Abell, A. A. Midland, J. S. Zawistowski, N. L. Johnson, D. A. Granger, N. V. Jordan, D. B. Darr, J. Usary, P. F. Kuan, D. M. Smalley, B. Major, X. He, K. A. Hoadley, B. Zhou, N. E. Sharpless, C. M. 
Perou, W. Y. Kim, S. M. Gomez, X. Chen, J. Jin, S. V. Frye, H. S. Earp, L. M. Graves, and G. L. Johnson. 2012. Dynamic reprogramming of the kinome in response to targeted MEK inhibition in triple-negative breast cancer. Cell 149:307-321.

12. Sun, T., N. Aceto, K. L. Meerbrey, J. D. Kessler, C. Zhou, I. Migliaccio, D. X. Nguyen, N. N. Pavlova, M. Botero, J. Huang, R. J. Bernardi, E. Schmitt, G. Hu, M. Z. Li, N. Dephoure, S. P. Gygi, M. Rao, C. J. Creighton, S. G. Hilsenbeck, C. A. Shaw, D. Muzny, R. A. Gibbs, D. A. Wheeler, C. K. Osborne, R. Schiff, M. tires-Alj, S. J. Elledge, and T. F. Westbrook. 2011. Activation of multiple proto-oncogenic tyrosine kinases in breast cancer via loss of the PTPN12 phosphatase. Cell 144:703-718.

13. Ritt, D. A., D. M. Monson, S. I. Specht, and D. K. Morrison. 2010. Impact of feedback phosphorylation and Raf heterodimerization on normal and mutant B-Raf signaling. Mol. Cell Biol. 30:806-819.

14. Nazarian, R., H. Shi, Q. Wang, X. Kong, R. C. Koya, H. Lee, Z. Chen, M. K. Lee, N. Attar, H. Sazegar, T. Chodon, S. F. Nelson, G. McArthur, J. A. Sosman, A. Ribas, and R. S. Lo. 2010. Melanomas acquire resistance to B-RAF(V600E) inhibition by RTK or NRAS upregulation. Nature 468:973-977.

15. Villanueva, J., J. R. Infante, C. Krepler, P. Reyes-Uribe, M. Samanta, H. Y. Chen, B. Li, R. K. Swoboda, M. Wilson, A. Vultur, M. Fukunaba-Kalabis, B. Wubbenhorst, T. Y. Chen, Q. Liu, K. Sproesser, D. J. DeMarini, T. M. Gilmer, A. M. Martin, R. Marmorstein, D. C. Schultz, D. W. Speicher, G. C. Karakousis, W. Xu, R. K. Amaravadi, X. Xu, L. M. Schuchter, M. Herlyn, and K. L. Nathanson. 2013. Concurrent MEK2 mutation and BRAF amplification confer resistance to BRAF and MEK inhibitors in melanoma. Cell Rep. 4:1090-1099.

16. Shi, H., A. Hong, X. Kong, R. C. Koya, C. Song, G. Moriceau, W. Hugo, C. C. Yu, C. Ng, T. Chodon, R. A. Scolyer, R. F. Kefford, A. Ribas, G. V. Long, and R. S. Lo. 2014. A novel AKT1 mutant amplifies an adaptive melanoma response to BRAF inhibition. Cancer Discov. 4:69-79.

17. Yu, C. F., Z. X. Liu, and L. G. Cantley. 2002. ERK negatively regulates the epidermal growth factor-mediated interaction of Gab1 and the phosphatidylinositol 3-kinase. J Biol. Chem. 277:19382-19388.

18. Sos, M. L., S. Fischer, R. Ullrich, M. Peifer, J. M. Heuckmann, M. Koker, S. Heynck, I. Stuckrath, J. Weiss, F. Fischer, K. Michel, A. Goel, L. Regales, K. A. Politi, S. Perera, M. Getlik, L. C. Heukamp, S. Ansen, T. Zander, R. Beroukhim, H. Kashkar, K. M. Shokat, W. R. Sellers, D. Rauh, C. Orr, K. P. Hoeflich, L. Friedman, K. K. Wong, W. Pao, and R. K. Thomas. 2009. Identifying genotype-dependent efficacy of single and combined. Proc. Natl. Acad. Sci. U. S. A 106:18351-18356.

19. Hoeflich, K. P., C. O'Brien, Z. Boyd, G. Cavet, S. Guerrero, K. Jung, T. Januario, H. Savage, E. Punnoose, T. Truong, W. Zhou, L. Berry, L. Murray, L. Amler, M. Belvin, L. S. Friedman, and M. R. Lackner. 2009. In vivo antitumor activity of MEK and 
phosphatidylinositol 3-kinase inhibitors in basal-like breast cancer models. Clin Cancer Res 15:4649-4664.

20. Mirzoeva, O. K., D. Das, L. M. Heiser, S. Bhattacharya, D. Siwak, R. Gendelman, N. Bayani, N. J. Wang, R. M. Neve, Y. Guan, Z. Hu, Z. Knight, H. S. Feiler, P. Gascard, B. Parvin, P. T. Spellman, K. M. Shokat, A. J. Wyrobek, M. J. Bissell, F. McCormick, W. L. Kuo, G. B. Mills, J. W. Gray, and W. M. Korn. 2009. Basal subtype and MAPK/ERK kinase (MEK)-phosphoinositide 3-kinase feedback signaling determine susceptibility of breast cancer cells to MEK inhibition. Cancer Res 69:565-572.

21. Sun, C., S. Hobor, A. Bertotti, D. Zecchin, S. Huang, F. Galimi, F. Cottino, A. Prahallad, W. Grernrum, A. Tzani, A. Schlicker, L. F. Wessels, E. F. Smit, E. Thunnissen, P. Halonen, C. Lieftink, R. L. Beijersbergen, N. F. Di, A. Bardelli, L. Trusolino, and R. Bernards. 2014. Intrinsic resistance to MEK inhibition in KRAS mutant lung and colon cancer through transcriptional induction of ERBB3. Cell Rep. 7:86-93.

22. Herschkowitz, J. I., K. Simin, V. J. Weigman, I. Mikaelian, J. Usary, Z. Hu, K. E. Rasmussen, L. P. Jones, S. Assefnia, S. Chandrasekharan, M. G. Backlund, Y. Yin, A. I. Khramtsov, R. Bastein, J. Quackenbush, R. I. Glazer, P. H. Brown, J. E. Green, L. Kopelovich, P. A. Furth, J. P. Palazzo, O. I. Olopade, P. S. Bernard, G. A. Churchill, D. T. Van, and C. M. Perou. 2007. Identification of conserved gene expression features between murine mammary carcinoma models and human breast tumors. Genome Biol. 8:R76.

23. Rakha, E. A., J. S. Reis-Filho, and I. O. Ellis. 2008. Basal-like breast cancer: a critical review. J Clin Oncol. 26:2568-2581.

24. Prat, A. and C. M. Perou. 2011. Deconstructing the molecular portraits of breast cancer. Mol. Oncol. 5:5-23.

25. Cancer Genome Atlas Network. 2012. Comprehensive molecular portraits of human breast tumours. Nature 490:61-70.

26. Sears, R., F. Nuckolls, E. Haura, Y. Taya, K. Tamai, and J. R. Nevins. 2000. Multiple Ras-dependent phosphorylation pathways regulate Myc protein stability. Genes Dev 14:2501-2514.

27. Oster, S. K., W. W. Marhin, C. Asker, L. M. Facchini, P. A. Dion, K. Funa, M. Post, J. M. Sedivy, and L. Z. Penn. 2000. Myc is an essential negative regulator of plateletderived growth factor beta receptor expression. Mol. Cell Biol. 20:6768-6778.

28. Akhavan, D., A. L. Pourzia, A. A. Nourian, K. J. Williams, D. Nathanson, I. Babic, G. R. Villa, K. Tanaka, A. Nael, H. Yang, J. Dang, H. V. Vinters, W. H. Yong, M. Flagg, F. Tamanoi, T. Sasayama, C. D. James, H. I. Kornblum, T. F. Cloughesy, W. K. Cavenee, S. J. Bensinger, and P. S. Mischel. 2013. De-repression of PDGFRbeta transcription promotes acquired resistance to EGFR tyrosine kinase inhibitors in glioblastoma patients. Cancer Discov. 3:534-547. 
29. Sharma, S. B., C. C. Lin, M. K. Farrugia, S. L. McLaughlin, E. J. Ellis, K. M. Brundage, M. A. Salkeni, and J. M. Ruppert. 2014. MicroRNAs 206 and 21 cooperate to promote RAS-extracellular signal-regulated kinase signaling by suppressing the translation of RASA1 and SPRED1. Mol. Cell Biol. 34:4143-4164.

30. McCormick, S. M., S. G. Eskin, L. V. McIntire, C. L. Teng, C. M. Lu, C. G. Russell, and K. K. Chittur. 2001. DNA microarray reveals changes in gene expression of shear stressed human umbilical vein endothelial cells. Proc. Natl. Acad. Sci. U. S. A 98:89558960.

31. Pedersen, T. X., C. Leethanakul, V. Patel, D. Mitola, L. R. Lund, K. Dano, M. Johnsen, J. S. Gutkind, and T. H. Bugge. 2003. Laser capture microdissection-based in vivo genomic profiling of wound keratinocytes identifies similarities and differences to squamous cell carcinoma. Oncogene 22:3964-3976.

32. Yoon, H. S., X. Chen, and V. W. Yang. 2003. Krüppel-like factor 4 mediates p53dependent G1/S cell cycle arrest in response to DNA damage. J Biol. Chem. 278:21012105.

33. Liu, Y., S. Sinha, O. G. McDonald, Y. Shang, M. H. Hoofnagle, and G. K. Owens. 2005. Krüppel-like factor 4 abrogates myocardin-induced activation of smooth muscle gene expression. J Biol. Chem. 280:9719-9727.

34. Takahashi, K. and S. Yamanaka. 2006. Induction of pluripotent stem cells from mouse embryonic and adult fibroblast cultures by defined factors. Cell 126:663-676.

35. Liu, Y., J. Wang, Y. Yi, H. Zhang, J. Liu, M. Liu, C. Yuan, D. Tang, I. J. Benjamin, and X. Xiao. 2006. Induction of KLF4 in response to heat stress. Cell Stress. Chaperones. 11:379-389.

36. Hamik, A., Z. Lin, A. Kumar, M. Balcells, S. Sinha, J. Katz, M. W. Feinberg, R. E. Gerzsten, E. R. Edelman, and M. K. Jain. 2007. Krüppel-like factor 4 regulates endothelial inflammation. J Biol. Chem. 282:13769-13779.

37. Ghaleb, A. M., J. P. Katz, K. H. Kaestner, J. X. Du, and V. W. Yang. 2007. Krüppel-like factor 4 exhibits antiapoptotic activity following gamma-radiation-induced DNA damage. Oncogene 26:2365-2373.

38. Liao, X., S. M. Haldar, Y. Lu, D. Jeyaraj, K. Paruchuri, M. Nahori, Y. Cui, K. H. Kaestner, and M. K. Jain. 2010. Krüppel-like factor 4 regulates pressure-induced cardiac hypertrophy. J Mol. Cell Cardiol. 49:334-338.

39. Pandya, A. Y., L. I. Talley, A. R. Frost, T. J. Fitzgerald, V. Trivedi, M. Chakravarthy, D. C. Chhieng, W. E. Grizzle, J. A. Engler, H. Krontiras, K. I. Bland, A. F. LoBuglio, S. M. Lobo-Ruppert, and J. M. Ruppert. 2004. Nuclear localization of KLF4 is associated with an aggressive phenotype in early-stage breast cancer. Clin Cancer Res 10:2709-2719. 
40. Chen, C. J., S. E. Lin, Y. M. Lin, S. H. Lin, D. R. Chen, and C. L. Chen. 2012. Association of expression of krüppel-like factor 4 and krüppel-like factor 5 with the clinical manifestations of breast cancer. Pathol. Oncol. Res 18:161-168.

41. Kamalakaran, S., V. Varadan, H. E. Giercksky Russnes, D. Levy, J. Kendall, A. Janevski, M. Riggs, N. Banerjee, M. Synnestvedt, E. Schlichting, R. Karesen, P. K. Shama, H. Rotti, R. Rao, L. Rao, M. H. Eric Tang, K. Satyamoorthy, R. Lucito, M. Wigler, N. Dimitrova, B. Naume, A. L. Borresen-Dale, and J. B. Hicks. 2011. DNA methylation patterns in luminal breast cancers differ from non-luminal subtypes and can identify relapse risk independent of other clinical variables. Mol. Oncol. 5:77-92.

42. Lin, C. C., L. Z. Liu, J. B. Addison, W. F. Wonderlin, A. V. Ivanov, and J. M. Ruppert. 2011. A KLF4-miRNA-206 autoregulatory feedback loop can promote or inhibit protein translation depending upon cell context. Mol. Cell Biol. 31:2513-2527.

43. Farrugia, M. K., S. B. Sharma, C. C. Lin, S. L. McLaughlin, D. B. Vanderbilt, A. G. Ammer, M. A. Salkeni, P. Stoilov, Y. M. Agazie, C. J. Creighton, and J. M. Ruppert. 2015. Regulation of anti-apoptotic signaling by Krüppel-like factors 4 and 5 mediates lapatinib resistance in breast cancer. Cell Death. Dis. 6:e1699.

44. Yang, X., J. S. Boehm, X. Yang, K. Salehi-Ashtiani, T. Hao, Y. Shen, R. Lubonja, S. R. Thomas, O. Alkan, T. Bhimdi, T. M. Green, C. M. Johannessen, S. J. Silver, C. Nguyen, R. R. Murray, H. Hieronymus, D. Balcha, C. Fan, C. Lin, L. Ghamsari, M. Vidal, W. C. Hahn, D. E. Hill, and D. E. Root. 2011. A public genome-scale lentiviral expression library of human ORFs. Nat. Methods 8:659-661.

45. Johannessen, C. M., J. S. Boehm, S. Y. Kim, S. R. Thomas, L. Wardwell, L. A. Johnson, C. M. Emery, N. Stransky, A. P. Cogdill, J. Barretina, G. Caponigro, H. Hieronymus, R. R. Murray, K. Salehi-Ashtiani, D. E. Hill, M. Vidal, J. J. Zhao, X. Yang, O. Alkan, S. Kim, J. L. Harris, C. J. Wilson, V. E. Myer, P. M. Finan, D. E. Root, T. M. Roberts, T. Golub, K. T. Flaherty, R. Dummer, B. L. Weber, W. R. Sellers, R. Schlegel, J. A. Wargo, W. C. Hahn, and L. A. Garraway. 2010. COT drives resistance to RAF inhibition through MAP kinase pathway reactivation. Nature 468:968-972.

46. Foster, K. W., Z. Liu, C. D. Nail, X. Li, T. J. Fitzgerald, S. K. Bailey, A. R. Frost, I. D. Louro, T. M. Townes, A. J. Paterson, J. E. Kudlow, S. M. Lobo-Ruppert, and J. M. Ruppert. 2005. Induction of KLF4 in basal keratinocytes blocks the proliferationdifferentiation switch and initiates squamous epithelial dysplasia. Oncogene 24:14911500 .

47. Mathelier, A., X. Zhao, A. W. Zhang, F. Parcy, R. Worsley-Hunt, D. J. Arenillas, S. Buchman, C. Y. Chen, A. Chou, H. Ienasescu, J. Lim, C. Shyr, G. Tan, M. Zhou, B. Lenhard, A. Sandelin, and W. W. Wasserman. 2014. JASPAR 2014: an extensively expanded and updated open-access database of transcription factor binding profiles. Nucleic Acids Res 42:D142-D147.

48. Boyd, K. E. and P. J. Farnham. 1999. Coexamination of site-specific transcription factor binding and promoter activity in living cells. Mol. Cell Biol. 19:8393-8399. 
49. Cerami, E., J. Gao, U. Dogrusoz, B. E. Gross, S. O. Sumer, B. A. Aksoy, A. Jacobsen, C. J. Byrne, M. L. Heuer, E. Larsson, Y. Antipin, B. Reva, A. P. Goldberg, C. Sander, and N. Schultz. 2012. The cBio cancer genomics portal: an open platform for exploring multidimensional cancer genomics data. Cancer Discov. 2:401-404.

50. Gao, J., B. A. Aksoy, U. Dogrusoz, G. Dresdner, B. Gross, S. O. Sumer, Y. Sun, A. Jacobsen, R. Sinha, E. Larsson, E. Cerami, C. Sander, and N. Schultz. 2013. Integrative analysis of complex cancer genomics and clinical profiles using the cBioPortal. Sci. Signal. 6:11.

51. Green, J. E., M. A. Shibata, K. Yoshidome, M. L. Liu, C. Jorcyk, M. R. Anver, J. Wigginton, R. Wiltrout, E. Shibata, S. Kaczmarczyk, W. Wang, Z. Y. Liu, A. Calvo, and C. Couldrey. 2000. The C3(1)/SV40 T-antigen transgenic mouse model of mammary cancer: ductal epithelial cell targeting with multistage progression to carcinoma. Oncogene 19:1020-1027.

52. Davies, B. R., A. Logie, J. S. McKay, P. Martin, S. Steele, R. Jenkins, M. Cockerill, S. Cartlidge, and P. D. Smith. 2007. AZD6244 (ARRY-142886), a potent inhibitor of mitogen-activated protein kinase/extracellular signal-regulated kinase kinase 1/2 kinases: mechanism of action in vivo, pharmacokinetic/pharmacodynamic relationship, and potential for combination in preclinical models. Mol. Cancer Ther 6:2209-2219.

53. Yeh, T. C., V. Marsh, B. A. Bernat, J. Ballard, H. Colwell, R. J. Evans, J. Parry, D. Smith, B. J. Brandhuber, S. Gross, A. Marlow, B. Hurley, J. Lyssikatos, P. A. Lee, J. D. Winkler, K. Koch, and E. Wallace. 2007. Biological characterization of ARRY-142886 (AZD6244), a potent, highly selective mitogen-activated protein kinase kinase 1/2 inhibitor. Clin Cancer Res 13:1576-1583.

54. Karin, M., Z. Liu, and E. Zandi. 1997. AP-1 function and regulation. Curr. Opin. Cell Biol. 9:240-246.

55. Shaulian, E. and M. Karin. 2002. AP-1 as a regulator of cell life and death. Nat. Cell Biol. 4:E131-E136.

56. Favata, M. F., K. Y. Horiuchi, E. J. Manos, A. J. Daulerio, D. A. Stradley, W. S. Feeser, D. E. Van Dyk, W. J. Pitts, R. A. Earl, F. Hobbs, R. A. Copeland, R. L. Magolda, P. A. Scherle, and J. M. Trzaskos. 1998. Identification of a novel inhibitor of mitogen-activated protein kinase kinase. J Biol. Chem. 273:18623-18632.

57. Jechlinger, M., A. Sommer, R. Moriggl, P. Seither, N. Kraut, P. Capodiecci, M. Donovan, C. Cordon-Cardo, H. Beug, and S. Grunert. 2006. Autocrine PDGFR signaling promotes mammary cancer metastasis. J Clin Invest 116:1561-1570.

58. Liu, J., S. Liao, Y. Huang, R. Samuel, T. Shi, K. Naxerova, P. Huang, W. Kamoun, R. K. Jain, D. Fukumura, and L. Xu. 2011. PDGF-D improves drug delivery and efficacy via vascular normalization, but promotes lymphatic metastasis by activating CXCR4 in breast cancer. Clin Cancer Res 17:3638-3648. 
59. Heldin, C. H. and B. Westermark. 1999. Mechanism of action and in vivo role of plateletderived growth factor. Physiol Rev. 79:1283-1316.

60. Andrae, J., R. Gallini, and C. Betsholtz. 2008. Role of platelet-derived growth factors in physiology and medicine. Genes Dev 22:1276-1312.

61. Heldin, C. H. 2013. Targeting the PDGF signaling pathway in tumor treatment. Cell Commun. Signal. 11:97.

62. Coltrera, M. D., J. Wang, P. L. Porter, and A. M. Gown. 1995. Expression of plateletderived growth factor B-chain and the platelet-derived growth factor receptor beta subunit in human breast tissue and breast carcinoma. Cancer Res 55:2703-2708.

63. Seymour, L. and W. R. Bezwoda. 1994. Positive immunostaining for platelet derived growth factor (PDGF) is an adverse prognostic factor in patients with advanced breast cancer. Breast Cancer Res Treat. 32:229-233.

64. Carvalho, I., F. Milanezi, A. Martins, R. M. Reis, and F. Schmitt. 2005. Overexpression of platelet-derived growth factor receptor alpha in breast cancer is associated with tumour progression. Breast Cancer Res 7:R788-R795.

65. Tam, W. L., H. Lu, J. Buikhuisen, B. S. Soh, E. Lim, F. Reinhardt, Z. J. Wu, J. A. Krall, B. Bierie, W. Guo, X. Chen, X. S. Liu, M. Brown, B. Lim, and R. A. Weinberg. 2013. Protein kinase $\mathrm{C}$ alpha is a central signaling node and therapeutic target for breast cancer stem cells. Cancer Cell 24:347-364.

66. Shi, H., X. Kong, A. Ribas, and R. S. Lo. 2011. Combinatorial treatments that overcome PDGFRbeta-driven resistance of melanoma cells to V600EB-RAF inhibition. Cancer Res 71:5067-5074.

67. Jurek, A., C. H. Heldin, and J. Lennartsson. 2011. Platelet-derived growth factor-induced signaling pathways interconnect to regulate the temporal pattern of Erk1/2 phosphorylation. Cell Signal. 23:280-287.

68. Heldin, C. H., A. Ostman, and L. Ronnstrand. 1998. Signal transduction via plateletderived growth factor receptors. Biochim. Biophys. Acta 1378:F79-113.

69. Fantl, W. J., J. A. Escobedo, G. A. Martin, C. W. Turck, R. M. del, F. McCormick, and L. T. Williams. 1992. Distinct phosphotyrosines on a growth factor receptor bind to specific molecules that mediate different signaling pathways. Cell 69:413-423.

70. Vigil, D., J. Cherfils, K. L. Rossman, and C. J. Der. 2010. Ras superfamily GEFs and GAPs: validated and tractable targets for cancer therapy? Nat. Rev. Cancer 10:842-857.

71. Bos, J. L., H. Rehmann, and A. Wittinghofer. 2007. GEFs and GAPs: critical elements in the control of small G proteins. Cell 129:865-877. 


\section{FIGURE LEGENDS}

Figure 1: KLF4 regulates PDGFRß expression in human and murine models of TNBC.

A. MDA-MB-231 and SUM159PT cells were transduced with lentiviral vectors encoding KLF4 shRNA or non- targeting control (Ctl). PDGFRB mRNA expression was analyzed by qRT-PCR (Columns, mean; bars, SEM; *, P < 0.05; **, $\mathrm{P}<0.01$; ***, $\mathrm{P}<0.001$ ).

B. The expression of the indicated proteins was analyzed in KLF4 knockdown cells and control cells by immunoblot. $\beta$-actin served as the loading control.

C. Quantitation of three independent experiments (immunoblots) performed as described in B (Columns, mean; bars, SD).

D. TNBC cells were transduced with a retroviral vector encoding KLF4 or else empty vector (Ctl). PDGRB mRNA levels were measured in the indicated cells.

E. Immunoblot analysis of protein expression in KLF4 overexpressing cells and control cells.

F. Quantitation of three independent experiments as performed as described in E.

G. Schematic of the human PDGFRB promoter indicating KLF4 consensus binding sites that were analyzed by chromatin immunoprecipitation (ChIP) analysis of MDA-MB-231 cells.

H. ChIP intensity levels at Site 1 and Site 2 were analyzed in MDA-MB-231 cells (left panel). To demonstrate specificity of the ChIP signal, Site $1 \mathrm{ChIP}$ intensity levels were measured in KLF4 knockdown cells and control MDA-MB-231 cells (right panel).

I. Immunoblot analysis of the expression of the indicated proteins in MDA-MB-468 and Hs578t expressing KLF4 shRNAs or a non-targeting control.

J. PDGFRB mRNA expression was analyzed in the indicated cells.

K. Spontaneoulsy arising mammary tumors were harvested from female $\mathrm{Klf}^{+/-}$- and $\mathrm{Klf}^{+/+}$C3(1)/TAg mice. Total RNA was extracted and the expression of Pdgfrb and Klf4 mRNA was analyzed. 
Figure 2: KLF4 is required for the time-dependent induction of PDGFR $\beta$ in response to MEK 1/2 inhibition.

A. TNBC cells were treated with AZD6244 $(5 \mu \mathrm{M})$ for the indicated time periods and protein expression was analyzed by immunoblot. ERK2 served as the loading control.

B. Cells treated with AZD6244 for 72 hours and PDGFRB mRNA levels were analyzed.

C. KLF4 knockdown cells and control cells were treated with AZD6244 for the indicated time periods. Protein expression was analyzed by immunoblot.

D. Similar to C, cells were treated with AZD6244 for either 0 hours or 72 hours and protein expression was analyzed by immunoblot.

E. Quantitation of PDGFR $\beta$ protein levels from three independent experiments that were performed as described in $\mathrm{C}$.

F. Quantitation of PDGFR $\beta$ expression from three independent experiments that were performed as described in D.

G. PDGFRB mRNA levels were analyzed in KLF4 knockdown cells and control cells treated with AZD6244 for the indicated time periods.

H. To analyze AP-1 transcription activity, a correlate of RAS-ERK signaling, TNBC cells were co-transfected with either an AP-1 responsive firefly luciferase (luc) construct or else a control firefly luc plasmid (vector), along with pRLTK Renilla luc, which served as an internal control. 24 hours post transfection, cells were treated with $5 \mu \mathrm{M}$ AZD6244 for the indicated time periods. Cell extracts were prepared and luc activity was analyzed as previously described $(29,42)$. 


\section{Figure 3: KLF4 opposes cMYC mediated repression of PDGFRß.}

A. ChIP analysis of cMYC in MDA-MB-231 cells on the human PDGFRB promoter.

B. The indicated cells that were treated with $5 \mu \mathrm{M}$ AZD6244 for 48 hours and ChIP analysis for cMYC (left panel) and KLF4 (right panel) was performed.

C. TNBC cells were transfected with cMYC siRNAs or a non-targeting control (siCtl). PDGFRB mRNA levels were analyzed 48 hours post-transfection.

D. The expression of the indicated proteins in siRNA transfected cells was analyzed by immunoblot.

E. KLF4 knockdown cells or control cells were transfected with the indicated cMYC siRNAs or control (denoted " -"). Protein expression was analyzed by immunoblot.

F. Quantitation of three independent experiments as performed as described in E.

G. PDGFRB mRNA levels were measured in transfected cells. 


\section{Figure 4: KLF4 promotes resistance to MEK 1/2 inhibition in TNBC cells.}

A. Cells were treated with the indicated doses of AZD6244 (top panels) or U0126 (bottom panels) for 72 hours. Viable cell number was determined using an ATP-based luminescence assay and normalized to the signal from cells treated with vehicle [(DMSO), denoted $0 \mu \mathrm{M}$ ]. The horizontal dotted line indicates $50 \%$ relative viability. Three independent experiments were performed in duplicate (bars, SEM).

B. Cells were treated with $5 \mu \mathrm{M}$ AZD6244 for 72 hours and live cell number was determined by counting. Three independent experiments were performed in duplicate (bars, SEM).

C. Cells were treated in a similar fashion as in B. Cell death was determined by trypan blue exclusion. Three independent experiments were performed in duplicate (bars, SEM). 
Figure 5: KLF4-PDGFRß regulation is critical for MEK 1/2 inhibitor resistance.

A. KLF4 knockdown cells, or shCtl cells, were transduced with a retroviral vector encoding PDGFR $\beta$, or else empty vector. Expression of the indicated proteins was analyzed by immunoblot.

B. Cells were treated with the indicated doses of AZD6244 for 72 hours and the viable cell number was determined by an ATP-based luminescence assay.

C. Cells were treated with $5 \mu \mathrm{M}$ AZD6244 for 72 hours. Cell death was analyzed by trypan blue exclusion. 


\section{Figure 6: KLF4 promotes MEK 1/2 inhibitor resistance through PDGFRß.}

The schematic shows the adaptive signaling that occurs upon MEK 1/2 inhibition in TNBC cells. When RAS-ERK signaling is not perturbed, pathway activity is maintained in a delicate homeostatic balance by ERK 1/2 dependent negative feedback loops and a basal level of RTK signaling (left panel). KLF4 opposes cMYC mediated repression and promotes PDGFR $\beta$ expression and steady-state RAS-ERK signaling (left panel and not shown). Upon MEK 1/2 inhibition, derepression of ERK 1/2 and cMYC signaling occurs, resulting in unopposed KLF4 regulation of PDGFR $\beta$ and activation of RTK signaling (right panel). These compensatory changes promote cell proliferation and survival upon MEK 1/2 inhibition. 


\section{FIGURES}

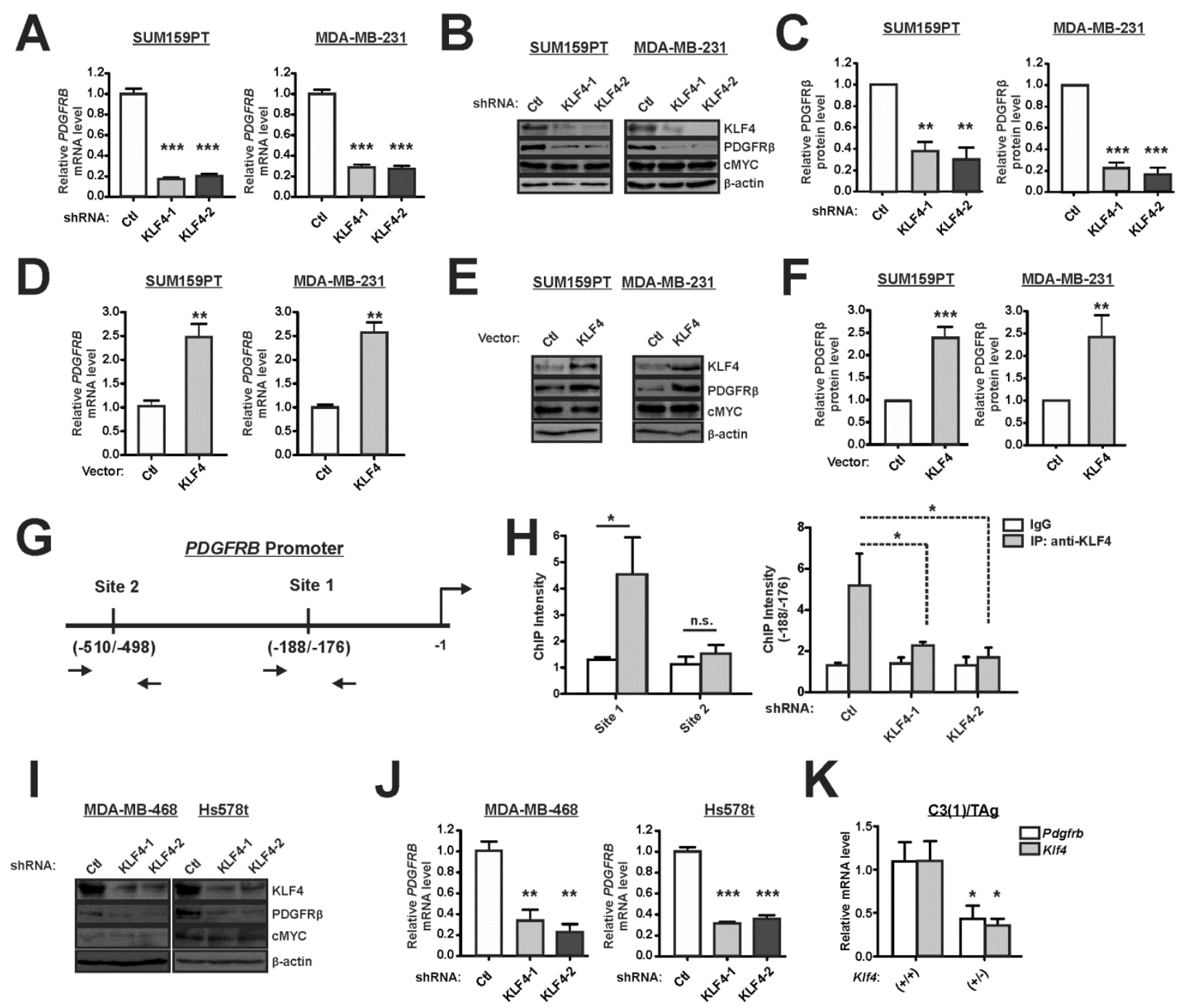

Figure 1 
A

Time (h): \begin{tabular}{llllllllllllll}
\multicolumn{1}{l}{ SUM159PT } & & \multicolumn{3}{c}{ MDA-MB-231 } \\
0 & 4 & 12 & 24 & 48 & 72 & 0 & 4 & 12 & 24 & 48 & 72
\end{tabular}

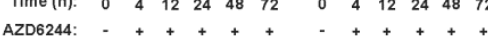

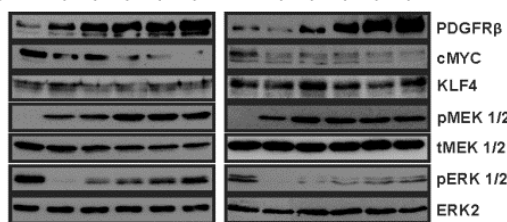

B

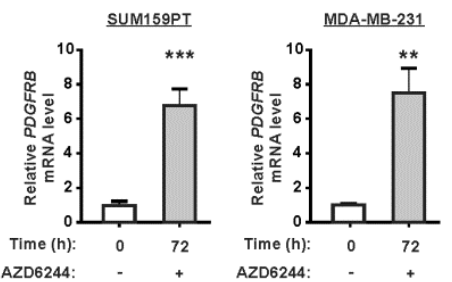

C

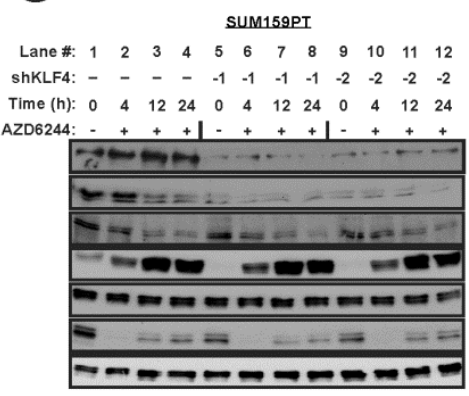

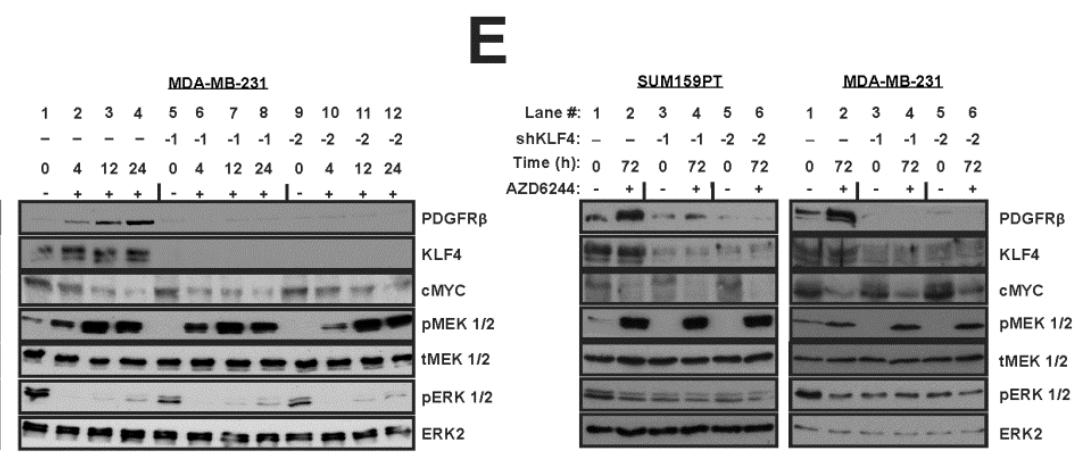

D

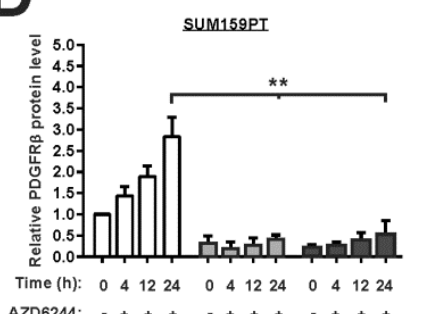

PDGFR

MEK $1 / 2$

MEK $1 / 2$ RK2
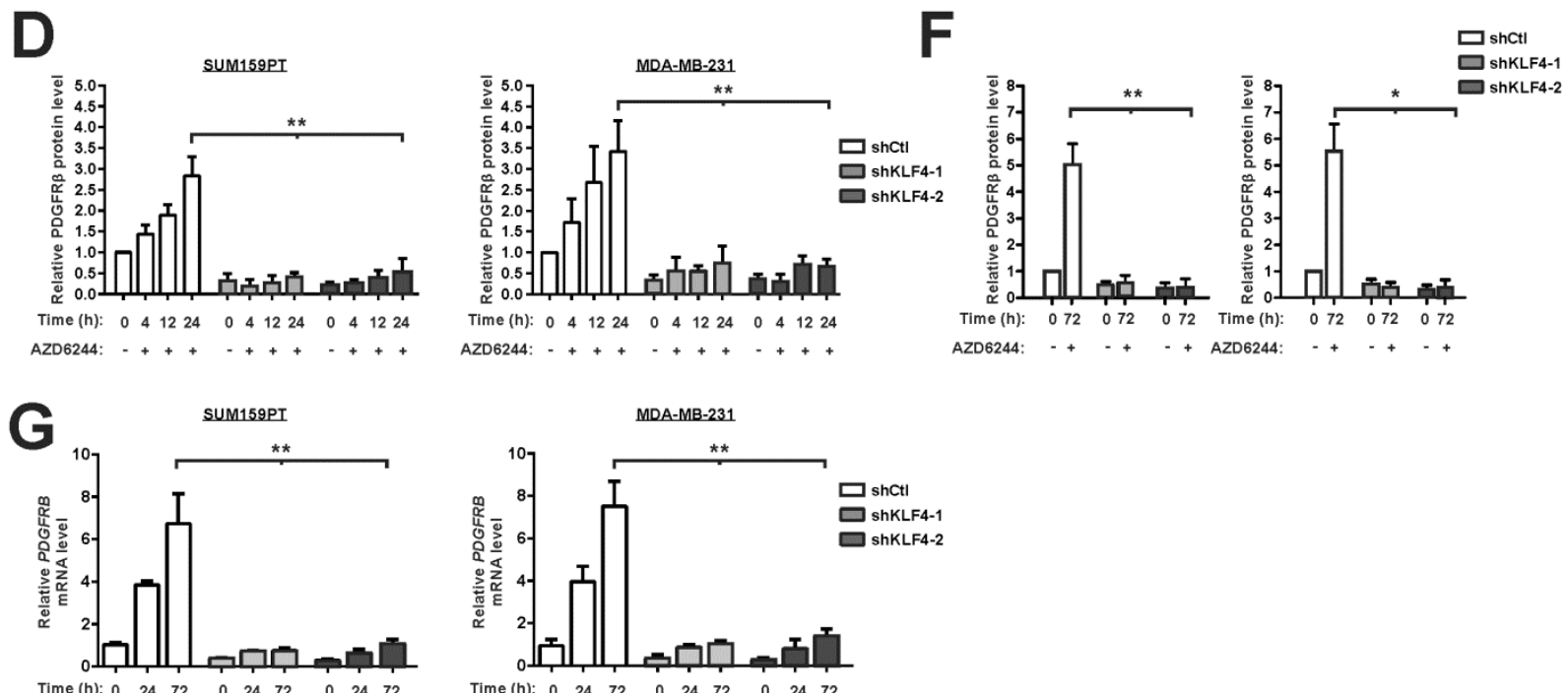

Time (h): $\begin{array}{lllllllll}0 & 24 & 72 & 0 & 24 & 72 & 0 & 24 & 72\end{array}$ $\begin{array}{cccccccccc}\text { Time (h): } & 0 & 24 & 72 & 0 & 24 & 72 & 0 & 24 & 72 \\ \text { AZD6244: } & - & + & + & - & + & + & - & + & +\end{array}$

1.
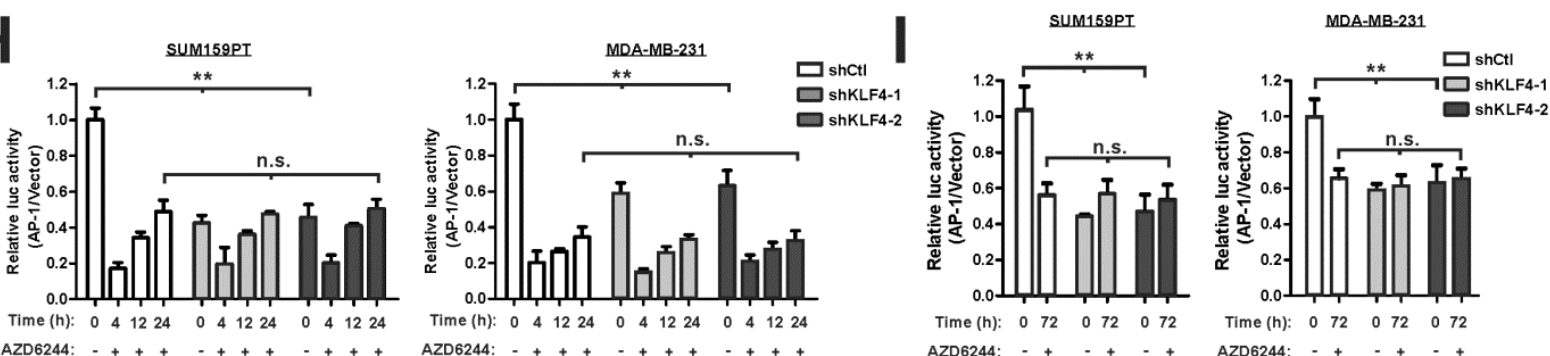

Figure 2 

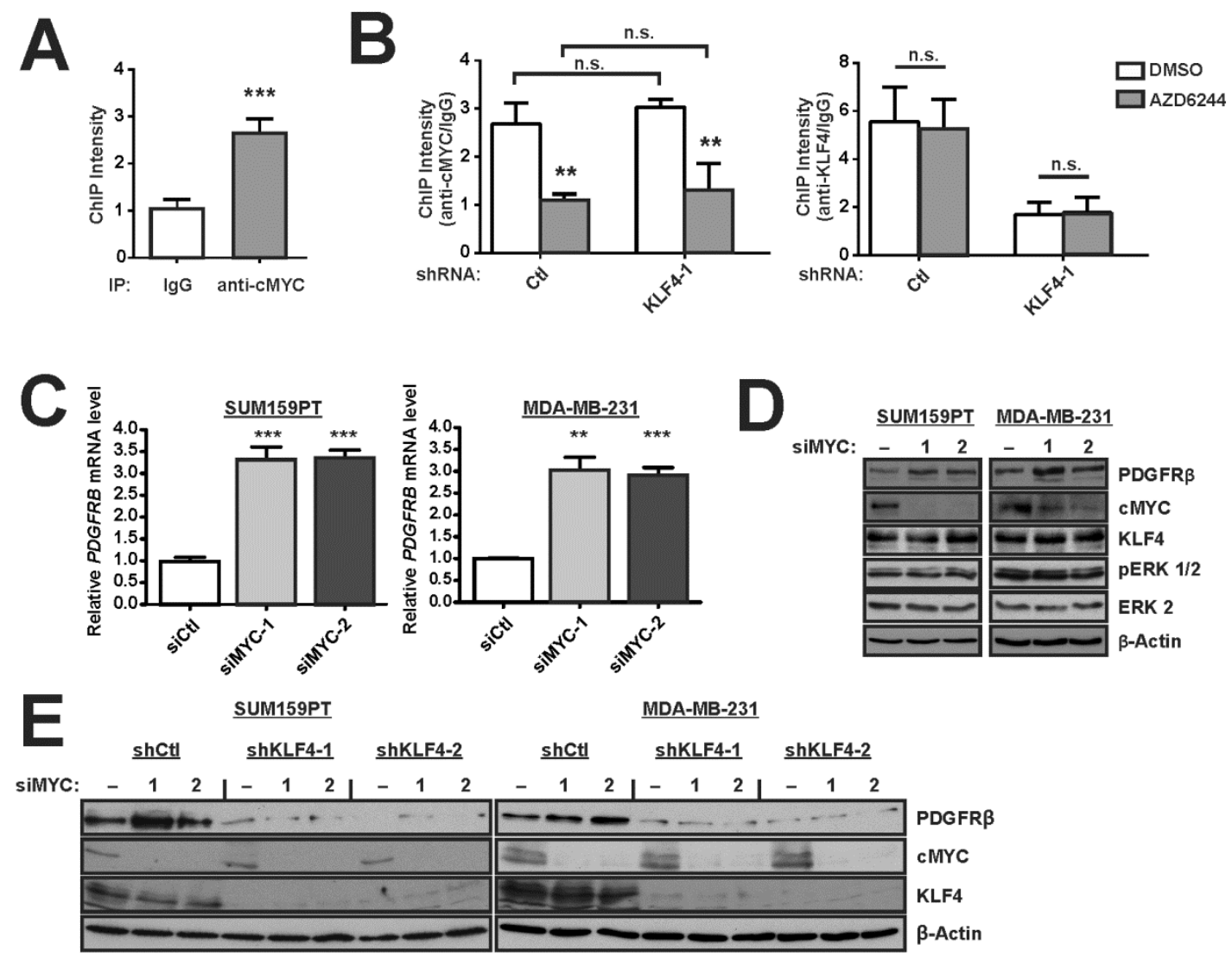

$F$
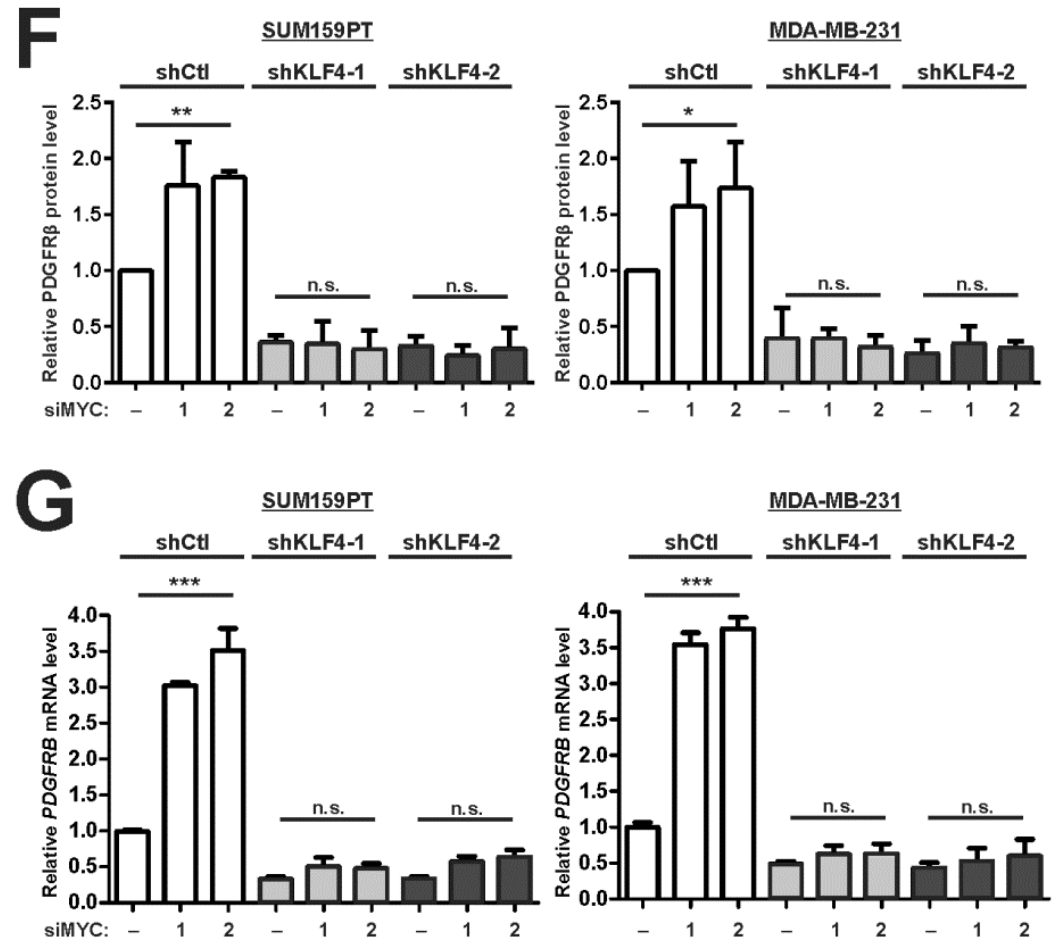

Figure 3 

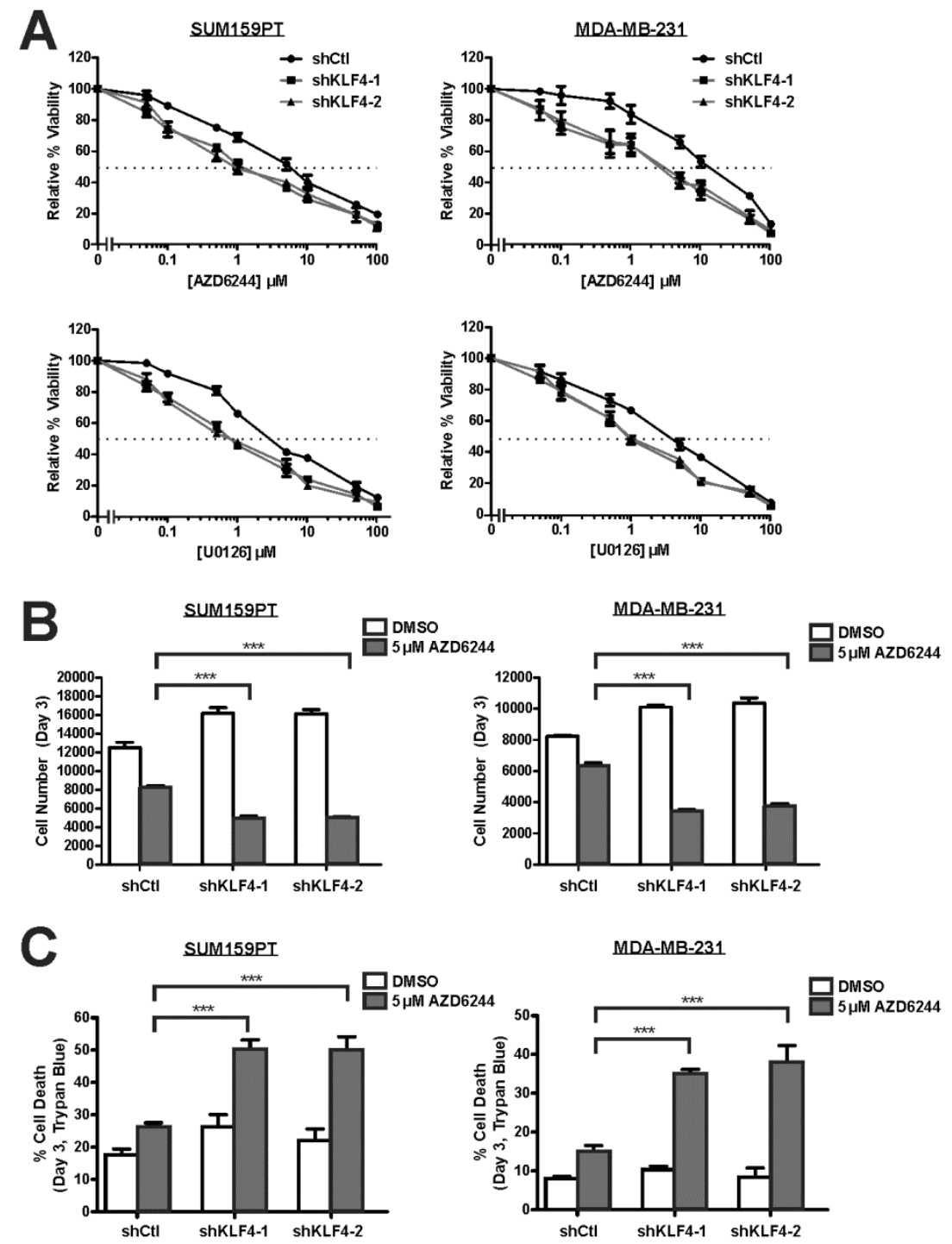

Figure 4 
A

SUM159PT

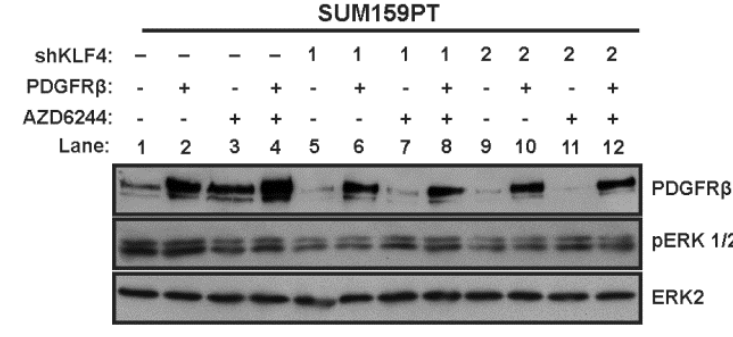

MDA-MB-231

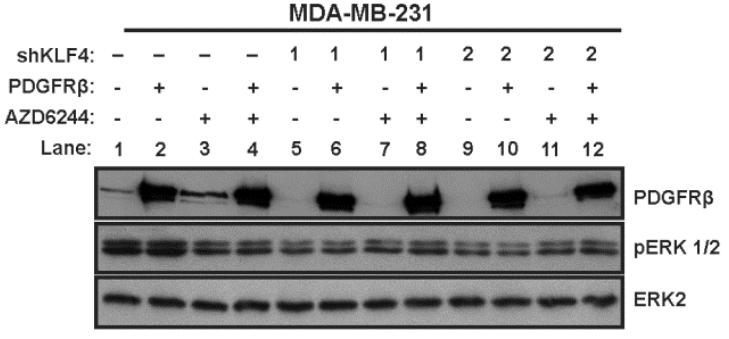

B

SUM159PT
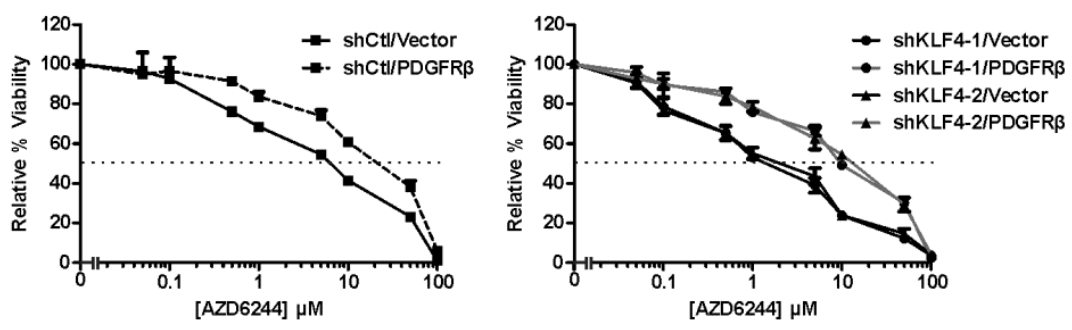

MDA-MB-231
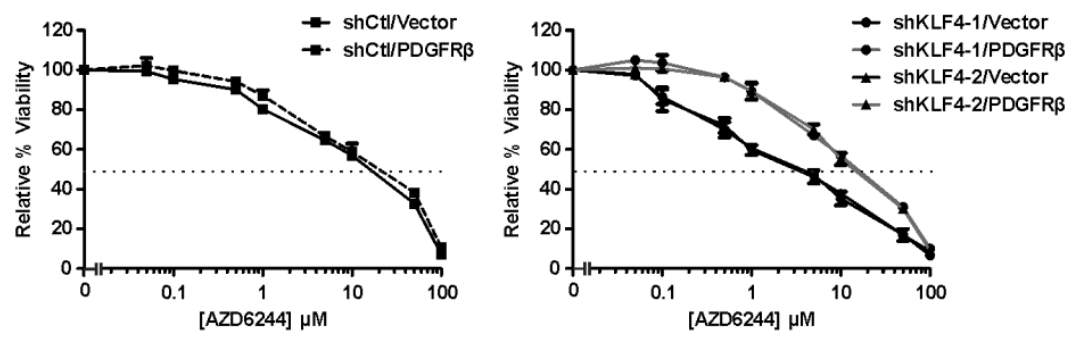

C
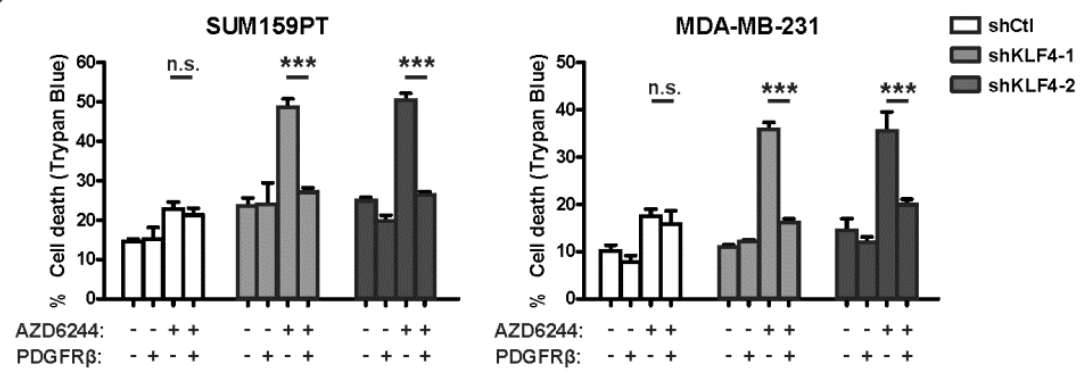

Figure 5 


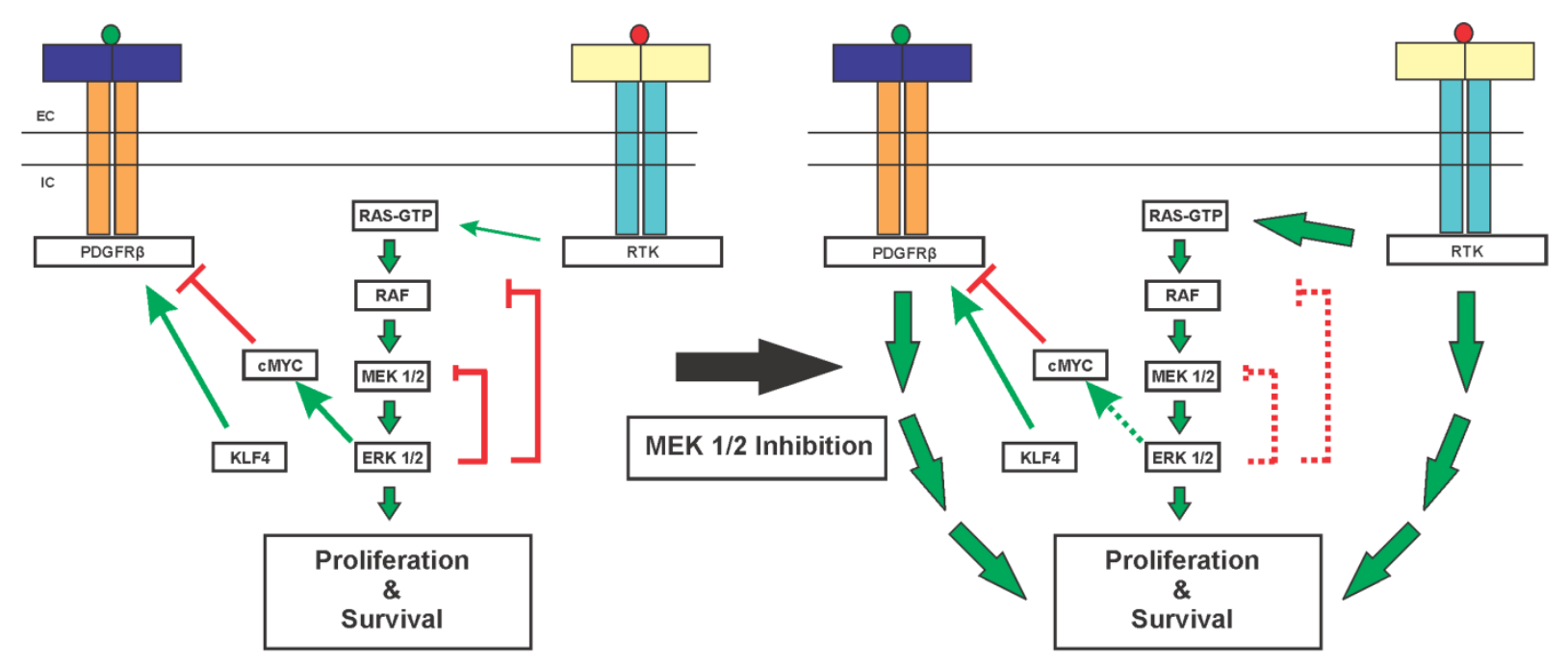

Figure 6 


\section{CHAPTER 5}

\section{SUMMARY \& DISCUSSION}

This dissertation identifies three KLF4 dependent signaling axes that strongly suggest a protumorigenic role for this zinc finger pluripotency factor in TNBC cells (Fig. 1). In addition to elucidating KLF4 function in this context, the studies presented in this dissertation provide insight into the regulation of RAS-ERK signaling by the action of GAPs (Chapter 2) (1), identify functional CSC markers in TNBCs that can potentially be therapeutically targeted (Chapter 3), and dissect the mechanisms of how resistance to targeted inhibition of MEK 1/2 arises (Chapter 4). This chapter consists of a brief summary of each of the studies presented in this dissertation, followed by a discussion of the implications that arise as a consequence of these studies. Furthermore, this chapter elaborates on how the findings of the presented studies may be extended to gain insight into designing effective anti-cancer therapies targeting the various facets of KLF4 driven protumorigenic signaling (i.e., RAS-ERK signaling, anti-apoptotic signaling).

In the first study (Chapter 2), we found that KLF4 regulates the expression of miR-206 and the well-known oncogenic miR-21 (collectively termed miR-206/21) to promote RAS-ERK signaling in TNBC cells. We identified co-targeting and suppression of the GAP, RASAl, and the NF1 GAP associated protein, SPRED1, by miR-206/21, which resulted in increased activation of the RAS-ERK signaling. Importantly, the inhibition of KLF4-miR-206/21 signaling potently suppresses RAS-ERK signaling in multiple RAS-mutant TNBC cells (MDA-MB-231$K R A S^{G 13 D}$, Hs578t $-H R A S^{G 12 D}$, and SUM159PT $-H R A S^{G 12 D}$ ) as well as in cells that exclusively harbor WT-RAS proteins. Similarly, the miR-mediated suppression of RASA1 and SPRED1 promoted RAS-ERK pathway activity regardless of RAS mutational status. These results present 
a novel role for RASA1 and SPRED1 in TNBC cells and are consistent with the newly emerging paradigm that the output of RAS-ERK signaling is dependent on the activation of WT-RAS (Fig. 2) (2-4). Therefore, this study uncovers a previously unappreciated role for GAP proteins in cells harboring RAS mutations.

Our study provides initial evidence for the protumorigenic potential of endogenous miR206 in TNBC cells. Whereas miR-206 is well characterized in regulating the differentiation of adult muscle stem cells $(5,6)$, the role of endogenous miR-206 in breast cancer is less well known. In contrast to miR-206, miR-21 is prominently upregulated in many malignancies, including in breast cancer, and promotes tumorigenesis by repressing the translation of multiple tumor suppressors, including negative regulators of RAS-ERK-AP-1 signaling (e.g., SPRY1, RASA1, and PDCD4), as well as RAS-PI3-K signaling (e.g., PTEN) (7-18). However, we found that manipulation of each miR singly yielded subtle effects on RAS-ERK signaling, thus identifying that the activity of both miR-206 and miR-21 was required for significant regulation of RAS-ERK signaling and dependent phenotypes. Extending this observation regarding collaborative signaling, the second study (Chapter 3) shows that miR-206 signaling suppresses the translation of pro-apoptotic proteins including the AP-1 inhibitor PDCD4, a well-established miR-21 targeted transcript (17-19). These studies collectively suggest that KLF4 and miR206/21 are critical regulators of cell survival in TNBC cells, especially by impacting RAS-ERK regulatory molecules. Consequently, KLF4-miR signaling promotes resistance to various cell stresses including matrix detachment, growth in immunocompromised mice, and treatment with chemotherapeutic agents.

In addition to regulating cell survival by promoting miR-206/21 expression, KLF4 endows TNBC cell resistance against targeted inhibition of RAS-ERK signaling by way of its 
transcriptional activity. KLF4 promotes the transcription of $P D G F R B$, which is functionally implicated in mediating cellular resistance against MEK 1/2 inhibitors. KLF4 antagonizes cMYC mediated repression of PDGFR $\beta$, and loss of cMYC upon RAS-ERK inhibition, results in unopposed KLF4 mediated transcription of PDGFRB. Suggesting a broader prosurvival role, we found that KLF4 collaborates with the KLF5 to promote the expression of the pro-apoptotic proteins MCL1 and BCL-XL and consequently resistance to anti-HER2 targeted therapies (20). These studies strongly suggest that KLF4 is a critical regulator of cell survival in breast cancer cells. These studies implicate that inhibiting KLF4 regulated downstream effectors such as miR206/21, PDGFR $\beta$, or proteins involved in apoptotic signaling could be beneficial in the treatment of TNBCs.

\section{INHIBITION OF miR-206/21 FOR THE TREATMENT OF TNBC}

The following sections evaluate the prospect of targeted inhibition of miR-206/21, and consequently the therapeutic re-expression of RASA1 and SPRED1, for the treatment of RASERK driven cancers such as TNBC. Additionally, the newly uncovered roles of RASA1 and SPRED1 in TNBC are presented in perspective of the expanding role of GAPs in cancer. Also considered is the therapeutic potential of targeting dysregulated GAP activity in cancer. The potential strategy of miR-206/21 inhibition for targeting RAS-ERK signaling in cancer is presented in light of another promising miR-based therapeutic strategy focused on let-7, which suppresses the translation of all three RAS isoforms. Finally, the potential for miR-206/21 
inhibition in the treatment of RAS-ERK driven cancers is discussed along with the necessary considerations required for designing effective anti-miR therapeutic agents.

\section{The expanding roles of GAPs in cancer:}

Our study shows that GAP activity mediated by RASA1 and SPRED1 (presumably through NF1-GAP) plays a tumor suppressive role in TNBC cells. These results are consistent with studies that have attributed an anti-tumorigenic role to RASA1 and SPRED1 in a variety of cancers (21-28). For example, RASA1 loss-of-function mutations occur in basal cell carcinomas (29), and loss of SPRED1 expression is observed in pediatric acute myeloid leukemia, juvenile myelomonocytic leukemia, and hepatocellular carcinoma (30-33). Of relevance to our work in TNBC, a recent study found that the downregulation of RASA1 is associated with a poor survival in patients with invasive ductal carcinoma of the breast (34). Furthermore, this study found that decreased RASA1 expression was a feature of a triple-negative phenotype, and this finding is corroborated by analysis of RNAseq data of breast tumors in which RASAl mRNA was lower in basal-like and claudin-low samples $(34,35)$. Compared to RASA1, the role of SPRED1 in breast cancer is unclear and further investigation is necessary. As SPRED1 is required for the proper membrane localization and GAP activity of NF1, it is possible that SPRED1 may function as a tumor suppressor in breast cancer $(1,28)$.

Our study supports the emerging notion that alterations in GAP expression and activity may be a critical mechanism by which RAS signaling is activated in many cancers. Indeed, germline alterations in GAP proteins result in hyperactivity of RAS dependent pathways in a variety of congenital developmental disorders (e.g., NF1 mutations in Neurofibromatosis 1, RASA1 mutations in capillary malformation-arteriovenous malformation syndrome [CM-AVM]) 
and cancer predisposition syndromes (36-41), and recent evidence suggests that somatic alterations of GAPs and/or deregulation GAP expression may be important for RAS dependent tumorigenesis, including in breast cancer $(35,42-47)$. For example, somatic mutations and/or inactivation of NF1 have been identified in a variety of cancers, including breast cancer, and found to promote resistance to targeted therapies against RAS-ERK signaling (48-51). Furthermore, epigenetic silencing of the GAP Disabled homolog 2-interacting protein (DAB2IP) frequently occurs in lung, prostate, gastrointestinal, and breast cancers, and a variety of loss-offunction alterations in RAS protein activator like 2 (RASAL2) GAP occur in luminal B subtype breast cancers and promotes mammary carcinogenesis and cancer cell metastasis in experimental models (52-57).

Additionally, GAP regulation of RAS signaling may be more complex than once expected and many questions remain to be answered. The human genome encodes at least 14 RAS GAP genes and the precise function of many of these genes is not well known $(58,59)$. Furthermore, RAS GAP genes are heterogenous in structure and these proteins share little similarity besides the GAP catalytic domain, suggesting the potential regulation of tissue and context specific processes by GAP proteins $(58,59)$. One example of this hetereogeneity is represented by the IQGAP-family of proteins, which contain a GAP domain that lacks a critical arginine residue necessary for catalysis, but nonetheless can impact RAS-ERK signaling by mediating the scaffolding of pathway components $(60,61)$. Interestingly, in contrast to the inhibitory action of other GAPs, IQGAP1 promotes RAS-ERK signaling by mediating the scaffolding of pathway components, and is thought to play a tumor promoting role in numerous cancers (62). 
Given the expanding roles of GAPs in cancer, much research focused on mechanism of the regulation of GAP expression and activity is necessary $(41,63,64)$. Unlike RAS proteins, which are mutationally activated, increasing evidence suggests that RAS GAPs are commonly dysregulated by non-genetic mechanisms, including epigenetic silencing, transcriptional repression, proteasome-mediated degradation $(41,63,64)$. Our results presented in Chapter 2 provide initial evidence that the expression of GAP proteins, and consequently GAP activity, are regulated by the action of miRs, and suggest that other non-coding RNAs could impact the expression of GAPs (1). Increasing support of this method of regulating GAPs in cancer is seen in recent studies which show that miR-mediated suppression of RASA1 promotes carcinogenesis in experimental models of colorectal and gastric cancer (65-67). In addition to these methods of GAP regulation, additional work investigating how post-translational modifications (e.g., phosphorylation of NF1) and protein-protein interactions of GAPs (e.g., the interaction of NF1 with SPRED1) affect the expression and activity of GAPs is required (41). Based on this evidence, it can be postulated that analysis of protein levels, rather than the genetic status of RAS GAPs, might more accurately indicate GAP inactivation/activation in cancer (41).

\section{Targeting RAS GAPs and RAS-ERK signaling in the treatment of cancers:}

As RAS GAP activity is either lost or rendered ineffective in cancer cells, and it is easier to develop small molecule antagonists of protein targets, rather than agonists, RAS GAP tumor suppressors are not considered classically druggable targets $(41,63,64)$. However, since RAS GAPs are often inactivated by non-mutational mechanism, there is an opportunity for developing therapeutic strategies that can restore GAP expression and activity (41). Furthermore, the novel paradigm that GEFs and GAPs can regulate the output of RAS signaling by modulating the activity of WT-RAS proteins irrespective of the presence of RAS-mutations in a single isoform 
indicates that therapeutic targeting GAPs could be effective in the treatment of RAS-signaling driven cancers $(1-4,68,69)$.

One potential method for restoring GAP activity could be through miR-based therapeutic strategies. As miR dysregulation is a prominent feature of many pathological states, the therapeutic targeting of miRs holds the promise of being a disease-specific treatment modality (70-75), including in the treatment of RAS-driven cancers where GAP expression is repressed. Furthermore, an increasing amount of evidence suggests that miRs are capable of regulating RAS-ERK signaling by targeting multiple pathway components as well as regulatory molecules $(1,10,15,18,24,76-78)$. Therefore, targeting RAS-ERK regulatory miRs could be an attractive strategy for inhibiting this important signaling pathway in cancers. Our identification of miR206/21 as critical regulators of RAS-ERK signaling and cell survival that suppress GAP activity and the expression of pro-apoptotic proteins points to in vivo silencing of these miRs as a potential therapeutic strategy in the treatment of TNBCs (1). The following sections discuss how miRs can regulate RAS-ERK signaling, and the how miR based strategies can be developed to therapeutically target this signaling pathway.

\section{Emerging paradigms in miR-regulation of RAS-ERK signaling:}

Over the past decade, we have gained considerable insight into the critical regulatory roles that miRs exert over key cancer relevant signaling networks such as the RAS-ERK pathway (70-75,79). Our knowledge of how miRs can modulate RAS-ERK pathway activation continues to grow as potential miR-mRNA regulatory networks are identified using a variety of strategies including in silico miR target prediction methods and profiling of the cellular transcriptome/proteome, and following subsequent experimental validation. Three major 
paradigms of miR-mediated RAS-ERK regulation have emerged from these studies. miRs can impact the translation of (i) core RAS-ERK pathway components (e.g., let-7 targets $H R A S$, $N R A S$, and $K R A S$ ) (76), (ii) critical pathway regulatory proteins that are required for the proper spatio-temporal control of RAS-ERK signaling (e.g., miR-206/21 collaboratively target RASAl, SPRED1, SPRY1) (1,15), and (iii) upstream drivers and downstream effector/regulatory molecules (e.g., miR-9-3p targets ITGB1, and miR-206/21 target PDCD4) (80). Recent studies have identified a large number of miRs that have been shown to regulate the RAS-ERK pathway activity in a variety of cancer contexts $(1,10,15,18,24,65,66,76-78)$.

Our work strongly suggests that miR-206/21 represents promising therapeutic targets that can be modulated in the treatment of RAS-ERK driven cancers as they regulate multiple components of this pathway (1). Therapeutic targeting of miRs for modulating RAS-ERK signaling in vivo is not unprecedented as studies focused on in vivo manipulation of let-7, a tumor suppressor miR which inhibits RAS-ERK signaling by repressing the translation of HRAS, NRAS, and KRAS and cMYC, have yielded promising results leading to the current development of let-7 based therapeutics (76,81-85). Interestingly, let-7 abrogates tumor development and RAS-ERK signaling in an autochthonous model of NSCLC driven by activated KRAS $\left(K R A S^{G 12 D}\right)(76)$. Thus, by suppressing RAS expression, let-7 can attenuate RAS-ERK signaling and dependent oncogenic phenotypes regardless of the RAS-mutation status of cancers. These studies suggest that let-7 can act as both a cancer-preventative and cancer-therapeutic agent, have led to the ongoing clinical development of let-7 supplements for the treatment of lung adenocarcinoma $(74,75)$. These studies serve as an encouraging proof of concept that miRs, such as miR-206/21 can be therapeutically targeted to modulate RAS-ERK signaling in the treatment of cancer. 


\section{The potential of in vivo inhibition of miR-206/21 for the treatment of cancer:}

Our study suggests that inhibition of miR-206/21 could be a promising therapeutic strategy in the treatment of TNBC and in other RAS-driven cancers (1). In vivo inhibition of miR-206/21 activity using anti-sense oligonucleotide based strategies could effectively attenuate RAS-ERK signaling by inducing GAP activity and repressing RAS activation and expression and upregulate the expression of the pro-apoptotic proteins PDCD4 and CX43 (Chapters 2 and 3). Furthermore, inhibition of these miRs could be effective in attenuating RAS signaling in both RAS-WT and RAS-mutant cancers.

Therapeutic targeting of miR-206/21 could offer an advantage over single kinase inhibition/small-molecule drug strategies, which do not yield durable responses due to the rapid emergence of inhibitor-resistant signaling (86-100). Suppression of miR-206/21 leads to inhibition of RAS-ERK activity, but may not impart the dramatic signaling effects of small molecule kinase inhibitors (i.e., BRAF inhibitors, MEK 1/2 inhibitors), which have been shown to induce cellular resistance mechanisms such as adaptive reprogramming of the kinome (91). miR-206/21 inhibition may not attenuate ERK 1/2 activity as acutely as MEK 1/2 inhibitors, allowing sufficient residual RAS-ERK activation to stave off the induction of cellular response mechanisms, but nevertheless potently inhibiting RAS-ERK dependent phenotypes (1).

Furthermore, in comparison to small-molecule agents, miRs are capable of regulating a wide variety targets (70-75). Therefore, another advantage of miR-206/21 inhibition would be the sensitization of cancer cells to the effects of other anti-cancer therapeutic modalities (e.g., chemotherapy, radiotherapy, etc.) and could potentiate the effects of conventional treatments. Therefore, this combinatorial approach could allow for the use of lower doses of toxic 
chemotherapeutics or radiation exposure and could potentially mitigate the adverse events associated with anti-cancer therapy.

However many more additional pre-clinical studies (both in vitro and in vivo) are required to assess the safety and efficacy of miR-206/21 inhibition in the treatment of RAS-ERK driven cancers. It will be interesting to see if anti-miR-206/21 therapy, either singly, or in combination with conventional chemotherapy, can inhibit tumorigenesis in in vitro and in vivo experimental models of TNBC. Additionally, these studies would demonstrate the dose limiting toxicities of anti-miR-206/21 therapy, and thus inform the design of future studies. Furthermore, the efficacy of inhibiting miR-206/21 in a variety of cancer models would be useful in broadening the therapeutic utility of this strategy. Future studies can also investigate other methods of inhibiting miR-206/21. For example, a recent study found that the inhibition of the nucleolar protein nucleolin in vivo, a protein which is required for the maturation of a certain subset of miRs and surprisingly is also present on the surface of breast cancer cells, using a Grich aptamer (AS1411) targeting cell surface nucleolin, could downregulate the expression of miR-21 and attenuate breast cancer cell metastasis (101). Therefore anti-miR-206 could be used in conjunction with AS1411 to therapeutically inhibit miR-206/21.

Ultimately the utility of the anti-sense oligonucleotide based therapeutic inhibition of miR-206/21 in these studies (and in future trials) hinges on ensuring the stability and the successful delivery of anti-miRs to the tumor (70-75). Fortunately, compared to small-molecule drugs, anti-sense oligonucleotide based therapeutics are amenable to many chemical modifications to enhance their pharmacokinetic/pharamcodynamic profile $(70,71,75)$. Therefore, concurrent development of synthetically modified anti-sense strategies and optimization of 
delivery methods is required for effective testing of the utility of anti-miR-206/21 in the treatment of TNBCs, and potentially, other cancers.

\section{Design of effective miR-based therapeutic strategies for targeting miRs in vivo:}

The use of anti-sense oligonucleotide-based approaches for targeting endogenous miRs (anti-miR) in vivo is an area of intense research and recently many anti-miR reagents have been developed. Generally, various chemical modifications are made to protect anti-miRs from ubiquiotously present nucleases and enhance stability $(70,71,75)$. These include (i) alterations to the 2'-carbon of the ribose sugar (i.e., substitution of 2'-OH with 2'-O-methyl [2'-O-Me], 2'-Omethoxyethyl [2'-O-MOE], or 2'-O-fluoro [2'-O-F]), (ii) selective substitution of phosphodiester bonds with phosphorothioate bonds, (iii) cholesterol/lipid conjugation to the 3 '-end of the synthetic RNA molecule (with the resulting anti-miR termed "antagomiR"), and (iv) tethering the 2'-oxygen of ribose to the 4'-carbon via a methylene bridge resulting in a locked sugar structure and thereby producing locked nucleic acids (LNAs) (Fig. 3) (70,71,75). Additionally, the ribose sugar backbone of the oligonucleotides may be replaced by a backbone comprised of either 2-(aminoethyl) glycine or six-membered morpholine rings producing peptide nucleic acids (PNAs) or morpholino antisense nucleic acids respectively, which exhibit excellent nuclease resistance and show great target specificity $(102,103)$. These examples of miR-based strategies and their chemical modifications represent the culmination of an enormous effort to discover methods to therapeutically modulate miRs. Though many of these strategies have shown promise in experimental models, methods that combine LNA or PNA technology with other chemical modifications show particular promise for clinical development, and thus may be used for antimiR-206/21 development (70,71,75). The following section describes additional considerations that must be undertaken for the development of effective miR-based therapeutics. 


\section{Obstacles that must be overcome for the successful utilization of anti-miR therapeutics:}

The successful implementation of anti-sense oligonucleotide based therapeutic strategies, such as anti-miR-206/21, in vivo is challenged several additional obstacles. First, the successful delivery of anti-miRs to the target tissue is limited by anatomical, pharmacokinetic, and pharmacodynamic barriers, which can be overcome in part by a variety of delivery methods which include (i) miR-cholesterol conjugation, (ii) liposome encapsulation, (iii) miRnanoparticle/polymer conjugation, and (iv) antibody-based methods (70,71,75). Second, antisense strategies have the potential for promiscuous miR inhibition, thereby causing off-target effects. Thus, anti-miRs must be able to discern between the many miRs may share identical/similar seed sequences with the intended target, but may target non-overlapping sets of mRNAs in a context-dependent manner $(70,71,75)$. Thus, careful consideration of target miRs must be undertaken to ensure that miR-based therapeutics can modulate the intended endogenous target with a high degree of specificity, and to minimize miR sequence associated off-target effects. Finally, the administration of anti-miRs and carrier molecules may cause deleterious consequences by miR sequence independent off-target effects. Anti-miR agents may be detected by both the innate and adaptive arms of the human immune systems, and further modification of the therapeutic components is necessary to avoid immunostimulatory off-target effects. Furthermore, anti-miR agents may alter physiological processes (e.g., blood coagulation, complement cascade activation) or may induce organ system dysfunction (e.g., hepatotoxicity, nephrotoxicity), owing to the accumulation, clearance, and excretion of these molecules $(70,71,75)$. Therefore, the consideration of these barriers is necessary for the effective utilization of anti-miR-206/21 therapy. 
A recent seminal study, which exemplifies a strategy that was developed with the aforementioned considerations in mind, described a novel miR-therapeutic delivery strategy that effectively targets tumor cells, and can be exploited for the development of anti-miR-206/21 therapy (104). This study focused on inhibiting the activity of the oncogenic miR-155 in diffuse large B-cell lymphoma (DLBCL) by using PNA anti-miRs conjugated via a derivatized cysteine residue to a $\mathrm{pH}$ dependent cell membrane penetrating peptide (i.e., $\mathrm{pH}$ low insertion peptide [pHLIP]), which preferentially inserts itself into the plasma membrane of cells that are present in acidic microenvironments, such as those associated with tumors. Upon membrane association, the reducing intracellular environment enables the dissociation of the PNA from the pHLIP peptide, resulting in free anti-miR-155, which can then target and inhibit miR-155. When administered intravenously, these modified anti-miRs were able to preferentially target and drastically inhibit the growth of DLBCL lymphoma xenografts and metastases in immunocompromised mice with comparable efficacy of the standard of care treatment for DLBCL (CHOP chemotherapy regimen). However, in contrast to conventional chemotherapy, pHLIP-anti-miR-155, did not induce myelosuppression, suggesting specificity of this new therapeutic strategy. Furthermore, pHLIP-anti-miR-155 administration was well tolerated both immunocompromised and immune competent mice and did not seem to cause immune suppression. This strategy of anti-miR delivery is currently being investigated for the therapeutic inhibition of additional miRs, including miR-21, and may aide in the development of miR206/21 inhibition strategies for the treatment of cancer. 


\section{THE USE OF SMALL MOLECULE INHIBITION OF RAS-ERK SIGNALING IN THE TREATMENT OF CANCER}

Over the last two decades there has been a tremendous effort to identify and modulate the activity of signaling networks that endow cancer cells with malignant properties. This large body of work has led to the development of potent kinase inhibitors targeting many of these cancer relevant signaling pathways, including the RAS-ERK pathway (105-111). However, the use of RAS-ERK inhibitors as single agents in the treatment of cancer has largely been unsuccessful in clinical trials as resistance develops rapidly through adaptive reprogramming of cell signaling, a phenomenon observed in response to inhibition of other signaling pathways (86-91). These failures have prompted the consideration of preemptive co-targeting of compensatory cell signaling (e.g., mediated by PI3-K-AKT, RTKs, anti-apoptotic proteins, etc.] to yield rational drug combinations that can produce durable therapeutic efficacy $(80,90,96,112-114)$. Furthermore, these studies have underscored a critical role for transcription factors and epigenetic regulators in functioning as sensors of cell signaling activity and effectors that can orchestrate resistance programs when certain pathways are inhibited (87-90).

The induction of PDGFR $\beta$ is a prominent prosurvival response that is observed upon MEK 1/2 inhibition in a variety of contexts, and co-targeting this RTK along with RAS-ERK signaling to extend therapeutic efficacy has been suggested $(90,94,115)$. In agreement with previous studies, we identified that PDGFR $\beta$ is a critical KLF4-dependent resistance factor that promotes TNBC cell survival against MEK 1/2 inhibition (Chapter 4). Though neither PDGFR $\beta$ nor KLF4 were required for the re-emergence of RAS-ERK signaling following MEK 1/2 
inhibition, both proteins were essential for TNBC cell survival following drug treatment in vitro. Therefore, our results further identify a prosurvival role for KLF4, and suggest that co-targeting KLF4 and/or PDGFR $\beta$ and RAS-ERK signaling might be efficacious compared to MEK 1/2 inhibition alone in the treatment of TNBC.

\section{Concerns for co-targeting resistance mechanisms along with RAS-ERK signaling:}

However, there are two salient concerns that arise from our results (Chapter 4) and similar studies that identify MEK 1/2 inhibitor resistance factors. First, the in vivo targeting of regulators of transcription in the treatment of cancer is in its infancy, and therefore do not represent "clinic-ready" therapeutic modalities. Even for factors that can be successfully targeted (e.g., some chromatin modifier proteins), pharmacological modulation of these factors might be extremely toxic and carry the potential for off-target effects, thus necessitating the establishment of therapeutic windows for the utilization of such agents. A recent study served as a proof of concept that co-inhibiting cellular epigenetic machinery (BET-bromodomains with the agent JQ1) along with HER2 signaling (with lapatinib) could effectively block the reflexive inhibitor induced reprogramming of the kinome in HER2 ${ }^{+}$breast cancer cells (113). A second concern is that co-inhibition of resistance factors such as RTKs, in addition to RAS-ERK signaling, could induce unique resistance programs that may require further tailoring of the therapeutic strategies. For example, combinatorial inhibition of PDGFR $\beta$ and MEK $1 / 2$ could result in novel/unexpected reprogramming of cellular signaling which could confer resistance $(91,113)$. It may not be feasible to constantly monitor tumors for driving signaling/genetic alterations that would confer resistance to these targeted therapeutic strategies. These concerns suggest the reevaluation of RAS-ERK inhibition (and more broadly, kinase inhibition) strategies in the treatment of cancers. 


\section{Using RAS-ERK inhibitors in combinatorial anti-cancer therapies:}

As an increasing amount of evidence suggests that single-kinase inhibition strategies are prone to failure despite the heavy reliance of cancer cells on key signaling pathways, we must reconsider the utilization of therapeutic regimens predominantly consisting of targeted smallmolecule agents $(90,91,113)$. One possible role for kinase inhibitors, such as RAS-ERK (and RAS-PI3-K) inhibitors, is as chemo- or radiosensitization agents. Numerous studies show that these pathways are critical for cell survival in response to cytotoxic therapies and radiotherapy, thus these agents could sensitize cancer cells to the effects of conventional therapeutic modalities and be beneficial in the treatment of a wide variety of cancers (116-122). This use of RAS-ERK pathway inhibitors could allow for the design of treatment regimens with lower doses of cytotoxic chemotherapy and/or radiotherapy and improve the manageability of anti-cancer regimens. As a consequence of potential additive/synergistic action, combinatorial therapeutic strategies could lower the dose of RAS-ERK inhibitors required, and thereby possibly reduce the onset/magnitude of inhibitor reprogrammed signaling, which is thought to be a function of inhibitor dose. Furthermore, these combination therapies may be used concurrently with agents directed at apoptotic signaling (e.g., inhibition of BCL-XL or MCL1) to further potentiate antitumor activity $(20,114)$. In addition to numerous published pre-clinical trials, the studies presented in Chapters 2 and 3 serve as proof of concept that combined targeting of RAS-ERK signaling, apoptotic signaling, and conventional chemotherapeutics can be a beneficial therapeutic strategy that should be further investigated. 


\section{The evaluation of the efficacy of RAS-ERK inhibitors in clinical trials:}

There are currently $\sim 180$ clinical trials in various phases that are evaluating the safety and efficacy of MEK $1 / 2$ and/or BRAF inhibitors in the treatment of cancers (https://clinicaltrials.gov/). Though a majority of these clinical trials are focused on the investigating the use of these agents as monotherapies, a growing number of trials have started to determine whether RAS-ERK inhibitors could be used in combination regimens - an idea in line with the potential chemo- /radio-sensitization role of these agents. However, the method of evaluating the therapeutic potential of RAS-ERK inhibitors in cancer treatment in current clinical trials may be flawed, and could underestimate utility of these agents. Current clinical trials test the safety (and then the efficacy) of targeted agents in patients with advanced stage disease that is refractory to the standard of care therapy, but this might not be the appropriate setting for the testing of these therapies (123-126). Furthermore, there is a strong possibility that RAS-ERK inhibitors would be more efficacious in the early stage setting, and thus, these agents need to be evaluated in neo-adjuvant chemotherapeutic trials, which offer advantages over trials conducted in the adjuvant setting (123-126). Importantly, neo-adjuvant trials offer a finite therapeutic window in which the efficacy of a treatment can be evaluated. Furthermore, compared to the current trial design, it is possible that in neo-adjuvant trials that enrolled patients would have lower stage disease, which would be more amenable to therapies that target cell proliferation (i.e., RAS-ERK inhibitors). Finally, in the neo-adjuvant setting, patient tissue can be harvested upon surgery can be used for monitoring the emergence of cancer cell resistance to the therapeutic agent, and may inform how the disease is managed in the adjuvant setting. Therefore, new methods of testing the efficacy of RAS-ERK inhibitors, and more generally, single-kinase 
inhibitors, may better allow for implementing these therapeutic strategies in the treatment of cancers.

\section{CONCLUSIONS}

The work presented in this dissertation identifies three examples of KLF4 dependent protumorigenic signaling in TNBCs. Endogenous KLF4 is a critical mediator of TNBC tumor cell survival by way of regulating the level of oncogenic miRs, pro-apoptotic proteins, and RASERK-AP-1 pathway activity. Our analysis of KLF4 dependent RAS-ERK signaling uncovers a novel role for GAP proteins in regulating the activation of WT-RAS proteins, and further extends an emerging paradigm for the regulation of RAS-ERK signaling in RAS-mutant cells. Collectively, these results strongly suggest a novel role for KLF4 in enforcing malignant properties in TNBC cells. Future research stemming from our findings should investigate whether KLF4 regulates the activity of other RAS-dependent prosurvival pathways (such as PI3K-AKT) and whether KLF4 modulates RAS-ERK signaling in other normal and disease contexts. Furthermore, the role of KLF4 in the generation of spontaneously arising breast tumors and promoting chemosensitivity in vivo must be further examined. Resolution of these questions would greatly broaden our understanding of the role of KLF4 in cancer. Our findings suggest that therapeutic targeting of KLF4 dependent signaling could be efficacious in the treatment of TNBCs as it would target critical protumorigenic signaling pathways. 


\section{FIGURES}

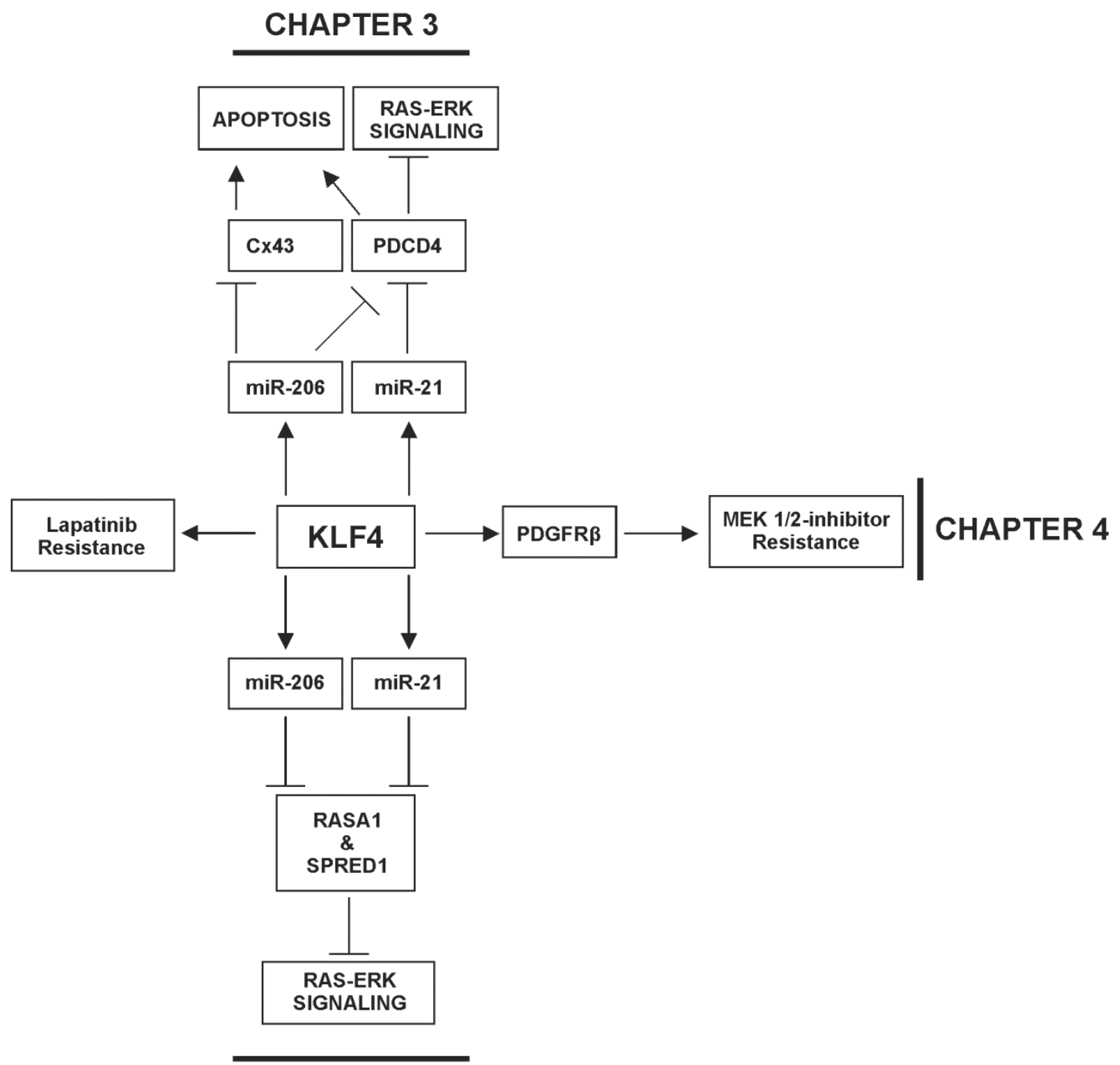

CHAPTER 2

Figure 1: This dissertation presents three studies which identify KLF4-mediated protumorigenic signaling in TNBC cells. KLF4 promotes the expression of miR-206 and miR-21 and RAS-ERK signaling through the miR-mediated repression of GAP activity by suppressing the translation RASA1 and SPRED1 (Chapter 2). Furthermore, miR-206 represses the translation of CX43 and the well-established miR-21 targeted transcript PDCD4 to confer resistance to cell death (Chapter 3). Finally, KLF4 promotes the transcription of PDGFR $\beta$, and functions as a prosurvival factor in response to MEK 1/2-inhibitor treatment (Chapter 4). Also shown is the prosurvival role of KLF4 in mediating lapatinib resistance (20) 

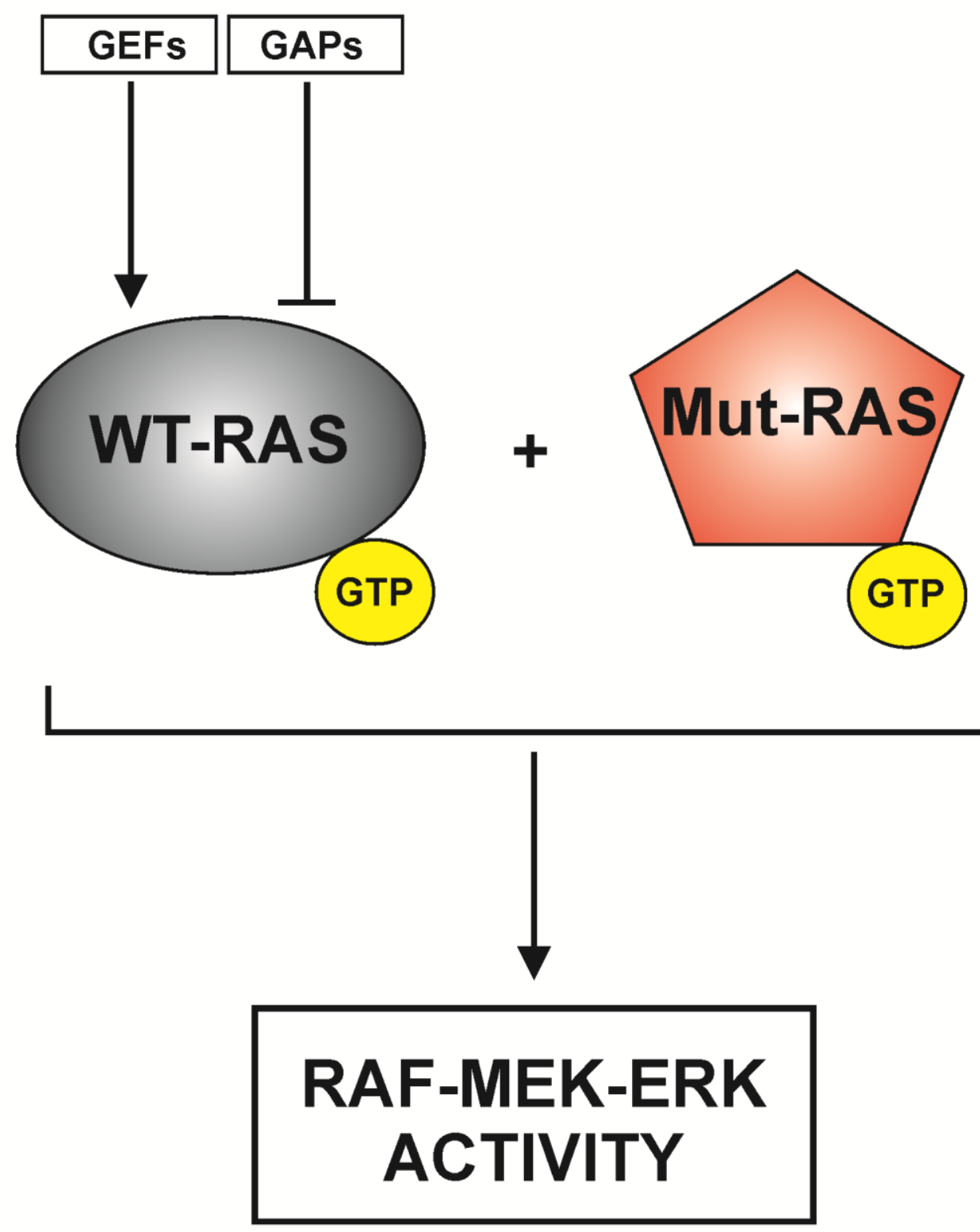

Figure 2: An emerging paradigm in RAS-signaling. The output of RAS-ERK signaling is determined by contributions from the activity of wild-type (WT-RAS) and mutant (Mut-RAS) proteins. Whereas, the activation (GTP-binding) of WT-RAS proteins is tightly regulated by GEFs and GAPs, the activation of Mut-RAS proteins is resistant to the action of these factors. By modulating WT-RAS-GTP levels, GEFs and GAPs can impact RAS-ERK signaling in cells harboring RAS mutations (1-4). 
a Chemical modifications

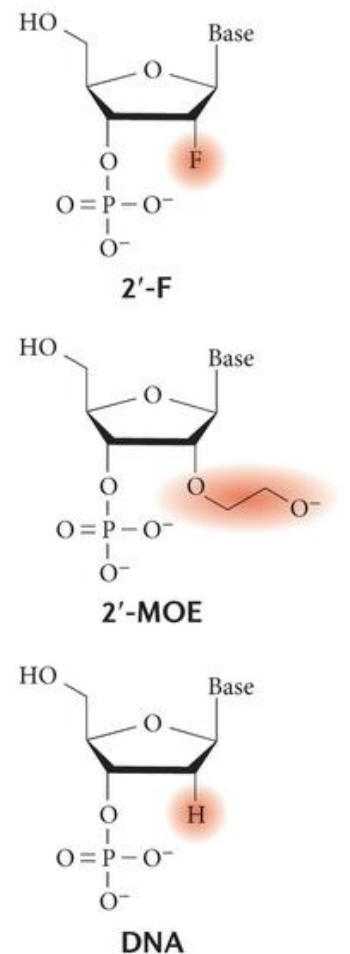

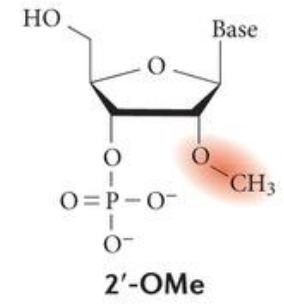

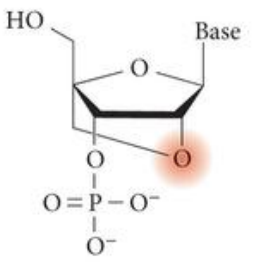

LNA

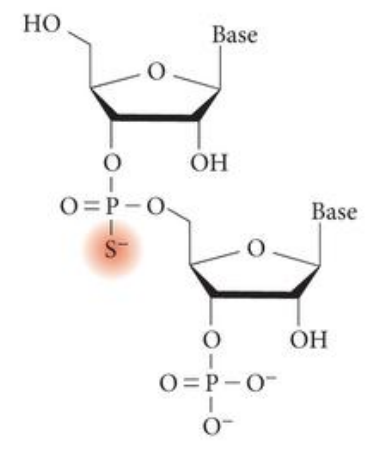

Phosphorothioate b Design of anti-miR
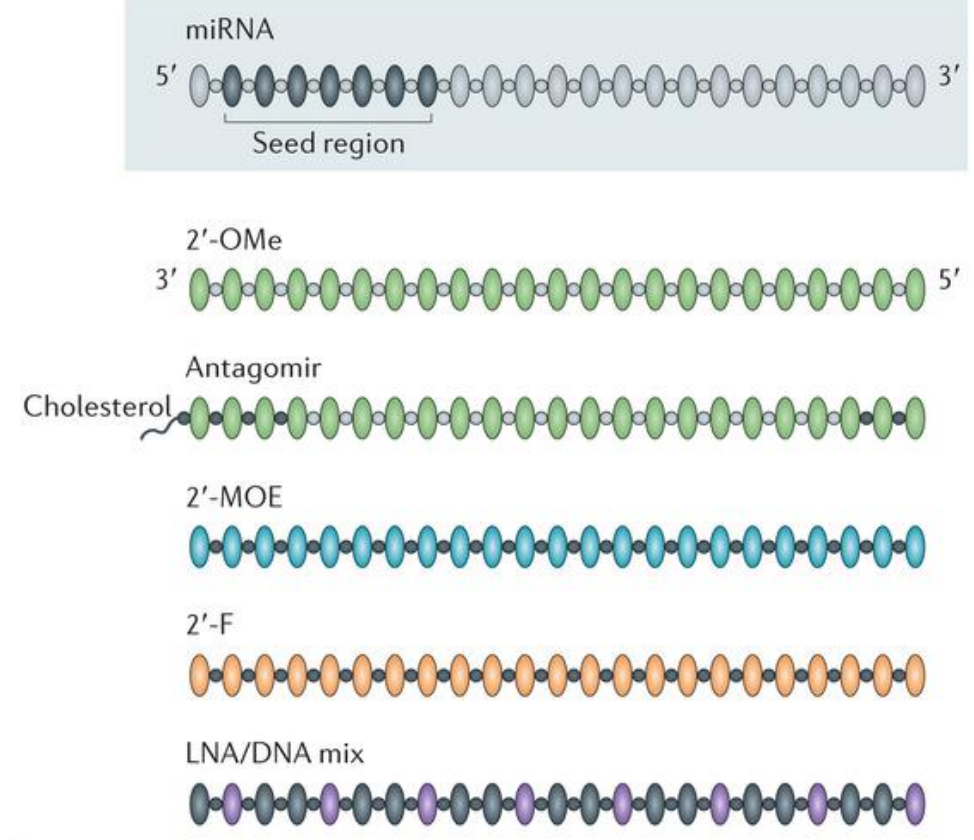

Tiny LNAs

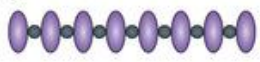

ODNA O2'-OMe O2'-MOE O2'-F O LNA - Phosphodiester bond • Phosphorothioate bond

Nature Reviews | Drug Discovery

Figure 3: Chemical modifications of anti-miR therapeutics. (A) Chemical structures show alterations to the 2'-carbon of the ribose sugar including substitution of 2'-fluoro (2'-F), 2'-OH with 2'-O-methyl (2'-O-Me), 2'-O-methoxyethyl (2'-O-MOE). Also shown are the locked nucleic acid (LNA) conformation - tethering the 2'-oxygen of ribose to the 4'-carbon via a methylene bridge, and structures of DNA and phosphorothioate linkages. (B) The design of numerous anti-miR therapeutics is shown. AntagomiRs are chemically modified anti-miRs that are conjugated to cholesterol at the 3'-end. Adapted from Li Z. and Rana T.M., Nat. Rev. Drug. Disc., 2014 (75) 


\section{REFERENCES}

1. Sharma, S. B., C. C. Lin, M. K. Farrugia, S. L. McLaughlin, E. J. Ellis, K. M. Brundage, M. A. Salkeni, and J. M. Ruppert. 2014. MicroRNAs 206 and 21 cooperate to promote RAS-extracellular signal-regulated kinase signaling by suppressing the translation of RASA1 and SPRED1. Mol. Cell Biol. 34:4143-4164.

2. Grabocka, E., Y. Pylayeva-Gupta, M. J. Jones, V. Lubkov, E. Yemanaberhan, L. Taylor, H. H. Jeng, and D. Bar-Sagi. 2014. Wild-type H- and N-Ras promote mutant K-Rasdriven tumorigenesis by modulating the DNA damage response. Cancer Cell 25:243-256.

3. Jeng, H. H., L. J. Taylor, and D. Bar-Sagi. 2012. Sos-mediated cross-activation of wildtype Ras by oncogenic Ras is essential for tumorigenesis. Nat. Commun. 3:1168.

4. Young, A., D. Lou, and F. Mccormick. 2013. Oncogenic and wild-type Ras play divergent roles in the regulation of mitogen-activated protein kinase signaling. Cancer Discov. 3:112-123.

5. Liu, N., A. H. Williams, J. M. Maxeiner, S. Bezprozvannaya, J. M. Shelton, J. A. Richardson, R. Bassel-Duby, and E. N. Olson. 2012. microRNA-206 promotes skeletal muscle regeneration and delays progression of Duchenne muscular dystrophy in mice. J. Clin. Invest 122:2054-2065.

6. Kim, H. K., Y. S. Lee, U. Sivaprasad, A. Malhotra, and A. Dutta. 2006. Muscle-specific microRNA miR-206 promotes muscle differentiation. J. Cell Biol. 174:677-687.

7. Zhu, S., M. L. Si, H. Wu, and Y. Y. Mo. 2007. MicroRNA-21 targets the tumor suppressor gene tropomyosin 1 (TPM1). J Biol Chem 282:14328-14336.

8. Zhu, S., H. Wu, F. Wu, D. Nie, S. Sheng, and Y. Y. Mo. 2008. MicroRNA-21 targets tumor suppressor genes in invasion and metastasis. Cell Res. 18:350-359.

9. Zhang, J. G., J. J. Wang, F. Zhao, Q. Liu, K. Jiang, and G. H. Yang. 2010. MicroRNA-21 (miR-21) represses tumor suppressor PTEN and promotes growth and invasion in nonsmall cell lung cancer (NSCLC). Clin. Chim. Acta 411:846-852.

10. Wickramasinghe, N. S., T. T. Manavalan, S. M. Dougherty, K. A. Riggs, Y. Li, and C. M. Klinge. 2009. Estradiol downregulates miR-21 expression and increases miR-21 target gene expression in MCF-7 breast cancer cells. Nucleic Acids Res. 37:2584-2595.

11. Volinia, S., G. A. Calin, C. G. Liu, S. Ambs, A. Cimmino, F. Petrocca, R. Visone, M. Iorio, C. Roldo, M. Ferracin, R. L. Prueitt, N. Yanaihara, G. Lanza, A. Scarpa, A. Vecchione, M. Negrini, C. C. Harris, and C. M. Croce. 2006. A microRNA expression signature of human solid tumors defines cancer gene targets. Proc Natl Acad Sci U S A 103:2257-2261. 
12. Meng, F., R. Henson, H. Wehbe-Janek, K. Ghoshal, S. T. Jacob, and T. Patel. 2007. MicroRNA-21 regulates expression of the PTEN tumor suppressor gene in human hepatocellular cancer. Gastroenterology 133:647-658.

13. Ma, X., M. Kumar, S. N. Choudhury, L. E. Becker Buscaglia, J. R. Barker, K. Kanakamedala, M. F. Liu, and Y. Li. 2011. Loss of the miR-21 allele elevates the expression of its target genes and reduces tumorigenesis. Proc. Natl. Acad. Sci. U. S. A 108:10144-10149.

14. Iorio, M. V., M. Ferracin, C. G. Liu, A. Veronese, R. Spizzo, S. Sabbioni, E. Magri, M. Pedriali, M. Fabbri, M. Campiglio, S. Menard, J. P. Palazzo, A. Rosenberg, P. Musiani, S. Volinia, I. Nenci, G. A. Calin, P. Querzoli, M. Negrini, and C. M. Croce. 2005. MicroRNA gene expression deregulation in human breast cancer. Cancer Res. 65:70657070.

15. Hatley, M. E., D. M. Patrick, M. R. Garcia, J. A. Richardson, R. Bassel-Duby, R. E. van, and E. N. Olson. 2010. Modulation of K-Ras-dependent lung tumorigenesis by MicroRNA-21. Cancer Cell 18:282-293.

16. Gabriely, G., T. Wurdinger, S. Kesari, C. C. Esau, J. Burchard, P. S. Linsley, and A. M. Krichevsky. 2008. MicroRNA 21 promotes glioma invasion by targeting matrix metalloproteinase regulators. Mol. Cell Biol. 28:5369-5380.

17. Frankel, L. B., N. R. Christoffersen, A. Jacobsen, M. Lindow, A. Krogh, and A. H. Lund. 2008. Programmed cell death 4 (PDCD4) is an important functional target of the microRNA miR-21 in breast cancer cells. J Biol. Chem. 283:1026-1033.

18. Asangani, I. A., S. A. Rasheed, D. A. Nikolova, J. H. Leupold, N. H. Colburn, S. Post, and H. Allgayer. 2008. MicroRNA-21 (miR-21) post-transcriptionally downregulates tumor suppressor Pdcd4 and stimulates invasion, intravasation and metastasis in colorectal cancer. Oncogene 27:2128-2136.

19. Bhatti, I., A. Lee, V. James, R. I. Hall, J. N. Lund, C. Tufarelli, D. N. Lobo, and M. Larvin. 2011. Knockdown of microRNA-21 inhibits proliferation and increases cell death by targeting programmed cell death 4 (PDCD4) in pancreatic ductal adenocarcinoma. J. Gastrointest. Surg. 15:199-208.

20. Farrugia, M. K., S. B. Sharma, C. C. Lin, S. L. McLaughlin, D. B. Vanderbilt, A. G. Ammer, M. A. Salkeni, P. Stoilov, Y. M. Agazie, C. J. Creighton, and J. M. Ruppert. 2015. Regulation of anti-apoptotic signaling by Krüppel-like factors 4 and 5 mediates lapatinib resistance in breast cancer. Cell Death. Dis. 6:e1699.

21. Calvisi, D. F., S. Ladu, E. A. Conner, D. Seo, J. T. Hsieh, V. M. Factor, and S. S. Thorgeirsson. 2011. Inactivation of Ras GTPase-activating proteins promotes unrestrained activity of wild-type Ras in human liver cancer. J. Hepatol. 54:311-319.

22. Hu, X., H. M. Stern, L. Ge, C. O'Brien, L. Haydu, C. D. Honchell, P. M. Haverty, B. A. Peters, T. D. Wu, L. C. Amler, J. Chant, D. Stokoe, M. R. Lackner, and G. Cavet. 2009. 
Genetic alterations and oncogenic pathways associated with breast cancer subtypes. Mol. Cancer Res. 7:511-522.

23. Johannsdottir, H. K., G. Jonsson, G. Johannesdottir, B. A. Agnarsson, H. Eerola, A. Arason, P. Heikkila, V. Egilsson, H. Olsson, O. T. Johannsson, H. Nevanlinna, A. Borg, and R. B. Barkardottir. 2006. Chromosome 5 imbalance mapping in breast tumors from BRCA1 and BRCA2 mutation carriers and sporadic breast tumors. Int. J. Cancer 119:1052-1060.

24. Sun, D., F. Yu, Y. Ma, R. Zhao, X. Chen, J. Zhu, C. Y. Zhang, J. Chen, and J. Zhang. 2013. MicroRNA-31 activates the RAS pathway and functions as an oncogenic MicroRNA in human colorectal cancer by repressing RAS p21 GTPase activating protein 1 (RASA1). J. Biol. Chem. 288:9508-9518.

25. Brems, H., M. Chmara, M. Sahbatou, E. Denayer, K. Taniguchi, R. Kato, R. Somers, L. Messiaen, S. S. De, J. P. Fryns, J. Cools, P. Marynen, G. Thomas, A. Yoshimura, and E. Legius. 2007. Germline loss-of-function mutations in SPRED1 cause a neurofibromatosis 1-like phenotype. Nat. Genet. 39:1120-1126.

26. McClatchey, A. I. and K. Cichowski. 2012. SPRED proteins provide a NF-ty link to Ras suppression. Genes Dev. 26:1515-1519.

27. Miyoshi, K., T. Wakioka, H. Nishinakamura, M. Kamio, L. Yang, M. Inoue, M. Hasegawa, Y. Yonemitsu, S. Komiya, and A. Yoshimura. 2004. The Sprouty-related protein, Spred, inhibits cell motility, metastasis, and Rho-mediated actin reorganization. Oncogene 23:5567-5576.

28. Stowe, I. B., E. L. Mercado, T. R. Stowe, E. L. Bell, J. A. Oses-Prieto, H. Hernandez, A. L. Burlingame, and F. Mccormick. 2012. A shared molecular mechanism underlies the human rasopathies Legius syndrome and Neurofibromatosis-1. Genes Dev. 26:14211426.

29. Friedman, E., P. V. Gejman, G. A. Martin, and F. Mccormick. 1993. Nonsense mutations in the C-terminal SH2 region of the GTPase activating protein (GAP) gene in human tumours. Nat. Genet. 5:242-247.

30. Pasmant, E., B. Gilbert-Dussardier, A. Petit, L. B. de, A. Luscan, A. Gruber, H. Lapillonne, C. Deswarte, P. Goussard, I. Laurendeau, B. Uzan, F. Pflumio, F. Brizard, P. Vabres, I. Naguibvena, S. Fasola, F. Millot, F. Porteu, D. Vidaud, J. Landman-Parker, and P. Ballerini. 2014. SPRED1, a RAS MAPK pathway inhibitor that causes Legius syndrome, is a tumour suppressor downregulated in paediatric acute myeloblastic leukaemia. Oncogene.

31. Yoshida, T., T. Hisamoto, J. Akiba, H. Koga, K. Nakamura, Y. Tokunaga, S. Hanada, H. Kumemura, M. Maeyama, M. Harada, H. Ogata, H. Yano, M. Kojiro, T. Ueno, A. Yoshimura, and M. Sata. 2006. Spreds, inhibitors of the Ras/ERK signal transduction, are dysregulated in human hepatocellular carcinoma and linked to the malignant phenotype of tumors. Oncogene 25:6056-6066. 
32. Batz, C., H. Hasle, E. Bergstrasser, van den Heuvel-Eibrink MM, M. Zecca, C. M. Niemeyer, and C. Flotho. 2010. Does SPRED1 contribute to leukemogenesis in juvenile myelomonocytic leukemia (JMML)? Blood 115:2557-2558.

33. Pasmant, E., P. Ballerini, H. Lapillonne, C. Perot, D. Vidaud, G. Leverger, and J. Landman-Parker. 2009. SPRED1 disorder and predisposition to leukemia in children. Blood 114:1131.

34. Liu, Y., T. Liu, Q. Sun, M. Niu, Y. Jiang, and D. Pang. 2015. Downregulation of Ras GTPaseactivating protein 1 is associated with poor survival of breast invasive ductal carcinoma patients. Oncol. Rep. 33:119-124.

35. Cancer Genome, A. N. 2012. Comprehensive molecular portraits of human breast tumours. Nature 490:61-70.

36. Eerola, I., L. M. Boon, J. B. Mulliken, P. E. Burrows, A. Dompmartin, S. Watanabe, R. Vanwijck, and M. Vikkula. 2003. Capillary malformation-arteriovenous malformation, a new clinical and genetic disorder caused by RASA1 mutations. Am. J Hum. Genet. 73:1240-1249.

37. Ballester, R., D. Marchuk, M. Boguski, A. Saulino, R. Letcher, M. Wigler, and F. Collins. 1990. The NF1 locus encodes a protein functionally related to mammalian GAP and yeast IRA proteins. Cell 63:851-859.

38. Martin, G. A., D. Viskochil, G. Bollag, P. C. McCabe, W. J. Crosier, H. Haubruck, L. Conroy, R. Clark, P. O'Connell, R. M. Cawthon, and . 1990. The GAP-related domain of the neurofibromatosis type 1 gene product interacts with ras p21. Cell 63:843-849.

39. Xu, G. F., B. Lin, K. Tanaka, D. Dunn, D. Wood, R. Gesteland, R. White, R. Weiss, and F. Tamanoi. 1990. The catalytic domain of the neurofibromatosis type 1 gene product stimulates ras GTPase and complements ira mutants of S. cerevisiae. Cell 63:835-841.

40. Xu, G. F., P. O'Connell, D. Viskochil, R. Cawthon, M. Robertson, M. Culver, D. Dunn, J. Stevens, R. Gesteland, R. White, and R. Weiss. 1990. The neurofibromatosis type 1 gene encodes a protein related to GAP. Cell 62:599-608.

41. Maertens, O. and K. Cichowski. 2014. An expanding role for RAS GTPase activating proteins (RAS GAPs) in cancer. Adv. Biol. Regul. 55:1-14.

42. Boudry-Labis, E., C. Roche-Lestienne, O. Nibourel, N. Boissel, C. Terre, C. Perot, V. Eclache, N. Gachard, I. Tigaud, G. Plessis, W. Cuccuini, S. Geffroy, C. Villenet, M. Figeac, F. Lepretre, A. Renneville, M. Cheok, J. Soulier, H. Dombret, and C. Preudhomme. 2013. Neurofibromatosis-1 gene deletions and mutations in de novo adult acute myeloid leukemia. Am. J Hematol. 88:306-311.

43. Imielinski, M., A. H. Berger, P. S. Hammerman, B. Hernandez, T. J. Pugh, E. Hodis, J. Cho, J. Suh, M. Capelletti, A. Sivachenko, C. Sougnez, D. Auclair, M. S. Lawrence, P. Stojanov, K. Cibulskis, K. Choi, W. L. de, T. Sharifnia, A. Brooks, H. Greulich, S. 
Banerji, T. Zander, D. Seidel, F. Leenders, S. Ansen, C. Ludwig, W. Engel-Riedel, E. Stoelben, J. Wolf, C. Goparju, K. Thompson, W. Winckler, D. Kwiatkowski, B. E. Johnson, P. A. Janne, V. A. Miller, W. Pao, W. D. Travis, H. I. Pass, S. B. Gabriel, E. S. Lander, R. K. Thomas, L. A. Garraway, G. Getz, and M. Meyerson. 2012. Mapping the hallmarks of lung adenocarcinoma with massively parallel sequencing. Cell 150:11071120.

44. Holzel, M., S. Huang, J. Koster, I. Ora, A. Lakeman, H. Caron, W. Nijkamp, J. Xie, T. Callens, S. Asgharzadeh, R. C. Seeger, L. Messiaen, R. Versteeg, and R. Bernards. 2010. NF1 is a tumor suppressor in neuroblastoma that determines retinoic acid response and disease outcome. Cell 142:218-229.

45. McGillicuddy, L. T., J. A. Fromm, P. E. Hollstein, S. Kubek, R. Beroukhim, R. T. De, B. W. Johnson, S. M. Williams, P. Nghiemphu, L. M. Liau, T. F. Cloughesy, P. S. Mischel, A. Parret, J. Seiler, G. Moldenhauer, K. Scheffzek, A. O. Stemmer-Rachamimov, C. L. Sawyers, C. Brennan, L. Messiaen, I. K. Mellinghoff, and K. Cichowski. 2009. Proteasomal and genetic inactivation of the NF1 tumor suppressor in gliomagenesis. Cancer Cell 16:44-54.

46. Ding, L., G. Getz, D. A. Wheeler, E. R. Mardis, M. D. McLellan, K. Cibulskis, C. Sougnez, H. Greulich, D. M. Muzny, M. B. Morgan, L. Fulton, R. S. Fulton, Q. Zhang, M. C. Wendl, M. S. Lawrence, D. E. Larson, K. Chen, D. J. Dooling, A. Sabo, A. C. Hawes, H. Shen, S. N. Jhangiani, L. R. Lewis, O. Hall, Y. Zhu, T. Mathew, Y. Ren, J. Yao, S. E. Scherer, K. Clerc, G. A. Metcalf, B. Ng, A. Milosavljevic, M. L. GonzalezGaray, J. R. Osborne, R. Meyer, X. Shi, Y. Tang, D. C. Koboldt, L. Lin, R. Abbott, T. L. Miner, C. Pohl, G. Fewell, C. Haipek, H. Schmidt, B. H. Dunford-Shore, A. Kraja, S. D. Crosby, C. S. Sawyer, T. Vickery, S. Sander, J. Robinson, W. Winckler, J. Baldwin, L. R. Chirieac, A. Dutt, T. Fennell, M. Hanna, B. E. Johnson, R. C. Onofrio, R. K. Thomas, G. Tonon, B. A. Weir, X. Zhao, L. Ziaugra, M. C. Zody, T. Giordano, M. B. Orringer, J. A. Roth, M. R. Spitz, I. I. Wistuba, B. Ozenberger, P. J. Good, A. C. Chang, D. G. Beer, M. A. Watson, M. Ladanyi, S. Broderick, A. Yoshizawa, W. D. Travis, W. Pao, M. A. Province, G. M. Weinstock, H. E. Varmus, S. B. Gabriel, E. S. Lander, R. A. Gibbs, M. Meyerson, and R. K. Wilson. 2008. Somatic mutations affect key pathways in lung adenocarcinoma. Nature 455:1069-1075.

47. Legius, E., D. A. Marchuk, F. S. Collins, and T. W. Glover. 1993. Somatic deletion of the neurofibromatosis type 1 gene in a neurofibrosarcoma supports a tumour suppressor gene hypothesis. Nat. Genet. 3:122-126.

48. Nissan, M. H., C. A. Pratilas, A. M. Jones, R. Ramirez, H. Won, C. Liu, S. Tiwari, L. Kong, A. J. Hanrahan, Z. Yao, T. Merghoub, A. Ribas, P. B. Chapman, R. Yaeger, B. S. Taylor, N. Schultz, M. F. Berger, N. Rosen, and D. B. Solit. 2014. Loss of NF1 in cutaneous melanoma is associated with RAS activation and MEK dependence. Cancer Res 74:2340-2350.

49. Wagle, N., E. M. Van Allen, D. J. Treacy, D. T. Frederick, Z. A. Cooper, A. TaylorWeiner, M. Rosenberg, E. M. Goetz, R. J. Sullivan, D. N. Farlow, D. C. Friedrich, K. 
Anderka, D. Perrin, C. M. Johannessen, A. McKenna, K. Cibulskis, G. Kryukov, E. Hodis, D. P. Lawrence, S. Fisher, G. Getz, S. B. Gabriel, S. L. Carter, K. T. Flaherty, J. A. Wargo, and L. A. Garraway. 2014. MAP kinase pathway alterations in BRAF-mutant melanoma patients with acquired resistance to combined RAF/MEK inhibition. Cancer Discov. 4:61-68.

50. Whittaker, S. R., J. P. Theurillat, A. E. Van, N. Wagle, J. Hsiao, G. S. Cowley, D. Schadendorf, D. E. Root, and L. A. Garraway. 2013. A genome-scale RNA interference screen implicates NF1 loss in resistance to RAF inhibition. Cancer Discov. 3:350-362.

51. Maertens, O., B. Johnson, P. Hollstein, D. T. Frederick, Z. A. Cooper, L. Messiaen, R. T. Bronson, M. McMahon, S. Granter, K. Flaherty, J. A. Wargo, R. Marais, and K.

Cichowski. 2013. Elucidating distinct roles for NF1 in melanomagenesis. Cancer Discov. 3:338-349.

52. Zhang, X., N. Li, X. Li, W. Zhao, Y. Qiao, L. Liang, and Y. Ding. 2012. Low expression of DAB2IP contributes to malignant development and poor prognosis in hepatocellular carcinoma. J Gastroenterol. Hepatol. 27:1117-1125.

53. Chen, H., S. W. Tu, and J. T. Hsieh. 2005. Down-regulation of human DAB2IP gene expression mediated by polycomb Ezh2 complex and histone deacetylase in prostate cancer. J Biol. Chem. 280:22437-22444.

54. Dote, H., S. Toyooka, K. Tsukuda, M. Yano, T. Ota, M. Murakami, M. Naito, M. Toyota, A. F. Gazdar, and N. Shimizu. 2005. Aberrant promoter methylation in human DAB2 interactive protein (hDAB2IP) gene in gastrointestinal tumour. Br. J Cancer 92:11171125.

55. Yano, M., S. Toyooka, K. Tsukuda, H. Dote, M. Ouchida, T. Hanabata, M. Aoe, H. Date, A. F. Gazdar, and N. Shimizu. 2005. Aberrant promoter methylation of human DAB2 interactive protein (hDAB2IP) gene in lung cancers. Int J Cancer 113:59-66.

56. Dote, H., S. Toyooka, K. Tsukuda, M. Yano, M. Ouchida, H. Doihara, M. Suzuki, H. Chen, J. T. Hsieh, A. F. Gazdar, and N. Shimizu. 2004. Aberrant promoter methylation in human DAB2 interactive protein (hDAB2IP) gene in breast cancer. Clin Cancer Res 10:2082-2089.

57. McLaughlin, S. K., S. N. Olsen, B. Dake, R. T. De, E. Lim, R. T. Bronson, R. Beroukhim, K. Polyak, M. Brown, C. Kuperwasser, and K. Cichowski. 2013. The RasGAP gene, RASAL2, is a tumor and metastasis suppressor. Cancer Cell 24:365-378.

58. Bernards, A. and J. Settleman. 2005. GAPs in growth factor signalling. Growth Factors 23:143-149.

59. Bernards, A. and J. Settleman. 2004. GAP control: regulating the regulators of small GTPases. Trends Cell Biol. 14:377-385. 
60. Wang, S., T. Watanabe, J. Noritake, M. Fukata, T. Yoshimura, N. Itoh, T. Harada, M. Nakagawa, Y. Matsuura, N. Arimura, and K. Kaibuchi. 2007. IQGAP3, a novel effector of Rac1 and Cdc42, regulates neurite outgrowth. J Cell Sci. 120:567-577.

61. Brill, S., S. Li, C. W. Lyman, D. M. Church, J. J. Wasmuth, L. Weissbach, A. Bernards, and A. J. Snijders. 1996. The Ras GTPase-activating-protein-related human protein IQGAP2 harbors a potential actin binding domain and interacts with calmodulin and Rho family GTPases. Mol. Cell Biol. 16:4869-4878.

62. Jameson, K. L., P. K. Mazur, A. M. Zehnder, J. Zhang, B. Zarnegar, J. Sage, and P. A. Khavari. 2013. IQGAP1 scaffold-kinase interaction blockade selectively targets RASMAP kinase-driven tumors. Nat. Med 19:626-630.

63. Bos, J. L., H. Rehmann, and A. Wittinghofer. 2007. GEFs and GAPs: critical elements in the control of small G proteins. Cell 129:865-877.

64. Vigil, D., J. Cherfils, K. L. Rossman, and C. J. Der. 2010. Ras superfamily GEFs and GAPs: validated and tractable targets for cancer therapy? Nat. Rev. Cancer 10:842-857.

65. Sun, D., C. Wang, S. Long, Y. Ma, Y. Guo, Z. Huang, X. Chen, C. Zhang, J. Chen, and J. Zhang. 2015. C/EBP-beta-activated microRNA-223 promotes tumour growth through targeting RASA1 in human colorectal cancer. Br. J Cancer.

66. Gong, B., W. W. Liu, W. J. Nie, D. F. Li, Z. J. Xie, C. Liu, Y. H. Liu, P. Mei, and Z. J. Li. 2015. MiR-21/RASA1 axis affects malignancy of colon cancer cells via RAS pathways. World J Gastroenterol. 21:1488-1497.

67. Li, Z., D. Li, G. Zhang, J. Xiong, Z. Jie, H. Cheng, Y. Cao, M. Jiang, L. Lin, Z. Le, S. Tan, W. Zou, B. Gong, S. Lin, and K. Yang. 2014. Methylation-associated silencing of MicroRNA-335 contributes tumor cell invasion and migration by interacting with RASA1 in gastric cancer. Am. J Cancer Res 4:648-662.

68. Cox, A. D., S. W. Fesik, A. C. Kimmelman, J. Luo, and C. J. Der. 2014. Drugging the undruggable RAS: Mission possible? Nat. Rev. Drug Discov. 13:828-851.

69. Stephen, A. G., D. Esposito, R. K. Bagni, and F. Mccormick. 2014. Dragging ras back in the ring. Cancer Cell 25:272-281.

70. Kasinski, A. L. and F. J. Slack. 2011. Epigenetics and genetics. MicroRNAs en route to the clinic: progress in validating and targeting microRNAs for cancer therapy. Nat. Rev. Cancer 11:849-864.

71. Ling, H., M. Fabbri, and G. A. Calin. 2013. MicroRNAs and other non-coding RNAs as targets for anticancer drug development. Nat. Rev. Drug Discov. 12:847-865.

72. Stenvang, J., A. Petri, M. Lindow, S. Obad, and S. Kauppinen. 2012. Inhibition of microRNA function by antimiR oligonucleotides. Silence. 3:1. 
73. van, R. E. and S. Kauppinen. 2014. Development of microRNA therapeutics is coming of age. EMBO Mol. Med 6:851-864.

74. Kasinski, A. L. and F. J. Slack. 2010. Potential microRNA therapies targeting Ras, NFkappaB and p53 signaling. Curr. Opin. Mol. Ther 12:147-157.

75. Li, Z. and T. M. Rana. 2014. Therapeutic targeting of microRNAs: current status and future challenges. Nat. Rev. Drug Discov. 13:622-638.

76. Johnson, S. M., H. Grosshans, J. Shingara, M. Byrom, R. Jarvis, A. Cheng, E. Labourier, K. L. Reinert, D. Brown, and F. J. Slack. 2005. RAS is regulated by the let-7 microRNA family. Cell 120:635-647.

77. Stinson, S., M. R. Lackner, A. T. Adai, N. Yu, H. J. Kim, C. O'Brien, J. Spoerke, S. Jhunjhunwala, Z. Boyd, T. Januario, R. J. Newman, P. Yue, R. Bourgon, Z. Modrusan, H. M. Stern, S. Warming, F. J. de Sauvage, L. Amler, R. F. Yeh, and D. Dornan. 2011. TRPS 1 targeting by miR-221/222 promotes the epithelial-to-mesenchymal transition in breast cancer. Sci Signal 4:ra41.

78. Talotta, F., A. Cimmino, M. R. Matarazzo, L. Casalino, V. G. De, M. D'Esposito, L. R. Di, and P. Verde. 2009. An autoregulatory loop mediated by miR-21 and PDCD4 controls the AP-1 activity in RAS transformation. Oncogene 28:73-84.

79. Inui, M., G. Martello, and S. Piccolo. 2010. MicroRNA control of signal transduction. Nat. Rev. Mol. Cell Biol. 11:252-263.

80. Zawistowski, J. S., K. Nakamura, J. S. Parker, D. A. Granger, B. T. Golitz, and G. L. Johnson. 2013. MicroRNA 9-3p targets beta1 integrin to sensitize claudin-low breast cancer cells to MEK inhibition. Mol. Cell Biol. 33:2260-2274.

81. Pillai, R. S., S. N. Bhattacharyya, C. G. Artus, T. Zoller, N. Cougot, E. Basyuk, E. Bertrand, and W. Filipowicz. 2005. Inhibition of translational initiation by Let-7 MicroRNA in human cells. Science 309:1573-1576.

82. Sampson, V. B., N. H. Rong, J. Han, Q. Yang, V. Aris, P. Soteropoulos, N. J. Petrelli, S. P. Dunn, and L. J. Krueger. 2007. MicroRNA let-7a down-regulates MYC and reverts MYC-induced growth in Burkitt lymphoma cells. Cancer Res. 67:9762-9770.

83. Zhang, B., X. Pan, G. P. Cobb, and T. A. Anderson. 2007. microRNAs as oncogenes and tumor suppressors. Dev Biol. 302:1-12.

84. Yu, F., H. Yao, P. Zhu, X. Zhang, Q. Pan, C. Gong, Y. Huang, X. Hu, F. Su, J. Lieberman, and E. Song. 2007. let-7 regulates self renewal and tumorigenicity of breast cancer cells. Cell 131:1109-1123.

85. Takamizawa, J., H. Konishi, K. Yanagisawa, S. Tomida, H. Osada, H. Endoh, T. Harano, Y. Yatabe, M. Nagino, Y. Nimura, T. Mitsudomi, and T. Takahashi. 2004. Reduced 
expression of the let-7 microRNAs in human lung cancers in association with shortened postoperative survival. Cancer Res. 64:3753-3756.

86. Rodrik-Outmezguine, V. S., S. Chandarlapaty, N. C. Pagano, P. I. Poulikakos, M. Scaltriti, E. Moskatel, J. Baselga, S. Guichard, and N. Rosen. 2011. mTOR kinase inhibition causes feedback-dependent biphasic regulation of AKT signaling. Cancer Discov. 1:248-259.

87. Garrett, J. T., M. G. Olivares, C. Rinehart, N. D. Granja-Ingram, V. Sanchez, A. Chakrabarty, B. Dave, R. S. Cook, W. Pao, E. McKinely, H. C. Manning, J. Chang, and C. L. Arteaga. 2011. Transcriptional and posttranslational up-regulation of HER3 (ErbB3) compensates for inhibition of the HER2 tyrosine kinase. Proc. Natl. Acad. Sci. U. S. A 108:5021-5026.

88. Chandarlapaty, S., A. Sawai, M. Scaltriti, V. Rodrik-Outmezguine, O. Grbovic-Huezo, V. Serra, P. K. Majumder, J. Baselga, and N. Rosen. 2011. AKT inhibition relieves feedback suppression of receptor tyrosine kinase expression and activity. Cancer Cell 19:58-71.

89. Engelman, J. A., K. Zejnullahu, T. Mitsudomi, Y. Song, C. Hyland, J. O. Park, N. Lindeman, C. M. Gale, X. Zhao, J. Christensen, T. Kosaka, A. J. Holmes, A. M. Rogers, F. Cappuzzo, T. Mok, C. Lee, B. E. Johnson, L. C. Cantley, and P. A. Janne. 2007. MET amplification leads to gefitinib resistance in lung cancer by activating ERBB3 signaling. Science 316:1039-1043.

90. Duncan, J. S., M. C. Whittle, K. Nakamura, A. N. Abell, A. A. Midland, J. S. Zawistowski, N. L. Johnson, D. A. Granger, N. V. Jordan, D. B. Darr, J. Usary, P. F. Kuan, D. M. Smalley, B. Major, X. He, K. A. Hoadley, B. Zhou, N. E. Sharpless, C. M. Perou, W. Y. Kim, S. M. Gomez, X. Chen, J. Jin, S. V. Frye, H. S. Earp, L. M. Graves, and G. L. Johnson. 2012. Dynamic reprogramming of the kinome in response to targeted MEK inhibition in triple-negative breast cancer. Cell 149:307-321.

91. Johnson, G. L., T. J. Stuhlmiller, S. P. Angus, J. S. Zawistowski, and L. M. Graves. 2014. Molecular pathways: adaptive kinome reprogramming in response to targeted inhibition of the BRAF-MEK-ERK pathway in cancer. Clin Cancer Res 20:2516-2522.

92. Villanueva, J., J. R. Infante, C. Krepler, P. Reyes-Uribe, M. Samanta, H. Y. Chen, B. Li, R. K. Swoboda, M. Wilson, A. Vultur, M. Fukunaba-Kalabis, B. Wubbenhorst, T. Y. Chen, Q. Liu, K. Sproesser, D. J. DeMarini, T. M. Gilmer, A. M. Martin, R. Marmorstein, D. C. Schultz, D. W. Speicher, G. C. Karakousis, W. Xu, R. K. Amaravadi, X. Xu, L. M. Schuchter, M. Herlyn, and K. L. Nathanson. 2013. Concurrent MEK2 mutation and BRAF amplification confer resistance to BRAF and MEK inhibitors in melanoma. Cell Rep. 4:1090-1099.

93. Shi, H., A. Hong, X. Kong, R. C. Koya, C. Song, G. Moriceau, W. Hugo, C. C. Yu, C. Ng, T. Chodon, R. A. Scolyer, R. F. Kefford, A. Ribas, G. V. Long, and R. S. Lo. 2014. A novel AKT1 mutant amplifies an adaptive melanoma response to BRAF inhibition. Cancer Discov. 4:69-79. 
94. Nazarian, R., H. Shi, Q. Wang, X. Kong, R. C. Koya, H. Lee, Z. Chen, M. K. Lee, N. Attar, H. Sazegar, T. Chodon, S. F. Nelson, G. McArthur, J. A. Sosman, A. Ribas, and R. S. Lo. 2010. Melanomas acquire resistance to B-RAF(V600E) inhibition by RTK or NRAS upregulation. Nature 468:973-977.

95. Sos, M. L., S. Fischer, R. Ullrich, M. Peifer, J. M. Heuckmann, M. Koker, S. Heynck, I. Stuckrath, J. Weiss, F. Fischer, K. Michel, A. Goel, L. Regales, K. A. Politi, S. Perera, M. Getlik, L. C. Heukamp, S. Ansen, T. Zander, R. Beroukhim, H. Kashkar, K. M. Shokat, W. R. Sellers, D. Rauh, C. Orr, K. P. Hoeflich, L. Friedman, K. K. Wong, W. Pao, and R. K. Thomas. 2009. Identifying genotype-dependent efficacy of single and combined. Proc. Natl. Acad. Sci. U. S. A 106:18351-18356.

96. Hoeflich, K. P., C. O'Brien, Z. Boyd, G. Cavet, S. Guerrero, K. Jung, T. Januario, H. Savage, E. Punnoose, T. Truong, W. Zhou, L. Berry, L. Murray, L. Amler, M. Belvin, L. S. Friedman, and M. R. Lackner. 2009. In vivo antitumor activity of MEK and phosphatidylinositol 3-kinase inhibitors in basal-like breast cancer models. Clin Cancer Res 15:4649-4664.

97. Sun, C., S. Hobor, A. Bertotti, D. Zecchin, S. Huang, F. Galimi, F. Cottino, A. Prahallad, W. Grernrum, A. Tzani, A. Schlicker, L. F. Wessels, E. F. Smit, E. Thunnissen, P. Halonen, C. Lieftink, R. L. Beijersbergen, N. F. Di, A. Bardelli, L. Trusolino, and R. Bernards. 2014. Intrinsic resistance to MEK inhibition in KRAS mutant lung and colon cancer through transcriptional induction of ERBB3. Cell Rep. 7:86-93.

98. Heidorn, S. J., C. Milagre, S. Whittaker, A. Nourry, I. Niculescu-Duvas, N. Dhomen, J. Hussain, J. S. Reis-Filho, C. J. Springer, C. Pritchard, and R. Marais. 2010. Kinase-dead BRAF and oncogenic RAS cooperate to drive tumor progression through CRAF. Cell 140:209-221.

99. Poulikakos, P. I., C. Zhang, G. Bollag, K. M. Shokat, and N. Rosen. 2010. RAF inhibitors transactivate RAF dimers and ERK signalling in cells with wild-type BRAF. Nature 464:427-430.

100. Hatzivassiliou, G., K. Song, I. Yen, B. J. Brandhuber, D. J. Anderson, R. Alvarado, M. J. Ludlam, D. Stokoe, S. L. Gloor, G. Vigers, T. Morales, I. Aliagas, B. Liu, S. Sideris, K. P. Hoeflich, B. S. Jaiswal, S. Seshagiri, H. Koeppen, M. Belvin, L. S. Friedman, and S. Malek. 2010. RAF inhibitors prime wild-type RAF to activate the MAPK pathway and enhance growth. Nature 464:431-435.

101. Pichiorri, F., D. Palmieri, L. L. De, J. Consiglio, J. You, A. Rocci, T. Talabere, C. Piovan, A. Lagana, L. Cascione, J. Guan, P. Gasparini, V. Balatti, G. Nuovo, V. Coppola, C. C. Hofmeister, G. Marcucci, J. C. Byrd, S. Volinia, C. L. Shapiro, M. A. Freitas, and C. M. Croce. 2013. In vivo NCL targeting affects breast cancer aggressiveness through miRNA regulation. J Exp. Med 210:951-968.

102. Summerton, J. 1999. Morpholino antisense oligomers: the case for an RNase Hindependent structural type. Biochim. Biophys. Acta 1489:141-158. 
103. Egholm, M., O. Buchardt, L. Christensen, C. Behrens, S. M. Freier, D. A. Driver, R. H. Berg, S. K. Kim, B. Norden, and P. E. Nielsen. 1993. PNA hybridizes to complementary oligonucleotides obeying the Watson-Crick hydrogen-bonding rules. Nature 365:566568.

104. Cheng, C. J., R. Bahal, I. A. Babar, Z. Pincus, F. Barrera, C. Liu, A. Svoronos, D. T. Braddock, P. M. Glazer, D. M. Engelman, W. M. Saltzman, and F. J. Slack. 2015. MicroRNA silencing for cancer therapy targeted to the tumour microenvironment. Nature 518:107-110.

105. Liu, Q., Y. Sabnis, Z. Zhao, T. Zhang, S. J. Buhrlage, L. H. Jones, and N. S. Gray. 2013. Developing irreversible inhibitors of the protein kinase cysteinome. Chem. Biol. 20:146159.

106. Gibney, G. T. and J. S. Zager. 2013. Clinical development of dabrafenib in BRAF mutant melanoma and other malignancies. Expert Opin. Drug Metab Toxicol. 9:893-899.

107. Bollag, G., P. Hirth, J. Tsai, J. Zhang, P. N. Ibrahim, H. Cho, W. Spevak, C. Zhang, Y. Zhang, G. Habets, E. A. Burton, B. Wong, G. Tsang, B. L. West, B. Powell, R. Shellooe, A. Marimuthu, H. Nguyen, K. Y. Zhang, D. R. Artis, J. Schlessinger, F. Su, B. Higgins, R. Iyer, K. D'Andrea, A. Koehler, M. Stumm, P. S. Lin, R. J. Lee, J. Grippo, I. Puzanov, K. B. Kim, A. Ribas, G. A. McArthur, J. A. Sosman, P. B. Chapman, K. T. Flaherty, X. Xu, K. L. Nathanson, and K. Nolop. 2010. Clinical efficacy of a RAF inhibitor needs broad target blockade in BRAF-mutant melanoma. Nature 467:596-599.

108. Flaherty, K. T., J. R. Infante, A. Daud, R. Gonzalez, R. F. Kefford, J. Sosman, O. Hamid, L. Schuchter, J. Cebon, N. Ibrahim, R. Kudchadkar, H. A. Burris, III, G. Falchook, A. Algazi, K. Lewis, G. V. Long, I. Puzanov, P. Lebowitz, A. Singh, S. Little, P. Sun, A. Allred, D. Ouellet, K. B. Kim, K. Patel, and J. Weber. 2012. Combined BRAF and MEK inhibition in melanoma with BRAF V600 mutations. N. Engl. J Med 367:1694-1703.

109. Davies, B. R., A. Logie, J. S. McKay, P. Martin, S. Steele, R. Jenkins, M. Cockerill, S. Cartlidge, and P. D. Smith. 2007. AZD6244 (ARRY-142886), a potent inhibitor of mitogen-activated protein kinase/extracellular signal-regulated kinase kinase 1/2 kinases: mechanism of action in vivo, pharmacokinetic/pharmacodynamic relationship, and potential for combination in preclinical models. Mol. Cancer Ther 6:2209-2219.

110. Yeh, T. C., V. Marsh, B. A. Bernat, J. Ballard, H. Colwell, R. J. Evans, J. Parry, D. Smith, B. J. Brandhuber, S. Gross, A. Marlow, B. Hurley, J. Lyssikatos, P. A. Lee, J. D. Winkler, K. Koch, and E. Wallace. 2007. Biological characterization of ARRY-142886 (AZD6244), a potent, highly selective mitogen-activated protein kinase kinase $1 / 2$ inhibitor. Clin Cancer Res 13:1576-1583.

111. Ohori, M., T. Kinoshita, M. Okubo, K. Sato, A. Yamazaki, H. Arakawa, S. Nishimura, N. Inamura, H. Nakajima, M. Neya, H. Miyake, and T. Fujii. 2005. Identification of a selective ERK inhibitor and structural determination of the inhibitor-ERK2 complex. Biochem. Biophys. Res Commun. 336:357-363. 
112. Mirzoeva, O. K., D. Das, L. M. Heiser, S. Bhattacharya, D. Siwak, R. Gendelman, N. Bayani, N. J. Wang, R. M. Neve, Y. Guan, Z. Hu, Z. Knight, H. S. Feiler, P. Gascard, B. Parvin, P. T. Spellman, K. M. Shokat, A. J. Wyrobek, M. J. Bissell, F. Mccormick, W. L. Kuo, G. B. Mills, J. W. Gray, and W. M. Korn. 2009. Basal subtype and MAPK/ERK kinase (MEK)-phosphoinositide 3-kinase feedback signaling determine susceptibility of breast cancer cells to MEK inhibition. Cancer Res 69:565-572.

113. Stuhlmiller, T. J., S. M. Miller, J. S. Zawistowski, K. Nakamura, A. S. Beltran, J. S. Duncan, S. P. Angus, K. A. Collins, D. A. Granger, R. A. Reuther, L. M. Graves, S. M. Gomez, P. F. Kuan, J. S. Parker, X. Chen, N. Sciaky, L. A. Carey, H. S. Earp, J. Jin, and G. L. Johnson. 2015. Inhibition of Lapatinib-Induced Kinome Reprogramming in ERBB2-Positive Breast Cancer by Targeting BET Family Bromodomains. Cell Rep. 11:390-404.

114. Corcoran, R. B., K. A. Cheng, A. N. Hata, A. C. Faber, H. Ebi, E. M. Coffee, P. Greninger, R. D. Brown, J. T. Godfrey, T. J. Cohoon, Y. Song, E. Lifshits, K. E. Hung, T. Shioda, D. as-Santagata, A. Singh, J. Settleman, C. H. Benes, M. Mino-Kenudson, K. K. Wong, and J. A. Engelman. 2013. Synthetic lethal interaction of combined BCL-XL and MEK inhibition promotes tumor regressions in KRAS mutant cancer models. Cancer Cell 23:121-128.

115. Akhavan, D., A. L. Pourzia, A. A. Nourian, K. J. Williams, D. Nathanson, I. Babic, G. R. Villa, K. Tanaka, A. Nael, H. Yang, J. Dang, H. V. Vinters, W. H. Yong, M. Flagg, F. Tamanoi, T. Sasayama, C. D. James, H. I. Kornblum, T. F. Cloughesy, W. K. Cavenee, S. J. Bensinger, and P. S. Mischel. 2013. De-repression of PDGFRbeta transcription promotes acquired resistance to EGFR tyrosine kinase inhibitors in glioblastoma patients. Cancer Discov. 3:534-547.

116. McCubrey, J. A., L. S. Steelman, S. L. Abrams, J. T. Lee, F. Chang, F. E. Bertrand, P. M. Navolanic, D. M. Terrian, R. A. Franklin, A. B. D'Assoro, J. L. Salisbury, M. C. Mazzarino, F. Stivala, and M. Libra. 2006. Roles of the RAF/MEK/ERK and PI3K/PTEN/AKT pathways in malignant transformation and drug resistance. Adv. Enzyme Regul. 46:249-279.

117. Young, A., J. Lyons, A. L. Miller, V. T. Phan, I. R. Alarcon, and F. Mccormick. 2009. Ras signaling and therapies. Adv. Cancer Res 102:1-17.

118. Roberts, P. J. and C. J. Der. 2007. Targeting the Raf-MEK-ERK mitogen-activated protein kinase cascade for the treatment of cancer. Oncogene 26:3291-3310.

119. Downward, J. 2003. Targeting RAS signalling pathways in cancer therapy. Nat. Rev. Cancer 3:11-22.

120. Karnoub, A. E. and R. A. Weinberg. 2008. Ras oncogenes: split personalities. Nat. Rev. Mol. Cell Biol. 9:517-531.

121. Cox, A. D. and C. J. Der. 2010. Ras history: The saga continues. Small GTPases. 1:2-27. 
122. Pylayeva-Gupta, Y., E. Grabocka, and D. Bar-Sagi. 2011. RAS oncogenes: weaving a tumorigenic web. Nat. Rev. Cancer 11:761-774.

123. Sledge, G. W., E. P. Mamounas, G. N. Hortobagyi, H. J. Burstein, P. J. Goodwin, and A. C. Wolff. 2014. Past, present, and future challenges in breast cancer treatment. J Clin Oncol. 32:1979-1986.

124. Gampenrieder, S. P., G. Rinnerthaler, and R. Greil. 2013. Neoadjuvant chemotherapy and targeted therapy in breast cancer: past, present, and future. J Oncol. 2013:732047.

125. Metzger-Filho, O. and A. E. de. 2011. Neoadjuvant chemotherapy and targeted therapies: a promising strategy. J Natl. Cancer Inst. Monogr 2011:116-119.

126. Ragaz, J. 2010. Randomized adjuvant trials in oncology: a necessity or time-consuming luxury? Breast Cancer Res 12 Suppl 4:S14. 
This page is intentionally left blank 
This page is intentionally left blank 


\section{APPENDIX}


This page is intentionally left blank 


\title{
PART I
}

\section{MicroRNA-based therapeutic strategies for targeting mutant and wild type RAS in cancer}

\author{
Sriganesh B. Sharma ${ }^{1,2}$ and J. Michael Ruppert ${ }^{1,3, *}$
}

${ }^{1}$ Department of Biochemistry, ${ }^{2}$ Program in Cancer Cell Biology, and ${ }^{3}$ The Mary Babb Randolph Cancer Center, West Virginia University, Morgantown, West Virginia, 26506

"To whom correspondence should be addressed: J. Michael Ruppert, Department of Biochemistry and The Mary Babb Randolph Cancer Center, Morgantown, West Virginia, 26505.

Email: mruppert@ @sc.wvu.edu

Running title: miR-therapy for targeting of wild type and mutant RAS

Keywords: microRNAs, RAS-ERK signaling, RAS-GTP, cancer

Note: The following manuscript is a submission for an invited review from Drug Development

Research 


\section{ABSTRACT}

microRNAs (miRs) have been causally implicated in the progression and development of a wide variety of cancers. miRs modulate the activity of key cell signaling networks by regulating the translation of pathway component proteins. Thus, the pharmacological targeting of miRs that regulate cancer cell signaling networks, either by promoting (using miRsupplementation) or by suppressing (using anti-sense oligonucleotide based strategies) miR activity is an area of intense research. The RAS-Extracellular signal regulated kinase (ERK) pathway represents a major miR-regulated signaling network that endows cells with some of the classical hallmarks of cancer, and is often inappropriately activated in malignancies by somatic genetic alteration through point mutation or alteration of gene copy number. In addition, recent progress indicates that many tumors may be deficient in GTPase activating proteins (GAPs) due to the collaborative action of oncogenic microRNAs. Recent studies also suggest that in tumors harboring a mutant RAS allele there is a critical role for wild type RAS proteins in determining overall RAS-ERK pathway activity. Together, these two advances comprise a new opportunity for therapeutic intervention. In this review, we evaluate miR-based therapeutic strategies for modulating RAS-ERK signaling in cancers, in particular for more direct modulation of RASGTP levels, with the potential to complement current strategies in order to yield more durable treatment responses. To this end, we discuss the potential for miR-based therapies focused on three prominent miRs including the pan-RAS regulator let-7 and the GAP regulator comprised of miR-206 and miR-21 (miR-206/21). 


\section{INTRODUCTION}

MicroRNAs (miRs) are genomically encoded single stranded noncoding RNAs that are typically 19-25 nucleotides (nt) in length and result from extensive processing of endogenous hairpin-shaped precursors (1-4). miRs were initially identified in Caenorhabidits elegans as gene products required for the regulation of proper developmental timing $(5,6)$. Subsequently, thousands of miRs and putative miR-encoding genes have been identified in a wide variety of organisms, including in plants and metazoans. Since their discovery, miRs have emerged as critical regulators of gene expression and cell signaling, and are functionally implicated in numerous cellular processes including development, differentiation, proliferation, and apoptosis (7-10). As a consequence of these critical roles, dysregulation of miR expression, activity, and signaling results in a multitude of pathological states, including the development and progression of cancers (11-13).

Perturbation of key signaling networks endows cells with many of the well-established hallmarks of cancer, such as enhanced cell proliferation, resistance to cell stress and death, and motility, and is implicated in the pathogenesis of virtually every type of human malignancy $(14,15)$. Attributed to elevated levels of membrane-associated RAS-GTP, signaling downstream of RAS proto-oncogenes through the RAF-MEK-ERK mitogen activated protein kinase (MAPK) pathway is often inappropriately activated in a wide variety of cancers, promoting several of the classical hallmarks of cancer (16-22). Activation of this hierarchically tiered signaling pathway can occur through a variety of ways, including in response to stimulation by upstream inputs (i.e., receptor tyrosine kinases (RTKs), integrins, ion channels, etc.), somatic mutation of pathway components such as RAS and RAF, and alteration of the expression of pathway 
regulators (23-28). In cancer cells, the activation of RAS-ERK signaling has been most prominently documented in the context of somatic acquisition of activating point mutations in RAS GTPase genes (e.g., KRAS, HRAS, NRAS). These mutations render the encoded gene products resistant to the inhibitory action of GTPase activating proteins (GAPs, which potently stimulate GTP hydrolysis by RAS) $(17,29-32)$. In addition to GAPs, numerous factors contribute to the proper spatio-temporal regulation of RAS-ERK signaling, including guanine nucleotide exchange factors (GEFs), which promote recycling to the active, GTP bound state by reducing the affinity of RAS proteins for GDP. In addition, other proteins function as scaffolds or adaptors for the proper localization of signaling molecules, such as SPRED1 which is critical for the membrane localization of NF1/GAP (17,33-37). More recently, analyses of tumor cells containing a RAS mutation indicated that the wild type proteins encoded by the remaining, unmutated RAS alleles play a critical role in pathway output, identifying these wild type proteins as a potential Achilles' heel for therapeutic targeting (38-41).

miRs represent yet another level of regulatory control of RAS-ERK signaling and, in certain tumor cells such as basal-like or triple-negative breast cancer (TNBC), can represent major regulators of RAS-ERK activity by impacting the translation of pathway components such as GAPs and/or GAP-associated scaffolding proteins such as SPRED1 (41-47). Unraveling how miRs impact RAS-ERK signaling in cancer has the potential to uncover novel therapeutic strategies which can complement conventional modalities and/or targeted therapies such as kinase inhibitors. In this review, we briefly describe miR biogenesis and how miRs can impact the pathogenesis of cancer by altering cell signaling. We discuss miR-based therapeutic strategies and necessary considerations for the successful use of in vivo miR-targeting agents. We then describe the structure of the circuitry of the RAS-ERK signaling pathway, and briefly 
review the utility of inhibiting this pathway in the treatment of cancers. We consider how miRs can regulate RAS-ERK signaling by targeting specific pathway components and critical regulatory proteins, including wild-type RAS proteins. We next evaluate the prospect of targeting miR-mediated regulation of RAS-ERK in the therapy of cancers and contrast this therapeutic modality with other pharmacological RAS-ERK inhibitory strategies. We conclude by proposing a therapeutic strategy for the more direct suppression of RAS-GTP levels, including the in vivo silencing of the cooperative GAP regulators, miR-206 and miR-21 (i.e., miR-206/21). Recent studies indicate that these two miRs maintain RAS-ERK signaling in breast cancer cells by limiting the translation of a major GAP termed RASA1 and the NF1/GAPassociated factor, SPRED1 (41). In TNBC cells the resulting suppression of GAP activities by endogenous miR-206/21 is critical for the maintenance of wild type RAS-GTP levels, RAS-ERK signaling and malignant properties not only for cancer cells harboring wild type RAS proteins, but similarly in tumor cells harboring a RAS mutation, further supporting a critical role of wild type RAS in cells harboring the mutant protein (38-41).

\section{MICRORNAS (miRs)}

\section{miR-biogenesis:}

In humans, the predominant miR biosynthetic route involves the transcription of miRencoding genes by RNA polymerase II (1-4). The resulting primary miR transcripts (pri-miRs) are processed in the nucleus by the RNAse-III enzyme Drosha, which exists as part of a heterodimeric complex with DGCR8 microprocessor complex unit (also known as "DiGeorge Syndrome Critical Region 8") to yield stem-loop hairpin structures of approximately $70 \mathrm{nt}$ in 
length, termed precursor miRs (pre-miRs) (48-52). Pre-miRs are exported out of the nucleus predominantly by the action of Ran-GTPase/exportin- 5 and further processed by the cytoplasmic RNAse-III enzyme Dicer, to yield RNA duplexes composed of the mature miRs of approximately $19-25 \mathrm{nt}$ in length (48,53-56). These are termed miR-miR* duplexes. Following strand selection, "miR" represents the more abundant strand of the duplex and the less abundant strand is denoted "miR*" $(57,58)$. In addition to this major route of biogenesis, some miRs arise from alternate synthetic pathways, including processes that do not utilize RNA polymerase II mediated transcription, or the action of Drosha/DGCR8 or Dicer proteins (59). For example, miR-genes located near Alu-repeat sequences or tRNA genes can be transcribed by RNA polymerase III (60). Furthermore, miRs can also arise from a Drosha/DGCR8 independent synthetic process from the splicing of intronic regions from mRNA transcripts and are termed miRtrons (61). Finally, miR-miR* duplexes may be produced by direct cleavage of RNA precursors by the endoribonuclease Argonaute-2 (AGO2) to yield mature miRs in a Dicer independent process (62).

\section{miR-mediated regulation of protein translation:}

Mature miRs typically repress the translation of mRNA transcripts by associating with the catalytic center (AGO endoribonucleases) of multiprotein complexes termed RNA-induced silencing complexes (RISCs) $(63,64)$. miR-miR* duplexes are subsequently unwound and a mature miR strand is retained in RISC based on the relative thermodynamic stability across the miR-miR* duplex. The miR* strand may subsequently either be cleaved or be ejected from the assembled complex. The mature RISC then scans target mRNA sequences. The selectivity for target mRNAs arises from miR sequence complementarity to portions of the target mRNAs, particularly in the 3' untranslated region (3' UTR). In mammals, sequence complementarity 
between bases 2-8 near the 5' end of the miR (termed the miR-seed sequence) and portions of the mRNA is the dominant factor that guides RISCs to target and to repress the translation of specific transcripts $(1,3,4,65)$. The resulting miR-mediated translational repression may occur by (i) blocking translation initiation, (ii) enhanced mRNA degradation, or by (iii) site specific cleavage of the target mRNA, though the latter process occurs infrequently in mammals $(1,3,4,65)$. Interestingly, though miRs typically repress the translation of target mRNAs, a few miRs have been documented to promote the translation of cognate transcripts, suggesting an additional level of complexity in this modality of regulating gene expression $(4,66-71)$.

miRs are capable of widespread regulation of gene expression, with more than $60 \%$ of protein coding mRNA transcripts possessing at least one evolutionarily conserved miR seed complementary sequence (72). Conversely, whereas the translation of a single mRNA transcript may be impacted by multiple miRs, individual miRs can potentially regulate the expression of hundreds of protein coding transcripts, each of which may have diverse cellular functions. Importantly, the collaborative action of miRs can potently modulate the activity of key signaling networks by targeting one or more pathway components $(1,3,4,65)$. miRs may also participate in reciprocal regulation of transcripts that are critical for miR biogenesis or maturation, and thus constitute components of feedback loops along with their target mRNAs.

These complex miR-mRNA interaction paradigms are essential for the maintenance of homeostasis of critical cell physiological processes and the perturbation of miR regulation of important cell signaling networks, such as the RAS-ERK pathway, contributes to a wide variety of pathological states, including cancer. Indeed, recent work suggests that miR signaling to RASERK can be responsible for maintaining low GAP activity that leads to high RAS-ERK pathway activity, regardless of the RAS mutational status (41). Furthermore, studies indicate that mutant 
RAS-GTP level does not on its own determine pathway activity, but instead that both the mutant and wild type RAS proteins must preferentially associate with GTP in order to maintain higher levels of pathway activity (38-41).

\section{miR-based therapeutic strategies:}

As miR dysregulation is a prominent feature of many pathological states, the therapeutic targeting of specific miRs holds the promise for therapy of various diseases $(7,73-78)$. miRbased therapeutics are defined as strategies that restore or inhibit miR function to counteract perturbations in miR-signaling. These strategies include (i) restoring miR function by supplementation of miR-mimics, (ii) inhibiting $\mathrm{miR}$ function by synthetic anti-sense oligonucleotide-based approaches targeting endogenous miRs (termed anti-miRs and antagomiRs) or by (iii) modulating miR function by non-oligonucleotide based methods including peptide nucleic acids (PNAs) $(7,76,78)$. Furthermore, oligonucleotide based strategies may feature various chemical modifications to enhance the stability and affinity of these therapeutic agents $(7,76,78)$.

Despite our extensive knowledge of attractive miR targets in various disease states, the successful utilization of miR-based therapeutic strategies in vivo is challenged by several obstacles $(7,76,78)$. First, the successful delivery of these agents to the target tissue is limited by physical, anatomical, pharmacokinetic, and pharmacodynamic barriers, which may be overcome in part by a variety of delivery methods and targeting strategies including miR-cholesterol conjugation, liposome encapsulation, miR-nanoparticle conjugation, and antibody- or aptamerbased targeting methods $(7,76,78)$. As miR-mimics and anti-miRs can be degraded by 
ubiquitously present nucleases in the human body, synthetic modification of these agents is necessary for their stability $(79,80)$.

Second, miR-based therapies, including anti-sense strategies, have the potential for promiscuous miR inhibition, yielding off-target effects $(7,76,78)$. miR-therapeutics must be able to discern between the many miRs that may share identical/similar seed sequences with the intended target. Thus, careful consideration of target miRs must be undertaken to ensure that miR-based therapeutics can modulate the intended endogenous target with a high degree of specificity, to minimize off-target effects.

Finally, the administration of miR-therapeutics and carrier vehicles such as targeted nanoparticles may cause deleterious consequences by miR sequence independent off-target effects $(7,76,78)$. miR agents may be detected by both the innate and adaptive arms of the human immune systems, and chemical modification to therapeutic components is necessary to avoid immunostimulatory off-target effects $(81,82)$. Furthermore, these agents may alter physiological processes (e.g., blood coagulation, complement cascade activation) or may induce organ system dysfunction (e.g., hepatotoxicity, nephrotoxicity), owing to the accumulation, clearance, and excretion of these molecules (83-85). Therefore, the consideration of these therapeutic barriers is necessary for the successful utilization of miR-based therapeutics. 


\section{RAS-ERK SIGNALING}

\section{Organization and regulation of the RAS-ERK signaling pathway:}

RAS-ERK signaling is a critical mediator of cell physiological processes including cell proliferation, differentiation and motility (21,22,24-28). Activation of this pathway occurs downstream of signaling inputs including receptor tyrosine kinases (RTKs), integrins, and ion channels, which are in turn activated by a variety of stimuli and cell stresses $(23,24,86)$. The RAS family of GTPases consists of four members (HRAS, NRAS, KRAS4A and KRAS4B [alternatively spliced variants]) that arise from three distinct genes (HRAS, NRAS, and KRAS) and occupy a critical position in relaying signaling from these diverse inputs to activate downstream effector pathways such as the RAF-MEK-ERK pathway, as well as the phosphoinositide 3-kinase (PI3-K)-AKT pathway $(21,87,88)$. RAS proteins fulfill this important role by functioning as binary switches that alternate between the GTP-bound "on" state (RASGTP), which enables RAS to engage downstream effector pathways, and the GDP-bound "off" state (RAS-GDP) $(17,89)$. The activation state of RAS is predominantly governed by critical accessory proteins that enable the transition between either of these states. Essential factors for proper RAS signaling include guanine nucleotide exchange factors (GEFs) which promote the formation of RAS-GTP, GAPs, which promote GTP hydrolysis, and scaffolding proteins such as SPRED1 which appears critical for membrane localization of NF1/GAP (37).

In humans, the activation of the RAF-MEK-ERK pathway is initiated by the preferential interaction of membrane-associated RAS-GTP with the RAS-binding domain of the RAF family of serine/threonine kinases $(19,22,90)$. Membrane-associated RAS proteins exist as dimers, and this dimerization may be critical for the activation of RAF kinases (composed of three paralogs: 
ARAF, BRAF, and CRAF/RAF-1), which occurs in a complex multi-step process (91-93). Activated RAF kinases phosphorylate and activate the dual specificity kinases MEK 1 and MEK 2 (MEK 1/2), which in turn phosphorylate and activate ERK 1 and ERK 2 (ERK 1/2), the terminal effector kinases of this pathway $(24,35,36)$. In contrast to the limited substrate specificity of RAF and MEK 1/2, ERK 1/2 are capable of phosphorylating and consequently modulating the activity of a wide variety of cytoplasmic and nuclear substrates. Importantly, the activity of ERK 1/2 responsive transcription factors is critical in orchestrating cell responses to numerous input stimuli that lie upstream of RAS GTPases $(35,36)$.

\section{Regulation of RAS-ERK pathway activity:}

The activation of RAS-ERK signaling is tightly regulated through a variety of means. RAS-ERK pathway activity is maintained by a delicate balance between factors that promote pathway activation (i.e., GEFs), factors that inhibit pathway activation (i.e., GAPs, DUSPs), and proteins that function as scaffolds, adaptors, and/or provide docking sites for pathway regulatory components $(17,30,33-36,89,90,94)$. These factors may confer signaling specificity to membrane subdomains, allowing distinct effects of the different RAS family members. Additionally, ERK 1/2 can directly phosphorylate and inhibit the activity of the GEF SOS1, CRAF, and MEK1, and thus attenuate signaling by feedback inhibition (95-97). Furthermore, ERK 1/2 can also regulate the transcription of upstream drivers of RAS-ERK signaling such as RTKs $(86,98-100)$.

Tightly controlled spatio-temporal regulation of RAS-ERK signaling is critical for the proper execution of cell physiological processes, and inappropriate regulation of the pathway results in a variety of disease states, including developmental disorders and cancer (17,33-36). For example, somatic activating point mutation of $R A S$ and $B R A F$ genes occur in approximately 
$15-30 \%$ and $7-8 \%$ of all cancers respectively $(18-22,25,90,101,102)$. Though some malignancies, such as pancreatic ductal adenocarcinomas, colorectal carcinomas, and melanomas feature a high proportion of activating RAS and RAF mutations $(16,22)$, other cancers such as TNBCs display RAS-ERK pathway activation despite the infrequent occurrence of somatic point mutations, thus implicating dysregulation of RAS-ERK through other means (103-105). Interestingly, cancers such as TNBCs frequently display genetic alterations such as gene copy number changes in pathway components and altered expression of pathway regulatory proteins (105-111). Remarkably, $32 \%$ of basal-like breast cancers display KRAS gene amplifications and $30 \%$ of cancers of this subtype harbor $B R A F$ gene amplifications (105).

\section{The emerging paradigm of a critical role for wild type RAS proteins in cells harboring RAS mutations:}

Early seminal studies identified the potent transforming ability of virally encoded $R A S$ genes and subsequently characterized these gene products as mutated versions of the human $R A S$ homologs (112-121). Furthermore, the observation of similarly mutated endogenous $R A S$ genes in human tumor samples was critical for our understanding of the molecular basis of carcinogenesis (122-124). These important early studies uncovered that RAS mutations predominantly occur in codons 12,13 , and 61 and that these mutant proteins were constitutively bound to GTP (125-128). Furthermore, mutant RAS possessed far less intrinsic GTPase activity compared to the non-mutant counterparts and were virtually resistant to the action of GAPs (29,129-134). The striking effect of activated RAS in these early experiments and the identification of $R A S$ mutations in cancers sparked several decades of research that has vastly broadened our knowledge of cell signaling and its role in neoplasia. 
But compelling questions regarding the regulation of RAS signaling still remain. Recently, how the activity of wild-type RAS proteins contributes to downstream pathway activation in the context of RAS-mutant cells was uncovered in a series of genetic and biochemical studies (38-40), including a study by our lab focused on the role of miR-regulated GAPs in RAS-mutant cells (41). These studies uncovered a previously unappreciated role for wild-type RAS proteins, as well as GEFs and GAPs, as critical signaling molecules in the context of mutant-RAS associated phenotypes. Possibly attributed to the formation of wild type/mutant RAS dimers, these studies found that GEFs and GAPs are critical regulators of tumorigenesis of RAS-mutant cells through their modulation of WT-RAS-GTP levels. These studies concluded that regardless of the RAS mutational status, the ultimate signaling output is likely determined by the ratio of RAS-GTP to RAS-GDP, where the pool of WT-RAS plays a major role, even in cells with mutant RAS. A critical difference between wild-type and mutant RAS proteins is the greater dependence of WT-RAS on GEFs and GAPs $(17,89)$. Consequently these new studies identify these regulators as critical therapeutic targets regardless of the RAS mutational status. These studies establish a new paradigm of how RAS signaling is regulated and highlight the potential of small molecule modulators of GAP or GEF activity.

\section{Therapeutic targeting of RAS-ERK signaling in cancers:}

Given the important role of RAS-ERK signaling in the development and progression of many cancers, the successful therapeutic inhibition of this pathway has been a long standing goal of the targeted chemotherapy era $(19,22,25,135,136)$. Numerous strategies to inhibit RAS-ERK signaling have been envisioned, including those that (i) directly target RAS, (ii) modulate factors that regulate RAS activity, and those that (iii) target downstream kinases (e.g. RAF, MEK, and ERK). One of the most promising therapeutic strategies, utilizing ATP analogues as allosteric or 
competitive inhibitors of RAF or MEK kinase activity has proceeded toward clinical utility, but with prolonged therapeutic responses limited by a variety of factors $(137,138)$. Despite the potent action of these compounds in vitro, therapeutic resistance emerges rapidly and hampers the successful use of these kinase inhibitors $(100,138-142)$.

Contributing to this resistance, acute loss of RAS-ERK signaling in cancer cells results in adaptive reprogramming, including reprogramming of the kinome, with upregulation of multiple (receptor tyrosine) kinases (RTKs) including PDGFR $\beta$, DDR1, and others (100,138-142). In addition, pathway inhibition by agents such as MEK inhibitor is thwarted by the loss of negative feedback regulation, including ERK 1/2 mediated inhibition of positive pathway regulators such as MEK1 and BRAF $(100,138)$. Another factor is that phosphorylation of MEK1 by cRAF results in reduced affinity of MEK $1 / 2$ allosteric inhibitors (96,143). Furthermore, and particularly in melanoma, long term treatment with RAS-ERK inhibitory compounds results in tumor cells acquiring somatic mutations in NRAS, MEK2, or AKT1 to counteract sustained inhibited signaling (144-146). Finally, through the induction of multiple RTKs, the activation of alternative signaling pathways (e.g., PI3-K-AKT) can compensate for the inhibited RAS-ERK signaling (100,146-149). Either singly or in combination, these adaptive changes ultimately circumvent blocked signaling and prevent sustained therapeutic responses. Thus, the development and optimization of more effective RAS-ERK pathway inhibitory strategies and counteracting the rapid emergence of resistant signaling represent critical obstacles to effective therapeutic intervention.

A major goal has been more direct methods for suppression of RAS-GTP levels $(135,136)$. Toward this end, the recent recognition that WT RAS proteins play a critical role in pathway output would appear to reenergize ongoing efforts to target GEFs and GAPs $(17,89)$. 
Other ongoing approaches include reovirus-based therapies and siRNA therapy against mutant KRAS (150-153).

\section{MICRORNA REGULATION OF MUTANT AND WILD TYPE RAS-GTP}

The past decade has provided considerable insight into the critical regulatory roles that miRs exert over key cancer relevant signaling networks such as the RAS-ERK pathway. Our knowledge of how miRs can modulate RAS-ERK pathway activation continues to grow as potential miR-mRNA regulatory networks are identified using a variety of strategies including in silico miR target prediction methods, profiling of the cellular transcriptome/proteome, and experimental validation of putative interactors that typically employs translational reporter assays.

Three major paradigms of miR-mediated RAS-ERK regulation have emerged from these studies. miRs can impact the translation of (i) core RAS-ERK pathway components (e.g., let-7 targets $H R A S, N R A S$, and $K R A S$ ) (42), (ii) critical pathway regulatory proteins that are required for the proper spatio-temporal control of RAS-ERK signaling (e.g., miR-206 and/or miR-21 collaboratively target $R A S A 1, S P R E D 1, S P R Y 1$; and miR-21 individually targets PTEN) (Fig. 1) $(41,45,154)$, and (iii) upstream drivers and downstream effector/regulatory molecules (e.g., miR9-3p targets ITGB1, and miR-206/21 co-target PDCD4) (155). Examples of miRs that regulate RAS-ERK pathway activity in a variety of cancer contexts are listed in Table 1 . Indeed, these miRs represent potential therapeutic substrates and targets that can be modulated in the treatment

of cancer. In the following sections, we evaluate the therapeutic potential of three miRs, let-7 and 
miR-206/21, that hold great promise as potential therapeutic targets in the treatment of cancers by impacting RAS-ERK signaling.

\section{let-7 represses the translation of the RAS family of GTPases:}

The let-7 gene was initially identified as an essential regulator of patterning development in the nematode $C$. elegans, and was among the first defined miRs $(5,6)$. Subsequent studies observed evolutionary conservation of let-7 and identified related paralogs in the genomes of multiple species, including humans $(156,157)$. Similarly to C. elegans, human let-7 is critical for epithelial cellular differentiation and proliferation $(158,159)$. Furthermore, reduced expression of let-7 in cancer occurs through genetic deletion, mutation, epigenetic silencing, and posttranscriptional regulation of let-7 biogenesis, and decreased let-7 expression has been implicated in pathogenesis of a wide variety of malignancies, including cancers of the lung, colon, ovary, and breast $(42,74,159)$. These studies suggest a tumor suppressive function for this miR.

Prominent mechanisms by which let-7 exerts a tumor suppressive role is by repressing the translation of the three RAS proteins (HRAS, NRAS, and KRAS) and cMYC, a downstream effector of RAS-ERK signaling $(42,160)$. Studies analyzing in vitro and in vivo models of nonsmall cell lung cancer (NSCLC), as well as human tumor samples, show that let-7 expression is inversely correlated with the expression of KRAS, a critical promoter of NSCLC tumorigenesis. Let-7 abrogates tumor development and RAS-ERK signaling in an autochthonous model of NSCLC driven by activated KRAS $\left(K R A S^{G 12 D}\right)(161,162)$. Consistent with this previous result, a tumor suppressive role for let-7 was observed in a study analyzing a xenograft model of NSCLC (160). Additionally, in a breast cancer context, let-7 antagonizes the maintenance, survival, and self-renewal of cancer stem-like cells (CSCs), and this suppressive activity was correlated with 
the reduced expression of RAS and HMGA2 (159). Thus, by suppressing RAS expression, let-7 can attenuate RAF-MEK-ERK signaling and dependent oncogenic phenotypes regardless of the RAS-mutation status of cancers. These studies suggest that let-7 can act as both a cancerpreventative and cancer-therapeutic agent, and point to let-7 supplementation as a promising strategy to target RAS-ERK signaling in the treatment of cancers.

miR-206/21 collaboratively repress the translation of $R A S A 1$ and SPRED1 and inhibit GAP activity:

Our laboratory recently identified two miRs, miR-206 and the well characterized oncogene miR-21 (collectively: miR-206/21), as critical regulators of RAS-ERK signaling in TNBC cells (Fig. 1) (41). Whereas miR-206 is well characterized in regulating the differentiation of adult muscle stem cells, the role of endogenous miR-206 in breast cancer is less well known (163-166). In contrast to miR-206, miR-21 is prominently upregulated in many malignancies, including in breast cancer, and promotes tumorigenesis by repressing the translation of multiple tumor suppressors, including negative regulators of RAS-ERK signaling (e.g., SPRY1, RASA1, and PDCD4), as well as RAS-PI3K signaling (e.g., PTEN) (44,167-174).

We found that the expression of miR-206/21 was dependent on the zinc-finger pluripotency factor Kruppel-like factor 4 (KLF4), which has most often been implicated as a poor prognostic factor in breast cancer (175-177). Whereas KLF4 and miR-206 are preferentially expressed in MaCSCs, miR-21 is similarly expressed in these two compartments, consistent with the "on/off” mode of miR-21 regulation by KLF4 (41). Furthermore, recent studies from our laboratory indicate that KLF4 and/or its dependent miRs are important regulators of anti- 
apoptotic signaling in breast cancer cells and promote survival against diverse forms of stress, including treatment with conventional cytotoxic or targeted chemotherapies (178).

The combined action of miR-206/21 promotes signaling by repressing the translation of multiple RAS-ERK pathway inhibitory proteins which act at various hierarchical levels in this signaling network (41). Interestingly, the manipulation of each individual miR did not yield large changes in pathway activity, suggesting that the collaborative action of miR-206/21 was required to achieve a substantial effect. Indeed, treatment of TNBC cells with anti-miR-206/21 was sufficient to suppress pathway activity by greater than $80 \%$. We found that miR-206/21 co-target and co-suppress the translation of the GAP RASA1, and the Neurofibromatosis 1 (NF1) GAP associated protein, SPRED1. This GAP-deficient state interferes with RAS inactivation (i.e., the formation of signaling-deficient WT-RAS-GDP), and consequently promotes RAS-ERK signaling, RAS dependent cell phenotypes, and TNBC tumorigenesis. Importantly, whereas inhibition of KLF4-miR-206/21 signaling potently suppresses RAS-ERK signaling in multiple RAS-mutant TNBC models (MDA-MB-231-KRAS $S^{G 13 D}$, Hs578t - HRAS $S^{G 12 D}$, and SUM159PT $H R A S^{G 12 D}$ ) as well as in cells that exclusively harbor WT-RAS proteins, stable shRNA-mediated suppression of RASA1 and SPRED1 promoted pathway activity on its own, and rendered cells virtually resistant to anti-miR-206/21. Consequently, TNBC cells and potentially many other tumor types are GAP-deficient owing to the collaborative action of miR-206/21 on RASA1 and SPRED1. Therefore the RASA1 and SPRED1 transcripts represent latent tumor suppressors with the potential for reactivation by anti-miR-206/21. Our analysis of GAP signaling and RAS-GTP levels in this study yielded results that were consistent with the newly emerging paradigm that the output of RAS-ERK signaling is critically dependent on the activation status of WT-RAS, 
and uncovered a previously unappreciated role for GAP proteins in cells harboring RAS mutations (38-41).

\section{The potential for in vivo inhibition of miR-206/21 for the treatment of cancer:}

In addition to the numerous studies that have elucidated the oncogenic role of miR-21, our analysis of KLF4-miR signaling suggests that inhibition of miR-206/21 has the potential to be a promising therapeutic strategy in the treatment of TNBC and in other cancers. In vivo silencing of miR-206/21 using anti-sense oligonucleotide based strategies could effectively attenuate RAS-ERK signaling by more directly suppressing RAS-GTP levels. Improved therapeutic effects may be achieved when used in combination with other pathway inhibitory strategies or in conjunction with cytotoxic chemotherapy. Furthermore, other strategies for suppression of oncogenic miRs hold promise, including aptamer-mediated inhibition of nucleolin (179), a cell surface protein required for the maturation of a specific subset of miRs, including miRs that promote RAS-ERK signaling (e.g., miR-21, miR-221).

Therapeutic targeting of these miRs in combination with other therapeutic modalities could offer an advantage over single kinase inhibition strategies. Due to the rapid adaptive reprogramming and the emergence of inhibitor-resistant signaling, these do not yield durable responses (100,138-142). Probably owing to the more physiologic enhancement of GAP activity, suppression of miR-206/21 leads to potent inhibition of RAS-ERK signaling and RAS-dependent phenotypes (41), but appears not to destabilize c-MYC or to induce the dramatic adaptive reprogramming response that results from kinase inhibitors (unpublished observations, SBS and JMR). Additionally, miR-206/21 suppression may sensitize cells to the effects of other anticancer therapeutic modalities. We have recently found that miR-206 represses the translation of 
the pro-apoptotic protein $P D C D 4$, a well established miR-21 target, to protect breast cancer cells from apoptosis in response to cytotoxic chemotherapy. Thus the combinatorial suppression of miR-206/21 could potentiate the effects of conventional treatments and thereby reduce the required dosage of these agents, potentially mitigating the adverse events associated with anticancer therapy.

While therapeutic windows are notoriously difficult to predict from the analysis of models, mice deficient in either miR-206 or miR-21 develop normally and appear healthy as adults, supporting the potential for dual inhibition as a therapeutic strategy. Thus, the therapeutic inhibition of miR-206/21 activity has the potential to target RAS-ERK signaling through the reexpression of GAP activity, and holds great promise for the treatment of RAS-driven cancers such as TNBC.

\section{CONCLUSIONS}

The RAS-ERK signaling pathway is critical in the development and progression of numerous malignancies, and more effective targeted pathway inhibition has the potential to greatly improve the treatment of cancers. Despite extensive work that has culminated in the development of numerous pathway inhibitory strategies, direct suppression of RAS-GTP levels has been difficult to achieve, and the successful utilization of existing kinase inhibitory agents is hampered by adaptive reprogramming of cell signaling. The consistent demonstration of the inefficacy of single kinase inhibition strategies has prompted the consideration of alternative routes of targeting RAS-ERK signaling components and pathway regulatory molecules. miRs represent one such set of important regulatory molecules that can be targeted for the therapeutic 
inhibition of pathway activity. These miR-based therapeutic strategies involve the supplementation of RAS-ERK inhibitory miRs using miR-mimics (e.g., let-7), or the in vivo silencing of miRs that promote pathway activity using anti-sense strategies (e.g., miR-206/21). Unlike single kinase inhibitors, these targeted miR-based therapeutic strategies may yield durable anti-tumor responses as they can collaboratively target multiple levels of this signaling pathway and regulate other cell physiologic processes that are critical to RAS-ERK mediated tumorigenesis. Though development and optimization of improved miR delivery methods is necessary, targeting RAS-ERK signaling by miR-based therapeutics holds great promise in the treatment of cancers that are reliant on this signaling pathway.

\section{ACKNOWLEDGEMENTS}

This work was supported by grants NCI RO1 CA127405 (to JMR), the Jo and Ben Statler Chair in Breast Cancer Research, and the Wilmer V. and Helen B. Morley Memorial Fund at the Mary Babb Randolph Cancer Center (MBRCC). 


\section{REFERENCES}

1. He, L. and G. J. Hannon. 2004. MicroRNAs: small RNAs with a big role in gene regulation. Nat. Rev. Genet. 5:522-531.

2. Kim, V. N. 2005. MicroRNA biogenesis: coordinated cropping and dicing. Nat. Rev. Mol. Cell Biol. 6:376-385.

3. Chen, K. and N. Rajewsky. 2007. The evolution of gene regulation by transcription factors and microRNAs. Nat. Rev. Genet. 8:93-103.

4. Ameres, S. L. and P. D. Zamore. 2013. Diversifying microRNA sequence and function. Nat. Rev. Mol. Cell Biol. 14:475-488.

5. Wightman, B., T. R. Burglin, J. Gatto, P. Arasu, and G. Ruvkun. 1991. Negative regulatory sequences in the lin-14 3'-untranslated region are necessary to generate a temporal switch during Caenorhabditis elegans development. Genes Dev. 5:1813-1824.

6. Lee, R. C., R. L. Feinbaum, and V. Ambros. 1993. The C. elegans heterochronic gene lin4 encodes small RNAs with antisense complementarity to lin-14. Cell 75:843-854.

7. Kasinski, A. L. and F. J. Slack. 2011. Epigenetics and genetics. MicroRNAs en route to the clinic: progress in validating and targeting microRNAs for cancer therapy. Nat. Rev. Cancer 11:849-864.

8. Sayed, D. and M. Abdellatif. 2011. MicroRNAs in development and disease. Physiol Rev. 91:827-887.

9. Iorio, M. V. and C. M. Croce. 2012. MicroRNA dysregulation in cancer: diagnostics, monitoring and therapeutics. A comprehensive review. EMBO Mol. Med. 4:143-159.

10. Sun, K. and E. C. Lai. 2013. Adult-specific functions of animal microRNAs. Nat. Rev. Genet. 14:535-548.

11. Calin, G. A. and C. M. Croce. 2006. MicroRNA-cancer connection: the beginning of a new tale. Cancer Res 66:7390-7394.

12. Calin, G. A. and C. M. Croce. 2006. MicroRNA signatures in human cancers. Nat. Rev. Cancer 6:857-866.

13. Esquela-Kerscher, A. and F. J. Slack. 2006. Oncomirs - microRNAs with a role in cancer. Nat. Rev. Cancer 6:259-269.

14. Hanahan, D. and R. A. Weinberg. 2000. The hallmarks of cancer. Cell 100:57-70. 
15. Weinberg, R. A. 2007. Growth Factors, Receptors, and Cancer, p. 119-158. In R. A. Weinberg (ed.), The Biology of Cancer. Garland Science, Taylor and Francis Group, LLC, New York.

16. Bos, J. L. 1989. RAS oncogenes in human cancer: a review. Cancer Res. 49:4682-4689.

17. Bos, J. L., H. Rehmann, and A. Wittinghofer. 2007. GEFs and GAPs: critical elements in the control of small G proteins. Cell 129:865-877.

18. Schubbert, S., K. Shannon, and G. Bollag. 2007. Hyperactive Ras in developmental disorders and cancer. Nat. Rev. Cancer 7:295-308.

19. Karnoub, A. E. and R. A. Weinberg. 2008. Ras oncogenes: split personalities. Nat. Rev. Mol. Cell Biol. 9:517-531.

20. Tidyman, W. E. and K. A. Rauen. 2009. The RASopathies: developmental syndromes of Ras/MAPK pathway dysregulation. Curr. Opin. Genet. Dev. 19:230-236.

21. Young, A., J. Lyons, A. L. Miller, V. T. Phan, I. R. Alarcon, and F. Mccormick. 2009. Ras signaling and therapies. Adv. Cancer Res 102:1-17.

22. Pylayeva-Gupta, Y., E. Grabocka, and D. Bar-Sagi. 2011. RAS oncogenes: weaving a tumorigenic web. Nat. Rev. Cancer 11:761-774.

23. Schlessinger, J. 2000. Cell signaling by receptor tyrosine kinases. Cell 103:211-225.

24. Johnson, G. L. and R. Lapadat. 2002. Mitogen-activated protein kinase pathways mediated by ERK, JNK, and p38 protein kinases. Science 298:1911-1912.

25. Downward, J. 2003. Targeting RAS signalling pathways in cancer therapy. Nat. Rev. Cancer 3:11-22.

26. Dhillon, A. S., S. Hagan, O. Rath, and W. Kolch. 2007. MAP kinase signalling pathways in cancer. Oncogene 26:3279-3290.

27. Kolch, W. 2005. Coordinating ERK/MAPK signalling through scaffolds and inhibitors. Nat. Rev. Mol. Cell Biol. 6:827-837.

28. Mebratu, Y. and Y. Tesfaigzi. 2009. How ERK1/2 activation controls cell proliferation and cell death: Is subcellular localization the answer? Cell Cycle 8:1168-1175.

29. Trahey, M. and F. Mccormick. 1987. A cytoplasmic protein stimulates normal N-ras p21 GTPase, but does not affect oncogenic mutants. Science 238:542-545.

30. Boguski, M. S. and F. Mccormick. 1993. Proteins regulating Ras and its relatives. Nature 366:643-654. 
31. Scheffzek, K., C. Klebe, K. Fritzwolf, W. Kabsch, and A. Wittinghofer. 1995. Crystal structure of the nuclear Ras-related protein Ran in its GDP-bound form. Nature 374:378381.

32. Scheffzek, K., M. R. Ahmadian, W. Kabsch, L. Wiesmuller, A. Lautwein, F. Schmitz, and A. Wittinghofer. 1997. The Ras-RasGAP complex: structural basis for GTPase activation and its loss in oncogenic Ras mutants. Science 277:333-338.

33. McKay, M. M. and D. K. Morrison. 2007. Integrating signals from RTKs to ERK/MAPK. Oncogene 26:3113-3121.

34. Wortzel, I. and R. Seger. 2011. The ERK Cascade: Distinct Functions within Various Subcellular Organelles. Genes Cancer 2:195-209.

35. Roskoski, R., Jr. 2012. ERK1/2 MAP kinases: structure, function, and regulation. Pharmacol. Res. 66:105-143.

36. Roskoski, R., Jr. 2012. MEK1/2 dual-specificity protein kinases: structure and regulation. Biochem. Biophys. Res. Commun. 417:5-10.

37. Stowe, I. B., E. L. Mercado, T. R. Stowe, E. L. Bell, J. A. Oses-Prieto, H. Hernandez, A. L. Burlingame, and F. Mccormick. 2012. A shared molecular mechanism underlies the human rasopathies Legius syndrome and Neurofibromatosis-1. Genes Dev. 26:14211426.

38. Jeng, H. H., L. J. Taylor, and D. Bar-Sagi. 2012. Sos-mediated cross-activation of wildtype Ras by oncogenic Ras is essential for tumorigenesis. Nat. Commun. 3:1168.

39. Young, A., D. Lou, and F. Mccormick. 2013. Oncogenic and wild-type Ras play divergent roles in the regulation of mitogen-activated protein kinase signaling. Cancer Discov. 3:112-123.

40. Grabocka, E., Y. Pylayeva-Gupta, M. J. Jones, V. Lubkov, E. Yemanaberhan, L. Taylor, H. H. Jeng, and D. Bar-Sagi. 2014. Wild-type H- and N-Ras promote mutant K-Rasdriven tumorigenesis by modulating the DNA damage response. Cancer Cell 25:243-256.

41. Sharma, S. B., C. C. Lin, M. K. Farrugia, S. L. McLaughlin, E. J. Ellis, K. M. Brundage, M. A. Salkeni, and J. M. Ruppert. 2014. MicroRNAs 206 and 21 cooperate to promote RAS-extracellular signal-regulated kinase signaling by suppressing the translation of RASA1 and SPRED1. Mol. Cell Biol. 34:4143-4164.

42. Johnson, S. M., H. Grosshans, J. Shingara, M. Byrom, R. Jarvis, A. Cheng, E. Labourier, K. L. Reinert, D. Brown, and F. J. Slack. 2005. RAS is regulated by the let-7 microRNA family. Cell 120:635-647.

43. Fish, J. E., M. M. Santoro, S. U. Morton, S. Yu, R. F. Yeh, J. D. Wythe, K. N. Ivey, B. G. Bruneau, D. Y. Stainier, and D. Srivastava. 2008. miR-126 regulates angiogenic signaling and vascular integrity. Dev Cell 15:272-284. 
44. Thum, T., C. Gross, J. Fiedler, T. Fischer, S. Kissler, M. Bussen, P. Galuppo, S. Just, W. Rottbauer, S. Frantz, M. Castoldi, J. Soutschek, V. Koteliansky, A. Rosenwald, M. A. Basson, J. D. Licht, J. T. Pena, S. H. Rouhanifard, M. U. Muckenthaler, T. Tuschl, G. R. Martin, J. Bauersachs, and S. Engelhardt. 2008. MicroRNA-21 contributes to myocardial disease by stimulating MAP kinase signalling in fibroblasts. Nature 456:980-984.

45. Hatley, M. E., D. M. Patrick, M. R. Garcia, J. A. Richardson, R. Bassel-Duby, R. E. van, and E. N. Olson. 2010. Modulation of K-Ras-dependent lung tumorigenesis by MicroRNA-21. Cancer Cell 18:282-293.

46. Sun, D., F. Yu, Y. Ma, R. Zhao, X. Chen, J. Zhu, C. Y. Zhang, J. Chen, and J. Zhang. 2013. MicroRNA-31 activates the RAS pathway and functions as an oncogenic MicroRNA in human colorectal cancer by repressing RAS p21 GTPase activating protein 1 (RASA1). J. Biol. Chem. 288:9508-9518.

47. Stark, M. S., V. F. Bonazzi, G. M. Boyle, J. M. Palmer, J. Symmons, C. M. Lanagan, C. W. Schmidt, A. C. Herington, R. Ballotti, P. M. Pollock, and N. K. Hayward. 2015. miR514a regulates the tumour suppressor NF1 and modulates BRAFi sensitivity in melanoma. Oncotarget.

48. Lee, Y., C. Ahn, J. Han, H. Choi, J. Kim, J. Yim, J. Lee, P. Provost, O. Radmark, S. Kim, and V. N. Kim. 2003. The nuclear RNase III Drosha initiates microRNA processing. Nature 425:415-419.

49. Denli, A. M., B. B. Tops, R. H. Plasterk, R. F. Ketting, and G. J. Hannon. 2004.

Processing of primary microRNAs by the Microprocessor complex. Nature 432:231-235.

50. Gregory, R. I., K. P. Yan, G. Amuthan, T. Chendrimada, B. Doratotaj, N. Cooch, and R. Shiekhattar. 2004. The Microprocessor complex mediates the genesis of microRNAs. Nature 432:235-240.

51. Landthaler, M., A. Yalcin, and T. Tuschl. 2004. The human DiGeorge syndrome critical region gene 8 and Its D. melanogaster homolog are required for miRNA biogenesis. Curr. Biol. 14:2162-2167.

52. Han, J., Y. Lee, K. H. Yeom, Y. K. Kim, H. Jin, and V. N. Kim. 2004. The DroshaDGCR8 complex in primary microRNA processing. Genes Dev. 18:3016-3027.

53. Bernstein, E., A. A. Caudy, S. M. Hammond, and G. J. Hannon. 2001. Role for a bidentate ribonuclease in the initiation step of RNA interference. Nature 409:363-366.

54. Ketting, R. F., S. E. Fischer, E. Bernstein, T. Sijen, G. J. Hannon, and R. H. Plasterk. 2001. Dicer functions in RNA interference and in synthesis of small RNA involved in developmental timing in C. elegans. Genes Dev. 15:2654-2659.

55. Provost, P., D. Dishart, J. Doucet, D. Frendewey, B. Samuelsson, and O. Radmark. 2002. Ribonuclease activity and RNA binding of recombinant human Dicer. EMBO J. 21:58645874. 
56. Lee, Y., K. Jeon, J. T. Lee, S. Kim, and V. N. Kim. 2002. MicroRNA maturation: stepwise processing and subcellular localization. EMBO J. 21:4663-4670.

57. Ambros, V., B. Bartel, D. P. Bartel, C. B. Burge, J. C. Carrington, X. Chen, G. Dreyfuss, S. R. Eddy, S. Griffiths-Jones, M. Marshall, M. Matzke, G. Ruvkun, and T. Tuschl. 2003. A uniform system for microRNA annotation. RNA. 9:277-279.

58. Griffiths-Jones, S., R. J. Grocock, S. van Dongen, A. Bateman, and A. J. Enright. 2006. miRBase: microRNA sequences, targets and gene nomenclature. Nucleic Acids Res. 34:D140-D144.

59. Yang, J. S. and E. C. Lai. 2011. Alternative miRNA biogenesis pathways and the interpretation of core miRNA pathway mutants. Mol. Cell 43:892-903.

60. Borchert, G. M., W. Lanier, and B. L. Davidson. 2006. RNA polymerase III transcribes human microRNAs. Nat. Struct. Mol. Biol. 13:1097-1101.

61. Okamura, K., J. W. Hagen, H. Duan, D. M. Tyler, and E. C. Lai. 2007. The mirtron pathway generates microRNA-class regulatory RNAs in Drosophila. Cell 130:89-100.

62. Yang, J. S., T. Maurin, N. Robine, K. D. Rasmussen, K. L. Jeffrey, R. Chandwani, E. P. Papapetrou, M. Sadelain, D. O'Carroll, and E. C. Lai. 2010. Conserved vertebrate mir451 provides a platform for Dicer-independent, Ago2-mediated microRNA biogenesis. Proc. Natl. Acad. Sci. U. S. A 107:15163-15168.

63. Bartel, D. P. 2009. MicroRNAs: target recognition and regulatory functions. Cell 136:215-233.

64. Czech, B. and G. J. Hannon. 2011. Small RNA sorting: matchmaking for Argonautes. Nat. Rev. Genet. 12:19-31.

65. Jackson, R. J., C. U. Hellen, and T. V. Pestova. 2010. The mechanism of eukaryotic translation initiation and principles of its regulation. Nat. Rev. Mol. Cell Biol. 11:113127.

66. Vasudevan, S. and J. A. Steitz. 2007. AU-rich-element-mediated upregulation of translation by FXR1 and Argonaute 2. Cell 128:1105-1118.

67. Vasudevan, S., Y. Tong, and J. A. Steitz. 2007. Switching from repression to activation: microRNAs can up-regulate translation. Science 318:1931-1934.

68. Vasudevan, S., Y. Tong, and J. A. Steitz. 2008. Cell-cycle control of microRNAmediated translation regulation. Cell Cycle 7:1545-1549.

69. Steitz, J. A. and S. Vasudevan. 2009. miRNPs: versatile regulators of gene expression in vertebrate cells. Biochem. Soc. Trans. 37:931-935. 
70. Jangra, R. K., M. Yi, and S. M. Lemon. 2010. DDX6 (Rck/p54) is required for efficient hepatitis $\mathrm{C}$ virus replication but not for internal ribosome entry site-directed translation. J. Virol. 84:6810-6824.

71. Lin, C. C., L. Z. Liu, J. B. Addison, A. V. Ivanov, and J. M. Ruppert. 2011. A KLF4miRNA-206 autoregulatory feedback loop can promote or inhibit protein translation depending upon cell context. Mol. Cell Biol. 31:2513-2527.

72. Ha, M. and V. N. Kim. 2014. Regulation of microRNA biogenesis. Nat. Rev. Mol. Cell Biol. 15:509-524.

73. Inui, M., G. Martello, and S. Piccolo. 2010. MicroRNA control of signal transduction. Nat. Rev. Mol. Cell Biol. 11:252-263.

74. Kasinski, A. L. and F. J. Slack. 2010. Potential microRNA therapies targeting Ras, NFkappaB and p53 signaling. Curr. Opin. Mol. Ther 12:147-157.

75. Stenvang, J., A. Petri, M. Lindow, S. Obad, and S. Kauppinen. 2012. Inhibition of microRNA function by antimiR oligonucleotides. Silence. 3:1.

76. Ling, H., M. Fabbri, and G. A. Calin. 2013. MicroRNAs and other non-coding RNAs as targets for anticancer drug development. Nat. Rev. Drug Discov. 12:847-865.

77. van, R. E. and S. Kauppinen. 2014. Development of microRNA therapeutics is coming of age. EMBO Mol. Med 6:851-864.

78. Li, Z. and T. M. Rana. 2014. Therapeutic targeting of microRNAs: current status and future challenges. Nat. Rev. Drug Discov. 13:622-638.

79. Lamond, A. I. and B. S. Sproat. 1993. Antisense oligonucleotides made of 2'-OalkylRNA: their properties and applications in RNA biochemistry. FEBS Lett. 325:123127.

80. Cummins, L. L., S. R. Owens, L. M. Risen, E. A. Lesnik, S. M. Freier, D. McGee, C. J. Guinosso, and P. D. Cook. 1995. Characterization of fully 2'-modified oligoribonucleotide hetero- and homoduplex hybridization and nuclease sensitivity. Nucleic Acids Res 23:2019-2024.

81. Judge, A. D., V. Sood, J. R. Shaw, D. Fang, K. McClintock, and I. MacLachlan. 2005. Sequence-dependent stimulation of the mammalian innate immune response by synthetic siRNA. Nat. Biotechnol. 23:457-462.

82. Hornung, V., M. Guenthner-Biller, C. Bourquin, A. Ablasser, M. Schlee, S. Uematsu, A. Noronha, M. Manoharan, S. Akira, F. A. de, S. Endres, and G. Hartmann. 2005. Sequence-specific potent induction of IFN-alpha by short interfering RNA in plasmacytoid dendritic cells through TLR7. Nat. Med 11:263-270. 
83. Galbraith, W. M., W. C. Hobson, P. C. Giclas, P. J. Schechter, and S. Agrawal. 1994. Complement activation and hemodynamic changes following intravenous administration of phosphorothioate oligonucleotides in the monkey. Antisense Res Dev 4:201-206.

84. Henry, S. P., W. Novotny, J. Leeds, C. Auletta, and D. J. Kornbrust. 1997. Inhibition of coagulation by a phosphorothioate oligonucleotide. Antisense Nucleic Acid Drug Dev 7:503-510.

85. Swayze, E. E., A. M. Siwkowski, E. V. Wancewicz, M. T. Migawa, T. K. Wyrzykiewicz, G. Hung, B. P. Monia, and C. F. Bennett. 2007. Antisense oligonucleotides containing locked nucleic acid improve potency but cause significant hepatotoxicity in animals. Nucleic Acids Res 35:687-700.

86. Lemmon, M. A. and J. Schlessinger. 2010. Cell signaling by receptor tyrosine kinases. Cell 141:1117-1134.

87. McCubrey, J. A., L. S. Steelman, S. L. Abrams, J. T. Lee, F. Chang, F. E. Bertrand, P. M. Navolanic, D. M. Terrian, R. A. Franklin, A. B. D'Assoro, J. L. Salisbury, M. C. Mazzarino, F. Stivala, and M. Libra. 2006. Roles of the RAF/MEK/ERK and PI3K/PTEN/AKT pathways in malignant transformation and drug resistance. Adv. Enzyme Regul. 46:249-279.

88. Roberts, P. J. and C. J. Der. 2007. Targeting the Raf-MEK-ERK mitogen-activated protein kinase cascade for the treatment of cancer. Oncogene 26:3291-3310.

89. Vigil, D., J. Cherfils, K. L. Rossman, and C. J. Der. 2010. Ras superfamily GEFs and GAPs: validated and tractable targets for cancer therapy? Nat. Rev. Cancer 10:842-857.

90. Cox, A. D. and C. J. Der. 2010. Ras history: The saga continues. Small GTPases. 1:2-27.

91. Inouye, K., S. Mizutani, H. Koide, and Y. Kaziro. 2000. Formation of the Ras dimer is essential for Raf-1 activation. J Biol. Chem. 275:3737-3740.

92. Guldenhaupt, J., T. Rudack, P. Bachler, D. Mann, G. Triola, H. Waldmann, C. Kotting, and K. Gerwert. 2012. N-Ras forms dimers at POPC membranes. Biophys. J 103:15851593.

93. Lin, W. C., L. Iversen, H. L. Tu, C. Rhodes, S. M. Christensen, J. S. Iwig, S. D. Hansen, W. Y. Huang, and J. T. Groves. 2014. H-Ras forms dimers on membrane surfaces via a protein-protein interface. Proc. Natl. Acad. Sci. U. S. A 111:2996-3001.

94. Yoshimura, A. 2009. Regulation of cytokine signaling by the SOCS and Spred family proteins. Keio J. Med. 58:73-83.

95. Rossomando, A. J., P. Dent, T. W. Sturgill, and D. R. Marshak. 1994. Mitogen-activated protein kinase kinase 1 (MKK1) is negatively regulated by threonine phosphorylation. Mol. Cell Biol. 14:1594-1602. 
96. Ueki, K., S. Matsuda, K. Tobe, Y. Gotoh, H. Tamemoto, M. Yachi, Y. Akanuma, Y. Yazaki, E. Nishida, and T. Kadowaki. 1994. Feedback regulation of mitogen-activated protein kinase kinase kinase activity of c-Raf-1 by insulin and phorbol ester stimulation. J Biol. Chem. 269:15756-15761.

97. Buday, L., P. H. Warne, and J. Downward. 1995. Downregulation of the Ras activation pathway by MAP kinase phosphorylation of Sos. Oncogene 11:1327-1331.

98. Sears, R., F. Nuckolls, E. Haura, Y. Taya, K. Tamai, and J. R. Nevins. 2000. Multiple Ras-dependent phosphorylation pathways regulate Myc protein stability. Genes Dev 14:2501-2514.

99. Amit, I., A. Citri, T. Shay, Y. Lu, M. Katz, F. Zhang, G. Tarcic, D. Siwak, J. Lahad, J. Jacob-Hirsch, N. Amariglio, N. Vaisman, E. Segal, G. Rechavi, U. Alon, G. B. Mills, E. Domany, and Y. Yarden. 2007. A module of negative feedback regulators defines growth factor signaling. Nat. Genet. 39:503-512.

100. Duncan, J. S., M. C. Whittle, K. Nakamura, A. N. Abell, A. A. Midland, J. S. Zawistowski, N. L. Johnson, D. A. Granger, N. V. Jordan, D. B. Darr, J. Usary, P. F. Kuan, D. M. Smalley, B. Major, X. He, K. A. Hoadley, B. Zhou, N. E. Sharpless, C. M. Perou, W. Y. Kim, S. M. Gomez, X. Chen, J. Jin, S. V. Frye, H. S. Earp, L. M. Graves, and G. L. Johnson. 2012. Dynamic reprogramming of the kinome in response to targeted MEK inhibition in triple-negative breast cancer. Cell 149:307-321.

101. Davies, H., G. R. Bignell, C. Cox, P. Stephens, S. Edkins, S. Clegg, J. Teague, H. Woffendin, M. J. Garnett, W. Bottomley, N. Davis, E. Dicks, R. Ewing, Y. Floyd, K. Gray, S. Hall, R. Hawes, J. Hughes, V. Kosmidou, A. Menzies, C. Mould, A. Parker, C. Stevens, S. Watt, S. Hooper, R. Wilson, H. Jayatilake, B. A. Gusterson, C. Cooper, J. Shipley, D. Hargrave, K. Pritchard-Jones, N. Maitland, G. Chenevix-Trench, G. J. Riggins, D. D. Bigner, G. Palmieri, A. Cossu, A. Flanagan, A. Nicholson, J. W. Ho, S. Y. Leung, S. T. Yuen, B. L. Weber, H. F. Seigler, T. L. Darrow, H. Paterson, R. Marais, C. J. Marshall, R. Wooster, M. R. Stratton, and P. A. Futreal. 2002. Mutations of the BRAF gene in human cancer. Nature 417:949-954.

102. Fernandez-Medarde, A. and E. Santos. 2011. Ras in cancer and developmental diseases. Genes Cancer 2:344-358.

103. Hoeflich, K. P., C. O'Brien, Z. Boyd, G. Cavet, S. Guerrero, K. Jung, T. Januario, H. Savage, E. Punnoose, T. Truong, W. Zhou, L. Berry, L. Murray, L. Amler, M. Belvin, L. S. Friedman, and M. R. Lackner. 2009. In vivo antitumor activity of MEK and phosphatidylinositol 3-kinase inhibitors in basal-like breast cancer models. Clin. Cancer Res. 15:4649-4664.

104. Mirzoeva, O. K., D. Das, L. M. Heiser, S. Bhattacharya, D. Siwak, R. Gendelman, N. Bayani, N. J. Wang, R. M. Neve, Y. Guan, Z. Hu, Z. Knight, H. S. Feiler, P. Gascard, B. Parvin, P. T. Spellman, K. M. Shokat, A. J. Wyrobek, M. J. Bissell, F. Mccormick, W. L. Kuo, G. B. Mills, J. W. Gray, and W. M. Korn. 2009. Basal subtype and MAPK/ERK 
kinase (MEK)-phosphoinositide 3-kinase feedback signaling determine susceptibility of breast cancer cells to MEK inhibition. Cancer Res 69:565-572.

105. Cancer Genome, A. N. 2012. Comprehensive molecular portraits of human breast tumours. Nature 490:61-70.

106. van Beers, E. H., W. T. van, L. F. Wessels, Y. Li, R. A. Oldenburg, P. Devilee, C. J. Cornelisse, S. Verhoef, F. B. Hogervorst, L. J. van't Veer, and P. M. Nederlof. 2005. Comparative genomic hybridization profiles in human BRCA1 and BRCA2 breast tumors highlight differential sets of genomic aberrations. Cancer Res 65:822-827.

107. Herschkowitz, J. I., K. Simin, V. J. Weigman, I. Mikaelian, J. Usary, Z. Hu, K. E. Rasmussen, L. P. Jones, S. Assefnia, S. Chandrasekharan, M. G. Backlund, Y. Yin, A. I. Khramtsov, R. Bastein, J. Quackenbush, R. I. Glazer, P. H. Brown, J. E. Green, L. Kopelovich, P. A. Furth, J. P. Palazzo, O. I. Olopade, P. S. Bernard, G. A. Churchill, D. T. Van, and C. M. Perou. 2007. Identification of conserved gene expression features between murine mammary carcinoma models and human breast tumors. Genome Biol. $8:$ R76

108. Rakha, E. A., J. S. Reis-Filho, and I. O. Ellis. 2008. Basal-like breast cancer: a critical review. J. Clin. Oncol. 26:2568-2581.

109. Hu, X., H. M. Stern, L. Ge, C. O'Brien, L. Haydu, C. D. Honchell, P. M. Haverty, B. A. Peters, T. D. Wu, L. C. Amler, J. Chant, D. Stokoe, M. R. Lackner, and G. Cavet. 2009. Genetic alterations and oncogenic pathways associated with breast cancer subtypes. Mol. Cancer Res. 7:511-522.

110. Balko, J. M., L. J. Schwarz, N. E. Bhola, R. Kurupi, P. Owens, T. W. Miller, H. Gomez, R. S. Cook, and C. L. Arteaga. 2013. Activation of MAPK pathways due to DUSP4 loss promotes cancer stem cell-like phenotypes in basal-like breast cancer. Cancer Res. 73:6346-6358.

111. Balko, J. M., R. S. Cook, D. B. Vaught, M. G. Kuba, T. W. Miller, N. E. Bhola, M. E. Sanders, N. M. Granja-Ingram, J. J. Smith, I. M. Meszoely, J. Salter, M. Dowsett, K. Stemke-Hale, A. M. Gonzalez-Angulo, G. B. Mills, J. A. Pinto, H. L. Gomez, and C. L. Arteaga. 2012. Profiling of residual breast cancers after neoadjuvant chemotherapy identifies DUSP4 deficiency as a mechanism of drug resistance. Nat. Med. 18:10521059.

112. HARVEY, J. J. 1964. An unidentified virus which causes the rapid production of tumors in mice. Nature 204:1104-1105.

113. Kirsten, W. H. and L. A. Mayer. 1967. Morphologic responses to a murine erythroblastosis virus. J. Natl. Cancer Inst. 39:311-335.

114. Scolnick, E. M., E. Rands, D. Williams, and W. P. Parks. 1973. Studies on the nucleic acid sequences of Kirsten sarcoma virus: a model for formation of a mammalian RNAcontaining sarcoma virus. J. Virol. 12:458-463. 
115. Scolnick, E. M. and W. P. Parks. 1974. Harvey sarcoma virus: a second murine type C sarcoma virus with rat genetic information. J. Virol. 13:1211-1219.

116. Krontiris, T. G. and G. M. Cooper. 1981. Transforming activity of human tumor DNAs. Proc. Natl. Acad. Sci. U. S. A 78:1181-1184.

117. Santos, E., S. R. Tronick, S. A. Aaronson, S. Pulciani, and M. Barbacid. 1982. T24 human bladder carcinoma oncogene is an activated form of the normal human homologue of of BALB- and Harvey-MSV transforming genes. Nature 298:343-347.

118. Chang, E. H., M. E. Furth, E. M. Scolnick, and D. R. Lowy. 1982. Tumorigenic transformation of mammalian cells induced by a normal human gene homologous to the oncogene of Harvey murine sarcoma virus. Nature 297:479-483.

119. Parada, L. F., C. J. Tabin, C. Shih, and R. A. Weinberg. 1982. Human EJ bladder carcinoma oncogene is homologue of Harvey sarcoma virus ras gene. Nature 297:474478.

120. Der, C. J., T. G. Krontiris, and G. M. Cooper. 1982. Transforming genes of human bladder and lung carcinoma cell lines are homologous to the ras genes of Harvey and Kirsten sarcoma viruses. Proc. Natl. Acad. Sci. U. S. A 79:3637-3640.

121. Shih, C., B. Z. Shilo, M. P. Goldfarb, A. Dannenberg, and R. A. Weinberg. 1979. Passage of phenotypes of chemically transformed cells via transfection of DNA and chromatin. Proc. Natl. Acad. Sci. U. S. A 76:5714-5718.

122. Capon, D. J., P. H. Seeburg, J. P. McGrath, J. S. Hayflick, U. Edman, A. D. Levinson, and D. V. Goeddel. 1983. Activation of Ki-ras2 gene in human colon and lung carcinomas by two different point mutations. Nature 304:507-513.

123. Feig, L. A., R. C. Bast, Jr., R. C. Knapp, and G. M. Cooper. 1984. Somatic activation of rasK gene in a human ovarian carcinoma. Science 223:698-701.

124. Santos, E., D. Martin-Zanca, E. P. Reddy, M. A. Pierotti, P. G. Della, and M. Barbacid. 1984. Malignant activation of a K-ras oncogene in lung carcinoma but not in normal tissue of the same patient. Science 223:661-664.

125. Taparowsky, E., Y. Suard, O. Fasano, K. Shimizu, M. Goldfarb, and M. Wigler. 1982. Activation of the T24 bladder carcinoma transforming gene is linked to a single amino acid change. Nature 300:762-765.

126. Reddy, E. P., R. K. Reynolds, E. Santos, and M. Barbacid. 1982. A point mutation is responsible for the acquisition of transforming properties by the T24 human bladder carcinoma oncogene. Nature 300:149-152.

127. Tabin, C. J., S. M. Bradley, C. I. Bargmann, R. A. Weinberg, A. G. Papageorge, E. M. Scolnick, R. Dhar, D. R. Lowy, and E. H. Chang. 1982. Mechanism of activation of a human oncogene. Nature 300:143-149. 
128. Hall, A., C. J. Marshall, N. K. Spurr, and R. A. Weiss. 1983. Identification of transforming gene in two human sarcoma cell lines as a new member of the ras gene family located on chromosome 1. Nature 303:396-400.

129. Sweet, R. W., S. Yokoyama, T. Kamata, J. R. Feramisco, M. Rosenberg, and M. Gross. 1984. The product of ras is a GTPase and the T24 oncogenic mutant is deficient in this activity. Nature 311:273-275.

130. Gibbs, J. B., I. S. Sigal, M. Poe, and E. M. Scolnick. 1984. Intrinsic GTPase activity distinguishes normal and oncogenic ras p21 molecules. Proc. Natl. Acad. Sci. U. S. A 81:5704-5708.

131. McGrath, J. P., D. J. Capon, D. V. Goeddel, and A. D. Levinson. 1984. Comparative biochemical properties of normal and activated human ras p21 protein. Nature 310:644649.

132. Manne, V., E. Bekesi, and H. F. Kung. 1985. Ha-ras proteins exhibit GTPase activity: point mutations that activate Ha-ras gene products result in decreased GTPase activity. Proc. Natl. Acad. Sci. U. S. A 82:376-380.

133. Mccormick, F., G. A. Martin, R. Clark, G. Bollag, and P. Polakis. 1991. Regulation of ras p21 by GTPase activating proteins. Cold Spring Harb. Symp. Quant. Biol. 56:237-241.

134. Mccormick, F. 1992. Coupling of ras p21 signalling and GTP hydrolysis by GTPase activating proteins. Philos. Trans. R. Soc. Lond B Biol. Sci. 336:43-47.

135. Gysin, S., M. Salt, A. Young, and F. Mccormick. 2011. Therapeutic strategies for targeting ras proteins. Genes Cancer 2:359-372.

136. Mattingly, R. R. 2013. Activated Ras as a Therapeutic Target: Constraints on Directly Targeting Ras Isoforms and Wild-Type versus Mutated Proteins. ISRN. Oncol. 2013:536529.

137. Liu, Q., Y. Sabnis, Z. Zhao, T. Zhang, S. J. Buhrlage, L. H. Jones, and N. S. Gray. 2013. Developing irreversible inhibitors of the protein kinase cysteinome. Chem. Biol. 20:146159.

138. Johnson, G. L., T. J. Stuhlmiller, S. P. Angus, J. S. Zawistowski, and L. M. Graves. 2014. Molecular pathways: adaptive kinome reprogramming in response to targeted inhibition of the BRAF-MEK-ERK pathway in cancer. Clin Cancer Res 20:2516-2522.

139. Engelman, J. A., K. Zejnullahu, T. Mitsudomi, Y. Song, C. Hyland, J. O. Park, N. Lindeman, C. M. Gale, X. Zhao, J. Christensen, T. Kosaka, A. J. Holmes, A. M. Rogers, F. Cappuzzo, T. Mok, C. Lee, B. E. Johnson, L. C. Cantley, and P. A. Janne. 2007. MET amplification leads to gefitinib resistance in lung cancer by activating ERBB3 signaling. Science 316:1039-1043. 
140. Garrett, J. T., M. G. Olivares, C. Rinehart, N. D. Granja-Ingram, V. Sanchez, A. Chakrabarty, B. Dave, R. S. Cook, W. Pao, E. McKinely, H. C. Manning, J. Chang, and C. L. Arteaga. 2011. Transcriptional and posttranslational up-regulation of HER3 (ErbB3) compensates for inhibition of the HER2 tyrosine kinase. Proc. Natl. Acad. Sci. U. S. A 108:5021-5026.

141. Chandarlapaty, S., A. Sawai, M. Scaltriti, V. Rodrik-Outmezguine, O. Grbovic-Huezo, V. Serra, P. K. Majumder, J. Baselga, and N. Rosen. 2011. AKT inhibition relieves feedback suppression of receptor tyrosine kinase expression and activity. Cancer Cell 19:58-71.

142. Stuhlmiller, T. J., S. M. Miller, J. S. Zawistowski, K. Nakamura, A. S. Beltran, J. S. Duncan, S. P. Angus, K. A. Collins, D. A. Granger, R. A. Reuther, L. M. Graves, S. M. Gomez, P. F. Kuan, J. S. Parker, X. Chen, N. Sciaky, L. A. Carey, H. S. Earp, J. Jin, and G. L. Johnson. 2015. Inhibition of Lapatinib-Induced Kinome Reprogramming in ERBB2-Positive Breast Cancer by Targeting BET Family Bromodomains. Cell Rep. 11:390-404.

143. Ritt, D. A., D. M. Monson, S. I. Specht, and D. K. Morrison. 2010. Impact of feedback phosphorylation and Raf heterodimerization on normal and mutant B-Raf signaling. Mol. Cell Biol. 30:806-819.

144. Villanueva, J., J. R. Infante, C. Krepler, P. Reyes-Uribe, M. Samanta, H. Y. Chen, B. Li, R. K. Swoboda, M. Wilson, A. Vultur, M. Fukunaba-Kalabis, B. Wubbenhorst, T. Y. Chen, Q. Liu, K. Sproesser, D. J. DeMarini, T. M. Gilmer, A. M. Martin, R. Marmorstein, D. C. Schultz, D. W. Speicher, G. C. Karakousis, W. Xu, R. K. Amaravadi, X. Xu, L. M. Schuchter, M. Herlyn, and K. L. Nathanson. 2013. Concurrent MEK2 mutation and BRAF amplification confer resistance to BRAF and MEK inhibitors in melanoma. Cell Rep. 4:1090-1099.

145. Shi, H., A. Hong, X. Kong, R. C. Koya, C. Song, G. Moriceau, W. Hugo, C. C. Yu, C. Ng, T. Chodon, R. A. Scolyer, R. F. Kefford, A. Ribas, G. V. Long, and R. S. Lo. 2014. A novel AKT1 mutant amplifies an adaptive melanoma response to BRAF inhibition. Cancer Discov. 4:69-79.

146. Nazarian, R., H. Shi, Q. Wang, X. Kong, R. C. Koya, H. Lee, Z. Chen, M. K. Lee, N. Attar, H. Sazegar, T. Chodon, S. F. Nelson, G. McArthur, J. A. Sosman, A. Ribas, and R. S. Lo. 2010. Melanomas acquire resistance to B-RAF(V600E) inhibition by RTK or NRAS upregulation. Nature 468:973-977.

147. Sos, M. L., S. Fischer, R. Ullrich, M. Peifer, J. M. Heuckmann, M. Koker, S. Heynck, I. Stuckrath, J. Weiss, F. Fischer, K. Michel, A. Goel, L. Regales, K. A. Politi, S. Perera, M. Getlik, L. C. Heukamp, S. Ansen, T. Zander, R. Beroukhim, H. Kashkar, K. M. Shokat, W. R. Sellers, D. Rauh, C. Orr, K. P. Hoeflich, L. Friedman, K. K. Wong, W. Pao, and R. K. Thomas. 2009. Identifying genotype-dependent efficacy of single and combined. Proc. Natl. Acad. Sci. U. S. A 106:18351-18356.

148. Hoeflich, K. P., C. O'Brien, Z. Boyd, G. Cavet, S. Guerrero, K. Jung, T. Januario, H. Savage, E. Punnoose, T. Truong, W. Zhou, L. Berry, L. Murray, L. Amler, M. Belvin, L. 
S. Friedman, and M. R. Lackner. 2009. In vivo antitumor activity of MEK and phosphatidylinositol 3-kinase inhibitors in basal-like breast cancer models. Clin Cancer Res 15:4649-4664.

149. Sun, C., S. Hobor, A. Bertotti, D. Zecchin, S. Huang, F. Galimi, F. Cottino, A. Prahallad, W. Grernrum, A. Tzani, A. Schlicker, L. F. Wessels, E. F. Smit, E. Thunnissen, P. Halonen, C. Lieftink, R. L. Beijersbergen, N. F. Di, A. Bardelli, L. Trusolino, and R. Bernards. 2014. Intrinsic resistance to MEK inhibition in KRAS mutant lung and colon cancer through transcriptional induction of ERBB3. Cell Rep. 7:86-93.

150. Golan, T., E. Z. Khvalevsky, A. Hubert, R. M. Gabai, N. Hen, A. Segal, A. Domb, G. Harari, E. B. David, S. Raskin, Y. Goldes, E. Goldin, R. Eliakim, M. Lahav, Y. Kopleman, A. Dancour, A. Shemi, and E. Galun. 2015. RNAi therapy targeting KRAS in combination with chemotherapy for locally advanced pancreatic cancer patients. Oncotarget.

151. Yuan, T. L., C. Fellmann, C. S. Lee, C. D. Ritchie, V. Thapar, L. C. Lee, D. J. Hsu, D. Grace, J. O. Carver, J. Zuber, J. Luo, F. Mccormick, and S. W. Lowe. 2014. Development of siRNA payloads to target KRAS-mutant cancer. Cancer Discov. 4:11821197.

152. Zorde, K. E., R. Gabai, I. H. Rachmut, E. Horwitz, Z. Brunschwig, A. Orbach, A. Shemi, T. Golan, A. J. Domb, E. Yavin, H. Giladi, L. Rivkin, A. Simerzin, R. Eliakim, A. Khalaileh, A. Hubert, M. Lahav, Y. Kopelman, E. Goldin, A. Dancour, Y. Hants, S. rbelAlon, R. Abramovitch, A. Shemi, and E. Galun. 2013. Mutant KRAS is a druggable target for pancreatic cancer. Proc. Natl. Acad. Sci. U. S. A 110:20723-20728.

153. Thirukkumaran, C. and D. G. Morris. 2009. Oncolytic viral therapy using reovirus. Methods Mol. Biol. 542:607-634.

154. Meng, F., R. Henson, H. Wehbe-Janek, K. Ghoshal, S. T. Jacob, and T. Patel. 2007. MicroRNA-21 regulates expression of the PTEN tumor suppressor gene in human hepatocellular cancer. Gastroenterology 133:647-658.

155. Zawistowski, J. S., K. Nakamura, J. S. Parker, D. A. Granger, B. T. Golitz, and G. L. Johnson. 2013. MicroRNA 9-3p targets beta1 integrin to sensitize claudin-low breast cancer cells to MEK inhibition. Mol. Cell Biol. 33:2260-2274.

156. Pasquinelli, A. E., B. J. Reinhart, F. Slack, M. Q. Martindale, M. I. Kuroda, B. Maller, D. C. Hayward, E. E. Ball, B. Degnan, P. Muller, J. Spring, A. Srinivasan, M. Fishman, J. Finnerty, J. Corbo, M. Levine, P. Leahy, E. Davidson, and G. Ruvkun. 2000. Conservation of the sequence and temporal expression of let-7 heterochronic regulatory RNA. Nature 408:86-89.

157. Reinhart, B. J., F. J. Slack, M. Basson, A. E. Pasquinelli, J. C. Bettinger, A. E. Rougvie, H. R. Horvitz, and G. Ruvkun. 2000. The 21-nucleotide let-7 RNA regulates developmental timing in Caenorhabditis elegans. Nature 403:901-906. 
158. Johnson, C. D., A. Esquela-Kerscher, G. Stefani, M. Byrom, K. Kelnar, D. Ovcharenko, M. Wilson, X. Wang, J. Shelton, J. Shingara, L. Chin, D. Brown, and F. J. Slack. 2007. The let-7 microRNA represses cell proliferation pathways in human cells. Cancer Res 67:7713-7722.

159. Yu, F., H. Yao, P. Zhu, X. Zhang, Q. Pan, C. Gong, Y. Huang, X. Hu, F. Su, J. Lieberman, and E. Song. 2007. let-7 regulates self renewal and tumorigenicity of breast cancer cells. Cell 131:1109-1123.

160. He, X. Y., J. X. Chen, Z. Zhang, C. L. Li, Q. L. Peng, and H. M. Peng. 2010. The let-7a microRNA protects from growth of lung carcinoma by suppression of $\mathrm{k}-\mathrm{Ras}$ and $\mathrm{c}-\mathrm{Myc}$ in nude mice. J Cancer Res Clin Oncol. 136:1023-1028.

161. Esquela-Kerscher, A., P. Trang, J. F. Wiggins, L. Patrawala, A. Cheng, L. Ford, J. B. Weidhaas, D. Brown, A. G. Bader, and F. J. Slack. 2008. The let-7 microRNA reduces tumor growth in mouse models of lung cancer. Cell Cycle 7:759-764.

162. Kumar, M. S., S. J. Erkeland, R. E. Pester, C. Y. Chen, M. S. Ebert, P. A. Sharp, and T. Jacks. 2008. Suppression of non-small cell lung tumor development by the let-7 microRNA family. Proc. Natl. Acad. Sci. U. S. A 105:3903-3908.

163. Liu, N., A. H. Williams, J. M. Maxeiner, S. Bezprozvannaya, J. M. Shelton, J. A. Richardson, R. Bassel-Duby, and E. N. Olson. 2012. microRNA-206 promotes skeletal muscle regeneration and delays progression of Duchenne muscular dystrophy in mice. $\mathrm{J}$. Clin. Invest 122:2054-2065.

164. Chen, J. F., Y. Tao, J. Li, Z. Deng, Z. Yan, X. Xiao, and D. Z. Wang. 2010. microRNA-1 and microRNA-206 regulate skeletal muscle satellite cell proliferation and differentiation by repressing Pax7. J. Cell Biol. 190:867-879.

165. Kim, H. K., Y. S. Lee, U. Sivaprasad, A. Malhotra, and A. Dutta. 2006. Muscle-specific microRNA miR-206 promotes muscle differentiation. J. Cell Biol. 174:677-687.

166. Dey, B. K., J. Gagan, and A. Dutta. 2011. miR-206 and -486 induce myoblast differentiation by downregulating Pax7. Mol. Cell Biol. 31:203-214.

167. Iorio, M. V., M. Ferracin, C. G. Liu, A. Veronese, R. Spizzo, S. Sabbioni, E. Magri, M. Pedriali, M. Fabbri, M. Campiglio, S. Menard, J. P. Palazzo, A. Rosenberg, P. Musiani, S. Volinia, I. Nenci, G. A. Calin, P. Querzoli, M. Negrini, and C. M. Croce. 2005. MicroRNA gene expression deregulation in human breast cancer. Cancer Res. 65:70657070 .

168. Volinia, S., G. A. Calin, C. G. Liu, S. Ambs, A. Cimmino, F. Petrocca, R. Visone, M. Iorio, C. Roldo, M. Ferracin, R. L. Prueitt, N. Yanaihara, G. Lanza, A. Scarpa, A. Vecchione, M. Negrini, C. C. Harris, and C. M. Croce. 2006. A microRNA expression signature of human solid tumors defines cancer gene targets. Proc Natl Acad Sci U S A 103:2257-2261. 
169. Frankel, L. B., N. R. Christoffersen, A. Jacobsen, M. Lindow, A. Krogh, and A. H. Lund. 2008. Programmed cell death 4 (PDCD4) is an important functional target of the microRNA miR-21 in breast cancer cells. J Biol. Chem. 283:1026-1033.

170. Jin, X. L., Q. S. Sun, F. Liu, H. W. Yang, M. Liu, H. X. Liu, W. Xu, and Y. Y. Jiang. 2013. microRNA 21-mediated suppression of Sprouty1 by Pokemon affects liver cancer cell growth and proliferation. J. Cell Biochem. 114:1625-1633.

171. Mei, Y., C. Bian, J. Li, Z. Du, H. Zhou, Z. Yang, and R. C. Zhao. 2013. miR-21 modulates the ERK-MAPK signaling pathway by regulating SPRY2 expression during human mesenchymal stem cell differentiation. J. Cell Biochem. 114:1374-1384.

172. Wickramasinghe, N. S., T. T. Manavalan, S. M. Dougherty, K. A. Riggs, Y. Li, and C. M. Klinge. 2009. Estradiol downregulates miR-21 expression and increases miR-21 target gene expression in MCF-7 breast cancer cells. Nucleic Acids Res. 37:2584-2595.

173. Asangani, I. A., S. A. Rasheed, D. A. Nikolova, J. H. Leupold, N. H. Colburn, S. Post, and H. Allgayer. 2008. MicroRNA-21 (miR-21) post-transcriptionally downregulates tumor suppressor Pdcd4 and stimulates invasion, intravasation and metastasis in colorectal cancer. Oncogene 27:2128-2136.

174. Meng, F., R. Henson, M. Lang, H. Wehbe, S. Maheshwari, J. T. Mendell, J. Jiang, T. D. Schmittgen, and T. Patel. 2006. Involvement of human micro-RNA in growth and response to chemotherapy in human cholangiocarcinoma cell lines. Gastroenterology 130:2113-2129.

175. Pandya, A. Y., L. I. Talley, A. R. Frost, T. J. Fitzgerald, V. Trivedi, M. Chakravarthy, D. C. Chhieng, W. E. Grizzle, J. A. Engler, H. Krontiras, K. I. Bland, A. F. Lobuglio, S. M. Lobo-Ruppert, and J. M. Ruppert. 2004. Nuclear localization of KLF4 is associated with an aggressive phenotype in early-stage breast cancer. Clin. Cancer Res. 10:2709-2719.

176. Kamalakaran, S., V. Varadan, H. E. Giercksky Russnes, D. Levy, J. Kendall, A. Janevski, M. Riggs, N. Banerjee, M. Synnestvedt, E. Schlichting, R. Karesen, P. K. Shama, H. Rotti, R. Rao, L. Rao, M. H. Eric Tang, K. Satyamoorthy, R. Lucito, M. Wigler, N. Dimitrova, B. Naume, A. L. Borresen-Dale, and J. B. Hicks. 2011. DNA methylation patterns in luminal breast cancers differ from non-luminal subtypes and can identify relapse risk independent of other clinical variables. Mol Oncol 5:77-92.

177. Chen, C. J., S. E. Lin, Y. M. Lin, S. H. Lin, D. R. Chen, and C. L. Chen. 2012. Association of Expression of Kruppel-like Factor 4 and Kruppel-like Factor 5 with the Clinical Manifestations of Breast Cancer. Pathol. Oncol Res 18:161-168.

178. Farrugia, M. K., S. B. Sharma, C. C. Lin, S. L. McLaughlin, D. B. Vanderbilt, A. G. Ammer, M. A. Salkeni, P. Stoilov, Y. M. Agazie, C. J. Creighton, and J. M. Ruppert. 2015. Regulation of anti-apoptotic signaling by Kruppel-like factors 4 and 5 mediates lapatinib resistance in breast cancer. Cell Death. Dis. 6:e1699. 
179. Pichiorri, F., D. Palmieri, L. L. De, J. Consiglio, J. You, A. Rocci, T. Talabere, C. Piovan, A. Lagana, L. Cascione, J. Guan, P. Gasparini, V. Balatti, G. Nuovo, V. Coppola, C. C. Hofmeister, G. Marcucci, J. C. Byrd, S. Volinia, C. L. Shapiro, M. A. Freitas, and C. M. Croce. 2013. In vivo NCL targeting affects breast cancer aggressiveness through miRNA regulation. J Exp. Med 210:951-968.

180. Wang, L., Z. M. Shi, C. F. Jiang, X. Liu, Q. D. Chen, X. Qian, D. M. Li, X. Ge, X. F. Wang, L. Z. Liu, Y. P. You, N. Liu, and B. H. Jiang. 2014. MiR-143 acts as a tumor suppressor by targeting N-RAS and enhances temozolomide-induced apoptosis in glioma. Oncotarget. 5:5416-5427.

181. Stark, M. S., V. F. Bonazzi, G. M. Boyle, J. M. Palmer, J. Symmons, C. M. Lanagan, C. W. Schmidt, A. C. Herington, R. Ballotti, P. M. Pollock, and N. K. Hayward. 2015. miR514a regulates the tumour suppressor NF1 and modulates BRAFi sensitivity in melanoma. Oncotarget.

182. Sun, D., C. Wang, S. Long, Y. Ma, Y. Guo, Z. Huang, X. Chen, C. Zhang, J. Chen, and J. Zhang. 2015. C/EBP-beta-activated microRNA-223 promotes tumour growth through targeting RASA1 in human colorectal cancer. Br. J Cancer 112:1491-1500.

183. Shin, K. H., S. D. Bae, H. S. Hong, R. H. Kim, M. K. Kang, and N. H. Park. 2011. miR181a shows tumor suppressive effect against oral squamous cell carcinoma cells by downregulating K-ras. Biochem. Biophys. Res Commun. 404:896-902.

184. Liu, S. M., J. Lu, H. C. Lee, F. H. Chung, and N. Ma. 2014. miR-524-5p suppresses the growth of oncogenic BRAF melanoma by targeting BRAF and ERK2. Oncotarget. 5:9444-9459.

185. Yu, S., Z. Lu, C. Liu, Y. Meng, Y. Ma, W. Zhao, J. Liu, J. Yu, and J. Chen. 2010. miRNA-96 suppresses KRAS and functions as a tumor suppressor gene in pancreatic cancer. Cancer Res 70:6015-6025.

186. Tanic, M., K. Yanowsky, C. Rodriguez-Antona, R. Andres, I. Marquez-Rodas, A. Osorio, J. Benitez, and B. Martinez-Delgado. 2012. Deregulated miRNAs in hereditary breast cancer revealed a role for miR-30c in regulating KRAS oncogene. PLoS. One. 7:e38847. 


\section{FIGURE LEGENDS}

Figure 1: miRs regulate RAS-ERK pathway activity by regulation of RAS-GTP. The schematic shows the organization of the RAS-ERK pathway. miR-206/21 co-targeted repressors of RAS-ERK signaling are indicated in ovals. The GAP protein NF1 is indicated as a likely catalytic partner of SPRED1 (37). The let-7 miR targets each of the RAS family GTPases including $K R A S, H R A S$, and NRAS. 


\section{FIGURES}

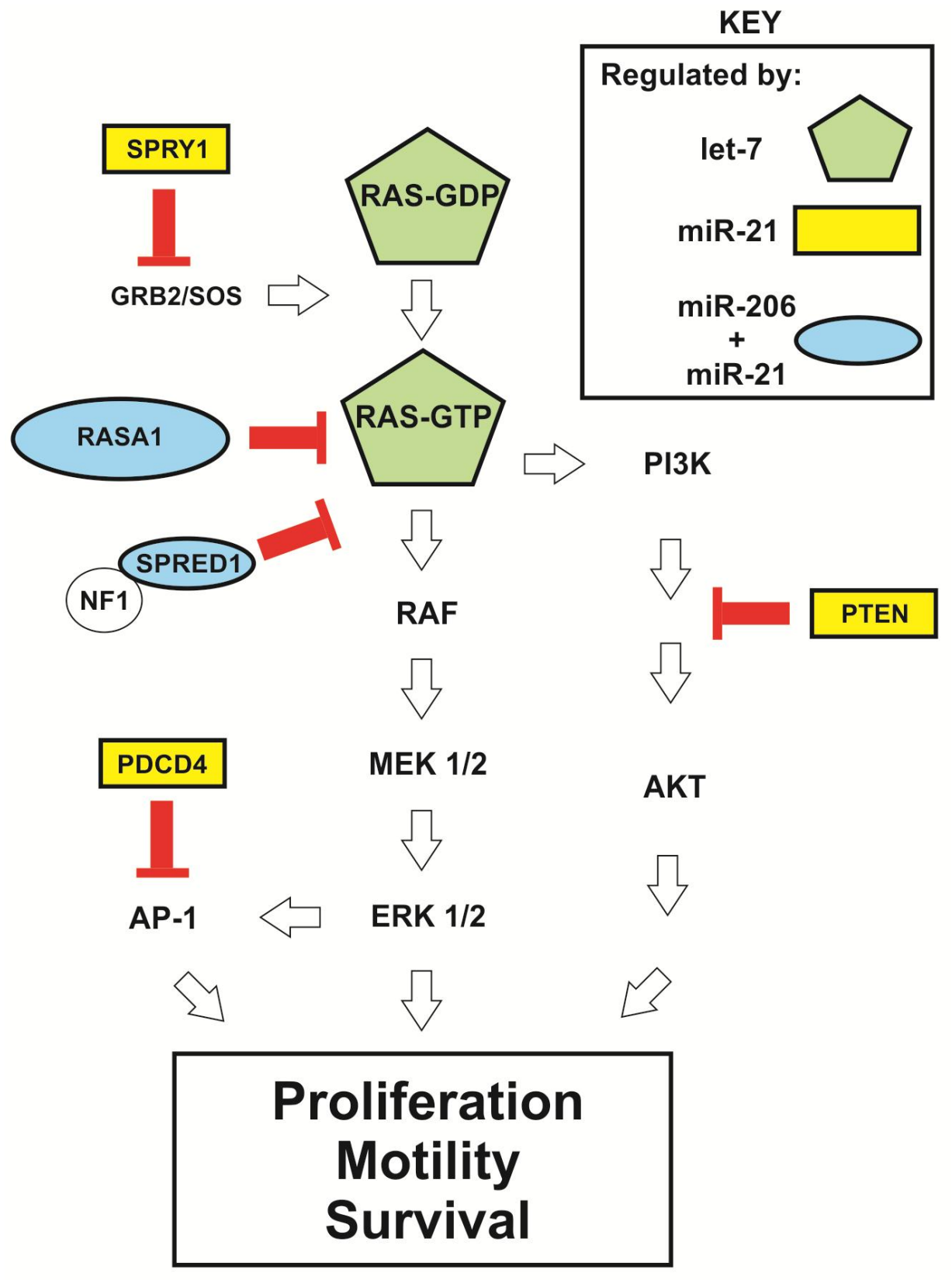

Figure 1 


\section{TABLES}

Table 1: miRs that regulate RAS-ERK pathway activity in a variety of cancer contexts

\begin{tabular}{|c|c|c|c|}
\hline miR & Target(s) & Disease contexts & References \\
\hline let-7 & $\begin{array}{l}\text { HRAS, NRAS, } \\
K R A S, c M Y C\end{array}$ & $\begin{array}{l}\text { Multiple cancer } \\
\text { contexts including } \\
\text { lung } \\
\text { adenocarcinoma }\end{array}$ & $\begin{array}{l}(42) \\
(158) \\
(159) \\
(74) \\
(160)\end{array}$ \\
\hline miR-21 & $\begin{array}{l}\text { RASA1, SPRED1, } \\
\text { SPRY1, SPRY2, } \\
\text { PTEN, PDCD4 }\end{array}$ & $\begin{array}{l}\text { Multiple cancer } \\
\text { contexts including } \\
\text { TNBCs }\end{array}$ & $\begin{array}{l}(167) \\
(168) \\
(169) \\
(170) \\
(171) \\
(172) \\
(173) \\
(174) \\
(41) \\
\end{array}$ \\
\hline miR-206 & $\begin{array}{l}\text { RASA1, SPRED1, } \\
\text { PDCD4 }\end{array}$ & TNBCs & (41) \\
\hline miR-31 & RASAl & $\begin{array}{l}\text { Colorectal } \\
\text { carcinoma }\end{array}$ & (46) \\
\hline miR-143 & $N R A S$ & Glioma & $(180)$ \\
\hline miR-514a & $N F 1$ & Melanoma & (181) \\
\hline miR-223 & RASAl & $\begin{array}{l}\text { Colorectal } \\
\text { adenocarcinoma }\end{array}$ & $(182)$ \\
\hline miR-181a & $K R A S$ & $\begin{array}{l}\text { Oral squamous cell } \\
\text { carcinoma }\end{array}$ & (183) \\
\hline miR-524-5p & $B R A F, E R K 2$ & Melanoma & (184) \\
\hline miR-96 & $K R A S$ & $\begin{array}{l}\text { Pancreatic } \\
\text { adenocarcinoma }\end{array}$ & $(185)$ \\
\hline miR-30c & $K R A S$ & Breast cancer & (186) \\
\hline
\end{tabular}




\section{PART II: CURRICULUM VITAE (CV)}


This page is intentionally left blank 


\title{
Sriganesh Balasubramanian Sharma
}

\author{
308 Morgan Pointe, Morgantown, WV, USA, 26505 \\ Tel: +1 (304)-293-0949 \\ Email: sbsharma@hsc.wvu.edu
}

\section{Personal Summary:}

I am currently an M.D.-Ph.D. dual degree scholar with aspirations of training as a physician/surgeon scientist and advancing both the basic science based understanding of cancer biology as well as patient care. I am very motivated and I believe in life-long and team-based learning. In my life, I strive for balance between work and family-life. I am very interested in surgical management of pathologies of the thoracic cavity as well as cell and cancer biology, cell signal transduction pathways, and rationally designed small molecule-based targeted chemotherapeutics. Outside of academics, I am very involved with my family, various outdoor recreational activities (i.e. sports), and in my community. I believe my educational and personal background give me a unique vantage point with which I envision melding the bench (basic science research) with the bedside (patient management) to better patient care.

\section{Education:}

Ph.D. M.D.-Ph.D. Dual degree scholar: 08/2009-05/2017

Anticipated degree date: Ph.D.: 05/2017

Time in Ph.D.: 08/201105/2015

M.D. M.D.-Ph.D. Dual degree scholar: 08/2009-05/2017

Anticipated degree date: M.D.: 05/2017

B.S. Chemistry: 08/2005-05/2009 (with honors)

Honors Thesis: 08/200605/2009

B.S.

(with honors) Biology: 08/2005-05/2009

\author{
West Virginia University, Morgantown, WV, USA \\ Department of Biochemistry, \\ Program in Cancer Cell Biology, \\ School of Medicine \\ Dissertation Title: Krüppel-like factor 4 (KLF4) \\ regulates protumorigenic signaling in triple-negative \\ breast cancer (TNBC) cells \\ Dissertation Advisor: Dr. J. Michael Ruppert, M.D., \\ Ph.D. \\ Dissertation Defense Date: 05/20/2015
}

West Virginia University, Morgantown, WV, USA School of Medicine

West Virginia University, Morgantown, WV, USA Department of Chemistry, Eberly School of Arts \& Sciences Honors Thesis Title: A short synthesis of Salviadione and Dillemaones A-C

Honors Thesis Advisor: Dr. Bjorn C.G. Soderberg

West Virginia University, Morgantown, WV, USA

Department of Biology,

Eberly School of Arts \& Sciences 


\section{Research Experience:}

2010-2015 Lab of Dr. J. Michael Ruppert, Department of Biochemistry, West Virginia University Investigating KLF4 and KLF4-dependent signaling pathways in breast cancer

2014-2015 Collaboration with the Lab of Dr. Robert Wysolmerski, Department of Anatomy, West Virginia University Investigating the role of KLF4-DDR1 signaling in mediating collagen stiffness

2006-2009 Lab of Dr. Bjorn C.G. Soderberg, Department of Chemistry, West Virginia University Organic synthesis of indole alkaloids and derivatives

2006-2007 Lab of Dr. Charles Jaffe, Department of Chemistry, West Virginia University Investigation and study of three-body problems in theoretical chemistry

\section{Techniques:}

Cell and Molecular Biology: Primary Cell Culture, PCR Primer Design, Molecular Cloning Techniques (Including sub-cloning, site directed mutagenesis, and plasmid preparation), RNA isolation (from primary tissues and cells), DNA purification, qRT-PCR, microRNA analysis, cell transfection, mammalian cell retroviral and lentiviral transduction, luciferase reporter assays, immunoblotting (western blot), immunoprecipitation, RAS-GTP affinity precipitation, phosphor-protein blotting, in vitro cell migration and invasion assays, anoikis assays, soft agar assays, working with murine models of mammary cancer, statistical analysis, 3D collagen assays, force measurements to determine elastic modulus of collagen matricies, two-photon microscopy

Organic Synthesis and Spectroscopy: General organic synthesis, silica gel column chromatography, HPLC, ${ }^{1} \mathrm{H}-\mathrm{NMR},{ }^{13} \mathrm{C}-\mathrm{NMR}$

Publications: (* denotes co-first authorship)

1. Lin CC, Sharma SB*, Farrugia MK, McLaughlin SL, Ice RJ, Loskutov Y, Pugacheva EN, Brundage KM, Chen D, Ruppert JM (2015). Krüppel-like Factor 4 (KLF4) signals through microRNA-206 to promote tumor initiation and cell survival. Oncogenesis - In press

2. Farrugia MK, Sharma SB, Lin CC, McLaughlin SL, Vanderbilt DB, Ammer AG, Salkeni, MA, Stoilov P, Agazie YM, Creighton CJ, Ruppert JM (2015). Regulation of anti-apoptotic signaling by Krüppel-like factors 4 and 5 mediates lapatinib resistance in breast cancer. Cell Death Dis. 2015 Mar 19;6:e1699. doi: 10.1038/cddis.2015.65.

3. Sharma SB*, Lin CC, Farrugia MK, McLaughlin SL, Ellis EJ, Brundage KM, Salkeni MA, Ruppert JM (2014). MicroRNAs 206 and 21 cooperate to promote RAS-extracellular signal-regulated kinase signaling by suppressing the translation of RASA1 and SPRED1. Mol Cell Biol. 2014 Nov 15;34(22):4143-64.

4. Sharma SB, Farrugia MK, Pifer PM, Lin CC, Ruppert JM (2015). Krüppel-like factor 4 (KLF4) promotes the expression of platelet-derived growth factor receptor beta polypeptide (PDGFR $\beta$ ) and resistance to MEK 1/2 inhibition in triple-negative breast cancers (TNBCs). Manuscript in submission to J. Biol. Chem.

5. Sharma SB, Ruppert JM. MicroRNA based therapeutic targeting of mutant and wild-type RAS in cancer. Invited Review - Manuscript in submission to Drug Dev. Res.

6. Vanderbilt DB, Sharma SB, Lin CC, Ruppert JM (2015). KLF4 suppresses discoidin domain receptor 1 (DDR1) and spontaneous metastasis of breast cancer cells. Manuscript in submission to Clin. Exp. Metastasis. 
7. Cummings MM, Clawson RW, Sharma SB, Byerly RA, Akhmedov NG, Soderberg BCG (2011). An expedient synthesis of salviadione. Tetrahedron 67 (26), 4753-4757.

\section{Abstracts/Posters:}

1. Sharma SB, Farrugia MK, Pifer PM, Lin CC, Ruppert JM. Krüppel-like factor 4 (KLF4) promotes the expression of platelet-derived growth factor receptor beta polypeptide (PDGFR $\beta$ ) and resistance to MEK 1/2 inhibition in triple-negative breast cancers (TNBCs) - Van Liere Research Convocation - West Virginia University, Morgantown, WV (2015)

2. Sharma SB, Lin CC, Farrugia MK, Ruppert JM. KLF4-regulated microRNAs promote RAS-ERK signaling by suppressing the translation of RASA1 and SPRED1. Van Liere Research Convocation - West Virgnia University, Morgantown, WV (2014) - First place winning poster

3. Sharma SB, Fisher KD, Soderberg BCG. The effect of nucleophilicity of aryl substituents on unsymmetrical cyclobutanediones in the synthesis of napthoquinones. WVU SURE symposium West Virginia University, Morgantown, WV (2009)

\section{Invited Presentations:}

1. Sharma SB. Krüppel-like factor 4 (KLF4) promotes RAS-ERK signaling and MEK $1 / 2$ inhibitor resistance in triple-negative breast cancer (TNBCs) - 2015 Van Liere Research Convocation one of ten invited seminars

2. Sharma SB, Farrugia MK. The MD-PhD career track: a student's perspective - (2013-2015) IDeA Network of Biomedical Research Excellence (INBRE) invited speaker

3. Sharma SB, Farrugia MK, Pilkerton CK, Turner RC. The Physician-Scientist career track: How to be a successful MD-PhD candidate - (2011-2013) WVU chapter of the American PhysicianScientist Association (APSA) sponsored talk

\section{Awards, Honors, and Membership:}

$2015 \quad$ WVU EJ Van Liere Research Convocation Invited Seminar

$2014 \quad$ WVU EJ Van Liere $-1^{\text {st }}$ place poster presentation

$2011 \quad$ WVU School of Medicine - Academic Excellence Recognition

2011 - current American Physician-Scientist Association (APSA)

2011 - current American Society for Clinical Oncology (ASCO) - student member

$2010 \quad$ WVU School of Medicine - Academic Excellence Recognition

2009 WVU Department of Chemistry - Outstanding Honors Thesis

2009 WVU Eberly College of Arts and Science - Department of Chemistry - Recognition of Excellence

$2008 \quad$ WVU Summer Undergraduate Research Experience fellowship

2008

2007

2007

2007

2007-2009

2006 WVU Junior Class Honorary WV Health Careers Opportunity Program (HCOP) Facilitating Entry (FE) participant WVU Sophomore Class Honorary WVU Eberly College of Arts and Sciences Academic Excellence Recognition WV Louis-Stokes Alliance for Minority Participation mentor Nominated by Dr. C.Q. Zhang

2005 WVU Presidential Scholarship recipient - WVU Honors College

2005

2005-2009

2001

Scholarship recipient

WVU Dean's List

Ashland Chemicals Young Scientist Award 


\section{Teaching Experience/Leadership:}

2013 WVU MD-PhD program Admissions Committee member

2012-2014 Graduate student mentor for BIOL-486 Honors Thesis Student

Student: Francesca Giovannone

Project: Anti-miR/Anti-miD therapy in breast cancer

2011-2014 President of the WVU Chapter of the American Physician Society (APSA)

2011-2012 Teaching Assistant (TA) for NBAN-703 Human Structure - Gross Anatomy Lab

2007-2009 Teaching Assistant (TA) and tutor for WVU Department of Chemistry

2006-2007 Residential Assistant for WVU - Dadisman Hall

Community Service/Extracurricular Activities:

2014 Student Liason for the Mary Babb Randolph Cancer Center - Legislative Day

2013 WVU Health Sciences Center Graduate Student Organization (GSO) volunteer

2010-2015 WV Habitat for Humanity

2010-2015 Ronald McDonald House volunteer

2009-2015 WVU SAFE Clinic volunteer

2009-current WVU MD-PhD Program Interview weekend volunteer

2009 WV Project MUSHROOM (Multidisciplinary unsheltered homeless relief outreach Morgantown) volunteer

2004-2011 Pittsburgh Barbell Olympic Weightlifting Club

2004-2011 USA Weightlifting member 
This page is intentionally left blank 